BEITRĀGE ZUR BEVÖLKERUNGSWISSENSCHAFT

Herausgegeben durch das Bundesinstitut for Bewolkerungsforschung

Norbert F. Schneider, Sabine Diabaté, Kerstin Ruckdeschel (Hrsg.]

\title{
Familienleitbilder in Deutschland
}

Kulturelle Vorstellungen zu

Partnerschaft, Elternschaft und Familienleben
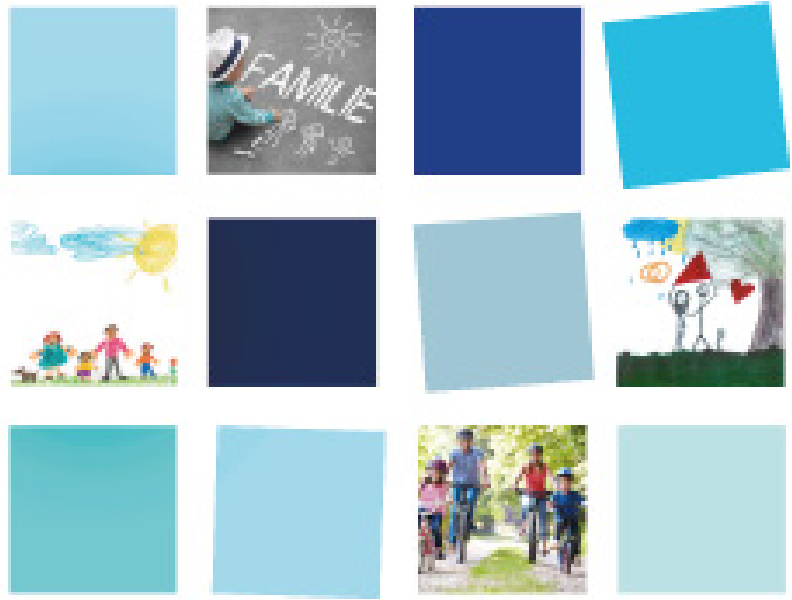


\title{
BEITRÄGE ZUR BEVÖLKERUNGSWISSENSCHAFT
}

\author{
Herausgegeben durch das \\ Bundesinstitut für Bevölkerungsforschung (BiB)
}

\author{
Band 48
}


Norbert F. Schneider

Sabine Diabaté

Kerstin Ruckdeschel (Hrsg.)

\section{Familienleitbilder in Deutschland}

Kulturelle Vorstellungen zu

Partnerschaft, Elternschaft und Familienleben

Verlag Barbara Budrich

Opladen • Berlin • Toronto 2015 
Bibliografische Information der Deutschen Nationalbibliothek

Die Deutsche Nationalbibliothek verzeichnet diese Publikation in der Deutschen Nationalbibliografie; detaillierte bibliografische Daten sind im Internet über

http://dnb.d-nb.de abrufbar.

(C) 2018 Dieses Werk ist beim Verlag Barbara Budrich erschienen und steht unter der Creative Commons Lizenz Attribution-ShareAlike 4.0 International (CC BY-SA 4.0):

https://creativecommons.org/licenses/by-sa/4.0/

Diese Lizenz erlaubt die Verbreitung, Speicherung, Vervielfältigung und Bearbeitung bei

Verwendung der gleichen CC-BY-SA 4.0-Lizenz und unter Angabe der UrheberInnen, Rechte, Änderungen und verwendeten Lizenz.

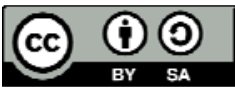

Dieses Buch steht im Open-Access-Bereich der Verlagsseite zum kostenlosen Download bereit (https://doi.org/10.3224/84740663).

Eine kostenpflichtige Druckversion (Printing on Demand) kann über den Verlag bezogen werden. Die Seitenzahlen in der Druck- und Onlineversion sind identisch.

$\begin{array}{ll}\text { ISBN } & 978-3-8474-0663-1 \text { (Paperback) } \\ \text { eISBN } & 978-3-8474-0809-3 \text { (eBook) } \\ \text { DOI } & 10.3224 / 84740663\end{array}$

Titelbildnachweis: (C) Monkey Business - Fotolia.com, (C) motorradcbr - Fotolia.com Umschlaggestaltung: Bettina Lehfeldt, Kleinmachnow - www.lehfeldtgraphic.de und BiB Schriftleitung: Dr. Martin Bujard, BiB

Satz: Sybille Steinmetz, BiB

Druck: Strauss GmbH, Mörlenbach

Printed in Europe 


\section{Inhaltsverzeichnis}

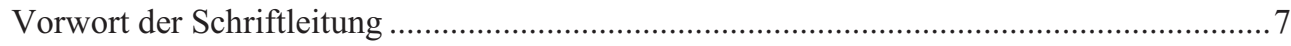

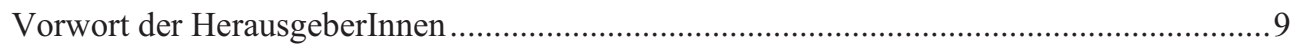

1 Leitbilder als ,missing link“ der Familienforschung:

Eine Einführung

Sabine Diabaté, Kerstin Ruckdeschel \& Norbert F. Schneider

2 Familienleitbilder:

Ein theoretisches Konzept

Detlev Lück \& Sabine Diabaté

3 Zur Messung von Familienleitbildern:

Studiendesign und Operationalisierung

Detlev Lück, Robert Naderi \& Kerstin Ruckdeschel

4 Gesellschaftliche Leitbilder:

Herkunft und Einflussfaktoren

Nadine Gies \& Dorothee Dietrich

5 Was ist Familie?

Familienleitbilder und ihre Vielfalt

Detlev Lück \& Kerstin Ruckdeschel

6 Partnerschaftsleitbilder heute:

Zwischen Fusion und Assoziation

Sabine Diabaté.

7 Moderne oder traditionelle Partnerschaftsleitbilder:

Welchen Einfluss haben Konfession und Religiosität?

Robert Naderi

8 Leitbild und Kinderlosigkeit:

Kulturelle Vorstellungen zum Leben ohne Kinder

Jürgen Dorbritz \& Sabine Diabaté

9 Heirat, Haus, Kinder?

Leitbilder der Familiengründung und der Familienerweiterung

Jürgen Dorbritz \& Kerstin Ruckdeschel.

10 Mütter in Ost- und Westdeutschland:

Wie wichtig sind regionalspezifische Leitbilder für Elternschaft?

Katrin Schiefer \& Robert Naderi

\section{Familie XXL:}

Leitbild Kinderreichtum?

Sabine Diabaté, Kerstin Ruckdeschel, Jürgen Dorbritz \& Linda Lux 
12 Verantwortete Elternschaft:

„Für die Kinder nur das Beste"

Kerstin Ruckdeschel

13 Mutterleitbilder:

Spagat zwischen Autonomie und Aufopferung

Sabine Diabaté.....

14 Vaterleitbilder:

Ernährer und Erzieher?

Detlev Lück

15 Leitbilder der Elternschaft:

Zwischen Kindeswohl und fairer Aufgabenteilung

Sabine Diabaté, Detlev Lück \& Norbert F. Schneider.

16 Leitbildforschung:

Befunde, Potenziale und Impulse

Sabine Diabaté, Kerstin Ruckdeschel \& Norbert F. Schneider 269

Autorinnen und Autoren. 


\section{Vorwort der Schriftleitung}

In der bevölkerungswissenschaftlichen Forschung sind ökonomische und strukturelle Erklärungsansätze stark verbreitet. Auch kulturelle Ansätze haben ein großes Potenzial, hierfür war jedoch die Datenlage in Deutschland bisher begrenzt. Durch die FamilienleitbilderStudie - eine neue, umfangreiche Befragung von jungen Menschen zwischen 20 und 39 Jahren - wurde diese Lücke geschlossen. Im vorliegenden Band werden die ersten Befunde dieser Studie zu den kulturellen Idealvorstellungen zum Familienleben und zur Familienentwicklung vorgestellt. Hierbei geht es um elementare Fragen: Wie sieht eine „richtige“ Familie aus? Welche Bedingungen müssen erfüllt sein, damit man sich für Kinder entscheiden kann? Wie teilen sich Männer und Frauen die Aufgaben von Erwerbstätigkeit und Familienarbeit am besten auf? Wie unterscheiden sich die Vorstellungen der jungen Generation von gesamtgesellschaftlichen Erwartungen?

In den Beiträgen des Bandes wird diesen Fragen nachgegangen. Dabei wurde eine Vielzahl von Antworten, Vorstellungen und Normen identifiziert, die bemerkenswert und auch für die breite mediale Öffentlichkeit spannend sind. Diese Studie geht dabei weit über klassische Meinungsumfragen hinaus, da sie auf einem profunden theoretischen Konzept gründet und die Ergebnisse systematisch gebündelt werden. Durch die Identifizierung von Leitbildern lässt sich der oftmals schwammig verwendete Begriff von Kultur empirisch messbar machen. Damit können die Autorinnen und Autoren eine Forschungslücke schließen und dem kulturellen Ansatz in der demografischen Forschung und der Familienforschung neue Impulse geben.

Mit der Schriftenreihe „Beiträge zur Bevölkerungswissenschaft“ verfolgt das Bundesinstitut für Bevölkerungsforschung $(\mathrm{BiB})$ das $\mathrm{Ziel}$, neue und vielversprechende demografische Studien einer breiten wissenschaftlichen Öffentlichkeit zu präsentieren. Das Buch ist für Forscherinnen und Forscher der Disziplinen Soziologie, Ökonomie, Demografie und Psychologie ebenso geeignet wie für Praktiker aus Sozialverbänden, Erwachsenenbildung und Politik.

Die Reihe „Beiträge zur Bevölkerungswissenschaft" hat sich der wissenschaftlichen Exzellenz und Qualitätssicherung verpflichtet. Alle Beiträge dieses Sammelbandes wurden einem Peer-Review-Prozess mit zweifacher Begutachtung unterzogen: einer internen Begutachtung durch die HerausgeberInnen bzw. AutorInnen sowie einem externen anonymen Peer-Review. Allen Gutachterinnen und Gutachtern sei für ihre kritischen Hinweise und Anregungen herzlich gedankt. Großer Dank geht auch an Evelyn Grünheid für das umsichtige Korrektorat, Sybille Steinmetz für den professionellen Satz der Manuskripte und die kompetente grafische Beratung. Ein Dankeschön geht auch an Beatriz Feiler-Fuchs für Unterstützung beim Satz und Christian Fiedler für das Buchcover.

Allen Leserinnen und Lesern wünsche ich eine anregende Lektüre.

Wiesbaden, Januar 2015

Martin Bujard 



\section{Vorwort der HerausgeberInnen}

Helikoptereltern, „Latte-Macchiato-Mütter“ und Wochenendväter - in der öffentlichen Debatte tauchen immer wieder neue Schlagwörter auf. Sie stehen symbolhaft für die vermeintliche Komplexität heutiger Elternschaft in Deutschland und vermitteln, dass Elternsein eine große, schwer zu bewältigende (Lebens-)Aufgabe ist, verantwortungsvoll und voraussetzungsreich. Der Diskurs in Deutschland suggeriert, dass nach einer Geburt die Karriere, das Kind, die Bedürfnisse der Eltern oder besser alles miteinander zu kurz kommen. In Deutschland dominiert eine Kultur des Bedenkens, Zweifelns und Sorgens im Hinblick auf Elternschaft.

Liegt in dieser Skepsis einer der Gründe, weshalb es für junge Menschen nicht mehr so erstrebenswert scheint, eine Familie zu gründen? Ist die Angst vor der Verantwortung zu groß? Oder vor der Verarmung? Sind die gesellschaftlichen Rahmenbedingungen, z. B. fehlende Betreuungsinfrastruktur und belastende Arbeitswelt so ungünstig, erst recht, wenn die Entscheidung für die Familie mit der Berufsentwicklung in der „Rushhour des Lebens“ kollidiert?

Während die Verbesserung struktureller und materieller Rahmenbedingungen längst Eingang in die politischen Strategien zur Erleichterung der Familiengründung und des Familienlebens gefunden hat, wird kulturellen Leitbildern und Erwartungshaltungen bislang kaum Bedeutung beigemessen. Die kulturelle Dimension ist in der Diskussion um den demografischen Wandel selten in den Mittelpunkt gerückt worden. An der Debatte um die möglichen Vorteile und Risiken einer externen Kinderbetreuung von unter 3-Jährigen wird jedoch deutlich, dass die Akzeptanz und die kulturellen Vorstellungen einer optimalen Kindheit stärker ins Blickfeld der Politik und Wissenschaft gelangen müssen.

Mit dieser Studie über die Leitbilder von jungen Menschen zwischen 20 und 39 Jahren leisten wir einen Beitrag für ein besseres Verständnis der kulturellen Dimension des generativen Handelns in Deutschland. Bislang ist wenig bekannt, welche Leitvorstellungen junge Menschen heute haben, wenn sie an Familie denken. Was halten sie für angemessen, was für abweichend? Wie bewerten sie die gesellschaftliche Stimmung zur Familie im Allgemeinen?

Wir möchten mit diesem Sammelband einen Überblick über die verschiedenen Blickwinkel auf das ,ideale“ Familienleben bieten und durch vornehmlich deskriptive Analysen und Befunde beleuchten. Mit neuen Methoden und Herangehensweisen wollen wir eine Forschungslücke zur Erklärung des generativen Geschehens in Deutschland ein stückweit schließen. Die bisherigen Erklärungen werden dabei um flankierende kulturelle Zugänge ergänzt. Auf diese Weise soll ein Beitrag zu einem besseren Verständnis der Entwicklung des Geburtengeschehens in Deutschland erbracht werden.

$\mathrm{Da}$ die vorliegenden Querschnittdaten keine Aussagen über Ursache-WirkungsMechanismen ermöglichen, ist geplant, die Studie „Familienleitbilder“ als Panel weiterzuführen, auch um Aussagen über die Stabilität von Familienleitbildern treffen zu können. Die Wiederholungsbefragung (FLB II) wird im Jahr 2015/2016 angestrebt.

Dieses Buch ist ein Gemeinschaftswerk, das ohne die tatkräftige Unterstützung einer Vielzahl von Personen nicht zu realisieren gewesen wäre. Sabine Wolfert, Monika Pupeter und ihren KollegInnen von Infratest danken wir für die engagierte Unterstützung. Evelyn Grünheid hat uns mit hilfreichen Kommentaren und konstruktiver Kritik bei der Überarbeitung der Kapitel sehr unterstützt. Desgleichen hat uns Martin Bujard als Schriftleiter der Buchreihe mit der Organisation des Begutachtungsprozesses und hilfreichen Anregungen sehr geholfen. Ein großer Dank gilt außerdem allen Kolleginnen und Kollegen am BIB, die direkt und indirekt zum Sammelband beigetragen haben, namentlich ganz besonders Sybille Steinmetz und Beatriz Feiler-Fuchs (Satz), Esther Thiel und Susanne Stock (Korrekturen) sowie Christian Fiedler (Covergestaltung). Außerdem möchten wir den Teilnehme- 
rinnen und Teilnehmern an unserem BiB-Leitbild-Workshop am 29. November 2011, namentlich Katharina D. Giesel, Brigitte Unger-Soyka, Harald Rost, Anna Dechant und Lena Corell, einen besonderen Dank aussprechen. Die Diskussionen über das Leitbild-Konzept waren sehr inspirierend für uns.

Die Beiträge des vorliegenden Bandes sind sämtlich einem wissenschaftlichen Begutachtungsverfahren zur Qualitätssicherung unterzogen worden, wie es bei wissenschaftlichen Fachzeitschriften üblich ist. Ein großer Dank gilt daher allen externen Gutachterinnen und Gutachtern. Sie haben mit ihren Kommentierungen einen wichtigen Beitrag zu diesem Buch geliefert.

Wiesbaden, Januar 2015

Norbert F. Schneider, Sabine Diabaté \& Kerstin Ruckdeschel 


\title{
Leitbilder als „,missing link“ der Familienforschung: Eine Einführung
}

\author{
Sabine Diabaté, Kerstin Ruckdeschel \& Norbert F. Schneider
}

\section{$1 \quad$ Einleitung}

Familienleitbilder haben eine starke Signalwirkung, wirken oftmals polarisierend, beeinflussen generative Entscheidungen und sind gerade deshalb familienpolitisch relevant: $\mathrm{Ob}$ die kontrovers geführte Diskussion über eine Krippenbetreuung im ersten Lebensjahr eines Kindes, das Betreuungsgeld, das Adoptionsrecht gleichgeschlechtlicher Partner oder auch über das Ehegattensplitting: Diese Themen polarisieren. Familienleitbilder verkörpern Normalitätsvorstellungen, wie etwa eine ideale Kindheit aussehen sollte, was eine „richtige" Familie ist und wie das Zusammenleben von Menschen am besten gestaltet werden sollte. Warum bergen diese Themen jedoch so viel (gesellschaftlichen und politischen) Zündstoff? Kulturelle Leitbilder zum Familienleben erscheinen im gesellschaftspolitischen Diskurs ideologisch hoch aufgeladen. Was als „normal“ gilt und was als wünschenswert, als akzeptabel und als abweichend, darüber scheiden sich die Geister teilweise grundsätzlich. Die Debatte um das, was im Leben, in der Liebe und in der Familie „richtig“, „selbstverständlich“ und „normal“ ist, betrifft jeden Menschen persönlich. Andersartige Vorstellungen von Dritten werden nicht selten als Affront gegen die eigenen Überzeugungen oder als Angriff auf die eigene Lebensweise empfunden.

Mit diesem Buch möchten wir einen Blick auf die Vielfalt und Widersprüchlichkeit der derzeit in Deutschland vorfindbaren Familienleitbilder werfen und damit auch ein stückweit zur Versachlichung der aufgewühlten Debatte um das „,adäquate Familienleben“ beitragen. Im Begriff „Leitbild“ stecken die Worte „leiten“ und „Bild“ und damit auch der Hinweis auf die Bedeutung von Familienleitbildern: Das Bild von Familie ist verbunden mit einer Handlungsmaxime. Es geht sowohl um etwas Sichtbares als auch um etwas Erlebbares sowie um die Wechselwirkung zwischen beidem. Nicht zuletzt geht es darum, die vielfältigen Lebensrealitäten und Normalitätsvorstellungen zum ,guten Familienleben“ von Menschen in Deutschland sichtbar zu machen, sie in ihrer Vielschichtigkeit und Ambivalenz ernst zu nehmen und so zu einer Weiterentwicklung der Familienpolitik beizutragen.

Ein weiteres Anliegen ist es, das seit Mitte der 1970er Jahre andauernde niedrige Geburtenniveau in Deutschland besser zu verstehen: Jede Generation von Kindern ist mittlerweile um etwa ein Drittel kleiner als ihre Elterngeneration - obwohl in Deutschland vielseitige politische Anstrengungen unternommen werden, das Land familienfreundlicher zu gestalten. Familiengründungs- und Familienerweiterungsprozesse von Individuen und Paaren werden in vielschichtiger Weise durch Natur, Struktur und Kultur beeinflusst. In diesem Buch soll es explizit um die kulturelle Beeinflussung in Form von Leitbildern gehen, da dieser Aspekt in der Forschung bislang eher selten berücksichtigt wurde. Wir möchten mit diesem Band zu einem tieferen Verständnis darüber beitragen, nach welchen Vorstellungen Menschen ihr Leben gestalten. Die Art und Weise, in der wir unser privates Leben gestalten, folgt bestimmten, zum Teil sehr konkreten und selten hinterfragten, kulturellen Leitbildern eines „,normalen”, „richtigen” und „guten” Zusammenlebens als Paar bzw. als Familie oder auch der Rolle als Mutter oder als Vater. Gesellschaftlich und poli- 
tisch relevant ist dieser Zusammenhang vor allem insofern, als die Leitbilder in Deutschland heute an Eltern, und insbesondere an Mütter, sehr hohe Anforderungen stellen, die nur schwer einzulösen sind (Schneider et al. 2014: 29).

Der vorliegende Sammelband soll dieses Feld erhellen und einen Beitrag dazu leisten, kulturelle Muster in der Gesellschaft zu entschlüsseln sowie ihre Relevanz für die private Lebensführung von Menschen besser und tiefgreifender verstehen zu können. Die in diesem Band bearbeiteten Fragestellungen basieren auf der bundesweiten repräsentativen Studie „Familienleitbilder in Deutschland“, die im Jahr 2012 im Auftrag des Bundesinstituts für Bevölkerungsforschung erhoben wurde. Bei der Konzeption dieser Studie wurde ein multimethodischer Ansatz verfolgt, wobei verschiedene quantitative und qualitative Vorstudien zur Verbesserung des Datenerhebungsinstruments eingesetzt wurden. Die einzelnen Beiträge in diesem Band widmen sich verschiedenen Teilaspekten von Familienleitbildern. In vielen bisherigen quantitativen Studien werden Meinungen und Einstellungen erhoben, weniger Vorstellungen eines optimalen, guten Familienlebens und deren Abgleich mit der gesellschaftlich vorherrschenden Meinung. Diese Lücke möchten wir mit diesem Band ein Stück weit schließen.

\section{$2 \quad$ Ausgangspunkt: Warum Leitbilder?}

Dieser Studie liegen drei Ausgangspunkte zugrunde: Erstens ist bisher noch nicht tiefgreifend erklärbar, warum Deutschland seit rund 40 Jahren weltweit zu den Niedrig-FertilitätsLändern gehört. Das anhaltend niedrige Geburtenniveau bleibt bisher trotz zum Teil großer politischer Bemühungen recht stabil. Zweitens können wir feststellen, dass sich die Einstellungen zur Kleinkindbetreuung in Westdeutschland im Vergleich zu vielen anderen europäischen Ländern stark unterscheiden: Frauen wird trotz der Bildungsgewinne immer noch die Hauptverantwortung für die Fürsorgearbeit zugeschrieben, es ist eine weit verbreitete Sichtweise in Westdeutschland, dass Kleinkinder unter der Berufstätigkeit der Mutter leiden (EVS 2008). Dabei ist die Auffassung verbreitet, dass Kinderbetreuung außerhalb des Elternhauses vornehmlich der „Aufbewahrung“ von Kindern diene und damit vor allem die Erwerbsbeteiligung der Mütter fördere, insbesondere vor dem Hintergrund des volkswirtschaftlichen Nutzens. Zudem fühlen sich Eltern in Deutschland im Erziehungsalltag öfter gestresst und erleben ihre Kinder häufiger als Belastung als in anderen Ländern (Quaiser-Pohl 2001: 301). Drittens beobachten wir, dass in Deutschland ein großes Ausmaß an Angst und Verunsicherung existiert (z. B. Kuschel 2001; Kuschel/Miller 2000; HenryHuthmacher/Borchard 2008), wie Elternschaft optimal gestaltet werden kann, so dass sich ein Kind gut entwickelt. Laut einer Forsa-Umfrage der Zeitschrift BRIGITTE (2006) schätzen Eltern die Gefahren für ihr Kind viel höher ein, als sie es tatsächlich sind - und schaden damit ihren Kindern, weil diese nicht lernen autonom zu werden. Die Folge: Durch die Zahl der überbesorgten Eltern steige die Zahl der Kinder, die therapeutische Hilfe benötigen (ebd.). Besonders Mütter sehen sich selbst massiv unter Druck, dem Perfektionszwang gerecht zu werden (Rheingold 2010). Außerdem wurde bei einem interkulturellen Vergleich (Nickel/Quaiser-Pohl 2001) zwischen verschiedenen Ländern Europas, Asiens und Nordamerikas festgestellt, dass junge Eltern aus Westdeutschland neben den Südkoreanern die größten Schwierigkeiten beim Übergang zur Elternschaft haben.

Betrachtet man die quantitative Forschung zu diesem Themenkomplex, dann fällt auf, dass besonders die Infrastruktur und die sozioökonomischen Rahmenbedingungen untersucht werden, während Kultur erst seit Kurzem eine größer werdende Rolle einnimmt (z. B. 
Billari et al. 2009; Pfau-Effinger et al. 2009). Dabei ist die Operationalisierung von kulturellen Vorstellungen zum Familienleben und zur Elternschaft bislang thematisch sehr eingeschränkt. Eine Identifikation der Vielfalt von kulturellen Normalitätsvorstellungen ist jedoch für ein besseres Verständnis des komplexen Zusammenspiels aus Kultur, Infrastruktur und Ökonomie nötig.

Wir schlagen dafür eine neuartige Operationalisierung vor, um die Kultur der Familie in Deutschland mittels Leitbildern messbar und einer empirischen Untersuchung zugänglich zu machen. Der Leitbildbegriff ist dabei hilfreich und auch notwendig, weil er die zum Familienleben vielseitig existierenden Rollenvorstellungen, Normen und Werthaltungen in sich bündelt. Leitbilder werden der Vielschichtigkeit des Familienlebens gerecht, weil sie all ihre Facetten (ob Akteure, Interaktionen oder Situationen, Handlungsabläufe) zu einer Normalitätsvorstellung in sich vereinen. Leitbilder sind mehr als bloße Normen, sie sind komplexe Programme, die mal mehr oder weniger bildhaft in den Köpfen von Menschen existieren und die notwendig sind, um daran das eigene Handeln auszurichten. Sie sind zentrale (bereits bewährte) Orientierungspunkte und dienen als Schema für das eigene Verhalten. Verschiedene Deutungsgeber sind an der Vermittlung tradierter, modifizierter und neuer Leitbilder beteiligt: Dazu zählen beispielsweise Parteien, Kirchen oder auch herausragende Personen des öffentlichen Lebens. Die Medien und der öffentliche Raum (z. B. Begegnungsorte wie Vereine) spielen in diesem Prozess eine wesentliche Rolle. Auf diese Weise entsteht ein Stimmungsbild zu den verschiedenen Leitbild-Themen, das jeder Einzelne subjektiv wahrnehmen kann. Wir unterstellen, dass diese Wahrnehmung der gesellschaftlichen Idealvorstellung zur Familie neben der persönlichen Normalitätsvorstellung einen eigenständigen Einfluss auf das individuelle Verhalten nimmt.

\section{Dimensionen des Leitbild-Begriffs}

Familienleitbilder beinhalten unterschiedliche Dimensionen des Familienlebens. Sie besitzen mehrere aufeinander bezogene Aspekte. In struktureller Hinsicht beinhalten Familienleitbilder Vorstellungen, wie Familie (oder ein Aspekt davon) im Allgemeinen idealerweise zu sein hat. Dazu zählen Vorstellungen zu einer ,guten“ Partnerschaft, die beispielsweise Kohabitation, eine bestimmte Aufgabenteilung zwischen den Partnern (im Haushalt oder bei der Kinderbetreuung) sowie eine bestimmte Familienorientierung (z. B. zur Ausgestaltung von Mütter- und Väterrollen) beinhaltet. In prozessualer Hinsicht beziehen sie sich auf die idealen Übergangszeiten und -zeitpunkte im Familienbildungsprozess, also das (,richtige") Alter beim Übergang zur Elternschaft in der Partnerschafts- und Erwerbsbiographie oder den („optimalen“) Geburtenabstand. Prozessuale Leitbilder sind vergleichbar mit dem Forschungsthema der Life Scripts, die seit Jahrzehnten in der Soziologie, Sozialpsychologie und Gerontologie untersucht werden (z. B. Janssen/Rubin 2011). Diese besagen, dass es eine Art „Normalbiografie“ gibt, die ein Mensch normalerweise durchlebt oder auch idealerweise durchleben soll. Insgesamt betrachtet hängen strukturelle und prozessuale Leitbilder zusammen und sind sehr komplex. Sie adressieren Vorstellungen von der (,richtigen“) Ausgestaltung des Familienlebens im Hinblick auf Partnerschaft, Ehe, Familiengründung, Elternschaft und Generationenbeziehungen. Innerhalb des vielschichtigen Leitbilds der Familie existieren diverse dazugehörige Leitbild-Elemente, die einzelne Aspekte, Akteure bzw. Bestandteile von Familie betreffen und deren Beschaffenheit genauer charakterisieren. Der Leitbild-Begriff enthält mithin mehrere Dimensionen, drei zentrale werden in diesem Band behandelt: Partnerschaft, Elternschaft und Familienleben. 


\section{$4 \quad$ Zielsetzungen}

Die Leitbildforschung verfolgt vielseitige Ziele: Zunächst geht es darum zu prüfen, ob die Existenz von Leitbildern überhaupt nachweisbar ist. Welche sind es? Auf welchen Ebenen wie z. B. Elternschaft oder Partnerschaft existieren sie? Wie sind sie inhaltlich gefüllt? Variieren sie sozialstrukturell? Korrespondieren individuelle Vorstellungen mit den in der Gesellschaft wahrgenommenen Leitbildern? Wie hängen Familienleitbilder und Geburtenentwicklung zusammen? Wie entstehen Leitbilder? Bleiben Leitbilder über die Generationen und im Lebensverlauf stabil? Mit den Daten der ersten Querschnitterhebung zu Familienleitbildern in Deutschland können wir den ersten Teil der Forschungsfragen bearbeiten. Fragen zum Einfluss auf die Geburtenentwicklung und zur Stabilität von Leitbildern können jedoch erst mit einer Wiederholungsbefragung analysiert werden. Daher ist das erklärte Ziel dieser Veröffentlichung, die vielschichtigen und disparaten Familienleitbilder erstmals sichtbar zu machen und ihre Variation über die verschiedenen Bereiche der Gesellschaft in Deutschland darzustellen. Dabei können auch Abgleiche zwischen Leitbildern und Wirklichkeit verdeutlichen, wie leitbild-konform Menschen heutzutage ihren Alltag gestalten.

\section{$5 \quad$ Aufbau des Sammelbandes}

Der Aufbau des Bandes orientiert sich eng an der dimensionalen Struktur des Familienbegriffs: Dabei wird zunächst das Leitbildkonzept erklärt (siehe Lück/Diabaté, Kap. 2). Mit diesem Beitrag wird das theoretische Fundament des Leitbildkonzeptes dargestellt, auf dem die folgenden Beiträge aufbauen.

Es folgen die detaillierte Beschreibung der Studie im Hinblick auf die methodischen Grundlagen, die Fragebogenkonzeption und ein Überblick über das Design und die Durchführung der Studie (siehe Lück/Ruckdeschel/Naderi, Kap. 3). Darin wird zudem erläutert, wie die verschiedenen Schwerpunkte des Themenbereichs „Familie“ operationalisiert wurden.

Anschließend wird eine kurze Einführung in den theoretischen Hintergrund der Analyse kollektiver Leitbilder gegeben (siehe Gies/Dietrich, Kap. 4). Dabei wird das methodische Vorgehen des Zusatzmoduls beschrieben, welches zur Validierung des Konstrukts „Allgemeinheit“ in den Fragebogen implementiert wurde. Es wird gezeigt, welche Orientierungsgeber den kollektiven Familienleitbildern überhaupt zugrunde liegen und welche Faktoren dabei die größte Bedeutung haben. Zudem ist von Interesse, wie sich einzelne Gruppen von Befragten in ihren Vorstellungen voneinander unterscheiden und welche persönlichen Eigenschaften einen Einfluss auf das Antwortverhalten ausüben.

Die darauf folgenden Beiträge zeichnen verschiedene zentrale Dimensionen des Familienlebens nach:

Die Beschreibung dessen, was heutzutage aus Sicht der 20- bis 39-Jährigen in Deutschland eine Familie definiert, bildet den Ausgangspunkt (siehe Lück/Ruckdeschel, Kap. 5). Anhand von vorgegebenen Lebensformen, für die erfragt wurde, ob es sich dabei jeweils um eine Familie handele, stimmen nahezu alle Befragten zu, dass eine Kernfamilie, bestehend aus einem zusammenwohnenden heterosexuellen Paar mit Kindern, eine Familie sei. Familie wird darüber hinaus vor allem dann als solche definiert, wenn Kinder dazu gehören. Die Bewertung variiert sowohl nach sozialem Umfeld als auch nach der Lebensform, in der die Befragten selbst leben. 
Ein weiterer Beitrag zielt auf die Partnerschaftsvorstellungen ab (siehe Diabaté, Kap. 6). Es wird geklärt, wie weit bestimmte kulturelle Normalitätsvorstellungen zur Partnerschaft verbreitet sind. Dabei werden individuelle und gesellschaftlich wahrgenommene Vorstellungen näher untersucht und miteinander verglichen. Insgesamt können verschiedene Partnerschaftsleitbilder identifiziert werden: Am häufigsten wird das assoziativ-moderne Partnerschaftsleitbild vertreten, gefolgt vom fusionsorientierten, bürgerlich-konventionellen und schließlich dem nichtehelichen-instabilen Partnerschaftsleitbild. Es bestätigt sich, dass junge Menschen eher assoziativ-orientierte Idealvorstellungen vertreten. Die öffentliche Meinung hingegen wird insgesamt etwas konventioneller und stärker polarisierend wahrgenommen.

Eine vertiefende Analyse der Partnerschaftsleitbilder erfolgt im Anschluss, indem die partnerschaftlichen Idealvorstellungen von Mitgliedern verschiedener Glaubensgemeinschaften und von Konfessionslosen untersucht werden (siehe Naderi, Kap. 7). Hinter dieser Fragestellung steht die übergeordnete Annahme, dass bestimmte Religionszugehörigkeiten und eine höhere Religiosität mit konventionelleren und auf höhere Stabilität ausgerichteten Vorstellungen zur Partnerschaft einhergehen. Tatsächlich zeigen die Ergebnisse auch einen Zusammenhang zwischen Religiosität, Konfessionszugehörigkeit und Partnerschaftsleitbildern.

Im Beitrag zur Kinderlosigkeit wird untersucht, wie gesellschaftliche Leitbilder und Kinderlosigkeit zusammenhängen (siehe Dorbritz/Diabaté, Kap. 8). Dazu wurden neben dem Kinderwunsch auch die Gründe der Kinderlosigkeit und die soziale Akzeptanz von Kinderlosigkeit untersucht. Festgestellt wurde, dass Kinderlosigkeit in Deutschland als „,normal“" gilt und Sanktionen gegenüber Kinderlosen keine Mehrheit finden. Trotz breiter Akzeptanz der Kinderlosigkeit wurde in der Altersgruppe der 20- bis 39-Jährigen nur eine kleine Gruppe identifiziert, die keine Kinder und auch keinen Kinderwunsch hat. Es konnten zwei gesellschaftliche Leitbilder aufgefunden werden, weshalb viele Menschen in Deutschland aus Sicht der Befragten keine Kinder bekommen: Das Leitbild der risikovermeidenden Elternschaft und das Leitbild der autonomiebetonten Kinderlosigkeit.

Ein weiterer Betrag thematisiert Vorstellungen dazu, wann die Familiengründung idealerweise stattfinden soll (siehe Dorbritz/Ruckdeschel, Kap. 9). Es werden der Beginn und der Verlauf der Familiengründung anhand der Gründe für die Geburt von Kindern, der idealen Kinderzahlen und des idealen Alters für die Geburt des ersten Kindes betrachtet. Herauszustellen ist, dass trotz aller Diskussionen um den Wandel der Familie Kinder zu haben immer noch als etwas ganz Normales angesehen wird (Leitbild der Selbstverständlichkeit des Kinderhabens). Es bestätigt sich der Trend zu einem Wiederanstieg des Kinderwunsches, der nach wie vor an der Zwei-Kind-Familie orientiert ist. Begrenzend auf den idealen Kinderwunsch wirken hohe persönliche Ansprüche an die Bedingungen von Elternschaft (Leitbild der materiell gesicherten Elternschaft). Als bemerkenswert ist hervorzuheben, dass das benannte ideale Alter für die Erstgeburt niedriger ist als das tatsächliche Erstgebäralter.

An die Vorstellungen zur Familiengründung anknüpfend wir danach der Zusammenhang zwischen kulturellen Vorstellungen und dem Geburtenverhalten im Ost-West-Vergleich untersucht (siehe Schiefer/Naderi, Kap. 10). Ausgangspunkt ist, dass in Ost- und Westdeutschland unterschiedliche Familienleitbilder vorherrschen und dass diese für die divergenten Fertilitätsregime mitverantwortlich sind. Es wurden kinderlose Frauen mit Müttern verglichen und nach soziodemografischen Merkmalen kontrolliert. Die Ergebnisse legen nahe, dass Leitbilder mitursächlich für Kinderlosigkeit und Elternschaft sind. Insgesamt konnte gezeigt werden, dass kulturelle Leitbilder neben den sozioökonomischen Merkmalen eine eigenständige Erklärungskraft entfalten.

Im danach folgenden Beitrag zum Kinderreichtum werden die persönlichen und allgemein wahrgenommenen Einstellungen gegenüber Großfamilien untersucht (siehe Diabaté/ Ruckdeschel/Dorbritz/Lux, Kap. 11). Die Ergebnisse zeigen, dass aus der individuellen Sicht 
der Befragten viele Kinder zu haben als etwas ganz Wundervolles gesehen wird. Andererseits wird in der Gesellschaft ein negatives Image von Großfamilien wahrgenommen. Bezüglich des Kinderreichtums konnten drei Leitbilder identifiziert werden. Das erste wurde mit dem Begriff der Distanz gegenüber Kinderreichen bezeichnet. Ein zweites Leitbild bringt eine gemischtgeschlechtliche Geschwisterorientierung zum Ausdruck. Ein drittes Leitbild entspricht einem positiv konnotiertem Kinderreichtum.

Mit dem Thema Kinderreichtum eng verbunden sind die Vorstellungen von einer guten, „verantworteten“ Elternschaft (siehe Ruckdeschel, Kap. 12). Das Leitbild Verantwortete Elternschaft ist in Deutschland stark präsent. Darin findet sich einerseits das Fördergebot, d. h. Kinder sollten beim Aufwachsen intensiv begleiten werden. Dieses Leitbild findet individuell breite Zustimmung und wird auch in der Gesellschaft häufig wahrgenommen. Es verbindet verschiedenste Elterntypen und wird auch als gesellschaftlicher Anspruch an Eltern empfunden. Insgesamt kann von einem stark in der Gesellschaft verankerten Leitbild gesprochen werden. Auf der anderen Seite steht das Gebot der Mutternähe und Aufopferung, das Fremdbetreuung von unter 3-Jährigen ablehnt und die Rückstellung eigener Bedürfnisse hinter die des Kindes fordert.

In weiteren Kapiteln wird in Anlehnung an die Verantwortete Elternschaft die Ausgestaltung der elterlichen Rollen näher beleuchtet. Daher werden die Leitbilder zur Mutterund zur Vaterschaft sowie zur verantworteten Elternschaft betrachtet: Die Ergebnisse des Beitrags zu den Mutterschaftsleitbildern spiegeln den hohen Qualitätsanspruch an Mütter und die Idealisierung der „sich aufopfernden Mutter“ wider (siehe Diabaté, Kap. 13). Gleichzeitig wird auch die Überfrachtung des allgemein als vorherrschend wahrgenommenen Mutterleitbildes mit seiner Widersprüchlichkeit und Komplexität deutlich. Herausgearbeitet werden konnten vier Mutterleitbilder: das berufs- und das kindorientierte Mutterleitbild sowie zwei Mischtypen, das moderate und das vereinbarkeitsorientierte Mutterleitbild. Sichtbar wird auch eine stärkere Kindorientierung bei in Westdeutschland lebenden Personen. Es zeigt sich, dass vor allem das kind- und das berufsorientierte Mutterleitbild eng mit dem jeweiligen Lebensmodell der Mütter der Befragten korrespondieren.

Entsprechend zur Mutterschaft setzt sich der nachfolgende Beitrag mit der Vaterschaft auseinander (siehe Lück, Kap. 14). Unter den Vorstellungen, wie ein Vater sein sollte, lassen sich zwei Leitbilder entdecken: zum einen das des Familienernährers, der für das Bestreiten des Haushaltseinkommens Verantwortung übernimmt und Familien-arbeit der Mutter überlässt. Dieses Leitbild ist auf gesellschaftlicher Ebene relevant: als Vorstellung davon, was andere denken. Zum zweiten lässt sich das Leitbild des aktiven Vaters identifizieren, der seine Erwerbsarbeit reduziert und sich aktiv in die Erziehung einbringt. Dieses ist unter den persönlichen Leitbildern dominant. Daneben gibt es eine relevante Gruppe, die beide Leitbilder verinnerlicht hat und sich vereinbarkeitsorientiert zeigt.

Im darauf folgenden Beitrag erfolgt eine Zusammenfassung der verschiedenen Aspekte zur Elternschaft durch eine synchrone Untersuchung der Vorstellungen zur Mutterschaft, Vaterschaft sowie der Verantworteten Elternschaft (siehe Diabaté/Lück/Schneider, Kap. 15). Die Vorstellungen, wie Elternschaft idealerweise gestaltet werden sollte, sind vielfältig. Mit den hier vorliegenden Befunden können auf individueller wie auf gesellschaftlicher Ebene jeweils zwei Elternschaftsleitbilder identifiziert werden. Im Kern des einen Leitbildes steht die Vorstellung einer komplementären Geschlechterrollenteilung, verbunden mit der Ablehnung externer Kinderbetreuung (Leitbild der komplementären Elternrollen). Das andere Leitbild ist durch ein Kürzertreten des Vaters im Beruf mit hohen Ansprüchen an eine Verantwortete Elternschaft gekennzeichnet (Leitbild der kind-zentrierten Erziehungsarbeit). Festzustellen ist insgesamt, dass es sowohl eine Ambivalenz als auch eine Verunsicherung hinsichtlich der idealen Ausgestaltung von Elternschaft in Deutschland gibt. 
Der abschließende Beitrag fasst die zentralen Ergebnisse der verschiedenen Kapitel zusammen, diskutiert die wesentlichen Erkenntnisse und beeinhaltet einen Ausblick auf künftige Forschungsfragen (siehe Diabaté/Ruckdeschel/Schneider, Kap. 16). Zudem werden Impulse für die gesellschaftliche Debatte und Implikationen für die politische Praxis skizziert: Bislang zeichnet sich die Familienpolitik in Deutschland durch eine Vielzahl an Maßnahmen aus, die im Wesentlichen auf den drei Handlungsebenen Zeit, Geld und Infrastruktur aufsetzen. „Kultur“ wurde bei der politischen Auseinandersetzung rund um das Thema „Familie“ bislang stark vernachlässigt. Die in diesem Buch dargestellten Ergebnisse verweisen jedoch darauf, dass bestimmbare Familienleitbilder existieren, die als kulturelle Barrieren wirken können und der Nutzung und Entwicklung institutioneller Rahmenbedingungen entgegenstehen. Die Zusammenhänge zwischen kulturellen Vorstellungen und Verhalten deuten darauf hin, dass sich die Politik noch stärker als bislang mit den in Deutschland vorherrschenden Ansichten und Wünschen von jungen Menschen in der Familiengründungs- und Familienerweiterungsphase auseinandersetzen sollte. Auf diese Weise kann die Wahlfreiheit der Lebensführung vergrößert und dadurch die Lebensqualität in Deutschland erhöht werden.

\section{Literatur}

Billari, Francesco C.; Philipov, Dimiter; Testa, Maria Rita (2009): Attitudes, norms and perceived behavioural control: Explaining fertility intentions in Bulgaria. In: European Journal of Population/ Revue européenne de Démographie, 25, 4, S. 439-465.

BRIGITTE Online-Artikel bei Presseportal (2006): [http://www.presseportal.de/pm/6788/797099/ eltern-heute-viel-zu-aengstlich-eine-brigitte-umfrage-ergab-eltern-schaetzen-die-gefahren-fuer-ihr, 30.09.2014].

EVS (European Values Study) (2008): [http://www.europeanvaluesstudy.eu/].

Henry-Huthmacher, Christine; Borchard, Michael (Hrsg.) (2008): Eltern unter Druck. Stuttgart: Lucius \& Lucius.

Janssen, Steve M. J.; Rubin, David C. (2011): Age Effects in Cultural Life Scripts. In: Applied Cognitive Psychology 25, S. 291-298.

Kuschel, Annett; Miller, Yvonne (2000): Prävention von oppositionellen und aggressiven Verhaltensstörungen bei Kindern: Triple P - ein Programm zu einer positiven Erziehung. In: Kindheit und Entwicklung 9, 1, S. 20-29.

Kuschel, Annett (2001): Psychische Auffälligkeiten bei Braunschweiger Kindergartenkindern. Dissertation an der Gemeinsamen Naturwissenschaftlichen Fakultät der Technischen Universität Braunschweig.

Nickel, Horst; Quaiser-Pohl, Claudia (2001): Junge Eltern im kulturellen Wandel: Untersuchungen zur Familiengründung im internationalen Vergleich. Weinheim und München: Beltz Juventa.

Quaiser-Pohl, Claudia (2001): Deutsche Eltern im interkulturellen Vergleich. In: Nickel, Horst; Quaiser-Pohl, Claudia (Hrsg.): Junge Eltern im kulturellen Wandel: Untersuchungen zur Familiengründung im internationalen Vergleich. Weinheim, München: Beltz Juventa, S. 301-311.

Rheingold (2010): Die deutsche Angst vorm Kinderkriegen. Pressemeldung vom 24.11.2010. Rheingold-Institut Köln.

Pfau-Effinger, Birgit; Flaquer, Lluis; Jensen, Per H. (2009): Formal and informal work in Europe. The hidden work regime. New York: Routledge.

Schneider, Norbert F.; Diabaté, Sabine; Lück, Detlev (2014): Familienleitbilder in Deutschland. Ihre Wirkung auf Familiengründung und Familienentwicklung. Herausgegeben von der KonradAdenauer-Stiftung e.V., Sankt Augustin, Berlin. 



\title{
Familienleitbilder: Ein theoretisches Konzept
}

\author{
Detlev Lück \& Sabine Diabaté*
}

\section{Zusammenfassung}

In diesem Beitrag wird das theoretische Fundament des Leitbildkonzeptes dargestellt, auf dem die folgenden Beiträge aufbauen. Unter einem Leitbild verstehen wir ,ein Bündel aus kollektiv geteilten bildhaften Vorstellungen des ,Normalen', das heißt von etwas Erstrebenswertem, sozial Erwünschtem und/oder mutmaßlich weit Verbreitetem, also Selbstverständlichem.“ Damit vereinigen Leitbilder in sich Eigenschaften von Werten, Normen und Frames. Sie sind komplexe Visualisierungen, die Menschen als Orientierung dienen. Leitbilder können als persönliche Leitbilder auf individueller Ebene vorgefunden werden, sie existieren aber auch als kulturelle Leitbilder auf der Meso- und Makro-Ebene, da sie intersubjektiv geteilt werden und für bestimmte Kollektive charakteristisch sind. In der Gesamtgesellschaft ist demnach eine gewisse Homogenität, innerhalb bestimmter sozialer Milieus, Generationen und Regionen ist eine graduell stärkere Homogenität individueller Leitbilder zu erwarten. Dennoch sind kulturelle Leitbilder nicht durch die Summe der individuellen Leitbilder innerhalb einer Gesellschaft bestimmt, sondern gewinnen durch Institutionalisierung eine eigene, unabhängige, sozial reproduzierte Realität. Manifestationen kultureller Leitbilder, die mit standardisierten Erhebungsinstrumenten erfassbar sind, können unter anderem in der statistischen Aggregation individueller Leitbilder sowie in der individuellen Wahrnehmung dessen gesehen werden, wie das in der Gesellschaft mehrheitlich verbreitete Leitbild aussieht.

\section{$1 \quad$ Einleitung}

Machen wir ein kleines Experiment: Bilden Sie einmal einen Satz, der mit „Es ist normal, dass ..." beginnt! Wenn nötig, lassen Sie sich einen Moment Zeit zum Überlegen! Der Satz sollte in Ihren Augen durchaus Sinn ergeben. Wenn Ihnen gleich mehrere Möglichkeiten einfallen, ist es auch gut.

Welchen Satz könnten Sie gebildet haben - oder welche Sätze? Vielleicht: „Es ist normal, dass in Deutschland deutsch gesprochen wird.“ Oder: „Es ist normal, dass im Winter Schnee fällt.“ Vielleicht auch: „Es ist normal, dass man sechs bis acht Stunden Schlaf braucht.“ Oder auch: „Es ist normal, dass Frauen nach einer Geburt für einige Monate ihre Erwerbsarbeit unterbrechen und mit dem Kind zuhause bleiben."

Was auch immer Ihnen eingefallen ist: Es könnte ein Naturgesetz sein, vielleicht auch eine soziale Norm. Häufig ist es auch (gleichzeitig) ein Beispiel für das, was wir mit dem Begriff Leitbild meinen oder zumindest ein Bestandteil davon. Dieses theoretische Konzept

\footnotetext{
* Wir danken der anonymen Gutachterin bzw. dem anonymen Gutachter für die wertvollen Hinweise und die konstruktive Kritik.
} 
(ausführlich siehe Diabaté/Lück 2014) soll im Folgenden vorgestellt und erläutert werden. Der Beitrag geht darauf ein, was die Begriffe Leitbild bzw. Familienleitbild genau bezeichnen, welche Eigenschaften Leitbilder und Familienleitbilder haben und warum sie für die Familien- und Bevölkerungsforschung relevant sind.

\section{Eine Begriffsbestimmung}

Der Begriff „Leitbild“ wird in verschiedenen sozialwissenschaftlichen Disziplinen sporadisch verwendet, jedoch mit unterschiedlichen Bedeutungen und meist ohne eine (klare) Begriffsdefinition oder -explikation (z. B. Busch/Scholz 2006; Heut 2004; Holetschek et al. 2000; Vaskovics 1997; Meyer 1990). Eine Ausnahme stellt die Arbeit von Giesel (2007) dar: Sie sichtet die Vielfalt der Verwendungen des Begriffs „Leitbild“, die in den Sozialwissenschaften kursieren, systematisch und schlägt als eine Synthese vor, Leitbilder derart zu definieren, dass sie ,sozial geteilte (mentale oder verbalisierte) Vorstellungen von einer erwünschten bzw. wünschenswerten und prinzipiell erreichbaren Zukunft [bündeln], die durch entsprechendes Handeln realisiert werden soll“ (Giesel 2007: 245).

Die Leitbildforschung am Bundesinstitut für Bevölkerungsforschung und die darin implementierte Studie Familienleitbilder (FLB) 2012 orientieren sich an dieser Definition, fassen den Begriff jedoch noch einmal unterschiedlich, im Bemühen ihn besser operationalisierbar und tragfähiger zu machen. Demnach ist „ein Leitbild ein Bündel aus kollektiv geteilten bildhaften Vorstellungen des ,Normalen', das heißt von etwas Erstrebenswertem, sozial Erwünschtem und/oder mutmaßlich weit Verbreitetem, also Selbstverständlichem“ (Diabaté/Lück 2014: 56).

Diese Definition lässt zum einen die Frage offen, ob Leitbilder praktisch handlungsleitend sind, um sie einer empirischen Überprüfung zu überantworten. Zum zweiten lässt sie $\mathrm{zu}$, dass sich ein Leitbild nicht nur auf Zukünftiges bezieht, sondern auf Gegenwärtiges bzw. Zeitloses. Dies erscheint sinnvoll, zumal kaum etwas, was sich Menschen für die Zukunft wünschen, nicht bereits - zumindest graduell - für manche Menschen in der Gegenwart Realität ist, und ein häufiger Wunsch für die Zukunft lautet, dass das, was ist, fortbestehen soll. Zum dritten erweitert die Definition die Charakterisierung von Leitbildern um eine weitere Facette: Neben Vorstellungen dessen, was persönlich als wünschenswert angesehen wird, und dem, was (mutmaßlich) durch das soziale Umfeld erwünscht wird, können sich die zum Leitbild gebündelten Vorstellungen auch auf etwas (mutmaßlich) weit Verbreitetes beziehen - auf etwas, was ,üblich ist“, was ,jeder so macht" und was „,schon immer so war". Damit rückt die Definition von Diabaté und Lück in die Nähe des wissenssoziologischen Begriffs des Alltagswissens und ähnelt unter anderem der Verwendung bei Birgit Pfau-Effinger, die Leitbilder im Familienkontext als ,typical societal ideal representations, norms and values regarding the family and the societal integration of women and men" (Pfau-Effinger 2004: 382) beschreibt.

Konsens ist: Leitbilder sind sozial geteilte Vorstellungen. Damit reihen sie sich ein in die lange Liste kultureller Phänomene, ähnlich wie etwa Werte, Normen oder Frames, welche in zahlreichen Theorien thematisiert werden, so z. B. in der Theory of Planned Behavior (Ajzen 1985), in der Rollentheorie (Dahrendorf 1958) oder in der Theorie der Frame-Selektion (Esser 2002, 1991, 1990). Unser Vorschlag für einen Leitbild-Ansatz knüpft daran an und übernimmt wesentliche Grundgedanken, die er zum Teil nur neu kombiniert. Im Unterschied zu Werten, Normen oder Frames sind Leitbilder Bündel, die mehrere miteinander verknüpfte, häufig bildhafte kulturelle Vorstellungen in sich vereinen. Diese 
Vorstellungen können jeweils einem Wert, einer Norm oder einem Frame entsprechen, je nachdem ob sie am ehesten den Charakter des (1) Gewünschten, (2) des Erwünschten oder (3) des als selbstverständlich Vorausgesetzten aufweisen. Als Regelfall unterstellt das Leitbild-Konzept, dass alle drei Eigenschaften gemeinsam auftreten: Das, was mutmaßlich ,alle“ „schon immer" so getan haben, wird typischerweise auch von vielen Menschen in der Gesellschaft als gut und richtig angesehen und als sozial erwünscht empfunden.

Aufgrund aller drei Eigenschaften, die sprachlich mit dem Begriff des „Normalen“ gebündelt werden, sind Normalitätsvorstellungen und die Leitbilder, die sich aus ihnen zusammensetzen, grundsätzlich geeignet, Handeln bzw. Verhalten zu beeinflussen: Menschen verhalten sich einem Leitbild entsprechend, weil sie dies per se wollen, weil sie negative Sanktionen zu vermeiden suchen oder weil sie in der hochkomplexen Vielfalt der Handlungsoptionen im Alltag vielfach auf unreflektierte, internalisierte Handlungsroutinen zurückgreifen müssen. In diesem Sinne sind Leitbilder immer auch normativ besetzt. Auf eine theoretisch-analytische Trennung oder Verengung dieser drei Einflüsse verzichtet das Leitbildkonzept, zumal sie im Regelfall auch empirisch nicht zu trennen sind. Leitbilder und Normalitätsvorstellungen sind als Handlungsorientierung konzipiert und daher keine utopischen Wunschvorstellungen, sondern an bereits bewährten oder zumindest vermeintlich bewährten Alltagserfahrungen orientiert und grundsätzlich realisierbar.

Offen lässt das Leitbildkonzept auch, ob das durch Leitbilder beeinflusste Handeln bzw. Verhalten bewusst oder unbewusst abläuft. Vorstellbar ist sowohl, dass sich Akteure aufgrund dessen, was sie für „normal“ halten, bewusst für eine bestimmte Handlungsoption entscheiden (etwa wenn eine Frau entscheidet, nach der Hochzeit den Nachnamen ihres Ehemannes anzunehmen), als auch, dass sie sich spontan und unreflektiert verhalten (etwa wenn ein Hotelangestellter die Partnerin eines Gastes, der sich als Herr „Müller“ ausweist, mit „Frau Müller“ anspricht). Vorstellbar sind zudem Mischformen wie zum Beispiel intuitive „Bauchentscheidungen“ oder Ad hoc-Entscheidungen unter Zeitdruck.

Komplementär zu den ,positiven“ Leitbildern im engeren Sinne (z. B. dem der „Guten Mutter") kann es auch „,negative Leitbilder“ geben (z. B. das der „Rabenmutter“), die als vermeidenswert und sozial unerwünscht wahrgenommen werden. Diese bieten indirekt ebenfalls Orientierung, indem Menschen versuchen, davon abzuweichen.

Leitbilder können Vorstellungen zu normalen Zuständen beinhalten: etwa zu einer „,normalen“ Kinderzahl. In dem Fall sprechen wir von strukturellen Leitbildern. Sie können aber auch Vorstellungen zu „,normalen“ Abläufen beinhalten: etwa zum ,normalen“ Alter von werdenden Eltern bei der Familiengründung. In dem Fall sprechen wir von prozessualen Leitbildern. Zu diesen gehören auch die sogenannten Life Scripts, die Janssen und Rubin (2011: 291) als ,culturally shared expectations about the timing of life events in an idealized life course" definieren. Wir gehen davon aus, dass Menschen beim Sich-Vorstellen von Normalität typischerweise in Bildern denken, weil es einfacher ist, komplexe Sachverhalte in visualisierter Form zu erfassen, in Erinnerung zu behalten und abzurufen. Für ihre Relevanz ist dieser Umstand jedoch nicht entscheidend.

\section{Charakteristika von Leitbildern}

Um die Interpretation der Daten bzw. die Hypothesenbildung zu unterstützen, sollen Leitbilder im Folgenden anhand von sechs Thesen genauer charakterisiert werden. Möglicherweise können Ihre Erfahrungen beim Bilden des Satzes („Es ist normal, dass ...“) diese Thesen plausibilisieren. 
These 1: Leitbilder sind Teil des Alltagswissens.

Es ist schwer, einen Satz mit „Es ist normal, dass...“ spontan zu formulieren. Meist müssen Menschen einen Moment darüber nachdenken, bis ihnen eine sinnvolle Formulierung einfällt. Normalitätsvorstellungen und Leitbilder sind demnach teilweise unbewusst und wenig reflektiert. Sie sind gewissermaßen so selbstverständlich, dass sie einem erst nach einigem Nachdenken bewusst werden. Das Wissen um die sozial konstruierten Wirklichkeiten, die in einer Gesellschaft geteilt und für so selbstverständlich wie Naturgesetze gehalten werden, wird in der Wissenssoziologie unter anderem als „Wissen in der Alltagswelt“ oder „Alltagswissen" bezeichnet (Berger/Luckmann 2001: 21f.). Es stiftet Orientierung und erleichtert die Interaktion mit anderen. Leitbilder sind integraler Bestandteil dieses Alltagswissens.

These 2: Leitbilder existieren für jeden Lebensbereich.

Mit einigem Nachdenken lassen sich „Es ist normal, dass...“-Sätze quasi zu allen Themen formulieren: zu Arbeit und Beruf, zu Schule und Bildung, zum Straßenverkehr - oder eben zum Familienleben. Demnach gibt es auch Leitbilder zu allen Lebensbereichen, oder zumindest zu allen, die im Alltag der Menschen in einer Gesellschaft eine Rolle spielen. Das macht insofern Sinn, als es in jeder Situation, die Menschen wiederholt erleben, möglich ist, auf Erfahrungen zurückzugreifen und Vorstellungen des Normalen zu konstruieren. Auf diese Weise können sich Menschen in solchen Situationen routiniert verhalten und die energie- und zeitaufwändige bewusste Reflexion auf neue Situationen beschränken.

These 3: Zu jedem Lebensbereich gibt es ein System aufeinander bezogener Leitbilder.

Die ersten „Es ist normal, dass...“-Sätze, die einem einfallen, werden sich typischerweise auf das gleiche Thema bzw. auf ähnliche Themen beziehen, denn oft lassen sich, nachdem man einen ersten Satz formuliert hat, sehr schnell mehrere andere Sätze zum gleichen Thema ergänzen: Es ist normal, dass ein Paar nach einigen Jahren zusammenzieht,... dass es viel Freizeit miteinander verbringt,... dass es gemeinsam in Urlaub fährt,... dass die Partner einander treu sind,... etc. Dieser Effekt spiegelt den Umstand wider, dass es zu jedem Themenbereich zahlreiche Vorstellungen gibt, die typischerweise miteinander assoziiert werden. Oft sind sie auch logisch miteinander verknüpft. Gemeinsam ergeben sie ein Leitbild, das als ganzheitliches komplexes Konzept erlernt und abgerufen wird.

These 4: Leitbilder sind hierarchisch strukturiert.

Dabei ist nicht unbedingt eindeutig bestimmbar, wo ein Themenbereich endet und ein anderer anfängt: Man kann Elternschaft und die Frage, wie sie normalerweise organisiert wird, als einen Themenbereich auffassen, zu dem es ein Leitbild gibt (Abbildung 1). Man kann Vaterschaft und Mutterschaft als zwei getrennte Themenbereiche mit je einem eigenen Leitbild auffassen. In gewisser Weise ist jedoch auch die Gestaltung von Familienleben ein übergeordnetes Leitbild, zu dem Elternschaft nur einen Teilaspekt darstellt.

Diese hierarchische Struktur verschiedener familienbezogener Leitbilder ist in Abbildung 1 exemplarisch dargestellt; weitere hier nicht aufgeführte Leitbilder und Ausdifferenzierungen sind denkbar. Auf diese Weise sind Leitbilder ineinander verschachtelt und miteinander assoziiert: Innerhalb jedes Leitbildes mit aufeinander bezogenen Vorstellungen finden sich typischerweise Teilaspekte, zu denen immer noch zahlreiche Vorstellungen existieren. Diese sind jeweils untereinander stärker verbunden als mit anderen Vorstellungen innerhalb des übergeordneten Leitbildes. Auch strukturelle und prozessuale Leitbilder (Diabaté/Lück 2014) hängen miteinander zusammen, sie bedingen einander gegenseitig und lassen gemeinsam nicht nur das übergeordnete Leitbild einer „normalen“ Familie, sondern auch das einer ,normalen“ Familienbiografie entstehen, also eine logische Abfolge familienbiografischer Phasen und Ereignisse. 
Abbildung 1: Hierarchische Struktur von Familienleitbildern

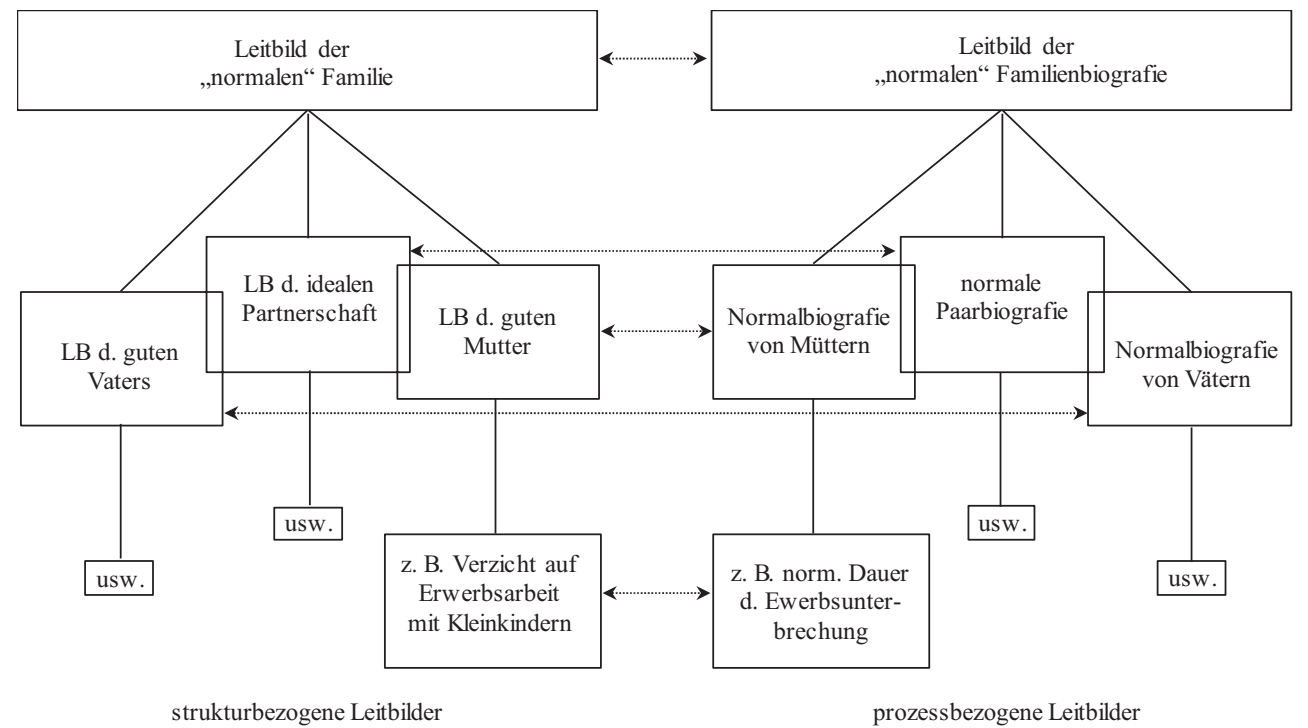

Quelle: Diabaté/Lück 2014: 58, eigene schematische Darstellung

Anm.: LB=Leitbild.

\section{These 5: Leitbilder sind sozial konstruiert und daher kulturspezifisch. Sie unterliegen sozialem Wandel und sind Gegenstand sozialer Konflikte.}

Zu manchen „Es ist normal, dass..."-Sätzen, die einem in den Sinn kommen, kommen einem im zweiten Augenblick auch Bedenken, ob man mit ihnen nicht bei anderen auf Kritik stoßen würde. Ist es zum Beispiel wirklich normal, dass die Mutter den größeren Teil der Kinderbetreuung übernimmt? Und selbst die Sätze, bei denen einem keine Bedenken kommen, erweisen sich, wenn man ein wenig darüber nachdenkt, meist als willkürliche Setzungen. Eine Gegenposition ist fast immer vorstellbar. Viele Sätze sind bestenfalls für unsere Gesellschaft und unsere Zeit unstrittig, möglicherweise aber auch nur für das eigene soziale Umfeld.

Hier zeigt sich, dass Leitbilder eben keine objektive Wirklichkeit im Sinne eines physikalischen Gesetzes darstellen, sondern eine soziale Konstruktion visualisieren. Sie werden vor dem Hintergrund von Alltagserfahrungen durch öffentliche Diskussionen, durch die Gestaltung institutioneller Rahmenbedingungen (z. B. Gesetzesänderungen) und durch die Interaktionen zwischen Menschen produziert und immer wieder reproduziert, so wie dies in zahlreichen interaktionistischen und konstruktivistischen Theorien beschrieben wird (Schier/Jurczyk 2007; West/Fenstermaker 1995; Berger/Kellner 1965). Daraus ergeben sich weitere Charakteristika:

- Erstens kann es, trotz einer überraschenden Stabilität vieler sozialer Konstruktionen, im Zuge ihrer Reproduktion zu sozialem Wandel und zur Veränderung von Leitbildern kommen: Was heute normal erscheint, war es zu einem anderen Zeitpunkt nicht. Das gilt beispielsweise für den Umstand, dass heute viele Paare ohne Trauschein zusammenleben, was noch Anfang der 1970er Jahre undenkbar schien und durch den „Kuppelei-Paragrafen“ verboten war. Das Beispiel macht deutlich, dass verschiedene Akteure zur Veränderung von Leitbildern beitragen. In diesem Fall hat eine 
Gesetzesänderung eine entscheidende Rolle gespielt. Es ist aber davon auszugehen, dass sowohl vor als auch nach der rechtlichen Änderung Individuen bzw. Paare teils durch ihr Verhalten, teils durch ihre (ablehnende, gleichgültige oder positive) Reaktion darauf die Vorstellungen von Normalität in dieser Hinsicht geprägt haben.

- Zweitens führt der Umstand, dass Leitbilder sozial konstruiert sind, dazu, dass sie auch kulturspezifisch sind: Die Vorstellungen der Menschen in Deutschland oder Mitteleuropa sind andere als die der Menschen in Südostasien oder Westafrika.

- Drittens zeigen sich bei genauer Betrachtung selbst zwischen verschiedenen Generationen, Regionen, sozialen Milieus und sozialen Lagen innerhalb einer Gesellschaft Unterschiede. Man denke etwa an die Frage nach der Normalität lesbischer und schwuler Partnerschaften.

- Viertens können - auch über sozialstrukturelle Grenzen hinweg - in einer Gesellschaft zu einem Thema zwei oder mehrere einander widersprechende Leitbilder existieren, an denen sich dann vermutlich soziale Konflikte festmachen.

- Der Wandel von Leitbildern kann fünftens auch auf individueller Ebene im Lebenslauf stattfinden, beispielsweise als Anpassung an biografische Ereignisse wie Scheidung oder Elternschaft.

- Sechstens muss unterstellt werden, dass auch auf individueller Ebene innere Konflikte auftreten können, weil eine Person in ihrem sozialen Umfeld mit unterschiedlichen, konkurrierenden Leitbildern konfrontiert wird, diese gleichermaßen verinnerlicht und sich selbst nicht eindeutig $\mathrm{zu}$ positionieren vermag. Allerdings muss auch angenommen werden, dass Menschen darum bemüht sind, solche inneren Konflikte im Sinne kognitiver Dissonanzen (Festinger 1957) aufzulösen.

\section{These 6: Leitbilder werden durch Prozesse der Institutionalisierung und der Objektivierung stabilisiert und verstetigt.}

Der oben angeführte „Kuppelei-Paragraf“ ist gleichzeitig ein Beispiel dafür, dass sich ein Leitbild in Form von Recht institutionalisieren kann. Institutionalisiert ist ein Leitbild immer, und zwar bereits dadurch, dass es „Allgemeingut“ und „für alle Mitglieder der jeweiligen gesellschaftlichen Gruppe erreichbar" (Berger/Luckmann 1966: 58) ist. Ein Gesetz ist jedoch ein Beispiel für eine vergleichsweise starre Form, die eine solche Institutionalisierung annehmen kann (ebd.). Neben dem „Kuppelei-Paragrafen“ wären aktuell unter anderem die Regelungen zum Betreuungsgeld und zum Elterngeld anzuführen, denen im einen Fall die Vorstellung zugrunde liegt, dass ein Kind bis zu drei Jahren zuhause betreut wird, und im anderen Fall die Vorstellung, dass beide Eltern nach einem Jahr wieder in den Beruf zurückkehren.

Neben einer solchen Manifestation in geltendem Recht kann es auch zu Objektivierungen von Leitbildern kommen: zu Manifestationen in Form von physischen Gegenständen oder Dingen. Beispiele für solche „zu Objekten gewordene[n] Sedimente“ (ebd.: 73) könnten zum Beispiel Anzahl und Größe der vorhandenen Einrichtungen zur öffentlichen Kinderbetreuung sein.

Solche Arten der Verstetigung - der Institutionalisierung in Form von Recht oder der Objektivierung in Form einer faktisch vorhandenen Infrastruktur - repräsentieren ihrerseits die Existenz und Gültigkeit der ihnen zugrundeliegenden kulturellen Leitbilder. Auf diese Weise werden Leitbilder vergleichsweise resistent gegenüber sozialem Wandel: Sie verändern sich schwerfälliger und langsamer, als es der Fall wäre, wenn sie nur durch die tägliche öffentliche Kommunikation und Interaktion zwischen Menschen reproduziert würden. Sie unterliegen zwar, wie These 5 besagt, sozialem Wandel, sie wandeln sich aber typischerweise nicht im Laufe einiger Wochen oder Monate, sondern eher über Jahrzehnte hinweg. 


\section{$4 \quad$ Individuelle Leitbilder, gesellschaftliche Leitbilder und Handlungsrelevanz}

Wie alle kulturellen Phänomene haben auch Leitbilder eine Doppelnatur zwischen individuellem und gesellschaftlichem Phänomen: Einerseits besitzen nur Menschen (und nicht Kollektive) die Fähigkeit, sich etwas vorzustellen. Ungeachtet dessen, ob Vorstellungen von mehreren Menschen geteilt werden, sind es zunächst immer (einzelne) Menschen, die sie verinnerlicht haben. Entsprechend kann man jedem Individuum ein individuelles Leitbild zuschreiben. In diesem Sinne sind Leitbilder ein Mikrophänomen.

Andererseits ist es für viele grundlegende Vorstellungen von unserem Leben typisch, dass wir sie mit den meisten unserer Mitmenschen teilen. Das ist notwendig, um Kommunikation und Interaktion mit Mitmenschen und das Zusammenleben in einer Gesellschaft zu ermöglichen. Entsprechend werden Leitbilder innerhalb einer bestimmten Gesellschaft durch Sozialisation und kollektive Konstruktionsprozesse relativ stark homogenisiert. Das macht sie zu einem kulturellen Phänomen. Leitbilder können charakteristisch sein für eine bestimmte Gesellschaft oder für ein bestimmtes soziales Milieu, für verschiedene Generationen oder auch Regionen. In diesem Sinne sind Leitbilder ein Meso- oder auch Makrophänomen.

Im Kern des Interesses der Leitbildforschung stehen kulturelle Leitbilder: also Bündel von Normalitätsvorstellungen, die innerhalb einer bestimmten sozialen Gruppe oder einer Gesellschaft intersubjektiv geteilt werden. Diese manifestieren sich auf unterschiedliche Weise. Sie spiegeln sich beispielsweise in der Gesetzgebung (z. B. im Ehegattensplitting oder im Elterngeld), in der Architektur (z. B. in der Größe von Kinderzimmern), in der Literatur, in anderen Kunstformen oder in der Werbung wider. Dabei ist ein kulturelles Leitbild mehr als ein statistisches Mittel (oder der Modus) aller individueller Leitbilder des entsprechenden Kollektivs. Zwar gibt auch die statistische Aggregation individueller Leitbilder Rückschlüsse auf das kollektive, kulturelle Leitbild. Doch letzteres wird nicht auf demokratischem Wege gebildet, sondern in öffentlicher Kommunikation und Interaktion, wobei sich nicht notwendigerweise die Vorstellung der Mehrheit durchsetzt (NoelleNeumann 1980). Unter anderem haben Massenmedien und deren Entscheidungsträger eine große Deutungs- und Definitionsmacht, aber auch alle in der Öffentlichkeit stehenden Personen sowie Menschen in hoher sozialer Position.

Zudem gewinnt ein kulturelles Leitbild durch Institutionalisierungsprozesse (etwa durch seine Manifestation in Form von Recht) zusätzlich ein Eigenleben - eine Realität „,sui generis“, wie Durkheim (1984: 109) formuliert. So kann ein kulturelles Leitbild fortbestehen und nachwirken, auch wenn sich alle individuellen Leitbilder der Gesellschaftsmitglieder bereits weiterentwickelt haben. Auch kann durch unter- oder überproportionale Medienpräsenz der Eindruck entstehen, dass hinter einem solchen kulturellen Leitbild ein kleinerer oder größerer Teil der Gesellschaft stünde, als es tatsächlich der Fall ist. Insofern gibt es neben der statistischen Aggregation individueller Leitbilder andere Manifestationen, die möglicherweise bessere oder zumindest ähnlich gute Rückschlüsse erlauben: beispielsweise die Interviewfrage im Leitbildsurvey, welche Vorstellungen (der Wahrnehmung des Befragten zufolge) die Mehrheit der Menschen in der Gesellschaft zu einem bestimmten Thema habe.

Zwischen individuellen und kulturellen Leitbildern müssen wechselseitige Einflüsse unterstellt werden: Einerseits bringen Individuen ihre persönlichen Leitbilder mit in die Kommunikationen und Interaktionen mit ihren Mitmenschen ein, aus denen kulturelle Leitbilder hervorgehen. Insofern beeinflussen individuelle Leitbilder in ihrer Summe den Konstruktionsprozess auf gesellschaftlicher Ebene. Andererseits prägen kulturelle Leitbilder die individuellen Leitbilder auf dem Wege der Sozialisation. 
Daraus ergeben sich (mindestens) zwei Wege der Einflussnahme kultureller Leitbilder auf Handeln bzw. auf Verhalten (vgl. Abbildung 2): Zum einen prägen sie, wie eben ausgeführt, die individuellen Leitbilder der Akteure auf dem Wege der Sozialisation. Diese schlagen sich im Handeln des Akteurs nieder, weil dieser seine persönlichen Überzeugungen in die Realität umsetzen will und weil er es häufig in einer unreflektierten, routinierten und automatisierten Weise tut, ähnlich wie es die Theorie der Frame-Selektion unterstellt (Esser 1990). Diese zweistufige Einflussnahme auf das Handeln wird über die individuellen Leitbilder des Akteurs vermittelt.

Abbildung 2: Modell zur Erklärung familialen Verhaltens

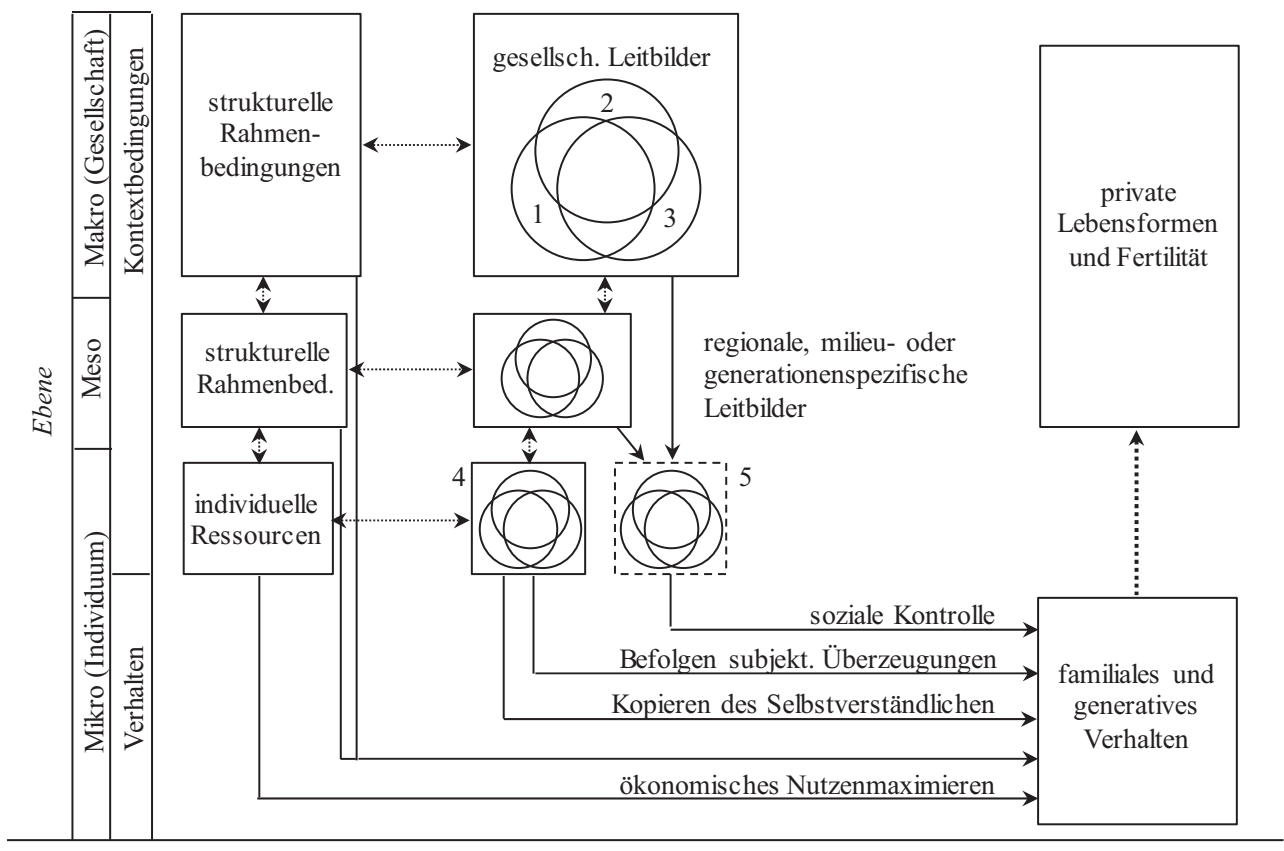

1) als selbstverständlich empfunden, 2) subjektiv gewünscht, 3) sozial erwünscht

4) individuelle Leitbilder, 5) subjektive Wahrnehmung kollektiver Leitbilder

Quelle: Diabaté/Lück 2014: 60, eigene schematische Darstellung

Zum anderen prägen sie das Handeln der Akteure - etwas unmittelbarer - dadurch, dass diese den (mutmaßlich) sozial erwünschten Vorstellungen entsprechen wollen, um negative Sanktionen zu vermeiden. Dieser Einfluss wird lediglich durch die subjektive Wahrnehmung des Akteurs von den mutmaßlichen kulturellen Leitbildern vermittelt.

Selbstverständlich stellen Leitbilder nicht den einzigen Einfluss auf Handeln und Verhalten dar - wahrscheinlich auch häufig nicht den maßgeblichsten. Vielmehr wirken sie parallel zu anderen, insbesondere strukturellen Rahmenbedingungen, die etwa für rationales nutzenmaximierendes Handeln entscheidende Ressourcen, Barrieren, Nutzen oder Kosten ausmachen. Allerdings wird hier unterstellt, dass der Einfluss der Leitbilder auf das Handeln nicht vernachlässigt werden darf, vor allem nicht für eine adäquate Erklärung familialen Verhaltens, d. h. hinsichtlich der privaten Lebensführung und der Entscheidung für eine Familiengründung oder -erweiterung. 


\section{$5 \quad$ Relevanz von Leitbildern im Kontext von Familie}

Wir nehmen an, dass es zu jedem Lebensbereich umfassende Leitbilder gibt, die Orientierungspunkte für menschliches Verhalten bieten. Insbesondere für das Familienleben ist diese Annahme plausibel; denn gerade das Zusammenleben in Partnerschaften, die Familienplanung und der Umgang mit Kindern sind Situationen, die sehr viele Menschen seit Generationen immer wieder auf ähnliche Weise erleben. Gerade diese Themen eignen sich also dafür, Vorstellungen einer Normalität zu entwickeln und zu pflegen. Gleichzeitig sind Menschen möglicherweise explizit in diesen Situationen auf Leitbilder angewiesen: Denn zum einen ist die private Lebensführung viel zu allgegenwärtig und zu facettenreich, als dass jedes Handeln stets bewusst rational entschieden werden könnte. Und zum zweiten verfügen Menschen gerade in familialen Fragen (,Ist er der ,Richtige“?“ „Werden wir mit einem Kind glücklicher sein?"“ etc.) nicht über die nötige Information, um eine rationale Entscheidung treffen zu können.

Tatsächlich haben Leitbilder bei der Erklärung von privater Lebensführung und von generativem Verhalten in der empirischen Forschung bisher eine sehr randständige Rolle gespielt. Stattdessen dominieren zuletzt ökonomische Ansätze - die allerdings die Realität nicht vollständig plausibel erklären können (vgl. ausführlicher Diabaté/Lück 2014). Beispielsweise ist die Fürsorgearbeit - ob für Kinder, für Kranke oder für ältere Menschen, ob privat oder beruflich - trotz der Bildungsgewinne der Frauen immer noch stark weiblich konnotiert. Das führt dazu, dass wider alle ökonomische Rationalität Männer durch die Familiengründung kaum Opportunitätskosten zu befürchten haben und dass Mütter sie pauschal in Kauf nehmen (Wengler et al. 2009; Lück 2009; Schmitt/Winkelmann 2005). Gerade die immer noch omnipräsenten Zuschreibungen dessen, was Frauen und Männer bzw. Mütter und Väter innerhalb der Familie für Kompetenzen haben und welche spezifische Verantwortung ihnen zukommt, zeigt, wie resistent kulturelle Normalitätsvorstellungen gegenüber anderen gesellschaftlichen Entwicklungen und wie einflussreich sie in Bezug auf das Verhalten im Familienkontext sein können. Daher erscheint es geboten, Kultur auch für die Erklärung familialen Verhaltens stärker als bislang in den Blick zu nehmen.

Dieses Anliegen ist grundsätzlich nicht neu, und die Entwicklung einer stärker kulturell unterfütterten Perspektive hat Tradition (vgl. Überblick bei Diabaté/Lück 2014: 51f.). Auch gibt es Beispiele für Studien, die kulturelle Deutungen erfolgreich belegen oder plausibel machen (Pfau-Effinger 1996, 2004; van Oorschot et al. 2008; Billari et al. 2009). Dennoch ist diese Perspektive in der empirischen Forschung heute unterrepräsentiert. Das könnte daran liegen, dass kulturelle Phänomene theoretisch schwerer zu fassen und methodisch schwieriger zu operationalisieren sind als die Rahmenbedingungen, mit denen ökonomische Erklärungen und Hypothesen argumentieren. Dieser Umstand soll hier als Herausforderung verstanden werden, den theoretischen Blick auf kulturelle Phänomene und ihren Einfluss auf familiales Verhalten zu schärfen und bestehende Konzepte weiterzuentwickeln.

\section{Literatur}

Ajzen, Icek (1985): From Intensions to Actions: A Theory of Planned Behavior. In: Kuhl, Julius; Beckmann, Jürgen (Hrsg.): Action Control: From Cognition to Behavior. Heidelberg: Springer, S. 11-39.

Berger, Peter L.; Luckmann, Thomas (2001, orig. 1966): Die gesellschaftliche Konstruktion der Wirklichkeit. Eine Theorie der Wissenssoziologie. Frankfurt a. M.: Fischer. 
Berger, Peter L.; Kellner, Hansfried (1965): Die Ehe und die Konstruktion der Wirklichkeit. Eine Abhandlung zur Mikrosoziologie des Wissens. In: Soziale Welt 16, 3, S. 220-235.

Billari, Francesco C.; Philipov, Dimiter; Testa, Maria R. (2009): Attitudes, Norms and Perceived Behavioural Control: Explaining Fertiltiy Intentions in Bulgaria. In: European Journal of Population/Revue européenne de Démographie 25, 4, S. 439-465.

Busch, Friedrich W.; Scholz, Wolf-Dieter (2006): Familienvorstellungen zwischen Fortschrittlichkeit und Beharrung. Ergebnisse einer empirischen Untersuchung von Ehe- und Familienvorstellungen Jugendlicher im internationalen Vergleich. Würzburg: Ergon Verlag.

Dahrendorf, Ralf (1977, orig. 1958): Homo Sociologicus. Ein Versuch zur Geschichte und Kritik der Kategorie der sozialen Rolle. Opladen: Westdeutscher Verlag.

Diabaté, Sabine; Lück, Detlev (2014): Familienleitbilder - Identifikation und Wirkungsweise auf generatives Verhalten. In: Zeitschrift für Familienforschung 26, 1, S. 49-69.

Durkheim, Émile (1984): Die Regeln der soziologischen Methode. Frankfurt a. M.: Suhrkamp.

Esser, Hartmut (1990): „Habits“, „Frames“ und „Rational Choice“. In: Zeitschrift für Soziologie, 4, S. 231-247.

Esser, Harmut (1991): Die Rationalität des Alltagshandelns. Eine Rekonstruktion der Handlungstheorie von Alfred Schütz. Zeitschrift für Soziologie, 20, 6, S. 430-445.

Esser, Harmut (2002): Ehekrisen: Das (Re-)Framing der Ehe und der Anstieg der Scheidungsraten. Zeitschrift für Soziologie, 31, 6, S. 472-496.

Festinger, Leon (1957): A Theory of Cognitive Dissonance. Stanford, California: Stanford University Press.

Giesel, Katharina D. (2007): Leitbilder in den Sozialwissenschaften. Wiesbaden: VS Verlag für Sozialwissenschaften.

Heut, Michael (2004): Familienleitbilder. Die sozialethische Dimension des Leitbildes für die Institution Familie. Hamburg: Verlag Dr. Kovac.

Holetschek, Klaus; Müller, Gerd; Ruck, Christian; Ferber, Markus (Hrsg.) (2000): Die neue Familie: Familienleitbilder - Familienrealitäten. München: Hanns-Seidel-Stiftung.

Janssen, Steve M. J.; Rubin, David C. (2011): Age Effects in Cultural Life Scripts. In: Applied Cognitive Psychology 25, S. 291-298.

Lück, Detlev (2009): Der zögernde Abschied vom Patriarchat. Der Wandel von Geschlechterrollen im internationalen Vergleich. Berlin: edition sigma.

Meyer, Birgit (1990): Frauenpolitiken und Frauenleitbilder der Parteien in der Bundesrepublik. In: Aus Politik und Zeitgeschichte 40, 34/35, S. 16-28.

Noelle-Neumann, Elisabeth (1980): Die Schweigespirale. Öffentliche Meinung - unsere soziale Haut. München: Langen Müller.

Pfau-Effinger, Birgit (1996): Analyse internationaler Differenzen in der Erwerbsbeteiligung von Frauen. Theoretischer Rahmen und empirische Ergebnisse. In: Kölner Zeitschrift für Soziologie und Sozialpsychologie, 48, 3, S. 462-492.

Pfau-Effinger, Birgit (2004): Socio-historical paths of the male breadwinner model - an explanation of cross-national differences. In: The British Journal of Sociology 55, 3, S. 377-399.

Schier, Michaela; Jurczyk, Karin (2007): „Familie als Herstellungsleistung“ in Zeiten der Entgrenzung. In: Aus Politik und Zeitgeschichte, 34, S. 10-17.

Schmitt, Christian; Winkelmann, Ulrike (2005): Wer bleibt kinderlos? Sozialstrukturelle Daten zur Kinderlosigkeit von Frauen und Männern. DIW Discussion Papers Nr. 473.

van Oorschot, Wim; Opielka, Michael; Pfau-Effinger, Birgit (Hrsg.) (2008): Culture and Welfare State. Values of Social Policy from a Comparative Perspective. Cheltenham, GB \& Northampton, USA: Edward Elgar.

Vaskovics, Laszlo A. (Hrsg.) (1997): Familienleitbilder und Familienrealitäten. Opladen: Leske + Budrich.

Wengler, Annelene; Trappe, Heike; Schmitt, Christian (2009): Alles wie gehabt? Zur Aufteilung von Hausarbeit und Elternaufgaben in Partnerschaften. In: Zeitschrift für Bevölkerungswissenschaft, 34, 1-2, S. 57-78.

West, Candace; Fenstermaker, Sarah (1995): Doing Difference. In: Gender \& Society, 9, S. 8-37. 


\title{
Zur Messung von Familienleitbildern: Studiendesign und Operationalisierung
}

\author{
Detlev Lück, Robert Naderi \& Kerstin Ruckdeschel*
}

\section{Zusammenfassung}

Mit der Studie Familienleitbilder (FLB) 2012 wurden persönliche und gesellschaftliche Leitbilder standardisiert erfasst. Dafür wurden neue Items konstruiert, die individuelle Vorstellungen davon messen, wie Familienleben normalerweise oder idealerweise aussieht, und die sich zu Leitbildern aggregieren lassen. Zusätzlich wurde ein Zugang geschaffen, um gesellschaftliche Leitbilder standardisiert zu erfassen. Dazu wurden die einzelnen Befragten aufgefordert, einzuschätzen, wie „die Allgemeinheit“ die Items beantworten würde, die sie zuvor persönlich beantwortet hatten. Die meisten Items auf persönlicher Ebene wurden in diesem Sinne für die gesellschaftliche Ebene ,gespiegelt“. Die Qualität der Daten wurde durch verschiedene Kontrollmaßnahmen sowie durch Vor- und Nachuntersuchungen sichergestellt. Das Konzept hat sich dabei als valide erwiesen.

\section{$1 \quad$ Einleitung}

Zwischen dem Vorsatz, Leitbildforschung zu betreiben, und seiner Umsetzung, steht die Herausforderung, Leitbilder empirisch zu erfassen und geeignete Daten zu generieren. Diese Aufgabe ist ebenso grundlegend wie anspruchsvoll, zumal es für Leitbilder, trotz einer verbreiteten Einstellungsforschung, deren Indikatoren unter anderem auch als Leitbilder interpretiert werden (z. B. Busch/Scholz 2005), keine etablierten Daten oder Messinstrumente gibt. Angesichts der theoretischen Überlegungen, wie das Konzept der Leitbilder von anderen kulturell-normativen Konzepten abzugrenzen sei (vgl. Lück/Diabaté, Kap. 2), erscheint es angemessen, auch methodisch eine Abgrenzung vorzunehmen. Dabei kann es ebenso wenig wie auf theoretischer Ebene darum gehen, Überschneidungen zu anderen Konzepten zu vermeiden. Jedoch muss die Operationalisierung vor dem Hintergrund der theoretischen Konzeption konsistent und begründbar sein.

Die Datengrundlage für die empirischen Beiträge in diesem Sammelband liefert die Studie Familienleitbilder (FLB) 2012 (Lück et al. 2013). Für diese wurden eine Befragungstechnik sowie viele konkrete Indikatoren neu entwickelt. Studie, Datensatz sowie die Operationalisierung von Leitbildern und anderen zentralen Konzepten, die für den gesamten Sammelband grundlegend sind, sollen im Folgenden vorgestellt werden. Darüber hinaus geht der Beitrag auf methodische Fragen ein, die sich bei der Analyse und Interpretation der Daten stellen, wobei sich auch hier die Relevanz auf den gesamten Sammelband bezieht.

\footnotetext{
* Wir danken der anonymen Gutachterin bzw. dem anonymen Gutachter für wertvolle Hinweise und konstruktive Kritik.
} 


\section{Die Studie „Familienleitbilder“ (FLB) 2012}

Die Studie Familienleitbilder (FLB) $2012^{1}$ ist eine repräsentative Befragung der deutschen Wohnbevölkerung im Alter von 20 bis 39 Jahren aus dem Jahr 2012, die eigens dafür entwickelt wurde, Leitbilder zum Familienleben zu erfassen. Durchgeführt wurde sie von TNS Infratest Sozialforschung im Auftrag des Bundesinstituts für Bevölkerungsforschung (BiB) in Wiesbaden.

Die realisierte Nettostichprobe umfasst exakt 5.000 Personen, die zwischen dem 27. August und dem 15. November 2012 mittels CATI-Verfahren ${ }^{2}$ telefonisch befragt wurden. Angesichts eines mittlerweile (gerade in jüngeren Altersgruppen) nicht mehr zu vernachlässigenden Anteils der Bevölkerung, der über keinen Festnetzanschluss verfügt, folgte die Stichprobenziehung dem Dual-Frame-Ansatz (Gabler/Ayhan 2007). Dieser bezieht in die randomisierte Auswahl von Telefonnummern ein bestimmtes Kontingent von Mobilfunknummern mit ein und stellt die repräsentative Verteilung hinsichtlich der Verfügbarkeit von Festnetz- und Mobilfunkanschlüssen über ein Designgewicht her. Innerhalb eines über Festnetztelefon kontaktierten Haushaltes erfolgte die Randomisierung der Kontaktperson mittels „Schwedenschlüssel“, also mittels einer computerbasierten Zufallsauswahl. Die Nettostichprobe des FLB 2012 setzt sich aus $\mathrm{N}_{1}=4.596$ Befragten auf Basis einer Festnetzstichprobe und $\mathrm{N}_{2}=404$ sogenannten „Mobile Only“-Zielpersonen zusammen, die nur über ein Mobilfunktelefon zu erreichen sind. Die Ausschöpfungsquote in der Festnetzstichprobe betrug 41,1 \%, die innerhalb der „Mobile Only“-Zielpersonen 56,5 \%. Es wurden 157 Interviewerinnen und Interviewer eingesetzt und intensiv geschult. Ein Interview dauerte im Mittel 32 Minuten.

Untersuchungsgegenstand der Studie sind Leitbilder im Kontext des Familienlebens. $\mathrm{Zu}$ neun Themenblöcken wurden die Befragten sowohl hinsichtlich ihrer eigenen Vorstellungen befragt als auch zu den Vorstellungen, die in ihren Augen in der Allgemeinheit verbreitet sind. Die Themenblöcke sind überschrieben mit

1. „Partnerschaft",

2. „Bedeutung von Familie“,

3. „Familiengründung und Familienerweiterung“,

4. „Kinderlosigkeit",

5. „Elternschaft“,

6. „Kinderreichtum“,

7. "Bedingungen für Elternschaft",

8. "Geschwisterkinder" und

9. „Eltern-Kind-Beziehung“.

Der letzte Themenblock enthält die Unterkapitel „Verantwortete Elternschaft“, „Mutterleitbilder“, „Vaterleitbilder“ und „Intensität des Eltern-Kind-Kontaktes“. Zusätzlich werden Informationen zur Verbreitung von Elternschaft bzw. Kinderlosigkeit im sozialen Umfeld der Befragten sowie zahlreiche „Standardmerkmale“ zur familialen, sozio-demografischen und sozio-ökonomischen Lage erfasst.

Für die Analyse der Daten wurde eine Gewichtungsvariable erstellt. Sie nimmt sowohl die Designgewichtung im Rahmen des Dual-Frame-Ansatzes als auch eine praxisübliche

Eine ausführlichere Beschreibung findet sich im Methodenbericht der Studie (Lück et al. 2013).

CATI steht für „computer-assisted telephone interview“, also für computergestützte telefonische Interviews. 
Redressmentgewichtung vor, die Verzerrungen aufgrund von systematischen Antwortverweigerungen korrigiert, indem sie die Stichprobenverteilungen an Eckdaten der amtlichen Statistik anpasst. In der Redressmentgewichtung wurden die Randverteilungen bezüglich Alter, Geschlecht, Familienstand, Bildung sowie die regionale Verteilung nach Bundesland und BIK-Gemeindetypen berücksichtigt (siehe Lück et al. 2013).

\section{$3 \quad$ Operationalisierung und Messung}

Zentraler Bestandteil des Erhebungsinstrumentes sind Item-Batterien zur Messung von Leitbildern und Items zur Messung von Normalitätsvorstellungen. Diese sind inhaltlich überwiegend neu entwickelt worden. Eine Besonderheit liegt in der Befragungstechnik anhand von zwei Ebenen: Zum einen wurden die Vorstellungen der Befragten selbst erfasst (persönliche Ebene), zum anderen die mutmaßlich in der Gesellschaft verbreiteten Vorstellungen, soweit sie der Befragte einschätzen kann (gesellschaftliche Ebene bzw. Ebene der Allgemeinheit). Die Operationalisierung des Leitbild-Konzeptes im FLB 2012 wird im Folgenden ausführlicher dargestellt (3.1). Darüber hinaus wird die Messung von Leitbildern (3.2) und von zentralen sozio-ökonomischen Standardmerkmalen (3.3) erläutert.

\subsection{Operationalisierung von persönlichen und gesellschaftlichen Vorstellungen zur Familie}

Leitbilder werden hier verstanden als „Bündel aus kollektiv geteilten bildhaften Vorstellungen des ,Normalen', das heißt von etwas Erstrebenswertem, sozial Erwünschtem und/oder mutmaßlich weit Verbreitetem, also Selbstverständlichem“ (Diabaté/Lück 2014: 56; vgl. auch Lück/Diabaté, Kap. 2). Anlehnend an diese zugrunde gelegte Definition erfolgt die empirische Messung von Familienleitbildern in (mindestens) zwei Schritten: in der Operationalisierung von Vorstellungen des „Normalen“ durch den FLB 2012, also durch die im Fragebogen enthaltenen Items, und in der anschließenden Operationalisierung von Leitbildern als „Bündel“ solcher Vorstellungen auf Basis des FLB 2012, das heißt durch eine Aufbereitung der fertig erhobenen Daten mittels multivariater statistischer Verfahren.

Vorstellungen des „Normalen“ sind im FLB 2012 überwiegend als Items formuliert, die eine Aussage beinhalten. Im Regelfall kann der Befragte diesen Aussagen jeweils auf einer vierstufigen Skala zustimmen bzw. sie ablehnen (,stimme voll und ganz zu“, „stimme eher zu“, ,stimme eher nicht zu“, ,stimme überhaupt nicht zu“), wobei bewusst auf Mittelkategorien wie „weder noch“" verzichtet wurde, um ausweichende Antworten zu vermeiden. ${ }^{3}$

Viele der Aussagen sind normativer Art und lassen sich unter anderen theoretischen Vorzeichen als Einstellungen interpretieren (z. B. „Es ist schlecht, wenn ein Kind als Einzelkind aufwächst“ oder „Wenn man als Paar dauerhaft zusammenlebt, sollte man heiraten"). Dies ist kein Widerspruch zur Theorie, zumal auch Einstellungen bzw. Vorstellungen „von etwas Erstrebenswertem“ (vgl. die Definition oben) als Normalitätsvorstellungen und somit als mögliche Bestandteile von Leitbildern gedeutet werden. Allerdings beinhalten Leitbilder auch Vorstellungen „,von etwas ... mutmaßlich weit Verbreite-

3 Abweichungen von diesem Frageschema werden in den empirischen Beiträgen in diesem Band dargestellt, die die entsprechenden Items verwenden. 
tem, also Selbstverständlichem“ (vgl. oben). Daher enthalten manche Items auch Aussagen empirischer Art (z. B. „Eine Familie ist ab ... Kindern kinderreich“). Häufig sind empirische Aussage und normative Bewertung im Sinne des Erstrebenswerten und des sozial Erwünschten bewusst vermischt, zumal sich auch im theoretischen Konzept des Leitbildes beides vermischt (z. B. „Eltern können bei der Erziehung vieles falsch machen, daher müssen sie sich gut informieren").

Ein Schwerpunkt der Studie ist auf kollektive kulturelle Leitbilder gerichtet. Diese lassen sich annäherungsweise durch die Aggregation individueller Haltungen messen (vgl. den nachfolgenden Abschnitt). Doch es ist davon auszugehen, dass kulturelle Leitbilder dadurch nicht hinreichend beschrieben sind: Erstens müssen bereits ihre soziale Konstruktion und Reproduktion nicht die Vorstellungen einer statistischen Bevölkerungsmehrheit abbilden, und zweitens kann ein kulturelles Leitbild durch Prozesse der Institutionalisierung (z. B. in Form von Recht) und der Objektivierung (z. B. in Form einer faktisch ausgebauten Infrastruktur zur Kinderbetreuung) eine Stabilität erhalten, die es die individuellen Vorstellungen in der Bevölkerung (und deren statistische Mehrheit) überdauern und von diesen abweichen lässt (vgl. Lück/Diabaté, Kap. 2). ${ }^{4}$ Aus diesem Grund wurde im FLB 2012 ein zweiter Zugang angelegt, um kulturelle Leitbilder zu messen: Er besteht darin, Befragte nicht (nur) nach ihrer eigenen Vorstellung, sondern (auch) nach der Haltung zu befragen, die - ihrer Wahrnehmung zufolge - in der Allgemeinheit verbreitet ist. Die Abbildung des kulturellen Leitbildes bleibt so zwar durch die subjektive Wahrnehmung der Befragten gefiltert, wird aber unmittelbarer erfasst und muss nicht erst durch die Aggregation aller Antworten im Datensatz rekonstruiert werden. Unterschiedliche Ergebnisse zwischen beiden Zugängen bieten zusätzliche Erklärungsmöglichkeiten sozialer Phänomene.

Die konkrete Umsetzung dieses zweiten Zugangs sieht so aus, dass viele Items ,gespiegelt“" wurden: Nachdem eine Item-Batterie einmal mit der Frage „Was denken Sie persönlich ...?“ eingeleitet wurde, wird sie ein zweites Mal wiederholt und dieses Mal mit der Frage „Und wie sieht das die Allgemeinheit ...?“ (oder ähnlich) überschrieben. Um die Befragung zeitlich nicht zu sehr in die Länge zu ziehen, konnten allerdings nicht immer alle Items gespiegelt werden.

Darauf, dass vom Befragten solche Einschätzungen gefordert werden, wurde er $\mathrm{zu}$ Beginn des Interviews jeweils vorbereitet. Gleichzeitig wurde erklärt, wie die Fragen nach der Allgemeinheit zu verstehen sind, wobei sich die Erläuterung der Allgemeinheit an dem Konzept des „,verallgemeinerten anderen“ (Mead 1973: 194f.) orientiert, der eine abstrahierte „Organisation der Haltungen all jener Personen ist, die in den gleichen Prozess eingeschaltet sind" (ebd.: 196), also in diesem Fall der Haltungen der gesamten Gesellschaft. So gibt der Interviewer im Zuge der Einführung in das Interview den Hinweis: „Darüber hinaus interessiert uns auch, was Sie glauben, was die Allgemeinheit dazu denkt. Damit meinen wir die vorherrschende Meinung in Deutschland, also was man im Alltag durch die Medien oder durch den Kontakt mit anderen Menschen besonders oft mitbekommt. Wichtig ist: Diese öffentliche Meinung kann sich durchaus von Ihrer ganz persönlichen Meinung unterscheiden!“

Dafür, dass diese Messung der gesellschaftlichen Ebene als valide angesehen werden kann, sprechen sowohl die Ergebnisse von Voruntersuchungen als auch die Auswertung eines Zusatzmoduls am Ende der Befragung (vgl. Abschnitt 4).

\footnotetext{
4 Das schließt weder aus, dass rechtliche oder strukturelle Rahmenbedingungen geschaffen werden, die keinem aktuell herrschenden kulturellen Leitbild entsprechen, noch, dass sich individuelle Leitbilder einem zuvor vorhandenen kulturellem Leitbild anpassen.
} 


\subsection{Messung von persönlichen und gesellschaftlichen Familienleitbildern}

Durch den FLB 2012 selbst werden zunächst einzelne Normalitätsvorstellungen gemessen, also Bestandteile von Leitbildern gemäß der hier zugrunde gelegten Definition. Ein Leitbild selbst ist definiert als ein Bündel solcher Normalitätsvorstellungen (vgl. die Definition oben). Es stellt sich also die Frage, wie - basierend auf den Daten des FLB 2012 - Leitbilder methodisch abgebildet werden können, sowohl für die persönliche als auch für die gesellschaftliche Ebene.

Als mögliche Verfahren, um Bündel aus miteinander assoziierten Vorstellungen zu identifizieren, bieten sich die Faktoren- oder die Hauptkomponentenanalyse an. ${ }^{5}$ Da Leitbilder themenspezifisch sind, ist es empfehlenswert, Analysen mit thematisch verwandten Items zu rechnen, beispielsweise mit allen Items, die Vorstellungen zum Thema Mutterschaft formulieren. Da die Normalitätsvorstellungen, die als konsistente Bestandteile des gleichen Leitbildes miteinander assoziiert werden, von den gleichen Menschen gleichzeitig verinnerlicht sein müssen, müssen die Zustimmungen bzw. Ablehnungen der entsprechenden Items untereinander statistisch korrelieren. Ein Leitbild sollte sich demnach in einer Korrelationsmatrix als eine Gruppe von Items abbilden, die untereinander relativ stark korrelieren. Übertragen auf die Hauptkomponentenanalyse bedeutet das: Ein Leitbild sollte sich als ein Faktor abbilden; Faktoren, die thematisch verwandte Normalitätsvorstellungen zusammenfassen, können jeweils als ein Leitbild gedeutet werden.

An dieser Stelle kann sich ein zentrales Interpretationsproblem stellen, nämlich das, welcher Pol der Dimension als Leitbild gedeutet wird: Die Hauptkomponentenanalyse gruppiert Items nach der Stärke ihrer Korrelation, wobei es keine Rolle spielt, ob die Items positiv oder negativ gepolt sind. Beispielsweise könnten die Aussagen (a) „Eine Mutter sollte auf keinen Fall erwerbstätig sein“ und (b) „Eine Mutter ist hauptverantwortlich für Haushalt und Kinder" miteinander korrelieren und als ein gemeinsamer Faktor identifiziert werden. Eine Interpretation dazu wäre, dass der Faktor das Leitbild der „klassischen“ Mutter misst, die zuhause bleibt und sich um Haushalt und Kinder kümmert. Gleichzeitig kann aber auch die Ablehnung des gleichen Faktors als ein (mit negativ gepolten Items gemessenes) Leitbild gedeutet werden, nämlich als das der emanzipierten Mutter, die ihr eigenes Geld verdient und ihren Partner bei der Haus- und Familienarbeit mit in die Verantwortung nimmt. ${ }^{6}$

Grundsätzlich können also beide Pole einer Dimension als Leitbild interpretiert werden. $\mathrm{Ob}$ beide Pole oder nur ein Pol als Leitbild gedeutet werden, ob das Leitbild im positiven oder im negativen Pol der Dimension gesehen wird, kann nur theoretisch bzw. inhaltlich begründet werden. Als Leitlinie sollte dabei gelten, dass ein Leitbild durch Aussagen (bzw. Zurückweisungen von Aussagen) markiert sein sollte, die möglichst ähnliche und möglichst konkrete Vorstellungen von der Realität zum Ausdruck bringen und somit wenig Raum für unterschiedliche Leitbilder oder für Indifferenz lassen. Das heißt, bei einem Item mit der Aussage „Eine Mutter sollte unter allen Umständen erwerbstätig sein“ sollte davon ausgegangen werden, dass die Zustimmung das Leitbild beschreibt, während bei der Aussage „Es ist schon in Ordnung, wenn eine Mutter erwerbstätig ist“ eher die Ablehnung Bestandteil eines Leitbildes sein dürfte. Im FLB 2012 (wie in den meisten

Die meisten Beiträge in diesem Band nehmen Hauptkomponentenanalysen mit Varimax-Rotation vor.

Anschaulich wird das Problem vor allem dann, wenn ein Faktor aus zwei unterschiedlich gepolten Items besteht, die negativ miteinander korrelieren, etwa das oben verwendete Beispiel-Item (a) mit einem Item (b') „Eine Mutter trägt für Haushalt und Kinder nicht mehr Verantwortung als der Vater“. In dem Fall stellt sich lediglich die Frage, welches der Items als negativ und welches als positiv gepolt interpretiert wird. 
anderen Erhebungen) ist die Mehrzahl der Items so formuliert, dass eine Zustimmung eine konkretere Aussage bedeutet als eine Ablehnung. Als Faustregel kann daher gelten, dass (bei überwiegend positiven Faktorladungen) im Zweifelsfall eher der positive Pol des Faktors als ein Leitbild interpretiert werden sollte.

Die verschiedenen Einzelbefunde, die sich aus der Hauptkomponentenanalyse ergeben, können ebenfalls inhaltlich interpretiert werden: Items mit hoher Faktorenladung sind eher als Kernbestandteile des Leitbildes zu werten, solche mit niedriger Faktorladung eher als Aspekte, die sich dem Leitbild am Rande zuordnen lassen. Faktoren mit hoher Reliabilität und hoher erklärter Varianz können als stark ausgeprägte, deutlich akzentuierte Leitbilder gedeutet werden, solche mit niedriger Reliabilität und niedriger erklärter Varianz eher als schwach ausgeprägt und nur vage vorhanden. Da Leitbilder, ähnlich wie andere sozial konstruierte Phänomene, sozial unscharf sind (Schulze 1993: 213f.), kann nicht nur ihre Beschaffenheit, sondern bereits ihre Existenz lediglich graduell bestimmt werden: Leitbilder können stärker oder schwächer ausgeprägt sein, an Kontur gewinnen oder verblassen. Insofern gibt es keinen Schwellenwert, den ein Faktor mindestens erreichen muss, um als ein Leitbild interpretiert werden zu können.

Ein besonderer Fall kann dann eintreten, wenn eine Normalitätsvorstellung oder ein kulturelles Leitbild tatsächlich gesellschaftsweit verbreitet ist und quasi von allen Mitgliedern der Gesellschaft einheitlich wahrgenommen wird. Das ist beispielsweise für die Vorstellung der Fall, dass ein verheiratetes Paar aus Frau und Mann, das mit Kind(ern) zusammenlebt, eine Familie ist (vgl. Lück/Ruckdeschel, Kap. 5). Normalitätsvorstellungen, die zu einem solchen Leitbild gehören, sollten Zustimmungswerte nahe $100 \%$ aufweisen. Somit könnte ein Item mit extremer Zustimmung Bestandteil eines gesellschaftsweit verbreiteten Leitbildes sein, mangels Varianz aber möglicherweise keine Korrelation zu anderen Items aufweisen. Die Frage, mit welchen anderen Vorstellungen es gemeinsam ein Leitbild abbildet, ist dann auf eine inhaltlich-theoretische Interpretation verwiesen.

Im Normalfall lassen sich individuelle Leitbilder operationalisieren als Zustimmung ${ }^{7}$ einer befragten Person zu einer Gruppe von Items, d. h. zu persönlichen Vorstellungen, die in einer Faktoren- oder Hauptkomponentenanalyse als eine gemeinsame inhaltliche Dimension identifiziert wurden. Die Aggregation dieser individuellen Leitbilder über alle Befragten kann als ein erster Anhaltspunkt für die Existenz und Verbreitung eines kulturellen Leitbildes gedeutet werden.

Ein zweiter Anhaltspunkt für die Existenz und Verbreitung eines kulturellen Leitbildes ist dessen direkte Wahrnehmung auf gesellschaftlicher Ebene. Die Operationalisierung erfolgt wieder als Zustimmung zu einer Gruppe von Items, die mittels Faktorenanalyse als eine gemeinsame Dimension identifiziert wurden; jedoch handelt es sich bei den Items um Aussagen über die (mutmaßlichen) Vorstellungen der Allgemeinheit. Dabei genügt schon ein kleiner Anteilswert, um zu unterstellen, dass ein kulturelles Leitbild existiert, denn durch die Items auf gesellschaftlicher Ebene wird jeder einzelne Befragte unmittelbar nach kollektiven Vorstellungen befragt. Haben nur wenige Befragte hohe Zustimmungswerte bei den Items einer Dimension, muss von einem Leitbild ausgegangen werden, das gesellschaftlich nicht sehr verbreitet und möglicherweise nur in einem bestimmten sozialen Milieu oder in einer bestimmten Region etabliert ist. Hat eine große Mehrheit der Befragten hohe Zustimmungswerte, muss von einem gesellschaftsweit dominanten Leitbild ausgegangen werden.

7 Die spezifische Messung von „Zustimmung“ hängt dabei von theoretischen und inhaltlichen Argumenten ab und obliegt dem jeweiligen Forscher. 
Die empirischen Analysen zeigen, dass die beiden methodischen Zugänge - die Aggregation individueller Leitbilder und die mehrheitliche Wahrnehmung dessen, welche Leitbilder die Allgemeinheit in der Gesellschaft hat - zu sehr unterschiedlichen Ergebnissen kommen können. Wie diese Diskrepanzen zu deuten sind und welcher Anhaltspunkt für die wahre Verbreitung kultureller Leitbilder aussagekräftiger ist, wird in Abschnitt 5 diskutiert.

\subsection{Operationalisierung weiterer Konzepte}

Sowohl für die differenzierte Beschreibung von Leitbildern als auch zur Erstellung von Modellen zur Erklärung von Leitbildern ist eine Erfassung verschiedener soziodemografischer und sozioökonomischer Merkmale der Befragten und ihrer Lebenssituation unabdingbar. Im Folgenden werden deshalb die Variablen erläutert, deren Messung entweder nicht auf den ersten Blick nachvollziehbar ist oder bei denen nachträglich Konstruktionen ergänzt wurden.

\section{Migrationshintergrund}

Alle Befragten wurden danach gefragt, ob sie in Deutschland geboren wurden und welche Staatangehörigkeit sie haben. Der genaue Geburtsort des Befragten wurde nicht erhoben. Der Migrationshintergrund wurde in Anlehnung an die Definition des Statistischen Bundesamtes (2013) erfasst. Dazu wurde zusätzlich gefragt, ob beide, ob einer oder ob keiner der beiden Elternteile die deutsche Staatsangehörigkeit besitzt. Darüber hinaus sind Informationen über das jeweilige Geburtsland der beiden Elternteile im Interview festgehalten worden. Aus diesen Informationen wurde für den Datennutzer eine dichotome Variable erstellt, welche differenziert, ob der Befragte einen Migrationshintergrund hat oder nicht. Dies trifft zu, wenn die Person entweder selbst nicht in Deutschland geboren wurde oder keine deutsche Staatsbürgerschaft aufweist oder wenn mindestens eines der Elternteile nicht in Deutschland geboren wurde oder keine deutsche Staatsangehörigkeit besitzt.

West- und Ostdeutschland

Auf Basis der theoretischen Vorüberlegungen erschien es bei der Entwicklung des Fragebogens sinnvoll, eine regionale Differenzierung nach West- und Ostdeutschland sowohl für den aktuellen Wohnort als auch für den Sozialisationsort zu ermöglichen. Bezogen auf den aktuellen Wohnort wird das Bundesland herangezogen, in dem der Befragte lebt. Um dem Datennutzer die Entscheidung offen zu halten, wie mit Berlin zu verfahren ist, wurden als Antwortvorgaben „Westberlin“ und „Ostberlin“ angeboten, so dass zusätzlich zwischen dem westlichen und östlichen Teil Berlins unterschieden werden kann. Auf die gleiche Weise wurde auch der Sozialisationsort erfragt. Die genaue Frageformulierung lautet: „In welchem Bundesland haben Sie den Großteil Ihrer Kindheit und Jugend verbracht?". Die Befragten sollten hierauf spontan antworten; die Kategorien wurden nur bei Bedarf vorgelesen. Außer den aktuell existierenden Bundesländern konnte die Nennung „ehemalige DDR“ erfasst werden (für den Fall, dass dem Befragten die Zuordnung nicht möglich war) sowie die Kategorien „europäisches“ und „nichteuropäisches Ausland“.

\section{Partnerschaftsform und Familienstand}

Partnerschaftsform und Familienstand stellen zentrale Variable des FLB 2012 dar. Die Erhebung war relativ komplex: Zunächst wurde die Frage nach dem Familienstand gestellt, wobei auch die eingetragene Lebenspartnerschaft in allen Variationen (zusammenlebend, getrennt, aufgehoben, Partner verstorben) als Ausprägung erfasst werden konnte. Des Weiteren wurden alle übrigen Personen gefragt, ob sie in einer festen Beziehung leben und welches Geschlecht ggf. dieser Partner hat. Unabhängig vom Familienstand wurde die 
Dauer der gegenwärtigen Beziehung erfragt, ebenso die Dauer der Ehe bzw. der eingetragenen Lebenspartnerschaft. Dabei konnte der Befragte entweder angeben, seit welchem Jahr oder alternativ seit wie vielen Jahren diese besteht. Diese Form der Erfassung wurde prinzipiell bei der Abfrage von Dauern verwendet, um fehlende Angaben zu reduzieren. Unabhängig vom Familienstand wurde schließlich auch gefragt, ob der Befragte mit seinem Partner einen gemeinsamen Haushalt führt und ggf. seit wann dies der Fall ist.

\section{Elternschaft}

Ähnlich wie bei der Partnerschaftsform wurden Fragen zur Elternschaft so umfangreich und komplex wie nötig in den Fragebogen integriert. Die Frage nach Kindern wurde allen Befragten mit dem Hinweis gestellt, dass sowohl „eigene“ Kinder als auch Adoptivkinder unabhängig von deren Wohnort gemeint sind. Pflege- und Stiefkinder wurden ausgenommen, wobei aber rekonstruiert werden kann, ob die Kinder aus der gegenwärtigen Beziehung stammen. Neben der Kinderzahl wurde das Geburtsjahr des ersten bzw. einzigen und des jüngsten Kindes erhoben.

\section{Kinderwunsch \\ Zur Erfassung des Kinderwunsches wurde in einem ersten Schritt erfragt, ob ein Kinder- wunsch vorliegt oder nicht. In einem zweiten Schritt konnte die gewünschte Kinderzahl angegeben werden. Um auch hier die Ausfälle zu minimieren, konnte sowohl eine absolute Zahl als auch eine Spanne (z. B. zwei bis drei Kinder) angegeben werden. Für die Nutzer des Datensatzes wurde aus den offenen Angaben und den Spannen jeweils eine Variable ergänzt, welche den Mittelwert der Spanne mit der genauen Angabe vereint. Hieraus wurde zudem der Gesamtkinderwunsch erstellt, welcher die bereits vorhandene Kinderzahl mit einbezieht (siehe auch Dorbritz/Naderi 2013; Ruckdeschel 2004).}

\section{Bildung (ISCED-Konstruktion, Befragter und Partner)}

Die Befragten gaben sowohl für sich selbst als auch für ihren jeweiligen Partner den höchsten allgemeinen Schulabschluss und den höchsten beruflichen Bildungsabschluss an. Orientiert an der Vorgehensweise des Statistischen Bundesamts (siehe schematische Darstellung in Schroedter et al. 2006: 22), wurde eine ISCED97-Kodierung durchgeführt und danach zu drei Kategorien zusammengefasst (niedrig, mittel, hoch). Die Kategorie „niedrig“ umfasst die Stufen 1 bis 2, welche Personen ohne schulischen oder beruflichen Bildungsabschluss bzw. diejenigen beinhalten, die höchstens die mittlere Reife, aber keine berufliche Ausbildung haben. Die Kategorie ,mittel“ umfasst die Stufen 3A bis 4A, welche die Fachhochschulreife ohne berufliche Qualifikation bis hin zum Abitur mit anschließender Lehre beinhalten. Die Kategorie „hoch“ beginnt bei Stufe 5B, d. h. mit einem Meisterabschluss, und beinhaltet außerdem Stufe 6 mit den höchsten akademischen Bildungsgraden.

\section{$4 \quad$ Entwicklung des Erhebungsinstruments und Validitätsprüfungen}

Nicht nur die Befragungstechnik anhand von zwei Ebenen, sondern auch die inhaltliche Formulierung der meisten Items zur Messung von Normalitätsvorstellungen sowie deren Zusammenstellung zu Item-Batterien sind im FLB 2012 neu entwickelt worden. Dabei wurde durch eine Reihe von Voruntersuchungen versucht, ein hohes Maß an Validität und Reliabilität zu gewährleisten. Diese Vorgehensweise wird im Folgenden beschrieben. 


\subsection{Entwicklung des Erhebungsinstruments}

Eine besondere Herausforderung der Leitbildforschung besteht darin, dass Leitbilder in standardisierten Befragungen nur theoriegeleitet deduktiv nachweisbar sind. Die Fülle der Normalitätsvorstellungen, die zum Familienleben grundsätzlich existieren könnten, ist quasi unendlich. Das Gleiche trifft auf die Fülle der Leitbilder zu, die diese Normalitätsvorstellungen bündeln könnten. Insofern ist es nicht sinnvoll, eine mehr oder minder zufällige Sammlung von Aussagen über das Familienleben abzufragen, um auf induktivem Wege darin Leitbilder zu entdecken. Um innerhalb einer begrenzten Befragungsdauer Leitbilder empirisch zu erfassen, muss sich die Formulierung der abgefragten Items an begründeten Annahmen orientieren, welche Leitbilder wahrscheinlich existieren.

An erster Stelle steht selbstverständlich die Sichtung des entsprechenden Forschungsstandes, um zu solchen Annahmen zu gelangen. Allerdings ist dieser zum Thema Familienleitbilder eher schwach besetzt. Daneben ist jeder Forscher selbst Teil der Gesellschaft und hat die existierenden Leitbilder über das Familienleben in gleicher Weise verinnerlicht wie ein Befragter. Auch auf Basis dieses Alltagswissens lassen sich Erwartungen formulieren, welche Normalitätsvorstellungen und welche Leitbilder wahrscheinlich existieren. Um dem Umstand Rechnung zu tragen, dass die Wahrnehmung jedes einzelnen Forschers von den existierenden kulturellen Leitbildern subjektiv bleibt, wurde die Auswahl und Formulierung der Items in einer Gruppe von acht Wissenschaftlern ausführlich diskutiert und gemeinsam entschieden.

Die Auswahl der Themen und inhaltlichen Aussagen orientierte sich maßgeblich an zwei qualitativen Voruntersuchungen. Die erste bestand in einer moderierten Fokusgruppendiskussion mit sechs Teilnehmern im November 2010 (Banavas 2010) und die zweite in leitfadengestützten qualitativen Interviews mit 29 befragten Personen, die selbst keine Wissenschaftler waren, im Winter 2010/2011 (vgl. Dietrich/Gies 2012). Beide Untersuchungen hatten das Ziel, durch ihre offene Befragungsweise unvoreingenommene Hinweise auf die Existenz und inhaltliche Zusammensetzung zentraler Leitbilder zum Familienleben zu erfassen, die später im FLB 2012 standardisiert erhoben werden sollten.

Weitere Hinweise auf verbreitete Normalitätsvorstellungen ergaben sich aus der Forschungsliteratur sowie aus der Sichtung anderer standardisierter Erhebungen und der darin erhobenen Einstellungsvariablen. Neben der Festlegung der zu erfassenden inhaltlichen Aussagen wurden die dort vorgefundenen Items auch als Inspiration oder auch als unmittelbare Vorlage für die konkrete Itemformulierung verwendet. Zudem bieten sie die Möglichkeit der Validierung des Fragebogens durch einen Vergleich der Ergebnisse identischer Fragen. Mit in die Sichtung einbezogen wurden der Generations and Gender Survey (GGS), die Population Policy Acceptance Study (PPAS), das International Social Survey Programme (ISSP) sowie die European Values Study (EVS).

Bis Anfang 2012 wurde ein vorläufiger Entwurf eines Fragebogens erstellt. Eine Auswahl von Items wurde daraufhin von GESIS (Gesellschaft Sozialwissenschaftlicher Infrastruktureinrichtungen) mit 20 befragten Personen in einem kognitiven Pretest (Porst et al. 2012) getestet, wobei sowohl die konkreten Itemformulierungen als auch die grundsätzliche Technik des Fragens nach der „Allgemeinheit“ überprüft wurde (vgl. ausführlich dazu Abschnitt 4.3). Der verbesserte Fragebogen wurde vom Befragungsinstitut TNS Infratest Sozialforschung in eine CATI-Programmierung umgewandelt und anschließend im BiB ausführlich auf korrekte Programmierung getestet. Kurz vor Feldbeginn wurden durch TNS Infratest Sozialforschung ein Pretest mit 50 Zielpersonen durchgeführt und anschließend letzte Kürzungen und Verbesserungen am Fragebogen vorgenommen. 


\subsection{Allgemeine Qualitätsprüfung}

Viele der geschilderten Vorarbeiten im Zuge der Entwicklung des Erhebungsinstrumentes verfolgen unter anderem das Ziel, dessen Qualität zu optimieren, und tragen somit dazu bei, eine hohe Datenqualität zu gewährleisten. Das gilt insbesondere für den kognitiven Pretest durch die GESIS, für die Überprüfung der CATI-Programmierung durch das BiB sowie für den Pretest durch TNS Infratest Sozialforschung. Für die Gewährleistung einer hohen Datenqualität ist es darüber hinaus notwendig, den korrekten Ablauf der Feldarbeit sicherzustellen. Hier setzten weitere Maßnahmen an.

Es wurden Plausibilitätsprüfungen in der CATI-Programmierung eingefügt, die auf widersprüchliche Werte unmittelbar nach der Eingabe hinweisen und dem Interviewer erlauben nachzufragen. Sowohl vor dem Pretest als auch vor Beginn der Haupterhebung führte die Projektleitung von TNS Infratest Sozialforschung unter der Beteiligung des BiB eine intensive Schulung der Interviewerinnen und Interviewer durch. Darüber hinaus wurde jeder Interviewerin und jedem Interviewer ein Interviewerhandbuch zur Verfügung gestellt, das den Umgang mit schwierigen Fragen schriftlich erläuterte.

Während der Haupterhebung fand ein Monitoring durch die Studioleitung der Telefonstudios statt. Standardprüfprogramme werteten Kriterien wie die interviewerspezifische Ausschöpfungsquote, die durchschnittliche Interviewdauer, die Zahl der durchgeführten Interviews pro Stunde und die Anzahl der benötigten Anrufe pro Interview bereits während der Feldarbeit aus, so dass auf Auffälligkeiten frühzeitig reagiert werden konnte. Außerdem kontrollierten Supervisoren stichprobenartig 791 (15,8\%) der Interviews und konnten so bei Defiziten die entsprechenden Interviewerinnen bzw. Interviewer umgehend in Feedbackgesprächen nachschulen.

Durch Infratest wurde das $\mathrm{BiB}$ regelmäßig über den Fortgang der Feldarbeit in den Telefonstudios unterrichtet. Auch wurde kurz nach Beginn der Erhebung ein vorläufiger Datensatz auf Basis der bereits geführten Interviews generiert, mit dem die Plausibilität der Daten und insbesondere die korrekte Filterführung überprüft wurden. Weitere Prüfungen von Plausibilität und Konsistenz sowie eine systematische Suche nach Interviewereffekten erfolgten nach Ende der Feldphase mit dem vollständigen Datensatz.

Insgesamt wurden im Zuge der Erhebung nur vergleichsweise unbedeutende Schwierigkeiten festgestellt. BiB und TNS Infratest Sozialforschung gehen daher beim FLB 2012 von einer hohen Datenqualität aus.

\subsection{Validitätsprüfung der Messung von Leitbildern}

Besondere Aufmerksamkeit und Anstrengung hat die Überprüfung der Validität des Erhebungsinstrumentes in Bezug auf die Messung von Familienleitbildern verdient.

Auch die Gewährleistung der Validität der Messung von Familienleitbildern beginnt mit der Entwicklung des Erhebungsinstrumentes. Dabei ist die geschilderte Auswahl der Themen und inhaltlichen Aussagen ebenso bedeutsam wie deren konkrete Formulierung (vgl. Abschnitt 4.1). Eine besondere Bedeutung kommt dem kognitiven Pretest durch GESIS zu (Porst et al. 2012). Darin wurden 20 nach einem Quotenschlüssel ausgewählte Zielpersonen zunächst anhand einer Auswahl von Items befragt, die für die Haupterhebung vorgesehen waren. Im Anschluss daran wurden dieselben Personen mittels offener Nachfragen gebeten, sowohl ihr Verständnis von den unterschiedlichen Items als auch ihre Beweggründe für ihr Antwortverhalten zu erläutern. Zwar zeigten sich zum Teil Schwierigkeiten beim korrekten Verständnis bestimmter Itemformulierungen. Jedoch konnten die 
Befragten grundsätzlich sehr gut zwischen ihren eigenen Vorstellungen und der Einschätzung der Vorstellungen der „Allgemeinheit“ differenzieren und verstanden gut, was mit der „Allgemeinheit" gemeint war.

Um das Verständnis der Befragten von der „Allgemeinheit“ und die Validität dieser Art der Frageformulierung zusätzlich zu prüfen, wurde in der Haupterhebung am Ende des Interviews ein Zusatzmodul erhoben (siehe ausführlich Gies/Dietrich, Kap. 4). Die dort enthaltenen Fragen wurden lediglich einer Unterstichprobe von 537 zufällig ausgewählten Befragungsteilnehmern gestellt. Gefragt wurde zunächst offen, „an wen oder was“ der Befragte bei der „Allgemeinheit“ gedacht habe, um dann im Anschluss zusätzlich sechs mögliche Quellen des Bildes von der „Allgemeinheit“ standardisiert zu erheben. Die Antworten ergeben ein heterogenes Bild: Genannt wurden vor allem Personen aus dem sozialen Nahumfeld (Freunde, Bekannte, Familie und Kollegen) aber auch Darstellungen in den Medien sowie ein bereits abstrahiertes Bild der Gesellschaft insgesamt, dessen Herkunft nicht mehr weiter spezifiziert werden kann. Der standardisierten Frage, ob der Befragte „bei den Fragen zur ,Allgemeinheit" immer dasselbe Bild vor Augen“ hatte, stimmen $67 \%$ $\mathrm{zu}$, der Frage, ob er „,das Gefühl [hatte], immer sagen zu können, was die ,Allgemeinheit‘ denkt", lediglich $43 \%$.

Auch die Interviewerinnen und Interviewer wurden zur Validität der Fragen nach der „Allgemeinheit" konsultiert. Nach Abschluss jedes Interviews beantworteten sie jeweils drei Fragen anhand einer vierstufigen Skala (,trifft voll und ganz zu“, „trifft eher zu“, „trifft eher nicht zu“, „trifft überhaupt nicht zu“): ob die Antworten bezüglich der Allgemeinheit spontan kamen, ob der Befragte genau sagen konnte, woher sein Bild von der „Allgemeinheit" stammt, und ob der Befragte ,eher überfordert mit der Aufgabe [war], genau zu sagen, was für ihn , die Allgemeinheit" ist". Die Angaben der Interviewerinnen und Interviewer lassen darauf schließen, dass die Fragen zur „Allgemeinheit“ bei etwa vier von fünf Interviews unproblematisch verliefen.

\section{Unterschiede zwischen persönlichen und gesellschaftlichen Leitbildern}

In der empirischen Analyse der Daten ergeben sich zum Teil sehr gegensätzliche Befunde $\mathrm{zu}$ persönlichen und $\mathrm{zu}$ gesellschaftlichen Leitbildern: So stimmen beispielsweise der Aussage „Ein Mann muss seine Familie allein ernähren können“ nur 28,9 \% persönlich zu; allerdings nehmen 75,4\% wahr, dass die Allgemeinheit bzw. die Mehrheit der Menschen in Deutschland dieser Aussage zustimmen würde (Lück, Kap. 14). Ähnliche Befunde zeigen sich unter anderem auch für Vorstellungen zu Mutterschaft (Diabaté, Kap. 13) oder zu kinderreichen Familien (Diabaté/Ruckdeschel/Dorbritz/Lux, Kap. 11). Wodurch sind diese Diskrepanzen zu erklären?

Die Deutlichkeit der Kontraste ist deshalb überraschend, weil beide Ebenen letztlich Zugänge zum gleichen Phänomen sind: zum kulturellen Leitbild (vgl. Abschnitt 3.1). Sowohl die Feststellung, dass ein individuelles Leitbild in einer Gesellschaft statistisch weit verbreitet ist, als auch die individuelle Einschätzung jedes einzelnen Befragten, welches das gesellschaftlich vorherrschende Leitbild sei, sind Wege, kulturelle Leitbilder abzubilden. Zwar ist eine gewisse Diskrepanz zwischen diesen beiden methodischen Zugängen auch theoretisch erwartbar, da das kulturelle Leitbild nicht durch statistische Mehrheiten zustande kommt, sondern dadurch, was sich im medienvermittelten öffentlichen Diskurs durchsetzt und durch Institutionalisierungsprozesse verfestigt. Insofern werden, streng 
genommen, doch zwei unterschiedliche Phänomene gemessen. Dennoch ist es überraschend, wie stark sich diese beiden doch eigentlich theoretisch verknüpften Phänomene unterscheiden können.

Als Erklärung für die teilweise große Diskrepanz bieten sich verschiedene Thesen an, die teils inhaltlich, teils methodisch argumentieren. Inwieweit diese zutreffen, lässt sich nur bedingt empirisch überprüfen. Insofern bleibt Raum für theoretische Reflexionen und Interpretation. Im Zweifelsfall ist davon auszugehen, dass alle genannten Argumente bis zu einem gewissen Grad mit zur Erklärung beitragen. Folgende Thesen lassen sich aufstellen:

1. Soziale Erwünschtheit: Die Befragten vermuten, dass von ihnen bestimmte, von ihren eigenen Überzeugungen abweichende Antworten erwartet werden und stellen ihre persönlichen Vorstellungen, gemessen daran, positiver dar als sie sind. Dadurch wird die Messung der persönlichen Leitbilder verzerrt (vgl. Schnell et al. 2005: 355f.) - in der vorliegenden Untersuchung in Richtung liberalerer Antworten.

2. Vorurteil gegenüber der Allgemeinheit: Der Befragte unterstellt der Mehrheit seiner Mitmenschen - beispielsweise aufgrund medienvermittelter Stereotype oder aufgrund persönlicher Vorurteile, die den Glauben an seine eigene moralische Überlegenheit stützen - dass sie weniger tolerant und daher ,rückständiger“ seien als der Befragte selbst. Als Konsequenz daraus nimmt er an, dass das Leitbild der Allgemeinheit ,rückständiger" sei als sein persönliches Leitbild, so dass das gesellschaftliche Leitbild verzerrt abgebildet wird.

Dieser Effekt zeigt sich ähnlich auch in vergleichbaren Befragungssituationen, etwa wenn Befragte auf der einen Seite ihre persönliche und auf der anderen Seite die allgemeine wirtschaftliche Lage beurteilen sollen. Hier wird der Befund so gedeutet, dass sich Menschen schwertun, die gesellschaftliche Situation zu erfassen, und in ihrer Einschätzung zum Teil zu Pessimismus neigen (Terwey 1990: 149-151).

3. Selektions- bzw. Alterseffekt: Die im FLB 2012 erhobene Stichprobe ist auf Befragte im Alter von 20 bis 39 begrenzt. Die persönlichen Leitbilder dieser Altersgruppe sind jedoch liberaler als die älterer Menschen in Deutschland, so dass die Aggregierung der persönlichen Leitbilder das allgemeine kulturelle Leitbild verzerrt abbildet. Die Einschätzungen der Vorstellung der Allgemeinheit durch die Befragten berücksichtigen dagegen auch die Auffassungen älterer Mitbürger und spiegeln das allgemeine kulturelle Leitbild adäquater wider.

Im Gegensatz zu den vorherigen Thesen lässt sich diese empirisch zumindest plausibilisieren: Obwohl die im FLB 2012 verwendeten Items in ihrer Überzahl neu entwickelt wurden, gibt es ähnliche Items in anderen Befragungen. Für diese lassen sich Befunde auch für ältere Kohorten generieren. So sind zum Beispiel im Rahmen des International Social Survey Programme (ISSP) 2012 einige Items erhoben worden, die mit Items aus dem FLB 2012 Ähnlichkeit haben. Abbildung 1 stellt die Zustimmung zu der im ISSP 2012 erhobenen Aussage „Menschen, die Kinder wollen, sollten heiraten“ differenziert nach Altersklassen dar und vergleicht sie mit der Zustimmung im FLB 2012 zu der Aussage „Welche Bedingungen müssen erfüllt sein, dass man überhaupt daran denken kann, Kinder zu bekommen? ... Das Paar muss verheiratet sein“. Diese ist einmal (links) im Sinne der persönlichen Vorstellung der Befragten und einmal (rechts, ohne Altersdifferenzierung) im Sinne der wahrgenommenen Vorstellung der Allgemeinheit abgebildet.

Die Befunde lassen sich nur eingeschränkt vergleichen. Dem Item im ISSP 2012 wird auf deutlich höherem Niveau zugestimmt. Zudem hatten Befragte im ISSP 2012 eine Skala mit fünf, die im FLB 2012 nur eine mit vier Antwortvorgaben. Dennoch zeigt die Analyse, dass die Altersgruppe der 20- bis 39-Jährigen tatsächlich auf einem niedrigeren 
(„liberaleren“) Niveau antwortet als die ältere Bevölkerung. Insbesondere zu den Befragten im Alter von 60 und darüber besteht ein deutlicher Kontrast, der zumindest einen Teil der Diskrepanz zwischen persönlicher und gesellschaftlicher Ebene der Items im FLB 2012 erklären kann. Zu ähnlichen Ergebnissen kommen Vergleiche anderer Items (Junck/Lück 2015).

4. Zeitlich versetzter sozialer Wandel: Von kulturellen Leitbildern ist anzunehmen, dass sie sich in verschiedenen Formen verstetigen, das heißt institutionalisieren und objektivieren (vgl. Abschnitt 3.1 bzw. Lück/Diabaté, Kap. 2). Bestimmte Formen der Institutionalisierung, wie etwa ein Gesetz, sowie bestimmte Formen der Objektivierung, wie etwa Anzahl und Größe der vorhandenen Einrichtungen zur öffentlichen Kinderbetreuung, repräsentieren die Existenz und Gültigkeit der ihnen zugrundeliegenden kulturellen Leitbilder und verleihen diesen auf diese Weise eine gewisse Stabilität und Resistenz gegenüber sozialem Wandel: Sie verändern sich schwerfälliger und langsamer als individuelle Leitbilder und deren Aggregation. Letztere gibt das kulturelle Leitbild demnach verzerrt wider, signalisiert aber immerhin, in welche Richtung sich das kulturelle Leitbild voraussichtlich mittelfristig entwickeln wird.

Abbildung 1: Altersunterschiede hinsichtlich der Vorstellung, Ehe sei eine Voraussetzung für die Familiengründung, in FLB und ISSP 2012

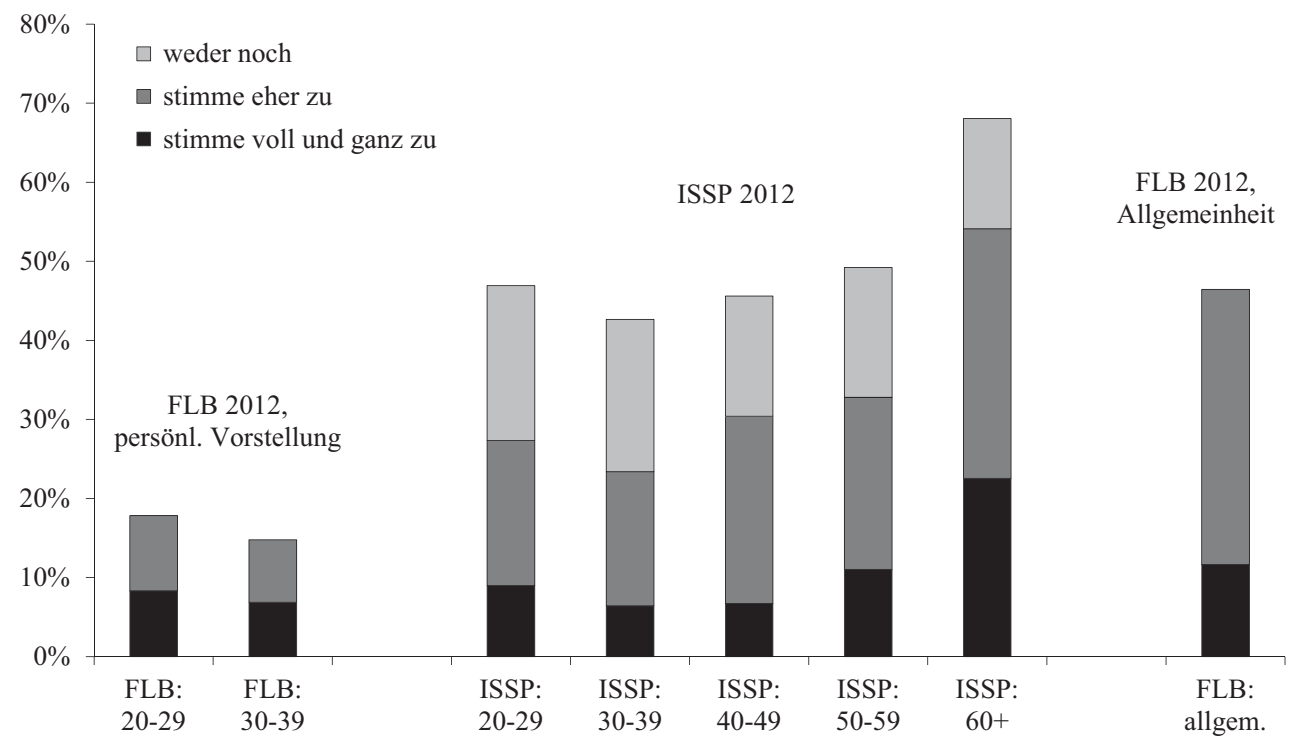

Quelle: FLB (2012) und ISSP 2012 (Deutschland), gewichtete Daten, eigene Berechnungen

Anm.: Items: a) „Welche Bedingungen müssen erfüllt sein, dass man überhaupt daran denken kann, Kinder zu bekommen? ... Das Paar muss verheiratet sein. (persönliche Meinung)“ (FLB 2012), b) „Menschen, die Kinder wollen, sollten heiraten.“ (ISSP 2012), c) „Welche Bedingungen müssen erfüllt sein, ... Wie sehen das die meisten Menschen in Deutschland“" (FLB 2012).

Die Thesen sprechen insgesamt eher dafür, dass die Operationalisierung über die gesellschaftliche Ebene einer Abbildung des wahren kulturellen Leitbildes näher kommt als die Aggregation der individuellen Leitbilder. Es darf aber angenommen werden, dass beide Zugänge Verzerrungen aufweisen. 


\section{Schlussbemerkungen}

Mit der Studie Familienleitbilder (FLB) 2012 wurde eine Datengrundlage gelegt, um persönliche und gesellschaftliche Leitbilder im Kontext der Familie systematisch quantitativ zu erforschen. Grundlage sind Items, die Vorstellungen zum normalen oder idealen Familienleben abbilden. Diesen konnten die Befragten einmal persönlich zustimmen und sie konnten angeben, ob sie annehmen, dass die Allgemeinheit der Menschen in Deutschland ihnen zustimmen würde. Diese Normalitätsvorstellungen lassen sich mit Hilfe multivariater Verfahren zu Leitbildern zusammenfassen, wobei im einen Fall persönliche und im anderen Fall gesellschaftliche Leitbilder gemessen werden.

Nicht nur die Fragen nach den Vorstellungen der Allgemeinheit bieten einen Zugang, um gesellschaftliche kulturelle Leitbilder abzubilden. Auch die Aggregation der persönlichen Leitbilder über alle Befragungsteilnehmerinnen und -teilnehmer ist grundsätzlich ein Anhaltspunkt dafür, welche Leitbilder in der Gesellschaft verankert sind. Die Ergebnisse der beiden Zugänge sind jedoch nicht deckungsgleich; in Einzelfällen weichen sie sogar stark voneinander $\mathrm{ab}$. Als Ursachen für diese unterschiedlichen Ergebnisse wurde unter anderem die Diskrepanz zwischen der befragten Altersgruppe und der Gesamtbevölkerung ausgemacht. Gerade solche Ergebnisse bieten den Rahmen für weitergehende Reflexionen über sozialen Wandel und die treibenden Kräfte in der Bevölkerung, die auch im Schlusskapitel dieses Sammelbandes nochmals angesprochen werden.

Insbesondere das direkte Erfragen (mutmaßlicher) gesellschaftlicher Leitbilder ist als methodischer Zugang bisher nicht etabliert. Doch das Erhebungsinstrument insgesamt ist bereits dadurch innovativ, dass die meisten der erhobenen Items neu konstruiert wurden. Mit Hilfe diverser Vor- und Nachuntersuchungen wurde versucht sicherzustellen, dass das Erhebungsinstrument in der Lage ist, valide Messungen kultureller Leitbilder vorzunehmen. Die bisher vorliegenden Befunde sprechen dafür, dass dies grundsätzlich gelungen ist. Dennoch wird letztlich die Forschungspraxis auf Basis der Daten erweisen müssen, wie gut die Messung welcher Leitbilder im Einzelnen gelingt und welchen Stellenwert der methodische Zugang für die Erforschung von Familienleitbildern insgesamt hat.

Wie die meisten Erhebungen muss auch die Studie Familienleitbilder einen Spagat leisten: Einerseits soll der Themenbereich Familie relativ breit abgedeckt werden, um den Datensatz für die Forschung ergiebig zu machen, andererseits sollen die verschiedenen Aspekte von Familie jeweils mit einer ausreichenden Anzahl von Items abgebildet werden. Die Studie ist in dieser Hinsicht vergleichsweise breit angelegt und bemüht sich um ein Höchstmaß an thematischer Vielfalt, ohne das nötige Mindestmaß an verfügbaren Items zu unterschreiten. Dennoch bleiben einzelne Themenfelder unberührt - beispielsweise die Generationenbeziehungen innerhalb der Familie.

Die Studie stellt zunächst eine Querschnittserhebung dar, und die Auswertung der Daten unterliegt allen Restriktionen, die damit verbunden sind. Allerdings lässt die Studie eine Erweiterung zu einem Panel zu: Die Befragten haben in großer Zahl ihr Einverständnis gegeben, dass ihre Kontaktdaten für eine eventuelle Wiederholungsbefragung gespeichert werden dürfen. Darin liegt mittelfristig sowohl die Chance, Veränderungen sowie kausale Wechselwirkungen im Längsschnitt zu untersuchen, als auch die Möglichkeit, zusätzliche Aspekte des Familienlebens abzubilden, die in der Erhebung 2012 nicht berücksichtigt werden konnten. 


\section{Literatur}

Banavas, Tanja (2010): Dokumentation und Ergebnisbericht zur Fokusgruppendiskussion im Rahmen der Leitbilderforschung. Unveröffentlichtes Manuskript (auf Nachfrage bei Autoren erhältlich).

Busch, Friedrich W.; Scholz, Wolf-Dieter (2005): Zwischen Bewahrung und Veränderung. Ehe-, Familien- und Erziehungsvorstellungen von Jugendlichen. In: Busch, Friedrich W.; Nave-Herz, Rosemarie (Hrsg.): Familie und Gesellschaft. Oldenburg: BIS-Verlag, S. 125-145.

Diabaté, Sabine; Lück, Detlev (2014): Familienleitbilder - Identifikation und Wirkungsweise auf generatives Verhalten. In: Zeitschrift für Familienforschung, 26, 1: S. 49-69.

Dietrich, Dorothee; Gies, Nadine (2012): Familienleitbilder. Ergebnisse einer qualitativen Leitfadenstudie. Wiesbaden. [http://www.bib-demografie.de/leitbild]

Dorbritz, Jürgen; Naderi, Robert (2013): Trendwende beim Kinderwunsch? In: Bevölkerungsforschung Aktuell, 34, 4, S. $2-7$

Gabler, Siegfried; Ayhan, Öztas (2007): Gewichtung bei Erhebungen im Festnetz und über Mobilfunk: Ein Dual Frame Ansatz. In: Gabler, Siegfried; Häder, Sabine (Hrsg.): Mobilfunktelefonie Eine Herausforderung für die Umfrageforschung. ZUMA-Nachrichten, Spezial-Band 13. Mannheim: GESIS-ZUMA, S. 39-45.

Junck, Sara; Lück, Detlev (2015): Die Älteren setzen den Standard. Untersuchung von Altersunterschieden in persönlichen Leitbildern als Ansatz zur Erklärung von Diskrepanzen zwischen den vorherrschenden gesellschaftlichen und den vorherrschenden persönlichen Leitbildern in der Studie „Familienleitbilder“ (FLB 2012). Wiesbaden: Bundesinstitut für Bevölkerungsforschung. [http://www.bib-demografie.de/leitbild]

Lück, Detlev; Gründler, Sabine; Naderi, Robert; Dorbritz, Jürgen; Schiefer, Katrin; Ruckdeschel, Kerstin; Hiebl, Johannes; Wolfert, Sabine; Stadler, Manuela; Pupeter, Monika (2013): Familienleitbilder 2012. Methodenbericht zur Studie. BiB Daten- und Methodenberichte 2/2013. Wiesbaden: Bundesinstitut für Bevölkerungsforschung.

Mead, George H. (1973): Geist, Identität und Gesellschaft. Aus der Sicht des Sozialbehaviorismus. Frankfurt a. M.: Suhrkamp.

Porst, Rolf; Lenzner, Timo; Bischof, Lisa-Marie (2012): Familienbezogene Leitbilder. Kognitiver Pretest. GESIS-Projektbericht. Mannheim: GESIS.

Ruckdeschel, Kerstin (2004): Determinanten des Kinderwunsches in Deutschland. In: Zeitschrift für Bevölkerungswissenschaft 29, 3-4, S. 363-386.

Schnell, Rainer; Hill, Paul B.; Esser, Elke (2005): Methoden der empirischen Sozialforschung. München: Oldenbourg (7. Auflage).

Schroedter, Julia H.; Lechert, Yvonne; Lüttinger, Paul (2006): Die Umsetzung der Bildungsskala ISCED-1997 für die Volkszählung 1970, die Mikrozensus-Zusatzerhebung 1971 und die Mikrozensen 1976-2004 (Version 1). ZUMA Methodenbericht 2006/08. Mannheim: ZUMA.

Schulze, Gerhard (1993): Die Erlebnisgesellschaft. Kultursoziologie der Gegenwart. Frankfurt a.M.: Campus.

Statistisches Bundesamt (2013): Bevölkerung und Erwerbstätigkeit. Bevölkerung mit Migrationshintergrund - Ergebnisse des Mikrozensus 2012. Fachserie 1 Reihe 2.2. Wiesbaden: Destatis.

Terwey, Michael (1990): Zur Wahrnehmung von wirtschaftlichen Lagen in der Bundesrepublik. In: Müller, Walter; Mohler, Peter Ph.; Erbslöh, Barbara; Wasmer, Martina (Hrsg.): Einstellungen und Verhalten der Bundesbürger. Opladen: Westdeutscher Verlag, S. 144-171.

TNS Infratest Sozialforschung (2012): Familienbezogene Leitbilder. Ergebnisse zum Pretest. München: TNS Infratest. 



\title{
Gesellschaftliche Leitbilder: Herkunft und Einflussfaktoren
}

\author{
Nadine Gies \& Dorothee Dietrich ${ }^{*}$
}

\section{Zusammenfassung}

Der Beitrag gibt eine kurze Einführung in den theoretischen Hintergrund der Analyse kollektiver Leitbilder und der öffentlichen Meinung sowie in das methodische Vorgehen des Zusatzmoduls, welches zur Validierung des Konstrukts „Allgemeinheit“ in den Fragebogen implementiert wurde. Dabei wird gezeigt, welche Orientierungsgeber den kollektiven Familienleitbildern überhaupt zugrunde liegen und welche Faktoren dabei die größte Bedeutung haben. Zudem ist von Interesse, wie sich einzelne Gruppen von Befragten in ihren Vorstellungen voneinander unterscheiden und welche persönlichen Eigenschaften einen Einfluss auf das Antwortverhalten ausüben. Das Zusatzmodul der Studie „Familienleitbilder" des BiB liefert nicht nur Angaben darüber, welche kulturellen und individuellen Leitbilder zur Familie in der Gesellschaft verbreitet sind, sondern kann darüber hinaus zeigen, von welchen Faktoren diese Idealvorstellungen abhängen. Insgesamt zeigt sich, dass Familienleitbilder aus Sicht der Befragten vor allem von Personen des direkten und weiteren persönlichen Umfeldes beeinflusst werden und man sich weniger an Institutionen orientiert.

\section{$1 \quad$ Einleitung}

Die meisten Menschen besitzen individuelle Vorstellungen davon, wie ihrer Meinung nach eine ideale Familie und eine ideale Partnerschaft aussehen sollten. Diese bildhaften Vorstellungen einer Gesellschaft bezeichnen wir als Familienleitbilder. Innerhalb eines Kulturkreises kann davon ausgegangen werden, dass viele Menschen bestimmte Familienleitbilder miteinander teilen und ihr eigenes Denken und Handeln innerhalb familienpolitischer und ökonomischer Rahmenbedingungen nach diesen Vorstellungen ausrichten. Der vorliegende Beitrag basiert auf einer Untersuchung der Forschungsgruppe „Familienleitbilder“ des Bundesinstituts für Bevölkerungsforschung (BiB) (vgl. Lück et al. 2013; Lück/Naderi/ Ruckdeschel, Kap. 3), welche zum Ziel hat, die individuellen Vorstellungen zu den als ideal empfundenen Formen der privaten Lebensführung zu erforschen. Neben der konkreten Ausgestaltung verschiedener handlungsrelevanter Leitbilder ist in diesem Zusammenhang die Analyse ihrer Herkunft und Beeinflussung durch andere Faktoren von besonderem Interesse.

Außer der Erhebung persönlicher Leitbilder der Familie wurden in der repräsentativen Studie daher ergänzend auch kulturell geteilte Leitbilder erfasst, welche durchaus von den individuellen Leitbildern, also den eigenen Idealvorstellungen von Familie, Partnerschaft

\footnotetext{
* Wir danken Kerstin Ruckdeschel für konstruktive Kritik und zahlreiche Hinweise und Anmerkungen.
} 
und Elternschaft abweichen können. ${ }^{1}$ Kulturelle Leitbilder beinhalten Wert- und Normvorstellungen einer Gesellschaft bzw. eines Teil-Kollektivs und werden als Verhaltenserwartung an deren Mitglieder herangetragen (vgl. Giesel 2007: 252). Im Bereich der Familie enthalten sie u.a. kollektiv geteilte Normalitätsvorstellungen der Partnerschaft, Elternschaft und Kindererziehung. In der modernen Gesellschaft herrscht dabei keine singuläre Vorstellung des Idealzustandes. Vielmehr zeigt sich im Übergang zur „Zweiten Moderne“ eine Pluralität miteinander konkurrierender gesellschaftlicher Leitbilder, an welchen sich das Individuum in seiner persönlichen Lebensplanung orientieren kann ${ }^{2}$ (vgl. Beck 1986). Hinzu kommt, dass sich die Idealvorstellungen meist nur noch auf Teilbereiche der Lebensführung beziehen und keine ganzheitlichen Verhaltensmuster zeichnen (vgl. Lukesch 1996: 153f.). Indem eine Auswahl unterschiedlicher allgemein geteilter Wertvorstellungen herrscht, unterscheiden sich die Individuen in ihrer Wahrnehmung und Akzeptanz kultureller Familienleitbilder und halten sich demnach an verschiedene Orientierungsgeber im direkten und indirekten persönlichen Umfeld. Vor allem die Variablen Alter, Bildung und Herkunftsland beeinflussen die Ansicht von der öffentlichen Meinung und die Orientierung an kollektiven Leitbildern. Um die Wahrnehmung der allgemein geteilten Leitbilder, an denen sich die individuellen Vorstellungen von Familie orientieren, sowie die Herkunft der beeinflussenden Faktoren zu analysieren, wurde ein Zusatzmodul der Studie „Familienleitbilder" des $\mathrm{BiB}$ angelegt. ${ }^{3}$ Dieses dient der Ermittlung der subjektiv empfundenen zugrundeliegenden Basis, auf welche sich die kulturell geteilten Familienleitbilder gründen, sowie der Validität des Konzeptes. Ziel ist es, diejenigen Personen und andere Faktoren (z. B. Medien) zu identifizieren, auf welchen die Annahme eines kollektiven Leitbildes beruht. In dem hier vorliegenden Beitrag soll gezeigt werden, an welchen Personen/-kreisen aus dem direkten oder indirekten sozialen Umfeld bzw. öffentlichen Medien sich die Individuen orientieren, wenn sie von der Meinung der Allgemeinheit sprechen.

Im Folgenden soll zunächst eine kurze Einführung in den theoretischen Hintergrund der Analyse kollektiver Leitbilder und der öffentlichen Meinung gegeben und das methodische Vorgehen des Zusatzmoduls erläutert werden. Anschließend werden die wichtigsten Ergebnisse der Erhebung dargestellt. Dabei wird gezeigt, welche Orientierungsgeber den kollektiven Familienleitbildern überhaupt zugrunde liegen und welche Faktoren dabei die größte Bedeutung haben. Zudem ist von Interesse, wie sich einzelne Gruppen von Befragten in ihren Vorstellungen voneinander unterscheiden und welche persönlichen Eigenschaften einen Einfluss auf das Antwortverhalten ausüben.

$1 \quad$ Kulturelle Leitbilder enthalten zwar gesellschaftlich geteilte Vorstellungen von einer als „ideal“ erachteten Familie, welche es anzustreben gilt. Da diese Bilder jedoch weitestgehend immateriell sind und nur in den Köpfen der Menschen existieren, kann dasselbe allgemeine Leitbild bei den einzelnen Individuen je nach Milieuzugehörigkeit und Sozialisationshintergrund zu einem gewissen Grad voneinander abweichen. Auch gibt es Unterschiede in den persönlichen Möglichkeiten der Realisierung gemeinschaftlicher Normvorstellungen. Zum Begriff des Leitbildes vgl. Diabaté/Lück 2014.

2 Diese Tendenz zur Pluralisierung der familialen Leitbilder hat bereits Wurzbacher in einer der ersten Studien zur Leitbildforschung innerhalb der Familiensoziologie festgestellt (vgl. Wurzbacher 1969: 250).

3 Die Ergebnisse des hier beschriebenen Zusatzmoduls entstammen einer deutschlandweit repräsentativen Telefonbefragung des Bundesinstituts für Bevölkerungsforschung von 5.000 Personen zwischen 20 und 39 Jahren zum Thema Familienleitbilder. 


\section{Theoretische Grundlagen}

\subsection{Definition der Begriffe „Allgemeinheit“ und „Öffentliche Meinung“}

Ziel der vorliegenden Studie war die Erfassung der individuell wahrgenommenen Meinung der Allgemeinheit, welche aus Sicht der sozialwissenschaftlichen Theorie die soziale Öffentlichkeit bzw. Gesamtheit darstellt, und von George Herbert Mead mit dem Begriff des „verallgemeinerten Anderen“ bezeichnet wird. ${ }^{4}$ In Bezug auf die von uns gewählte Fragestellung repräsentiert die Allgemeinheit damit das direkte und indirekte soziale Umfeld, in dem sich der Befragte befindet. Werden die Interviewten gefragt, was ihrer Ansicht nach die Meinung der „Allgemeinheit“ darstellt, so werden sie gebeten, die vermutete allgemein geteilte Meinung bzw. den allgemeinen gesellschaftlichen Konsens zu einem bestimmten Thema aus ihrer Sicht wiederzugeben. Die Allgemeinheit ist sowohl undifferenziert als auch unbestimmt. Das bedeutet, sie ist per se als Gruppe nicht klar abzugrenzen bzw. zu bestimmen. Jeder Interviewteilnehmer hat jedoch eine mehr oder weniger genaue Vorstellung von ihrer Zusammensetzung, da er sich in einem bestimmten sozialen Umfeld aufhält und von diesem in seinen Vorstellungen und Handlungen beeinflusst werden kann.

Mit dem Begriff der Allgemeinheit eng verknüpft ist der Begriff der „öffentlichen Meinung“. Sie wird definiert als „Ausdruck der Ansichten, Werturteile oder Willensneigungen des allgemeinen oder speziellen Publikums“ (Hillmann 1994). Zwar bezeichnet auch die öffentliche Meinung den allgemein geteilten Konsens zu einem bestimmten Thema. Für die Allgemeinheit wird aber zusätzlich auch die Bildung der öffentlichen Meinung und deren Regulierung näher beleuchtet. Die Herausbildung der öffentlichen Meinung wird hierbei eng mit den öffentlichen Medien verknüpft. Diese können Meinungsbildungsprozesse durch Art und Wahl der dargestellten Themen lenken. Durch die „veröffentlichten Meinungen" in Zeitungen, Zeitschriften, Fernsehen, Internet usw. wird somit die konkrete Ausgestaltung der öffentlichen Meinung in der Gesellschaft gesteuert. Gleichzeitig beeinflusst wiederum das in einer Gesellschaft wahrgenommene Meinungsbild die Berichterstattung in den Medien, so dass diese beiden Faktoren sich immer wechselseitig beeinflussen. Öffentliche Meinung darf dabei nicht mit der Summe aller Einzelmeinungen gleichgesetzt werden.

„Öffentliche Meinung ist nicht die Summe aller öffentlich geäußerten Meinungen von Öffentlichkeitsakteuren, sondern ein kollektives Produkt von Kommunikationen, das sich zwischen den Sprechern als ,herrschende“ Meinung darstellt“" (Neidhardt 1994: 26).

Stattdessen ist sie die Meinung, die aktuell den stärksten Zuspruch unter allen Gesellschaftsmitgliedern erfährt bzw. am wirksamsten verbreitet und somit als allgemein geltender Konsens durchgesetzt wird. Die tatsächlichen privaten Meinungen können jedoch teils erheblich von der zugeschriebenen öffentlichen Meinung abweichen. Sozialwissenschaftler haben sich bereits vielfältig mit der Entstehung der öffentlichen Meinung in Meinungsbildungs- bzw. Einfluss-Prozessen auseinandergesetzt. Im Folgenden soll die Theorie der

$4 \quad$ Mead erklärt den Begriff anhand der Identitätsbildung bei Kindern. Um in der Gesellschaft bestimmte Rollen übernehmen zu können, muss sich das Kind in die Verhaltensweisen und Erwartungshaltungen der Mitmenschen hineinversetzen und sein eigenes Verhalten danach ausrichten. Es „,versetzt sich an die Stelle des verallgemeinerten Anderen, der die organisierten Reaktionen aller Mitglieder der Gruppe repräsentiert.“ Der bzw. das „verallgemeinerte Andere“ können sowohl Personen als auch Gegenstände sein, an welchen man sich orientiert und die damit einen Einfluss auf die eigene Handlungsweise ausüben (vgl. Mead 1968: 194ff. sowie Berger/Luckmann 2007: 139ff.). 
Schweigespirale von Elisabeth Noelle-Neumann zur Erklärung der Entstehung öffentlicher Meinung herangezogen werden.

\subsection{Die Theorie der öffentlichen Meinung von Elisabeth Noelle-Neumann}

Die kommunikationswissenschaftliche Theorie der öffentlichen Meinung von Elisabeth Noelle-Neumann entstand im Zusammenhang mit der These der „Schweigespirale“ zu Beginn der 1970er Jahre. Bis heute stellt sie eine kontrovers diskutierte Annahme dar, welche die Meinungsbildung innerhalb der Bevölkerung zu erklären versucht. Sie besitzt vor allem deshalb bis heute Aktualität, weil Noelle-Neumann in Abgrenzung zu anderen Theoretikern ihrer Zeit als zentralen Faktor des Meinungsbildungsprozesses die Rolle der Medien hervorhebt.

Ihren Ausführungen zufolge beobachten Individuen mit Hilfe eines „quasi-statistischen Wahrnehmungsorgans" fortwährend und unbewusst ihre soziale Umwelt, um Aufschluss über das gerade herrschende Meinungsklima im Kollektiv zu erlangen. Dabei kann die reale öffentliche Meinung von der individuell wahrgenommenen abweichen (Roessing 2009: 124f.). Die aktuelle öffentliche Meinung kann auf zwei Arten erfasst werden. Zum einen dient der direkte Kontakt mit dem sozialen Umfeld der Feststellung des Meinungsklimas. Das Verhalten von Freunden und Bekannten hilft beispielsweise, die öffentliche Meinung in der Bevölkerung einschätzen zu können. Darüber hinaus werden die in den Massenmedien vermittelten Diskurse wahrgenommen und ermöglichen somit ebenfalls Rückschlüsse auf die öffentliche Meinung. Dabei kommt den Medien die so genannte „Artikulationsfunktion“ zu. Massenmedien sind immer gezwungen, alle aktuellen Vorkommnisse und Meinungen zu einem jeweiligen Thema darzustellen, wobei auch hier eine Verzerrung in der Wahrnehmung der tatsächlichen öffentlichen Meinung eintreten kann, indem eine inhaltliche Wertung stattfindet. Beispielsweise kann für eine bestimmte Meinungspartei die Initiative ergriffen, ausschließlich deren Meinung weiterverbreitet und andere Meinungen unberücksichtigt gelassen werden. An dieser Stelle setzt die „Schweigespirale“ ein (NoelleNeumann 1996).

Menschen, die der Ansicht sind, ihre Meinung werde eher von einer Minderheit der Bevölkerung geteilt, tendieren aus Angst vor sozialer Isolation zum Schweigen, also zur NichtArtikulation der eigenen Meinung. Dies bedeutet nicht zwangsläufig, dass eine Person ihre eigene Meinung abändert, die Meinung wird lediglich vor anderen Gesellschaftsmitgliedern verheimlicht, da sie als nicht dem allgemeinen Konsens entsprechend betrachtet wird. Auch die vermuteten Zukunftsaussichten einer bestimmten Auffassung sind wichtig für die Entwicklung der Kommunikationsbereitschaft. Ist ein Gesellschaftsmitglied der Auffassung, dass seine Meinung in Zukunft einen stärkeren Zuspruch erfahren wird, so steigt die Bereitschaft, diese zu kommunizieren. Ein sich selbst verstärkender Effekt entsteht, indem die vermuteten Minderheitsmeinungen somit fortwährend tatsächlich immer seltener öffentlich geäußert werden, wohingegen Vertreter der Mehrheitsmeinung weiterhin zur Kommunikation ihrer Meinung animiert werden. Somit entsteht ein Bevölkerungsteil, der sich stärker und öffentlich dominanter gibt, als er in der Realität ist, während die übrige Bevölkerung mit entgegengesetzten Auffassungen zunehmend in Schweigen verfällt und sich zurückzieht $^{5}$ (Hillmann 1994). Dieser Prozess wird durch die Massenmedien zusätzlich verstärkt. Sie reduzieren die komplexe Realität auf die wesentlichen Nachrichten, so dass Meinungs-

5 Noelle-Neumann sieht in ihrer Theorie zwar vor, dass es einzelne Individuen gibt, die sich dem sozialen Druck entziehen und sich entgegen der Schweigespirale äußern, diese Fälle gelten bei ihr aber als Ausnahme. 
lager übersichtlicher werden und somit von den einzelnen Gesellschaftsmitgliedern leichter bewertet werden können. Gleichzeitig können sie die Wahrnehmung der öffentlichen Meinung durch selektive Berichterstattung steuern (Noelle-Neumann 1994). Der Einfluss der Medien ist dabei umso stärker, je gleichförmiger die Inhalte an das Publikum vermittelt werden und diese einer konsonanten Berichterstattung folgen (Neidhardt 1994: 28).

Trotz ihrer allgemeinen Anerkennung kann Noelle-Neumanns Theorie nicht alle Aspekte im Prozess der Meinungsbildung ausreichend erklären. So können neben der genannten Isolationsfurcht auch andere Aspekte dazu beitragen, dass ein Mensch seine Meinung nicht öffentlich äußert. Ergänzend müssen auch persönliche Charaktereigenschaften der Individuen (wie z. B. das Peinlichkeitsempfinden) sowie die persönliche Betroffenheit von oder das Interesse an dem behandelten Thema für die Einschätzung der Redebereitschaft bedacht werden (vgl. Schulz/Rössler 2013: 32).

In den letzten Jahren hat es diverse Erweiterungen der Theorie der öffentlichen Meinung gegeben, die sich bemühten, problematische Gesichtspunkte der grundlegenden Theorie zu lösen. Für die vorliegende Studie ist hierbei besonders die Arbeit Helmut Scherers zu nennen. Er konnte in einer empirischen Untersuchung nachweisen, dass der Einfluss des sozialen Umfelds auf die Kommunikationsbereitschaft eines Individuums in der Arbeit Noelle-Neumanns tendenziell unterschätzt wurde. Noelle-Neumann ging davon aus, dass das unmittelbare soziale Umfeld kaum einen Einfluss auf Meinungsbildungsprozesse ausübt und stattdessen vor allem die Medien und das in der anonymen Gesamtgesellschaft wahrgenommene Meinungsklima von Bedeutung sind. Scherer konnte dies in seiner Studie widerlegen. Er fand heraus, dass Personen, die der Auffassung sind, ihre Meinung sei innerhalb des eigenen Bekanntenkreises in der Mehrheit, deutlich redebereiter sind, als diejenigen, die innerhalb ihres Bekanntenkreises eine Minderheitsmeinung vertreten. Die soziale Bezugsgruppe stellt somit einen der Faktoren dar, der die Redebereitschaft am maßgeblichsten mit beeinflusst und ist laut Scherer daher als bedeutender zu bewerten als beispielsweise der Einfluss der Massenmedien (vgl. Scherer 1990). Auch Schenk und Rössler kommen anhand einer Medienwirkungsstudie in den 1990er Jahren zu dem Ergebnis, dass die interpersonale Kommunikation im Alltag für die Meinungsbildung in der Forschung bis dahin unterschätzt, während der Medieneinfluss hingegen überschätzt wurde (Schenk/ Rössler 1994). So schreiben die Autoren den öffentlichen Medien vor allem eine Thematisierungsfunktion zu. Sie liefern zunächst einmal Informationen und relevante, öffentlich zu verhandelnde Gegenstände. Die Meinungsbildung der Adressaten über diese Themen findet anschließend vor allem im persönlichen Gespräch mit Verwandten, Freunden, Bekannten und Kollegen statt. Allerdings gibt es daneben viele weitere Faktoren, die bei der individuellen Meinungsbildung mit einfließen. So ist die Wahrscheinlichkeit einer vergleichsweise geringen Medienbeeinflussung vor allem bei solchen Personen gegeben, die über ein großes und heterogenes soziales Umfeld verfügen, in denen unterschiedliche Sichtweisen kommuniziert werden ${ }^{6}$. Bei Personen, die sich dagegen vor allem innerhalb ihrer Primärgruppen aufhalten, welche eher kongruente Meinungen aufweisen, ist der Medieneffekt stärker, da besonders über diese Kanäle Informationen bezogen werden (vgl. Schenk/Rössler 1994: 268f.). Dieser Eindruck kann auch in der von uns durchgeführten Studie bestätigt werden (vgl. Abschnitt 3).

Die Theorie der Schweigespirale und ihre Ergänzungen sind somit in der Lage, die wesentlichen Einflussfaktoren zu benennen, welche die öffentliche Meinung bestimmen. Für die vorliegende Studie ist sie von besonderer Relevanz, da sie Meinungsbildungsprozesse sowohl von dem direkten sozialen Umfeld einer Person abhängig macht als auch

6 Die Autoren sehen diese Konstellation vor allem bei jüngeren Personen gegeben. 
von den in den Massenmedien behandelten und vermittelten Themen und Botschaften. Außerdem zeigt sie, dass der Prozess der Aushandlung von kulturellen Leitbildern dynamisch verläuft, und stets andere Gruppen von Akteuren dabei an Deutungsmacht gewinnen können. Somit unterliegen kulturelle Leitbilder einem gesellschaftlichen Wandel oder gelten jeweils nur für bestimmte Bevölkerungsgruppen (vgl. Pfau-Effinger 2009: 120f.). Die Theorie der öffentlichen Meinung und der Schweigespirale verfolgt wie diese Studie den Ansatz, dass die Bildung und Wahrnehmung der öffentlichen Meinung von einem komplexen Zusammenspiel unterschiedlicher Faktoren abhängig ist. Noelle-Neumann kann schlüssig darlegen, warum die öffentliche Meinung nicht mit der Summe aller Individualmeinungen übereinstimmt. Die Theorie der Schweigespirale erklärt, warum es einzelnen Lagern gelingt, meinungsführend $\mathrm{zu}$ werden und ihre Meinung in der Öffentlichkeit zu artikulieren, während die Vertreter anderer Auffassungen teils unabhängig von deren tatsächlicher Verbreitung und Zustimmung innerhalb der Bevölkerung in Schweigen verfallen. Vor allem die theoretische Erweiterung zum Einfluss des sozialen Umfelds von Helmut Scherer ist in Zusammenhang mit dieser Studie sehr wertvoll und kann weitere wichtige Aspekte der Meinungsbildung beleuchten.

\section{Methodische Vorgehensweise}

Die Studie „Familienleitbilder“ des BiB erfasst in der Haupterhebung das persönliche Verständnis der Teilnehmer von individuellen und gesellschaftlichen Leitbildern an sich sowie innerhalb eines Zusatzmoduls auch deren Auffassung von gesellschaftlichen Orientierungsgebern, welche die gemeinsamen Vorstellungen verbreiten. Die Meinung, an welchen Personen oder öffentlichen Medien man sich bei der Angabe der geteilten Leitbilder orientiert bzw. welche Faktoren dabei einen höheren Stellenwert einnehmen, wurden innerhalb eines Zusatzmoduls der Studie „Familienleitbilder“ erfragt. Mittels einer zufallsbasierten Teilstichprobe wurden 537 ausgewählte Befragte der insgesamt 5.000 Teilnehmer im Anschluss an die Haupterhebung nach ihrem persönlichen Verständnis vom Begriff des Orientierungsgebers „Allgemeinheit“ und nach Schwierigkeiten im Umgang mit dieser Vorgehensweise befragt. ${ }^{7}$

Zur Operationalisierung des Forschungsgegenstandes wurde in der gesamten Befragung der Begriff „Allgemeinheit“ genutzt. Um den Befragten den inhaltlichen Unterschied in der Fragestellung zu verdeutlichen, wurde während des Interviews die Formulierung „Was denken Sie persönlich ...?“ bzw. „Was denkt die Allgemeinheit ...?“ gewählt, um die persönlichen Meinungen von der vermuteten Meinung der Gesamtgesellschaft zu trennen. Die angenommenen Meinungen der „Allgemeinheit“ bilden einen zentralen Aspekt der Untersuchung, an welchem sich die Individualmeinungen durch (partielle) Zustimmung oder Ablehnung orientieren können. Dabei war in der Zusatzerhebung von Interesse, an wen oder was die Befragten denken, wenn sie die vermutete öffentliche Meinung wiedergeben und wem folglich eine Bedeutung bei der Orientierung an gesellschaftlich geteilten Meinungen zukommt. Gegenstand dieser Befragung ist außerdem, ob es den Teilnehmern Probleme bereitet hat, sich in die vermuteten Meinungen eines Kollektivs einzufühlen und diese Vorstellung zu kommunizieren.

7 Für die Ergebnisse der Analyse des Zusatzmoduls siehe auch Dietrich/Gies 2012. 
Um eine Beeinflussung der Teilnehmer-Aussagen weitestgehend zu vermeiden, wurde in der Gesamtbefragung von Seiten der Interviewer der Begriff „Allgemeinheit“ lediglich zu Beginn des Interviews mit dem Hinweis auf „die vorherrschende Meinung in Deutschland, also das, was man im Alltag durch die Medien oder den Kontakt zu anderen Menschen vermittelt bekommt", umschrieben. In einer offenen Fragestellung sollten die Befragten der Zusatzerhebung zunächst erläutern, ,,an wen oder was sie bei der Frage nach der Allgemeinheit gedacht haben", also welche Personenkreise oder öffentliche Medien ihre Vorstellungen von kollektiven Familienleitbildern beeinflussen, um zunächst völlig unvoreingenommen durch die Interview-Situation spontane Orientierungsgeber zu benennen. Diese Angaben wurden inhaltsanalytisch erfasst und mit der Antwortverteilung auf die standardisierten Fragen verglichen, welche im Anschluss an die offene Fragestellung folgten. Die vorgegebenen Antwortkategorien umfassten mögliche Vorbilder, wobei die Zustimmung erhoben wurde, ob diese Orientierungsgeber dem Bild des Befragten von der „Allgemeinheit“ zugrunde liegen, oder nicht. ${ }^{8}$ Hierdurch wurden die Befragten eventuell auf weitere Einflussfaktoren hingewiesen, an welche sie in der offenen Antwortmöglichkeit nicht gedacht haben. Zur Bewertung der Zuverlässigkeit der Angaben der Befragten bzw. der Schwierigkeiten bei der Reflexion der Herkunft kollektiver Familienleitbilder wurde abschließend um eine Selbsteinschätzung seitens der Teilnehmer sowie eine Bewertung des Antwortverhaltens seitens der Interviewer gebeten.

In Vorbereitung auf die Datenauswertung mussten zunächst einige Variablen umcodiert werden. So wurden das Alter, das monatliche Haushaltseinkommen sowie die höchsten erreichten Schul- und Berufsabschlüsse der Befragten klassiert, um die Häufigkeiten der einzelnen Variablenausprägungen für die anschließenden statistischen Berechnungen darstellen zu können. Mittels Kreuztabellen wurde überprüft, welche soziodemografischen Merkmale das Antwortverhalten der Befragten beeinflussen. Es sollte beispielsweise geklärt werden, ob jüngere Personen bei bestimmten Einflussfaktoren häufiger zustimmen als ältere. Weitere Kontrollvariablen sind $\mathrm{u}$. a. das Einkommen, die schulische und berufliche Bildung sowie das Geschlecht der Befragten. Ein Chi-Quadrat-Test zeigte an, ob ein Zusammenhang zwischen den unabhängigen und den abhängigen Variablen besteht. Hier wurde das Zusammenhangsmaß „Exakter Test nach Fisher“ berechnet, da die erwarteten Zellhäufigkeiten teilweise unter dem Wert 5 lagen und fast ausschließlich nominal skalierte Variablen vorlagen. Um die Stärke der festgestellten Zusammenhänge zu messen, wurde der Korrelationswert Cramers-V gewählt.

\section{Die öffentliche Meinung als Orientierungsgeber für die persönliche Vorstellung von Familie}

\subsection{Befunde des Zusatzmoduls}

Im Folgenden sollen die zentralen Ergebnisse des Zusatzmoduls vorgestellt werden. Von den 537 interviewten Personen wurden in der vorangestellten offenen Frage nach der per-

Folgende Einflussfaktoren wurden abgefragt: fremde Menschen, denen man im Alltag begegnet; die Herkunftsfamilie; Personen aus dem näheren persönlichen Umfeld (Freunde, Kollegen und Bekannte, vom Stammtisch oder aus Vereinen); sowie die öffentlichen Medien (unterschieden wurde nach Printmedien und dem Fernsehen einschließlich der Werbung). 
sonellen Zusammensetzung des den Leitbildvorstellungen zugrundeliegendem „generalisierten Anderen“ insgesamt 876 Personen oder Orientierungsgeber" genannt, welche mithilfe der qualitativen Inhaltsanalyse ermittelt wurden. ${ }^{10}$ Abbildung 1 zeigt die vorliegende Antwortverteilung auf die einzelnen Über- bzw. Unterkategorien. Mit 49,1 \% aller Nennungen und damit am häufigsten wurde das direkte persönliche Umfeld der Befragten genannt. Hierzu zählen die eigene Herkunftsfamilie, Verwandte, Freunde, Bekannte und Arbeitskollegen. Danach folgt das weitere persönliche Umfeld der Teilnehmer mit 29,0 \% aller genannten Faktoren. In dieser Kategorie befinden sich fremde Personen, denen der Befragte im Alltag begegnet, bestimmte soziale Gruppen (wie z. B. Arbeitslose, junge Menschen oder Besserverdienende) sowie ganz allgemein die gesamte Bevölkerung in Deutschland. Die öffentlichen Medien (Printmedien, Hörfunk, Fernsehen und Internet) werden in 20,3\% aller Zitate erwähnt und sonstige Einflussfaktoren bilden schließlich eine Gruppe von $1,6 \%$ aller Äußerungen.

Abbildung 1: Ergebnisse der quantitativen Inhaltsanalyse zur offenen Frage nach der Allgemeinheit

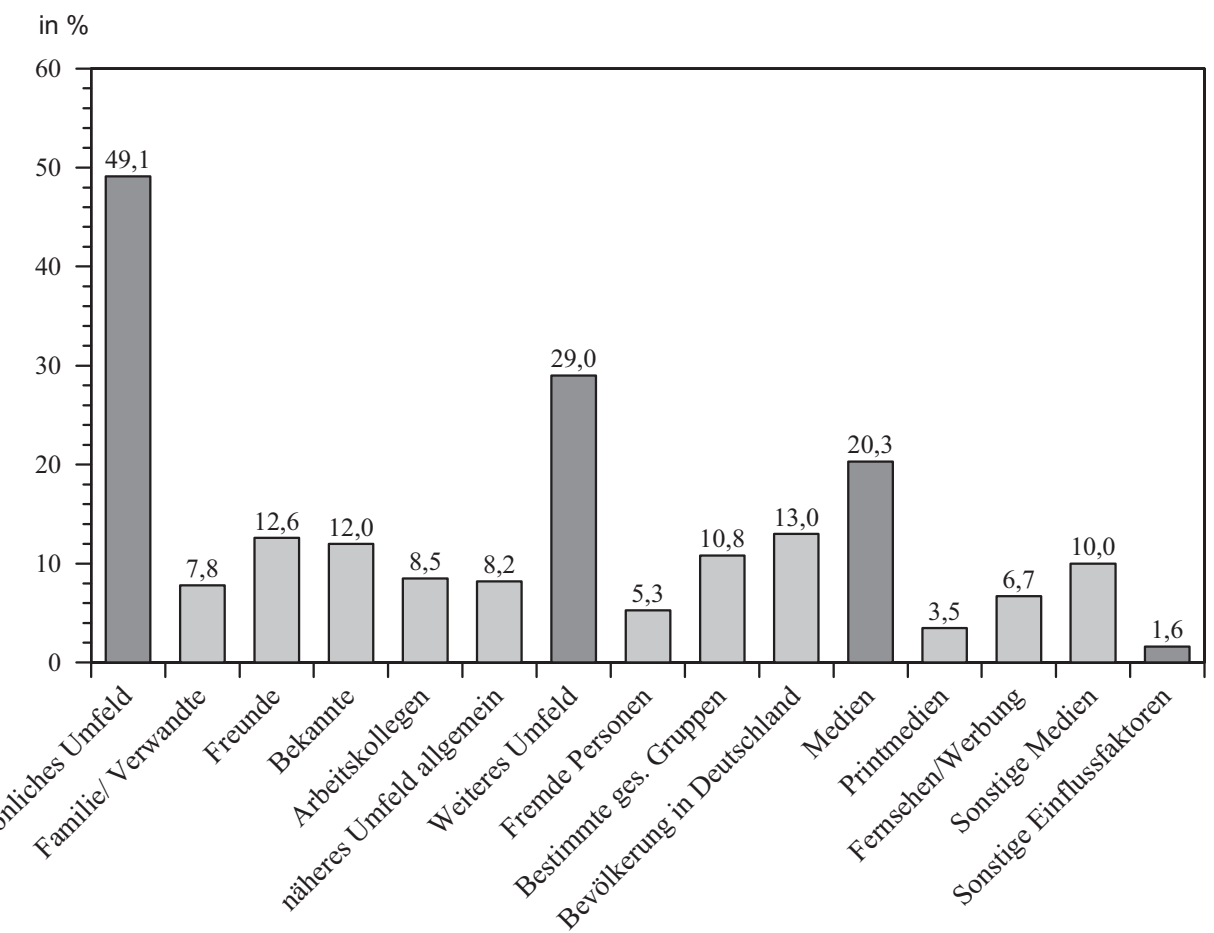

Quelle: FLB (2012), gewichtete Daten, eigene Berechnungen

9 Dabei handelt es sich um Personen oder Orientierungsgeber, welche von den befragten Personen als prägend für deren Vorstellungen von der Allgemeinheit empfunden werden. Von den Befragten genannt wurden einzelne Orientierungsgeber aus dem persönlichen oder weiteren sozialen Umfeld sowie den Medien.

10 Für eine detaillierte Darstellung der Forschungsergebnisse vgl. Dietrich/Gies 2013. 
In einer standardisierten Kernfrage des Zusatzmoduls wurde anschließend erhoben, welche der den Befragten vorgegebenen sozialen Gruppen und gesellschaftlichen Einflussfaktoren nach ihrer Meinung das Bild von der Allgemeinheit beeinflussen. Die Interviewten konnten hierbei zustimmen oder ablehnen, ob die ihnen genannten Faktoren ihrem Bild von der Allgemeinheit zugrunde liegen (vgl. Abbildung 2). Der hier verwendete Chi-Quadrat-Test hat ergeben, dass der Beobachtung (fremder) Mitmenschen mit einer Zustimmungsquote von 91,4\% der größte Einfluss auf die individuellen Leitbilder zugesprochen wird. Am zweitbedeutendsten wird mit 86,8 \% die Rolle von Freunden, Bekannten und Kollegen eingeschätzt. Es zeigt sich eine Differenz im Antwortverhalten zwischen der offenen und der standardisierten Frage. Das direkte persönliche Umfeld nimmt bei den spontan gegebenen Antworten den deutlich größeren Anteil vor dem weiteren Umfeld ein, es ist demnach ohne direkte Nachfrage des Interviewers präsenter. Werden jedoch verschiedene Antwortvorgaben gemacht, so ist der Abstand zwischen beiden Faktoren viel geringer. Kontakte zum weiteren persönlichen Umfeld sind zwar meist zahlreicher im Alltag vorhanden, dafür sind die Kontakte zum Freundes-, Bekannten- und Familienkreis intensiver, was letztlich zu der Einschätzung führt, dass beiden eine ähnlich große Bedeutung für die Handlungsorientierung zukommt.

Es folgt mit einer Zustimmungsrate von 68,7 \% die Rolle der Herkunftsfamilie, welche in dieser Interviewfrage separat erwähnt wurde. Der Einfluss der Medien wird mit einer Zustimmungsrate von 58,5\% in Bezug auf die Printmedien und mit 39,7 \% in Bezug auf Fernsehen und Werbung analog zur offenen Frage als weniger stark bewertet. Die geringste Bedeutung kommt nach Meinung der Teilnehmer der Religion zu. Diese weist lediglich eine durchschnittliche Zustimmungsrate von 9,3\% auf und wurde in den spontanen Antworten überhaupt nicht erwähnt.

Abbildung 2: Ranking der Einflussfaktoren gemessen an der Zustimmungshäufigkeit zur Frage „Woher, denken Sie, kommt Ihr Bild von der Allgemeinheit?“

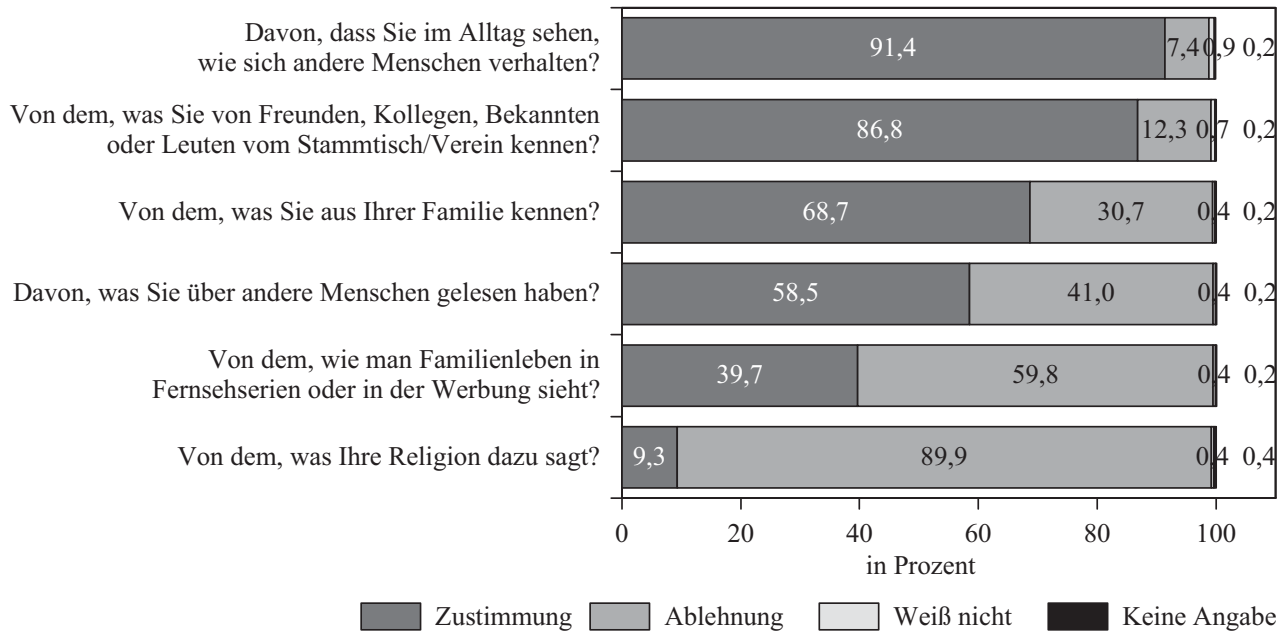

Quelle: FLB (2012), gewichtete Daten, eigene Berechnungen

Die Analyseergebnisse verdeutlichen die theoretische Annahme Scherers sowie Schenk und Rösslers (vgl. Abschnitt 2), dass dem sozialen Umfeld (bestehend aus den Mitmenschen, denen man im Alltag begegnet, den Freunden, Bekannten und Kollegen sowie der 
Herkunftsfamilie) der größte Einfluss auf die Bildung kultureller Leitbilder zugeschrieben wird. Auch Berger und Luckmann weisen auf die bedeutende Rolle der ,signifikanten Anderen“ hin (Berger/Luckmann 2007: 162). Ihre Deutungen der Wirklichkeit, der gesellschaftlichen Normen und Werte sind sehr viel stärker im Bewusstsein der Menschen verankert als diejenigen Wirklichkeitsauffassungen der restlichen Gesellschaft, mit denen man sich weniger stark identifiziert. Eine deutlich geringere Bedeutung kommt demgegenüber den institutionellen Orientierungsgebern öffentliche Medien sowie der Religion zu.

Im Folgenden war es für die Auswertung der Daten von Interesse, ob das Antwortverhalten der Befragten von deren soziodemografischen Merkmalen beeinflusst wird. So konnte z. B. vermutet werden, dass nur solche Befragte, die einer Religionsgemeinschaft angehören, auch einem Einfluss dieser auf die individuellen Leitbilder zustimmen oder dass vor allem solche Befragte einen Einfluss der Printmedien erkennen, welche ein gewisses Bildungsniveau aufweisen. Daher wurden die wichtigsten soziodemografischen Merkmale der Befragten mit deren Antwortverhalten abgeglichen. Als Maßzahl zum Nachweis eines Einflusses des jeweiligen betrachteten soziodemografischen Merkmals auf das Antwortverhalten wurde ein Chi-Quadrat-Test durchgeführt (vgl. Abschnitt 3). Es kann bestätigt werden, dass das Antwortverhalten der Befragten mit deren individuellen soziodemografischen Merkmalen zusammenhängt. Die wichtigsten Ergebnisse dieser Analyse sollen im Folgenden gesondert für jeden einzelnen Einflussfaktor dargestellt werden.

\subsection{Die Bedeutung des sozialen Umfelds}

Die beiden Faktoren, die am stärksten das Bild der Befragten von der öffentlichen Meinung beeinflussen, sind die Beobachtung (fremder) Mitmenschen sowie der Einfluss von Freunden, Bekannten und Kollegen. Es folgt der Einfluss durch die Herkunftsfamilie als am dritthäufigsten genannter Faktor. Das unmittelbare soziale Umfeld der Befragten wird somit allgemein als am bedeutsamsten für die Bildung sozialer Leitbilder eingeschätzt. Die große Bedeutung des sozialen Umfelds überrascht nicht, führt man sich die Vielzahl an nahezu täglichen flüchtigen Kontakten sowie den regelmäßigen Kontakt zu Freunden, Bekannten und Kollegen vor Augen, die ein Mensch im sozialen und gesellschaftlichen Umfeld erlebt. Der Kontakt zu (fremden) Mitmenschen unterscheidet sich dabei in Qualität und Quantität zu dem Umgang mit Freunden, Kollegen und Bekannten und der Herkunftsfamilie. Letztere stellen bewusst wahrgenommene Kontakte dar, die von den Betroffenen in der Regel initiiert werden und eine intensivere Form des sozialen Umgangs bedeuten als der Kontakt zu fremden Mitmenschen. Ihnen begegnet man im Alltag in großer Zahl, wobei das Zusammentreffen jedoch weniger nachhaltig ist. Umso bemerkenswerter erscheint es, dass gerade der Kontakt zu fremden Mitmenschen, der als unbewusst wirkender Orientierungsgeber angenommen wurde, als bedeutendster Faktor noch vor dem direkten sozialen Umfeld der Befragten bewertet wurde. Bedingt durch die explizite Nennung seitens der Interviewer, scheint den Befragten die Bedeutung dieses Einflussfaktors bewusst zu werden, so dass sie ihn entsprechend bewerten.

Von den soziodemografischen Merkmalen beeinflussen solche persönlichen Eigenschaften das Antwortverhalten der Befragten, die sich auf die Einbindung der Personen in das soziale Kollektiv auswirken. Das Maß an sozialer Eingebundenheit eines Individuums wurde anhand unterschiedlicher Variablen gemessen. Eine steigende Gemeindegröße bedeutet eine Zunahme der individuellen Freiheitsgrade, während die Integration in ein Kollektiv abnimmt (vgl. Bühlmann 2010: 218). Weiterhin bewirkt ein zunehmendes Alter der Befragten eine stärkere Einbindung. Auch das Einkommen besitzt einen Einfluss, da eine regelmäßige Teilnahme an gemeinsamen Aktivitäten einer Gruppe mit steigenden 
finanziellen Ressourcen leichter zu realisieren ist (vgl. Gerlach et al. 1993). So kann in Bezug auf die Beobachtung fremder Mitmenschen ein geringer Einfluss des Haushaltseinkommens und des Alters festgestellt werden, in Bezug auf die Bewertung des Einflusses von Freunden, Bekannten und Kollegen sind ebenfalls das Alter sowie die Gemeindegröße, in der die Befragten leben, und deren Erwerbssituation ausschlaggebend. Die Bewertung der Bedeutung der Herkunftsfamilie schließlich hängt ebenfalls von den Faktoren Gemeindegröße, Haushaltseinkommen und weiterhin dem Schulabschluss der Befragten ab. Der Grad der sozialen Integration ist abhängig von der biografischen Phase, in welcher sich die Befragten befinden, deren materieller Situation und somit der Möglichkeit der gesellschaftlichen Partizipation sowie dem individuellen Zugehörigkeitsgefühl zu einer Gemeinschaft. Mit abnehmender sozialer Eingebundenheit (z. B. bedingt durch einen geringeren Zusammenhalt in größeren Gemeinden und Städten, in denen mehr Möglichkeiten zur persönlichen Autonomie gegeben sind) nimmt demgegenüber die Zustimmungsneigung der Befragten ab. Generell bleibt jedoch festzustellen, dass der Einfluss der soziodemografischen Merkmale auf die Faktoren näheres sowie weiteres soziales Umfeld schwächer zu werten ist als ihr Einfluss auf andere abgefragte mögliche Einflussfaktoren. Dies erklärt sich vermutlich durch die Tatsache, dass jeder Mensch in irgendeiner Form in ein gesellschaftliches Kollektiv eingebunden ist, so dass ein Einfluss, ob positiv oder negativ ausgerichtet $^{11}$, fast automatisch festgestellt werden kann.

\subsection{Die Bedeutung der öffentlichen Medien}

Aus Sicht der Befragten kommt den öffentlichen Medien weniger Bedeutung zu, als Noelle-Neumann in der Theorie der Schweigespirale angenommen hat. Es zeigt sich aber ein Unterschied in der Art der Mediennutzung. Während knapp 60 \% der Befragten einen Einfluss der Printmedien auf ihre Vorstellung von der „Allgemeinheit“ erkennen, sind nur ca. $40 \%$ der Meinung, dass sie sich selbst am Fernsehen und der Werbung orientieren. Die Bewertung der Rolle der öffentlichen Medien spiegelt im Allgemeinen auch den persönlichen Zugang zu den verschiedenen Medienarten wider. Das Antwortverhalten wird vor allem durch den Bildungsabschluss und das Alter der Teilnehmer bestimmt. Personen mit einem höheren Bildungsniveau und in Deutschland geborene Personen, welche vermutlich über eine vergleichsweise hohe Lesekompetenz und damit einhergehende Lesefreude verfügen (vgl. Rammstedt 2013: 96ff.), bejahen den Einfluss von dem, was sie über Andere gelesen haben, auf ihre eigenen Vorstellungen. Das Lebensalter wirkt sich dahingehend aus, dass Ältere zwar häufiger Fernsehsendungen konsumieren, dabei aber weniger Einfluss auf die eigenen Leitbilder feststellen können. ${ }^{12}$

\subsection{Die Bedeutung der Religion}

Der Religion wird von den Befragten der geringste Einfluss auf ihr Bild von der „Allgemeinheit“ zugewiesen. Nur 9,3\% aller Befragten können bei einer direkten Nachfrage

11 Leitbilder können sowohl positiv im Sinne einer Übernahme gesellschaftlich verbreiteter Vorstellungen wirken, als auch im Sinne eines Negativ-Vorbildes, welches man gerade nicht für das eigene Leben realisieren möchte und daher zu vermeiden versucht. In beiden Fällen ist jedoch eine Orientierung an der „Allgemeinheit“ feststellbar (vgl. Diabaté/Lück 2014).

12 Vgl. graphische Darstellung der täglichen Fernsehdauer in Deutschland nach Altersgruppen im 1. Quartal 2011 und 2012 aus einem Fernsehpanel der AGF/GfK-Fernsehforschung: Quelle: www.statista.com. 
einen Einfluss erkennen, demgegenüber geben $89,9 \%$ an, keinen Einfluss auf die eigenen Vorstellungen von einer idealen Familie feststellen zu können. Die geringe Bedeutung zeigt sich weiterhin in der Tatsache, dass in der zuvor gestellten offenen Frage keiner der Befragten von sich aus religiöse Einflussgeber nennt.

Das Antwortverhalten wird vor allem von solchen soziodemografischen Merkmalen beeinflusst, die einen Einfluss auf die Religiosität eines Menschen ausüben. So stimmen Befragte, die einer Religionsgemeinschaft angehören, einer Orientierung an deren Inhalten eher zu als Konfessionslose. Weiterhin nimmt auch mit steigender Religiosität die Zustimmungsneigung zu. Schließlich ist ein Zusammenhang zwischen Gemeindegröße und Antwortverhalten erkennbar. Je kleiner die Gemeinde ist, in der ein Befragter lebt, desto eher stimmt er einer Beeinflussung individueller familiärer Leitbilder durch die Religion zu. Dieser Befund ist möglicherweise durch einen allgemein höheren Stellenwert der Kirche und ihrer Aktivitäten im ländlichen Raum zu erklären. Demgegenüber konkurriert die Kirche in Städten mit einer Vielzahl anderer Institutionen und Aktivitäten, so dass ihr in diesem Kontext eine geringere Bedeutung zugeschrieben werden muss.

Insgesamt hängt somit die Zustimmungsneigung von der Religiosität der Befragten und der Stärke des Einflusses der Kirche in deren Wohngebiet ab. Trotzdem bleibt festzustellen, dass bei allen Befragten (auch bei solchen Befragten, die sich als sehr religiös einschätzen) der Religion die geringste Bedeutung für ihre individuellen Leitbilder unter allen abgefragten Faktoren zugeschrieben wird. Andere Faktoren (wie zum Beispiel das Beobachten fremder Personen, die Herkunftsfamilie oder die Medien) werden in allen Befragtengruppen als bedeutender für die Bildung der individuellen Leitbilder eingestuft.

\subsection{Das Bewusstsein von der Handlungsorientierung an der öffentlichen Meinung}

Die Wiedergabe impliziter Leitbilder und deren gesellschaftlicher Beeinflussung ist keinesfalls eine leichte Aufgabe für die Studienteilnehmer. Das zu Grunde liegende Thema der Befragung ist zwar ein alltägliches, welches Jede und Jeden betrifft. Jedoch wirken gesellschaftliche Idealvorstellungen von Familie und Partnerschaft vor allem unbewusst auf biografische Entscheidungen und das Verhalten in Bezug auf andere Familienmitglieder, so dass einigen Studienteilnehmern deren Einflussnahme weniger präsent ist als anderen.

Etwas mehr als die Hälfte der Befragten (56,2 \%) gibt daher an, dass sie unsicher ist, ob sie treffend beschreiben kann, welche Meinung die Allgemeinheit vertritt. ${ }^{13}$ Die Ergebnisse der offenen Fragestellung ergaben, dass die Teilnehmer versuchen, sich bildlich vorzustellen, wie ihre Mitmenschen auf die vorliegenden Fragen antworten würden. Da die Vorstellung der den gesellschaftlichen Leitbildern zugrundeliegenden „Allgemeinheit“ zum Teil sehr heterogen ausgeprägt ist und verschiedene Personengruppen und Medienarten beinhaltet, ist es für die Befragten schwierig, die Wert- und Normvorstellungen einer solch uneinheitlichen Gruppe wiederzugeben. ${ }^{14}$ Eine heterogene Vorstellung der „Allgemeinheit“ weisen vor allem Personen mit höherem Bildungsabschluss auf, während Personen mit niedrigerem Bildungsabschluss kleinere Bezugsrahmen wählen. Daneben ist auch von Be-

13 Wortlaut der Frage im Interview: „Wenn wir Sie nach der Meinung der Allgemeinheit gefragt haben, hatten Sie da das Gefühl, immer sagen zu können, was die ,Allgemeinheit‘ denkt, oder nicht?““

14 Im Rahmen einer Vorstudie mittels leitfadengestützter qualitativer Interviews mit 29 Teilnehmern gaben die meisten Befragten an, dass es eine Übereinstimmung der eigenen Familienleitbilder mit denen der Freunde und Bekannten gebe. Darüber hinaus äußerten sich zwei Befragte aber auch dahingehend, dass sie die Leitbilder der Anderen nicht einschätzen könnten, da es keinen gegenseitigen Austausch über derartige Meinungen und Vorstellungen gebe (Dietrich/Gies 2012). 
deutung, wie geläufig Familienthematiken sind und wie sehr man sich über die eigene Position bereits Gedanken gemacht hat. So geben Eltern häufiger an, dass sie das Bild von der „Allgemeinheit“ beschreiben können im Vergleich zu jüngeren, kinderlosen Befragten, die mit dem Thema weniger vertraut sind und ihre Antworten daher aus Sicht der Interviewer auch weniger spontan geben. Jüngere, sich in Ausbildung befindliche Personen und niedriger Gebildete weisen darüber hinaus auch ein inkonsistenteres Bild von der öffentlichen Meinung auf. ${ }^{15}$ Was die Orientierung an gesellschaftlichen Idealvorstellungen betrifft, wechseln ihre Bezugspersonen häufiger als bei älteren Befragten, wodurch sie mit unterschiedlichen kulturell geteilten Idealvorstellungen konfrontiert werden können. Bei der überwiegenden Mehrheit der Befragten (65,9\%) bleiben die Einflussgeber auf die persönlichen Leitbilder hingegen nach eigener Ansicht konstant.

Auch wenn die Benennung gesellschaftlicher Orientierungsgeber - im Gegensatz zur Wiedergabe der eigenen Meinung über Familienleitbilder - keine sehr leichte Aufgabe darstellt, geben dennoch 86,9\% der Interviewpartner spontane Antworten auf die entsprechenden Fragen, welche Meinung die gesellschaftliche Öffentlichkeit vertritt. ${ }^{16}$ Ein geschlechtsbezogener Unterschied zeigt sich dahingehend, dass die Angaben von Frauen häufiger als unvermittelt eingeschätzt wurden im Vergleich zu denen der Männer. Eine Erklärung hierfür könnte sein, dass Frauen stärker in den familialen Lebensbereich involviert sind und sich dadurch häufiger mit familialen Themen beschäftigen, was ein spontanes Antworten ermöglicht. Auch eine Betrachtung der Aussagen aus der offenen Fragestellung nach der „Allgemeinheit“ zeigt, dass die meisten Teilnehmer eine konkrete Vorstellung hiervon haben. Der Anteil derjenigen, die sich überhaupt nicht äußern können oder wollen, liegt nur bei $3 \%$.

\section{$5 \quad$ Fazit}

Im vorliegenden Beitrag haben wir dargestellt, welchen Einfluss Faktoren aus dem persönlichen sozialen Umfeld, der Medienlandschaft und der Religion auf die Vorstellung von Familie haben. Die größte Bedeutung für die Bildung kollektiver Leitbilder haben aus Sicht der Befragten (fremde) Mitmenschen, denen man im Alltag begegnet, die Herkunftsfamilie sowie Freunde, Bekannte und Kollegen. Diese Faktoren werden am häufigsten genannt und zwar unabhängig von den persönlichen Eigenschaften der Studienteilnehmer. Häufig wird ein bestimmter Personenkreis mit der öffentlichen Meinung verbunden, bestehend aus verschiedenen Gruppen des sozialen Umfelds, wie beispielsweise Verwandte, Freunde und Bekannte oder Arbeitskollegen. Die Einflussnahme der öffentlichen Medien folgt neben dem direkten und dem weiteren persönlichen Umfeld an dritter Stelle. Hierbei differenzieren die interviewten Personen seltener nach bestimmten Medienarten. Stattdessen wird das Zusammenspiel aller Medien im Allgemeinen als prägender Einflussfaktor für das Bild von der „Allgemeinheit“ verstanden.

Die Bewertung des persönlichen Umfelds hängt von soziodemografischen Merkmalen ab, welche die soziale Teilhabe bzw. das Maß an Autonomie eines Menschen bestimmen, wie zum Beispiel die schulische und berufliche Bildung oder das Einkommen. Personen,

15 Wortlaut der Frage im Interview: "Hatten Sie bei den Fragen zur ,Allgemeinheit" immer dasselbe Bild vor Augen, oder nicht?"“

16 Aus Sicht der Interviewer. 
die einen niedrigen Bildungsgrad sowie ein geringes Einkommen aufweisen, lassen sich vor allem von anderen Menschen, denen sie im Alltag begegnen, der Herkunftsfamilie, Freunden, Bekannten und Kollegen beeinflussen.

Die Rolle medialer Einflussfaktoren wird hingegen von persönlichen Merkmalen beeinflusst, die den Zugang zu und den Umgang mit Medien beeinflussen. Hier sind vor allem die persönliche Freizeit neben der Berufstätigkeit zu nennen bzw. kulturelle Sprachbarrieren. Wer sich durch die Printmedien beeinflussen lässt, bezieht häufig ein höheres Einkommen, ist in Deutschland geboren und weist einen hohen Bildungsstand auf. Die Orientierung am Fernsehen und an der Werbung wird hingegen vor allem von den jüngeren Befragten, die sich noch in der Ausbildung befinden, sowie von Personen mit Migrationshintergrund bejaht.

Den Stellenwert der Religion und der Religiosität verstärken schließlich diejenigen soziodemografischen Eigenschaften, die das Zugehörigkeitsgefühl zu einer Religionsgemeinschaft wahrscheinlicher machen, wie z. B. das Leben in kleinen Gemeinden. Je stärker die Religiosität, desto höher fallen die Zustimmungsraten aus. Weniger religiöse Menschen lassen sich in ihren Meinungen und Handlungen vorwiegend von anderen Mitmenschen im Alltag sowie der Herkunftsfamilie beeinflussen. Jedoch kommt der Religion grundsätzlich die geringste Bedeutung für die individuelle Handlungsorientierung zu. Selbst bei Personen, die sich selbst als sehr religiös einschätzen, dominieren alle anderen genannten Faktoren.

Die Fähigkeit zur Reflexion gesellschaftlicher Leitbilder und deren Herkunft ist generell unterschiedlich ausgeprägt. Einigen Befragten fällt es leichter, ihre Vorstellungen von der Allgemeinheit abzurufen, als anderen. Vor allem solche Personen, die in ihrem eigenen Leben häufiger mit familialen Themen in Kontakt kommen, können ihr Bild von der Allgemeinheit spontan wiedergeben. So zeigt sich ein Geschlechterunterschied mit einer schnelleren Abrufbarkeit des Bildes von der Allgemeinheit auf Seiten der Frauen. Die Befragung konnte weiterhin zeigen, dass es einem Teil der Interviewten deshalb schwerfällt, ihr Bild von der Allgemeinheit abzurufen, weil sie sich der Heterogenität der gesellschaftliche Wert- und Normvorstellungen über Familie bewusst sind und daher glauben, nicht mit Sicherheit sagen zu können, was die Allgemeinheit denkt. Dabei sind es vor allem Menschen mit einem hohen Bildungsabschluss, die ein komplexes Wertgefüge der Allgemeinheit vor Augen haben und ihre Angaben dadurch mit Unsicherheiten behaftet sehen.

Das Zusatzmodul der Studie „Familienleitbilder“ des BiB lieferte somit nicht nur Angaben darüber, welche kulturellen und individuellen Leitbilder der Familie in der Gesellschaft verbreitet sind, sondern konnte darüber hinaus zeigen, von welchen Faktoren diese Idealvorstellungen abhängen. Insgesamt kann festgestellt werden, dass Familienleitbilder aus Sicht der Befragten vor allem von Personen des direkten und indirekten persönlichen Umfeldes beeinflusst werden und man sich weniger an Institutionen orientiert. Entgegen der Meinung Noelle-Neumanns über die Entstehung der öffentlichen Meinung sind die Diskurse in den öffentlichen Medien von geringerer Bedeutung. Dies ist ein Befund, der erstaunt, angesichts der Allgegenwart der öffentlichen Medien, welche in der modernen Gesellschaft tagtäglich gesellschaftliche Normen und Werte verbreiten. Ihnen kommt zwar eine Thematisierungsfunktion $\mathrm{zu}$ und sie reduzieren Komplexität, indem die öffentliche Meinung auf verschiedene Meinungslager der einzelnen Parteien aufgeteilt wird, was es dem Einzelnen erleichtert, sich einen eigenen Standpunkt zu bilden. Das Thema Familie und Partnerschaft ist jedoch ein sehr persönliches, weshalb menschliche Kontakte und der Dialog bei der Meinungsbildung und Entscheidungsfindung im Vordergrund stehen und mediale Einflüsse weniger präsent sind. Es ist allerdings durchaus möglich, dass es sich bei den öffentlichen Medien um einen unbewussten Einflussfaktor handelt, der auf die Entste- 
hung der individuellen Leitbilder einwirkt, von den Befragten jedoch nicht als solcher realisiert wird. Die größte Rolle für die bewusste Handlungsorientierung spielen hingegen das direkte und das indirekte persönliche Umfeld, in welchen bestimmte Leitvorstellungen vorgelebt und miteinander besprochen werden.

\section{Literatur}

Beck, Ulrich (1986): Risikogesellschaft. Auf dem Weg in eine andere Moderne. Frankfurt am Main: Suhrkamp.

Berger, Peter L.; Luckmann, Thomas (2007): Die gesellschaftliche Konstruktion der Wirklichkeit. Eine Theorie der Wissenssoziologie. 21. Auflage. Frankfurt am Main: Fischer Taschenbuch Verlag.

Bühlmann, Marc (2010): Kommunale Identität. Eine Mehrebenenanalyse der Determinanten individueller Verbundenheit mit der Gemeinde. In: Zeitschrift für vergleichende Politikwissenschaft 4, S. 203-231.

Diabaté, Sabine; Lück, Detlev (2014): Familienleitbilder. Identifikation und Wirkungsweise auf generatives Verhalten. In: Zeitschrift für Familienforschung 26, S. 49-69.

Dietrich, Dorothee; Gies, Nadine (2012): Familienleitbilder. Ergebnisse einer qualitativen Leitfadenstudie. Projektbezogene Publikation. Wiesbaden: Bundesinstitut für Bevölkerungsforschung.

Dietrich, Dorothee; Gies, Nadine (2013): Woher stammen Familienleitbilder? Eine Analyse des Zusatzmoduls der Studie „Familienleitbilder“ (FLB) unter besonderer Beachtung der Orientierung an der öffentlichen Meinung. Projektbezogene Publikation. Wiesbaden: Bundesinstitut für Bevölkerungsforschung.

Gerlach, Frank; Kronauer, Martin; Vogel, Berthold (1993): Im Schatten der Arbeitsgesellschaft. Arbeitslose und die Dynamik sozialer Ausgrenzung. Frankfurt am Main, New York: Campus.

Giesel, Katharina D. (2007): Leitbilder in den Sozialwissenschaften. Begriffe, Theorien und Forschungskonzepte. Wiesbaden: VS Verlag für Sozialwissenschaften.

Hillmann, Karl-Heinz (Hrsg.) (1994): Wörterbuch der Soziologie. Stuttgart: Kröner.

Lück, Detlev; Gründler, Sabine; Naderi, Robert; Dorbritz, Jürgen; Schiefer, Katrin; Ruckdeschel, Kerstin; Hiebl, Johannes; Wolfert, Sabine; Stadler, Manuela; Pupeter, Monika (2013): Familienleitbilder 2012. Methodenbericht zur Studie. BiB Daten- und Methodenberichte 2/2013. Wiesbaden: Bundesinstitut für Bevölkerungsforschung.

Lukesch, Helmut (1996): Leitbilder in der Familienerziehung. In: Vaskovics, Laszlo A.; Lipinski, Heike (Hrsg.): Ehe und Familie im sozialen Wandel. Familiale Lebenswelten und Bildungsarbeit. Interdisziplinäre Bestandsaufnahme 1. Opladen: Leske + Budrich, S. 153-184.

Mead, George Herbert (1968): Geist, Identität und Gesellschaft aus Sicht des Sozialbehaviorismus. Frankfurt am Main: Suhrkamp.

Neidhardt, Friedhelm (1994): Öffentlichkeit, öffentliche Meinung, soziale Bewegungen. In: Neidhardt, Friedhelm (Hrsg.): Öffentlichkeit, öffentliche Meinung, soziale Bewegungen. Kölner Zeitschrift für Soziologie und Sozialpsychologie, Sonderheft 34. Opladen: Westdeutscher Verlag, S. 7-41.

Noelle-Neumann, Elisabeth (1994): Wirkung der Massenmedien auf die Meinungsbildung. In: Noelle-Neumann, Elisabeth; Schulz, Winfried; Wilke, Jürgen (Hrsg.): Publizistik. Massenkommunikation. Frankfurt am Main. S. 518-571.

Noelle-Neumann, Elisabeth (1996): Öffentliche Meinung. Die Entdeckung der Schweigespirale. Erweiterte Ausgabe. Frankfurt/Main: Ullstein.

Pfau-Effinger, Birgit (2009): Unterschiede in der Kinderbetreuung im Ländervergleich im Rahmen kultureller und familienpolitischer Kontextbedingungen. In: Kapella, Olaf; Rille-Pfeiffer, Christiane; Rupp, Marina; Schneider, Norbert F. (Hrsg.): Die Vielfalt der Familie. Wiesbaden: VS Verlag für Sozialwissenschaften, S. 113-130.

Rammstedt, Beatrice (Hrsg.) (2013): Grundlegende Kompetenzen Erwachsener im internationalen Vergleich. Ergebnisse von PIAAC 2012. Münster, New York, München, Berlin: Waxmann. 
Roessing, Thomas (2009): Öffentliche Meinung - die Erforschung der Schweigespirale. Baden-Baden: Nomos.

Schenk, Michael; Rössler, Patrick (1994): Das unterschätzte Publikum. Wie Themenbewusstsein und politische Meinungsbildung im Alltag von Massenmedien und interpersonaler Kommunikation beeinflusst werden. In: Neidhardt, Friedhelm (Hrsg.): Öffentlichkeit, öffentliche Meinung, soziale Bewegungen. Kölner Zeitschrift für Soziologie und Sozialpsychologie, Sonderheft 34. Opladen: Westdeutscher Verlag, S. 261-295.

Scherer, Helmut (1990): Massenmedien, Meinungsklima und Einstellung. Eine Untersuchung zur Theorie der Schweigespirale. Studien zur Sozialwissenschaft, Bd. 101. Opladen: Westdeutscher Verlag.

Schulz, Anne; Rössler, Patrick (2013): Schweigespirale online. Die Theorie der öffentlichen Meinung und das Internet. Baden-Baden: Nomos.

Wurzbacher, Gerhard (1969): Leitbilder gegenwärtigen deutschen Familienlebens. Methoden, Ergebnisse und sozialpädagogische Forderungen einer soziologischen Analyse von 164 Familienmonographien. Stuttgart: Ferdinand Enke-Verlag. 


\title{
Was ist Familie? Familienleitbilder und ihre Vielfalt
}

\author{
Detlev Lück \& Kerstin Ruckdeschel
}

\section{Zusammenfassung}

Hinsichtlich der Frage, was eine Familie sei, haben die Menschen ein im Kern einheitliches und in seiner äußeren Abgrenzung sehr heterogenes Leitbild. Anhand von vorgegebenen Lebensformen, für die Befragungsteilnehmer der Studie Familienleitbilder (FLB) 2012 angeben sollten, ob es sich dabei jeweils um eine Familie handele, stimmen nahezu alle Befragten zu, dass eine Kernfamilie, bestehend aus einem zusammenwohnenden heterosexuellen Paar mit Kindern, eine Familie sei. Die Beurteilung der übrigen Lebensformen scheint sich an ihrer Ähnlichkeit zur Kernfamilie zu orientieren. Allerdings gelten für die meisten Menschen auch viele nichtkonventionelle (d. h. von der Kernfamilie abweichende) Lebensformen als Familie, vor allem dann, wenn Kinder darin leben. Ein knappes Drittel sieht in jeder Lebensgemeinschaft eine Familie, auch ohne Kinder und ohne Trauschein. Die Bewertung variiert nach der sozialen Lage der Befragten. Außerdem neigen Menschen dazu, die Lebensform, in der sie selbst leben, eher als Familie wahrzunehmen, als andere Menschen dies tun.

\section{$1 \quad$ Einleitung}

Was ist eine Familie? Geht man vom Konzept der Familienleitbilder aus, demzufolge Menschen bildhafte komplexe Vorstellungen verinnerlicht haben, wie Familienleben „normalerweise“ aussieht (Lück/Diabaté, Kap. 2), muss angenommen werden, dass wir auch Bilder von der „normalen“ strukturellen Zusammensetzung einer Familie im Kopf haben: davon, wie die Personengruppe aussieht, die eine Familie bildet. Ist das der Fall? Und wenn ja, welche Personengruppe oder welche Personengruppen haben wir dabei vor Augen? Bis vor einigen Jahrzehnten war davon auszugehen, dass es sich bei diesem Bild um die „klassische“ bürgerliche Kernfamilie im Parson'schen Sinne handeln müsste - mit Vater, Mutter und ungefähr zwei Kindern (Parsons 1954: 109f.). Trifft das in Zeiten der Individualisierung und der Pluralisierung der Lebensformen immer noch zu? Oder mischen sich heute andere Lebensformen darunter? Welche Vorstellungen von Familie gibt es derzeit, und von wie vielen werden sie jeweils geteilt? Diesen Fragen soll im Folgenden mit Hilfe der Daten der Studie Familienleitbilder (FLB) 2012 (vgl. Lück/Naderi/Ruckdeschel, Kap. 3) nachgegangen werden, in der 5.000 20- bis 39-Jährige telefonisch zu ihren Familienleitbildern befragt wurden. Darüber hinaus wird die Passung zwischen Leitbild und Realität untersucht: Gibt es wechselseitige Einflüsse zwischen der Lebensform, in der man lebt, und dem Leitbild dessen, wie eine Familie aussieht?

\footnotetext{
* Wir danken der anonymen Gutachterin bzw. dem anonymen Gutachter für wertvolle Hinweise.
} 


\section{Forschungsstand und Vorüberlegungen}

Die Bedeutung des Begriffs Familie hat sich historisch mehrfach verändert - ebenso wie die Lebensformen, in denen Menschen typischerweise gelebt haben bzw. leben.

\subsection{Historische Entwicklung von Familie und Familienbegriff}

In vorindustrieller Zeit (bis ins 18. Jahrhundert) waren die Familienformen in hohem Maße durch Erfordernisse der Existenzsicherung bestimmt (Rosenbaum 2014: 21f.; Mitterauer 1980a: 20; Sieder 1980: 145). Der Begriff Familie meinte weniger einen Verwandtschaftszusammenhang als einen Haushalt, das ,ganze Haus“, in dem nicht nur zusammen gelebt, sondern auch zusammen produziert wurde (Mitterauer 1980a: 21f., Sieder 1980: 143). Meist handelte es sich dabei um einen landwirtschaftlichen Betrieb. In diesem lebte als Familie zusammen, wer auch den Hof gemeinsam bewirtschaftete: neben einem Ehepaar mit eigenen Kindern typischerweise auch andere unverheiratete Verwandte sowie Gesinde. Gleichzeitig konnten eigene (biologische) Kinder als Mägde oder Knechte auf anderen Höfen leben (Rosenbaum 2014: 20f.; Mitterauer 1980b: 43f., 59f.). Entscheidend für die Haushaltsgröße war in erster Linie die Größe des Hofes, die einerseits ein bestimmtes Minimum an Arbeitskräften zu seiner Bewirtschaftung erfordert und andererseits ein bestimmtes Maximum an Menschen versorgen konnte. Kinder durften legitim nur in einer Ehe geboren werden; das Recht zu heiraten wurde demjenigen zugesprochen, der eine Familie ernähren konnte - wer beispielsweise einen Handwerksbetrieb in der Stadt oder vom Vater den Hof vererbt bekommen hatte. Somit diente die Ehe als Regulativ, um zu gewährleisten, dass nur Kinder geboren werden, für die auch materiell gesorgt ist (Rosenbaum 2014: 22f.; Mitterauer 1980b: 54f.; Sieder 1980: 145). Der Familienvater, der den Hof besaß, von dem jeder existentiell abhängig war, genoß absolute Autorität (Mitterauer 1980a: 20).

Die Industrialisierung (gegen Ende des 19. und Anfang des 20. Jahrhunderts) brachte einige Verschiebungen mit sich. Die wichtigste neue Rahmenbedingung war die Trennung von Wohn- und Arbeitsstätte (Rosenbaum 2014: 35; Gestrich 2003: 387f.). Wer seinen Lebensunterhalt mit Fabrikarbeit bestritt, hatte wenig Verwendung für Mägde und Knechte im privaten Haushalt. Auch mussten unverheiratete Verwandte nicht mitversorgt werden; im Gegenzug mussten Verwitwete oder Geschiedene nicht neu heiraten, um eine Produktivkraft zu ersetzen. So schrumpfte der private Haushalt typischerweise zu einer Kernfamilie oder gar zur Ein-Eltern-Familien zusammen (Mitterauer 1980b: 57f.). Die Säuglingsund Kindersterblichkeit sank; zeitlich versetzt dazu sank auch die Geburtenrate (Mitterauer 1980b: 61). Gleichzeitig erhielt der Haushalt den Charakter eines privaten Rückzugsortes von der Arbeitswelt (Gestrich 2003: 391). Die gravierenden sozialen Umbrüche lösten Ängste aus, auf die die Gesellschaft mit einer romantischen Überhöhung der Familie reagierte. Hier entstand das Bild der Familie als Kernfamilie mit ihrer positiven Konnotation (Gestrich 2003: 381f.). In der bürgerlichen Oberschicht entstand die komplementäre Geschlechterrollenteilung zwischen Ehefrau und Ehemann (zunächst nur als wenig praktiziertes normatives Leitbild) als Lösung für das Vereinbarkeitsproblem, das durch die Trennung von Wohn- und Arbeitsstätte aufkam (Rosenbaum 2014: 34; Gestrich 2003: 391f.). Weite Verbreitung in der Praxis fand die komplementäre Geschlechterrollenteilung allerdings erst in den Jahren des wirtschaftlichen Aufschwungs nach dem Zweiten Weltkrieg, in denen es sich tatsächlich viele Familien leisten konnten, auf ein zweites Erwerbseinkommen zu verzichten (Nave-Herz 2006: 48f.). 
In der Wohlstandsgesellschaft der 1960er Jahre kam es zum Wertewandel vom Materialismus zum Postmaterialismus (Inglehart 1990), und damit zum zweiten demografischen Übergang (Lesthaeghe 1992). Infolgedessen setzte eine graduelle Pluralisierung der Lebensformen ein, die bis heute anhält (Kuhnt/Steinbach 2014: 45f.; Nave-Herz 2012: 13f.; Wagner 2008; Brüderl 2003). Der Automatismus, nach dem Menschen mit dem Eintritt ins Berufsleben einen Partner gesucht, geheiratet und Familien gegründet haben, wird hinterfragt. Lebensform und Familienbiografie werden der individuellen Entscheidung überlassen und an dem Kriterium gemessen, dass der Einzelne damit glücklich werde. Vielfalt wird zunehmend akzeptiert und gelebt (ebd.). Nichteheliches Zusammenleben wird nicht nur geduldet, sondern im Sinne einer vorehelichen Probephase für eine Beziehung zu einer neuen Norm. Scheidung wird nicht nur enttabuisiert; sie wird von jenen, die in einer Beziehung unglücklich sind, erwartet. Homosexualität wird nicht nur entkriminalisiert; sie kann in Form einer eingetragenen Lebenspartnerschaft formalisiert werden. Auch wenn die Kernfamilie die häufigste Lebensform bleibt und andere Lebensformen in den meisten Biografien „nur“ im Sinne neuer Lebensphasen vor und nach dem ehelichen Zusammenleben mit Kindern hinzukommen, so wird die Normalität des Familienlebens doch zumindest graduell vielfältiger (ebd.).

In der Familienforschung wird diese Entwicklung von einer Erweiterung des Familienbegriffes begleitet. Orientierte sich der Familienbegriff in den ersten Nachkriegsdekaden noch an der Ehe als maßgeblichem Kriterium, gilt seit dem späten 20. Jahrhundert jede Verantwortungsgemeinschaft zwischen Eltern und Kindern als Familie (Schneider 2008: 12). Ausschlaggebend ist nicht Blutsverwandtschaft, sondern die soziale Beziehung zwischen Menschen zweier Generationen - Stief-, Pflege- und Adoptivelternschaften mit eingeschlossen. In den vergangenen zwei Jahrzehnten etabliert sich zunehmend ein dritter Familienbegriff: die Definition der Familie als Solidarbeziehung. Familie ist demnach eine exklusive Solidargemeinschaft zwischen zwei oder mehreren Personen, die auf relative Dauer ausgerichtet ist, unabhängig davon, aus wie vielen Generationen sie besteht. Somit werden zunehmend mehr Lebensformen auch in den Familienbegriff eingeschlossen, bis wenig mehr als Alleinstehende übrig bleiben (Schneider 2008: 12f.).

\subsection{Vorstellungen von Familie in der Gegenwart}

Es ist davon auszugehen, dass mit einer veränderten Normalität, die die Menschen im Alltag erleben, auch ihre Normalitätsvorstellungen und Leitbilder andere werden. $\mathrm{Zu}$ der Frage, inwieweit das tatsächlich der Fall ist, gibt es ambivalente Befunde, die überwiegend keine grundlegende Ausweitung der Vorstellungen von Familie erkennen lassen. Für ein verändertes Leitbild spricht, dass die Akzeptanz nichtkonventioneller Lebensformen - also von Lebensformen jenseits der Kernfamilie - von der Einstellungsforschung seit geraumer Zeit als sehr hoch festgestellt wird; das gilt ganz besonders für nichteheliche Lebensgemeinschaften (Matthias-Bleck 2005; Busch/Scholz 2005: 132f.). Zudem geben Menschen, befragt danach, wen sie zu ihrer eigenen Familie zählen, sehr unterschiedliche und heterogene Netzwerke an, die entferntere Verwandte, Freunde, Nachbarn und Haustiere beinhalten können und die mit der Konstellation einer Kernfamilie selten übereinstimmen (Widmer 2010: 17f.). Dafür, dass trotz der Akzeptanz von Vielfalt die Vorstellungen dessen, was normal, optimal oder richtig sei, weiterhin stark an Ehe und Kernfamilie ausgerichtet sind, spricht, dass Abweichungen von der Kernfamilie dennoch oft negativ konnotiert werden (Tazi-Preve 2003: 445f.), zumal sie auch nicht in jedem Fall frei gewählt sind. Ehe oder zumindest Partnerschaft und Familiengründung spielen in der Lebensplanung der meisten Menschen auch heute eine zentrale Rolle (Busch/Scholz 2005: 132f.; 
Nave-Herz 2002: 62f.). So selbstverständlich davon ausgegangen wird, dass eine Biografie Phasen des Alleinlebens, des Getrenntwohnens, des nichtehelichen und des kinderlosen Zusammenlebens enthält, so deutlich scheint für viele Menschen dennoch das Leben mit (Ehe-)Partner und Kindern den Zielpunkt zu markieren, auf den eine solche Lebensplanung zuläuft und mit dem sie ihre Erfüllung findet. Die Kernfamilie wird als ein Ideal empfunden, das Stabilität und Funktionalität gewährleistet, und mit Attributen wie „normal“, „richtig“, „komplett“ oder „optimal“ beschrieben (Zartler 2012: 75). Scheidung wird zwar als gesellschaftlich akzeptiert wahrgenommen, aber dennoch von vielen als Auflösung der Familie gesehen (ebd.: 72f.), die „,eine defizitäre Lebensform“ zurücklässt (ebd.: 76).

Auf Basis dieser Befunde erscheint ein Resümee, wie das Leitbild einer Familie heute aussieht, auf den ersten Blick schwierig. Es ist möglich, dass Menschen im Hinblick auf die Frage, was eine Familie sei, differenzierte Leitbilder haben, die die Kernfamilie als Optimum und Koordinatenbezugspunkt und daneben eine Vielfalt unterschiedlicher Lebenskonstellationen als relevanten und annehmbaren Teil der Lebenswirklichkeit umfassen.

\section{Ergebnisse}

Für die obigen Fragestellungen bietet sich die Studie Familienleitbilder (FLB) 2012 (vgl. Lück/Naderi/Ruckdeschel, Kap. 3) als Datengrundlage an, da sie sowohl Fragen zur Bewertung verschiedener Lebensformen als Familie enthält, als auch Informationen, aus denen sich die tatsächlich gelebte Lebensform rekonstruieren lässt. Um zu erfassen, was in den Augen der befragten Person eine Familie sei, wurden ihr sieben verschiedene konkrete Lebensformen beschrieben, die sie jeweils mit „Ja, das ist eine Familie“ oder „Nein, das ist keine Familie“ einstufen sollte. Die Befragten hatten keine Möglichkeit, ihre Antworten abzustufen. Im Folgenden werden zunächst die Antworten auf die Frage dargestellt, was als Familie bewertet wird. Im Anschluss daran werden die Ergebnisse nach verschiedenen sozialstrukturellen Merkmalen der Befragten differenziert analysiert. In einem dritten Teil werden Leitbilder und tatsächliche Lebensform der Befragungsteilnehmer einander gegenübergestellt.

\subsection{Welche Vorstellungen von Familie sind wie weit verbreitet?}

In Abbildung 1 ist die Zustimmung, dass bestimmte vorgegebene Lebensformen jeweils eine „Familie“ seien, in absteigender Reihenfolge in Prozent abgetragen. Dabei sieht man zunächst, dass ein verheiratetes, heterosexuelles, zusammenwohnendes Paar mit Kindern - also die Kernfamilie - von so gut wie allen Befragten (99,9\%) als Familie angesehen wird. Es ist zu vermuten, dass diese „klassische“ Familie als eine Art „Normalfolie“ gilt, an der andere Lebensformen gemessen werden. Diese werden dann möglicherweise anhand des Grades ihrer Ähnlichkeit mit der Kernfamilie als Familie eingestuft - oder nicht. Aufgrund der gestiegenen sozialen Akzeptanz unterschiedlicher Lebensformen (vgl. Abschnitt 2) lässt sich vermuten, dass auch bei der Einschätzung, was eine Familie sei, heute Abweichungen von der Kernfamilie mit einer größeren Toleranz bewertet werden als vor einigen Jahrzehnten. So macht es für die Akzeptanz einer Lebensform als Familie nahezu keinen Unterschied mehr, ob ein Elternpaar verheiratet ist oder nicht, denn bei einem zusammenlebenden Paar mit Kindern handelt es in den Augen fast aller Befragten $(97,4 \%)$ auch ohne Trauschein um eine Familie. 
Abbildung 1: Was ist Familie? Beurteilung vorgegebener Lebensformen (Zustimmung in Prozent)

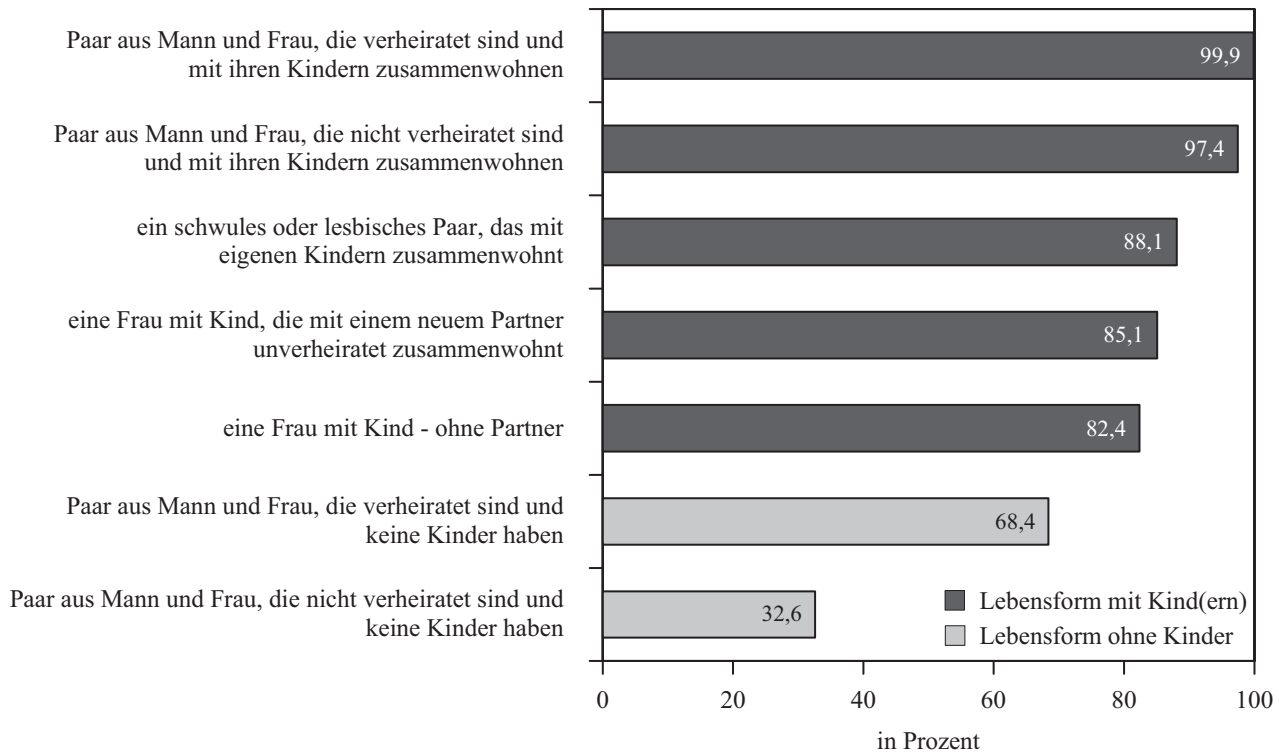

Quelle: FLB (2012), gewichtete Daten, eigene Berechnungen

Anm.: Die sieben dargestellten Lebensformen wurden mit dem genauen Wortlaut („Stellen Sie sich [ein Paar] vor [aus Mann und Frau, die verheiratet sind ...]") standardisierst abgefragt. Antwortmöglichkeiten waren „Ja, das ist eine Familie“ (in der Grafik dargestellt) und „Nein, das ist keine Familie“.

Schon deutlicher ist der Unterschied zu anderen nichtkonventionellen Konstellationen: zu homosexuellen Paaren mit Kindern $(88,1 \%)$, zu Stieffamilien, in denen eine Mutter mit Kind und neuem Partner unverheiratet zusammenlebt, $(85,1 \%)$ und zu Alleinerziehenden $(82,4 \%)$. Hier sind die Zustimmungswerte immer noch sehr hoch, aber „nur“ noch zwischen 80 und 90 Prozent und somit zehn Prozentpunkte unterhalb der ersten beiden Familienformen. Auf den ersten Blick erstaunt es, dass gleichgeschlechtliche Lebensgemeinschaften mit Kindern häufiger als Familie gewertet werden als Stieffamilien oder Alleinerziehende, obwohl sie in der Realität wesentlich seltener vorkommen (BiB 2014). Erklären lässt sich dies möglicherweise dadurch, dass der abwesende Vater bei Stieffamilien und Alleinerziehenden als eine größere Abweichung von der Normalfolie der Kernfamilie angesehen wird als die Gleichgeschlechtlichkeit eines Elternpaares. So ließe sich auch die Abstufung zwischen Stieffamilie und Alleinerziehenden erklären, denn bei Stieffamilien kann der neue Partner der Frau immerhin als „Vaterersatz“ gesehen werden; bei Alleinerziehenden ist so eine Substitution schlicht nicht möglich.

Ein wesentlich größerer Rückgang der Zustimmung, dass es sich hier um Familien handelt, findet sich bei den letzten beiden (farblich hervorgehobenen) Lebensformen: dem kinderlosen Ehepaar $(68,4 \%)$ und der kinderlosen nichtehelichen Lebensgemeinschaft $(32,6 \%)$. Das hier mutmaßlich ausschlaggebende Kriterium ist die fehlende Elternschaft: Alle bisher genannten Konstellationen waren Lebensformen mit Kindern - diese beiden nicht. Damit sinkt die Wahrnehmung dieser Lebensformen als Familie auf unter 70 bzw. auf unter 40 Prozent. Demnach hat das Zusammenleben mit Kindern eine überragende Bedeutung bei der Wahrnehmung einer Lebensform als Familie. 
Damit einher geht auch eine unterschiedliche Wahrnehmung der Bedeutung der Ehe. Während für die Bewertung heterosexueller Paare mit Kindern der Trauschein praktisch keine Rolle mehr spielt, macht er in der Gruppe der Lebensformen ohne Kinder einen extremen Unterschied: Für mehr als zwei Drittel ist eine kinderlose Ehe durchaus eine Familie; bei kinderlosen nichtehelichen Lebensgemeinschaften ist die Zustimmung weniger als halb so groß. Somit ist die Ehe kein notwendiges, aber für viele ein als Alternative zur Elternschaft hinreichendes Kriterium. Möglicherweise wird mit der Eheschließung auch eine baldige Familiengründung assoziiert, zumal Schwangerschaft ein häufiger Heiratsgrund ist (Nave-Herz 1989: 216).

Die in Abbildung 1 dargestellten Befunde geben Auskunft über die Beurteilung jeder einzelnen durch den Fragebogen vorgegebenen Lebensform. Sie lassen jedoch kaum Rückschlüsse über itemübergreifende Antwortmuster der Befragten zu. Eine Zusammenfassung und Typologisierung dieser Antwortmuster ist in der folgenden Abbildung 2 dargestellt.

Zunächst wurden die Beurteilungen der sieben einzelnen Lebensformen mit Hilfe einer Hauptkomponentenanalyse nach einer dimensionalen Struktur hin untersucht, also nach Gruppen von Lebensformen, die von der gleichen Person typischerweise gleich eingestuft werden. Zwei solche Gruppen lassen sich identifizieren: Zum einen werden die vier nichtehelichen Konstellationen mit Kindern als gemeinsame Dimension ausgewiesen (Cronbach's Alpha $=, 48 ; 23,1 \%$ erklärte Varianz), zum anderen die beiden Konstellationen ohne Kinder (Cronbach's Alpha $=, 62 ; 21,6 \%$ erklärte Varianz). ${ }^{1}$ Das heißt: Wer eine Lebensform mit Kind als eine Familie wahrnimmt, neigt dazu, auch in den übrigen Lebensformen mit Kind eine Familie zu sehen. Wer eine Lebensform ohne Kind als eine Familie wahrnimmt, nimmt mit einer höheren Wahrscheinlich auch die zweite Lebensform ohne Kinder als Familie wahr.

Auf Basis der ersten dieser beiden Dimensionen wurde ein Indikator gebildet. Dieser weist eine Zustimmung aus, dass Lebensformen mit Kind (generell) Familien sind, wenn mindestens drei der vier nichtehelichen Lebensformen mit Kind als Familie bezeichnet wurden. Dieser Index fließt mit den übrigen drei Items - der Beurteilung der Kernfamilie, der kinderlosen Ehe und der kinderlosen nichtehelichen Lebensgemeinschaft - in die in Abbildung 2 dargestellte Typologie ein.

Die Kreuzung der vier Indikatoren liefert bemerkenswerte Ergebnisse. Demnach lassen sich die Antwortmuster fast aller Befragten (98,1\%) auf relativ einfache Prinzipien zurückführen: etwa darauf, dass jede Lebensform oder jede Lebensform mit Kind als Familie bewertet wird. Das macht die Annahme plausibel, dass Menschen tatsächlich relativ konkrete Vorstellungen davon haben, was eine Familie ist. Bemerkenswert ist außerdem, dass sich die Antwortmuster der meisten Befragten (93,9 \%) als Teilmengen voneinander hierarchisch ordnen lassen: Demnach sieht jeder, der nichteheliche Lebensgemeinschaften ohne Kinder als Familie empfindet, auch in allen übrigen Konstellationen eine Familie. Jeder, der eine kinderlose Ehe als Familie ansieht, urteilt genauso für alle Lebensgemeinschaften mit Kind. Jeder, der eine nichteheliche Lebensgemeinschaft mit Kind als Familie einstuft, tut dies auch mit der Kernfamilie. Genauso lässt sich die Hierarchie umgekehrt lesen: Wer in nichtehelichen Lebensformen mit Kind keine Familie sieht, sieht auch in kinderlosen Lebensformen keine Familie. Wer eine kinderlose Ehe nicht als Familie bezeichnet, verweigert auch dem nichtehelichen kinderlosen Paar diesen Status. Nur 6,1 \% der Befragten lassen sich nicht in diese Hierarchie aus Teilmengen einordnen.

1 Das Ehepaar mit Kindern wird keiner der beiden Gruppen zugeordnet. 
Abbildung 2: Was ist Familie? Häufigkeit von Antwortmustern (Zustimmung in Prozent)
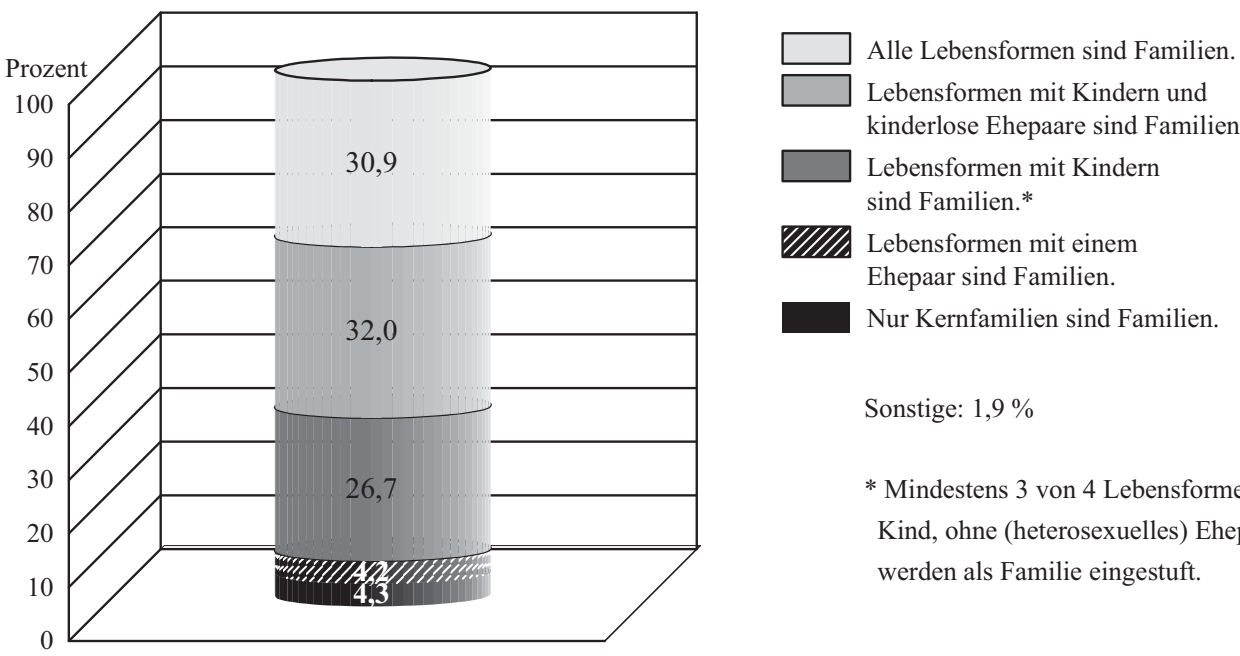

Sonstige: $1,9 \%$

* Mindestens 3 von 4 Lebensformen mit Kind, ohne (heterosexuelles) Ehepaar werden als Familie eingestuft.

Quelle: FLB (2012), gewichtete Daten, eigene Berechnungen

In dieser hierarchischen Ordnung spiegelt sich die Rangfolge in den Zustimmungswerten aus Abbildung 1 wieder. Sie ist ein weiteres Indiz für die bereits formulierte Vermutung, dass es einen Kern und eine Peripherie des Leitbildes der Familie gibt. Der Kern besteht in der Kernfamilie: Bei ihr sind sich alle Menschen in Deutschland einig, dass es sich dabei um eine Familie handelt - sie ist der Musterfall. Die Peripherie bilden die diversen nichtkonventionellen Lebensformen: Sie gehören zum Bild einer „normalen“ Familie für mehr oder weniger große Teile der Gesellschaft auch mit dazu.

Der Begriff „Peripherie“ darf nicht darüber hinwegtäuschen, dass es große Teile der Gesellschaft sind, die nichtkonventionelle Lebensformen in ihr Leitbild einer Familie einschließen. Die in Abbildung 2 dargestellten Antwortmuster zeichnen ein Bild, das der Pluralisierung der Lebensformen in hohem Maße Rechnung trägt. Eine sehr kleine Gruppe von Menschen (4,3\%) urteilt, dass nur die Kernfamilie - das zusammenlebende heterosexuelle Ehepaar mit Kind - eine Familie sei, und verweigert allen anderen Konstellationen diese Zuschreibung. Eine ähnlich kleine Gruppe (4,2 \%) bezeichnet Ehepaare als Familie, unabhängig davon, ob sie ein Kind haben oder nicht. Gemeinsam ergibt sich somit ein Anteil von $8,5 \%$, für den die Ehe eine zentrale Bedingung ist, um von „Familie“ zu sprechen.

Eine weitaus größere Gruppe, die mehr als ein Viertel der 20- bis 39-Jährigen umfasst, orientiert sich dagegen an dem Prinzip, Familie sei dort, wo Kinder sind: 26,7\% sehen nicht nur in der Kernfamilie, sondern (fast) in jeder Lebensform mit Kind eine Familie (und stimmen dem bei mindestens drei der vier Eltern-Kind-Gemeinschaften ohne heterosexuelles Ehepaar zu). Doch die Mehrheit der Befragten ist in ihrem Urteil noch offener: Ein knappes Drittel (31,9\%) spricht nicht nur jeder Lebensform mit Kind, sondern zusätzlich auch jeder kinderlosen Ehe zu, eine Familie zu sein. Und für ein weiteres knappes Drittel $(30,9 \%)$ wäre selbst ein unverheiratetes Paar ohne Kind eine Familie. Somit hat selbst die derzeit weiteste Definition einer Familie, die in der Familiensoziologie gängig ist - die 
Auffassung, jede Lebensform, in der Menschen miteinander auf Dauer solidarisch zusammenleben - eine breite Zustimmung in der (jungen erwachsenen) Bevölkerung. ${ }^{2}$

Insgesamt lässt sich aus diesen Befunden ein ambivalentes Fazit ziehen: Einerseits sind die Menschen heute sehr offen in ihrer Vorstellung, welche Lebensformen zu einer Familie zählen, und schließen - wenn auch in unterschiedlichen Graden - einen sehr großen Teil der nichtkonventionellen Lebensformen in ihr Leitbild einer ,,normalen“ Familienkonstellation mit ein. Andererseits zeichnet sich als Maßstab zur Einstufung von Lebensformen als „Familie“ die Ähnlichkeit einer Lebensform mit der Kernfamilie ab, so dass die „klassische" Familienkonstellation eines zusammenlebenden heterosexuellen Ehepaares mit Kind(ern) als Ideal einer Familie, und somit als ein Leitbild für Familie im engeren Sinne, gelten kann.

\subsection{Wer teilt welche Vorstellung von Familie?}

Wie Abbildung 2 ergeben hat, ist die Bevölkerung hinsichtlich des Grades ihrer Offenheit, welche Lebensform noch als Familie zu bezeichnen sei, in vier bzw. fünf Lager geteilt:

1. in jene, für die eine Ehe oder auch erst eine Ehe mit Kindern eine Familie ausmacht,

2. in jene, die alle Lebensformen mit Kindern als Familie ansehen,

3. in jene, für die außerdem eine kinderlose Ehe Familie ist, und

4. in jene, die quasi jedes Zusammenleben von Menschen als Familie bezeichnen.

Sind diese Vorstellungen quer über alle sozialen Gruppen verbreitet oder lassen sich bestimmte Teile der Gesellschaft ausmachen, in denen bestimmte Vorstellungen dominieren? Ein Blick auf die Ergebnisse in Tabelle 1 zeigt zunächst, dass sich in keiner der dort unterschiedenen Teilpopulationen etwas an der grundsätzlichen Rangordnung ändert. Unterschiede liegen im Detail, jedoch nicht im Grundsatz dessen, was zuvor für die Bevölkerung im Alter von 20 bis 39 Jahre allgemein beschrieben wurde: Die klassische Familienkonstellation wird auch über verschiedene Gruppen hinweg durchweg als Familie anerkannt, ähnlich verhält es sich mit unverheirateten Paaren mit Kindern. Die Zustimmung zu anderen Lebensformen liegt mehr oder minder in allen sozialen Gruppen in ähnlichen Größenordnungen.

Allerdings zeigen sich unter den nichtkonventionellen Lebensformen auch Unterschiede, die im Einzelfall bis zu zehn Prozentpunkte umfassen können. Homosexuelle Paare mit eigenen Kindern beispielsweise polarisieren stark zwischen Männern und Frauen, wobei Frauen in ihnen wesentlich häufiger eine Familie sehen $(92,4 \%)$ als Männer (83,8 \%). Darüber hinaus steigt die Anerkennung dieser Lebensform als Familie mit dem Bildungsgrad: von 83,8 \% unter den niedrig Gebildeten auf 91,9\% unter den hoch Gebildeten. Außerdem ist die Zustimmung unter den Jüngeren $(90,0 \%)$ etwas höher als unter den Älteren $(86,1 \%)$, unter den Kinderlosen $(89,7 \%$ ) etwas höher als unter den Eltern $(85,7 \%)$ und in Ostdeutschland $(91,4 \%)$ höher als in Westdeutschland $(87,2 \%)$.

2 Ein weiteres relevantes Antwortmuster innerhalb der Kategorie „Sonstige“ sieht in Kernfamilien, in Ehepaaren ohne Kinder und in nichtehelichen Lebensgemeinschaften ohne Kinder eine Familie, nicht aber jeder Lebensgemeinschaft mit Kind (1,3\%). Alle anderen Antwortkombinationen bleiben unterhalb von 0,5\%. Fälle, für die mindestens eine Angabe fehlt, wurden für die Prozentuierung nicht berücksichtigt. 
Tabelle 1: Was ist Familie? Beurteilung nach soziodemografischen Merkmalen (Zustimmung in Prozent)

\begin{tabular}{|c|c|c|c|c|c|}
\hline & $\begin{array}{l}\text { Paar aus Mann und } \\
\text { Frau, die verheiratet } \\
\text { sind und mit ihren } \\
\text { Kindern zusammen- } \\
\text { wohnen }\end{array}$ & $\begin{array}{l}\text { Paar aus Mann und } \\
\text { t Frau, die nicht } \\
\text { verheiratet sind und } \\
\text { - mit ihren Kindern } \\
\text { zusammenwohnen }\end{array}$ & $\begin{array}{l}\text { Schwules oder } \\
\text { lesbisches Paar, das } \\
\text { mit eigenen Kindern } \\
\text { zusammenwohnt }\end{array}$ & \multicolumn{2}{|c|}{$\begin{array}{l}\text { Frau mit Kind, die } \\
\text { mit einem neuen } \\
\text { Partner unverheirate } \\
\text { zusammenwohnt }\end{array}$} \\
\hline Männer & 99,8 & $96,6^{* * *}$ & $83,8^{* * *}$ & \multicolumn{2}{|c|}{84,1} \\
\hline Frauen & 99,9 & $98,3^{* * *}$ & $92,4^{* * *}$ & \multicolumn{2}{|c|}{86,1} \\
\hline $20-29$ & 99,9 & 97,4 & $90,0^{* * *}$ & \multicolumn{2}{|c|}{$83,8^{* *}$} \\
\hline $30-39$ & 99,8 & 97,5 & $86,1^{* * *}$ & \multicolumn{2}{|c|}{$86,3^{* *}$} \\
\hline Kinderlose & 99,9 & 97,2 & $89,7^{* * *}$ & \multicolumn{2}{|c|}{85,0} \\
\hline Eltern & 99,8 & 97,8 & $85,7^{* * *}$ & \multicolumn{2}{|c|}{85,1} \\
\hline \multicolumn{6}{|l|}{ Bildung $^{1}$} \\
\hline Niedrig & 100,0 & 95,8 & $83,8^{* * *}$ & \multicolumn{2}{|c|}{$82,4^{* * *}$} \\
\hline Mittel & 99,9 & 97,5 & $87,0^{* * *}$ & \multicolumn{2}{|c|}{$83,6^{* * *}$} \\
\hline Hoch & 99,8 & 97,9 & $91,9^{* * *}$ & \multicolumn{2}{|c|}{$90,1^{* * *}$} \\
\hline West & 99,8 & 97,0 & $87,2^{* * *}$ & \multicolumn{2}{|c|}{$83,7^{* * *}$} \\
\hline Ost & 100,0 & 99,0 & $91,4^{* * *}$ & \multicolumn{2}{|c|}{$90,6^{* * *}$} \\
\hline Gesamt & 99,9 & 97,4 & 88,1 & \multicolumn{2}{|c|}{85,1} \\
\hline & $\begin{array}{l}\text { Frau mit Kind } \\
\text { ohne Partner }\end{array}$ & $\begin{array}{l}\text { Paar aus Mann und Frau } \\
\text { die verheiratet sind, } \\
\text { zusammenwohnen und } \\
\text { keine Kinder haben }\end{array}$ & \multicolumn{2}{|c|}{$\begin{array}{l}\text { Paar aus Mann und Frau, } \\
\text { die nicht verheiratet sind, } \\
\text { zusammenwohnen und } \\
\text { keine Kinder haben }\end{array}$} & $\mathrm{N}$ \\
\hline Männer & $78,0^{* * *}$ & $63,5^{* * *}$ & $28,0^{* * *}$ & & 2.531 \\
\hline Frauen & $86,8^{* * *}$ & $73,5^{* * *}$ & $37,3^{* * *}$ & & 2.468 \\
\hline $20-29$ & 83,6 & 69,6 & 31,4 & & 2.514 \\
\hline $30-39$ & 81,1 & 67,2 & 33,8 & & 2.484 \\
\hline Kinderlose & 81,5 & 68,8 & 31,6 & & 2.984 \\
\hline Eltern & 83,6 & 67,9 & 34,0 & & 2.012 \\
\hline \multicolumn{6}{|l|}{ Bildung ${ }^{1}$} \\
\hline Niedrig & $73,2^{* * *}$ & $69,2^{* * *}$ & $29,5^{* *}$ & & 346 \\
\hline Mittel & $83,6^{* * *}$ & $70,9^{* * *}$ & $34,3^{* *}$ & & 3.249 \\
\hline Hoch & $82,3^{* * *}$ & $62,0^{* * *}$ & $29,0^{* *}$ & & 1.309 \\
\hline West & 82,0 & $67,5^{* *}$ & $30,8^{* * *}$ & & 3.985 \\
\hline Ost & 83,8 & $72,2^{* *}$ & $39,7^{* * *}$ & & 1.014 \\
\hline Gesamt & 82,4 & 68,4 & 32,6 & & 5.000 \\
\hline
\end{tabular}

Quelle: FLB (2012), gewichtete Daten, eigene Berechnungen

Anm.: Die Unterschiede der Antworten in den verschiedenen Teilgruppen wurden mittels $\mathrm{Chi}^{2}$-Tests berechnet, ${ }^{* *} \leq 1 \%,{ }^{* * *} \leq 1 \%$ o. ${ }^{1}$ Bildung: ,niedrig" $=$ kein Abschluss, Haupt-, Realschulabschluss oder Polytechnische Oberschule (POS) ohne beruflichen Bildungsabschluss, „mittel“ $=$ (Fach-) Hochschulreife oder Abitur ohne beruflichen Bildungsabschluss, Lehre oder Berufsfachschule, „hoch“ $=$ (Fach-) Hochschulabschluss, Promotion, Meister/Techniker. 
Der gleiche, starke Bildungseffekt findet sich auch bei der Beurteilung von Stieffamilien und Alleinerziehenden. Hinsichtlich der Stieffamilien zeigt sich außerdem ein signifikanter Ost-West-Unterschied: In den neuen Bundesländern, in denen diese Konstellation häufiger vorkommt (Steinbach 2008: 172), wird sie auch öfter als Familie bezeichnet.

Bezüglich der beiden Lebensformen ohne Kinder finden sich zum einen Geschlechtereffekte: Frauen sehen in kinderlosen Partnerschaften eher eine Familie als Männer. Zum anderen sind auch hier wieder deutliche Ost-West-Unterschiede vorhanden, wobei erneut Befragte in den alten Bundesländern den Begriff Familie restriktiver fassen als Befragte in den neuen Bundesländern.

Zusammenfassend lässt sich festhalten, dass keine wesentlichen Effekte von Alter oder Elternschaft zu finden sind. Dagegen zeigen sich signifikante Geschlechtereffekte, wobei Frauen im Hinblick auf die Anerkennung einer Lebensform als Familie grundsätzlich toleranter sind als Männer, vor allem in Bezug auf Paare ohne Kinder. Männer sind dagegen stärker an traditionellen Leitbildern orientiert, d. h. sowohl das Kriterium Elternschaft als auch das Kriterium Ehe sind ihnen wichtiger bei der Definition von Familie. Es finden sich auch signifikante Ost-West-Unterschiede, wobei Befragte aus den neuen Bundesländern durchgängig toleranter in ihren Urteilen sind. Das gilt vor allem für die Ehe als (nicht notwendige) Voraussetzung, um von einer Familie zu sprechen, in geringerem Maße aber auch für Elternschaft. Schließlich sind starke Bildungseffekte zu erkennen, wiederum sowohl bei der Bedeutung von Ehe als auch in Bezug auf Elternschaft. Dabei gilt, dass mit steigender Bildung die Toleranz steigt, eine Lebensform unabhängig von ihrem Institutionalisierungsgrad als Familie zu bezeichnen, dass also mit steigender Bildung die Bedeutung von Ehe abnimmt.

Bezogen auf die Typologisierung der Antwortmuster (wie in Abbildung 2) finden sich äquivalente Unterschiede. Statt einer Übersicht präsentiert die Abbildung 3 zwei „Extrempole" der Verteilung, indem sie auf der einen Seite die Antwortmuster hoch gebildeter Frauen in Ostdeutschland (mit Abitur oder Hochschulabschluss) und auf der anderen Seite die der niedrig gebildeten Männer in Westdeutschland (mit Real- oder Hauptschulabschluss) vergleicht.

Die hoch gebildeten Frauen in Ostdeutschland zeichnen sich dabei durch ein besonders offenes Bild einer Familie aus: Für 42,7 \% ist jede Lebensgemeinschaft eine Familie, für eine große Mehrheit von insgesamt $77,2 \%$ gilt das zumindest für Lebensformen mit Kindern sowie für kinderlose Ehepaare. Die niedrig gebildeten Männer in Westdeutschland antworten vergleichsweise restriktiv: Hier sehen lediglich 24,3\% alle Lebensgemeinschaften als Familie an. Nimmt man jene hinzu, die zumindest Lebensformen mit Kindern und kinderlosen Ehepaaren diesen Status zubilligen, ist dies mit 55,5 \% eine knappe Mehrheit. 
Abbildung 3: Was ist Familie? Antwortmuster verschiedener sozialer Gruppen (Zustimmung in Prozent)

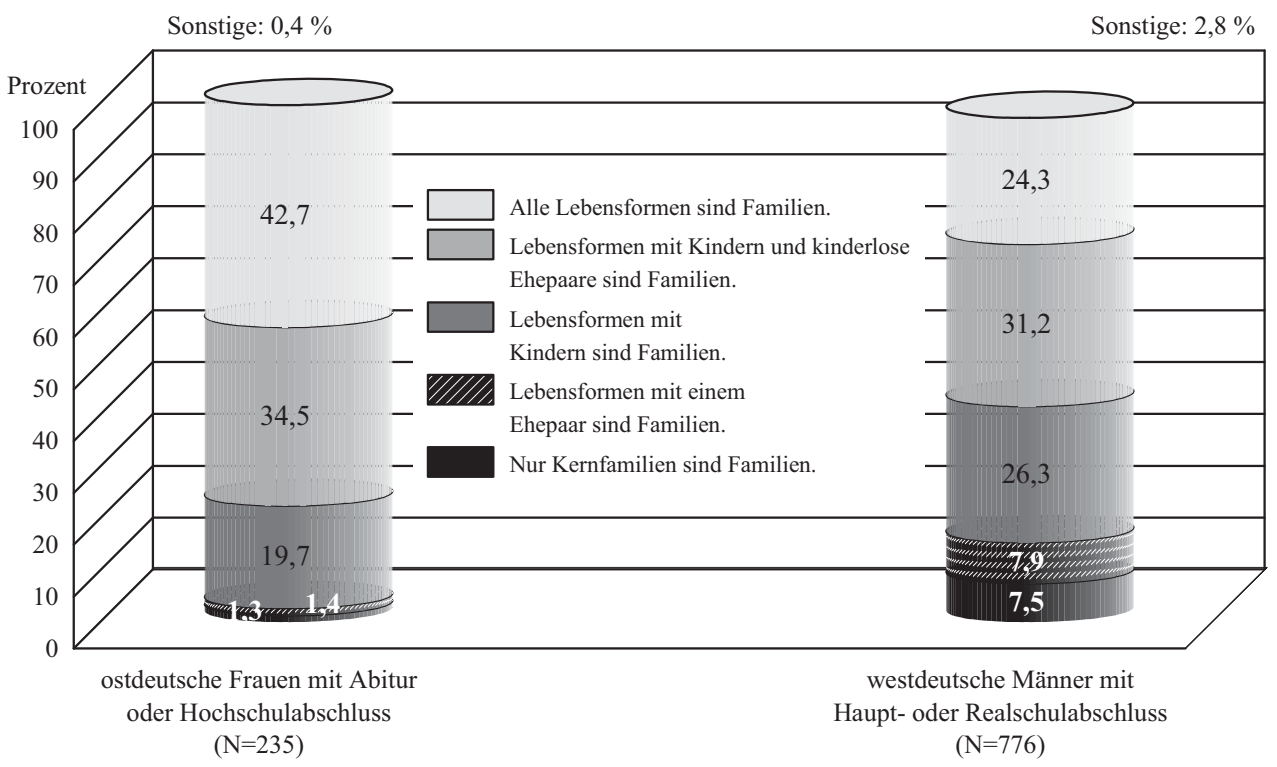

Quelle: FLB (2012), gewichtete Daten, eigene Berechnungen

\subsection{Zusammenhänge zwischen Leitbild und Lebensrealität}

Im Zusammenhang mit der Bewertung von Lebensformen ist natürlich auch die Frage nach einem möglichen Zusammenhang mit der tatsächlichen Partnerschafts- und Familiensituation der Befragten interessant. Um dieser Frage nachzugehen, wurde zunächst die Familiensituation der Befragten rekonstruiert (vgl. Tabelle 2).

Fast ein Drittel $(31,8 \%)$ hat zum Befragungszeitpunkt keinen Partner. Ebenso viele $(31,7 \%)$ sind verheiratet und leben als heterosexuelles Paar mit ihrem Partner in einem gemeinsamen Haushalt. Während aber von den Befragten ohne Partner nur ungefähr ein Achtel $(13,3 \%)$ eigene Kinder hat ${ }^{3}$ und somit alleinerziehend ist, hat bei den Verheirateten die überwiegende Mehrheit (83,5\%) Kinder. Ehepaare, die in getrennten Haushalten leben (ohne im Sinne des Familienstandes getrennt zu sein), sind sehr selten $(0,3 \%)$. Von ihnen sind etwas weniger als die Hälfte $(38,5 \%)$ Eltern.

In heterosexuellen nichtehelichen Partnerschaften lebt ein weiteres Drittel der Befragten $(34,7 \%)$. Diese lassen sich differenzieren in $18,7 \%$ der Befragten, die mit ihrem Partner im selben Haushalt als nichteheliche Lebensgemeinschaft zusammenleben, und in $16 \%$, die getrennte Haushalte haben. Von den zusammenlebenden unverheirateten Paaren haben $38,9 \%$ Kinder, von jenen ohne gemeinsamen Haushalt nur $11,1 \%$.

3 Gefragt wurde nach leiblichen Kindern oder Adoptivkindern, ungeachtet, ob diese im Haushalt wohnen oder nicht. 
Tabelle 2: Verteilung der Lebensformen, gesamt und nach Elternschaft

\begin{tabular}{lrrr}
\hline & \multicolumn{2}{c}{ Gesamt } & davon Eltern \\
Partnerschaftsform & \multicolumn{1}{c}{ N } & \multicolumn{1}{c}{$\%$} & \multicolumn{1}{c}{$\%$} \\
\hline Ohne Partner & 1.588 & 31,8 & 13,3 \\
Ehepaar, heterosexuell, zusammenlebend & 1.585 & 31,7 & 83,5 \\
Ehepaar, heterosexuell, bilokal & 13 & 0,3 & 38,5 \\
Nichteheliches Paar, heterosexuell, zusammenlebend & 931 & 18,7 & 38,9 \\
Nichteheliches Paar, heterosexuell, bilokal & 800 & 16,0 & 11,1 \\
Nichteheliches Paar, homosexuell, zusammenlebend & 36 & 0,7 & 35,1 \\
Nichteheliches Paar, homosexuell, bilokal & 29 & 0,6 & 10,3 \\
Eingetragene Partnerschaft, homosexuell, zusammenlebend & 9 & 0,2 & 44,4 \\
\hline
\end{tabular}

Quelle: FLB (2012), gewichtete Daten, eigene Berechnungen

Gleichgeschlechtliche Lebensformen sind relativ selten im Datensatz vertreten: Insgesamt $1,5 \%$ geben an, einen gleichgeschlechtlichen Partner zu haben. ${ }^{4}$ Trotz der geringen Fallzahlen soll auch ihre Verteilung auf die verschiedenen Lebensformen dargestellt werden, da sie für die Definition des Familienbegriffs eine Rolle spielt. Die meisten homosexuellen Paare haben keine eingetragene Partnerschaft $(1,3 \%) ; 0,7 \%$ leben als nichteheliche Lebensgemeinschaft mit gemeinsamen Haushalt und $0,6 \%$ in getrennten Haushalten. Eine eingetragene Partnerschaft haben 0,2 \%. Eltern finden sich am häufigsten (44,4\%) unter jenen, die ihre Beziehung formalisiert haben, aber auch bei etwa jedem Dritten $(35,1 \%)$, der mit Partner im gleichen Haushalt lebt. Unter jenen, die in einer gleichgeschlechtlichen Partnerschaft ohne gemeinsamen Haushalt leben, haben lediglich 10,3\% Kinder.

Diese 16 verschiedenen Lebensformen werden aufgrund der teilweise sehr geringen Fallzahlen für die weiteren Analysen zu neun Formen zusammengefasst (vgl. Tabelle 3). Dabei werden Befragte in gleichgeschlechtlichen Partnerschaften als eine Kategorie ausgewiesen, unabhängig davon, ob sie zusammenwohnen, Kinder haben oder eine eingetragene Lebenspartnerschaft sind. Heterosexuelle Ehepaare mit getrennten Haushalten werden von jenen mit gemeinsamem Haushalt nicht unterschieden.

Betrachtet man die Bewertung von Lebensformen nach der tatsächlichen Partnerschafts- und Familiensituation (Tabelle 3), so zeigt sich in fast allen Teilgruppen die gleiche Rangfolge der Häufigkeiten, mit denen die unterschiedlichen Lebensformen als Familie angesehen werden, wie in der Gesamtpopulation. Lediglich unter kinderlosen Ehepaaren, unter nichtehelichen Lebensgemeinschaften mit Kindern, unter bilokalen Lebensgemeinschaften mit Kindern sowie unter Alleinerziehenden gibt es leichte Abweichungen: In allen vier Fällen tauschen jeweils zwei Lebensformen ihre Plätze im Ranking. In zwei Fällen (bei kinderlosen Ehepaaren und bei Alleinerziehenden) wird dabei jeweils die eigene Lebensform höher platziert. In den übrigen beiden Fällen (bei kohabitierenden NELs und bei bilokalen nichtehelichen Paaren mit Kindern) kommt der Tausch einer Lebensform zugute, die der von den Befragten gelebten Lebensform zumindest ähnlich ist: der Frau mit Kind, die mit einem neuen Partner unverheiratet zusammenwohnt.

$4 \quad$ Bei Singles wurde nicht nach der sexuellen Orientierung gefragt. 
Tabelle 3: Was ist Familie? Beurteilung nach Lebensform des Befragten (Zustimmung in Prozent)

\begin{tabular}{|c|c|c|c|c|c|c|c|}
\hline \multirow[b]{2}{*}{ Eigene Lebensform } & \multicolumn{7}{|c|}{ Als „Familie“ wahrgenommene Lebensformen } \\
\hline & $\begin{array}{l}\text { Ehepaar } \\
\text { mit Kind }\end{array}$ & $\begin{array}{l}\text { NEL } \\
\text { mit Kind }\end{array}$ & $\begin{array}{c}\text { Gleichge- } \\
\text { schlechtliches } \\
\text { Paar mit Kind }\end{array}$ & $\begin{array}{l}\text { Stief- } \\
\text { familie }^{\mathrm{a}}\end{array}$ & $\begin{array}{l}\text { Alleiner- } \\
\text { ziehende }\end{array}$ & $\begin{array}{c}\text { Ehepaar } \\
\text { ohne Kind }\end{array}$ & $\begin{array}{l}\text { NEL } \\
\text { ohne } \\
\text { Kind }\end{array}$ \\
\hline Ehepaar mit Kind(ern) & 99,8 & 97,3 & 83,6 & 82,9 & 82,4 & 65,5 & 27,3 \\
\hline Ehepaar ohne Kind & 100,0 & 96,3 & 89,0 & 87,0 & 84,4 & 86,2 & 35,3 \\
\hline NEL mit Kind(ern) & 100,0 & 99,2 & 90,4 & 93,1 & 84,3 & 71,3 & 48,3 \\
\hline NEL ohne Kind & 100,0 & 98,2 & 93,8 & 85,7 & 83,3 & 68,4 & 40,2 \\
\hline bilokale NEL mit Kind(ern) & 100,0 & 98,9 & 87,6 & 93,3 & 86,7 & 66,3 & 44,3 \\
\hline bilokale NEL ohne Kind & 99,9 & 96,3 & 89,2 & 84,9 & 81,7 & 66,1 & 28,6 \\
\hline Gleichgeschlechtliches Paar & 100,0 & 96,0 & 94,7 & 90,7 & 81,1 & 60,0 & 48,0 \\
\hline Alleinerziehende(r) & 99,5 & 98,6 & 89,9 & 81,4 & 89,6 & 77,3 & 43,8 \\
\hline Single & 99,8 & 97,3 & 88,0 & 84,3 & 80,2 & 67,4 & 28,3 \\
\hline Gesamt & 99,9 & 97,4 & 88,1 & 85,1 & 82,4 & 68,4 & 32,6 \\
\hline
\end{tabular}

Quelle: FLB (2012), gewichtete Daten, eigene Berechnungen

Anm.: Die genauen Beschreibungen der Lebensformen, von denen die Befragungsteilnehmer beurteilen sollten, ob es ,Familie“ sind, finden sich in Abbildung 1.

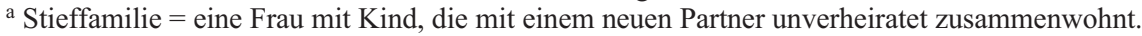

Dieser Befund hängt damit zusammen, dass generell eine Neigung besteht, die Lebensform, in der man selbst lebt, eher als Familie zu bewerten, als andere das tun. Fünf der sechs gemessenen (und in Tabelle 3 mit Kästchen hervorgehobenen) Bewertungen der eigenen Lebensform fallen höher aus, als die Bewertung der Lebensform insgesamt. Besonders extrem zeigt sich die eigene „Betroffenheit“ bei Ehepaaren ohne Kinder, von denen 86,2 \% ihre Lebensform als Familie bewerten - und somit ein um 17 Prozentpunkte höherer Anteil als in der Gesamtpopulation (mit 68,4\%). Einzig das Ehepaar mit Kind, das ohnehin von nahezu jedem als Familie bewertet wird, sieht sich selbst nicht noch häufiger als Familie.

In diesen Befunden kann ein Bedürfnis gesehen werden, selbst in einer Familie leben zu wollen. Offen bleiben muss die Frage der Kausalität: Entweder führt die Erfahrung, in einer bestimmten Lebensform zu leben, dazu, in dieser Lebensform auch eine Familie zu sehen. Oder das vorher bereits vorhandene Leitbild einer Familie führt dazu, dass man sich für eine bestimmte Lebensform entscheidet bzw. sich mit ihr arrangiert (zumal Lebensformen oft nicht frei gewählt sind). Wahrscheinlich bestehen wechselseitige Einflüsse.

Die differenzierte Analyse zeigt, dass für die Bewertungen der Lebensformen Ehepaar mit Kindern und Nichteheliche Lebensgemeinschaft mit Kindern kaum Varianz zwischen den gelebten Lebensformen der Befragten besteht. Das ist zumindest auch dem Umstand geschuldet, dass eine durchschnittliche Zustimmung nahe $100 \%$ generell wenig Varianz aufweisen kann. Homosexuelle Paare mit Kindern werden etwas seltener von Ehepaaren mit Kindern als Familie anerkannt (83,6\%), etwas häufiger von homosexuellen Paaren selbst $(94,7 \%)$, aber auch von kinderlosen nichtehelichen Lebensgemeinschaften (93,8 \%). Stieffamilien in der Form, dass Frauen mit eigenen Kindern und einem neuen Partner unverheiratet zusammenleben, werden vor allem von anderen nichtkonventionellen Lebensformen überdurchschnittlich häufig als Familien anerkannt: von nichtehelichen Lebensgemeinschaften mit Kindern (93,1\%), von bilokalen Lebensgemeinschaften mit Kindern (93,3 \%) 
sowie von homosexuellen Paaren (90,7\%). Ähnlich verhält es sich mit Alleinerziehenden, die überdurchschnittlich häufig von Alleinerziehenden selbst $(89,6 \%)$, aber auch von Personen in bilokalen Lebensgemeinschaften $(86,7 \%)$ als Familie angesehen werden.

Am stärksten polarisieren die Lebensformen, die am seltensten als Familie wahrgenommen werden: kinderlose Ehepaare und, noch stärker, die nichtehelichen Lebensgemeinschaften ohne Kinder. Diese erhalten die stärkste Zustimmung von den nichtehelichen Lebensgemeinschaften mit Kindern (48,3\%), dicht gefolgt von homosexuellen Paaren $(48,0 \%)$, bilokalen Lebensgemeinschaften mit Kind (44,3\%) und Alleinerziehenden $(43,8 \%)$. Alleinstehende und Ehepaare mit Kindern werten diese Lebensform unterdurchschnittlich häufig als Familie.

Für Ehepaare mit Kindern ist eine unterdurchschnittliche Zustimmung generell charakteristisch, auch in Bezug auf andere Lebensformen. In Analogie zu dem Ergebnis, dass Menschen ihre eigene Lebensform besonders häufig als Familie wahrnehmen, lässt sich mutmaßen, dass entweder die Erfahrung, in einer nichtkonventionellen Lebensform zu leben, die Tendenz fördert, generell auch in anderen nichtkonventionellen Lebensformen eine Familie zu sehen, oder dass das vorher vorhandene umfassendere Leitbild dazu führt, dass man sich eher auf eine nichtkonventionelle Lebensform einlässt.

\section{$4 \quad$ Fazit}

Das Leitbild davon, was eine Familie ausmacht bzw. wie sie sich „normalerweise“ zusammensetzt, lässt sich nicht auf eine Formel bringen, weil seine Konturen unscharf sind. Es hat einen eindeutig bestimmbaren und gesellschaftsweit einheitlichen Kern: die Kernfamilie. Diese wird quasi von jedem als Familie wahrgenommen. Sie ist das Musterbeispiel einer Familie, und je mehr eine andere Lebensform ihr in ihrer Zusammensetzung gleicht, desto eher wird auch in dieser eine Familie gesehen.

Sehr uneinheitlich sind die Vorstellungen davon, wie weit eine Lebensform von der Idealform der Kernfamilie abweichen darf, um noch eine Familie zu sein. Dabei herrscht zwar generell eine große Offenheit bzw. Toleranz, die der vorangeschrittenen Pluralisierung der Lebensformen durchaus Rechnung trägt. Doch in dieser Offenheit überbieten sich verschiedene gesellschaftliche Gruppen: Ein Viertel der 20- bis 39-Jährigen schließt alle Lebensformen ein, in denen Kinder leben. Dies gilt beispielsweise für Alleinerziehende und für gleichgeschlechtliche Paare mit Kind. Eine weitere Gruppe, die ungefähr ein Drittel der Population umfasst, zählt außerdem kinderlose Ehepaare zur Familie. Ein weiteres knappes Drittel schließlich nimmt jede Lebensformen von mindestens zwei Personen als Familie wahr, auch nichteheliche Lebensgemeinschaften ohne Kinder. Menschen, die ausschließlich Kernfamilien oder Lebensgemeinschaften mit einem Ehepaar als Familie wahrnehmen, sind vergleichsweise selten und machen jeweils knapp unter fünf Prozent aus. Andere Antwortmuster sind vernachlässigbar.

Die Offenheit für die Anerkennung von Lebensformen als Familie variiert über verschiedene Regionen und soziale Milieus. In Ostdeutschland wird eine nichtkonventionelle Lebensform eher als Familie wahrgenommen als in Westdeutschland, unter höher Gebildeten eher als unter Menschen mit niedriger Bildung. Die Unterschiede nach Alter und Elternschaft fallen vergleichsweise gering aus, so dass es wenige Hinweise auf eine Anpassung des Leitbildes im Lebenslauf gibt. Allein ein deutlicher Geschlechterunterschied kann in diese Richtung gedeutet werden: Frauen zeigen eine größere Offenheit, nichtkonventionelle Lebensformen als Familie anzusehen als Männer. Diese könnte dadurch begründet sein, 
dass Frauen, bedingt durch ihre stärkere Einbindung in Familienarbeit, eine pragmatischere Sicht darauf haben oder erhalten, was Familie ist bzw. wo Familie ,stattfindet“".

Die Wahrnehmung dessen, was eine Familie ist, variiert auch danach, in welcher Lebensform eine Person selbst lebt. Menschen, die in einer „klassischen“ Kernfamilie leben, sind in der Hinsicht weniger offen als Menschen in nichtkonventionellen Lebensformen. Bei der Lebensform, in der man selbst lebt, ist man eher geneigt, sie als Familie wahrzunehmen, als andere Menschen. Auch hier könnte die eigene Erfahrung das Leitbild prägen: Das persönliche Erleben, dass sich der Alltag in einer alternativen Lebensform wie „Familie“ anfühlt, könnte dazu führen, dass sich die Vorstellung von Familie erweitert. Allerdings sind auch Effekte in umgekehrter Richtung möglich: Möglicherweise haben Menschen die Lebensform, in der sie leben, aufgrund ihres Leitbildes bewusst gewählt oder sich damit zumindest leichter arrangiert. Eine dritte Möglichkeit sind Effekte sozialer Erwünschtheit. Unabhängig davon, welche kausale Beziehung genau dahinter steht, spricht die Angleichung zwischen eigener Lebensform und eigenem Begriff von Familie dafür, dass Familie grundsätzlich als etwas Positives und Erstrebenswertes angesehen wird.

\section{Literatur}

BiB (2014): Zahlen und Fakten - Familien und Lebensformen. Online-Publikation unter www.bibdemografie.de.

Brüderl, Josef (2003): Die Pluralisierung partnerschaftlicher Lebensformen. In: Aus Politik und Zeitgeschichte 19, S. 3-10.

Busch, Friedrich W.; Scholz, Wolf-Dieter (2005): Zwischen Bewahrung und Veränderung. Ehe-, Familien- und Erziehungsvorstellungen von Jugendlichen. In: Busch, Friedrich W.; Nave-Herz, Rosemarie (Hrsg.): Familie und Gesellschaft. Oldenburg: BIS-Verlag, S. 125-145.

Gestrich, Andreas (2003): Neuzeit. In: Gestrich, Andreas; Krause, Jens-Uwe; Mitterauer, Michael (Hrsg.): Geschichte der Familie. Stuttgart: Kröner, S. 364-405.

Inglehart, Ronald (1990): Culture Shift in Advanced Industrial Society. Princeton: Princeton University Press.

Kuhnt, Anne-Kristin; Steinbach, Anja (2014): Diversität von Familie in Deutschland. In: Steinbach, Anja; Hennig, Marina; Arranz Becker, Oliver (Hrsg.): Familie im Fokus der Wissenschaft. Wiesbaden: Springer VS, S. 41-70.

Lesthaeghe, Ron (1992): Der zweite demographische Übergang in den westlichen Ländern: Eine Deutung. In: Zeitschrift für Bevölkerungswissenschaft 18, 3, S. 313-354.

Matthias-Bleck, Heike (2005): Die gesellschaftliche Etablierung der nichtehelichen Lebensgemeinschaft. In: Busch, Friedrich W.; Nave-Herz, Rosemarie (Hrsg.): Familie und Gesellschaft. Oldenburg: BIS-Verlag, S. 53-76.

Mitterauer, Michael (1980a): Die Familie als historische Sozialform. In: Mitterauer, Michael; Sieder, Reinhard (Hrsg.): Vom Patriarchat zur Partnerschaft. Zum Strukturwandel der Familie. München: C. H. Beck, S. 13-37.

Mitterauer, Michael (1980b): Der Mythos der vorindustriellen Großfamilie. In: Mitterauer, Michael; Sieder, Reinhard (Hrsg.): Vom Patriarchat zur Partnerschaft. Zum Strukturwandel der Familie. München: C. H. Beck, S. 38-63.

Nave-Herz, Rosemarie (1989): Zeitgeschichtlicher Bedeutungswandel von Ehe und Familie in der Bundesrepublik Deutschland. In: Nave-Herz, Rosemarie; Markefka, Manfred (Hrsg.): Handbuch der Familien- und Jugendforschung. Band 1: Familienforschung. Neuwied: Luchterhand, S. 211-222.

Nave-Herz, Rosemarie (2002): Wandel und Kontinuität in der Bedeutung, in der Struktur und Stabilität von Ehe und Familie in Deutschland. In: Nave-Herz, Rosemarie (Hrsg.): Kontinuität und Wandel der Familie in Deutschland. Stuttgart: Lucius und Lucius, S. 45-70. 
Nave-Herz, Rosemarie (2006): Ehe- und Familiensoziologie. Eine Einführung in Geschichte, theoretische Ansätze und empirische Befunde. Weinheim: Juventa.

Nave-Herz, Rosemarie (2012): Familie heute. Wandel der Familienstrukturen und Folgen für die Erziehung. Darmstadt: Primus.

Parsons, Talcott (1973 orig. 1954): Beiträge zur soziologischen Theorie. hrsg. von D. Rüschemeyer. Neuwied: Luchterhand.

Rosenbaum, Heidi (2014): Familienformen im historischen Wandel. In: Steinbach, Anja; Hennig, Marina; Arranz Becker, Oliver (Hrsg.): Familie im Fokus der Wissenschaft. Wiesbaden: Springer VS, S. 19-39.

Schneider, Norbert F. (2008): Grundlagen der sozialwissenschaftlichen Familienforschung - einführende Betrachtungen. In: Schneider, Norbert F. (Hrsg.): Lehrbuch Moderne Familiensoziologie. Opladen: Budrich (UTB), S. 9-21.

Sieder, Reinhard (1980): Ehe, Fortpflanzung und Sexualität. In: Mitterauer, Michael; Sieder, Reinhard (Hrsg.): Vom Patriarchat zur Partnerschaft. Zum Strukturwandel der Familie. München: C. H. Beck, S. 141-161.

Steinbach, Anja (2008): Stieffamilien in Deutschland. Ergebnisse des „Generations and Gender Survey“ 2005. In: Zeitschrift für Bevölkerungswissenschaft 33, 2, S. 153-180.

Tazi-Preve, Irene Marjam (2003): Einstellungen der ÖsterreicherInnen zu Familie, Familienpolitik und dem Geschlechterverhältnis. Forschungsergebnisse des „Population Policy Acceptance Survey“ (PPA). In: Österreichische Zeitschrift für Politikwissenschaf, 32, 4, S. 443-455.

Wagner, Michael (2008): Entwicklung und Vielfalt der Lebensformen. In: Schneider, Norbert F. (Hrsg.): Lehrbuch Moderne Familiensoziologie. Opladen: Budrich (UTB), S. 99-120.

Widmer, Eric D. (2010): Family Configurations. A Structural Approach to Family Diversity. London: Ashgate.

Zartler, Ulrike (2012): Die Kernfamilie als Ideal. In: Zeitschrift für Familienforschung 24, 1, S. 67-84. 


\title{
Partnerschaftsleitbilder heute: Zwischen Fusion und Assoziation
}

\author{
Sabine Diabaté*
}

\section{Zusammenfassung}

Der Beitrag präsentiert Auswertungen der Erhebung „Familienleitbilder in Deutschland“ und fokussiert hierbei auf partnerschaftsbezogene Idealvorstellungen. Im Detail geht es a) um die Verbreitung bestimmter Normalitätsvorstellungen von Partnerschaft unter der jüngeren deutschen Bevölkerung, b) um die Antizipation gesellschaftlicher Partnerschaftsnormen und die Divergenzen zwischen den antizipierten gesellschaftlichen und den subjektivindividuellen Vorstellungen sowie c) um die Korrelation der subjektiven Partnerschaftsvorstellungen und die daraus ableitbare Bündelung zu bestimmten Typen von Partnerschaftsleitbildern mithilfe von Hauptkomponentenanalysen. Es bestätigt sich die Annahme, dass junge Menschen eher assoziativ-orientierte Vorstellungen vertreten und ein optimistisch anmutendes Bild von Partnerschaft haben. Es zeigt sich aber auch, dass die öffentliche Meinung insgesamt etwas konventioneller wahrgenommen und in der Gesellschaft ein eher instabileres pessimistisches Bild von Partnerschaft gesehen wird. Zudem gibt es einige Vorstellungen, die bei vielen jungen Erwachsenen sowohl individuell, als auch gesellschaftlich als gültig eingestuft werden: Zum Beispiel finden Menschen laut einem Großteil der Befragten nur in festen und stabilen Partnerschaften ihr Glück. Gegenseitige Liebe, erfüllte Sexualität, Freiraum lassen und finanzielle Absicherung bilden ein in der jungen Bevölkerung weit verbreitetes Werte-Fundament an Leitbildelementen für das Funktionieren einer Partnerschaft. Es konnten Partnerschaftsleitbilder identifiziert werden, die als assoziativ-modern, fusionsorientiert, bürgerlich-konventionell und als eheablehnend-instabil bezeichnet werden.

\section{$1 \quad$ Einleitung}

Ewige Liebe und dauerhaftes Zusammensein - zumeist wünschen sich das verliebte Paare am Anfang ihrer Beziehung und hoffen, dass sich ihre Erwartungen von der Partnerschaft bis ans Lebensende realisieren lassen. Die Idealvorstellungen zur Partnerschaft in Industrieländern umfassen heutzutage stärker emotionale Aspekte: Der Versorgungsaspekt steht häufig nicht mehr an erster Stelle, da die Frauen in den vergangenen Jahrzehnten durch die Bildungsgewinne bessere Chancen auf dem Arbeitsmarkt haben und daher finanziell unabhängig sein können. Eine glückliche Partnerschaft gilt als ein zentrales Fundament für die Lebenszufriedenheit (Bodenmann 2001: 85), häufige Konflikte jedoch senken die Zufrie-

Ich möchte meiner Kollegin Kerstin Ruckdeschel sowie dem/der anonymen Gutachter/in für die hilfreichen Anregungen danken. 
denheit (Wagner/Weiß 2010; Weiß/Wagner 2008) und hängen mit einem Trennungs- bzw. Scheidungsrisiko zusammen (Arránz Becker 2008). Aber was genau macht eine glückliche Partnerschaft aus? Sollte man, wenn man dauerhaft zusammen bleiben will, heiraten und eine Familie gründen? Welche Erwartungen hat die Gesellschaft an den Einzelnen - was nehmen wir in der Öffentlichkeit als „richtig“ wahr - wie lautet die vermutete „Mehrheitsmeinung“" zu den Idealvorstellungen von Partnerschaft? Ist das noch normal bzw. zeitgemäß, wenn eine Frau bei der Eheschließung den Nachnamen ihres Mannes annimmt? Welche Partnerschaftsleitbilder gibt es aktuell bei jüngeren Menschen? Und wie sind diese in verschiedenen sozialen Milieus verbreitet? Diese Fragen skizzieren beispielhaft die Vielfalt des Forschungsfeldes und deren Lücken: Kulturelle - insbesondere in der Gesellschaft wahrgenommene - Vorstellungen zur Partnerschaft sind in der empirischen Sozialforschung noch immer untererforscht.

In der Familiensoziologie hat die Paarforschung eine lange Tradition. Vielfach untersucht wurden die persönlichen Vorstellungen und hohen Erwartungen zur Partnerschaft, dabei auch häufig die Determinanten von Partnerschaftszufriedenheit, gilt doch eine „glückliche Beziehung“ als die Idealvorstellung. Ungeklärt ist jedoch, welche PartnerschaftsNormen Menschen in ihrem Alltag wahrnehmen - beispielsweise könnte man vermuten, dass sexuelle Treue innerhalb der Partnerschaft einen hohen gesellschaftlichen Wert hat. Und inwiefern korrespondieren solche gesellschaftliche Einstellungen mit den individuellen? Ausgangspunkt dieses Beitrags ist, dass das Beziehungsleben nicht nur von individuellen Überzeugungen und Vorstellungen, sondern auch von gesellschaftlichen Leitbildern beeinflusst wird, das heißt von solchen, die der Einzelne in seiner Umgebung wahrnimmt.

Im Zentrum dieses Beitrags steht erstens, wie weit zentrale kulturelle Normalitätsvorstellungen zur Partnerschaft auf Individualebene unter jungen Menschen in Deutschland verbreitet sind. Dabei wird auch untersucht, welche gesellschaftlichen Partnerschaftsnormen wahrgenommen werden und wie diese mit den individuellen Vorstellungen korrespondieren. Zweitens soll analysiert werden, ob es Partnerschaftsleitbilder gibt, d. h. ob sich die einzelnen Vorstellungen zur Partnerschaft zu verschiedenen Leitbildern gruppieren lassen, und das sowohl auf individueller Ebene als auch auf gesellschaftlicher Ebene. Falls dies möglich ist, soll geprüft werden, wie weit sie verbreitet sind und ob sie nach sozialstrukturellen Merkmalen differieren. Dabei wird außerdem untersucht, inwiefern persönliche und in der Gesellschaft wahrgenommene Partnerschaftsleitbilder deckungsgleich sind oder nicht.

Die Studie „Familienleitbilder in Deutschland“ bietet für die Analyse dieser Forschungsfragen eine geeignete Datengrundlage (Lück/Naderi/Ruckdeschel, Kap. 3). In mehreren Item-Batterien wurden zentrale Aspekte der Partnerschaftsgestaltung abgefragt und die subjektiven Kriterien von Partnerschaftserfolg (,Eine Partnerschaft funktioniert, wenn ...") ermittelt. Zur Erfassung partnerschaftlicher Leitbilder stellen die Daten des Familienleitbildsurveys demnach eine breite Palette dar, die es ermöglicht, bislang nicht untersuchte Zusammenhänge von subjektiven und gesellschaftlich wahrgenommenen Vorstellungen zu einer "normalen" und "guten" Partnerschaft aufzudecken.

\section{Stand der Forschung und theoretische Grundlagen}

In der Paarforschung gibt es vielfältige Herangehensweisen, um partnerschaftliche Erwartungen, das Verhalten und die damit in Wechselwirkung stehende Partnerschaftszufriedenheit zu erklären. Aus dem aktuellen Forschungsstand zur Beziehungsqualität geht hervor, 
dass sowohl paar-interne (endogene, d. h. innerhalb der Beziehung existierende bzw. entstehende) als auch paar-externe Faktoren (exogene, d. h. von außen kommende) die Partnerschaft beeinflussen. Dass das partnerschaftliche Konfliktverhalten (Merkmale der Interaktion in der Partnerschaft) und die Häufigkeit von Paarkonflikten maßgeblich mit der Partnerschaftszufriedenheit zusammenhängen, ist ein häufig verifiziertes Ergebnis. ${ }^{1}$ Zudem gibt es in der sozialpsychologischen Forschung neuere Ansätze, die die Bedeutung von Stress und Spannungen durch Alltagsbelastungen ${ }^{2}$ in der Beziehung als zentrale negative Prädiktoren für die Paarqualität und -stabilität in den Mittelpunkt rücken. ${ }^{3}$ Jedoch ist teilweise noch ungeklärt, welche kulturellen Vorstellungen zu einer ,idealen Partnerschaft“ auf Individualebene existieren. Bekannt sind bislang vor allem Erwartungen und Ansprüche an eine Partnerschaft (vgl. z. B. Wunderer 2005, Waller/McLanahan 2005), besonders hinsichtlich der Arbeitsteilung, nämlich die Wahrnehmung von Fairness, Austausch, Gerechtigkeit und Gleichberechtigung (Equity-Theorie, z. B. bei Grau et al. 2010, Mikula 1992). In diesem Kontext sind auch Ansätze zur Machtverteilung und Entscheidungsmacht in Partnerschaften zu nennen (z. B. Nave-Herz 20044). Gleichermaßen belegt ist die Bedeutung der Gestaltung von gemeinsamer Zeit (z. B. Crawford et al. 2002) oder aber der positive Einfluss von Ähnlichkeit, d. h. der Homogamie bezüglich der Bildung, des Alters und der normativen Einstellungen (z. B. Arránz Becker/Hill 2008, Hill 2004).

Es ist jedoch kaum bekannt, welche kulturellen Vorstellungen in der Gesellschaft wahrgenommen werden, wie zwei Menschen langfristig miteinander glücklich werden können und wie eine Partnerschaft dauerhaft funktioniert. Zudem wird häufig vermutet, dass stabile Partnerschaften zwar kaum weniger gewünscht werden als in den vergangenen Jahrzehnten, dass heute aber andere, und zwar voraussetzungsvollere Ansprüche an Partnerschaften bestehen. Prominenter Vertreter dieser These ist u. a. Giddens (1992) in seinen Überlegungen zu den „pure relationships“. Die theoretische Handlungsrelevanz einer fehlenden Übereinstimmung zwischen Partnerschaftsanspruch und -realität lässt sich auch mit der Frame-Selektions-Theorie umschreiben (Eckhard 2014). Ausgangspunkt ist die Annahme, dass vor jeder Handlung eine Deutung der Situation steht, die sich innerhalb eines gewissen kulturellen Rahmens bewegt, der durch kulturelle Leitbilder gekennzeichnet ist (Esser 2001: 259).

Dieser Beitrag möchte diese Forschungslücke bearbeiten: Es gibt bislang kaum empirische Erkenntnisse darüber, welche Idealvorstellungen zur Partnerschaft in der Bevölkerung vorherrschen. Angesichts der vielen und auch häufig diskutierten theoretischen Spekulationen über Partnerschaftsleitbilder (neben Giddens 1992: 49-65; z. B. auch in Becks „Risikogesellschaft“, 1986; oder in Tyrells These der „Deinstitutionalisierung von Ehe und Familie“, 1988; oder in Meyers“ These über den „Monopolverlust von Familie“, 1993) erscheint es lohnenswert, diese auf individueller und gesellschaftlicher Ebene zu untersuchen.

1 Zum Beispiel: Weiß/Wagner 2008, Arránz Becker et al. 2005, Schneewind/Wunderer 2003, Grau/Bierhoff 2003, Hill/Kopp 2002: 265, Überblick über den Forschungsstand der 1990er Jahre bei Bradbury et al. 2000, Karney/Bradbury 1995, Gottman/Krokoff 1989, Hahlweg 1986, Lewin 1948.

2 Es konnte in diversen Studien belegt werden (z. B. Reichle/Dette-Hagenmeyer 2008), dass besonders der partnerschaftliche Umgang (dyadisches Coping bzw. Stressbewältigung) für eine günstige Prognose der Paarbeziehung entscheidend ist (vgl. Überblick: Bodenmann 2003) und Konflikte daher nicht nur negativ, sondern auch positiv für die Weiterentwicklung einer Partnerschaft sein können (Wagner/Weiß 2010).

3 Weitere vor allem in der Sozialpsychologie und klinischen Psychologie behandelte Einflussgrößen der Paarbeziehung wie Attributionen/ Selbst- und Partnerwahrnehmung (z. B. Felser 2003), Persönlichkeitsmerkmale wie z. B. das Ausmaß an Maskulinität oder Neurotizismus (vgl. Bender/Lösel 2003) sowie das Bindungsverhalten und die Partnerschaftsrepräsentation (z. B. Stöcker et al. 2003) bzw. die emotionale Nähe (z. B. Grau 2003) werden hier nicht behandelt.

4 Überblick zu Befunden und soziologischen Theorien der Machtstrukturen bei Nave-Herz (2004: 157ff.). 
Des Weiteren ist in diesem Kontext bedeutsam zu klären, ob die eigenen Idealvorstellungen zur Partnerschaft und die in der Gesellschaft wahrgenommenen kongruent sind und inwiefern sie einen Einfluss auf die private Lebensführung nehmen. Beispielsweise ist bekannt, dass von außen wahrgenommene Erwartungen an die Partnerschaft, wie die Erwartung eine Familie zu gründen, einen Einfluss auf das Verhalten von Paaren nehmen. Es gibt Normen des Timings für zentrale Lebensereignisse, die sich auf ein bestimmtes Lebensalter beziehen, in dem Teilübergänge erfolgen sollten und auch, wie viel Zeit zwischen Übergangsereignissen idealerweise liegen soll (Dommermuth 2008; Billari/Wilson 2001). Daraus folgt, dass innerhalb jeder Gesellschaft besondere kulturelle Normen existieren, die die Partnerschaftsgestaltung von Menschen beeinflussen.

Schwerpunkt dieses Beitrages bildet daher die Identifikation partnerschaftlicher Leitbilder auf individueller und gesellschaftlicher Ebene. Neu daran ist, dass bereits z. T. bekannte Einstellungsdimensionen zur Partnerschaft zusammengefasst werden sollen, um individuelle und gesellschaftlich wahrgenommene Leitbilder zu entschlüsseln. Dieser Ansatz dient dazu, der Komplexität und Bildhaftigkeit von kulturellen Vorstellungen zur privaten Lebensführung besser als bisher gerecht werden (siehe Lück/Diabaté, Kap. 2).

Für die Analysen wird vermutet, dass sich viele junge Menschen (insbesondere Frauen) vor allem aufgrund der „Modernisierungsgewinne“ in Deutschland eher egalitäre Partnerschaftsmodelle vorstellen.

Ausgangspunkt für die empirische Suche nach Partnerschaftsleitbildern bildet die Annahme, dass sie auf einer Skala von traditionell bis egalitär zu verorten sind. Traditionell heißt, dem Mann die Entscheider- und Ernährerrolle (Stauder 2002: 23) zuzuweisen und eher das Modell des Fusionspaares (Schneider et al. 2002) zu präferieren, in dem die beiden Partner zu einer Einheit verschmelzen. Ein egalitäres Partnerschaftsmodell hingegen bedeutet, dass die Aufgabenverteilung geschlechtsunspezifisch funktioniert (Künzler/Walter 2001) und dass hier die Idealvorstellung des Assoziationspaares (Schneider et al. 2002) vorherrscht. Dahinter steht ein Beziehungsideal, in dem die Eigenständigkeit trotz Zweisamkeit stärker betont wird. Auch hier ließe sich vermuten, dass ein Großteil der jungen Bevölkerung in Deutschland eher assoziativ bis moderat fusions-orientiert eingestellt ist.

\section{Ergebnisse: Idealvorstellungen der Partnerschaft}

Um die erste Forschungsfrage, die Verbreitung zentraler Idealvorstellungen zu Partnerschaft ${ }^{5}$ auf Individual- und Allgemeinheitsebene zu untersuchen, werden zunächst verschiedene Facetten der Partnerschaft erläutert (3.1), beispielsweise der Stellenwert von Partnerschaft oder deren Dauerhaftigkeit. Danach werden die Eheorientierung und der Institutionalisierungsgrad (3.2) thematisiert sowie im Anschluss daran die Bedingungen zum Gelingen einer Partnerschaft beschrieben (3.3). Diese Beschreibungen sind deskriptiver und bivariater Natur und sollen zeigen, welche Elemente später in die Bildung möglicher Partnerschaftsleitbilder eingebunden werden könnten.

\footnotetext{
5 Partnerschaften werden in den nachfolgenden Analysen als intime und heterosexuelle Zweierbeziehungen operationalisiert.
} 
Die Analysen basieren auf dem Partnerschaftsmodul innerhalb der Befragung „Familienleitbilder in Deutschland“. Es sind Individualdaten", so dass leider an dieser Stelle keine Vorstellungen des Partners bzw. der Partnerin vorliegen. Die Grundgesamtheit besteht aus 5.000 Personen im Alter zwischen 20 und 39 Jahren, die in Deutschland leben. Die Erhebungs- und Messmethoden werden ausführlich bei Lück et al. (Kap. 3) beschrieben.

\subsection{Welche Idealvorstellungen existieren zur Partnerschaft?}

Um zu verstehen, von welchen Idealvorstellungen sich Menschen hauptsächlich leiten lassen könnten, werden im Folgenden verschiedene Aussagen zu Partnerschaften dargestellt (Tabelle 1). Zunächst ist festzustellen, dass eine überwiegende Mehrheit (65,1\%) davon überzeugt ist, dass Menschen lediglich in festen und stabilen Partnerschaften ${ }^{7}$ glücklich sein können. ${ }^{8}$ Lediglich 13,2 \% sind davon überzeugt, dass diese nur begrenzt funktionieren können. Jedoch wird in der Allgemeinheit häufig ( $\approx 40 \%)$ ein brüchigeres Bild von Partnerschaften wahrgenommen. Es gibt in der jüngeren Generation einen hohen Glauben an deren Dauerhaftigkeit, ungeachtet der gesellschaftlich eher gegenteiligen Meinung. Weit verbreitet ist auf persönlicher Ebene auch die Überzeugung, dass die Ziele des Paares wichtiger sind als die des Einzelnen (59,7\%). Damit ist gemeint, dass sowohl die eigenen Ziele als auch die des/der Partners/in den gemeinsamen Partnerschaftszielen untergeordnet werden sollen. Die Vorstellung des miteinander Verschmelzens ist offenbar eine Facette heutiger Paarbeziehungen jüngerer Menschen in Deutschland.

Tabelle 1: Zustimmung auf persönlicher und Allgemeinheits-Ebene zu verschiedenen Dimensionen der Partnerschaft (in Prozent)

\begin{tabular}{lcc}
\hline & $\begin{array}{c}\text { Persönliche } \\
\text { Zustimmung }\end{array}$ & $\begin{array}{c}\text { Wahrgenommene } \\
\text { Zustimmung in der } \\
\text { Allgemeinheit }\end{array}$ \\
\hline $\begin{array}{l}\text { Menschen können nur in einer festen und stabilen } \\
\text { Partnerschaft glücklich sein. }\end{array}$ & 65,1 & $\mathrm{x}$ \\
Partnerschaften können nur zeitlich begrenzt funktionieren. & 13,2 & 39,6 \\
$\begin{array}{l}\text { Die Ziele des Paares sind wichtiger als die des Einzelnen. } \\
\text { Es ist schlecht, wenn der Mann eine niedrigere Bildung hat } \\
\text { als die Frau. }\end{array}$ & 59,7 & $\mathrm{x}$ \\
\hline
\end{tabular}

Quelle: FLB (2012), gewichtete Daten, eigene Berechnungen

Anm.: $\mathrm{x}=$ Frage wurde nicht gestellt.

6 Für eine weitere Welle wäre es interessant, auch die Vorstellungen des/der Partners/in zu erfragen, um ähnlich zur Studie PAIRFAM (Panel Analysis of Intimate Relationships and Family Dynamics) auf dyadischer Ebene Analysen durchführen zu können.

7 Dieses Item wurde in ähnlicher Form z. B. auch im European Value Survey (2008) erhoben, die Ergebnisse variieren um wenige Prozentpunkte.

8 Alle in diesem Beitrag verwendeten Items wurden über eine vierstufige Zustimmungsskala ohne Mitte erhoben. Die Antwortkategorien wurden für sämtliche Tabellen dichotomisiert, d. h. 1=,Stimme voll und ganz zu“ und 2=,Stimme eher zu“ sind zusammengefasst zum Wert 1, auf den Wert 0 umkodiert wurden die Antwortkategorien 3="Stimme eher nicht zu" sowie 4="Stimme überhaupt nicht zu". 
Stark signifikant unterscheiden sich die persönliche und allgemeine Ebene hinsichtlich der Bewertung von Bildungsunterschieden in Partnerschaften: Während individuell lediglich 16,2 \% der 20- bis 39-Jährigen der Ansicht sind, dass es schlecht ist, wenn der Mann eine niedrigere Bildung hat als seine Frau, glaubt über die Hälfte der Befragten (63,3\%), dass ein solcher Bildungsunterschied innerhalb der Gesellschaft als negativ bewertet wird. Vermutlich auch, weil in der Realität Frauen häufiger mit status-höheren Männern zusammen sind (Hassebrauck/Küpper 2003) und das umgekehrte Muster eine Abweichung davon darstellt. Hinsichtlich der Partnerwahl, die nach dem Muster ,gleiche Bildung“ (Bildungshomogamie) oder „Mann höhere Bildung als Frau“ (Bildungshypergamie) abläuft, stellt sich die Frage, inwieweit der externe normative Druck der Gesellschaft immer noch eine Rolle spielt. Dies zu untersuchen, könnte hinsichtlich der weiteren Erforschung von Partnerwahlverhalten zusätzliche Erkenntnisse bringen.

In einem weiteren Schritt werden die beschriebenen Facetten des Partnerschaftsleitbildes nach sozialstrukturellen Merkmalen in Prozent dargestellt (Tabelle 2).

Signifikante Geschlechterunterschiede gibt es hinsichtlich der Orientierung an partnerschaftlichen Zielen, bei denen Männer (68,2 \%) deutlich häufiger die Interessen des Paares wichtiger finden als Frauen (50,9\%) und bei Bildungsunterschieden in der Beziehung (Mann geringere Bildung als Frau), die Frauen etwas häufiger ablehnen.

Unterschiede nach alten und neuen Bundesländern sind nicht $\mathrm{zu}$ verzeichnen. Hingegen führt die Betrachtung nach Bildung zu einem Erkenntnisgewinn: Am häufigsten sind niedrig Gebildete vom Partnerglück überzeugt (73,6 \%), höher Gebildete deutlich seltener $(61,4 \%)$. Außerdem nehmen hoch Gebildete $(71,7 \%)$ häufiger als mittel oder geringer Qualifizierte (58,7\%) in der Gesellschaft wahr, dass ein höheres Bildungsniveau der Frau gegenüber ihrem Partner negativ gesehen wird.

Betrachtet man Differenzen zwischen Eltern und Kinderlosen, wird deutlich, dass Befragte mit Kindern signifikant häufiger davon überzeugt sind, dass Partnerschaften für das Lebensglück zentral sind und die Ziele des Paares wichtiger als die des Einzelnen finden. Allerdings ergibt sich hier ein Kausalitätsproblem, weil Personen mit Kindern auch häufiger in Partnerschaften leben als Kinderlose. In der Allgemeinheit nehmen Eltern häufiger wahr, dass Partnerschaften als zeitlich begrenzt gesehen werden. Umkehrt wiederum sehen Kinderlose in der Gesellschaft häufiger, dass Bildungsunterschiede zuungunsten des Mannes schlecht sind.

Personen mit Migrationshintergrund sind wesentlich häufiger der Meinung, dass Partnerschaften glücklich machen als solche ohne Migrationshintergrund. Gleichermaßen zweifeln sie aber auch etwas häufiger an deren Dauerhaftigkeit (17,3\% vs. 12,0 \%). Und Menschen mit Migrationshintergrund sind persönlich eher der Ansicht, dass Bildungsunterschiede ungünstig sind. In der Gesellschaft nehmen sie seltener wahr, dass unterschiedliche Bildungsniveaus negativ bewertet werden.

Weitere signifikante Differenzen finden sich beim Partnerschaftsstatus. Bei der Differenzierung nach Verheirateten und Ledigen wird deutlich, dass Erstere sich selbst für eine Institutionalisierung der Partnerschaft entschieden haben und dies wahrscheinlich auch Einfluss auf ihre Leitbilder hat bzw. andere Leitbilder voraussetzen sollte: Mit steigendem Institutionalisierungsgrad wächst die Zustimmung, dass nur Partnerschaften glücklich machen können und partnerschaftliche Ziele über den individuellen stehen sollten. Auch nehmen Verheiratete am häufigsten wahr, dass die Allgemeinheit die Dauerhaftigkeit von Partnerschaften anzweifelt, gefolgt von Personen in bilokalen Partnerschaften. Das Ergebnis basiert vermutlich auf alltäglichen Erfahrungen, dass die Eheschließung angesichts der Scheidungszahlen als Risiko beurteilt wird bzw. auf der Meinung, dass Partnerschaften in getrennten Haushalten instabiler seien. 
Tabelle 2: Zustimmung auf persönlicher und Allgemeinheits-Ebene zu verschiedenen Dimensionen der Partnerschaft nach sozialstrukturellen Merkmalen (in Prozent)

\begin{tabular}{|c|c|c|c|c|c|c|c|}
\hline \multirow[t]{2}{*}{ Merkmal } & \multirow[t]{2}{*}{ Ausprägung } & \multirow[t]{2}{*}{$\begin{array}{l}\text { Menschen können } \\
\text { nur in einer festen } \\
\text { und stabilen } \\
\text { Partnerschaft } \\
\text { glücklich sein. } \\
\text { Pers. }\end{array}$} & \multirow[t]{2}{*}{$\begin{array}{c}\text { Die Ziele des } \\
\text { Paares sind } \\
\text { wichtiger als } \\
\text { die des } \\
\text { Einzelnen. } \\
\text { Pers. }\end{array}$} & \multicolumn{2}{|c|}{$\begin{array}{l}\text { Partnerschaften } \\
\text { können nur } \\
\text { zeitlich begrenzt } \\
\text { funktionieren. }\end{array}$} & \multicolumn{2}{|c|}{$\begin{array}{c}\text { Es ist schlecht, } \\
\text { wenn der Mann } \\
\text { eine niedrigere } \\
\text { Bildung hat als } \\
\text { die Frau. }\end{array}$} \\
\hline & & & & Pers. & Allg. & Pers. & Allg. \\
\hline \multirow[t]{2}{*}{ Geschlecht } & Männlich & 65,4 & $68,2^{* * *}$ & 14,3 & 38,3 & $13,0^{* * *}$ & 62,4 \\
\hline & Weiblich & 64,7 & $50,9^{* * *}$ & 12,1 & 40,9 & $19,4^{* * *}$ & 64,2 \\
\hline \multirow[t]{2}{*}{ Wohnort ${ }^{\mathrm{a}}$} & West & 65,0 & 59,3 & 13,1 & 39,7 & 62,4 & 63,9 \\
\hline & Ost (inkl. Berlin) & 65,3 & 61,4 & 14,0 & 39,2 & 64,2 & 60,7 \\
\hline \multirow[t]{3}{*}{ Bildung $^{\mathrm{b}}$} & Niedrig & $73,6^{* * *}$ & 62,2 & 15,4 & $37,5^{*}$ & 17,4 & $58,7^{* * * *}$ \\
\hline & Mittel & $65,5^{* * *}$ & 59,8 & 13,2 & $40,7^{*}$ & 15,3 & $60,6^{* * * *}$ \\
\hline & Hoch & $61,4^{* * *}$ & 58,3 & 12,2 & $36,5^{*}$ & 17,8 & $71,7^{* * * *}$ \\
\hline \multirow[t]{2}{*}{ Kind $^{c}$} & $\mathrm{Ja}$ & $69,6^{* * *}$ & $63,8^{* * *}$ & 13,8 & $42,5^{* * *}$ & 16,1 & $60,5^{* * * *}$ \\
\hline & Nein & $62,0^{* * *}$ & $56,9^{* * *}$ & 12,8 & $37,6^{* * *}$ & 16,1 & $65,2^{* * * *}$ \\
\hline \multirow{2}{*}{$\begin{array}{l}\text { Migrations- } \\
\text { hintergrund }^{d}\end{array}$} & $\mathrm{Ja}$ & $71,4^{* * *}$ & 62,2 & $17,3^{* * *}$ & 41,6 & $19,8^{* * *}$ & $58,8^{* * * *}$ \\
\hline & Nein & $63,0^{* * *}$ & 58,9 & $12,0^{* * *}$ & 38,9 & $15,0^{* * * *}$ & $64,7^{* * * *}$ \\
\hline \multirow[t]{4}{*}{ Partnerschaft ${ }^{\mathrm{e}}$} & Ehe/ZL & $71,9^{* * *}$ & $67,5^{* * *}$ & 11,8 & $45,4^{* * *}$ & 16,3 & $61,3^{*}$ \\
\hline & NEL & $66,1^{* * *}$ & $56,4^{* * *}$ & 12,7 & $33,8^{* * *}$ & 15,5 & $66,4^{*}$ \\
\hline & NEBP & $65,3^{* * *}$ & $52,7^{* * *}$ & 12,5 & $40,0^{* * *}$ & 17,8 & $66,2^{*}$ \\
\hline & Single & $57,3^{* * *}$ & $57,4^{* * *}$ & 15,4 & $36,8^{* * *}$ & 15,6 & $61,8^{*}$ \\
\hline \multirow[t]{3}{*}{ Religiosität } & Wenig/nicht religiös & $61,1^{* * *}$ & $58,4^{* * *}$ & 13,5 & $36,1^{* * *}$ & 14,0 & $64,1^{*}$ \\
\hline & Mittel religiös & $70,4^{* * *}$ & $58,6^{* * *}$ & 12,9 & $44,5^{* * *}$ & 19,1 & $60,3^{*}$ \\
\hline & (Sehr) religiös & $72,1^{* * *}$ & $65,7^{* * *}$ & 12,4 & $45,0^{* * *}$ & 19,5 & $65,0^{*}$ \\
\hline
\end{tabular}

Quelle: FLB (2012), gewichtete Daten, eigene Berechnungen

Anm.: ${ }^{* * *} \mathrm{p} \leq 0,001 ;{ }^{* *} \mathrm{p} \leq 0,01 ;{ }^{*} \mathrm{p} \leq 0,05$ (Signifikanzen nach $\mathrm{Chi}^{2}$-Test); Pers.: Persönliche Zustimmung zur Aussage; Allg.: Die von der Allgemeinheit erwartete Zustimmung zur Aussage; ${ }^{\text {a West: Befragte }}$ Person wohnt in alten Bundesländern; Ost: Befragte Person wohnt in neuen Bundesländern inklusive Berlin; ${ }^{b}$ Bildungs-niveau nach ISCED97, Klassifikation nach UNESCO (2002): Die Zuordnung erfolgt nach: Low (1-2), Medium (3A-4A), High (5B-6); ${ }^{\mathrm{c}}$ „Haben Sie Kinder? Ich meine sowohl Ihre eigenen Kinder als auch Adoptivkinder, egal, wo sie momentan wohnen.“; ${ }^{\mathrm{d}}$ Personen, die selbst eingewandert sind und/oder deren Elternteil(e) eingewandert sind bzw. Personen mit ausländischer Staatsbürgerschaft (zur Erfassung wurde das Geburtsland des Befragten, dessen Staatsangehörigkeit sowie das Geburtsland der Eltern und deren Staatsangehörigkeit hinzugezogen). Demnach sind in dieser Kategorie Personen mit Migrationshintergrund, zugewanderte Ausländer, in Deutschland geborene Ausländer, eingebürgerte Ausländer, Spätaussiedler und Personen mit zumindest einem Elternteil, der eines der genannten Merkmale erfüllt; ${ }^{\mathrm{e}} \mathrm{ZL}=\mathrm{Zusammenlebend,}$ $\mathrm{NEL}=$ Nicht-eheliche Lebensgemeinschaften, NEBP=Nichteheliche-bilokale Partnerschaften.

Schließlich spielt auch die eigene Einschätzung der Religiosität eine Rolle: Mit steigender Religiosität nimmt auch die persönliche Überzeugung der Befragten zu, dass Partnerschaften der Ort des größten Glücks sind. Auch die Orientierung an partnerschaftlichen Lebenszielen und die Zurückstellung individueller Ziele sind bei religiösen jungen Menschen stärker ausgeprägt. Auch hier zeigt sich wieder ein Erfahrungseffekt oder ein Effekt selektiver Wahrnehmung: Genau die (Mittel bis Hoch-)Religiösen, die aufgrund ihrer Religiosität eher an die Dauerhaftigkeit ihrer Partnerschaft bzw. Ehe glauben (Lambert/Dollahite 2006), 
sehen wesentlich häufiger einen gesellschaftlichen Pessimismus gegenüber der Beständigkeit $(45 \%)$ als die wenig oder nicht Religiösen (36,1\%).

Signifikante Altersunterschiede bei den partnerschaftlichen Einstellungen auf persönlicher Ebene gab es hingegen kaum (in Tabelle 2 nicht abgebildet), beobachtet werden kann nur ein leichter Erfahrungseffekt bei den 30- bis 39-Jährigen, die etwas häufiger daran glauben, dass eine Beziehung nur zeitlich begrenzt funktionieren kann (14,7 \% vs. 11,8\%). Hinsichtlich der Allgemeinheit existiert lediglich ein hoch signifikanter Unterschied: 20bis 29-Jährige nehmen deutlich häufiger als Ältere (67,2 \% vs. 59,7\%) in der Gesellschaft wahr, dass man langfristig auch heiraten sollte.

\subsection{Ehe, nichteheliche oder nichtehelich-bilokale Paare?}

Eine weitere Annahme ist, dass sich partnerschaftliche Leitbilder und der Institutionalisierungsgrad der Partnerschaft gegenseitig beeinflussen. Beispielsweise ist anzunehmen, dass zunächst eine Mehrheit junger Menschen davon ausgeht, dass Partnerschaften und Ehen dauerhaft halten können. Machen sie die Erfahrung des Scheiterns, wird das teilweise ihre Idealvorstellung (vorübergehend) verändern. Jedoch ist das Eingehen einer neuen Beziehung oder die Wiederverheiratung ein starker Beleg dafür, dass Partnerschaftsleitbilder unabhängig von der gemachten Erfahrung bei vielen Menschen bestehen bleiben können und häufig am Ideal der Dauerhaftigkeit von Beziehungen festgehalten wird. Ein weiterer Beleg dafür, dass Leitbilder und Lebensführung zusammenhängen, zeigt die Untersuchung von bilokalen Paaren bei Dorbritz und Naderi (2012).

Diese Überlegungen führen dazu, dass ein zentraler Aspekt der Partnerschaftsentwicklung, der Institutionalisierungsgrad, untersucht werden soll. Daher wird die Eheorientierung thematisiert. Die Gültigkeit der Institution Ehe wurde über „Die Ehe ist eine überholte Einrichtung "9 erfasst (Tabelle 3). Rund ein Drittel stimmt für sich selbst dieser Aussage (eher/voll und ganz) zu. Das bedeutet, dass für immerhin zwei Drittel der Befragten die Ehe noch von Bedeutung ist. Innerhalb der Gesellschaft hingegen nimmt die Hälfte der Befragten wahr, dass die Ehe als nicht mehr zeitgemäß gilt. Ein Drittel findet außerdem, dass eine Frau nach der Hochzeit den Namen ihres Mannes annehmen soll. Noch häufiger, so beobachten es die Befragten, wird dies von der Allgemeinheit befürwortet (58,4\%). Dass bei dauerhaftem Zusammenleben irgendwann die Eheschließung folgt, heißen 43,3 \% persönlich für gut. Dem gegenüber steht eine weit verbreitet wahrgenommene gesellschaftliche Norm $(63,5 \%)$.

Gleichermaßen signifikant verschieden ist die Ansicht, zu bilokal lebenden Paaren. Immerhin knapp ein Drittel (31,5\%) stimmt der Aussage zu, dass bilokal lebende Paare keine ,richtigen“ Paare sind, deutlich weiter verbreitet ist dies aus Sicht der Befragten innerhalb der Gesellschaft (48,6 \%). Es gibt offenbar nicht flächendeckend eine soziale Anerkennung für Paare mit getrennten Haushalten.

Zusätzlich wurden zwei Aussagen untersucht, die lediglich auf der AllgemeinheitsEbene abgefragt wurden: Hierbei wird eine große Offenheit gegenüber Paaren wahrgenommen, die dauerhaft unverheiratet zusammenleben $(70,0 \%)$. Sie steht auf den ersten Blick im Widerspruch zu der zuvor beschriebenen Norm, die auch wahrgenommen wird, nämlich dass dauerhaftes Zusammenleben auch standesamtlich legitimiert werden sollte. Dazu ist festzustellen, dass hier zwei unterschiedliche Stimuli verwendet wurden: Bei der

9 Dieses Item wurde auch in anderen Surveys häufig verwendet. Im Vergleich zum European Value Survey (2008) unterscheidet sich das Ergebnis kaum. 
ersten Variable wurde eine Soll-Aussage formuliert „,wenn man als Paar dauerhaft zusammenlebt, sollte man heiraten". In der zweiten Aussage geht es um die Bewertung von Paaren, die dauerhaft unverheiratet zusammenleben, ob dies ,in Ordnung“ sei. Hier gibt es eine große Akzeptanz, die jedoch nicht gleichzusetzen ist mit dem, was als Ideal in der Gesellschaft wahrgenommen wird. Außerdem gibt es in der Allgemeinheit eine sehr weit verbreitete Norm der Probeehe (84,9\%). Es stellt sich zudem die Frage, ob die zuvor beschriebenen Komponenten des Eheleitbildes nach soziodemografischen Merkmalen variieren.

Tabelle 3: Zustimmung auf persönlicher und Allgemeinheits-Ebene zu verschiedenen Dimensionen der Eheorientierung und des Institutionalisierungsgrades (in Prozent)

\begin{tabular}{lcc}
\hline & $\begin{array}{c}\text { Persönliche } \\
\text { Zustimmung }\end{array}$ & $\begin{array}{c}\text { Wahrgenommene } \\
\text { Zustimmung in der } \\
\text { Allgemeinheit }\end{array}$ \\
\hline Die Ehe ist eine überholte Einrichtung. & 35,1 & 49,7 \\
$\begin{array}{l}\text { Eine Frau sollte nach der Heirat den Namen } \\
\text { ihres Mannes annehmen. }\end{array}$ & 34,5 & 58,4 \\
$\begin{array}{l}\text { Wenn man als Paar dauerhaft zusammenlebt, } \\
\text { sollte man heiraten. }\end{array}$ & 43,3 & 63,5 \\
$\begin{array}{l}\text { Paare mit getrennten Haushalten sind keine } \\
\text { richtigen Paare. }\end{array}$ & 31,5 & 48,6 \\
$\begin{array}{l}\text { Es ist in Ordnung, wenn ein Paar dauerhaft ohne } \\
\text { Trauschein zusammenlebt. }\end{array}$ & $\mathrm{x}$ & 70,0 \\
$\begin{array}{l}\text { Ein Paar sollte erst ein paar Jahre unverheiratet } \\
\text { zusammenleben, bevor es heiratet. }\end{array}$ & $\mathrm{x}$ & 84,9 \\
\hline
\end{tabular}

Quelle: FLB (2012), gewichtete Daten, eigene Berechnungen

Anm.: $\mathrm{x}=$ Frage wurde nicht gestellt.

In Tabelle 4 werden die Items zur Eheorientierung abgebildet, unterschieden nach soziodemografischen Merkmalen ${ }^{10}$. Beschrieben werden signifikante Differenzen:

Männer finden etwas häufiger als Frauen, dass die Partnerin den Namen des Ehemannes annehmen sollte. Auch nehmen Männer in der Allgemeinheit etwas seltener wahr, dass dauerhafte nichteheliche Lebensgemeinschaften akzeptiert werden.

Ost-West-Unterschiede gibt es hingegen kaum, Befragte in den alten Bundesländern sind lediglich häufiger der Ansicht, dass Frauen den Nachnamen bei der Trauung abgeben sollten und dass bilokale Paare keine richtigen Paare sind.

Bildungsunterschiede sind im Gegensatz dazu ausschlaggebend für eine Reihe von beachtenswerten Unterschieden: Die Bedeutung der Ehe nimmt mit zunehmender Bildung zu, gleiches gilt für die Wahrnehmung in der Allgemeinheit. Zudem sind Personen mit mittlerem Bildungsabschluss persönlich am häufigsten der Meinung, dass Frauen nach der Heirat den Partnernamen tragen sollten (36,6\%). Etwas seltener ist dies bei niedrig Gebildeten

10 Die in der Tabelle nicht abgebildete Analyse nach Altersgruppen ergab, dass Jüngere (20- bis 29-Jährige im Vergleich zu 30- bis 39-Jährigen) persönlich eine geringere Eheorientierung haben, gleichzeitig aber auch z. T. traditioneller sind (Frauen sollen den Partnernamen annehmen). In der Allgemeinheit sehen sie häufiger die Probe-Ehe und die Namensübername durch die Frau als Ideal verbreitet und sie glauben, dass bilokale Partnerschaften weniger als Paar akzeptiert werden. 
(32,8 \%) und noch seltener bei den hoch Gebildeten $(29,9 \%)$ der Fall. In der Öffentlichkeit nehmen höher Gebildete etwas häufiger wahr, dass der Nachname des Mannes der Familienname sein sollte. Menschen mit hohen Bildungsabschlüssen stehen auch bilokalen Partnerschaften etwas toleranter gegenüber als solche mit mittleren und niedrigen, was vermutlich ein Erfahrungseffekt ist, da höher Qualifizierte berufsbedingt häufiger pendeln müssen und daher eher einen Zweitwohnsitz haben (Ruppenthal/Lück 2009). Dementsprechend signifikant häufiger machen höher Qualifizierte auch die Erfahrung in ihrer Umgebung, dass Paare mit getrennten Haushalten nicht als ,richtige“ Paare bewertet werden $(55,9 \%$ im Vergleich zu 42,2 \% bei niedrig Qualifizierten). Die Befürwortung der Probeehe wird von Menschen mit niedrigen Bildungsabschlüssen signifikant häufiger in der Allgemeinheit beobachtet $(90,5 \%)$.

Tabelle 4: Zustimmung auf persönlicher und Allgemeinheits-Ebene zu verschiedenen Dimensionen der Eheorientierung nach sozialstrukturellen Merkmalen (in Prozent)

\begin{tabular}{|c|c|c|c|c|c|c|c|c|c|}
\hline \multirow[t]{2}{*}{ Merkmal } & \multirow[t]{2}{*}{ Ausprägung } & \multicolumn{2}{|c|}{$\begin{array}{l}\text { Die Ehe ist } \\
\text { eine überholte } \\
\text { Einrichtung. }\end{array}$} & \multicolumn{2}{|c|}{$\begin{array}{c}\text { Eine Frau sollte } \\
\text { nach der Heirat } \\
\text { den Namen } \\
\text { ihres Mannes } \\
\text { annehmen. }\end{array}$} & \multicolumn{2}{|c|}{$\begin{array}{c}\text { Paare mit } \\
\text { getrennten } \\
\text { Haushalten sind } \\
\text { keine richtigen } \\
\text { Paare. } \\
\end{array}$} & \multirow{2}{*}{$\begin{array}{l}\text { Es ist in Ord- } \\
\text { nung, wenn ein } \\
\text { Paar dauerhaft } \\
\text { ohne Trauschein } \\
\text { zusammenlebt. } \\
\text { Allg. }\end{array}$} & \multirow{2}{*}{$\begin{array}{c}\text { Ein Paar sollte } \\
\text { erst ein paar Jahre } \\
\text { unverheiratet } \\
\text { zusammenleben, } \\
\text { bevor es heiratet. } \\
\text { Allg. }\end{array}$} \\
\hline & & Pers. & Allg. & Pers. & Allg. & Pers. & Allg. & & \\
\hline \multirow[t]{2}{*}{ Geschlecht } & Männlich & $37,1^{* *}$ & 48,4 & $37,2^{* * *}$ & $60,6^{* *}$ & 31,6 & 49,6 & $67,3^{* * *}$ & 84,9 \\
\hline & Weiblich & $33,1^{* *}$ & 51,0 & $31,8^{* * *}$ & $56,2^{* *}$ & 31,3 & 47,6 & $72,9^{* * *}$ & 84,8 \\
\hline \multirow[t]{2}{*}{ Wohnort $^{\mathrm{a}}$} & West & 34,6 & 49,3 & $35,8^{* * *}$ & 58,6 & $32,7^{* * *}$ & 48,8 & 69,7 & $85,4^{*}$ \\
\hline & Ost (inkl. Berlin) & 37,0 & 51,1 & $29,5^{* * *}$ & 57,7 & $26,7^{* * *}$ & 47,8 & 71,1 & $82,6^{*}$ \\
\hline \multirow[t]{3}{*}{ Bildung $^{b}$} & Niedrig & $42,1^{* * *}$ & $54,3^{* * *}$ & $32,8^{* * *}$ & $51,9^{* * *}$ & $32,1^{* * *}$ & $42,2^{* * *}$ & $67,3^{*}$ & $90,5^{* * *}$ \\
\hline & Mittel & $35,8^{* * *}$ & $52,9^{* * *}$ & $36,6^{* * *}$ & $57,4^{* * *}$ & $32,8^{* * *}$ & $46,8^{* * *}$ & $71,1^{*}$ & $84,8^{* * *}$ \\
\hline & Hoch & $30,5^{* * *}$ & $41,2^{* * *}$ & $29,9^{* * *}$ & $63,8^{* * *}$ & $28,1^{* * *}$ & $55,9^{* * *}$ & $67,5^{*}$ & $83,9^{* * *}$ \\
\hline \multirow[t]{2}{*}{ Kind $^{c}$} & $\mathrm{Ja}$ & $30,2^{* * *}$ & $54,8^{* *}$ & 33,9 & $51,5^{* * *}$ & $36,5^{* * *}$ & 47,2 & $75,4^{* * *}$ & $82,8^{* * *}$ \\
\hline & Nein & $38,4^{* * *}$ & $46,3^{* *}$ & 35,0 & $63,1^{* * * *}$ & $28,0^{* * *}$ & 49,6 & $66,4^{* * *}$ & $86,3^{* * *}$ \\
\hline \multirow{2}{*}{$\begin{array}{l}\text { Migrations- } \\
\text { hintergrund }^{d}\end{array}$} & $\mathrm{Ja}$ & 37,5 & $54,7^{* * * *}$ & $41,6^{* * *}$ & $54,3^{* * *}$ & $43,9^{* * *}$ & 46,3 & 71,1 & 86,3 \\
\hline & Nein & 34,3 & $48,0^{* * *}$ & $32,4^{* * *}$ & $59,7^{* * *}$ & $27,6^{* * *}$ & 49,3 & 69,8 & 84,5 \\
\hline \multirow[t]{4}{*}{ Partnerschaft ${ }^{\mathrm{e}}$} & Ehe/ZL & $18,3^{* * *}$ & $58,4^{* * *}$ & $37,0^{* * *}$ & $49,5^{* * *}$ & $40,3^{* * *}$ & $45,4^{* * *}$ & $77,7^{* * *}$ & 84,6 \\
\hline & NEL & $45,5^{* * *}$ & $45,8^{* * *}$ & $30,6^{* * *}$ & $62,4^{* * *}$ & $30,0^{* * *}$ & $54,1^{* * *}$ & $65,2^{* * *}$ & 83,7 \\
\hline & NEBP & $40,6^{* * *}$ & $47,4^{* * *}$ & $37,7^{* * *}$ & $64,9^{* * *}$ & $24,6^{* * *}$ & $45,6^{* * *}$ & $68,5^{* * *}$ & 85,7 \\
\hline & Single & $42,5^{* * *}$ & $44,7^{* * *}$ & $33,1^{* * *}$ & $61,2^{* * *}$ & $27,3^{* * *}$ & $49,7^{* * *}$ & $66,0^{* * *}$ & 85,3 \\
\hline \multirow[t]{3}{*}{ Religiosität } & Wenig/nicht religiös & $43,0^{* * *}$ & $44,1^{* * *}$ & $29,9^{* * *}$ & $62,0^{* * *}$ & $27,7^{* * *}$ & $51,6^{* * *}$ & $66,0^{* * *}$ & 84,5 \\
\hline & Mittel religiös & $27,2^{* * *}$ & $58,3^{* * *}$ & $39,3^{* * *}$ & $53,2^{* * *}$ & $31,6^{* * *}$ & $42,8^{* * *}$ & $76,1^{* * *}$ & 84,5 \\
\hline & (Sehr) religiös & $17,4^{* * *}$ & $58,1^{* * * *}$ & $44,7^{* * *}$ & $52,6^{* * *}$ & $44,9^{* * *}$ & $45,7^{* * *}$ & $76,4^{* * *}$ & 85,9 \\
\hline
\end{tabular}

Quelle: FLB (2012), gewichtete Daten, eigene Berechnungen

Anm.: ${ }^{* * *} \mathrm{p} \leq 0,001 ;{ }^{* *} \mathrm{p} \leq 0,01 ;{ }^{*} \mathrm{p} \leq 0,05$ (Signifikanzen nach $\mathrm{Chi}^{2}$-Test); Erläuterungen siehe Anmerkungen von Tabelle 2.

Weitere Unterschiede ergeben sich bei der Betrachtung von Personen mit oder ohne Kinder: Eltern denken weniger oft (30,2 \% vs. 38,4\%), dass die Ehe überholt ist. Sie nehmen auch häufiger als Kinderlose wahr, dass die Allgemeinheit der Ehe keine Bedeutung mehr beimisst (54,8 \% vs. 46,3\%). Befragte ohne Nachwuchs sehen in der Gesellschaft die Norm des Übernehmens des Männernamens als weit verbreiteter an (63,1 \%vs. 51,5 \%). Bilokale 
Paare werden von Eltern seltener als ,richtige“ Paare akzeptiert als von Kinderlosen, unter denen es auch deutlich mehr Personen gibt, die Partnerschaften in getrennten Haushalten führen (Dorbritz 2009). Auch hier ist das eigene aktuelle Lebensmodell mit der Bewertung verknüpft. Eltern sehen in der Öffentlichkeit häufiger als Kinderlose, dass auch ein Zusammenleben ohne Heirat in Ordnung ist $(75,4 \%$ vs. $66,4 \%)$. Kaum verschieden ist die Wahrnehmung hinsichtlich der Befürwortung der Ehe auf Probe, die von beiden Gruppen ähnlich oft innerhalb der Gesellschaft gesehen wird.

Außerdem spielt der Migrationshintergrund eine Rolle: Personen mit Migrationshintergrund finden eher, dass die Allgemeinheit der Ehe keine Bedeutung mehr beimisst. Sie beurteilen es persönlich wesentlich häufiger als wichtig, wenn ein Ehepaar den Nachnamen des Mannes trägt, sie nehmen dies jedoch etwas seltener in der Allgemeinheit wahr. Und ihre Akzeptanz gegenüber getrennt lebenden Paaren ist wesentlich geringer ausgeprägt $(43,9 \%$ vs. $27,6 \%)$.

Bei der Betrachtung nach Partnerschaftsstatus fällt auf, dass Verheiratete sehr viel seltener die Ehe als überholt einstufen als die Unverheirateten (18,3\% vs. ca. $40 \%$ bei den anderen drei Partnerschaftsformen), auch nehmen sie häufiger wahr, dass die Gesellschaft die Ehe als nicht mehr zeitgemäß bewertet. Die Diskrepanz zwischen eigener Bewertung und Allgemeinheitswahrnehmung ist bei den Verheirateten am größten. Ähnlich ist hingegen die persönliche Einstellung zur Namensänderung über die vier Gruppen hinweg. Verheiratete nehmen aber auch signifikant seltener $(49,5 \%$ im Vergleich zu ca. $60 \%$ bei den anderen Gruppen) wahr, dass die Öffentlichkeit eine Namensänderung von Frauen gutheißt. Dies liegt sicherlich daran, dass viele Verheiratete einen gemeinsamen Nachnamen (i. d. R. den des Mannes) tragen. Daher ist an dieser Stelle auch der Unterschied zwischen persönlicher und Allgemeinheits-Meinung weniger groß als bei den anderen drei Lebensformen. Hinsichtlich der Einstellung zur Bilokalität von Paaren sind die Verheirateten am wenigsten tolerant (40,3\%, ähnlich dazu auch 45,4\% Allgemeinheit). Von den nicht Verheirateten befürworten erwartungsgemäß die selbst Betroffenen, $d$. h. die unverheirateten Personen, die eine Partnerschaft mit getrennten Haushalten führen, bilokale Partnerschaften am ehesten als „richtige“ Paare. Jedoch bewertet immerhin ein Viertel derjenigen, die selbst in einer bilokalen Partnerschaft leben, das eigene Partnerschaftsmodell als nicht adäquat. Und wie werden getrennt lebende Paare gesellschaftlich gesehen? Befragte aus nichtehelichen Lebensgemeinschaften nehmen von allen vier Lebensform-Gruppen am häufigsten wahr (54,1 \%), dass getrennt lebende Paare nicht als vollwertige Beziehungen eingestuft werden. Hinsichtlich der öffentlichen Akzeptanz von nichtehelichen Lebensgemeinschaften sehen Verheiratete die Gesellschaft am tolerantesten (77,7\%). Vermutlich sind diese auch im Alltag im Vergleich zu den anderen nicht mit der Frage konfrontiert, ob und wann sie heiraten.

Abschließend wird die Stärke der subjektiven Religiosität untersucht: Es zeigt sich deutlich, dass Religiosität und die persönliche Wertschätzung der Institution Ehe ${ }^{11}$ stark zusammenhängen (lediglich 17,4 \% halten die Ehe für überholt; wenig/nicht Religiöse 43,0 \%). Umgekehrt nehmen mittel bis sehr religiöse in der Gesellschaft eher eine verbreitete ablehnende Haltung gegenüber der Ehe wahr. Mit zunehmender Religiosität steigt auch die individuelle Zustimmung für eine Namensänderung. Wenig oder nicht Religiöse stehen bilokalen Partnerschaften toleranter gegenüber $(27,7 \%)$ als (sehr) Religiöse $(44,9 \%)$ und sie sehen in der Allgemeinheit häufiger Intoleranz gegenüber dieser Lebensform. Dies könnte daran liegen, dass nicht Religiöse in der Stichprobe tendenziell seltener verheiratet sind und auch generell in den abrahamitischen ${ }^{12}$ Religionen die Institution Ehe für Paare

11 Das Commitment zur Ehe ist bei Religiösen stärker ausgeprägt (Lambert/Dollahite 2006).

12 Christentum, Judentum und Islam. 
eine größere Bedeutung hat. Die Bewertung von nichtehelichem dauerhaftem Zusammenleben sehen wenig bzw. nicht Religiöse auch dementsprechend seltener positiv in der Gesellschaft eingeordnet. Wie genau die Zugehörigkeit zu einer spezifischen Konfession bzw. Konfessionslosigkeit und die selbst eingeschätzte Religiosität mit Partnerschaftsleitbildern zusammenhängen, wird in diesem Band (vgl. Naderi, Kap. 7) näher erläutert.

\subsection{Wie kann Partnerschaft gelingen?}

Für die Messung von Partnerschaftserfolg wurden sechs Aussagen zusammengestellt. Nahe$\mathrm{zu}$ alle Befragten stimmen auf persönlicher Ebene zu, dass Liebe zentral ist (Tabelle 5). Gleichermaßen wichtig sind für die meisten $(97,8 \%)$ die Freiräume, allerdings wird dies in der Gesellschaft etwas seltener so wahrgenommen (89,2\%).

Tabelle 5: Zustimmung auf persönlicher Ebene zu verschiedenen Dimensionen des Partnerschaftserfolges (in Prozent)

\begin{tabular}{lcc}
\hline Partnerschaft funktioniert, wenn man ... & $\begin{array}{c}\text { Persönliche } \\
\text { Zustimmung }\end{array}$ & $\begin{array}{c}\text { Wahrgenommene } \\
\text { Zustimmung in der } \\
\text { Allgemeinheit }\end{array}$ \\
\hline sich liebt. & 97,8 & $\mathrm{x}$ \\
dem anderen Freiräume lässt. & 97,8 & 89,2 \\
eine erfüllte Sexualität miteinander hat. & 93,2 & 93,3 \\
finanziell abgesichert ist. & 86,1 & 93,0 \\
gemeinsame Kinder hat. & 62,5 & 70,7 \\
im Zweifelsfall den Mann entscheiden lässt, wo es langgeht. & 22,7 & 47,4 \\
\hline
\end{tabular}

Quelle: FLB (2012), gewichtete Daten, eigene Berechnungen

Anm.: $\quad \mathrm{x}=$ Frage wurde nicht gestellt.

Auch erfüllte Sexualität ist sowohl auf persönlicher Ebene (93,2 \%) als auch auf allgemeiner Ebene (93,3 \%) von großer Bedeutung. Finanzielle Absicherung kommt direkt im Anschluss mit 86,1\%, die sogar gesellschaftlich noch stärker als Erfolgsgarant gesehen wird $(93,0 \%)$. Während gemeinsame Kinder $62,5 \%$ wichtig finden, wird dies in der Öffentlichkeit noch stärker bedeutsam wahrgenommen (70,7 \%). Interessanterweise gibt immerhin ein Fünftel der Befragten an (22,7\%), dass es für Partnerschaften gut sei, wenn der Mann im Zweifelsfall entscheidet. In der Gesellschaft sehen fast die Hälfte (47,4 \%), dass dies für das Funktionieren einer Partnerschaft bedeutsam ist.

\section{Ergebnisse zur Hauptkomponentenanalyse: Welche Partnerschaftsleitbilder gibt es?}

Die zweite Forschungsfrage wird anhand der zuvor beschriebenen Variablen mittels Hauptkomponentenanalyse untersucht, ob sich dahinter inhaltliche Dimensionen verbergen und sich indexbasierte Partnerschaftsleitbilder daraus ableiten lassen. Diese werden schließlich nach verschiedenen soziodemografischen Merkmalen betrachtet, um zu prüfen, welche Personengruppen welche Partnerschaftsleitbilder verinnerlicht haben. Im Folgenden werden 
in einem ersten Schritt die individuellen und danach die gesellschaftlichen Partnerschaftsleitbilder getrennt voneinander untersucht.

\subsection{Individuelle Partnerschaftsleitbilder}

Ziel ist es, zunächst zu analysieren, ob sich die zuvor beschriebenen Aspekte der Partnerschaft zu einem Gesamtleitbild der idealen Partnerschaft gruppieren lassen. Dazu wurden alle Items zur Partnerschaft einbezogen (Tabelle 6). Der erste Faktor erklärt knapp $19 \%$ der Gesamtvarianz, 10,4 \% werden durch den zweiten Faktor, 9,4\% durch den dritten sowie 7,5\% durch den vierten aufgenommenen Faktor erklärt.

1. Die Variablen ergeben insgesamt vier verschiedene inhaltliche Dimensionen: Die erste Dimension ${ }^{13}$ wird das fusionsorientierte Partnerschaftsleitbild genannt, da sie den Glauben an das Lebensglück durch Partnerschaft umfasst, eine hohe Eheorientierung, die Verfolgung gemeinsamer Ziele sowie die Familiengründung als Stabilitätsgarant für die Partnerschaft. Dahinter steckt die Vorstellung, dass zwei Menschen zu einem „Wir“ fusionieren.

2. Eine weitere Komponente ${ }^{14}$ wird als bürgerlich-konventionelles Partnerschaftsleitbild bezeichnet (vgl. Naderi, Kap. 7): Hierbei ist gleichermaßen die Eheschließung wichtig, jedoch lehnen Personen mit diesem Leitbild dann Partnerschaften ab, wenn die Frau höher gebildet ist als der Partner. Und sie befürworten es, wenn Männer die Entscheidungsmacht haben und es finanzielle Sicherheit gibt. Zudem beinhaltet dieses Partnerschaftsleitbild, dass Frauen nach der Hochzeit den Namen ihres Mannes tragen sollten und dass Paare ohne gemeinsamen Haushalt keine richtigen Paare sind. Die Bezeichnung „bürgerlich-konventionell“" bezieht sich im weiteren Sinne auf die Definition der bürgerlichen Familie nach Rosenbaum (1978), wenngleich jedoch im Hinblick auf die Arbeitsteilung zwischen den Geschlechtern hier wichtige Items fehlen, um das Leitbild der Rosenbaum'schen bürgerlichen Familie genau abzubilden. Die Bedeutung der männlichen Entscheidungsmacht und der Namensänderung bei der Heirat lassen jedoch darauf schließen, dass es sich um ein geschlechterasymmetrisches Partnerschaftsleitbild handelt, welches der Partnerschaft innerhalb der bürgerlichen Familie zumindest in Teilen ähnelt.

3. Als drittes Leitbild konnte das assoziativ-moderne Partnerschaftsleitbild herausgefiltert werden ${ }^{15}$. Personen dieser Orientierung gehen davon aus, dass Partnerschaften funktionieren, wenn man sich liebt, eine erfüllte Sexualität miteinander hat, dem anderen auch Freiräume lässt, beide Partner assoziativ Seite an Seite leben (durch Wahrung ihrer Autonomie) und finanziell abgesichert sind.

4. Ein viertes Leitbild ${ }^{16}$ ergibt sich aus einer geringen Eheorientierung und aus dem Pessimismus gegenüber der Dauerhaftigkeit von Partnerschaften generell. Dieses wird im Folgenden eheablehnendes-instabiles Partnerschaftsleitbild genannt.

In einem weiteren Schritt wurden basierend auf den Ergebnissen der Hauptkomponentenanalyse vier Summenindizes ${ }^{17}$ gebildet, die wiederum dichotomisiert wurden nach Zustim-

Die interne Konsistenz der erfassten Items beträgt Alpha $=, 585$.

Die interne Konsistenz der erfassten Items beträgt Alpha $=, 474$.

Die interne Konsistenz der erfassten Items beträgt Alpha $=, 403$.

Die interne Konsistenz der erfassten Items beträgt Alpha $=, 413$.

Der Index wurde durch eine Addition der Items und Division durch ihre Anzahl berechnet. 
mung oder Ablehnung, um sie kreuztabellarisch im Hinblick auf mögliche sozialstrukturelle Unterschiede ${ }^{18} \mathrm{zu}$ untersuchen. Es wird zunächst deutlich, dass die 20- bis 39-Jährigen zum Teil mehrere Partnerschaftsleitbilder gleichzeitig vertreten, es wird nicht immer ein Partnerschaftsleitbild konsistent bzw. ausschließlich befürwortet. Am weitesten auf individueller Ebene verbreitet ist das assoziativ-moderne Partnerschaftsleitbild, fast alle Befragten befürworten die damit verbundenen Vorstellungen. Jedoch gibt es diverse Schnittmengen zwischen den Leitbildern, einige Items laden auf mehreren Faktoren (Ehenorm und finanzielle Absicherung). Am zweithäufigsten ist das ,fusionsorientierte Partnerschaftsleitbild“ (54,1\%), gefolgt vom „,bürgerlich-konventionellen“ $(34,7 \%)$ und schließlich dem „nichtehelichen-instabilen Partnerschaftsleitbild“ (29,7 \%).

Tabelle 6: Hauptkomponentenanalyse auf individueller Ebene zu verschiedenen Dimensionen der Partnerschaft

\begin{tabular}{|c|c|c|c|c|}
\hline & \multicolumn{4}{|c|}{ Komponente } \\
\hline & 1 & 2 & 3 & 4 \\
\hline Cronbach's $\alpha$ & ,585 & 474 & 403 & ,413 \\
\hline Anteil erklärter Varianz (in Prozent) & 18,9 & 10,4 & 9,4 & 7,5 \\
\hline $\begin{array}{l}\text { Menschen können nur in einer festen und stabilen } \\
\text { Partnerschaft glücklich sein. }\end{array}$ & ,662 & & & \\
\hline $\begin{array}{l}\text { Wenn man als Paar dauerhaft zusammenlebt, } \\
\text { sollte man heiraten. }\end{array}$ & ,513 & ,404 & &,- 437 \\
\hline Partnerschaften können nur zeitlich begrenzt funktionieren. & & & & ,679 \\
\hline Die Ziele des Paares sind wichtiger als die des Einzelnen. & 687 & & & \\
\hline $\begin{array}{l}\text { Es ist schlecht, wenn der Mann eine niedrigere } \\
\text { Bildung hat als die Frau. }\end{array}$ & & ,691 & & \\
\hline Partnerschaft funktioniert, wenn man sich liebt. & & &, 572 & \\
\hline $\begin{array}{l}\text { Partnerschaft funktioniert, wenn man eine erfüllte } \\
\text { Sexualität miteinander hat. }\end{array}$ & & & ,690 & \\
\hline $\begin{array}{l}\text { Partnerschaft funktioniert, wenn man dem anderen } \\
\text { Freiräume lässt. }\end{array}$ & & & ,625 & \\
\hline $\begin{array}{l}\text { Partnerschaft funktioniert, wenn man im Zweifelsfall } \\
\text { den Mann entscheiden lässt, wo es langgeht. }\end{array}$ & & ,492 & & \\
\hline $\begin{array}{l}\text { Partnerschaft funktioniert, wenn man finanziell } \\
\text { abgesichert ist. }\end{array}$ & & ,406 & ,421 & \\
\hline $\begin{array}{l}\text { Partnerschaft funktioniert, wenn man gemeinsame } \\
\text { Kinder hat. }\end{array}$ & ,438 & & & \\
\hline Die Ehe ist eine überholte Einrichtung. & & & & ,762 \\
\hline $\begin{array}{l}\text { Eine Frau sollte nach der Heirat den Namen ihres } \\
\text { Mannes annehmen. }\end{array}$ & & ,575 & & \\
\hline Paare mit getrennten Haushalten sind keine richtigen Paare. & & ,392 & & \\
\hline
\end{tabular}

Quelle: FLB (2012), gewichtete Daten, eigene Berechnungen

Anm.: Varimax, rotierte Komponentenmatrix; Werte ab 0,4 werden angezeigt; die ersten vier Komponenten erklären $46 \%$ der Gesamtvarianz.

18 Es wurden $\mathrm{Chi}^{2}$-Tests durchgeführt. 
Das „fusionsorientierte Partnerschaftsleitbild“ befürworten signifikant mehr Männer als Frauen und deutlich seltener hoch Qualifizierte (48,7\% vs. $56 \%$ mittel bzw. 57,2 \% niedrig). Gleichermaßen befinden sich unter den Befürwortern auch viel mehr Eltern $(62,5 \%)$ als Kinderlose (48,4 \%) und auch häufiger Personen mit Migrationshintergrund (62,4\% vs. $51,6 \%$ ). Betrachtet man signifikante Differenzen beim „,bürgerlich-konventionellen Partnerschaftsleitbild“, lassen sich weitere Unterschiede feststellen: Fast die Hälfte aller niedrig Qualifizierten vertreten dieses Leitbild (40,2\%), mit zunehmender Bildung sinkt die Zustimmung (mittel Gebildete: 35,8\%, hoch Gebildete: 29,5\%). Auch sind in dieser Gruppe mehr Eltern vertreten (38,6\% vs. 32,1\%) sowie deutlich mehr Befragte mit Migrationserfahrung (45,2 \% vs. 31,5\%). Da nahezu alle Befragten das ,assoziativ-moderne Partnerschaftsleitbild" befürworten, gibt es keine Varianz hinsichtlich sozialstruktureller Merkmale. Auch beim „eheablehnenden-instabilen Partnerschaftsleitbild“ finden sich wenig Unterschiede, lediglich Elternschaft führt zu signifikanten Unterschieden: Kinderlose (31,7\%) sind etwas häufiger Träger dieses Leitbildes als Eltern (26,6 \%).

\subsection{Gesellschaftliche Partnerschaftsleitbilder}

Wie bei den Bestandteilen der persönlichen Leitbilder wurde auch für die Allgemeinheitsleitbilder eine Hauptkomponentenanalyse durchgeführt (Tabelle 7).

Tabelle 7: Hauptkomponentenanalyse auf Allgemeinheits-Ebene zu verschiedenen Dimensionen der Partnerschaft

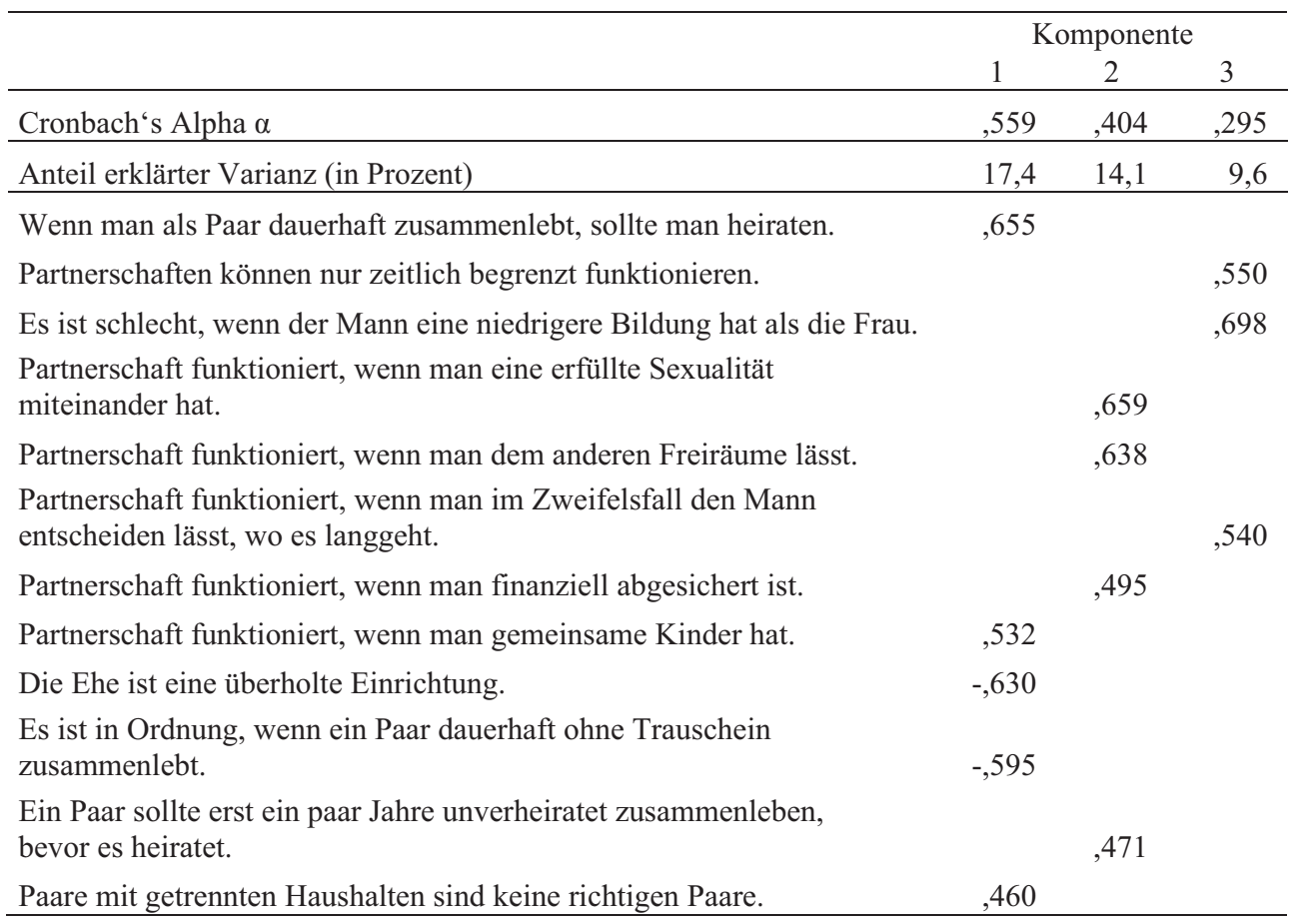

Quelle: FLB (2012), gewichtete Daten, eigene Berechnungen

Anm.: Varimax, rotierte Komponentenmatrix; Werte ab 0,4 werden angezeigt. 
Die einbezogenen Variablen sind jedoch nicht vollkommen deckungsgleich mit denen der ersten Berechnung, da nicht alle Aussagen auf gesellschaftlicher Ebene gespiegelt abgefragt worden sind. Daher kann an dieser Stelle kein Vergleich erfolgen. Jedoch kann hier festgestellt werden, dass in der Allgemeinheit drei dominante Partnerschaftsleitbilder wahrgenommen werden: Die erste Dimension könnte als bürgerlich-konventionelles Partnerschaftsleitbild innerhalb der Allgemeinheit bezeichnet werden, da es der Ehe, Familiengründung und dem Zusammenleben eine hohe Bedeutung beimisst. Ein weiteres Partnerschaftsleitbild, welches assoziativ-entwicklungsorientiert ist, thematisiert Partnerschaftserfolg durch erfüllte Sexualität, Freiräume, ökonomische Absicherung und durch die Probeehe. Es könnte als assoziativ-modernes Partnerschaftsleitbild bezeichnet werden, es ähnelt dem auf individueller Ebene gefundenem. Des Weiteren hängen Zweifel an der Dauerhaftigkeit von Partnerschaften, die Ablehnung von Bildungsheterogamie zugunsten der Frau sowie die Befürwortung männlicher Entscheidungsmacht eng zusammen. Dies wird als stabilitätsablehnendgeschlechterasymmetrisches Partnerschaftsleitbild bezeichnet.

\subsection{Zusammenfassung der Hauptkomponentenanalyse}

Insgesamt wurden vier verschiedene Partnerschaftsleitbilder auf individueller Ebene identifiziert, die teilweise Schnittmengen aufweisen aufgrund des weit verbreiteten Wunsches nach finanzieller Absicherung und nach einer Eheschließung bei dauerhaftem Zusammenleben: fusionsorientiert, bürgerlich-konventionell, assoziativ-modern und eheablehnendinstabil. Wie zu erwarten sinkt mit zunehmender Bildung die Zustimmung zu einem „bürgerlich-konventionellen Partnerschaftsleitbild“. Allerdings haben dessen Befürworter häufiger Kinder und einen Migrationshintergrund. Fusionsorientiert eingestellt sind häufiger Männer, niedriger Qualifizierte, Eltern und Befragte mit Migrationshintergrund. Bei „,assoziativ-modernen Leitbildern“ gab es keine Unterschiede, da hier nahezu alle Befragten hohe Zustimmung für die einbezogenen Variablen zeigten. Ebenfalls kaum Unterschiede nach Sozialstruktur finden sich bei den eheablehnenden-instabilen Leitbildern.

Des Weiteren konnten in der Gesellschaft drei wahrgenommene Leitbilder manifestiert werden, die den individuellen in einigen Aspekten ähneln und dementsprechend auch von ihrer Bezeichnung her angepasst wurden: bürgerlich-konventionell, assoziativ-modern und stabilitätsablehnend-geschlechterasymmetrisch.

\section{$5 \quad$ Diskussion und Fazit}

Erstens wurde untersucht, wie weit bestimmte kulturelle Idealvorstellungen zur Partnerschaft innerhalb der 20- bis 39-jährigen Bevölkerung in Deutschland verbreitet sind. Dabei wurden sowohl individuelle als auch gesellschaftlich wahrgenommene Vorstellungen näher untersucht und danach miteinander verglichen. Es wurde deutlich, dass es eine Reihe von Idealvorstellungen gibt, die in breiten Teilen der Gesellschaft sowohl individuell, als auch gesellschaftlich als gültig eingestuft werden: Dabei wird deutlich, dass das Leit(d)bild des Singles als Negativfolie bei einigen jungen Erwachsenen präsent ist. Menschen finden laut einem Großteil der Befragten nur in festen und stabilen Partnerschaften ihr Glück. Wenngleich eine überwiegende Mehrheit immer noch daran glaubt, ist immerhin ein Drittel der Meinung, dass dies nicht zutrifft. Daraus lässt sich schließen, dass Partnerschaft zwar einen hohen Stellenwert im Leben vieler junger Menschen hat, jedoch nicht von allen als alleiniger 
Glücksbringer gesehen wird. Ein Zehntel der Befragten glaubt, dass Partnerschaften nur zeitlich begrenzt funktionieren können. Entgegen der Individualisierungstendenzen in der Gesellschaft halten immer noch mehr als die Hälfte der jungen Erwachsenen die Ziele des Paares für wichtiger als die des Einzelnen.

Werden dazu die subjektiven Wahrnehmungen innerhalb der Gesellschaft gegenübergestellt, zeigen sich interessante Kontraste: Mehr als ein Drittel der Befragten sagt, dass die Gesellschaft Partnerschaften nur eine zeitlich begrenzte Gültigkeit einräumt. Auch nehmen mehr als die Hälfte wahr, dass die Allgemeinheit es schlecht findet, wenn ein Mann niedriger qualifiziert ist als seine Partnerin. Hinsichtlich der Bildungsheterogamie in Partnerschaften bleibt zu klären, ob sich diese gesellschaftliche Wahrnehmung auf die individuelle Partnerwahl (insbesondere bei höher qualifizierten Frauen) auswirkt, denn persönlich scheinen die Befragten damit kein Problem zu haben. Jedoch befürchten sie vielleicht eine Stigmatisierung.

Bei der Unterscheidung nach soziodemografischen Merkmalen zeigen sich verschiedene signifikante Unterschiede: Beispielweise sind Männern partnerschaftliche Ziele wichtiger als Frauen, und Frauen sehen etwas häufiger Probleme, wenn in der Partnerschaft die Frau höher qualifiziert ist. Hier könnten vermutlich antizipierte Effekte von Vereinbarkeitsproblemen hineinspielen, jedoch auch eher komplementäre Rollenverteilungen, die innerhalb der Öffentlichkeit teilweise noch wahrgenommen werden.

Bei der Untersuchung der Eheorientierung und des Institutionalisierungsgrades zeigt sich insgesamt, dass innerhalb der Gesellschaft der Institution Ehe eine weniger große Bedeutung zugestanden wird als auf persönlicher Ebene. Gleichzeitig gibt es aber im Vergleich zu den individuellen Ansichten einen wahrgenommenen stärkeren gesellschaftlichnormativen Druck auf Paare, dass sie den Nachnamen des Mannes tragen und bei dauerhaftem Zusammenleben heiraten. Auch werden bilokale Paare innerhalb der Öffentlichkeit als weniger akzeptiert angesehen, zumindest aus Sicht der Befragten. Das bedeutet, dass das subjektive Bild von der Allgemeinheit eher bürgerlich-konventionell und geschlechterasymmetrisch eingeschätzt wird. Gleichzeitig aber auch gibt es eine vielfach in der Gesellschaft gesehene Erwartung einer Probeehe. Hier gab es einige Geschlechterdifferenzen und auch leichte regionale Unterschiede: Befragte der neuen Bundesländer denken etwas weniger konventionell als die aus den alten Bundesländern. Auch höher Qualifizierte sind weniger konventionell genauso wie Kinderlose, weitere Unterschiede ergaben sich durch die Herkunft, den Partnerschaftsstatus und durch die Religiosität. Insgesamt zeigt sich bei den Einstellungen zur Partnerschaft, dass häufig ein Erfahrungseffekt hinter den Unterschieden nach sozialstrukturellen Merkmalen stecken könnte. Dies müssten weitere Analysen vertiefend prüfen. Es ist dabei fraglich, ob das Leitbild die Lebensführung oder die Lebensführung das Leitbild formt. Im Sinne der Sozialisation könnte man davon ausgehen, dass Leitbilder zur Partnerschaft zuerst da sind (durch das Lernen am Modell der Eltern), im Laufe des Lebens mit den gesammelten Erfahrungen abgeglichen werden und gegebenenfalls eine Anpassung erfahren, um kognitive Dissonanzen zu vermeiden. Zum Beispiel kann es sein, dass Geschiedene oder Befragte mit geschiedenen Eltern ${ }^{19}$ wesentlich häufiger finden, dass die Ehe eine überholte Einrichtung ist. Es wäre daher hilfreich, bei einer Wiederbefragung Informationen zu den Eltern der Befragten zu erheben, um so die Wirkungsrichtung von Leitbildern und Erfahrungen entschlüsseln zu können.

19 Im Leitbildsurvey sind keine Informationen enthalten, die die Untersuchung von intergenerationaler Scheidungstransmission erlauben. 
Einen weiteren Bestandteil der kulturellen Vorstellungen bilden die Kriterien für das Gelingen einer Partnerschaft. Die wichtigsten Kriterien für den Partnerschaftserfolg sind gegenseitige Liebe, gefolgt von erfüllter Sexualität, Freiraum lassen und finanzieller Absicherung. Diese Kriterien bilden ein in der jungen Bevölkerung weit verbreitetes WerteFundament an Leitbildelementen für das Funktionieren einer Partnerschaft. Kinderhaben hingegen erscheint in der Gesellschaft etwas wichtiger bewertet zu sein als auf Individualebene. Schließlich haben ein Fünftel der Studienteilnehmer zugestimmt, dass eine Partnerschaft funktioniert, wenn man im Zweifelsfall der Mann entscheiden lässt, wo es langgeht. Doppelt so viele Befragte sehen in der Gesellschaft die Befürwortung von gelegentlicher männlicher Entscheidungsmacht in Partnerschaften. Auch hier schimmert das etwas konservativere wahrgenommene Bild der Gesellschaft durch.

Insgesamt lässt sich beim Vergleich zwischen individuellen Vorstellungen und den in der Gesellschaft wahrgenommenen feststellen, dass es zum Teil starke Unterschiede zwischen persönlichen Vorstellungen und Gesellschaftswahrnehmung gibt: In der Gesellschaft wird ein eher instabileres pessimistischeres Bild einer Partnerschaft gesehen, während die persönliche Einschätzung durchaus optimistischer erscheint.

Zweitens sollte überprüft werden, ob sich Leitbilder der Partnerschaft identifizieren lassen und falls ja, wie weit verbreitet sie sind und ob sie auch nach soziodemografischen Merkmalen zu differenzieren sind. Insgesamt konnten auf persönlicher Ebene vier und auf der gesellschaftlich wahrgenommenen Ebene drei Partnerschaftsleitbilder identifiziert werden.

Fast alle befürworten auf Individualebene das ,assoziativ-moderne Partnerschaftsleitbild“. Über die persönlichen Leitbilder hinweg bedeutsam sind die Ehenorm und die finanzielle Absicherung. Am zweithäufigsten wird das „fusionsorientierte“ Partnerschaftsleitbild favorisiert, gefolgt vom „bürgerlich-konventionellen“ und schließlich dem „,nichtehelicheninstabilen Partnerschaftsleitbild“. Es bestätigt sich die eingangs gemachte Annahme, dass junge Menschen eher assoziativ-orientierte Idealvorstellungen vertreten. Jedoch besteht offenbar eine gewisse Inkonsistenz hinsichtlich anderer fusions- und auch stabilitäts-bezogener Dimensionen von Partnerschaft, die parallel zu assoziativen Aspekten befürwortet werden. Vielleicht spiegeln sich darin die in familien- und sozialpolitischen Regelungen latent enthaltenen inhärenten Partnerschaftsvorstellungen („male breadwinner“ vs. Eigenverantwortung von Frauen, siehe BMFSFJ 2012: 53ff.) und Rollenbilder wider. Beispielsweise die Reform des Unterhaltsrechts von 2008 jedoch auch andere gesetzliche Regelungen für verheiratete Paare (wie z. B. Ehegattensplittung, kostenlose Mitversicherung des nicht erwerbstätigen Ehepartners in gesetzlicher Krankenkasse) erzeugen häufig widersprüchliche Impulse und verstärken bestimmte Partnerschaftsleitbilder zuungunsten anderer (vgl. dazu auch BMFSFJ 2012). Insgesamt ist vorstellbar, dass dies zur Verunsicherung von jungen Erwachsenen in ihrer Gestaltung der Partnerschafts- und Erwerbsbiografie führt, welche Partnerschaftsleitbilder als passend und praktikabel für den Alltag erscheinen.

Innerhalb der Gesellschaft werden drei Leitbilder wahrgenommen: das „,bürgerlichkonventionelle“, das ,assoziativ-moderne“ und das „stabilitätsablehnend-geschlechterasymmetrische" Partnerschaftsleitbild. Welchen Einfluss die Wahrnehmung dieser Leitbilder auf die Entscheidung von Einzelnen nimmt, dazu ist weitere Forschung nötig. Wahrgenommener sozialer Druck jedoch könnte vermutlich eine Rolle spielen, um beispielsweise bestimmte Prozesse der Partnerwahl und Partnerschaftsentwicklung besser zu verstehen.

Es zeigt sich aber auch, dass die öffentliche Meinung insgesamt etwas konventioneller wahrgenommen wird. Persönliche und (aus Sicht der Befragten vermeintlich bestehende) allgemeine Partnerschaftsleitbilder sind daher teilweise nicht deckungsgleich. Vermutlich besonders dann nicht, wenn sie einen Kontrast zur eigenen Einstellung darstellen. Insgesamt wird deutlich, dass Aspekte der Sozialisation, nämlich Herkunft und Bildung, einen 
großen Einfluss auf die Ausgestaltung partnerschaftlicher Leitbilder nehmen. Eine ähnliche Rolle spielt die Lebenssituation: Elternschaft steht im engeren Bezug zu bürgerlichen Leitbildelementen wie zur finanziellen Absicherung und zur Ehenorm.

Künftig gilt es zu testen, inwiefern die gefundenen Partnerschaftsleitbilder mit bestimmten Mustern der Familiengründung und -erweiterung zusammenhängen und hierbei sowohl regionalspezifische als auch sozialstrukturelle Besonderheiten aufzuspüren.

\section{Literatur}

Arránz Becker, Oliver (2008): Was hält Partnerschaften zusammen? Psychologische und soziologische Erklärungsansätze zum Erfolg von Paarbeziehungen. 1. Auflage Wiesbaden: VS Verlag für Sozialwissenschaften.

Arránz Becker, Oliver; Hill, Paul B. (2008): Bildungshomogamie und Partnerschaftserfolg - eine dyadische Analyse. In: Huinink, Johannes; Felser, Georg (Hrsg.): Neuere Entwicklungen in der Beziehungs- und Familienforschung. Vorstudien zum Beziehungs- und Familienentwicklungspanel (PAIRFAM). Würzburg: Ergon Verlag.

Arránz Becker, Oliver; Rüssmann, Kerstin; Hill, Paul B. (2005): Wahrnehmung und Bewältigung von Konflikten und die Stabilität von Partnerschaften. In: Zeitschrift für Familienforschung 17, S. 251-278.

Beck, Ulrich (1986): Auf dem Weg in eine andere Moderne. Frankfurt: Suhrkamp.

Bender, Doris; Lösel, Friedrich (2003): Kohärenzsinn und andere Persönlichkeitsmerkmale als protektive Faktoren der Ehequalität. In: Grau, Ina; Bierhoff, Hans-Werner (Hrsg.): Sozialpsychologie der Partnerschaft. Berlin, New York: Springer, S. 405-427.

Billari, Francesco C.; Wilson, Chris (2001): Convergence towards diversity? Cohort dynamics in the transition to adulthood in contemporary Western Europe. In: MPIDR Working Papers WP-2001039. Rostock: Max Planck Institute for Demographic Research.

BMFSFJ (Bundesministerium für Familie, Senioren, Frauen und Jugend) (Hrsg.) (2012): Neue Wege - gleiche Chancen. Gleichstellung von Frauen und Männern im Lebensverlauf. Drucksache 17/6240. Stand: Dezember 2012, 3. Auflage.

Bodenmann, Guy (2001): Psychologische Risikofaktoren für Scheidung: Ein Überblick. In: Psychologische Rundschau 52, S. 85-95.

Bodenmann, Guy (2003): Die Bedeutung von Stress für die Partnerschaft. In: Grau, Ina; Bierhoff, HansWerner (Hrsg.): Sozialpsychologie der Partnerschaft. Berlin, New York: Springer, S. 481-504.

Bradbury, Thomas N.; Fincham, Frank D.; Beach, Steven R.H. (2000): Research on the Nature and Determinants of Marital Satisfaction: A Decade in Review. In: Journal of Marriage and Family 62, S. 964-980.

Crawford, Duane W.; Houts, Renate M.; Huston, Ted L.; George, Laura J. (2002): Compatibility, Leisure, and Satisfaction in Marital Relationships. In: Journal of Marriage and Family, S. 433-449.

Dommermuth, Lars (2008): Wege ins Erwachsenenalter in Europa. Italien, Westdeutschland und Schweden im Vergleich. Wiesbaden: VS Verlag für Sozialwissenschaften.

Dorbritz, Jürgen (2009): Bilokale Paarbeziehungen - die Bedeutung und Vielfalt einer Lebensform. In: Comparative Population Studies -Zeitschrift für Bevölkerungswissenschaft 34, 1-2, S. 31-56.

Dorbritz, Jürgen; Naderi, Robert (2012): Stabilität bilokaler Paarbeziehungen - Rahmenbedingungen und Entwicklungspfade. Eine Analyse der ersten und zweiten Welle von pairfam. In: Comparative Population Studies - Zeitschrift für Bevölkerungswissenschaft 37, 3-4, S. 393-428.

Eckhard, Jan (2014): Theoretische Erklärungen der zunehmenden Kinderlosigkeit - Divergierende Ansätze und das Integrationspotenzial der Frame-Selektions-Theorie. In: Comparative Population Studies - Zeitschrift für Bevölkerungswissenschaft. 39, 1, S. 23-48.

Esser, Hartmut (2001): Soziologie. Spezielle Grundlagen. Band 6: Sinn und Kultur, Frankfurt am Main: Campus.

European Value Survey (1978-2008): [http://www.europeanvaluesstudy.eu/] 
Felser, Georg (2003): Wahrnehmung und Kognitionen in Partnerschaften. In: Grau, Ina; Bierhoff, HansWerner (Hrsg.): Sozialpsychologie der Partnerschaft. Berlin, New York: Springer, S. 343-376.

Giddens, Anthony (1992): The Transformation of Intimacy. Stanford: Stanford University Press.

Gottman, John M.; Krokoff, Lowell J. (1989): Marital Interaction and Satisfaction. A Longitudinal View. In: Journal of Consulting and Clinical Psychology 57, S. 47-52.

Grau, Ina (2003): Emotionale Nähe. In: Grau, Ina; Bierhoff, Hans-Werner (Hrsg.): Sozialpsychologie der Partnerschaft. Berlin, New York: Springer, S. 285-314.

Grau, Ina; Bierhoff, Hans-Werner (2003): Sozialpsychologie der Partnerschaft. Berlin, New York: Springer.

Grau, Ina; Penning, Ravena; Andreß, Hans-Jürgen (2010): Gleichberechtigung und Beziehungszufriedenheit In: Beckers, Tilo; Birkelbach, Klaus; Hagenah, Jörg; Rosar, Ulrich (Hrsg.): Komparative empirische Sozialforschung. Wiesbaden: VS Verlag für Sozialwissenschaften, S. 199-227.

Hahlweg, Kurt (1986): Partnerschaftliche Interaktion. Empirische Untersuchungen zur Analyse und Modifikation von Beziehungsstörungen. München: Röttger.

Hassebrauck, Manfred; Küpper, Beate (2003): Warum wir aufeinander fliegen. Die Gesetze der Partnerwahl. Reinbek bei Hamburg: Rowohlt-Taschenbuch-Verlag.

Hill, Paul B.; Kopp, Johannes (2002): Familiensoziologie. Grundlagen und theoretische Perspektiven. 2. überarb. Auflage Wiesbaden: Westdeutscher Verlag.

Hill, Paul B. (2004): Interaktion und Kommunikation. Eine empirische Studie zu Alltagsinteraktionen, Konflikten und Zufriedenheit in Partnerschaften. Würzburg: Ergon Verlag.

Karney, Benjamin R.; Bradbury, Thomas N. (1995): The Longitudinal Course of Marital Quality and Stability: A Review of Theory, Method, and Research. In: Psychological Bulletin 118, 1, S. 3-34.

Künzler, Jan; Walter, Wolfgang (2001): Arbeitsteilung in Partnerschaften: Theoretische Ansätze und empirische Befunde. In: Huinink, Johannes; Strohmeier, Klaus P.; Wagner, Michael (Hrsg.): Solidarität in Partnerschaft und Familie. Zum Stand familiensoziologischer Theoriebildung. Würzburg: Ergon Verlag, S. 185-217.

Lambert, Nathaniel M.; Dollahite, David C. (2006): How Religiosity Helps Couples Prevent, Resolve, and Overcome Marital Conflict. In: Family Relations 55, S. 439-449.

Lewin, Kurt (1948): The Background of Conflict in Marriage. In: Lewin, Kurt (Hrsg.): Resolving Social Conflicts. Selected Papers on Group Dynamics. New York: Harper und Brothers, S. 84-102.

Meyer, Thomas (1993): Der Monopolverlust der Familie - vom Teilsystem Familie zum Teilsystem privater Lebensformen. In: Kölner Zeitschrift für Soziologie und Sozialpsychologie 45,1, S. 23-40.

Mikula, Gerold (1992): Austausch und Gerechtigkeit in Freundschaft, Partnerschaft und Ehe: Ein Überblickt über den aktuellen Forschungsstand. In: Psychologische Rundschau 43, S. 62-82.

Nave-Herz, Rosemarie (2004): Ehe- und Familiensoziologie. Eine Einführung in Geschichte, theoretische Ansätze und empirische Befunde. 1. Auflage Weinheim: Juventa-Verlag.

PAIRFAM (2008-2022): Beziehungs- und Familienpanel - Panel Analysis of Intimate Relationships and Family Dynamics. [http://PAIRFAM.de/]

Reichle, Barbara; Dette-Hagenmeyer, Dorothea (2008): Individuelle und dyadische Bewältigung von Alltagskonflikten: Themen, Copingstrategien und Partnerschaftsqualität. In: Feldhaus, Michael; Huinink, Johannes (Hrsg.): Neuere Entwicklungen in der Beziehungs- und Familienforschung. Schriftenreihe des Beziehungs- und Familienentwicklungspanels, Bd. 1.Würzburg: Ergon Verlag, S. 229-256.

Rosenbaum, Heidi (1978): Familie als Gegenstruktur zur Gesellschaft - Kritik grundlegender theoretischer Ansätze der westdeutschen Familiensoziologie. Stuttgart: Lucius \& Lucius.

Ruppenthal, Silvia; Lück, Detlev (2009): Jeder fünfte Erwerbstätige ist aus beruflichen Gründen mobil. Berufsbedingte räumliche Mobilität im Vergleich. In: Informationsdienst Soziale Indikatoren 42 , S. 1-5.

Schneewind, Klaus A.; Wunderer, Eva (2003): Bedingungen von „wahrgenommener Positivität“ und „Konfliktkompetenz“ in Ehebeziehungen. In: Zeitschrift für Familienforschung 15, S. 191-219.

Schneider, Norbert F.; Limmer, Ruth; Ruckdeschel, Kerstin (2002): Mobil, flexibel, gebunden. Beruf und Familie in der mobilen Gesellschaft. Frankfurt am Main: Campus. 
Stauder, Johannes (2002): Eheliche Arbeitsteilung und Ehestabilität. Eine Untersuchung mit den Daten der Mannheimer Scheidungsstudie 1996 unter Verwendung ereignisanalytischer Verfahren. Würzburg: Ergon Verlag.

Stöcker, Kerstin; Strasser, Karin; Winter, Monika (2003): Bindung und Partnerschaftsrepräsentation. In: Grau, Ina; Bierhoff, Hans-Werner (Hrsg.): Sozialpsychologie der Partnerschaft. Berlin, New York: Springer, S. 137-163.

Tyrell, Hartmann (1988): Ehe und Familie - Institutionalisierung und Deinstitutionalisierung. In: Lüscher, Kurt; Schultheis, Franz; Wehrspaun, Michael (Hrsg.): Die ,postmoderne' Familie. Konstanz: Universitätsverlag, S. 145-156.

Wagner, Michael; Bernd Weiß (2010): Erwerbssituation und Partnerschaft: Deutsche und türkische Paare im Vergleich. In: Beckers, Tilo; Birkelbach, Klaus; Hagenah, Jörg; Rosar, Ulrich (Hrsg.): Komparative empirische Sozialforschung. Wiesbaden: VS Verlag für Sozialwissenschaften, S. 169-198.

Waller, Maureen R.; McLanahan, Sara S. (2005): „His” and “Her” Marriage Expectations: Determinants and Consequences. In: Journal of Marriage and Family 67, S. 53-67.

Weiß, Bernd; Wagner, Michael (2008): Stehen Konflikte einer guten Partnerschaft entgegen? Eine empirische Analyse auf Grundlage dyadischer Daten. In: Feldhaus, Michael; Huinink, Johannes (Hrsg.): Neuere Entwicklungen in der Beziehungs- und Familienforschung. Würzburg: Ergon Verlag, S. 187-227.

Wunderer, Eva (2005): Partnerschaft zwischen Anspruch und Wirklichkeit: Anforderungen von Ehepartnern und ihre Wirkung in der Ehebeziehung. In: Zeitschrift für Familienforschung 17, S. 308-332. 



\title{
Moderne oder traditionelle Partnerschaftsleitbilder: Welchen Einfluss haben Konfession und Religiosität?
}

\author{
Robert Naderi*
}

\section{Zusammenfassung}

Vorstellungen zur Partnerschaft auf individueller Ebene, sei es die Frage, ob man verheiratet sein will, oder welche Arbeitsteilung im Haushalt präferiert wird, sind zweifellos nicht nur durch persönliche Erfahrungen gewachsen, sondern in verschiedener Weise auch institutionell sozialisiert. Naheliegend ist hier vor allem die normative Wirkung der Religion, mit der sich ein Mensch identifiziert. In diesem Beitrag soll der Frage nachgegangen werden, inwieweit Zugehörigkeit zu einer spezifischen Konfession bzw. Konfessionslosigkeit und die selbst eingeschätzte Religiosität Zusammenhänge mit der persönlichen Haltung gegenüber verschiedenen Aspekten der Partnerschaft aufweisen. Hierfür wird der vom BiB erhobene Survey zu Familienleitbildern in Deutschland analysiert. Hinter dieser Fragestellung steht die These, dass je nach Religionszugehörigkeit und bei einer stärker ausgeprägten Religiosität, häufiger traditionelle Vorstellungen zur Partnerschaft vorliegen. Für die Analyse wurden verschiedene Items zu einem Index zusammengefasst und nach einer Dichotomisierung als abhängige Variable in einer logistischen Regression verwendet. Die Ergebnisse zeigen einen Zusammenhang zwischen Partnerschaftsleitbildern und der Religiosität bzw. Konfessionszugehörigkeit. Zudem deuten sie darauf hin, dass sie wichtiger für die Erklärung der Hinwendung zu einem traditionellerem Partnerschaftsleitbild sind als andere wichtige individuelle Merkmale. Ob die Konfessionszugehörigkeit oder die Religiosität wichtiger ist, hängt von der jeweiligen Konfession ab.

\section{$1 \quad$ Einleitung}

Die individuelle Definition einer ,guten Partnerschaft“ entwickelt sich durch ein komplexes Zusammenwirken verschiedener Einflussfaktoren: Zentral sind auf der einen Seite persönliche Erfahrungen, die Frauen oder Männer in ihrem Leben sammeln, auf der anderen Seite persönliche Wünsche und Bedürfnisse. Zusätzlich gibt es von außen mehr oder weniger gut erkennbare Merkmale, aber auch individuell verbalisierte Vorstellungen darüber, wie eine Partnerschaft sein kann oder sein sollte, die von wissenschaftlichem Interesse sind. In diesem Beitrag wird der Frage nachgegangen, inwieweit sich traditionelle oder anders ausgedrückt bürgerlich-konventionelle ${ }^{1}$ Vorstellungen bezüglich Partnerschaft im Zusammenhang mit Religion erklären lassen.

Nach einem Blick auf ausgewählte Ergebnisse aus der Literatur zu diesem Bereich im zweiten Abschnitt, welches auch die theoretischen Überlegungen und Hypothesen enthält, wird in Abschnitt 3 die methodische Vorgehensweise geklärt. Außerdem werden dort zentrale

\footnotetext{
* Ich danke der anonymen Gutachterin bzw. dem anonymen Gutachter für wertvolle Hinweise.

1 Siehe zur Definition Diabaté, Kap. 6.
} 
Variablen und ihre Verteilung dargestellt. Abschnitt 4 zeigt die Ergebnisse vor allem der multivariaten Analyse. Am Ende werden die Schlussfolgerungen dargelegt.

\section{Forschungsstand, theoretische Überlegungen und Hypothesen}

Warum hier Religion in den Vordergrund gestellt wird, hat drei Gründe: Erstens ist sie insgesamt nachweislich ein Bestandteil der Programmierung bestimmter Leitbilder und ihrer Komponenten, weil diese u. a. von ,religiösen Deutungsgebern“ (Diabaté/Lück 2014: 60) vermittelt werden. Daher stellt sie eine Erklärungsmöglichkeit für die Hinwendung zu bestimmten persönlichen Leitbildelementen dar. Zweitens: Bei Partnerschaften zeigt sich ein besonderer normativer Bezug alleine schon durch die religiöse Fundierung der Institution Ehe - auch wenn sie hierdurch nicht zwangsläufig vor Trennung schützt (Schnor 2012: 230, 252), wie auch durch die konfessionsübergreifende Vorstellung von Stabilität. Bekanntlich sinkt diese mit fortschreitender gesellschaftlicher Säkularisierung, einhergehend mit Steigerung des relativen Wohlstandes bei nicht allzu gravierenden Ungleichheiten und höherer allgemeiner Bildung (Kröhnert/Klingholz 2010:3). Dadurch geht die Bedeutung der Ehe insgesamt zurück - auch wenn sie nach wie vor die beliebteste Partnerschaftsform ist. Entsprechende Befunde wurden bereits in den frühen 1990er Jahren publiziert (Höllinger 1992). Dabei sei aber auch auf den Begriff der Desäkularisierung und auf die aktuellen religionssoziologischen Erkenntnisse und Debatte im Allgemeinen hingewiesen: nach Peter L. Berger schließlich sei die Säkularisierungtheorie empirisch kaum haltbar, insofern der Blick über die westliche Welt und die intellektuellen Eliten hinausgeht (Berger 2013: 3-4). In der modernen Welt ist aus seiner Sicht die Gleichzeitigkeit vom Säkularen und Religiösen - sogar in einer Person - möglich bzw. Realität (Berger 2013: 4). Und selbst wenn es einen Rückgang beispielsweise in der Häufigkeit von Kirchgängen gibt, so scheint Ersatz „,...) durch neue, nicht-institutionalisierte Formen der Religiosität (z. B. Astrologie, Okkultismus, Yoga, New Age) (...)“ (Lois 2011: 84) gesucht zu werden. Dies sollte für die Interpretation der Ergebnisse in diesem Beitrag berücksichtigt werden, wenn man feststellt, dass entsprechend beide Einflüsse existieren und daher auch niemals ein eindeutiges Ergebnis in Richtung Erklärungskraft von Religion auf traditionelle Partnerschaftsleitbilder zu erwarten ist.

Es stellt sich also die Frage nach der individuellen Einstellung zur Ehe und der Ausgestaltung von Partnerschaft und nach dem Einfluss der ebenfalls individuellen Religiosität, was zu einem dritten Grund für die hier vorliegende Untersuchung und ihre vergleichende Perspektive führt: Je stärker auf individueller Ebene religiösen Vorstellungen bzw. Vorgaben oder Auslegungen gefolgt wird, desto eher kann mit Einstellungen gerechnet werden, die in Richtung Dauerhaftigkeit von Partnerschaft sowie ihrer ,sittsamen“ Ausgestaltung, aber auch auf eine Ablehnung von dauerhafter Nichtehelichkeit, hindeuten. Die Religionszugehörigkeit ist für sich genommen dafür erst mal nicht maßgeblich. Trotzdem kann doch für Muslime in Deutschland insgesamt eine höhere Religiosität bzw. eine höhere Orientierung an bestimmten Prinzipien des Familienlebens und der Familienentwicklung im Vergleich zu der Mehrheit der Deutschen mit christlicher Religionszugehörigkeit festgestellt werden. So zeigt eine Studie von Sagel und Althammer (2013) neben weiteren Befunden, dass die Einstellung von katholischen Jugendlichen zur Ehe den modernen Vorstellungen entspricht (Sagel/Althammer 2013: 111).

Im folgenden Beitrag wird zwischen der Zugehörigkeit zu einer Religionsgemeinschaft, die auf persönlicher Ebene noch nicht mit individuellen Handlungsweisen, Werten und Perspektiven verknüpft sein muss und der Religiosität, welche sicher handlungsrele- 
vant ist, unterschieden. In anderen Studien wird statt dem abstrakten Konstrukt der Religiosität vielmehr die religiöse Praxis verwendet (z. B. van Tubergen 2006).

Ein weiteres Anliegen dieses Kapitels ist es, einen Beitrag dafür zu leisten, zu verstehen, warum bei vielen Menschen mit türkischem Migrationshintergund die Empirie auf eine höhere Eheorientierung und häufiger traditionelle Vorstellungen zu Partnerschaft im Vergleich zu Personen ohne Migrationshintergrund hindeutet. Eine These lautet, dass dies zum Teil auf die Religion zurückgeführt werden kann, worauf Ergebnisse des deutschen Generations Gender Surveys hinweisen (Naderi 2008: 445). Personen mit türkischen Wurzeln sind überwiegend Muslime und ihre Religiosität hängt stark mit dem türkischen Migrationshintergrund zusammen (Gründler 2012: 249). Es gilt dabei als sichere Tatsache, dass die Religiosität türkischer Migranten höher ist als bei Deutschen ohne Migrationshintergrund (Diehl/König 2009: 301). Das gilt auch für die Folgegeneration, unter Berücksichtigung unterschiedlicher Altersstrukturen. (ebenda.: 309) Dies hängt mit der elterlichen Transmission von Religiosität (Arránz Becker et al. 2014: 433) zusammen und scheint auch stärker als spätere Einflüsse durch den Partner zu sein. (ebenda: 438) Die Stabilität der Religiosität durch die elterliche Prägung ist nach Arránz Becker, Lois und Steinbach wahrscheinlicher, wenn eine durch ,emotionale Nähe und häufige Kontakte geprägte, intergenerationale Beziehung“" (ebenda: 436) zu den Eltern vorliegt.

Van Tubergen zeigt in seinem Artikel von 2006, dass bei Migranten (und hierbei auch bei türkischen Migranten) die Religionszugehörigkeit mit dem Herkunftsland zusammenhängt, während für die religiöse Praxis der Aufnahmekontext wichtiger ist (Van Tubergen 2006: 17). Milewski kann hingegen bezüglich der Einstellungen zu Geschlechterrollen bei Türkinnen der zweiten gegenüber der ersten Generation einen Wandel feststellen, indem deutlich weniger Frauen die Betreuung der Eltern als ihre alleinige Aufgabe sehen, was zudem mit einer höheren Erwerbsbeteiligung der Frauen, die eine entsprechende Haltung vertreten, korrespondiert (Milewski 2013: 64, 69).

Einschränkend sei generell darauf hingewiesen, dass es, bei einer Analyse wie dieser hier, ein Problem ist, beim islamischen Glauben von einer grundsätzlichen Einheitlichkeit auszugehen (El-Menouar 2014: 54). Die Vielseitigkeit konnte allerdings der Survey „Familienleitbilder in Deutschland“" (FLB) nicht erfassen.

Eine weitere Einschränkung ist, dass die hier zugrunde liegenden Daten derzeit nur einen Blick auf einen Ist-Zustand und nicht auf eine Entwicklung im Lebenslauf ermöglichen. Das heißt, dass nicht nur der eingangs erwähnte gesellschaftliche Wandel hin zum Säkularen oder nicht, sondern auch Veränderungen im Lebenszyklus des Individuums berücksichtigt werden müssten (im Überblick über diese beiden Effekttypen siehe Lois 2011: 86f.). Auch wenn Ergebnisse auf einen Rückgang religiöser Praxis insgesamt hindeuten, so ist immer noch ein gegenläufiger Alterseffekt erkennbar (Lois 2011: 106). Dies kann in diesem Beitrag nicht beleuchtet werden, sollte aber bei der Interpretation berücksichtigt werden, vor allem weil auch mit Alterseffekten bei der Einstellung zur Partnerschaft gerechnet werden kann und das Sample bei einem Alter von 39 Jahren endet.

\section{Vorgehensweise und Beschreibung der Variablen}

Im FLB 2012 ist eine Vielzahl an Fragen zur Partnerschaft enthalten (siehe Methodenbericht: Lück et al. 2013: 43-103). Wie in den anderen Abschnitten des Fragebogens, wurde ein großer Teil der Variablen in Richtung der Perspektive „Allgemeinheit“ bzw. „Persönlich“ gespiegelt (siehe Lück/Naderi/Ruckdeschel, Kap. 3). Für die hier vorgenommenen Analysen 
wurde allerdings nur die persönliche Ebene verwendet (allgemeine Partnerschaftsleitbilder siehe Diabaté, Kap. 6).

Für die Auswertungen wurden alle Befragten mit einbezogen, weil aus methodischer Hinsicht bezüglich der Fragestellung eine Einschränkung z. B. der Altersgruppe nicht notwendig erscheint. Auch die Reduzierung auf Personen mit Paarbeziehung wäre aufgrund der theoretischen Definition des Leitbildbegriffs nicht nötig, zumal es um die Vorstellung von Partnerschaft geht. Die Frage der Lebensform wird als unabhängige Variable verwendet. Die Fallzahl beträgt daher 5.000 Personen, diese wird aber durch fehlende Angaben von Befragten reduziert.

Die zentralen erklärenden Variablen sind, entsprechend der oben dargelegten Fragestellung, die Religionszugehörigkeit und die selbsteingeschätzte Religiosität. Beides beruht auf jeweils einer Variablen. Die Angabe zur Religionszugehörigkeit ist: „Darf ich Sie fragen, ob Sie einer Religionsgemeinschaft angehören?" Die Antwortmöglichkeiten wurden zunächst mit einem „Ja,..." eingeleitet, gefolgt von „der römisch-katholischen Kirche“, „der evangelischen Kirche (ohne Freikirchen)“, „dem Islam“, „,dem Judentum“, „einer anderen christlichen Religionsgemeinschaft" ${ }^{\text {"2 }}$ und „einer anderen nicht-christlichen Religionsgemeinschaft“. ${ }^{3}$ Konfessionslose wurden der Kategorie „Keine Religionsgemeinschaft" zugeordnet. Insgesamt gibt es hierzu 4.970 gültige Angaben, 20 Personen wollten nicht antworten und 10 wussten keine Antwort. Für die folgenden Analysen wurden Juden, andere christliche Religionsgemeinschaften und andere nicht-christliche Religionen in eine Kategorie („Andere“) zusammengefasst. Die Heterogenität dieser Gruppe wird in den Ergebnisinterpretationen berücksichtigt. Es muss dabei noch angemerkt werden, dass 121 von den 146 Befragten in dieser Gruppe anderen christlichen Religionsgemeinschaften entstammen.

Hieraus ergibt sich unter Einbeziehung des Gewichtungsfaktors (siehe Lück/Naderi/ Ruckdeschel, Kap. 3) eine Verteilung der hier betrachteten Konfessionen: 38,4 \% Konfessionslose, 28,5 \% Protestanten, 26,4 \% Katholiken, 3,1 \% Andere, 3,0 \% Muslime und $0,6 \%$ sind fehlend. Da auch die Gruppe der Muslime insgesamt sehr heterogen ist, aber keine weitere Differenzierung in verschiedene Glaubensrichtungen aufgrund des Fragebogens und der Fallzahl möglich ist, sollen in Tabelle 1 wesentliche Merkmale der Gruppe im FLB gezeigt werden, weil sie für die Untersuchung besonders relevant ist.

Die meisten Befragten muslimischen Glaubens haben einen Migrationshintergrund. Nur ein kleiner Teil weist weder eigene noch indirekte Migrationserfahrung auf. Von den Personen mit Migrationshintergrund insgesamt sind 51,4 \%, von den Muslimen mit Migrationshintergrund 48,3\% nicht in Deutschland geboren. Folglich besteht ein ausgeglichenes Verhältnis zwischen erster und folgenden Migrantengenerationen im Datensatz.

Die Formulierung der Frage nach der Religiosität lautet: „Als wie religiös würden Sie sich selbst bezeichnen?“ Hier gab es die Antwortkategorien „Sehr religiös“, „Religiös“, „Mittel religiös“, „Wenig religiös“ und „Nicht religiös“. Für die weiteren Auswertungen wurden die Kategorien 1 und 2 als „sehr/hoch“, sowie 4 und 5 „niedrig/nicht“ zusammengefasst. Diese Frage wurde nur den Personen gestellt, die nicht explizit die Antwort zur Religionsgemeinschaft verweigert haben. Unter Berücksichtigung weiterer Antwortausfälle ergibt sich für diese Variable eine Zahl von 4.972 Fällen. Die Religiosität verteilt sich auf die Kategorien 16,0\% „hoch“, 23,8\% „mittel“ und 59,6\% „niedrig/nicht“. Beide Variablen sind erwartungsgemäß sehr stark miteinander assoziiert, besonders bezüglich der Konfessionslosigkeit (Cramers V von 0,34).

2 Auf Nachfrage hätte der Interviewer Beispiele genannt: „evangelikale Freikirchen, griechisch-orthodox, russisch-orthodox, Adventisten, Zeugen Jehovas".

3 Auch hier wurde nur auf Anfrage das Beispiel „Buddhisten“ genannt. 
Tabelle 1: Verteilung verschiedener Merkmale in der Gruppe der Personen mit muslimischem Glauben im Vergleich zur Gesamtzahl aller Befragten im Alter von 20 bis 39 Jahren (Anteile in Prozent)

\begin{tabular}{lcc}
\hline & Muslime & Insgesamt \\
\hline Geschlecht & & \\
Männlich & 42,0 & 50,7 \\
Weiblich & 58,0 & 49,3 \\
Altersgruppen & & \\
20 bis 29 & 63,1 & 50,2 \\
30 bis 39 & 36,9 & 49,8 \\
Wohnort Bundesländer & & \\
Alte Bundesländer & 92,0 & 79,7 \\
Neue Bundesländer & 5,3 & 15,2 \\
Berlin & 2,7 & 5,1 \\
Wohnort Größe & & \\
Bis 4.999 Einwohner & 0,7 & 12,7 \\
5.000 bis 19.999 Einwohner & 18,0 & 23,6 \\
20.000 bis 99.999 Einwohner & 30,7 & 25,6 \\
100.000 Einwohner und mehr & 50,7 & 38,1 \\
Erwerbssituation & & \\
Erwerbstätig & 51,3 & 69,7 \\
Hausfrau/Hausmann & 5,3 & 5,2 \\
Nicht erwerbstätig & 43,3 & 25,2 \\
Migrationshintergrund & & \\
Ja & 97,3 & 23,7 \\
Nein & 2,7 & 76,3 \\
Türkische Herkunft & & \\
Ja & 49,7 & \\
Nein & 50,3 & \\
Bildungsstand (nach ISCED97) & & \\
Niedrig (1-2) & 59,2 & \\
Mittel (3A-4A) & 12,9 & \\
Hoch (5B-6) & & \\
\hline
\end{tabular}

Quelle: FLB (2012), gewichtete Daten, eigene Berechnungen

Zu berücksichtigen ist, dass sowohl die Zugehörigkeit zu einer Religionsgemeinschaft als vor allem auch die Religiosität solche Aspekte wie Praktiken, Häufigkeit ihrer Ausübung und Ähnliches offenlassen. Im Datensatz sind hierzu keine weiteren Informationen enthalten. Somit stellt die Frage nach der subjektiv eingeschätzten Religiosität zwar bei Weitem keine perfekte Messung, aber einen Indikator dar, um zumindest die persönliche Einschätzung, die all das vereinen könnte, zu verwenden. Selbstverständlich liegt eine weitere Limitation bei der Selbsteinschätzung darin begründet, dass nur schwer die Relationen zwischen den Religionen ausgemacht werden können. Es liegt auf der Hand, dass die 
eigene Religiosität beispielsweise von einem Buddhisten in ihrem Grad anders eingeschätzt werden könnte, als von einem Mitglied der griechisch-orthodoxen Kirche. Dies hängt sicher auch mit der Praxis der jeweiligen Religion zusammen, wodurch unterschiedliche Maßstäbe entstehen können. Auf der anderen Seite ermöglicht die persönliche Einschätzung die Perspektive einer privaten Religiosität, die bei dem einen oder anderen vielleicht als hoch eingeschätzt wird, ohne dass überhaupt irgendwelche Rituale und Praktiken verfolgt werden.

Für die zu erklärende Variable ist ein Index aus sieben Variablen mit einer 4er-Skala aus dem FLB-Modul Partnerschaftsleitbilder des Fragebogens erstellt worden. Diese Items wurden inhaltlich ausgewählt, wobei zur Unterstützung eine Hauptkomponentenanalyse durchgeführt wurde, basierend auf der Vermutung, dass es traditionelle Haltungen auf der einen Seite und moderne Einstellungen auf der anderen Seite geben kann. Dies hat sich durch die Hauptkomponentenanalyse, in die 12 Items aus dem Befragungsmodul Partnerschaft (persönliche Ebene) eingeflossen sind, relativ gut bestätigt. ${ }^{4}$ Die ausgewählten Variablen sind in Tabelle 2 dargelegt. Nach einer Reliabilitätsanalyse ergibt sich ein Wert für Cronbach's Alpha von rund 0,65. Final wurde der Index durch eine Addition der Items und Division durch ihre Anzahl berechnet und anschließend in zwei Gruppen kategorisiert. Über die rein technische und rechnerische Seite der Zusammengehörigkeit der hier verwendeten Variablen hinaus lässt sich bereits durch die Formulierung erkennen, wie die Pole sinnvoll bezeichnet werden können. Der Wertebereich 1,00 bis 2,49 wurde dabei als bürgerlich-konventionell (36\%) und der Bereich 2,50 bis 4,00 als modern (64 \%) definiert. Für den Beitrag werden folgende Arbeitshypothesen überprüft:

- (H1) Bürgerlich-konventionelle Partnerschaftsleitbilder sind bei Konfessionslosen seltener vorzufinden.

- (H2) Die Stärke der Religiosität wirkt sich deutlicher als die Zugehörigkeit aus.

Tabelle 2: Einstellungen zur Paarbeziehung (Variablen sind Teile des Index)

\begin{tabular}{lc}
\hline Variable im Index & $\begin{array}{c}\text { Zustimmung } \\
\text { in Prozent }\end{array}$ \\
\hline $\begin{array}{l}\text { Menschen können nur in einer festen und stabilen Partnerschaft } \\
\text { glücklich sein. }\end{array}$ & 65,1 \\
Wenn man als Paar dauerhaft zusammenlebt, sollte man heiraten. & 43,4 \\
Es ist schlecht, wenn der Mann eine niedrigere Bildung hat als die Frau. & 16,1 \\
Eine Partnerschaft funktioniert gut, wenn man im Zweifelsfall & 22,8 \\
den Mann entscheiden lässt, wo es lang geht. & 62,7 \\
Eine Partnerschaft funktioniert gut, wenn man gemeinsame Kinder hat. & 34,5 \\
Eine Frau sollte nach der Heirat den Namen ihres Mannes annehmen. & 31,5 \\
\hline Paare mit getrennten Haushalten sind keine richtigen Paare. &
\end{tabular}

Quelle: FLB (2012), gewichtete Daten, eigene Berechnungen

Die Analyse stützt sich auf den Vergleich von zwei logistischen Regressionsmodellen und eines Gesamtmodells mit allen verwendeten unabhängigen Variablen. Um den Effekt der Religion beurteilen zu können, werden in einem Modell die Religionszugehörigkeit und

4 Die genauen Ergebnisse und Tabellen hierzu können beim Autor erfragt werden. 
Religiosität überprüft. Im anderen Modell werden Geschlecht, Alter, Wohnort, Kinderzahl, Paarsituation (partnerlos, nichtehelich oder verheiratet), Einkommen und Bildung zusammen betrachtet.

\section{$4 \quad$ Ergebnisse}

$\mathrm{Zu}$ Beginn ist die Verteilung der wie oben beschriebenen zusammengefügten Partnerschaftsleitbild-Elemente nach Konfessionszugehörigkeit und Religiosität von Interesse. In Abbildung 1 ist diese ablesbar, indem die Prozentwerte die Hinwendung zu einem eher bürgerlich-konventionellen Partnerschaftsleitbild darstellen. Zur Veranschaulichung wurde hier auch der Anteil der Ablehnung dargestellt.

Abbildung 1: Bürgerlich-konventionelles Partnerschaftsleitbild nach Religionszugehörigkeit (in Prozent)

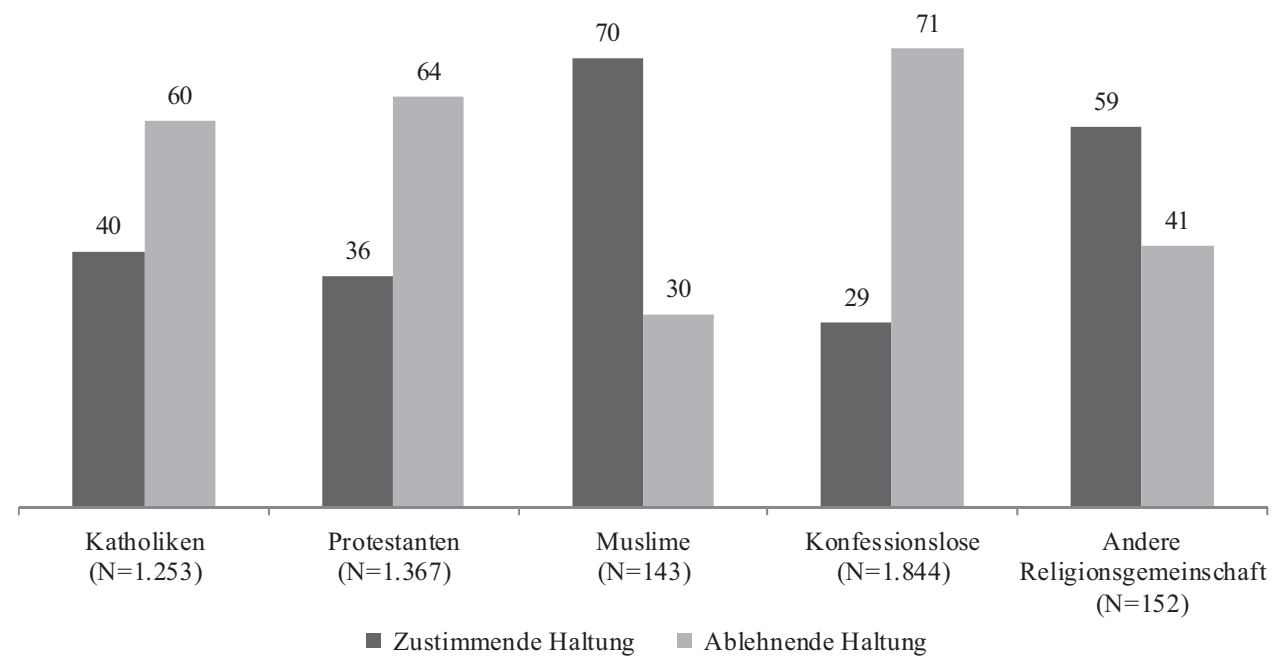

Quelle: FLB (2012), gewichtete Daten, eigene Berechnungen

Zunächst kann festgehalten werden, dass bei den Muslimen und den anderen, nicht genauer spezifizierten Religionsgemeinschaften jeweils eine deutliche Mehrheit mit bürgerlich-konventionellen Haltungen gegenüber Partnerschaft zu finden ist. Konfessionslose und katholische bzw. protestantische Christen lehnen entsprechende Vorstellungen zur Partnerschaft häufiger ab. Der Großteil der ,anderen Religionsgemeinschaften“ ist den anderen christlichen Gemeinschaften zuzuordnen. Zwischen Katholiken und Protestanten gibt es indes so gut wie keinen Unterschied - der größte Abstand in der Hinwendung zu einem bürgerlichkonventionellem Leitbild besteht zwischen Konfessionslosen und Muslimen. Auf Basis der eingangs beleuchteten Forschungsergebnisse und theoretischen Vorüberlegungen bezüglich der Rolle der Religion für persönliche Werte ist dieses Ergebnis, wenn auch nicht in dieser Deutlichkeit, zu erwarten gewesen. 
Grund hierfür könnte weniger die Konfession an sich als die höhere Religiosität sein, welche Einfluss auf die Werthaltung gegenüber der Paarbeziehung hat. Die Rolle der Konfession würde in den Hintergrund rücken, sofern man die Religiosität oder, wie an anderen Stellen, die religiöse Praxis einbezieht. Die selbsteingeschätzte Religiosität und ihr Zusammenspiel mit der Neigung zu bürgerlich-konventionellen Leitbild-Elementen wird in Abbildung 2 gezeigt. Als Hintergrundinformation ist für die Deutung wichtig, dass nur ein kleinerer Teil der Muslime von 21,3 \% sich als wenig oder nicht religiös bezeichnen würde, während dieses von den Protestanten mit 48,9 \% fast die Hälfte sind. Bei den Katholiken handelt es sich ebenfalls um einen hohen Anteil von 42,5\%. Die Konfessionslosen weisen erwartungsgemäß den höchsten Prozentwert gering oder gar nicht religiöser Befragter mit $86,5 \%$ auf - von den restlichen anderen Religionen sind es wiederum nur 20,0 \%. Zu berücksichtigen ist aber auch die hohe Subjektivität der Einschätzung, die wiederum zu einer starken Relativierung der eigenen Religiosität führen könnte. Werden andere beispielsweise aufgrund einer (vermeintlich) häufigeren Teilnahme an religiösen Veranstaltungen als engagierter wahrgenommen, so könnte die eigene Religiosität niedriger eingestuft werden.

\section{Abbildung 2: Bürgerlich-Konventionelles Partnerschaftsleitbild nach selbsteingeschätzter Religiosität (in Prozent)}

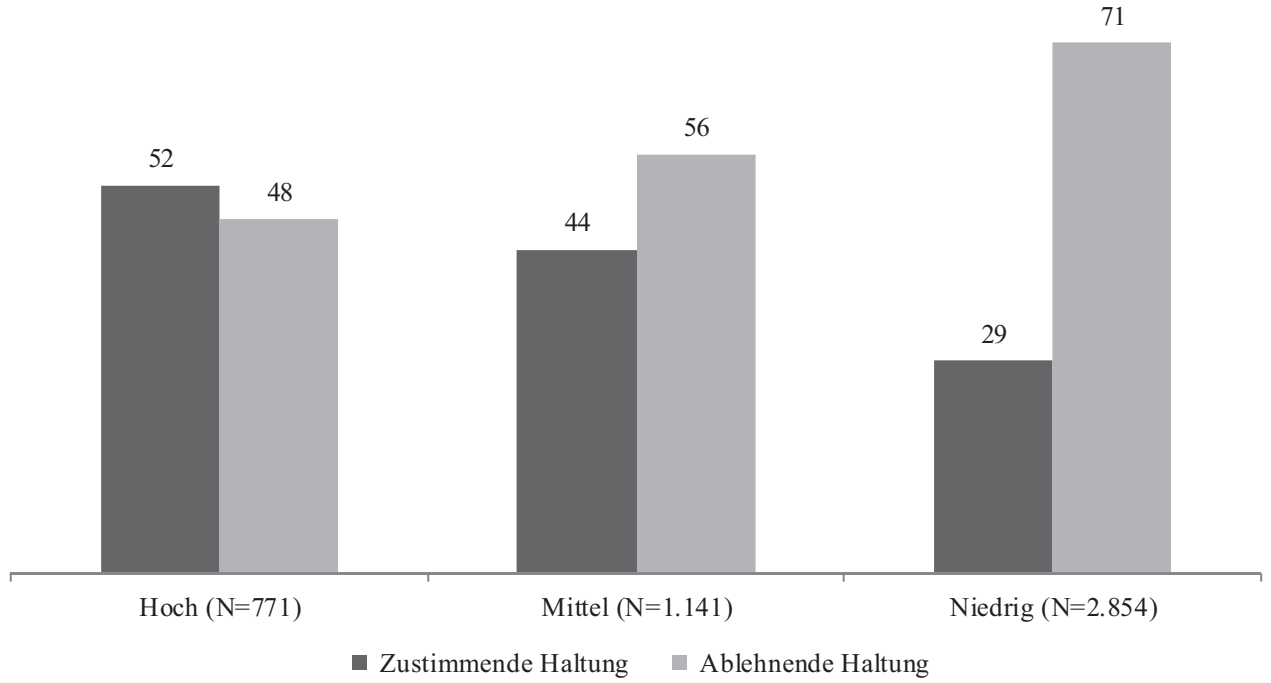

Quelle: FLB (2012), gewichtete Daten, eigene Berechnungen

Die Ergebnisse sind weniger deutlich als zu erwarten wäre. Der Unterschied zwischen zustimmender und ablehnender Haltung eines bürgerlich-konventionellen Partnerschaftsleitbildes ist bei hoher subjektiver Religiosität gering, sogar nahezu ausgeglichen. Allerdings sinkt die Zustimmung mit abnehmender Einschätzung der eigenen Religiosität. Offensichtlich sind Personen, die sich als sehr religiös bezeichnen, nicht zwangsläufig konventionell bezüglich der Partnerschaft eingestellt, aber es findet sich das traditionelle Leitbild häufiger im Vergleich zu weniger oder gar nicht religiösen Menschen. Die genaue Übereinstimmung der Prozentwerte der niedrigen Religiosität mit denen der Konfessionslosen ist ein Zufall, zeigt aber schon auf dieser Ebene die Bedeutung von Religion an sich für die 
Erklärung, warum sich Personen mehr oder weniger einem bürgerlich-konventionellem Leitbild bezüglich Partnerschaft zuordnen.

Diese bivariate Perspektive hat natürlich ihre Grenzen. Was passiert, wenn man beide Religions-Variablen gemeinsam bezüglich des Zusammenhangs mit dem Partnerschaftsleitbild betrachtet und wie bedeutend ist dies letztlich im Vergleich zu anderen Variablen? Hierfür ist eine multivariate Analyse notwendig, deren Ergebnisse in Tabelle 4 dargestellt sind. In Tabelle 3 wird zunächst die Verteilung der einzelnen in der Regression verwendeten Merkmale nach den Kategorien der abhängigen Variablen dargelegt. Auffällig an diesen Verteilungen ist, dass bei keiner der anderen Variablen die 50 \%-Marke bei der zustimmenden Haltung in einer der jeweiligen Kategorien, überschritten wird, wie dies bei den Religions-Variablen der Fall ist. Somit ist hiermit noch ein weiteres Indiz für ihre Bedeutung gegeben.

Zur Beurteilung der Regressionsmodelle sollen hier zunächst die jeweiligen Pseudo $\mathrm{R}^{2}$ herangezogen werden: Modell 1 und Modell 2 sind von ihrem Erklärungsgehalt nahezu gleich niedrig mit einem Wert von jeweils rund 0,07. Dies ist vor allem deswegen bemerkenswert, weil hier nur zwei Variable gegen acht durchaus wichtige individuelle Merkmale antreten. Auf Basis dieses Ergebnisses kann Religion folglich als nicht unwesentlich für die Hinwendung zu einem bürgerlich-konventionellem Partnerschaftsleitbild angesehen werden. Die Effekte im Detail lassen sich an den Odds Ratios ablesen und sollen im Folgenden genauer erläutert werden.

In Modell 1 weisen alle Variablen bis auf die Bildung statistisch signifikante Kategorien auf. Zusammenfassend lässt sich daher festhalten, dass eine Orientierung an einem bürgerlich-konventionellem Leitbild eher bei Männern, bei Jüngeren, bei Verheirateten und bei Kinderreichen vorzufinden ist. Bis auf den Alterseffekt sind diese Ergebnisse allgemein nachvollziehbar. Hier zeigt sich, dass alle gewählten Altersgruppen im Vergleich zur jüngsten Gruppe der 20- bis 24-Jährigen eine niedrigere Chance aufweisen dem bürgerlichkonventionellen Leitbild zugeordnet zu werden. Bei einer gröberen Einteilung von 20 bis 29 Jahren, würde dieses Phänomen nicht auffallen und die Zustimmung ist insgesamt niedriger im Vergleich zu den Älteren. Eine Vermutung wäre, dass sich die jüngsten Befragten stärker an einem Bild stabiler Partnerschaften und in Richtung Ehe orientieren. Dies kann unter anderem mit einer stärkeren Idealisierung aufgrund geringerer Beziehungs- und Lebenserfahrung in diesem Alter zusammenhängen. Ob ein kohortenbezogener Wertewandel vorliegt, kann hier nicht überprüft werden. Weiter zeigt sich, dass die Chance sinkt, wenn die Befragten in Großstädten über 100.000 Einwohner und insbesondere in Berlin leben sowie, wenn sie ihre eigene ökonomische Lage positiv beurteilen. Für dieses Modell ist letztlich noch die Tatsache relevant, dass besonders die Lebensform die Haltung zur Partnerschaft mitbestimmt - oder umgekehrt. Verheiratete haben eine deutlich höhere Chance einem bürgerlich-konventionellen Leitbild zu folgen. Dieser Effekt ist im Vergleich zu allen anderen am stärksten. 
Tabelle 3: Zustimmung zum bürgerlich-konventionellen Partnerschaftsleitbild

\begin{tabular}{|c|c|c|}
\hline & Zustimmung in Prozent & $\mathrm{N}$ \\
\hline \multicolumn{3}{|l|}{ Geschlecht } \\
\hline Männlich & 39,1 & 2.417 \\
\hline Weiblich & 32,8 & 2.372 \\
\hline \multicolumn{3}{|l|}{ Altersgruppen } \\
\hline 20 bis 24 & 38,4 & 1.207 \\
\hline 25 bis 29 & 31,7 & 1.202 \\
\hline 30 bis 34 & 36,9 & 1.190 \\
\hline 35 bis 39 & 36,8 & 1.189 \\
\hline \multicolumn{3}{|l|}{ Wohnort Bundesländer } \\
\hline Alte Bundesländer & 37,0 & 3.803 \\
\hline Neue Bundesländer & 35,3 & 743 \\
\hline Berlin & 22,2 & 243 \\
\hline \multicolumn{3}{|l|}{ Wohnort Größe } \\
\hline Bis 4.999 Einwohner & 43,7 & 609 \\
\hline 5.000 bis 19.999 Einwohner & 37,9 & 1.142 \\
\hline 20.000 bis 99.999 Einwohner & 37,3 & 1.232 \\
\hline 100.000 Einwohner und mehr & 31,3 & 1.806 \\
\hline \multicolumn{3}{|l|}{ Zahl eigener Kinder } \\
\hline Kinderlos & 32,1 & 2.841 \\
\hline Ein Kind & 38,8 & 806 \\
\hline Zwei Kinder & 42,9 & 793 \\
\hline Drei und mehr Kinder & 45,5 & 347 \\
\hline \multicolumn{3}{|c|}{ Selbsteingeschätzte Einkommenssituation } \\
\hline Schlecht & 42,2 & 347 \\
\hline Einigermaßen & 39,8 & 1.634 \\
\hline Gut & 32,8 & 2.750 \\
\hline \multicolumn{3}{|l|}{ Partnerschaftssituation } \\
\hline Partnerlos & 29,7 & 1.506 \\
\hline Verheiratet & 46,6 & 1.553 \\
\hline Unverheiratet mit Partner & 31,9 & 1.723 \\
\hline \multicolumn{3}{|l|}{ Bildungsstand (nach ISCED97) } \\
\hline Niedrig (1-2) & 41,5 & 330 \\
\hline Mittel (3A-4A) & 38,0 & 3.130 \\
\hline Hoch (5B-6) & 29,1 & 1.239 \\
\hline Insgesamt & 36,0 & 4.789 \\
\hline
\end{tabular}

Quelle: FLB (2012), gewichtete Daten, eigene Berechnungen 
Tabelle 4: Binär logistische Regression Index Leitbild: Bürgerlich-Konventionelle Paarbeziehung, $\mathrm{N}=4.683$, Odds Ratios

\begin{tabular}{|c|c|c|c|}
\hline & Modell 1 & Modell 2 & Komplett \\
\hline \multicolumn{4}{|l|}{ Geschlecht (Ref.: Männlich) } \\
\hline Weiblich & $0,671^{* * *}$ & & $0,591^{* * *}$ \\
\hline \multicolumn{4}{|l|}{ Altersgruppen (Ref.: 20 bis 24$)$} \\
\hline 25 bis 29 & $0,634^{* * *}$ & & $0,695^{* *}$ \\
\hline 30 bis 34 & $0,571^{* * *}$ & & $0,627^{* * *}$ \\
\hline 35 bis 39 & $0,505^{* * *}$ & & $0,529^{* * *}$ \\
\hline \multicolumn{4}{|c|}{ Wohnort Bundesländer (Ref.: Alte Bundesländer) } \\
\hline Neue Bundesländer & 0,981 & & $1,320^{* *}$ \\
\hline Berlin & $0,605^{* *}$ & & 0,756 \\
\hline \multicolumn{4}{|c|}{ Wohnort Größe (Ref.: Bis 4.999 Einwohner) } \\
\hline 5.000 bis 19.999 Einwohner & 0,864 & & 0,856 \\
\hline 20.000 bis 99.999 Einwohner & 0,912 & & 0,902 \\
\hline 100.000 Einwohner und mehr & $0,770^{*}$ & & $0,770^{*}$ \\
\hline \multicolumn{4}{|l|}{ Zahl eigener Kinder (Ref.: Kinderlos) } \\
\hline Ein Kind & $1,292^{*}$ & & $1,374^{* *}$ \\
\hline Zwei Kinder & $1,244^{*}$ & & $1,284^{*}$ \\
\hline Drei und mehr Kinder & $1,667^{* * *}$ & & $1,533^{* *}$ \\
\hline \multicolumn{4}{|c|}{$\begin{array}{l}\text { Selbsteingeschätzte Einkommenssituation } \\
\text { (Ref.: Schlecht) }\end{array}$} \\
\hline Einigermaßen & 0,853 & & 0,937 \\
\hline Gut & $0,762^{*}$ & & 0,831 \\
\hline \multicolumn{4}{|c|}{ Partnerschaftssituation (Ref.: Partnerlos) } \\
\hline Verheiratet & $2,213^{* * *}$ & & $2,053^{* * *}$ \\
\hline Unverheiratet mit Partner & 1,133 & & 1,173 \\
\hline \multicolumn{4}{|c|}{ Bildungsstand (nach ISCED97) (Ref.: Niedrig (1-2)) } \\
\hline Mittel (3A-4A) & 0,971 & & 1,018 \\
\hline $\operatorname{Hoch}(5 \mathrm{~B}-6)$ & 0,813 & & 0,797 \\
\hline \multicolumn{4}{|c|}{ Religionszugehörigkeit (Ref.: Katholiken) } \\
\hline Protestanten & & 0,890 & 0,887 \\
\hline Muslime & & $3,038^{* * *}$ & $2,859^{* * *}$ \\
\hline Konfessionslose & & $0,836^{*}$ & 0,840 \\
\hline Andere Religionsgemeinschaft & & $1,975^{* * *}$ & $2,065^{* * *}$ \\
\hline \multicolumn{4}{|c|}{ Selbsteingeschätzte Religiosität (Ref.: Niedrig) } \\
\hline Mittel & & $1,672^{* * *}$ & $1,730^{* * *}$ \\
\hline Hoch & & $2,540^{* * *}$ & $2,684^{* * *}$ \\
\hline Pseudo R ${ }^{2}$ (Nagelkerke) & 0,069 & 0,071 & 0,131 \\
\hline
\end{tabular}

Quelle: (FLB 2012), gewichtete Daten, eigene Berechnungen

Anm.: ${ }^{* * *} \mathrm{p} \leq 0,001 ;{ }^{* *} \mathrm{p} \leq 0,01 ;{ }^{*} \mathrm{p} \leq 0,05$. 
Modell 2 zeigt, wie bereits erwähnt, insgesamt eine ähnliche, sogar leicht höhere Modellgüte als das vorherige. Hier weisen fast alle Kategorien signifikante Ergebnisse auf. Bei der Konfession sind Katholiken als Referenz gewählt worden. Die Protestanten weisen hierzu keinen signifikanten Unterschied auf, $d$. h. es kann zuverlässig keine niedrigere Chance der Zuwendung zu einem bürgerlich-konventionellem Leitbild behauptet werden, auch wenn sie vom Maß her gegeben ist. Konfessionslose weisen einen leicht signifikanten negativen Effekt auf. Sofern man alle Odds Ratios miteinander vergleicht, so findet man den höchsten Wert beim Verhältnis von Muslimen und Katholiken. Erstere haben eine über dreifach höhere Chance, einem bürgerlich-konventionellen Leitbild zu folgen. Ebenfalls hoch, ist das bei den anderen Religionsgemeinschaften. Die bloße Zugehörigkeit zum Islam ist folglich ein wesentlicher Erklärungsfaktor dafür, ob man stabile, auf Dauer angelegte (in der Regel eheliche) Paarbeziehungen und dabei auch konventionelle Vorstellungen zu deren Ausgestaltung präferiert. Die Selbsteinschätzung der Religiosität korrespondiert ebenfalls hiermit. Ist sie hoch, so steigt die Chance um ein Zweieinhalbfaches gegenüber einer niedrigen Religiosität.

In der Gesamtbetrachtung zeigt sich eine relativ niedrige Modellgüte mit dem Nagelkerke $\mathrm{R}^{2}$ von 0,131 . Insgesamt werden nur $67,8 \%$ der Angaben mit dem Modell richtig vorhergesagt. Für die Einzeleffekte ergeben sich im Gesamtmodell folgende Änderungen: Die Kategorie zum Wohnort in den neuen Bundesländern wird signifikant und weist nun eine 1,3-fach höhere Chance auf, ein konventionelles Partnerschaftsleitbild zu bevorzugen im Vergleich zu einem Wohnort in einem westlichen Bundesland. Dabei handelt es sich zunächst um ein überraschendes Ergebnis. Erstens widerspricht es der Grundannahme und anderen Befunden, dass insgesamt „modernere“ Familienleitbilder in Ostdeutschland vorherrschen als im Westen. Zweitens ist in Tabelle 3 auf bivariater Ebene zu erkennen, dass die Zustimmung leicht geringer ist, als in Westdeutschland. Hierzu können Vermutungen angestellt werden: z. B. In Ostdeutschland leben als Folge der Politik des DDRRegimes, in der religiöse Praktiken weitgehend unterdrückt werden sollten und somit kein Teil der Sozialisation werden konnten (Arránz Becker 2010: 40), ein hoher Anteil Konfessionsloser und nichtreligiöser Menschen. Diejenigen hingegen, die sich als religiös bezeichnen, könnten als Gegenreaktion einer besonders konformen Auslegung der Religion folgen, die ggf. auch an die Kinder weitergegeben wurde. Mit dieser Konformität könnte ein „traditionelleres“ Partnerschaftsleitbild einhergehen. Zumindest kann das Ergebnis als ein weiteres Indiz für die Bedeutung der Religion bei der Erklärung der Hinwendung in die eine oder andere Richtung eines Partnerschaftsleitbildes gedeutet werden. Außerdem verliert die selbsteingeschätzte ökonomische Lage an statistischer Bedeutung, während sich sonst keine gravierenden Änderungen bei den einzelnen Effekten zeigen.

\section{$5 \quad$ Fazit}

Ziel dieses Beitrags war es, sich mit der Verbreitung eines potentiell bürgerlich-konventionellen Partnerschaftsleitbildes zu beschäftigen und dabei die Rolle der Religion zu beleuchten. Hierfür wurde ein Index aus verschiedenen Items gebildet, welche die persönliche Perspektive auf Partnerschaft und insbesondere die Bedeutung der Ehe widerspiegeln.

Folgende Ergebnisse sind zentral: Befragte, die ihre eigene Religiosität als hoch einstufen, weisen eine deutlich höhere Chance auf, ein bürgerlich-konventionelles Leitbild persönlich zu vertreten. Die Zugehörigkeit zum Islam oder zu anderen Religionsgemeinschaften, von denen der Großteil andere christliche Glaubensgemeinschaften sind, zeigt einen noch stärkeren 
Effekt in diese Richtung (erstere am stärksten). Zwischen Protestanten und Katholiken besteht kein signifikanter Unterschied. Das Religionsmodell ist etwas aussagekräftiger als alle anderen Variablen insgesamt. Im anderen Modell spielen vor allem das Geschlecht (Männer stärker konventionell), Alter (steigend mit dem Alter, mit der Ausnahme 20-24 Jahre), Kinderzahl (mit steigender Zahl eher konventionell) und Familienstand (Verheiratete eher konventionell im Vergleich zu Partnerlosen - nichtehelich allerdings nicht signifikant) eine Rolle.

Partnerschaftsleitbilder werden durch Religionszugehörigkeit und Religiosität mitbestimmt. Hier wurde zudem der direkt messbare Zusammenhang betrachtet - die latente Bedeutung der Religion für die jeweils prägende Kultur kann letztlich gar nicht so unmittelbar gemessen werden. Die beiden Religionsvariablen sind erklärungskräftiger als die Kombination wesentlicher demografischer und sozioökonomischer Variablen. Für die aufgestellten Arbeitshypothesen bedeutet dies:

Hypothese 1 „Bürgerlich-konventionelle Partnerschaftsleitbilder sind bei Konfessionslosen seltener vorzufinden“ kann bestätigt werden. Hypothese 2 „Die Stärke der Religiosität wirkt sich dabei deutlicher als die Zugehörigkeit zu einer Religionsgemeinschaft aus" kann nicht ohne Bedenken bestätigt werden, da auf bivariater Ebene bei einem Prozentwertvergleich bei Muslimen eine nahezu entgegengesetzte Verteilung im Vergleich zu Konfessionslosen erkennbar ist. Dies spräche dafür, dass die Zugehörigkeit wesentlicher wäre und sich die Religiosität bei Protestanten und Katholiken als wichtiger zeigen würde.

Im Zuge weiterer Säkularisierung oder der Verschiebung individueller Religiosität ins Private bzw. in weniger institutionalisierte Formen werden die im bisherigen historisch-gesellschaftlichen Kontext in Deutschland (v. a. Westdeutschland, da in Ostdeutschland traditionell eine geringe konfessionelle Bindung existiert) als konventionell-bürgerlich bezüglich der Paarbeziehung zu bezeichnende Leitbilder wahrscheinlich unbedeutender. Dies gilt insbesondere, weil selbst bei denen, die sich als religiös bezeichnen, bei Weitem keine eindeutige Hinwendung hierzu feststellbar ist. Dies ist ein Hinweis für die Parallelität von Säkularisierung und Religiosität, von der Peter L. Berger spricht (vgl. Abschnitt 2).

Einschränkend für die Beurteilung ist die betrachtete Altersgruppe und somit die mangelnde Berücksichtigung verschiedener Lebensphasen oder Kohorten. Es ist zu vermuten, dass in älteren Gruppen eine stärkere Hinwendung zu bürgerlich-konventionellen Leitbildern beobachtet werden könnte, was aber empirisch mit den FLB-Daten derzeit nicht überprüft werden kann. Befunde aus anderen Studien zeigen einen Alterseffekt, aber auch einen weiteren Rückgang der Religiosität insgesamt $-\mathrm{d}$. h. dass die Älteren von morgen auch weniger religiös sein werden im Vergleich zu den Älteren von gestern

\section{Literatur}

Arránz Becker, Oliver; Lois, Daniel; Nauck, Bernhard (2010): Unterschiede in den Fertilitätsmustern zwischen ost- und westdeutschen Frauen. Differenzierung der Rollen des kulturellen Hintergrunds und des Transformationsprozesses. In: Comparative Population Studies - Zeitschrift für Bevölkerungswissenschaft 35, 1, S. 35-64.

Arránz Becker, Oliver; Lois, Daniel; Steinbach, Anja (2014): Kontexteffekte in Familien - Angleichung von Paaren und intergenerationale Transmission am Beispiel Religiosität. In: Kölner Zeitschrift für Soziologie und Sozialpsychologie 66, 1, S. 417-444.

Berger, Peter L. (2013): Nach dem Niedergang der Säkularisierungstheorie. Wissen, leben. 1. Aufl. Münster: Centrum für Religion und Moderne.

Diabaté, Sabine; Lück, Detlev (2014): Familienleitbilder - Identifikation und Wirkungsweise auf generatives Verhalten. In: Zeitschrift für Familienforschung 26, 1, S. 49-69. 
Diehl, Claudia; König, Matthias (2009): Religiosität türkischer Migranten im Generationenverlauf: Ein Befund und einige Erklärungsversuche. In: Zeitschrift für Soziologie 38, 4, S. 300-319.

El-Menouar, Yasemin (2014): The Five Dimensions of Muslim Religiosity. Results of an Empirical Study. In: methods, data, analyses 8, 1, S. 53-78.

Gründler, Sabine (2012): Partnerschaftszufriedenheit von Deutschen und türkischen Migranten. Der Einfluss soziologischer und sozialpsychologischer Determinanten auf Partnerschaften. Wiesbaden: Springer VS.

Höllinger, Franz (1992): Verfall der christlich-bürgerlichen Ehemoral. Einstellungen zu Ehe und Scheidung im interkulturellen Vergleich. In: Zeitschrift für Familienforschung 4, 4, S. 197-220

Kröhnert, Steffen; Klingholz, Reiner (2010): Glaube, Macht und Kinder: Erobern religiöse Menschen mit vielen Nachkommen die Welt? Berlin: Institut für Bevölkerung und Entwicklung.

Lois, Daniel (2011): Wie verändert sich die Religiosität im Lebensverlauf? Eine Panelanalyse unter Berücksichtigung von Ost-West-Unterschieden. In: Kölner Zeitschrift für Soziologie und Sozialpsychologie 63, 1, S. 83-110.

Lück, Detlev et al. (2013): Familienleitbilder 2012. Methodenbericht zur Studie. BiB Daten- und Methodenberichte 2/2013. Wiesbaden: Bundesinstitut für Bevölkerungsforschung.

Milewski, Nadja (2013): Erwerbsbeteiligung und Einstellungen zur Familie von türkischen Migrantinnen im Generationenvergleich. In: Zeitschrift für Familienforschung 25, 1, S. 53-74.

Naderi, Robert (2008): Ehen und nichteheliche Lebensgemeinschaften im Lebensverlauf von Deutschen und türkischen Staatsbürgern in Deutschland. In: Zeitschrift für Bevölkerungswissenschaft 33, 3-4, S. 433-448.

Sagel, Irina; Althammer, Jörg (2013): Einstellung Heranwachsender zu Ehe und Familie. Die Einstellung junger Katholikinnen und Katholiken zu tradierten Familienleitbildern. In: Boos-Nünning, Ursula; Stein, Margit (Hrsg.): Familie als Ort von Erziehung, Bildung und Sozialisation. Münster: Waxmann, S. 95-122.

Schnor, Christine (2012): Trennungsrisiko von Paaren mit Kindern: Der Einfluss der Religion in West- und Ostdeutschland. In: Zeitschrift für Familienforschung - Sonderheft 2012, S. 229-256.

Van Tubergen, Frank (2006): Religious Affiliation and Attendance Among Immigrants in Eight Western Countries: Individual and Contextual Effects. In: Journal for the Scientific Study of Religion 45, 1, S. $1-22$. 


\title{
Leitbild und Kinderlosigkeit: Kulturelle Vorstellungen zum Leben ohne Kinder
}

\author{
Jürgen Dorbritz \& Sabine Diabaté*
}

\section{Zusammenfassung}

In Deutschland befindet sich die Kinderlosigkeit seit längerem auf einem sehr hohen Niveau. Bekannt ist, dass Zeit-, Geld- und Strukturprobleme dazu beitragen, dass weniger Kinder geboren werden. In dem vorgelegten Beitrag soll untersucht werden, welche kulturellen Leitbilder zur Kinderlosigkeit existieren und wie diese mit verschiedenen sozialstrukturellen Faktoren zusammenhängen. Dazu wurden neben dem Kinderwunsch auch die Gründe der Kinderlosigkeit und die soziale Akzeptanz von Kinderlosigkeit untersucht. Festgestellt wurde, dass Kinderlosigkeit in Deutschland als akzeptiert gilt und Sanktionen gegenüber Kinderlosen keine Mehrheit finden. Trotz breiter Akzeptanz der Kinderlosigkeit konnte in der Altersgruppe der 20- bis 39-Jährigen nur eine kleine Gruppe identifiziert werden, die keine Kinder und auch keinen Kinderwunsch hat. Es wurden zwei Leitbilder gefunden, die die Kinderlosigkeit in Deutschland aus Sicht der Befragten erklären: Erstens das Leitbild der risikovermeidenden Elternschaft. Darin sind Sorgen enthalten, den Kindern in der Zukunft nicht genug bieten zu können, den hohen Erziehungsanforderungen nicht gerecht zu werden und die Alltagsbelastungen nicht bewältigen zu können. Zweitens das Leitbild der autonomiebetonten Kinderlosigkeit. Darin sind Selbstbestimmung und Autonomie der Kinderlosen betont, die ihre private Freiheit genießen und sich auf die berufliche Karriere konzentrieren können.

\section{$1 \quad$ Einleitung}

Dauerhafte Kinderlosigkeit ist in den vergangenen Jahrzehnten zu einem verbreiteten Phänomen in Deutschland geworden. Oft wird angenommen, dass dahinter überwiegend nicht realisierte Kinderwünsche stehen. Aber ist in Deutschland eine Kultur der Kinderlosigkeit entstanden?

„Wenn dieser Ausdruck mehr sein soll als eine wohlfeile Provokation, dann heißt das: Kinderlosigkeit hat eine gewisse Attraktivität erlangt, sie wird nicht mehr als Problem oder Defizit von Paaren und Individuen gesehen sondern als kultureller Wert.“ (Burkart 2007: 401)

Kinderlosigkeit wäre demnach nicht mehr nur ein persönliches Schicksal von Einzelnen, sondern immer häufiger auch Ausdruck eines bewusst gewählten Lebensentwurfs. Es muss davon ausgegangen werden, dass der Sinn einer Partnerschaft für viele Paare nicht oder nur nachrangig über die Familiengründung definiert ist: Dies wird deutlich an der Entkoppelung von der Institution Ehe und Familie: Es gibt viele unverheiratete Paare mit Kindern, jedoch auch kinderlose Ehen. Darüber hinaus gibt es auch viele jüngere Menschen in bilokalen

Wir danken der anonymen Gutachterin bzw. dem anonymen Gutachter für die wertvollen Hinweise und die hilfreiche Kritik. Ein besonderer Dank geht an Robert Herter-Eschweiler für die Mikrozensusauswertungen. 
Partnerschaftsformen (Dorbritz/Naderi 2012), welche seltener Kinderwünsche haben, teilweise eben auch, weil sie Bilokalität nicht als temporäres notgedrungenes „Übel“ betrachten, sondern weil sie darin eine Möglichkeit sehen, frei und selbstbestimmt zu leben. Kinder wären in diesem Lebensentwurf genauso unpassend wie ein Lebenspartner im Haushalt, da sie die Autonomie des Einzelnen einschränken könnten. Inwieweit ein Leben ohne Kinder (besonders bei Männern) Teil eines neuen Lebensstils geworden ist und wie Kinderlosigkeit innerhalb der Gesellschaft wahrgenommen wird, ist bislang selten Untersuchungsgegenstand gewesen. Angesichts des seit 40 Jahren niedrigen Geburtenniveaus in Deutschland, das zu einem beachtlichen Ausmaß durch die hohe Kinderlosigkeit getragen wird, erscheint es vielversprechend der Frage nachzugehen, inwieweit Kinderlosigkeit ein durch kulturelle Leitbilder orientiertes Muster der privaten Lebensführung geworden ist.

\section{Forschungsstand, Theorie und Dimensionen von Kinderlosigkeit}

\subsection{Stand der Forschung und theoretische Grundlagen}

Zur Erklärung von Kinderlosigkeit werden beispielsweise auf Basis der Rational-ChoiceTheorie strukturell-ökonomische Faktoren verantwortlich gemacht (Überblick bei Hill/Kopp 2006). Weitere Ansätze thematisieren soziokulturelle und institutionelle Prozesse (Überblick bei Kreyenfeld/Konietzka 2013) oder auch sozio-kulturelle Leitbilder in Wechselwirkung mit strukturellen Anreizen auf Basis eines Frame-Selektions-Modells (Eckhard 2014). Ein Ausgangspunkt der Überlegungen zum Leitbild „Kinderlosigkeit“ in diesem Beitrag ist die These von Lutz und Milewski (2004), dass der Wandel in den Kinderzahlen nicht anhielte, wenn er nicht auf einem entsprechenden kulturellen Wandel basierte: Die Geburtenraten sinken und damit verschwinden Kinder immer mehr aus unserem Lebensalltag, so dass uns ihre Abwesenheit normal erscheint.

„Weitergedacht könnte dies sogar zu einer negativen Spirale führen: niedrige Geburtenraten bewirken mit Zeitverzögerung niedrigere Ideale, ein Sinken der Ideale führt zu noch niedrigeren Geburtenraten.“ (Lutz/Milewski 2004: 2)

Diese These wird dadurch gestützt, dass Kinderlosigkeit inzwischen ein Phänomen ist, das bereits in einer ganzen Reihe von Geburtsjahrgängen mit einer immer höheren Ausprägung beobachtet werden kann. Gegen die These spricht, dass etwa seit dem Jahr 2000 in Deutschland ein Wiederanstieg des Kinderwunsches (differenziertere Betrachtung der Kinderwunsch-Forschung: Dorbritz/Ruckdeschel, Kap. 9) zu beobachten ist und es Anzeichen dafür gibt, dass der Anstieg der Kinderlosigkeit bei Akademikerinnen gestoppt zu sein scheint. Analysen des Kinderwunsches anhand verschiedener Surveys wie dem Familienleitbildsurvey, pairfam, dem Eurobarometer oder der Population Policy Acceptance Study zeigen einen Anstieg des Kinderwunsches in allen seinen Messdimensionen (Dorbritz/ Naderi 2013). So ist der ideale Kinderwunsch von Werten um 1,8 bis 1,9 auf durchschnittlich 2,22 bis 2,26 gewünschte Kinder angewachsen. Ähnliche Trends finden sich, wenn auch auf niedrigerem Niveau, beim Kinderwunschleitbild ${ }^{1}$ und dem realistischen Kinder-

1 „Es wird also gefragt, wie viele Kinder sich der Befragte wünscht, ohne einen Zeitraum für eine geplante Geburt des Kindes anzugeben (aktueller Kinderwunsch) und ohne auf die konkreten Lebensumstände hinzuweisen. Damit können auch jüngere Menschen befragt werden, für die eine Familiengründung noch in einer ferneren Zukunft liegt.“ (Dorbritz/Naderi 2013: 2) 
wunsch (vgl. verschiedene Ebenen der Kinderwunschmessung bei Dorbritz/Naderi 2013). Den Hochqualifizierten wird in der Familienforschung attestiert, Vorreiter für Veränderungen im generativen Verhalten zu sein. Bujard (2012: 23) hat dazu festgestellt, dass der Geburtenrückgang bei Akademikerinnen gestoppt ist. Der Anteil von kinderlosen hochqualifizierten Frauen im Alter von 40 Jahren (ohne Kinder im Haushalt) hat sich von 33,8 \% (2007) auf 31,5\% (2011) leicht verringert. Diese Ergebnisse stützen die These u. a. von Goldstein et al. (2012: 10), dass das Geburtenniveau in Deutschland nicht dauerhaft niedrig bleiben wird und sich ein Wiederanstieg abzeichnet. Begründet wird die These mit der Veränderung der familienpolitischen Rahmenbedingungen in Deutschland, insbesondere der verbesserten Vereinbarkeit durch den Ausbau der Kinderbetreuungseinrichtungen und mit einem Einstellungswandel, der zu einer erhöhten Akzeptanz der Frauenerwerbstätigkeit und der außerhäuslichen Kinderbetreuung geführt hat.

Aus diesen Überlegungen und Befunden leiten sich die zentralen Fragestellungen dieses Beitrags ab. Zu untersuchen ist einerseits, ob sich in Deutschland eine Kultur der Kinderlosigkeit etabliert hat und wie die Einstellungen zur Kinderlosigkeit konkret aussehen.

Dazu werden in einem ersten Schritt (2.2) die Dimensionen der Kinderlosigkeit beschrieben. Im Anschluss daran werden in Abschnitt 2.3 der Begriff Kinderlosigkeit definiert sowie die zentralen Forschungsfragen zu diesem Beitrag näher erläutert. Abschnitt 3 beschäftigt sich mit der Frage, wer die Kinderlosen in Deutschland sind. Zudem wird untersucht, welche inhaltlichen Dimensionen Kinderlosigkeit in Deutschland kennzeichnen (3.1), welche Gründe für Kinderlosigkeit im Allgemeinen eine Rolle spielen (3.2) und inwiefern Kinderlosigkeit mit dem persönlichen Umfeld zusammenhängt. Abschließend (Abschnitt 4) werden die Ergebnisse im Hinblick auf die eingangs gestellt Forschungsfrage zusammengefasst und interpretiert sowie weitere Forschungspotenziale zur Kinderlosigkeit auf Basis des Leitbildsurveys skizziert.

\subsection{Dimensionen der Kinderlosigkeit}

Bevor eine leitbildgeprägte Sicht auf die Kinderlosigkeit entwickelt wird, soll ein Eindruck über die Dimensionen des Phänomens Kinderlosigkeit und damit über die soziale Relevanz vermittelt werden. Dazu eignet sich der Mikrozensus des Jahres 2012, in dem allerdings nur an Frauen und auf freiwilliger Basis die Frage nach der Zahl der geborenen Kinder gestellt worden ist.

In Tabelle 1 werden die Anteile kinderloser Frauen nach Geburtsjahrgängen, die Entwicklungstrends und die sozialstrukturellen Differenzierungen aufgeführt. Die Daten belegen die Ausbreitung der Kinderlosigkeit in Deutschland zu einem Massenphänomen. Waren im Geburtsjahrgang 1935 noch 10,6 \% der Frauen kinderlos, so hat sich ihr Anteil im Geburtsjahrgang 1970 auf 24,7 \% erhöht. Beim Trend zur Kinderlosigkeit sind erhebliche West-Ost-Unterschiede auffindbar: Der schnelle Anstieg des Anteils kinderloser Frauen in Gesamtdeutschland ist zu einem überwiegenden Teil auf den Trend in Westdeutschland zurückzuführen, wo ein Anstieg von 10,8 \% auf 26,0 \% zu beobachten war. Im Osten ist die Kinderlosigkeit zunächst auf einem sehr niedrigen Niveau verblieben. Erst bei den Jahrgängen, die in den 1960er Jahren zur Welt gekommen sind, sind moderate Anstiege zu verzeichnen. 
Tabelle 1: Anteile kinderloser Frauen nach Geburtsjahrgängen und Region in Deutschland (in Prozent)

\begin{tabular}{lccc}
\hline Geburtsjahrgang & Region & Ost \\
\hline 1935 & 10,6 & West & 7,7 \\
1940 & 10,5 & 10,8 & 7,0 \\
1945 & 12,9 & 11,2 & 7,3 \\
1950 & 13,0 & 13,9 & 6,7 \\
1955 & 16,6 & 13,9 & 6,2 \\
1960 & 17,8 & 18,8 & 7,0 \\
1965 & 21,6 & 19,9 & 11,1 \\
1970 & 24,7 & 23,4 & 13,2 \\
\hline
\end{tabular}

Quelle: Statistisches Bundesamt, Mikrozensus 2012, eigene Berechnungen

Wird nach Bildung und Familienstand unterschieden, werden weitere Differenzierungen offensichtlich (vgl. Tabelle 2). Der Anstieg der Kinderlosigkeit im Westen steht in einem unmittelbaren Verhältnis zur hohen Kinderlosigkeit unter den Akademikerinnen. Sie ist auch bei den anderen Bildungsgruppen angestiegen, allerdings deutlich langsamer als bei den Hochqualifizierten. Generell existiert in Westdeutschland ein enger Zusammenhang zwischen Bildung und generativem Verhalten: Je höher der Bildungsabschluss ist, desto öfter bleiben die Frauen ohne Kinder. Schon in den 1930er Jahren geborene westdeutsche Frauen mit einem hohen Bildungsniveau waren häufiger kinderlos als niedriger Qualifizierte. In beiden Gruppen ist die Kinderlosigkeit angewachsen, bei den Frauen mit Volksoder Hauptschulabschluss sogar schneller als bei denen mit einem Fach- oder Hochschulabschluss. In den Geburtsjahrgängen 1964 bis 1968 beträgt der Anteil der hochqualifizierten kinderlosen Frauen 30,9 \%, bei den gering qualifizierten kinderlosen Frauen sind es nur 18,7\%. Diese Differenzierung findet sich nicht in den neuen Bundesländern. Hochqualifizierte Frauen sind im Osten nicht häufiger kinderlos als die Frauen mit anderen Bildungsabschlüssen.

Tabelle 2: Anteile kinderloser Frauen nach Geburtsjahrgängen und sozialstrukturellen Merkmalen in Westdeutschland (in Prozent)

\begin{tabular}{lrrrr}
\hline Geburtsjahrgänge & \multicolumn{2}{c}{ Bildung } & \multicolumn{2}{c}{ Familienstand } \\
& hoch $^{1}$ & niedrig $^{2}$ & verheiratet & ledig \\
\hline $1933-38$ & 23,0 & 9,5 & 7,7 & 88,3 \\
$1939-43$ & 22,2 & 9,3 & 8,2 & 87,3 \\
$1944-48$ & 22,6 & 10,8 & 10,0 & 82,7 \\
$1949-53$ & 24,3 & 13,0 & 11,3 & 83,5 \\
$1954-58$ & 26,8 & 15,0 & 11,6 & 80,5 \\
$1959-63$ & 28,8 & 15,6 & 11,1 & 82,1 \\
$1964-68$ & 30,9 & 18,7 & 11,4 & 78,0 \\
\hline
\end{tabular}

Quelle: Statistisches Bundesamt, Mikrozensus 2012, eigene Berechnungen

Anm.: ${ }^{1}=$ Fach- oder Hochschulabschluss, ${ }^{2}=$ Haupt- oder Volksschulabschluss. 
Noch deutlichere Unterschiede finden sich zwischen den Familienständen: Verheiratet sein und Kinder haben sind zwei eng verknüpfte Verhaltensmuster. Die Kinderlosigkeit bei den verheirateten Frauen ist in den Geburtsjahrgängen 1964 bis 1968 mit 11,4\% außerordentlich niedrig. Die Anstiege seit den Jahrgängen 1933 bis 1938 sind geringfügig. Der Sinnzusammenhang ,wenn Ehe, dann Kinder“" ist zumindest in Westdeutschland erhalten geblieben. Lediglich der Umfang der Verheiratung hat sich verringert. Daneben gilt ebenso deutlich, dass Ledigsein mit Kinderlosigkeit verknüpft ist. In den Jahrgängen 1933 bis 1938 war der Kinderlosenanteil unter den ledigen Frauen mit 88,3\% extrem hoch und ist bis zu den Jahrgängen 1964 bis 1968 nur wenig auf 78,0 \% gesunken.

\subsection{Definition und Forschungsfragen}

Die Frage nach dem, was Kinderlosigkeit ausmacht, kann zunächst einfach beantwortet werden. Kinderlos sind diejenigen, die niemals in ihrem Leben Mutter bzw. Vater geworden sind. Dies ist zum Beispiel in den Mikrozensen 2008 und 2012 erfragt worden. Im FLB (Familienleitbilder in Deutschland, 2012) sind jüngere Befragte vertreten, die ihren Kinderwunsch teilweise noch nicht erfüllt haben, langfristig aber möglicherweise die gleichen Familienbiografien aufweisen werden wie die Eltern im Survey. Analysiert man den FLB, so wird deutlich, dass nur dauerhafte Kinderlosigkeit eine theoretisch relevante Kategorie ist und dass Kinderlosigkeit im Rahmen solcher Surveys auf einem anderen Weg operationalisiert werden muss. Als Kinderlose werden daher diejenigen angesehen, die keine Kinder haben und sich auch keine Kinder wünschen, ${ }^{2}$ so dass eine dauerhafte Kinderlosigkeit zumindest wahrscheinlich ist. Damit sind aber auch zwei Probleme verbunden, auf die hinzuweisen ist. Einerseits kann nicht der Situationsabhängigkeit des Kinderwunsches entsprochen werden. Eine neue Partnerschaft oder eine Verbesserung der individuellen Lebensumstände können dazu führen, dass Kinderwünsche entstehen. Andererseits wird mit der Vorgehensweise soziale Elternschaft etwa in Stieffamilien ausgeblendet.

Im Familienleitbildsurvey ist der Kinderwunsch aus der bereits realisierten Kinderzahl und der Zahl weiterer gewünschter Kinder unter Beachtung bestehender Schwangerschaften gebildet worden. Dabei ist ein möglicher Zeithorizont für die Erfüllung des Kinderwunsches nicht vorgegeben worden. Gemessen wurde damit das sogenannte Kinderwunschleitbild (eine ausführliche Diskussion zur Messung des Kinderwunsches findet sich bei Dorbritz/ Ruckdeschel, Kap. 9). Die Kontrollgruppe setzt sich dann aus den Befragten zusammen, die bereits Eltern sind und denen, die einen konkreten Kinderwunsch in der Befragung geäußert haben. Allerdings steht nicht fest, dass die Personen ohne Kinder und ohne Kinderwunsch wirklich kinderlos bleiben und diejenigen ohne Kinder aber mit Kinderwunsch auch tatsächlich Eltern werden. Insofern handelt es sich bei unserem Indikator um eine Proxy-Variable.

Die Forschungsfragen sind von folgenden Situationen um die Kinderlosigkeit abgeleitet: Erstens ist die Kinderlosigkeit insbesondere in Westdeutschland ein häufig vorkommendes Phänomen. Zu fragen ist anhand des Familienleitbildsurveys, ob dieses Phänomen unter den jüngeren Menschen sozial akzeptiert ist und ob angesichts der deutlichen West-OstUnterschiede spezifische Leitbildkonstellationen auffindbar sind, die als ursächlich gelten könnten.

2 Allen Kinderlosen wurde die Frage gestellt: „Möchten Sie Kinder?“ Die Antwortkategorien lauteten: 1 - Sicher nicht; 2 - Eher nicht, 3 - Eher schon, 4 - Ja, auf jeden Fall. Dabei wurden auch Schwangerschaften erfasst sowie nach der gewünschten Kinderzahl gefragt („Wie viele Kinder möchten Sie?“). Eltern wurden gleichermaßen befragt mit den Items „Möchten Sie noch weitere Kinder? und „Wie viele Kinder möchten Sie noch?“ 
Zweitens konnte mit den Mikrozensusanalysen festgestellt werden, dass Kinderlosigkeit in starkem $\mathrm{Ma} ß$ unter den Akademikerinnen und nicht verheirateten Frauen verbreitet ist. Das wiederum führt zu der Frage, ob soziale Gruppierungen ausgemacht werden können, in denen sich das Leitbild der Kinderlosigkeit besonders verfestigt hat. Dazu gehört auch die Fragestellung, inwieweit es sozialstrukturelle Unterschiede in der Akzeptanz von Kinderlosigkeit gibt.

\section{$3 \quad$ Kinderlosigkeit in der sozialstrukturellen Differenzierung - wer sind die Kinderlosen?}

Bereits die Analysen des Mikrozensus haben gezeigt, dass sowohl Kinderlosigkeit als auch die Paritätsstruktur insgesamt sozialstrukturell, regional und hinsichtlich der regionalen Herkunft deutliche Differenzierungen aufweisen. Bekannt sind die West-Ost-Unterschiede sowie die nach der Bildung, der paarspezifischen Erwerbssituation, dem Migrationshintergrund bzw. der Migrationserfahrung oder der Lebensform. In den FLB-basierten Analysen wird nun zunächst zwischen kinderlosen Befragten ohne Kinderwunsch (im Folgenden „Kinderlose“), Kinderlosen mit Kinderwunsch sowie Eltern (darunter auch Schwangere) unterschieden, um darzustellen, inwiefern diese Gruppen sozialstrukturell verteilt sind (vgl. Tabelle 3). Der Wunsch, kinderlos zu bleiben, zeigt eine Abhängigkeit vom Familienstand, dem Einkommen und der Bildung. Verheiratet Zusammenlebende möchten nur zu 3,0 \% keine Kinder haben, während kinderlose Singles dies zu 15,8 \% bleiben möchten. Daneben wollen Personen mit einem niedrigeren Einkommen (2.000 Euro oder weniger) und einer niedrigeren Bildung häufiger als die Vergleichsgruppen keine Kinder haben. Auffällig ist, dass die de facto höhere Kinderlosigkeit nicht in einem hohen Wunsch angelegt ist, kinderlos zu bleiben. Werden diejenigen betrachtet, die keine Kinder haben, aber Kinder einmal haben möchten, dann sind Unterschiede nach Geschlecht, Alter, Einkommen, Erwerbstätigkeit und Familienstand zu konstatieren. Nicht überraschend ist, dass Jüngere (20-29 Jahre) und Männer, deren Familiengründung später beginnt, häufiger keine Kinder haben, aber über einen Kinderwunsch verfügen. Diejenigen mit niedrigem Einkommen und Nichterwerbstätige, die zum großen Teil noch in der Ausbildungsphase sein dürften, haben ebenfalls öfter einen Kinderwunsch und noch keine Kinder. Vergleichsweise deutlich fallen die Differenzierungen hinsichtlich des Familienstandes und der Partnersituation aus. Verheiratet zusammenlebende Paare sind zu $86,4 \%$ bereits Eltern. Singles und Paare in einer bilokalen Beziehung sind dagegen fast alle noch kinderlos.

In einem ersten Schritt werden die zu untersuchenden Gruppen nach ihren Bevölkerungsanteilen unter den 20- bis 39-Jährigen betrachtet. 9,4\% der Befragten (458 Fälle) haben keine Kinder und sagen, dass sie auch keine Kinder möchten (sicher nicht bzw. eher nicht). Die überwiegende Mehrheit von $\approx 90,7 \%$ hat Kinder oder möchte wenigstens zukünftig ein Kind haben. Betrachtet man diese Gruppe genauer, befinden sich 49,0\% $(\mathrm{N}=2.399)$ der Personen in der Gruppe ohne Kinder mit Kinderwunsch, die restlichen 41,7 \% ( $\mathrm{N}=2.040)$ sind Eltern ${ }^{3}$ und werdende Eltern, die zuvor kinderlos waren (insgesamt 1,4 \%, N=72).

3 Hierunter fallen auch 1,6\% Personen $(\mathrm{N}=80)$, die Eltern sind und ein weiteres Kind erwarten. 
Tabelle 3: Soziodemografische Gruppen nach Kinderwunsch bzw. Vorhandensein von Kindern (in Prozent)

\begin{tabular}{|c|c|c|c|c|c|}
\hline Merkmal & Kategorie & $\begin{array}{c}\text { Kinderlose ohne } \\
\text { Kinderwunsch } \\
\%\end{array}$ & $\begin{array}{c}\text { Kinderlose mit } \\
\text { Kinderwunsch } \\
\%\end{array}$ & $\begin{array}{c}\text { Eltern } \\
\% \\
\end{array}$ & $\begin{array}{c}\text { Gesamt } \\
\mathrm{N} \\
\end{array}$ \\
\hline \multirow[t]{2}{*}{ Geschlecht } & Männlich & 10,5 & 56,1 & 33,3 & 2.480 \\
\hline & Weiblich & 8,2 & 41,7 & 50,1 & 2.417 \\
\hline \multirow{3}{*}{$\begin{array}{l}\text { Bildung ISCED97 } \\
\text { (2002) }\end{array}$} & Niedrig & 12,1 & 54,7 & 33,1 & 338 \\
\hline & Mittel & 9,5 & 47,9 & 42,6 & 3.180 \\
\hline & Hoch & 7,7 & 51,2 & 41,1 & 1.289 \\
\hline \multirow[t]{2}{*}{ Alter } & 20-29 Jahre & 10,1 & 71,2 & 18,7 & 2.471 \\
\hline & 30-39 Jahre & 8,6 & 26,3 & 65,1 & 2.426 \\
\hline \multirow[t]{2}{*}{ Einkommen } & $\leq 2.000$ Euro & 12,1 & 58,0 & 29,9 & 1.961 \\
\hline & $\geq 3.000$ Euro & 7,2 & 37,4 & 55,4 & 2.350 \\
\hline \multirow[t]{2}{*}{ Region/Wohnort } & West & 9,8 & 50,2 & 40,0 & 3.896 \\
\hline & Ost (inkl. Berlin) & 7,6 & 44,2 & 48,2 & 1.000 \\
\hline \multirow[t]{4}{*}{ Familienstand } & Ehe/ZL & 3,0 & 10,6 & 86,4 & 1.565 \\
\hline & NEL & 8,7 & 50,9 & 40,4 & 955 \\
\hline & NEBP & 10,3 & 78,1 & 11,6 & 807 \\
\hline & Single & 15,8 & 71,2 & 13,0 & 1.551 \\
\hline \multirow[t]{2}{*}{ Erwerbssituation } & Nicht erwerbstätig & 12,7 & 50,2 & 37,1 & 229 \\
\hline & Erwerbstätig & 9,6 & 47,2 & 43,1 & 3.723 \\
\hline \multirow{2}{*}{$\begin{array}{l}\text { Migrations- } \\
\text { hintergrund }\end{array}$} & $\mathrm{Ja}$ & 7,8 & 49,6 & 42,6 & 1.162 \\
\hline & Nein & 9,8 & 48,8 & 41,3 & 3.726 \\
\hline Gesamt & & 9,4 & 49,0 & 41,7 & $5.000^{\mathrm{b}}$ \\
\hline
\end{tabular}

Quelle: FLB (2012), gewichtete Daten, eigene Berechnungen

Anm.: $\quad Z L=Z u s a m m e n l e b e n d, ~ N E L=$ Nichteheliche Lebensgemeinschaften, NEBP=Nichteheliche bilokale Partnerschaften. ${ }^{a}$ Die Zuordnung erfolgt nach: Low (1-2), Medium (3A-4A), High (5B-6).

${ }^{\mathrm{b}}$ Fehlende Werte: 2,1 \% (,,weiß nicht“", keine Angabe).

Im nächsten Schritt wird gefragt, aus welchen sozialen Gruppierungen die „Kinderlosen“ stammen. Dazu wird zwischen den Frauen und Männern ohne Kinder und ohne Kinderwunsch einerseits und denen mit Kindern oder mit Kinderwunsch („Eltern“) andererseits unterschieden. Die Gruppen „Eltern“ und „Kinderlose“ wurden dafür in einer Vierfeldertafel jeweils mit verschiedenen sozialstrukturellen Merkmalen verknüpft und die OddsRatios (Quotenverhältnis) berechnet. Damit liegen Angaben über die Stärke des Zusammenhangs vor. Untersucht werden die Zusammenhänge mit dem Geschlecht, der Bildung, dem Einkommen, der Erwerbssituation, dem Familienstand, der Partnerschaftssituation, der Region, der Religion, dem Alter und dem Migrationshintergrund (vgl. Tabelle 4). Die Zusammenhänge zwischen den sozialstrukturellen Merkmalen und der Chance, zur Gruppe Kinderlose bzw. Elternschaft zu gehören, sind in fast allen Fällen signifikant (in Tabelle 4 werden lediglich die signifikanten Werte dargestellt). Die stärksten Zusammenhänge bestehen zwischen der Kinderlosigkeit sowie den Variablen Familienstand und Partnersituation. 
Ledige haben eine viereinhalb Mal größere Chance zu den Kinderlosen zu gehören als Verheiratete. Bei denen, die ohne Partner im Haushalt leben, ist es die dreifache. Die wesentliche Bedingung für die Erfüllung des Kinderwunsches ist das Vorhandensein eines Partners, mit dem man in einem Haushalt leben kann. Vergleichsweise groß ist auch der Einfluss der Erwerbssituation: Wer erwerbstätig ist, hat eine 2,2fach größere Chance, zur Gruppe der Kinderlosen zu gehören als Nichterwerbstätige. Dieser Befund verstärkt sich dadurch, dass in der Gruppe der Erwerbslosen auch nichterwerbstätige Mütter enthalten sind.

Tabelle 4: Zusammenhang zwischen Kinderlosigkeit und Elternschaft nach ausgewählten sozialstrukturellen Merkmalen ${ }^{4}$

\begin{tabular}{lcc}
\hline Sozialstrukturelle Merkmale & Odds-Ratios & Signifikanzen \\
\hline Bildung (niedrig/hoch) & 1,637 & 0,008 \\
Geschlecht (männlich/weiblich) & 1,309 & 0,004 \\
Einkommen (bis 2.000 Euro/3.000 Euro und mehr) & 1,726 & 0,000 \\
Region (West/Ost) & 1,301 & 0,034 \\
Familienstand (ledig/verheiratet) & 4,563 & 0,000 \\
Partnerschaftssituation (ohne Partner/mit Partner im Haushalt) & 3,065 & 0,000 \\
Religion (keine Religionsgemeinschaft/katholisch oder & & \\
evangelisch) & 1,738 & 0,000 \\
Erwerbssituation (Erwerbstätige/Nichterwerbstätige) & 2,239 & 0,011 \\
\hline
\end{tabular}

Quelle: FLB (2012), gewichtete Daten, eigene Berechnungen

Anm.: Hohe Bildung: Akademischer Abschluss oder Meister-/Techniker- bzw. Fachschulabschluss. Niedrige Bildung: Haupt-/Realabschluss, Polytechnische Oberschule und ohne beruflichen Abschluss bzw. ohne Bildungsabschluss.

Daher ist davon auszugehen, dass die als unzureichend eingestuften Vereinbarkeitsbedingungen von Erwerbstätigkeit und Elternschaft diesen Effekt verursachen. Auch der Einfluss der Religion zeigt den erwarteten Zusammenhang: Bei Personen, die keiner Religionsgemeinschaft angehören, ist die Wahrscheinlichkeit der Kinderlosigkeit um das 1,7fache höher im Vergleich zu Katholiken und Protestanten. Ähnlich starke Zusammenhänge finden sich nach Bildung, Einkommen und Region. Niedrigere Bildung und ein niedrigeres Einkommen erhöhen die Chance, zunächst zur Gruppe der Kinderlosen zu gehören, um das Zweieinhalb-

4 Die sozialstrukturellen Merkmale wurden folgendermaßen dichotomisiert: Bei der Bildung wurden ausgehend von der nach Destatis zusammengefassten ISCED97-Klassifikation (basierend auf UNESCO-Klassifizierung) die Gruppen mit der höheren und der niedrigeren Bildung gegenübergestellt. Die beiden Einkommensgruppen werden von denjenigen mit einem Haushaltsnettoeinkommen unter 2.000 Euro sowie 3.000 Euro und mehr gebildet. In der Betrachtung nach dem Familienstand wurde in ledig und verheiratet und bei der Partnersituation in mit einem Partner im Haushalt zusammenlebend (Ehepaare und nichteheliche Lebensgemeinschaften) und allein in einem Haushalt lebend (Singles und bilokale Paarbeziehungen) dichotomisiert. Bei der Variable „Erwerbssituation“ stehen auf der einen Seite die Erwerbstätigen und auf der anderen Seite die Nichterwerbstätigen (Arbeitslose, Arbeitssuchende, Praktikanten, Hausfrauen und -männer, Vorruheständler, Berufsunfähige und Frührentner). In der Analyse nach der Religion wird unterschieden zwischen denen, die keiner Religionsgemeinschaft angehören und denen, die römisch-katholisch und evangelisch sind. Andere Religionsgemeinschaften sind nicht in die Analyse eingeflossen (Analysen zum Einfluss der Religiosität auf Leitbilder finden sich bei Naderi). Die Variablen Region (West/Ost) und Geschlecht sind naturgemäß dichotom. 
fache. Sobotka und Testa (2008: 206) gelangen aufgrund der Ergebnisse der Population Policy Acceptance Study zu dem gleichen Ergebnis.

„..., we also found that women with a low educational level frequently intend to remain childless, whereas women with a higher educational attainment do not necessarily express a high preference for intended childlessness."

Das bedeutet insbesondere für Personen mit einer hohen Bildung, dass Kinderlosigkeit zunächst nicht angestrebt ist, die Kombination von starker Berufsorientierung und Distanz gegenüber traditionellen Geschlechterrollen sowie die bestehenden strukturellen Hürden letztlich aber zu einer höheren tatsächlichen Kinderlosigkeit führen.

Geschlecht und Region weisen die schwächsten Zusammenhänge zur Kinderlosigkeit auf. Für Männer und für in Westdeutschland Lebende ist das Risiko der Kinderlosigkeit jeweils um das 1,3fache gegenüber Frauen bzw. Ostdeutschen erhöht. Die Zusammenhänge zum Migrationshintergrund (ja/nein) und zum Alter (Altersgruppen: 20-29/30-39) sind nicht signifikant und daher nicht dargestellt.

\section{$4 \quad$ Einstellungen zur Kinderlosigkeit}

Im Familienleitbildsurvey wurden mehrere Fragen integriert, um die Einstellungen zur Kinderlosigkeit zu erfassen. In den nachfolgenden Abschnitten werden die verschiedenen Aspekte thematisiert, darunter Akzeptanz (4.1), Egoismus und Sozialabgaben (4.2) sowie Gründe für Kinderlosigkeit (4.3).

\subsection{Akzeptanz}

Die Akzeptanz von Kinderlosigkeit ist mit der Frage „Heutzutage ist es etwas ganz normales, keine Kinder zu haben“" ermittelt worden. Es wurde auf zwei Ebenen gemessen: Die persönliche Meinung und diejenige, die in der Gesellschaft wahrgenommen wird. Über die Hälfte, 58,6 \% der Befragten, haben dieser Aussage aus ihrer persönlichen Sicht zugestimmt, davon haben $24,9 \%$ voll und ganz und 33,7 \% eher zugestimmt. In der Gesellschaft nehmen $64,8 \%$ der Befragten wahr, dass Kinderlosigkeit als etwas ganz normales angesehen wird. Nach diesen Ergebnissen ist kinderlos sein in Deutschland unter den 20bis 39-Jährigen sozial akzeptiert. Die hohe festgestellte tatsächliche Kinderlosigkeit entspricht in einem erheblichen Ausmaß den geltenden Leitbildern.

Signifikante Unterschiede in der persönlichen Bewertung von Kinderlosigkeit bestehen nach der Bildung (ISCED97-Klassifikation), der Kinderzahl, dem Kinderwunsch und der Lebensform. Folgende Zusammenhänge wurden aufgefunden:

- Je höher die Bildung, desto häufiger wird der Aussage zugestimmt, dass Kinderlosigkeit etwas ganz Normales ist. In der Gruppe mit niedriger Bildung lag der Grad der Zustimmung (,voll und ganz" und „eher") bei 48,2\% und in der mit einer hohen Bildung bei 63,5\%. Der Bildungszusammenhang wird durch internationale Studien bestätigt. Merz und Liefbroer (2012) zeigen in einer europäisch vergleichenden Studie ebenfalls, dass mit einem höheren Bildungsstand eine höhere Akzeptanz von Kinderlosigkeit einhergeht. 
- Für den Zusammenhang nach der Kinderzahl gilt die erwartete Entwicklung, nach der die Akzeptanz von Kinderlosigkeit bei denjenigen höher ist, die keine Kinder oder nur ein Kind haben. Diejenigen, die keine Kinder haben, stimmen der Aussage mit 62,2 \% zu, diejenigen mit nur einem Kind zu 59,4\%. Bei zwei Kindern sinkt der Zustimmungsgrad auf 49,7 \% und bei drei Kindern auf 45,9\%.

- Hinsichtlich des Kinderwunsches ist das Ergebnis ähnlich, aber deutlicher ausgeprägt. Hier findet sich wiederum die mit Abstand höchste Akzeptanz (85,7\%) bei den Frauen und Männern, die keine Kinder haben und sich auch keine Kinder wünschen. Wer sich ein Kind wünscht, akzeptiert die Aussage „Kinderlosigkeit ist etwas ganz Normales“ noch zu 67,3\%, bei zwei gewünschten Kindern sind es dann nur noch 56,2 \%. Bemerkenswert ist, dass ungefähr die Hälfte der Befragten, die Kinder haben bzw. sich Kinder wünschen, dennoch der Aussage zustimmen.

- Wer verheiratet ist, empfindet Kinderlosigkeit seltener als etwas ganz Normales. Aber immerhin stimmt mit 52,1\% noch mehr als die Hälfte der Aussage zu. Bei nichtehelichen Lebensformen sind die Zustimmungswerte höher (nichteheliche Lebensgemeinschaften: 65,3\%, bilokale Paarbeziehungen: 59,2\%, Singles: 60,0 \%). Es ist davon auszugehen, dass Verheiratetsein und Kinderhaben bzw. Kinderwollen zwei eng verknüpfte Verhaltensmuster sind, die mit einer vergleichsweise geringeren Akzeptanz der Kinderlosigkeit korrelieren.

Ein überraschendes Ergebnis dieser Analyse ist, dass weder West-Ost- ${ }^{5}$ noch Altersunterschiede aufgefunden wurden. Es war angenommen worden, dass die höheren Anteile tatsächlich kinderloser Frauen im Westen mit einer höheren Akzeptanz der Kinderlosigkeit einhergehen. Dass dies nicht eingetreten ist, lässt sich aus dem vergleichsweise hohen Anteil von Frauen mit nur einem Kind im Osten erklären, die die Normalität von Kinderlosigkeit ähnlich beurteilen wie die Befragten ohne Kinder. Hinsichtlich des Alters wurde vermutet, dass jüngere Befragte in der Altersgruppe der 20- bis 24-Jährigen höhere Akzeptanzwerte aufweisen. Es konnten aber keine signifikanten Unterschiede nachgewiesen werden, so dass sich bei den 20- bis 39-Jährigen ein homogenes Bild bei der Sicht auf die Kinderlosigkeit bietet.

\subsection{Egoismus und Sozialabgaben}

Die Dimension Egoismus wurde mit dem Item „Kinderlose verhalten sich egoistisch, weil sie ein bequemes Leben führen wollen" abgebildet. Kinderlosen wird in Deutschland von etwas mehr als einem Viertel der Bevölkerung $(28,5 \%)$ egoistisches Verhalten vorgeworfen. Signifikante Unterschiede in der Bewertung finden sich ähnlich wie bei der Akzeptanz von Kinderlosigkeit nach der Bildung, der Kinderzahl und dem Kinderwunsch. Zusätzlich signifikant ist die Bewertung nach dem Alter geworden.

- Die niedriger Gebildeten werfen den Kinderlosen häufiger Egoismus vor als die höher Gebildeten. Sie stimmen der Aussage zu 33,0 \% zu, während es bei den Hochqualifizierten, die häufiger und aufgrund der Lebensumstände oftmals ungewollt kinderlos bleiben, nur 23,4 \% sind. Es kann davon ausgegangen werden, dass die Erfahrungen der Hochqualifizierten zu einer größeren Toleranz gegenüber Kinderlosigkeit führen.

5 Die Unterschiede waren jeweils nicht signifikant, unabhängig davon ob West- und Ostberlin einbezogen wurden oder nicht. 
- Befragte ohne Kinder sehen eine kinderlose Lebensführung deutlich seltener (Anteil der Zustimmung: 22,2 \%) als egoistisch an als Eltern. Mit steigender Kinderzahl steigt die Zustimmung und beträgt beispielsweise bei Befragten mit drei Kindern 55,9 \%.

- Ähnlich deutlich sind die Unterschiede nach dem Kinderwunsch. Diejenigen ohne Kinder und ohne Kinderwunsch stimmen nur zu 11,7 \% zu. Die Zustimmungsrate bei Personen mit einem Kinderwunsch ${ }^{6}$ von 3 beträgt beispielsweise 37,9 \%.

- Im Gegensatz zur Akzeptanz von Kinderlosigkeit finden die „Älteren“ in der Stichprobe (Altersgruppe 35 bis 39 Jahre) mit 34,6\% häufiger, dass ein egoistisches Verhalten vorliegt. Die Jüngeren (20 bis 24 Jahre) sehen dies nur zu 25,0 \% so.

Von der Forderung nach Sanktionen für Kinderlose (Frage: „Kinderlose sollten höhere Steuern und Abgaben leisten als Eltern") distanzierte sich die Mehrheit der Befragten $(59,0 \%)$. Immerhin sprachen sich aber 41,0 \% für höhere Steuern und Abgaben aus. Geht es um die Meinung, die in der Allgemeinheit wahrgenommen wird, dann stimmt sogar eine Mehrheit von 60,9\% dieser Aussage zu. Signifikante Unterschiede in der Bewertung konnten für Bildung, Geschlecht, Altersgruppen, Einkommen und Kinderzahl ermittelt werden. Wiederum haben sich die West- und Ostdeutschen bei der Zustimmung bzw. Ablehnung nicht unterschieden. Folgende Unterschiede wurden ermittelt:

- Männer (45,8 \%) stimmen häufiger als Frauen (36,0 \%) zu, dass Kinderlose höhere Abgaben zahlen sollen.

- Je niedriger der Bildungsabschluss, desto weniger wird der Aussage zugestimmt. In der niedrigen Bildungsstufe sind dies 32,6\%, in der mittleren Stufe 39,8\% und in der hohen Stufe 47,6 \%.

- In der Tendenz stimmen die Älteren häufiger für Steuern und Abgaben als die Jüngeren (Altersgruppe 20 bis 24 Jahre: 38,5\%, 35 bis 39 Jahre: 46,2 \%).

- Wer keine Kinder oder nur ein Kind hat, vertritt seltener die Position, dass höhere Abgaben geleistet werden sollten. Die Anteile der Zustimmung betragen bei keinem Kind $35,7 \%$ und steigen dann vom ersten zum zweiten sowie vom dritten zum vierten Kind stark an: Bei einem Kind 37,4\%, bei zwei Kindern 55,4\%, bei drei Kindern $51,0 \%$ und bei vier Kindern 70,5\%.

\subsection{Gründe der Kinderlosigkeit}

Warum bleiben Menschen kinderlos? Scheitern Kinderlose an den Bedingungen zur Realisierung ihrer Kinderwünsche oder setzen sie einen angestrebten Lebensentwurf um, indem sie ihren verinnerlichten Leitbildern folgen? An dieser Stelle wird zunächst auf der Basis von Mittelwertberechnungen eine Rangfolge der allgemeinen Gründe für Kinderlosigkeit aufgestellt. Diese sind nicht derart abgefragt worden, dass es um die persönliche Meinung ging. Vielmehr ging es darum, solche zu evaluieren, die von den Befragten für allgemein gesellschaftlich relevant eingeschätzt werden. Ausgangspunkt für diese Art der Messung war die Überlegung, dass allgemein wahrgenommene Gründe der Kinderlosigkeit gesellschaftliche Leitbilder zum Ausdruck bringen, die neben den individuellen Vorstellungen handlungsleitend sein können.

6 Dazu zählen z. B. Befragte ohne Kinder und einem Wunsch nach drei Kindern, Befragte, die drei Kinder haben und kein weiteres Kind wollen oder auch Befragte, die ein Kind haben und noch zwei weitere wollen. 
Als wichtigste Ursache für die Kinderlosigkeit haben sich die Vereinbarkeitsprobleme herausgestellt (vgl. Tabelle 5). Ein Großteil der Befragten (85,2 \%) hat der Aussage „Viele Menschen bekommen keine Kinder, weil Kinder und Beruf so schlecht vereinbar sind" zugestimmt $^{7}$ (Mittelwert: 1,83). Der Familienleitbildsurvey bestätigt damit die Bedeutung der Vereinbarkeitsbedingungen für die Erfüllung des Kinderwunsches, die bereits in zahlreichen Studien herausgearbeitet wurde. Eichhorst et al. (2007) stellen anhand eines internationalen Vergleichs fest, dass es in Deutschland bei einem traditionellen Verständnis der Geschlechterrollen schwieriger ist, Berufstätigkeit und Elternschaft $\mathrm{zu}$ vereinbaren. Bertram et al. (2005) weisen dabei auf den Dreiklang von Geld-, Zeit- und Infrastrukturpolitik hin.

Nur knapp unwichtiger war der Grund, dass sich Kinderlose sehr viel mehr leisten können (80,2 \%, Mittelwert: 1,89). Als drittwichtigste Ursache wird gesehen, dass Kinderlose so leben können, wie es ihnen gefällt (77,1\%, Mittelwert: 2,01), womit die Furcht ausgedrückt wird, durch Kinder in der privaten Lebensführung eingeschränkt zu werden. Auf den Rangplätzen vier und fünf befinden sich zwei Aussagen, die die lebenslange Verantwortung eines Lebens mit Kindern (58,2 \%) und den Respekt vor der Kompliziertheit $(38,9 \%)$ abbilden. Relativ unwichtig sind dazu im Vergleich die Angst vor einer Trennung (32,6\%) und die Belastung der Partnerschaft durch das Leben mit Kindern (26,2 \%). Damit haben sich die zentralen Annahmen zu den Ursachen der Kinderlosigkeit in der Literatur bestätigt (z. B. Konietzka/Kreyenfeld 2013, 2007): 1. Vereinbarkeitsprobleme, 2. die finanzielle Situation, 3. Autonomieprobleme und 4. Überforderungssorgen.

Tabelle 5: Gründe für Kinderlosigkeit in Deutschland

\begin{tabular}{lcc}
\hline $\begin{array}{l}\text { Gründe } \\
\text { Viele Menschen bekommen keine Kinder, .. }\end{array}$ & Mittelwerte & $\begin{array}{c}\text { Zustimmung in Prozent } \\
\text { (voll und ganz + eher) }\end{array}$ \\
\hline $\begin{array}{l}\text { 1. weil Kinder und Beruf schlecht zu } \\
\text { vereinbaren sind. }\end{array}$ & 1,83 & 85,2 \\
$\begin{array}{l}\text { 2. weil man sich ohne Kinder viel mehr } \\
\text { leisten kann. }\end{array}$ & 1,89 & 80,2 \\
$\begin{array}{l}\text { 3. weil Kinderlose so leben können, } \\
\quad \text { wie es ihnen gefällt. }\end{array}$ & 2,01 & 77,1 \\
$\begin{array}{l}\text { 4. weil viele vor der lebenslangen Verantwortung } \\
\text { zurückschrecken. }\end{array}$ & 2,32 & 58,2 \\
$\begin{array}{l}\text { 5. weil es viel zu kompliziert ist, } \\
\text { Kinder großzuziehen. }\end{array}$ & 2,64 & 38,9 \\
$\begin{array}{l}\text { 6. aus Angst vor einer Trennung. } \\
\text { 7. weil Kinder die Partnerschaft belasten. }\end{array}$ & 2,70 & 32,6 \\
\hline
\end{tabular}

Quelle: FLB (2012), gewichtete Daten, eigene Berechnungen

1. Vereinbarkeitsprobleme: Für Westdeutschland wurde schon immer angenommen, dass die hohe Kinderlosigkeit unter den Akademikerinnen aus einem Vereinbarkeitsproblem herrührt.

7 Die Antwortskala ist vierstufig: $1=$ stimme voll und ganz zu; $2=$ stimme eher zu; $3=$ stimme eher nicht $\mathrm{zu}$; 4=stimme überhaupt nicht zu. 
„Die zentrale These lautet, dass die niedrige Kinderlosigkeit bei ostdeutschen Frauen mit Hochschulabschluss auf einen höheren strukturellen Defamilialismus im Osten (bessere außerfamiliale Kinderbetreuungsangebote) bei gleichzeitig höherem kulturellem Familialismus (ausgeprägtere Familienwerte), der zudem eine geringere Bildungsgradierung als im Westen aufweist, zurückzuführen ist" (Boehnke 2007: 312).

Daraus leitet sich, wie bereits in den Hypothesen formuliert, die Annahme ab, dass für westdeutsche Frauen, insbesondere die Hochqualifizierten, die Vereinbarkeitsproblematik eine besondere Bedeutung besitzt. Der Unterschied in der Bewertung der Vereinbarkeitsbedingungen als Ursache für die Kinderlosigkeit nach Bildungsstufen ist signifikant. Je höher die Bildung ist, desto häufiger wird der Aussage zugestimmt, dass die schlechten Vereinbarkeitsbedingungen bei der Entscheidung gegen Kinder eine Rolle spielen.

Bei einer generell hohen Zustimmung sind es die Hochqualifizierten, die mit 90,5\% die höchsten Werte aufweisen. Auf der mittleren Bildungsstufe erreichen die Zustimmungsanteile 84,1 \% und auf der niedrigen Bildungsstufe 76,5 \%. Bei dieser Fragestellung bietet sich eine geschlechtsspezifische Betrachtung an. Dabei zeigt sich, dass die Bewertungsunterschiede bei den Frauen nicht signifikant sind. In allen drei Bildungsgruppen wird dem Grund sehr häufig zugestimmt. Bei den Männern hingegen sind die Sichtweisen deutlich abweichend. Der Zustimmungsanteil bei den niedriger Qualifizierten beträgt 70,8 \%, bei der mittleren Bildungsgruppe 82,6 \% und bei den Hochgebildeten 90,4 \%. Während die hochqualifizierten Frauen und Männer die Vereinbarkeitssituation ähnlich beurteilen, wird sie bei den niedrig qualifizierten Männern weniger kritisch gesehen. Die Betrachtung nach Altersgruppen, Kinderzahl, Haushaltsnettoeinkommen und West-Ost hat keine weiteren interpretierbaren Unterschiede erbracht.

2. Ökonomische Sorgen: Als zweitwichtigster Grund wurden finanzielle Gründe identifiziert. 80,2 \% haben voll und ganz bzw. eher zugestimmt, dass man sich ohne Kinder mehr leisten kann. Fehlende ökonomische Sicherheiten und auch eine geringe Stabilität des Lebensstandards werden von der überwiegenden Mehrheit als Risiken gesehen, die mit der Familiengründung einhergehen. Kontrolliert nach Alter und Bildung, zeigt sich, dass mit steigendem Alter die Zustimmung zu der Aussage steigt und niedriger Gebildete eine höhere Zustimmung aufweisen.

3. Sorge um Autonomie: Mit 77,1 \% stimmt eine Mehrheit der Befragten der Aussage $\mathrm{zu}$, dass viele Menschen keine Kinder bekommen, weil Kinderlose so leben können, wie es ihnen gefällt. Hinter dieser Aussage steckt eine ganze Reihe von Aspekten, die mit der Kinderlosigkeit einhergehen. Auf drei soll aufmerksam gemacht werden: Erstens ist eine starke Berufs- und Karriereorientierung ohne Kinder leichter realisierbar. Zweitens können manche Paare auch aufgrund ihrer Lebensumstände nur in getrennten Haushalten wohnen, was sehr häufig mit Kinderlosigkeit einhergeht. Und drittens gehört Kinderlosigkeit zu den Lebensformen, die auch durch gewollte Partnerlosigkeit oder die Orientierung auf Autonomie und Distanz zum Partner gekennzeichnet sind.

4. Sorge vor Überforderung: Die Mehrheit (58,2\%) gibt an, dass Menschen keine Kinder bekommen, weil sie vor der lebenslangen Verantwortung zurückschrecken. Hier spielt auch die gesellschaftliche Norm der verantworteten Elternschaft hinein. Schneider (2012: 1) betont,

„(...) dass Elternschaft mit der Etablierung neuer Leitbilder, etwa den ,Neuen Vätern', der ,Gelingenden Erziehung' oder der ,Verantworteten Elternschaft' heute deutlich voraussetzungsreicher und anspruchsvoller ist als noch vor wenigen Jahrzehnten. Daraus und auch aus der Tatsache, dass auf die Belange von Eltern gesellschaftlich wenig Rücksicht genommen wird, resultieren Probleme, die die Realisierung vorhandener Kinderwünsche verhindern oder dazu führen, dass ein steigender Anteil junger Menschen keine Kinderwünsche entwickelt." 
Kaufmann (2006: 26) weist mit Blick auf die Akademiker auf einen weiteren Aspekt hin:

„Soziologisch lautet die Antwort auf die Frage, warum Akademiker in Deutschland keine Kinder bekommen: Sie leiden unter einer bedrückenden Lebensängstlichkeit, die nicht im Materiellen wurzelt.“

Letztlich beinhaltet das Leitbild der verantworteten Elternschaft (siehe Ruckdeschel, Kap. 12), dass man sich erst für Kinder entscheiden soll, wenn man sich in der Lage sieht, emotional und finanziell für Kinder sorgen zu können. Es ist anzunehmen, dass ein Teil der jungen potentiellen Eltern sich nicht bereit fühlt, die damit verbundenen Normen zu erfüllen.

Geschlechterunterschiede

Bislang wurden aus Fallzahlgründen die Kinderlosen nicht getrennt nach dem Geschlecht analysiert. Aus der bisherigen Forschung zum Thema ist aber bekannt, dass das Phänomen der Kinderlosigkeit vor allem unter Frauen untersucht wird und dass Frauen und Männer aus besonderen Motivlagen heraus kinderlos bleiben. Die Orientierung der Forschung auf die weibliche Kinderlosigkeit hat zwei Gründe: Einerseits ist es für Männer schwieriger, das Ende der fertilen Lebensphase zu bestimmen. Zweitens kommt darin ein gewisser Traditionalismus zum Ausdruck, der auch in Statistik und Forschung zur Verbindung von Kinderlosigkeit und Frauen führt (Schmitt/Winkelmann 2005: 1).

Den Forschungen von Schmitt (2004) anhand der SOEP-Daten ist es zu verdanken, dass Erkenntnisse über geschlechtsspezifische Unterschiede vorliegen. $\mathrm{Zu}$ den wichtigen Ergebnissen zählt, dass es Unterschiede bei den Gründen gibt, die Kinderlosigkeit begünstigen. Für die Männer wird festgestellt, dass das Male-Breadwinner-Prinzip noch immer eine hohe Gültigkeit besitzt.

„Die vorliegenden Daten liefern aber vor allem starke Hinweise darauf, dass für Männer eine Familiengründung erst dann in Betracht kommt, wenn die ökonomische Absicherung einer Familie gewährleistet werden kann ...". (Schmitt 2004: 15)

Damit verzögert sich die Familiengründung und erhöht die Chance auf Kinderlosigkeit. Bei den Frauen steigt die Kinderlosigkeit mit der Bildung und damit rücken Vereinbarkeitsprobleme als Ursache in den Vordergrund.

Die Daten des Leitbildsurveys bestätigen dieses Ergebnis in Ansätzen. Hinsichtlich der Gründe für Kinderlosigkeit sehen Frauen und Männer Vereinbarkeitsprobleme gleichermaßen als wichtigsten Grund für Kinderlosigkeit an. Dies ist bei den Frauen (Mittelwert: 1,79) etwas stärker ausgeprägt als bei den Männern (Mittelwert: 1,87). Die Unterschiede sind allerdings nicht signifikant. Wird die Bildung mit einbezogen, schärft sich das Bild. Die hochqualifizierten Frauen und Männer stimmen zu ca. 90 \% zu, dass sich Menschen bei der Entscheidung gegen Kinder von schlechten Vereinbarkeitsbedingungen leiten lassen. Bei den niedrigqualifizierten Frauen sind es $84,7 \%$ und bei den Männern dieses Bildungsniveaus $70,8 \%$.

\section{$5 \quad$ Kinderlosigkeit und persönliches Umfeld}

Die vorherigen Analysen haben gezeigt, dass die gesellschaftliche Akzeptanz von Kinderlosigkeit für einen großen Teil der Bevölkerung in Deutschland gegeben ist. Die nachfolgenden Analysen sollen ermitteln, ob Kinderlosigkeit mit dem persönlichen Umfeld, den Verwandten, den Arbeitskollegen und dem Freundes- und Bekanntenkreis zusammenhängt. 
Nach Richter et al. (2012: 99ff.) sind hinsichtlich der Rolle sozialer Netzwerke bei Fertilitätsentscheidungen noch der soziale Druck und die Unterstützungsleistungen aus dem Netzwerk zu beachten.

„Der Prozess der sozialen Ansteckung kann als eine Art Anpassung an das soziale Umfeld bzw. als Nachahmungsverhalten definiert werden, welches nicht durch direkten sozialen Druck oder Sozialkapitalanreize hervorgerufen wird." (Richter et al. 2012: 100)

Geburten im Freundes-, Kollegen- oder Familienkreis führen dazu, dass der bisherige Lebensstil und damit die Handlungsoption „Familiengründung“ einer genaueren Prüfung unterzogen werden. Sind Familiengründungen in den privaten Netzwerken selten, wird die Möglichkeit einer Familiengründung weniger überdacht und es werden kaum Nachahmungseffekte ausgelöst. Wer ein kinderloses Umfeld hat, empfindet Kinderlosigkeit daher als etwas Normales.

„Dabei spielen familialistische Normen, die im Netzwerk gelten, eine wichtige Rolle: Sind die Angehörigen des Freundschaftsnetzwerkes z. B. überwiegend gewünscht kinderlos und unverheiratet, werden Familiengründung und Eheschließung für eine Person wenig instrumentell zur Erzielung von Anerkennung sein.“(ebd.)

Die Ergebnisse des Familienleitbildsurveys bestätigen die Annahme, dass Kinderlosigkeit dann häufiger vorkommt, wenn Verwandte, Arbeitskollegen oder Freunde ebenfalls keine Kinder haben. Der stärkste Zusammenhang besteht beim Freundes- und Bekanntenkreis: Sind die meisten Personen im Freundeskreis kinderlos, ist die Chance, selbst kinderlos zu bleiben, um das 2,5fache höher, als wenn die meisten Kinder haben. Dies gilt auch für die Familie und die Verwandtschaft. Hier ist die Chance um das 1,5fache höher, kinderlos zu sein. Der schwächste Zusammenhang ist im Kreis der Arbeitskollegen zu beobachten: Haben die Arbeitskollegen häufiger keine Kinder, ist die Wahrscheinlichkeit, ebenfalls kinderlos zu bleiben, um das 1,3fache höher gegenüber der Gruppe, bei denen die Arbeitskollegen häufiger Kinder haben.

An dieser Stelle ist wieder zu fragen, in welchen sozialen Gruppen der Zusammenhang am stärksten ausgebildet ist, wobei hier keine Aussagen über die Wirkrichtung getroffen werden können. In folgenden Punkten bestehen auffällig starke Verbindungen:

- Bei Frauen besteht ein stärkerer Zusammenhang zum Bekanntenkreis als bei Männern. Gibt es viele Bekannte ohne Kinder, so ist die Chance kinderlos zu bleiben, für Frauen um das 3,3fache höher. Bei den Verwandten und den Arbeitskollegen bestehen keine signifikanten Ergebnisse.

- In ihrer Arbeit zu Netzwerkseinflüssen bei Fertilitätsentscheidungen haben Richter et al. (2012: 95) herausgefunden, dass die Einflüsse im Osten tendenziell stärker sind als im Westen. Für die Entscheidung zur Kinderlosigkeit gilt dies nach den Ergebnissen des Leitbildsurveys nicht. Die Zusammenhänge sind signifikant, aber spiegelverkehrt. Bei den Bekannten ist im Westen die Chance um das 2,7fache, bei den Verwandten um das 1,5fache und bei den Kollegen um das 1,3fache höher. Im Osten ist nur der Zusammenhang zu den Freunden und Bekannten signifikant. Haben diese seltener Kinder, ist die Chance auf eigene Kinderlosigkeit um das Zweifache erhöht.

- Zwischen den niedriger und höher Gebildeten bestehen deutliche Unterschiede. Bei einer niedrigeren Bildung ist der Zusammenhang mit den Arbeitskollegen sehr hoch: Wenn die Arbeitskollegen häufig keine Kinder haben, ist das Risiko für eine eigene Kinderlosigkeit um das 3,5fache erhöht. Bei den höher Qualifizierten gibt es dagegen einen stärkeren Einfluss der Familie und der Verwandtschaft. Die Chance auf Kinderlosigkeit erhöht sich um das 2,1 fache. 
- Hinsichtlich des Haushaltseinkommens sind die Effekte gleich. Signifikant ist nur die Wechselwirkung mit Freunden und Bekannten. Die Chance kinderlos zu bleiben, ist bei denen mit niedrigem Einkommen ebenso wie bei denen mit einem hohen Einkommen um das 2,6fache erhöht.

Generell ist festzustellen, dass es möglicherweise Abfärbeffekte aus den individuellen Netzwerken gibt, ob jedoch die soziale Umgebung zur Kinderlosigkeit beiträgt oder ob Kinderlose sich in selektiver Weise bestimmte Netzwerke aufbauen, kann mittels Querschnittsdaten nicht geklärt werden.

\section{$6 \quad$ Welche Leitbilder tragen zur Kinderlosigkeit bei?}

In einem weiteren Schritt soll untersucht werden, ob Leitbilder identifiziert werden können, die innerhalb der Gesellschaft als ursächlich für Kinderlosigkeit wahrgenommen werden. Bei der Betrachtung der Ergebnisse der Hauptkomponentenanalyse laden die ersten drei Items hoch auf der zweiten Dimension (vgl. Tabelle 6: Komponente 2). Die restlichen vier Items laden auf der ersten Dimension am stärksten (vgl. Komponente 1). Bei der Überprüfung der Reliabilität der ersten drei Items ergibt sich ein Alpha-Wert von ,507. Die interne Konsistenz der letzten vier Items beträgt ,612. Hinter den beiden Dimensionen stecken inhaltlich zwei Konstrukte: Die erste Komponente thematisiert generell eine Ängstlichkeit und Unsicherheit gegenüber einem Leben mit Kindern. In der zweiten Komponente schwingt ein gewisser Hedonismus mit, einhergehend mit finanziellen und arbeitsmarktbedingten Vorteilen der Kinderlosigkeit für den Einzelnen. Die beiden Leitbilder in der Gruppe der Kinderlosen zeigen, dass die Befragten zwei Hauptmotive sehen, weshalb Menschen keine Kinder bekommen wollen: Ein gesellschaftliches Leitbild der risikovermeidenden Kinderlosigkeit und das Leitbild der autonomiebetonten Kinderlosigkeit. Kaufmann (2006: 26) stellt dazu fest:

„Je nervtötender die Forderung durch die Medien tönt, die Kinderlosen mögen endlich für die Zukunftssicherung des deutschen Volkes zur Zeugung schreiten, desto abschreckender wird diese Vorstellung. Ich kann das in diesem Fall sogar verstehen. Die Nebenwirkung der wiederkehrenden familienpolitischen Hysterie ist, dass Kinder als Problem wahrgenommen werden. Als etwas, was Zeit und Geld kostet, das unsexy macht, unfrei. Gerade Männer bekommen den Eindruck, der Spaß des Lebens sei mit dem ersten Kind schlagartig vorbei."

Um weitere soziodemografische Unterschiede zu untersuchen, wurden hauptkomponentenbasierte Summenindizes gebildet. Hierbei zeigen sich für beide Leitbilder der Kinderlosigkeit keine Geschlechterunterschiede, jedoch andere interessante Differenzen: Das Leitbild der autonomiebetonten Kinderlosigkeit sehen signifikant mehr Personen ohne Migrationshintergrund $(90,1 \%$ vs. $85,9 \%)$ in der Gesellschaft verbreitet als solche mit Migrationshintergrund. Auch Bildungsunterschiede lassen sich feststellen: Die Wahrnehmung der autonomiebetonten Kinderlosigkeit in der Öffentlichkeit nimmt zu, je höher der Bildungsabschluss ist $(81,7 \%=$ niedrig, $89,3 \%=$ mittel, 91,4 \% = hoch). Dies liegt vermutlich an der höheren Karriereorientierung von besser ausgebildeten, die sich wiederum eher als geringer Qualifizierte in Alltagskontexten bewegen, in denen der Autonomieverlust durch die Familiengründung verbreitet ist, da die Opportunitätskosten der Entscheidung für Kinder in diesen Milieus größer sind. Das Leitbild der risikovermeidenden Kinderlosigkeit sehen signifikant mehr Personen mit Migrationshintergrund als Grund, weshalb Menschen 
in Deutschland keine Kinder bekommen als solche ohne (36,6\% vs. 24,5\%). Genauso geben dies auch deutlich mehr Eltern (31,9\%) als Kinderlose (24,3\%) an. Die Zustimmung zur Risikovermeidung als gesellschaftliche Ursache für Kinderlosigkeit sinkt mit höherer Bildung $(36,4 \%=$ niedrig, $29,7 \%=$ mittel, 18,8 \% $=$ hoch) und ist bei Westdeutschen $(28,6 \%)$ stärker ausgeprägt als bei Ostdeutschen (22,3\%). Weitere Untersuchungen müssen klären, ob diese soziodemografischen Differenzen durch Selektionseffekte erklärt werden können, nämlich dass Personen mit bestimmten Leitbildern auch dazu passende Erfahrungen machen. Oder ob die gesellschaftlichen Leitbilder der Kinderlosigkeit vor allem durch die sozial nahräumliche Umgebung der Befragten geprägt sind (siehe Gies/ Dietrich, Kap. 4).

Tabelle 6: Hauptkomponentenanalyse für allgemeine Gründe gegen Kinder

\begin{tabular}{lcc}
\hline Allgemeiner Grund gegen Kinder: & \multicolumn{2}{c}{ Komponente } \\
Viele Menschen bekommen keine Kinder,... & 1 & 2 \\
\hline$\alpha$ & &, 507 \\
\hline weil Kinderlose so leben können, wie es ihnen gefällt. &, 718 \\
weil Kinder und Beruf schlecht zu vereinbaren sind. &, 669 \\
weil man sich ohne Kinder viel mehr leisten kann. &, 694 \\
weil Kinder die Partnerschaft belasten. &, 665 & \\
weil es viel zu kompliziert ist, Kinder großzuziehen. &, 703 \\
weil viele vor der lebenslangen Verantwortung zurückschrecken. &, 703 \\
aus Angst vor einer Trennung. &, 598 & \\
\hline
\end{tabular}

Quelle: FLB (2012), gewichtete Daten, eigene Berechnungen

Anm.: Varimax, rotierte Komponentenmatrix; Werte ab 0,4 werden angezeigt; die ersten beiden Faktoren erklären $49 \%$ der Gesamtvarianz.

\section{$7 \quad$ Zusammenfassung}

Im Mittelpunkt stand es, zu klären, welche allgemein wahrgenommenen Leitbilder zur Kinderlosigkeit beitragen und inwieweit diese in Deutschland kulturell verankert sind. Außerdem sollte untersucht werden, ob soziale Gruppierungen ausgemacht werden können, in denen sich Kinderlosigkeit besonders verfestigt hat. Dazu gehörte auch die Fragestellung, inwieweit es sozialstrukturelle Unterschiede in der Akzeptanz von Kinderlosigkeit gibt.

Es konnte eine spezielle Gruppe in der Gesellschaft identifiziert werden (9,4\%), die keine Kinder hat und sich auch keine Kinder wünscht, die Kinderlosigkeit als Lebensentwurf konsequent verinnerlicht hat. Laut der Leitbildstudie möchten vor allem Ledige ohne Partner/in sowie erwerbstätige und religionslose Personen, aber auch niedrig gebildete mit geringem Einkommen häufiger keine Kinder. Auch für die Hochqualifizierten ist festzustellen, dass sie häufiger kinderlos bleiben, als sie sich das wünschen. Am Ende des gebärfähigen Alters sind die Hochqualifizierten daher häufiger kinderlos als die Niedrigqualifizierten. Anzunehmen ist, dass die höhere Akzeptanz der Kinderlosigkeit den Aufschub der Familiengründung und schließlich den Übergang in die dauerhafte Kinderlosigkeit begünstigt. 
Dazu, warum Menschen keine Kinder bekommen, wurden verschiedene Gründe ausgemacht. Es wurden zwei Leitbilder identifiziert, die aus Sicht der Befragten zur Kinderlosigkeit vieler Menschen beitragen: Ein „Leitbild der risikovermeidenden Kinderlosigkeit“ und das „Leitbild der autonomiebetonten Kinderlosigkeit“. Das erste beinhaltet vor allem Ängste gegenüber der Zukunft und gegenüber der Verantwortung und generell gegenüber der Belastung, die ein Kind im Alltag bedeutet. Die Überfrachtung der Vorstellung eines Lebens mit Kind führt zur Ablehnung einer eigenen Familie. Diese Idee hängt mit dem Phänomen der „Helikopter“-Eltern zusammen, die sich zwar für ein Kind entschieden haben, sich selbst jedoch mit überhöhten Erwartungen überfrachten und ihr Kind unter starken Leistungsdruck setzen. Die Beobachtung solcher Eltern durch Kinderlose führt wiederum dazu, dass ein Leben mit Kindern unattraktiv aussieht bzw. wirkt. Dieses Leitbild wurde häufiger bei Personen mit Migrationshintergrund, bei Eltern und in den niedrigeren Bildungsstufen aufgefunden. Das zweite Leitbild betont die Selbstbestimmung bzw. die Autonomie der Kinderlosen, die durch ihre Kinderlosigkeit in der beruflichen und privaten Lebenswelt komplette Freiheit genießen und einen anderen Lebensstil pflegen können als Eltern. Beruf und Familie sind, besonders dort wo die Kinderbetreuungsinfrastruktur wenig ausgebaut und aufgrund Qualitätsbedenken wenig akzeptiert ist, noch schlechter vereinbar, teurer und einschränkender, je höher die Ansprüche an Elternschaft sind. Träger dieses Leitbildes sind in stärkerem Maße Hochgebildete und Personen ohne Migrationshintergrund.

Die Akzeptanz der Kinderlosigkeit ist in Deutschland relativ weit verbreitet. Es ist immerhin etwas mehr als die Hälfte in der Altersgruppe der 20- bis 39-Jährigen, die Kinderlosigkeit als eine normale Lebenssituation ansieht. Und auch Personen mit mehreren Kindern oder solche, die sich Kinder wünschen, empfinden Kinderlosigkeit immer noch zu einem hohen Anteil als normal, wenngleich sie sich von den Kinderlosen etwas abheben. Bemerkenswert ist, dass hierbei weder signifikante regionale noch altersbedingte Unterschiede gefunden wurden. Man könnte daher resümieren, dass in weiten Teilen der jüngeren Gesellschaft Kinderlosigkeit als selbstverständlicher Bestandteil des Lebens wahrgenommen wird. Insgesamt verwundert dies nicht, gehört Deutschland doch zu den Ländern Europas, in denen Kinderlosigkeit am weitesten verbreitet ist (Kreyenfeld/Konietzka 2013).

Das Thema Kinderlosigkeit wird in Deutschland ambivalent thematisiert: Einerseits erfährt Kinderlosigkeit eine große Akzeptanz, andererseits verurteilt eine große Gruppe der Befragten (vor allem Eltern, besonders die von drei und mehr Kindern) die Kinderlosen als egoistisch und sehen sie deshalb in der Pflicht, höhere Abgaben zu leisten. Demnach werden Kinderlose einerseits als die Gruppe gesehen, die an den sozialen Sicherungssystemen partizipiert, aber die Generation zukünftiger Beitragszahler ausfallen lässt. Andererseits gelten Kinderlose als beruflich engagiert und leistungsfähig zum Nutzen des Gemeinwesens.

Zahlreiche Gründe für Kinderlosigkeit sind laut der Befragten im Allgemeinen bedeutsam, zentral sind dabei vor allem die mangelnde Vereinbarkeit, die Verschlechterung der finanziellen Situation sowie der Verlust von Autonomie. Die Befunde der Leitbildstudie zeigen dabei, dass mangelnde Vereinbarkeit als zentraler Grund für Kinderlosigkeit kein spezifisch weibliches Problem mehr ist. Auch junge Männer antizipieren die hohen Opportunitätskosten einer Familiengründung für sich selbst, insbesondere die hochgebildeten. Hintergrund könnte sein, dass es einen Wandel gibt hin zu einer Verbreitung des Leitbildes der ,aktiven Vaterschaft“ (siehe Lück, Kap. 14), welches gleichermaßen mit Mutterleitbildern einhergeht, die seltener am Ideal der „Hausfrauenehe“ angelehnt sind (siehe Diabaté, Kap. 13). Dies führt dazu, dass Familienarbeit schrittweise immer häufiger als gemeinsame partnerschaftliche und etwas seltener als vorrangig mütterliche Aufgabe gesehen wird. 
Des Weiteren sollte untersucht werden, welcher Einfluss der soziale Kontext der Befragten auf deren Kinderlosigkeit hat: Es konnten dabei signifikante Zusammenhänge mit den individuellen Netzwerken nachgewiesen werden, die sich im Längsschnitt möglicherweise als Abfärbeffekte herausstellen könnten. Das gilt stärker für den Freundes- und Bekanntenkreis als für Familie und Verwandte oder die Arbeitskollegen. Inwiefern sich „gleich und gleich“ gern gesellt, bleibt zu klären, schließlich werden der Freundes- und Bekanntenkreis im Vergleich zu den anderen Gruppen selbst ausgesucht.

Als das generelle Fazit kann gezogen werden, dass Kinderlosigkeit in Deutschland inzwischen weitgehend akzeptiert ist. Kinderlosigkeit ist für einen, wenn auch kleinen Teil der jüngeren Bevölkerung, attraktiv geworden und wird als Lebensoption angestrebt. Sie wird kaum noch als Defizit wahrgenommen. Es ist demnach von einer kulturellen Verankerung der Kinderlosigkeit in Deutschland auszugehen. Von einer Kultur der Kinderlosigkeit im Sinne von Leitkultur möchten die Autoren ausdrücklich nicht sprechen, gewollte Kinderlosigkeit ist eher selten, wenn auch präsent. Von einer Kultur von Kinderlosigkeit - wie eingangs postuliert - kann nicht die Rede sein, eher von einer Subkultur bzw. einem Milieu. Eine breite gesellschaftliche Akzeptanz von Kinderlosigkeit bedeutet nicht, dass Kinderlosigkeit für weite Teile der Bevölkerung auch handlungsleitend geworden ist. Letztlich zeigt die Differenz zwischen realer und angestrebter Kinderlosigkeit, dass häufiger die Umstände als der tatsächliche Wunsch Elternschaft verhindern.

Die Analysen haben aber auch gezeigt, dass sich Kinderlosigkeit nicht allein mit einem kulturellen Ansatz erklären lässt. Kinderlosigkeit erscheint häufiger als ein Vereinbarkeitsproblem, Geldproblem, Strukturproblem oder Medizinproblem. Da Kinderlosigkeit aufgrund der gesellschaftlichen Umstände inzwischen seit mehr als 20 Jahren relevant für Deutschland ist, hat es seinen kulturellen Niederschlag gefunden.

Insgesamt wird deutlich, dass Vorstellungen zur Kinderlosigkeit und die individuelle private Lebensführung zusammenhängen. Es erscheint im Hinblick auf weitere multivariate Analysen aussichtsreich, diese kulturellen Komponenten einzubeziehen, um den Einfluss auf generative Entscheidungen in Zukunft noch tiefgreifender untersuchen zu können.

\section{Literatur}

Bertram, Hans; Rösler, Wiebke; Ehlert, Nancy (2005): Nachhaltige Familienpolitik. Zukunftssicherung durch einen Dreiklang von Zeitpolitik, finanzieller Transferpolitik und Infrastrukturpolitik. Gutachten im Auftrag des Bundesministeriums für Familie, Senioren, Frauen und Jugend, Berlin.

Boehnke, Mandy (2007): Hochschulbildung und Kinderlosigkeit: Deutsch-deutsche Unterschiede. In: Konietzka, Dirk; Kreyenfeld, Michaela (Hrsg.): Ein Leben ohne Kinder. Wiesbaden: VS Verlag für Sozialwissenschaften, S. 295-315.

Bujard, Martin (2012): Talsohle bei Akademikerinnen durchschritten? BiB Working Paper 4/2012. Wiesbaden: Bundesinstitut für Bevölkerungsforschung.

Burkart, Günter S. (2007): Eine Kultur des Zweifels: Kinderlosigkeit und die Zukunft der Familie. In: Konietzka, Dirk; Kreyenfeld, Michaela (Hrsg.): Ein Leben ohne Kinder. Wiesbaden: VS Verlag für Sozialwissenschaften, S. 401-420.

Dorbritz, Jürgen; Naderi, Robert (2013): Trendwende beim Kinderwunsch? In: Bevölkerungsforschung Aktuell 3/2013, S. 2-7.

Dorbritz, Jürgen; Naderi, Robert (2012): Stabilität bilokaler Paarbeziehungen - Rahmenbedingungen und Entwicklungspfade: eine Analyse der ersten und zweiten Welle von pairfam. In: Comparative Population Studies 37, 3-4, S. 393-428. 
Eichhorst, Werner; Kaiser, Lutz C.; Thode, Eric; Tobsch, Verena (2007): Vereinbarkeit von Familie und Beruf im internationalen Vergleich. Zwischen Paradigma und Praxis. Gütersloh: Bertelsmann Stiftung.

Eckhard, Jan (2014): Theoretische Erklärungen der zunehmenden Kinderlosigkeit - Divergierende Ansätze und das Integrationspotenzial der Frame-Selektions-Theorie. In: Comparative Population Studies 39, 1, S. 23-48.

Goldstein, Joshua; Kreyenfeld, Michaela; Rößger, Felix (2012): Gibt es eine Trendumkehr in der Kinderzahl nach Geburtsjahrgängen in Deutschland? Berliner Demografie Forum, Working Paper Ausgabe 4.

Hill, Paul B.; Kopp, Johannes (2006): Familiensoziologie: Grundlagen und theoretische Perspektiven. Studienskripte zur Soziologie. Wiesbaden: Springer VS.

Kaufmann, Tobias (2006): Die Angst vor dem Leben. In: FrauenRat 3/06.

Konietzka, Dirk; Kreyenfeld, Michaela (Hrsg.) (2007): Ein Leben ohne Kinder. Kinderlosigkeit in Deutschland. 2. Aufl. Wiesbaden: VS Verlag für Sozialwissenschaften.

Kreyenfeld, Michaela; Konietzka, Dirk (2013): Kinderlosigkeit in Deutschland. Theoretische Probleme und empirische Ergebnisse. In: Konietzka, Dirk; Kreyenfeld, Michaela, (Hrsg.): Ein Leben ohne Kinder. Ausmaß, Strukturen und Ursachen von Kinderlosigkeit. 2. Aufl. Wiesbaden: VS Verlag für Sozialwissenschaften, S. 13-47.

Merz, Eva-Maria; Liefbroer, Aart C. (2012): The attitude toward voluntary childlessness in Europe: cultural and institutional explanations. In: Journal of Marriage and Family 74, 3, S. 587-600.

Lutz, Wolfgang; Milewski, Nadja (2004): Als ideal angesehene Kinderzahl sinkt unter zwei. In: Demografische Forschung aus Erster Hand, Jahrgang 1, Nr. 2.

Richter, Nico; Lois, Daniel; Arránz Becker, Oliver; Kopp, Johannes (2012): Mechanismen des Netzwerkeinflusses auf Fertilitätsentscheidungen in Ost- und Westdeutschland. In: Huinink, Johannes; Kreyenfeld, Michaela; Trappe, Heike: Familie und Partnerschaft in Ost- und Westdeutschland. Ähnlich und doch immer noch anders. Sonderheft Zeitschrift für Familienforschung, 9, S. 95-118.

Schmitt, Christian (2004): Kinderlose Männer in Deutschland - Eine sozialstrukturelle Bestimmung auf der Basis des Sozio-oekonomischen Panels (SOEP), DIW Berlin, Materialien 34.

Schmitt, Christian; Winkelmann, Ulrike (2005): Wer bleibt kinderlos? Sozialstrukturelle Daten zur Kinderlosigkeit von Frauen und Männern. DIW Berlin, Discussion Papers 473.

Schneider, Norbert F. (2012): Die familiendemografische Entwicklung in Deutschland. Bundeszentrale für Politische Bildung. [http:/www.bpb.de/politik/grundfragen/deutsche-verhaeltnisse-einesozialkunde/138030/die-familiendemografische-entwicklung-in-deutschland? $\mathrm{p}=$ all, 30.09.2014]

Sobotka, Tomas; Testa, Maria Rita (2008): Attitudes and Intentions Toward Childlessness in Europe. In: Höhn, Charlotte; Avramov, Dragana; Kotowska, Irena E. (Hrsg.): People, Population Change and Policies, 16/1: Springer Netherlands (European studies of population), S. 177-211. 


\title{
Heirat, Haus, Kinder? Leitbilder der Familiengründung und der Familienerweiterung
}

\author{
Jürgen Dorbritz \& Kerstin Ruckdeschel ${ }^{*}$
}

\section{Zusammenfassung}

Die familiendemografische Situation in Deutschland ist durch einen späteren Beginn und damit eine Verkürzung der reproduktiven Lebensphase, einen Verzicht auf Familienerweiterung oder -gründung und eine noch immer enge Verknüpfung von Ehe und Familienerweiterung gekennzeichnet. In diesem demografischen Kontext werden in dem folgenden Beitrag der Beginn und der Verlauf der Familiengründung anhand der Gründe für die Geburt von Kindern, der idealen Kinderzahl und des idealen Alters für die Geburt des ersten Kindes betrachtet.

Dabei wurde das Leitbild der Selbstverständlichkeit des Kinderhabens formuliert, da trotz aller Diskussionen um den Wandel der Familie in Deutschland Kinder zu haben immer noch als etwas ganz Normales angesehen wird. Im Leitbild der idealen Familiengröße übersteigen die gemessenen idealen Kinderwünsche den Wert zwei. Begrenzend auf den idealen Kinderwunsch wirken hohe persönliche Ansprüche an die materielle Sicherung, die erfüllt sein müssen, um sich für die Geburt eines Kindes zu entscheiden, weshalb auf dieser Basis das Leitbild der materiell gesicherten Elternschaft formuliert wurde. Als bemerkenswert ist hervorzuheben, dass das benannte ideale Alter für die Geburt eines Kindes niedriger ist als das tatsächliche Erstgebäralter. Frühe und späte Geburten vor dem 20. bzw. bereits nach dem 35. Lebensjahr werden kaum akzeptiert. Insgesamt lassen sich vier Familiengründungstypen unterscheiden, die sich nach ihren Ansprüchen an die materielle Sicherung einer Familiengründung und nach der Bedeutung der beruflichen Eigenständigkeit der Frau unterscheiden. Während die materiellen Ansprüche den Kinderwunsch direkt senken, geschieht dies bei der Forderung nach beruflicher Eigenständigkeit der Frau vermittelt über ein höheres ideales Familiengründungsalter, das wiederum den Kinderwunsch senkt.

\section{$1 \quad$ Einleitung}

Heiraten, Haus bauen, Kinderkriegen, möglichst zwei, möglichst einen Jungen und ein Mädchen - lange Zeit sah so die ideale Abfolge im Lebenslauf aus. Im letzten Jahrhundert bis hinein in die frühen 1960er Jahre war es normal im Leben eine Familie zu gründen, über 90 \% der Männer und Frauen hatten damals Kinder. Angesichts der demografischen und gesellschaftlichen Entwicklung stellt sich nun die Frage, ob Familie immer noch ein Lebensziel ist, das von der Mehrheit der Deutschen angestrebt wird. Wie wichtig ist es, eine Familie zu haben, warum sollte man noch Kinder bekommen und wie viele sind ideal? Die eingangs

Wir danken der anonymen Gutachterin bzw. dem anonymen Gutachter sowie Sabine Diabaté für die wertvollen Hinweise und die hilfreiche Kritik. Ein besonderer Dank geht an Robert Herter-Eschweiler für die Mikrozensusauswertungen. 
zitierte Abfolge impliziert auch noch etwas anderes, nämlich dass Familiengründung voraussetzungsvoll ist. Außer der Heirat und dem Hausbau könnte man z. B. auch noch die Etablierung im Beruf anführen, die nach Meinung der Menschen erfüllt sein müsste, bevor man idealerweise ans Kinderbekommen denken sollte. Welche Bedingungen sollten also vor der Familiengründung idealerweise erledigt sein und gibt es in der Vorstellung der Deutschen ein ideales Alter, bis zu dessen Erreichen das alles geschehen sein muss?

Diese Fragen werden relevant, wenn man sich dem Leitbild von Familiengründung und -erweiterung annähern will und kulturelle Gründe des niedrigen Geburtenniveaus in Deutschland näher in den Blick nehmen möchte. Einsichten in die Beschaffenheit dieses Leitbildes helfen weiter, um zu verstehen, wie wichtig Familie für den Einzelnen heute überhaupt noch ist und ob eventuell Vorstellungen von einer idealen Abfolge bis zu einem idealen Alter zu einer Art Mismatch mit der Realität führen können und Familiengründung verzögern oder verhindern. In diesem Sinne werden nach einem Überblick über die aktuelle demografische Lage und über den Forschungsstand im Folgenden die Bedeutung von Kinderhaben, der Kinderwunsch und Gründe, die für Kinder sprechen, analysiert. Der ideale Zeitpunkt wird durch eine Analyse des Idealalters und der Bedingungen, die in den Augen der Befragten vor Geburt des ersten Kindes erfüllt sein müssen, in die Untersuchung einbezogen.

\section{Demografische Lage und theoretische Grundlagen}

\subsection{Demografische Daten zur Familiengründung und-entwicklung ${ }^{1}$}

Die Familiengründung und -entwicklung in Deutschland ist durch sechs grundlegende Merkmale geprägt. Erstens streben längst nicht mehr alle Frauen und Männer eines Geburtsjahrgangs eine Familiengründung an. Das kommt in den wachsenden Anteilen an Kinderlosen zum Ausdruck. Von den Frauen des 1970er Geburtsjahrgangs wird angenommen, dass sie zu 21,9\% keine Kinder haben werden (siehe auch Dorbritz/Diabaté, Kap. 8). Der Ausstieg aus der Familiengründung erfolgt geschlechtsspezifisch, sozialstrukturell und regional differenziert. Vor allem durch die Forschungen von Schmitt (2004) ist bekannt, dass der Anteil kinderloser Männer mit ca. $29 \%$ in der Altersgruppe der 40- bis 45-Jährigen höher ist als der der Frauen. Da auf Männern der Erwartungsdruck als Familienernährer lastet, können schlechte berufliche Aussichten eine Familiengründung verhindern (Tölke/ Diewald 2003). Als weitere Gründe werden die niedrigeren Kinderwünsche genannt, außerdem die höhere Ledigenquote, das höhere Erstheiratsalter und die geringere Bereitschaft der Männer, sich zu einem Kind zu bekennen, vor allem wenn es sich um eine nichteheliche Geburt handelt (z. B. Klein/Eckhard 2006). Aus der sozialstrukturellen Sicht ist in erster Linie auf die hohen Anteile an kinderlosen Akademikerinnen zu verweisen (Geburtsjahrgänge 1969-1973: 30,3 \%), aber auch bei der Bevölkerung ohne Migrationshintergrund, bei Vollzeit erwerbstätigen Frauen oder bei bilokalen Paarbeziehungen erfolgt seltener eine Familiengründung (Dorbritz 2010: 49). Im Hinblick auf regionale Differenzierungen wird immer wieder der West-Ost-Unterschied thematisiert. Nach wie vor kommt es im Osten häufiger zu Familiengründungen als im Westen. Die westdeutschen Frauen des Geburts-

$1 \quad$ Mit Ausnahme der altersspezifischen Geburtenziffern und des Durchschnittsalters bei der Erstgeburt basieren alle übrigen Daten auf dem Mikrozensus 2012. 
jahrgangs 1970 bleiben zu 22,9\% und die ostdeutschen zu 15,0 \% ohne Kinder. Allerdings ist in den neuen Bundesländern bei den Jahrgängen aus der zweiten Hälfte der 1960er Jahre diesbezüglich ein steigender Trend zu beobachten. Die höhere Bereitschaft zur Familiengründung in den älteren Kohorten wird aus den Besonderheiten der DDR-Gesellschaft abgeleitet (Huinink 1995a). Erklärt wird dies u. a. aus der Betonung des Privaten als Abgrenzung von der DDR-Öffentlichkeit, der eingeschränkten Vielfalt an Lebensoptionen, dem Bestreben, sich Ressourcen über die Familiengründung zu sichern (Wohnungen) und den relativ hohen Sicherheiten für die weitere Lebensplanung.

Zweitens gibt es einen noch immer anhaltenden Trend zu einem späteren Beginn der Familiengründung. Im Jahr 2013 waren Frauen in Deutschland bei der Geburt des ersten Kindes 29,3 Jahre alt. Bei verheirateten Frauen betrug der Wert 30,5 Jahre (Statistisches Bundesamt 2014). Das Durchschnittsalter bei der Geburt des ersten Kindes in einer Ehe ist seit 1980 um 5,3 Jahre angewachsen. Als Ursache für die verzögerte Familienentwicklung nennt Peuckert (2008: 122), dass die ,gestiegenen Qualifikationsanforderungen (...) dazu geführt (haben, d. A.), dass ökonomische Selbständigkeit und eine von den Eltern unabhängige Lebensführung in einem eigenen Haushalt von einem immer größeren Teil der jungen Erwachsenen immer später zu erwarten sind“".

Drittens hat dieser Trend zur Folge, dass sich die Lebensspanne, in der Kinder geboren werden, verkürzt hat. Abbildung 1 zeigt anhand des Verlaufs der altersspezifischen Geburtenziffern 1990 und 2012, wie sich der Anteil der Geburten in den Lebensabschnitt zwischen dem 30. und 35. Lebensjahr verschoben hat. Nach dem 40. Lebensjahr werden kaum noch Kinder geboren. Die Geburt des dritten Kindes erfolgt durchschnittlich im Alter von 33,0 und der vierten und weiteren Kinder im Alter von 34,2 Jahren, Mütter in Mehrkindfamilien fangen durchschnittlich viel früher mit der Familienphase an als Mütter in Ein- und Zwei-Kind-Familien.

Die Verkürzung der reproduktiven Lebensphase durch den Aufschub der ersten Geburt gilt inzwischen als einer der Faktoren, die Kinderlosigkeit begünstigen und die Chance auf weitere Geburten verringern (Statistisches Bundesamt 2007). Genannt werden in der Literatur als Gründe für die immer spätere Geburt der Kinder die langen Ausbildungswege, was vor allem die Hochqualifizierten betrifft, unsichere Beschäftigungsverhältnisse, berufliche Mobilität, und die hohen Ansprüche an die Erziehung und Betreuung der Kinder², so dass sich Paare erst für die Erfüllung des Kinderwunsches entscheiden, wenn die Bedingungen dafür ihren Anforderungen gerecht werden (siehe z. B. Konietzka/Kreyenfeld 2013).

Viertens wird auch auf Familienerweiterungen verzichtet, was in Deutschland auf ein Viertel aller Frauen zutrifft. Der Anteil der Frauen mit nur einem Kind ist in Ostdeutschland $(35,9 \%)$ deutlich höher als im Westen $(22,8 \%)$. Verantwortlich dafür dürfte die spezifische Verknüpfung von strukturellen und kulturellen Faktoren sein. In den neuen Bundesländern gehen Einstellungen wie die Erwerbsorientierungen der Frauen und die Akzeptanz der außerhäuslichen Kinderbetreuung auch mit höheren Betreuungsquoten einher (Schneider/ Dorbritz 2011: 30). Dadurch kann die geringere Kinderlosigkeit und die Geburt erster Kinder erklärt werden. Die ausbleibende Familienerweiterung steht dann eher im Zusammenhang mit Zukunftssorgen oder Jobunsicherheit.

2 Kaufmann (1990) hat dies als Norm der „Verantworteten Elternschaft“ bezeichnet, siehe auch Ruckdeschel, Kap. 12. 
Abbildung 1: Altersspezifische Geburtenziffern in Deutschland, 1990 und 2012

Geburten je 1.000 Frauen im jeweiligen Alter

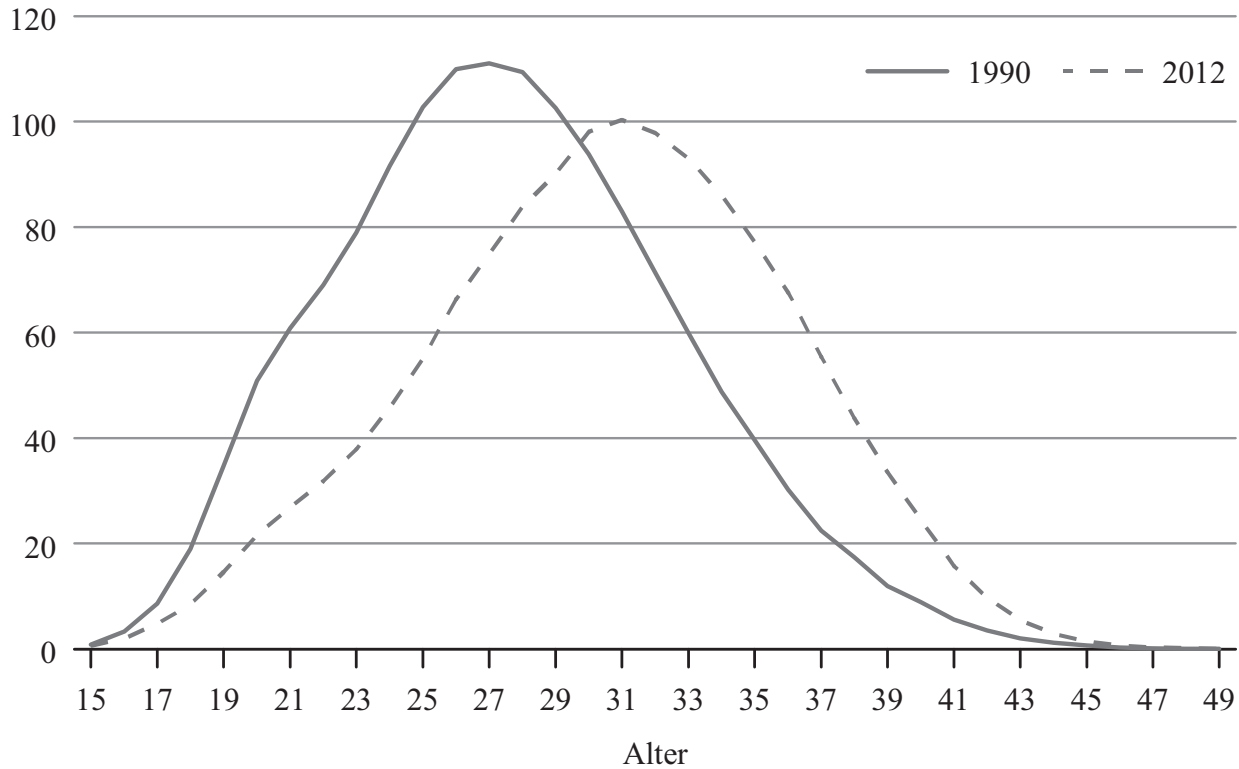

Quelle: Statistisches Bundesamt, grafische Darstellung: BiB

Fünftens hat eine Familienerweiterung über das dritte Kind hinaus inzwischen einen Seltenheitswert erlangt. Der Anteil vierter und weiterer Kinder beträgt im Geburtsjahrgang 1970 nur noch $4,8 \%$.

Sechstens ist die Familiengründung und -erweiterung noch immer an die Ehe gebunden, der Bezug ist aber abgeschwächt. Dennoch gilt: Verheiratete sind deutlich seltener kinderlos und haben auch häufiger drei und mehr Kinder. Von den verheirateten Frauen der Geburtsjahrgänge 1969 bis 1973 hatten bis zum Jahr 2012 nur 11,8 \% keine Kinder. Wer zum Zeitpunkt der Mikrozensusbefragung in einer nichtehelichen Lebensgemeinschaft oder ohne Partner im Haushalt lebte, war zu 37,5 \% bzw. zu 46,1 \% kinderlos. Umgekehrt haben $19,9 \%$ der Verheirateten drei oder mehr Kinder, während der Anteil Kinderreicher bei den nichtehelichen Lebensgemeinschaften und den Frauen ohne Partner weniger als $10 \%$ beträgt.

Anzeichen für eine Änderung dieser Merkmale der Familiengründung und -entwicklung gibt es derzeit kaum. Die Kinderlosigkeit scheint weiter zuzunehmen, auch wenn es Anzeichen dafür gibt, dass sich der Anstieg zumindest bei den Hochqualifizierten nicht fortsetzen wird. Begründet werden kann die Annahme eines weiteren Anstiegs mit den empirischen Beobachtungen, nach denen die Kinderlosigkeit in den neuen Bundesländern, bei niedriger Qualifizierten und bei Frauen mit einem Migrationshintergrund weiter wächst (Statistisches Bundesamt 2013).

Zudem gilt als äußerst unwahrscheinlich, dass es zukünftig wieder mehr Kinderreiche geben wird. Fortsetzen dürfte sich auch der Trend der verringerten Bedeutung der Ehe für die Familiengründung und -erweiterung. Im Bereich des Möglichen liegen häufigere Übergänge vom ersten zum zweiten Kind vor allem in den neuen Bundesländern und eine Verlängerung der generativen Lebensphase. Beides würde sich positiv auf das Geburten- 
niveau auswirken. Vor allem ein früherer Beginn der Familiengründung dürfte nachhaltig wirken, denn für die Kinderreichen hat es sich gezeigt, dass sie ihr erstes Kind früher bekommen.

\subsection{Forschungsstand und theoretische Grundlagen}

Aus den dargestellten Befunden ergeben sich für die Leitbildforschung folgende relevante Themen auf dem Gebiet der Familiengründung und Familienerweiterung:

- Wichtigkeit von Kindern,

- Gründe für Kinder,

- Kinderwunsch,

- Idealalter für die Geburt des Kindes und

- Bedingungen, die gegeben sein müssen, damit eine Entscheidung für die Erfüllung des Kinderwunsches getroffen wird.

In allen diesen Themenbereichen bieten gesellschaftliche Leitbilder Orientierungsrahmen für konkretes Handeln. Im Folgenden wird deshalb der Forschungsstand zu diesen Punkten dargestellt, um anschließend Forschungsfragen zu entwickeln.

\section{Wichtigkeit von Kindern und Gründe für Kinder}

Gemeinsames Ergebnis aller Surveys zu Familienthemen ist die hohe allgemeine Bedeutung von Kindern und Familie. Diese leitet sich trotz aller Diskussionen um den Funktionsverlust bzw. Funktionswandel der Familie immer noch daraus ab, welchen Nutzen Kinder für die Eltern darstellen und welche Kosten sie verursachen. Dies wird vor allem über die Value-Of-Children-Theorie abgebildet, die auf Hoffman und Hoffman (1973) zurückgeht. Kinder werden in der VOC-Theorie als „Zwischengüter“ gesehen, die zur Erreichung menschlicher Grundbedürfnisse beitragen und sich damit auf die Vorstellungen über Familiengröße oder Erziehungsstile auswirken. Nauck (2001) unterscheidet zwischen einer ökonomischen, einer psychologischen und einer soziokulturellen Nutzendimension. Generell wird angenommen, dass es eine Verlagerung von ökonomischem und sozio-kulturellem Nutzen zu psychologischem Nutzen gibt (Huinink/Konietzka 2007). Letztlich lassen sich die Dimensionen des Wertes von Kindern in immanente und instrumentelle Werte unterteilen, wobei für die instrumentellen Werte nochmals zwischen traditional-instrumentellen (Statussicherung, ökonomische Absicherung des Alters) und modern-instrumentellen (sozialer Status, Ehestabilität, Lebenssinn) unterschieden werden kann (Meulemann 2007). Der immanente Wert von Kindern liegt in der Eltern-Kind-Beziehung selbst, z. B. in der Freude am gelingenden Heranwachsen eigener Kinder, zu der es keine keine Alternativen gibt. Dagegen liegen instrumentelle Werte jenseits der Eltern-Kind-Beziehung und können durch andere soziale Beziehungen oder Institutionen ersetzt werden, z. B. durch Freunde, Therapeuten oder Religion (modern-instrumentelle Werte) bzw. Sozialversicherungen (traditionell-instrumentelle Werte) (Meulemann 2007: 31). Der Wert von Kindern hat sich in seiner Bedeutung weg von instrumentellen Werten verschoben, bleibt aber auch in modernen Gesellschaften hoch (Trommsdorf/Nauck 2005; Meulemann 2007).

Erhoben wurde der Nutzen von Kindern in den letzten Jahren z. B. in pairfam (Panel Analysis of Intimate Relationships and Family Dynamics) (Fitzner et al. 2007), wobei die Ergebnisse in der ersten und der zweiten Welle relativ übereinstimmend zeigen, dass die Erwartungen an das Leben mit Kindern an erster Stelle auf eine besonders emotionale 
Beziehung, auf die Hilfe in Notfällen und die Anregungen, die durch Kinder gegeben werden, gerichtet sind. Der GGS (Generations and Gender Survey, Ruckdeschel et al. 2006) brachte dabei eher ernüchternde Ergebnisse zum Vorschein. Es zeigt sich auch in dieser Studie, dass Kinder das Gefühl geben gebraucht zu werden, und dass über zwei Drittel der Auffassung sind, dass die engste Beziehung die zum eigenen Kind ist oder dass es genossen wird, Kinder um sich zu haben. Es denken aber auch etwas mehr als die Hälfte, dass man auch ohne Kinder glücklich sein kann. Die Mehrheit der Befragten erwartet von der Geburt eines (weiteren) Kindes deutliche Veränderungen, allerdings ins Negative: bei der Selbstbestimmung, bei den Beschäftigungschancen und bei der finanziellen Situation. Dieser Auffassung waren vor allem diejenigen, die bereits Eltern waren. Die Kinderlosen waren an dieser Stelle deutlich optimistischer. Sie sind deutlich öfter davon ausgegangen, dass die Lebensfreude steigt und sich die Qualität der Partnerschaft verbessert (Höhn et al. 2006). Hinsichtlich der Lebensfreude, der Enge der Beziehung, der Sicherheit im Alter, dem Sexualleben oder dem öffentlichen Ansehen werden mit der Geburt eines Kindes mehrheitlich keine Veränderungen erwartet.

\section{Kinderwunsch}

Die Ergebnisse der Kinderwunschforschung werden im Rahmen der an harten Fakten orientierten demografischen Forschung oftmals auf ihre Validität hin diskutiert (z. B. Philipov/Bernardi 2011, Miller 2011). Dennoch ist es in den familiensoziologischen Surveys Standard, den Kinderwunsch in dieser oder jener Form zu erheben. Die Kritiker an der Kinderwunschforschung bemängeln vor allem die Abhängigkeit von der gesellschaftlichen Situation und den verwendeten Messinstrumenten. Gerade diese Abhängigkeit von gesellschaftlichen Rahmenbedingungen erlaubt aber, die Rolle dieser Rahmenbedingungen für eine intendierte Elternschaft genauer zu spezifizieren und zu bewerten (Ruckdeschel 2007), also auch die Rolle von Leitbildern. Zudem hängen die Antworten auf Kinderwunschfragen stark von der Frageformulierung ab. Philipov und Bernardi (2011) unterscheiden in diesem Zusammenhang Fertilitätsideale und Fertilitätsabsichten, die unterschiedliche Realisierungschancen haben. Dorbritz und Naderi (2013) haben zusätzlich eine dritte Ebene zwischen Ideal und Absicht eingeführt, das Kinderwunschleitbild und kommen zu folgender Typologie:

\section{Idealer Kinderwunsch - gesellschaftlich, familienbezogen oder individuell}

Hier wird die ideale Kinderzahl für ein Land, eine Familie oder eine Person erhoben. Dabei wird stark von der Realsituation abstrahiert. Unter der Annahme idealer Lebensumstände wird nach der Zahl der gewünschten Kinder gefragt.

2. Kinderwunschleitbild

Bei der Frage nach dem Kinderwunschleitbild werden weder die idealen noch die konkreten Lebensumstände in Betracht gezogen, es liegt in Bezug auf die Realisierung also dazwischen und trägt insofern einen Leitbildcharakter. Darüber hinaus wird kein konkreter Zeitrahmen für die Erfüllung des Kinderwunsches vorgegeben.

3. Realistischer Kinderwunsch

Bei der realistischen Kinderzahl wird von den Befragten erwartet, dass sie die tatsächlichen Lebensumstände und ihre Veränderung in einem vorgegebenen Zeitraum bei der Angabe über die gewünschte Kinderzahl berücksichtigen.

Seit dem Jahr 2001 sind Kinderwünsche 14-mal in fünf verschiedenen Surveys gemessen worden. Dazu zählen das Eurobarometer, die PPAS (Population Policy Acceptance Study, vgl. dazu: Höhn, Avramov und Kotowska 2008), der GGS, die FLB (Familienleitbildstudie 
des BiB) und pairfam. In Abhängigkeit von der Fragestellung sind dabei sehr unterschiedliche Ergebnisse erzielt worden. Wird nach der idealen Kinderzahl, gesellschaftlich oder individuell, gefragt, sind die Kinderwünsche relativ hoch, da sehr stark von der realen Lebenssituation abstrahiert wird. Die aktuellen Messungen im Zeitraum 2006 bis 2012 zeigen Werte in einem Bereich von 2,06 bis 2,26. Beim Kinderwunschleitbild wird abgefragt, wie viele Kinder man sich tatsächlich wünscht, ohne aber einen Zeithorizont vorzugeben oder auf die konkreten Lebensumstände zu verweisen. Die so ermittelten Kinderwünsche sind niedriger als die idealen Kinderwünsche. Die Messergebnisse umfassen einen Bereich von 1,90 bis 2,07 gewünschte Kinder. Mit dem realistischen Kinderwunsch wird die Zahl der gewünschten Kinder abgebildet, die Frauen oder Männer unter Berücksichtigung ihrer konkreten Lebensumstände einmal haben möchten. Abgefragt wurde er in der jüngsten Vergangenheit nur in pairfam bei besonderen Altersgruppen. Die erzielten Ergebnisse sind 1,75 und 1,77 .

Allen Ergebnissen gemeinsam ist, dass es seit dem Jahr 2000 einen Anstieg der Kinderwünsche auf allen Messebenen gegeben hat. Geht man bis in die 1990er Jahre zurück, zeigt sich eine U-förmige Verteilung mit sehr niedrigen Werten um das Jahr 2000.

Idealalter und Bedingungen für die Geburt von Kindern

Der Forschungsstand zum Timing der Geburt von Kindern im Lebensverlauf gilt in Deutschland als unbefriedigend (Zerle et al. 2012). Eine Allensbach-Studie im Auftrag des Familienministeriums hat jedoch ergeben, dass die Mehrheit der 18- bis 44-Jährigen in Deutschland der Meinung ist, dass das günstigste Alter für eigene Kinder zwischen Anfang 20 und Anfang 30 liegt (BMFSFJ 2005: 25), also bevor für viele die tatsächliche Familiengründung einsetzt. Zerle et al. (2012) verweisen darauf, dass es mehr Untersuchungen zur frühen als zur späten Elternschaft gibt. Der am häufigsten hervorgehobene Befund ist, dass in Deutschland sehr frühe Geburten (Teenagergeburten) mit einem Negativbild verknüpft sind. Teenagergeburten werden als problematisch betrachtet, weil sie mit hohen Gesundheitsrisiken verknüpft sind und in einem Zusammenhang mit Schulabbrüchen, fehlender Qualifikation für die Berufstätigkeit und in dessen Folge für schlecht bezahlte Arbeit oder die Abhängigkeit von Sozialleistungen stehen (Block/Schmidt 2009). Inwieweit dies eher für Frauen als für Männer gilt, kann nicht beurteilt werden, da für Männer sowohl hinsichtlich der frühen als auch der späten Vaterschaft fast keine Untersuchungen vorliegen. Auf eine größere Akzeptanz der späten Vaterschaft deutet ein Befund aus der amerikanischen Forschung hin, auf den Fthenakis (1999) verweist: Ältere Väter leben in stabileren Paarbeziehungen und sind ökonomisch besser in der Lage, ihre Kinder abzusichern. Für Frauen hingegen wird die späte Mutterschaft im Kontext gesundheitlicher Risiken diskutiert.

Einen Beitrag zur Erklärung des ansteigenden Alters liefert der Theorieansatz Value Of Children (VOC). Dabei wird vom psychischen Nutzen von Elternschaft ausgegangen. Danach erfolgt die Entscheidung für ein Kind, wenn die Überzeugung vorhanden ist, dass der erwartete psychische Nutzen auch eintreten wird.

„Befindet sich jemand hingegen in einer Lebensphase oder einer in Bezug auf Elternschaft strukturell problematischen Lebenslage, wird er oder sie Geburtenkontrolle üben, weil Elternschaft nicht nur mit geringem psychischen Nutzen, sondern auch mit erheblichen psychischen und materiellen Kosten verbunden wäre.“ (Huinink/Konietzka 2007: 156)

Kinderhaben ist also an verschiedene Bedingungen bzw. Voraussetzungen geknüpft und dies umso mehr, als sich in Deutschland der Normkomplex der verantworteten Elternschaft (Kaufmann 1990) etabliert hat. Dieser besagt, dass man Kinder nur dann zur Welt bringen sollte, wenn man glaubt, der entsprechenden Verantwortung auch gerecht werden zu 
können und stellt damit hohe Anforderungen an Eltern (siehe auch Ruckdeschel, Kap. 12). Die Folge dieser Ansprüche ist, dass in den Augen der Betroffenen zunächst bestimmte Voraussetzungen erfüllt sein müssen, bevor eine Familiengründung überhaupt in Erwägung gezogen werden kann, Huinink (1995b) spricht hier vom Ressourcenproblem, das gelöst sein muss. Entsprechend sollten nach Meinung der meisten Befragten der bereits erwähnten Allensbach-Studie (BMFSFJ 2005) beide Partner ihre Berufsausbildung abgeschlossen haben $(69 \%)$, mindestens ein Partner beruflich etabliert sein $(67 \%)$ und ein Einkommen für eine Familie ausreichen (59\%). Diese Anspruchshaltung hat letztlich zur Etablierung eines Mehrphasen-Modells der Familiengründung geführt, in dem vor der Familiengründung eine Phase der beruflichen und finanziellen Etablierung kommt, in der ein Übergang zur Elternschaft sehr selten eintritt (z. B. Schaeper 2013: 51; Schröder/Brüderl 2008; Blossfeld/Rohwer 1995).

\section{Leitbilder als Erklärungsfaktor}

Die empirische Fertilitätsforschung richtet ihr Hauptaugenmerk auf die faktischen Übergänge und deren Timing und versucht sie mit strukturellen Rahmenbedingungen und individuellen Einstellungen zu erklären. Da trotz der beiden Erklärungsstränge Verständnislücken bleiben, sollen im Folgenden als ergänzende Erklärung Leitbilder ${ }^{3}$ zur Familiengründung und -erweiterung näher untersucht werden. Dafür gehen wir davon aus, dass sich Mitglieder einer Gesellschaft bei ihrer Lebensplanung auch an spezifischen Leitbildern orientieren. Deren Wirkung auf eine konkrete Entscheidung, z. B. für oder wider eine Familiengründung kann angelehnt an die Theory of planned Behaviour (Ajzen 1985), beschrieben werden. Diese besagt, dass das Handeln eines Individuums - vermittelt durch seine Handlungsintention - durch seine individuellen Einstellungen gegenüber den Handlungsoptionen sowie durch die von ihm subjektiv wahrgenommenen Erwartungen seines sozialen Umfeldes bestimmt ist. Hinzu kommt seine subjektiv wahrgenommene Verhaltenskontrolle, d. h. die Überzeugung, eine Handlungsoption tatsächlich umsetzen zu können (Ajzen/Klobas 2013; vgl. auch Diabaté/Lück 2014).

In diesem Sinne orientiert sich Handeln also auch an wahrgenommenen Leitbildern, die weiter ausdifferenziert werden können: Einerseits geben strukturelle Leitbilder an, wie viele Kinder man z. B. haben sollte oder welche Bedingungen für eine Familiengründung erfüllt sein sollten. Daneben existieren prozessuale Leitbilder (Diabaté/Lück 2014), die angelehnt an cultural life scripts (Janssen/Rubin 2011; Berntsen/Rubin 2002) definiert wurden und die auf zeitliche Aspekte von Familienleitbildern fokussieren, wie z. B. den Übergang zur Elternschaft oder den Übergang in die Ehe. Solche Leitbilder geben vor, in welchem Alter bzw. welcher Lebensphase in einer Gesellschaft der Übergang von einem Zustand in den anderen „,normalerweise“ stattfinden, sollte also eine Art „Normalbiografie", an der man sich mit der eigenen Lebensplanung orientiert (Diabaté/Lück 2014). Cultural life scripts bezeichnen somit ,,culturally shared expectations about the timing of life events in an idealized life course" (Janssen/Rubin 2011: 291). Innerhalb eines solchen standardisierten Lebenslaufes bestehen chronologisch aufeinanderfolgende Zeitpunkte, die von vielen Menschen in dieser Abfolge als ideal erachtet werden. Ist eine Person „on time“ heißt das, dass zum Beispiel die Familiengründung genau in die als kollektiv für passend erachtete Lebensphase fällt, d. h. dass individuelle Biografie und Normalbiografie deckungsgleich sind.

3 Nach Diabate'und Lück (2014: 56) definiert als ,kollektiv geteilte bildhafte Vorstellungen des „Normalen“. 
Interessant werden die Übergänge zu neuen Lebensereignissen, wenn sich gesellschaftliche Erwartungen z. B. aufgrund struktureller Rahmenbedingungen, wie verlängerten Ausbildungszeiten, zu weit von der Lebensrealität der Betroffenen entfernen, ein Phänomen, das unter dem Stichwort „Rush hour des Lebens“ bekannt geworden ist (Bertram 2012). In diesem Fall können normative Altersgrenzen möglicherweise nicht gehalten werden, was zu einem Aufschub oder zu einem Verzicht auf Familiengründung oder -erweiterung führen kann. In der Life-Skript-Forschung wird in diesem Kontext vom „being off time“ gesprochen. Wenn beispielsweise Elternschaft und Schulzeit in denselben Lebensabschnitt fallen, entspricht das nicht der weit verbreiteten Vorstellung zu einem idealen „Timing“ dieser beiden Aspekte. Dazu existiert in der Literatur auch das Concept of cultural age norms (Neugarten et al. 1965), welches thematisiert, dass bestimmte Lebensereignisse zu einem normativ festgelegten Lebensalter eintreten sollten. Die beschriebenen prozessorientierten Leitbilder hängen wiederum von strukturbezogenen ab. Der optimale Zeitpunkt oder das ideale Alter zur Familiengründung etwa hängt auch damit zusammen, wie eine Partnerschaft (ohne Kinder) ,idealerweise“ aus Sicht der Akteure auszusehen hat und was Elternschaft im Gegensatz dazu zu leisten hat, z. B. hinsichtlich der Etabliertheit oder Stabilität der Beziehung.

Bei der Betrachtung der Familiengründung und -erweiterung spielen beide Arten von Leitbildern eine Rolle. So soll im Folgenden zunächst grundsätzlich untersucht werden, wie wichtig Familie überhaupt noch ist und zwar sowohl für das einzelne Individuum als auch in der öffentlichen Wahrnehmung. Warum möchte man in Deutschland heute noch Kinder haben? Und wenn Kinder gewünscht werden, wie viele? Im Anschluss werden Fragestellungen untersucht, die auf ein Leitbild der Familiengründung abzielen, d. h. es werden sowohl das in den Augen der Befragten ideale Alter für eine Familiengründung analysiert, als auch die Bedingungen, die nach Meinung der Menschen erfüllt sein müssen, um eine Familie gründen zu können.

\section{Empirische Ergebnisse}

Im Folgenden sollen die einzelnen empirischen Analysen zu den Fragen nach der Bedeutung von Familie, dem Kinderwunsch, dem idealen Familiengründungsalter und den Bedingungen zur Familiengründung aus methodischer Sicht zu einer Typisierung von Familiengründungsmustern verdichtet werden. Gefragt wird, ob es beispielsweise Gruppen gibt, die einen hohen Kinderwunsch haben, ihre Kinder früh bekommen wollen und sich nicht am Leitbild der materiell gesicherten Elternschaft orientieren. Komplementär wäre dann eine Gruppe mit einem hohen Sicherheitsdenken, niedrigen Kinderwünschen und der Vorstellung zu vermuten, dass man seine Kinder besser etwas später bekommt.

\subsection{Wichtigkeit von Kindern und Kinderwunsch}

Familiengründung kann letztlich nur stattfinden, wenn Familie als wichtiges Lebensziel anerkannt wird. Deshalb soll zunächst dargestellt werden, wie wichtig es den Befragten ist, eigene Kinder zu haben. Es geht also nicht um die Bedeutung von Kindern allgemein, sondern um eigene Kinder. Im FLB wurde gefragt, „Finden Sie es selbst für sich wichtig, eigene Kinder zu haben?“, Der überwiegende Teil der Befragten $(85,1 \%)$ beantwortete die Frage für sich persönlich mit wichtig bzw. sehr wichtig (vgl. Tabelle 1). Differenzierter 
betrachtet sind es mehr Frauen als Männer (88,0 \% zu 82,2 \%), während es kaum Altersunterschiede gibt. Eltern nach der Bedeutung eigener Kinder zu fragen, ist fast überflüssig und entsprechend bejahen mit 97,5\% fast alle die persönliche Bedeutung von Kindern für sie. Interessanter sind hier Kinderlose, von denen drei Viertel $(76,6 \%)$ eigene Kinder wichtig finden. Beachtenswert ist auch, dass die Bedeutung eigener Kinder mit zunehmender Bildung ebenfalls zunimmt.

Nach dem Kinderwunsch wurden Kinderlose mit „Möchten Sie Kinder?“ und Eltern mit „Möchten Sie noch weitere Kinder?“ gefragt. Die überwiegende Mehrheit der Befragten möchte Kinder oder hat Kinder. Nur etwa $10 \%$ geben an, keine Kinder und auch keinen Kinderwunsch zu haben. Die Unterschiede zwischen den sozialen Gruppen sind gering. In der Tendenz wollen Frauen etwas häufiger Kinder als Männer, Ostdeutsche etwas häufiger als Westdeutsche und Hochgebildete häufiger als niedrig Gebildete. Dieses Ergebnis ist beachtenswert, denn es sind in Westdeutschland die Hochgebildeten, die mit ca. $30 \%$ besonders oft kinderlos bleiben. Es scheint also nicht die Distanz gegenüber Kindern zu sein, sondern es scheinen die Umstände zu sein, die in dieser Gruppe die Umsetzung der Kinderwünsche verhindern.

Von den zum Befragungszeitraum Kinderlosen möchten 82,8 \% später einmal Kinder haben. Auffällig ist der Unterschied zwischen den Altersgruppen 20 bis 29 und 30 bis 39 Jahre. Von den jüngeren Kinderlosen wollen noch $86,6 \%$ Kinder haben, bei den älteren sind es nur noch 74,5\%. Wer also über 30 ist und noch kinderlos ist, möchte signifikant seltener Kinder haben. Dieses Ergebnis entspricht einer der Thesen über die Wege in die Kinderlosigkeit. Kinderlos wird man u. a. durch das wiederholte Aufschieben des Kinderwunsches so lange, bis sich ein kinderloser Lebensstil verfestigt hat, sich das biologische Zeitfenster zu schließen beginnt oder nicht der für eine Familiengründung erwünschte Partner gefunden werden konnte (z. B. Konietzka/Kreyenfeld 2013; Rupp 2005, Schmitt/ Winkelmann 2005).

Nach der im Abschnitt zum Forschungsstand vorgestellten Klassifikation der verschiedenen Kinderwunschmessungen wurden im Familienleitbildsurvey das Kinderwunschleitbild (Zahl der geborenen Kinder + Schwangerschaft + noch gewünschte Kinder) und die ideale Kinderzahl für Familien in Deutschland gemessen (vgl. Abschnitt 2.2). Da bei beiden Messungen weder nach einem Zeithorizont gefragt noch ein Bezug zu den aktuellen Lebensumständen vorgenommen wird, fallen die Messergebnisse relativ hoch aus. Für das Kinderwunschleitbild wurde ein durchschnittlicher Wert von 2,04 Kindern ermittelt. Der ideale Kinderwunsch wurde mit 2,23 Kindern berechnet. Diese Werte entsprechen den Ergebnissen, wie sie beispielsweise in pairfam (3. Welle 2011) angegeben werden. Das so gefundene Leitbild der idealen Familiengröße übersteigt also in beiden Dimensionen zumindest leicht den Wert 2.

Wird das Kinderwunschleitbild nach Paritäten betrachtet, dann zeigt sich eindeutig die bereits bekannte Orientierung auf die Zwei-Kind-Familie. Die Hälfte, d. h. rund 53,2 \% der Befragten möchte zwei Kinder haben. Ein beachtenswertes Ergebnis ist, dass 25,2 \% kinderreich (3 Kinder oder mehr) sein möchten. Gewünschte Kinderlosigkeit liegt wie bereits erwähnt bei 10,2 \% und auch die Ein-Kind-Familie wird mit 11,4\% relativ selten angestrebt. Trotz der im Paritätenvergleich geringen Orientierung auf Kinderlosigkeit ist festzustellen, dass es in Deutschland eine kleine Gruppe in der jüngeren Bevölkerung gibt, die dem Leitbild Kinderlosigkeit folgen (siehe dazu Dorbritz/Diabaté, Kap. 8).

Im Bezug zur realen demografischen Entwicklung ist auffällig, dass die gewünschte Paritätsstruktur deutlich von der abweicht, die die Geburtsjahrgänge am Ende ihrer Familiengründungsphase realisiert haben. Als Bezugskohorte fungiert der Jahrgang 1967, der im Jahr des Leitbildsurveys 2012 das 45. Lebensjahr erreichte und der seine fertile Lebens- 
phase quasi beendet hat. In der Realität werden dabei mehr als doppelt so viele Frauen gefunden, die kinderlos sind oder nur ein Kind haben (Statistisches Bundesamt 2013), als dies gewünscht ist. Dagegen werden Wünsche nach zwei und drei oder mehr Kindern nicht realisiert. Beispielsweise möchten 25,2\% der Befragten drei oder mehr Kinder haben (FLB), tatsächlich haben dies im Jahrgang 1967 aber nur 15,8 \%.

Tabelle 1: Wichtigkeit von Kindern, Kinderwunsch und ideale Kinderzahl nach sozialstrukturellen Merkmalen (Prozent/Mittelwerte)

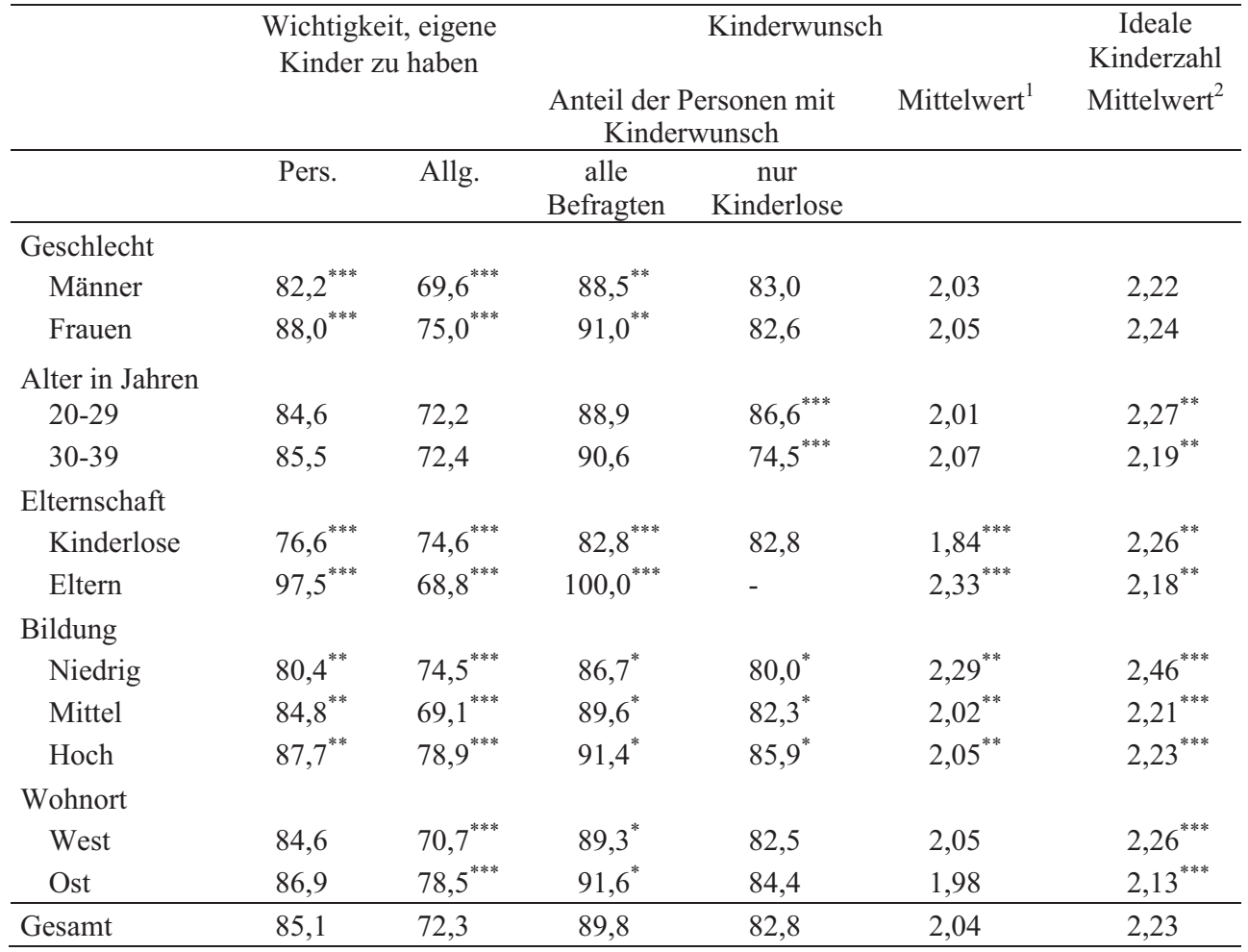

Quelle: FLB (2012), gewichtete Daten, eigene Berechnungen

Anm.: Signifikante Differenzen $\mathrm{Chi}^{2}$-Test für Anteile und T-Test bzw. ANOVA (Bildung) für Mittelwerte, Signifikanzniveau: ${ }^{* * *} \mathrm{p} \leq 0,001 ;{ }^{* *} \mathrm{p} \leq 0,01 ;{ }^{*} \mathrm{p} \leq 0,05$. Pers.: Persönliche Sicht; Allg.: Wahrgenommene Sicht in der Gesellschaft. ${ }^{1}$ Kinderwunsch inklusive realisierter Kinderwünsche, d. h. definiert als bereits geborene Kinder + zusätzlich gewünschte Kinder + Schwangerschaft $(+1) ;{ }^{2}$ Modus immer 2 .

\subsection{Gründe für Kinder}

Bei den Gründen für Kinder sollte in Erfahrung gebracht werden, wovon sich die meisten Menschen leiten lassen, wenn sie sich für Kinder entscheiden. Die Gründe wurden nur auf der allgemeinen Ebene abgefragt, da hier vor allem die wahrgenommene Meinung der Allgemeinheit interessiert und weniger individuelle Gründe, wie z. B. Krankheit oder Partnerlosigkeit. 
Hoffman und Hoffman sind ursprünglich von neun Wertegruppen ausgegangen, in der FLB-Studie wurden aber ausschließlich immanente und modern-instrumentelle Gründe erfragt (vgl. Abschnitt 2.2): Erwachsenenstatus und soziale Identität (Kinder gehören zum Leben dazu), Suchen neuer Erfahrungen (das Leben wird vielfältiger), Fortleben der eigenen Person (in Kindern über den Tod hinaus weiterleben) und familiäre Bindungen (nicht einsam im Alter sein, Stabilität der Partnerschaft durch Kinder). Von den fünf zu bewertenden Items haben sich zwei als besonders bedeutsam herauskristallisiert: Die höchste Zustimmung (93,8 \%; stimme voll und ganz zu, stimme eher zu) hat die Aussage erfahren, dass das Leben durch Kinder bunter und vielfältiger wird (vgl. Tabelle 2). Die zweithöchste Zustimmung mit 90,5 \% erlangte das Item "weil Kinder einfach zum Leben dazugehören". Diese Ergebnisse können in einer einfachen Aussage auf den Punkt gebracht werden: Es gibt nichts Schöneres als eigene Kinder. Dies gilt, obwohl dieser Sachverhalt aus einer ganzen Reihe von Untersuchungen bekannt ist, in Zeiten von hoher Kinderlosigkeit, veränderten Elternrollen oder dem Wandel der Lebensformen als ein heraushebenswertes Ergebnis.

Eine mittlere Bedeutung mit 71,5 \% wurde dem Grund, dass man ohne Kinder im Alter einsam sein wird, zugewiesen. Eine deutlich niedrigere, aber dennoch statistisch relevante Zustimmung erlangten die Aussagen „,weil Kinder eine Partnerschaft stabiler machen“ $(58,6 \%)$ und ,weil man in den Kindern über den Tod hinaus lebt (54,0 \%).

Tabelle 2: Gründe für Kinder - allgemeine Ebene (Zustimmung in Prozent)

\begin{tabular}{|c|c|c|c|c|c|}
\hline & $\begin{array}{c}\text {... weil Kinder } \\
\text { einfach zum } \\
\text { Leben } \\
\text { dazugehören. }\end{array}$ & $\begin{array}{c}\text {... weil das } \\
\text { Leben durch } \\
\text { Kinder bunter } \\
\text { und viel- } \\
\text { fältiger wird. }\end{array}$ & $\begin{array}{c}\text {... weil man } \\
\text { ohne Kinder } \\
\text { im Alter } \\
\text { einsam sein } \\
\quad \text { wird. } \\
\end{array}$ & $\begin{array}{l}\text {... weil Kinder } \\
\text { eine } \\
\text { Partnerschaft } \\
\text { stabiler } \\
\text { machen. } \\
\end{array}$ & $\begin{array}{c}\text {... weil man in } \\
\text { den Kindern } \\
\text { über den Tod } \\
\text { hinaus } \\
\text { weiterlebt. }\end{array}$ \\
\hline \multicolumn{6}{|l|}{ Geschlecht } \\
\hline Männer & 89,8 & $92,9^{* *}$ & $68,9^{* * *}$ & $64,7^{* * *}$ & $52,2^{* *}$ \\
\hline Frauen & 91,2 & $94,8^{* *}$ & $74,2^{* * *}$ & $52,3^{* * *}$ & $55,9^{* *}$ \\
\hline \multicolumn{6}{|l|}{ Alter in Jahren } \\
\hline $20-29$ & 90,7 & $92,9^{* *}$ & $72,8^{*}$ & $61,5^{* * *}$ & $52,0^{* *}$ \\
\hline $30-39$ & 90,3 & $94,8^{* *}$ & $70,2^{*}$ & $55,7^{* * *}$ & $56,1^{* *}$ \\
\hline \multicolumn{6}{|l|}{ Elternschaft } \\
\hline Kinderlose & 90,5 & 93,7 & $72,9^{* *}$ & $61,7^{* * *}$ & 54,1 \\
\hline Eltern & 90,5 & 94,0 & $69,5^{* *}$ & $54,1^{* * *}$ & 53,9 \\
\hline \multicolumn{6}{|l|}{ Bildung } \\
\hline Niedrig & $90,8^{* * *}$ & 93,2 & 72,8 & $65,2^{* * *}$ & $62,0^{* *}$ \\
\hline Mittel & $89,5^{* * *}$ & 93,7 & 71,2 & $59,5^{* * *}$ & $54,0^{* *}$ \\
\hline Hoch & $93,4^{* * *}$ & 94,4 & 71,6 & $54,3^{* * *}$ & $51,3^{* *}$ \\
\hline \multicolumn{6}{|l|}{ Wohnort } \\
\hline West & $89,9^{* *}$ & 93,6 & 71,4 & 58,2 & 53,5 \\
\hline Ost & $92,7^{* *}$ & 94,6 & 71,8 & 60,3 & 56,2 \\
\hline Gesamt & 90,5 & 93,8 & 71,5 & 58,6 & 54,0 \\
\hline
\end{tabular}

Quelle: FLB (2012), gewichtete Daten, eigene Berechnungen

Anm.: Zustimmung zusammengefasst „Stimme sehr zu“ und „Stimme zu“ in \%; Chi²-Test, Signifikanzniveau: ${ }^{* * *} \mathrm{p} \leq 0,001 ;{ }^{* *} \mathrm{p} \leq 0,01 ;{ }^{*} \mathrm{p} \leq 0,05$. 
Die Unterschiede in der Bewertung nach sozialstrukturellen Merkmalen sind schwach ausgeprägt. Deutliche Differenzierungen finden sich vor allem bei der Einschätzung, ob Kinder auf die Partnerschaft stabilisierend wirken. Dies denken Jüngere (20-29 Jahre) häufiger als Ältere (30-39 Jahre), niedriger Gebildete häufiger als höher Gebildete und Kinderlose häufiger als Eltern. Dass man in den Kindern über den Tod hinaus lebt, davon gehen eher die Älteren und diejenigen mit einer niedrigeren Bildung aus.

Versucht man mittels einer Hauptkomponentenanalyse verschiedene Dimensionen der Gründe für Kinder zu finden, dann erhält man nur einen einzigen Faktor. Ein Ergebnis, das auch Meulemann (2007: 49) so findet:

„In modernen Gesellschaften sind also immanenter und instrumenteller Wert zwei Seiten derselben Medaille: des Werts von Kindern.“

Trotzdem kann aufgrund der herausragenden Bedeutung der beiden am häufigsten genannten Items aus Sicht der Leitbildforschung als erster Schluss gezogen werden, dass ein positiv besetztes Leitbild des Kinderhabens existiert, das zum einen die Bereicherung des eigenen Lebens durch Kinder als Bestandteil hat. Hinzu kommt, dass kulturell immer noch die Vorstellung verankert ist, dass man einfach Kinder hat und dies völlig normal ist. Man könnte diese Dimension des Leitbildes als die Normalität, Kinder zu haben, bezeichnen. Das Leitbild selbst bezeichnen wir in Anlehnung an die zweite Dimension als Leitbild der Selbstverständlichkeit, Kinder zu haben.

\subsection{Ideales Alter bei der Geburt des ersten Kindes}

Anhand der demografischen Fakten konnte gezeigt werden, dass das Durchschnittsalter bei der ersten Geburt seit längerem kontinuierlich ansteigt und sich dadurch der reproduktive Lebensabschnitt verkürzt. In der Familienleitbildstudie ist nach dem idealen Alter für Frauen und Männer bei der Geburt des ersten Kindes gefragt worden. Die Ergebnisse zeigen durchaus Überraschendes (vgl. Tabelle 3). Das ideale Gebäralter für Frauen und Männer wird im Alter von 26,95 bzw. 28,90 Jahren gesehen. Das tatsächliche Erstgebäralter im Jahr 2012 wird vom Statistischen Bundesamt mit 29,2 Jahren angegeben und liegt damit deutlich höher als das in der Familienleitbildstudie ermittelte Idealalter. Frauen sehen für sich das ideale Alter bei 27,10 und Männer für sich bei 28,20 Jahren. Frauen bezeichnen dabei das ideale Alter für die Männer mit 29,61 Jahren höher als es die Männer für sich selbst sehen. Es ist anzunehmen, dass damit die Tatsache zum Ausdruck gebracht wird, dass in Paarbeziehungen die Männer im Normalfall älter als die Frauen sind, was unter anderem auch damit zusammenhängt, dass Frauen ältere und damit beruflich etabliertere Partner vorziehen (Buss 1989; Hasenkamp et al. 2005).

Weitere Differenzierungen sind zwischen Eltern und Kinderlosen, nach der Bildung und zwischen West- und Ostdeutschen aufgefunden worden:

- Für Eltern ist das ideale Erstgebäralter niedriger als für Kinderlose. Kinderlose geben für Frauen 27,49 (Eltern: 26,16) und für Männer 29,26 (Eltern: 28,36) Jahre an.

- Mit steigender Bildung steigt das Idealalter für die erste Geburt leicht an. Befragte mit einer niedrigen Bildung sehen als Idealalter 26,29 für Frauen und 28,09 für Männer an. Bei den Hochgebildeten, die ihre Kinder auch tatsächlich später bekommen, sind es 27,72 (Frauen) bzw. 29,49 (Männer) Jahre.

- In den neuen Bundesländern, in denen bis vor einigen Jahren die Kinder noch sehr früh geboren wurden, wird das Idealalter für Frauen (26,28 Jahre) und für Männer (27,96 Jahre) niedriger beziffert als im früheren Bundesgebiet (Frauen: 27,12, Männer: 29,14). 
Tabelle 3: Ideales Alter bei der Geburt des ersten Kindes für Frauen und Männer

\begin{tabular}{lllll}
\hline & \multicolumn{2}{c}{ Frauen } & \multicolumn{2}{c}{ Männer } \\
& Mittelwert & Modus & Mittelwert & Modus \\
\hline Geschlecht & & & & \\
Männer & $26,80^{* *}$ & 25 & $28,20^{* * *}$ & 30 \\
Frauen & $27,10^{* *}$ & 25 & $29,61^{* * *}$ & 30 \\
Alter in Jahren & & & $28,72^{* * *}$ & 30 \\
20-29 & 27,00 & 25 & $29,08^{* * *}$ & 30 \\
30-39 & 26,90 & 25 & & \\
Elternschaft & & & $29,26^{* * *}$ & 30 \\
Kinderlose & $27,49^{* * *}$ & 25 & $28,36^{* * *}$ & 30 \\
Eltern & $26,16^{* * *}$ & 25 & & \\
Bildung & & & $28,09^{* *}$ & 30 \\
Niedrig & $26,29^{* * *}$ & 25 & $28,76^{* *}$ & 30 \\
Mittel & $26,74^{* * *}$ & 25 & $29,49^{* *}$ & 30 \\
Hoch & $27,72^{* * *}$ & 30 & & \\
Wohnort & & & $29,14^{* * *}$ & 30 \\
West & $27,12^{* * *}$ & 25 & $27,96^{* * *}$ & 30 \\
Ost & $26,28^{* * *}$ & 25 & 28,90 & 30 \\
\hline Gesamt & 26,95 & 25 &
\end{tabular}

Quelle: FLB (2012), gewichtete Daten, eigene Berechnungen

Anm.: T-Test bzw. ANOVA (Bildung); Signifikanzniveau: ${ }^{* * *} \mathrm{p} \leq 0,001 ;{ }^{* *} \mathrm{p} \leq 0,01 ;{ }^{*} \mathrm{p} \leq 0,05$.

In der prozentualen Verteilung werden weitere Dimensionen des günstigsten Zeitpunktes für die Geburt des ersten Kindes sichtbar und es eröffnet sich eine differenzierte Leitbildperspektive. Erstens ist festzustellen, dass eine sehr frühe Geburt der Kinder nicht erwünscht ist. Nur $0,8 \%$ der Befragten gaben für Frauen und $0,3 \%$ der Befragten für Männer ein Idealalter von unter 20 Jahren für die erste Elternschaft an. Dieses Ergebnis deutet auf ein Leitbild der Ablehnung von Teenagergeburten hin, wovon das ideale Alter zur Familiengründung ein Teil ist. Diesbezüglich ist eine weitgehende Zustimmung zu verzeichnen, die auch offiziell gestützt wird. Das zeigt z. B. eine Stellungnahme der Bundeszentrale für gesundheitliche Aufklärung (2009) gegen Teenagergeburten, in der als Ursache für TeenagerSchwangerschaften, neben mangelndem Wissen und fehlerhafter Anwendung von Verhütungsmitteln, fehlende Berufs- und Zukunftsperspektiven junger Mädchen genannt werden.

Gleichermaßen wie die sehr frühe Geburt wird auch die sehr späte Geburt von Kindern nach dem 35. Lebensjahr nicht als Idealfall angesehen. Mit 1,7\% der Befragten sehen nur sehr wenige das Idealalter für Frauen nach dem 35. Lebensjahr an. Für Männer stellt sich dies etwas anders dar. Es denken 8,8 \%, dass das Idealalter für die erste Vaterschaft nach dem 35. Lebensjahr liegt. Für Frauen wird eindeutig die Altersphase zwischen dem 25. und 29. Lebensjahr als ideal für die Geburt des ersten Kindes angesehen. 62,1\% favorisieren diese Altersgruppe. Für die Männer sind zwei Altersgruppen relevant. 43,1\% sehen das Idealalter zwischen dem 25 . und 29. und 41,8 \% zwischen dem 30. und 34. Lebensjahr. 


\subsection{Bedingungen für die Geburt von Kindern}

Die Bedingungen, die erfüllt sein müssen, bevor die Entscheidung für die Geburt eines Kindes getroffen wird, sind über drei Items abgebildet worden (vgl. Tabelle 4). Eindeutig die höchste Zustimmung hat die Aussage erreicht, dass genügend Geld vorhanden sein muss. 78,8 \% sind persönlich dieser Meinung und 86,8 \% gehen davon aus, dass die Allgemeinheit dies ebenso sieht. Ebenfalls relativ wichtig ist den Befragten, dass die Frau unabhängig von der Erwerbssituation des Partners im Beruf Fuß gefasst haben muss. In der persönlichen Sicht sind es 60,4\%, die dem voll und ganz bzw. eher zustimmen. Wird die Frage aus der Perspektive der gesellschaftlichen Allgemeinheit beantwortet, stimmen dem nur noch 51,6 \% zu. Hier wird unterstellt, dass die Mehrheit noch immer traditioneller eingestellt ist und damit die gesicherte berufliche Position der Frau nicht so wichtig ist, da es den männlichen Familienernährer gibt. Die Ehe als Voraussetzung für Kinder ist aus der individuellen Perspektive unbedeutend. Nur 16,3\% stimmen dem zu. Auffällig ist der große Unterschied zur allgemeinen Sicht, von der zu 46,5 \% angenommen wird, dass Ehe eine wichtige Voraussetzung ist, um Kinder zu bekommen. Auch hier ist wieder das Phänomen zu beobachten, wonach in der Gesellschaft traditionellere Leitbilder vermutet werden, als man sie selbst besitzt.

Die Zustimmungswerte zu den einzelnen Items sind vornehmlich auf der individuellen Ebene, die nachfolgend ausschließlich betrachtet wird, deutlich differenziert. Hinsichtlich des Items „Es muss genügend Geld da sein“ bestehen Unterschiede nach Altersgruppen und Elternschaft. Jüngere (Altersgruppe 20-29 Jahre) und Kinderlose stimmen dieser Aussage deutlich häufiger zu als die jeweiligen Vergleichsgruppen (vgl. Tabelle 4). Die Voraussetzung, dass die Frau im Beruf Fuß gefasst haben muss, zeigt starke Differenzierungen nach Geschlecht und Bildung. Frauen stimmen dem viel häufiger zu als Männer und die Hochgebildeten sind öfter dieser Meinung als die Niedriggebildeten. Schwächere Zusammenhänge bestehen nach Elternschaft und Alter. Es sind die Kinderlosen und die Jüngeren, die stärker zustimmen. Bei der Bedingung, verheiratet sein“ wurde nur ein Bildungsunterschied festgestellt. Diejenigen mit einem niedrigeren Bildungsabschluss sehen die Ehe öfter als Voraussetzung für die Geburt eines Kindes an als diejenigen in den Bildungsgruppen mittel und hoch.

Aus den drei ausgewählten Items lassen sich keine verschiedenen Dimensionen ableiten, aber aufgrund der sehr hohen Bedeutung der monetären Bedingungen und auch der gesicherten beruflichen Stellung der Frau als Voraussetzungen für die Geburt eines Kindes, die hoch miteinander korrelieren, ${ }^{4}$ kann auch hier die Existenz eines Leitbildes der materiell gesicherten Familiengründung angenommen werden. Das Ergebnis passt zu dem Leitbild der verantworteten Elternschaft (siehe Ruckdeschel, Kap. 12). Die Entscheidung für die Geburt von Kindern fällt erst dann, wenn die Umstände als günstig eingeschätzt und dem Kind eine gesicherte Perspektive geboten werden kann.

$4 \quad$ Kendalls-Tau-b ,301; Signifikanzniveau: ,000. 
Tabelle 4: Bedingungen, die erfüllt sein müssen, um Kinder bekommen zu können

\begin{tabular}{|c|c|c|c|c|c|c|}
\hline & \multicolumn{2}{|c|}{$\begin{array}{c}\text { Das Paar muss } \\
\text { verheiratet sein. }\end{array}$} & \multicolumn{2}{|c|}{$\begin{array}{l}\text { Es muss genügend } \\
\text { Geld da sein. }\end{array}$} & \multicolumn{2}{|c|}{$\begin{array}{l}\text { Die Frau muss im Beruf } \\
\text { Fuß gefasst haben, } \\
\text { unabhängig davon, ob } \\
\text { ihr Partner arbeiten geht. }\end{array}$} \\
\hline & Pers. & Allg. & Pers. & Allg. & Pers. & Allg. \\
\hline \multicolumn{7}{|l|}{ Geschlecht } \\
\hline Männer & $17,4^{*}$ & 47,5 & 79,1 & 87,0 & $51,1^{* * *}$ & $48,8^{* * *}$ \\
\hline Frauen & $15,2^{*}$ & 45,4 & 78,6 & 86,6 & $69,9^{* * *}$ & $54,5^{* * *}$ \\
\hline \multicolumn{7}{|l|}{ Alter in Jahren } \\
\hline 20-29 & $17,8^{* *}$ & 47,8 & $84,7^{* * *}$ & $87,7^{*}$ & $62,7^{* *}$ & 50,9 \\
\hline $30-39$ & $14,8^{* *}$ & 45,1 & $72,9^{* * *}$ & $85,8^{*}$ & $58,1^{* *}$ & 52,3 \\
\hline \multicolumn{7}{|l|}{ Elternschaft } \\
\hline Kinderlose & 16,4 & $50,7^{* * *}$ & $85,3^{* * *}$ & 87,2 & $62,3^{* *}$ & $46,7^{* * *}$ \\
\hline Eltern & 16,2 & $40,1^{* * *}$ & $69,2^{* * *}$ & 86,2 & $57,7^{* *}$ & $58,9^{* * *}$ \\
\hline \multicolumn{7}{|l|}{ Bildung } \\
\hline Niedrig & $25,5^{* * *}$ & $53,0^{* *}$ & 78,4 & 86,2 & $53,9^{*}$ & $64,2^{* * *}$ \\
\hline Mittel & $16,8^{* * *}$ & $44,8^{* *}$ & 79,5 & 86,9 & $60,5^{*}$ & $54,1^{* * *}$ \\
\hline Hoch & $13,0^{* * *}$ & $49,1^{* *}$ & 77,7 & 86,6 & $62,1^{*}$ & $41,7^{* * *}$ \\
\hline \multicolumn{7}{|l|}{ Wohnort } \\
\hline West & $17,9^{* * *}$ & $48,5^{* * *}$ & $79,8^{* *}$ & 86,8 & 60,4 & 51,9 \\
\hline Ost & $10,1^{* * *}$ & $38,3^{* * *}$ & $75,1^{* *}$ & 86,6 & 60,5 & 50,4 \\
\hline Gesamt & 16,3 & 46,5 & 78,8 & 86,8 & 60,4 & 51,6 \\
\hline
\end{tabular}

Quelle: FLB (2012), gewichtete Daten, eigene Berechnungen

Anm.: Zustimmung zusammengefasst „Stimme sehr zu“ und „Stimme zu“ in \%; Chi²-Test, Signifikanzniveau: ${ }^{* * *} \mathrm{p} \leq 0,001 ;{ }^{* *} \mathrm{p} \leq 0,01 ;{ }^{*} \mathrm{p} \leq 0,05$. Pers.: Persönliche Sicht; Allg.: Wahrgenommene Sicht in der Gesellschaft.

\subsection{Familiengründungstypen}

Abschließend wird eine Typisierung vorgestellt, die von den vorherigen empirischen Analysen ausgeht. Einbezogen werden die Bedingungen, die in den Augen der Befragten erfüllt sein müssen, damit ein Kind geboren werden kann. Anhand der Items des Leitbildes der materiell gesicherten Elternschaft „es muss genügend Geld vorhanden sein“ und „die Frau muss im Beruf Fuß gefasst haben“", wurden vier Anspruchstypen gebildet und hinsichtlich ihrer soziodemografischen Merkmale, aber auch hinsichtlich ihres Kinderwunsches bzw. Kinderwunschleitbildes (vgl. Abschnitt 2.2) und ihres Idealalters für die Familiengründung verglichen. Dies geschieht im vorliegenden Beitrag auf rein deskriptiver Ebene (vgl. Tabelle 5).

1. Anspruchsvolle: Zum anspruchsvollen Typus, für den vor der Familiengründung sowohl die finanzielle Situation gesichert als auch die berufliche Etablierung der Frau erfolgt sein muss, gehören über $50 \%$ der Befragten. Die Gruppe zeichnet sich durch viele Frauen, überwiegend Jüngere und Kinderlose aus. Entsprechend den hohen Ansprüchen hat diese Gruppe von allen Befragten den niedrigsten durchschnittlichen Kinderwunsch. Ebenfalls in Übereinstimmung mit den vielen Bedingungen, die vor einer Familien- 
gründung erfüllt sein müssen, nennt diese Gruppe sowohl für Frauen als auch für Männer jeweils das höchste Idealalter zur Familiengründung (27,26 Jahre für Frauen bzw. 29,30 Jahre für Männer).

2. Anspruchsvoll-Pragmatische: Der anspruchsvoll-pragmatische Typus umfasst ein gutes Viertel $(26,9 \%)$ der 20- bis 39-Jährigen. Anspruchsvoll-Pragmatische unterscheiden sich vom rein anspruchsvollen Typ vor allem dadurch, dass kein besonderer Wert auf die berufliche Etablierung der Frau vor der Familiengründung gelegt wird. Dies kann auf zwei mögliche Ursachen zurückzuführen sein: Einmal könnte es sich um eine pragmatische Herangehensweise an die Familiengründung in dem Sinne handeln, dass es egal ist, wer von beiden Partnern für die finanzielle Sicherung der Familie sorgt - danach ist der Typ auch benannt. Es könnte sich aber auch um eine eher traditionelle Sichtweise handeln, die ausschließlich dem Mann die Rolle des Familienernährers zuschreibt. Es ist davon auszugehen, dass der anspruchsvoll-pragmatische Typ beide Gruppen umfasst, aufgrund der Datenlage ist aber eine weitergehende Differenzierung an dieser Stelle nicht möglich. Es kann jedoch festgehalten werden, dass dieser Typ sich vom anspruchsvollen vor allem darin unterscheidet, dass ihm signifikant mehr Männer angehören. Ansonsten zeichnet auch er sich durch eher Jüngere und mehr Kinderlose aus. Der Kinderwunsch ist etwas höher als bei den Anspruchsvollen und das jeweilige Idealfamiliengründungsalter für Männer und Frauen etwas niedriger.

3. Gelassen-Egalitäre: Der dritte Typus wurde als gelassen-egalitär bezeichnet, weil die finanzielle Absicherung der Familiengründung als weniger wichtig erachtet wird, gleichzeitig aber die berufliche Etablierung der Frau in den Fokus gerückt wird. In dieser Gruppe, die mit 8,4\% die kleinste darstellt, finden sich sehr viele Frauen, eher Ältere, überwiegend Eltern und höher Gebildete. Im Gegensatz zu den anderen beiden Gruppen lassen sich auch signifikante Ost-West-Unterschiede ausmachen, d. h. man findet häufiger Befragte aus den neuen Bundesländern. Mit den sinkenden finanziellen Anforderungen steigt die durchschnittlich gewünschte Kinderzahl auf 2,2 Kinder, während das ideale Familiengründungsalter bei 27,04 Jahren für Frauen und 29,03 Jahren für Männer liegt. Beide Angaben zum Familiengründungsalter liegen zwischen Anspruchsvollen und Anspruchsvoll-Praktischen, was insofern stimmig ist, als weniger Voraussetzungen eine frühere Familiengründung ermöglichen, eine berufliche Etablierung der Frau aber eine Familiengründung wiederum hinauszögern kann.

4. Gelassen: Der vierte und letzte Typus wurde als gelassen umschrieben, was die niedrigen Anforderungen sowohl an die finanzielle Absicherung als auch an die berufliche Etablierung der Frau vor einer Familiengründung ausdrücken soll. Mit 12,7\% ist er stärker vertreten als die Gelassen-Egalitären, wobei er sich durch mehr Männer, mehr Eltern und eher Ältere auszeichnet. Der gelassene Familiengründungstyp weist den höchsten Kinderwunsch auf, kombiniert mit dem jeweils niedrigsten Idealalter sowohl für Männer als auch für Frauen.

Die Häufigkeit des anspruchsvollen Familiengründungstyps' bestätigt die Existenz eines Mehrphasen-Modells, in dem vor der Familiengründung eine Phase der beruflichen und finanziellen Etablierung kommt (vgl. Abschnitt 2.2), was am stärksten dem Leitbild der materiell gesicherten Elternschaft entspricht. Über die Hälfte der Befragten folgt diesem „Wenn-dann“ Modell, das entsprechend anfällig für Verzögerungen ist. Dies gilt umso mehr, als in den Augen der Befragten nicht nur eine, sondern zwei Partner beruflich etabliert sein sollten. Im Kinderwunsch scheint die durch diese Ansprüche verkürzte Phase der 
Familiengründung und -erweiterung schon mit berücksichtigt zu sein, da gerade in dieser Gruppe das Idealalter zur Familiengründung am höchsten und die gewünschte Kinderzahl am niedrigsten ist. Sobald die Fokussierung auf die berufliche Etablierung der Frau wegfällt, sinkt das Idealalter und steigt die gewünschte Kinderzahl, allerdings nur in beschränktem Ausmaß. Erst eine relative Unabhängigkeit der Familienplanung von der finanziellen Absicherung führt zu signifikant höheren gewünschten Kinderzahlen und zu einem niedrigeren Idealalter. Dabei spielt die berufliche Etablierung der Frau für die gewünschte Kinderzahl keine herausragende Rolle, wohl aber für das Idealalter zur Familiengründung. Dieses erhöht sich mit den Ansprüchen an einen erfolgreichen Berufsstart, d. h. die Befragten sind der Meinung, dass dies nicht gleichzeitig, sondern nacheinander erfolgen sollte. Interessant ist, dass das Leitbild der materiell gesicherten Elternschaft besonders für die Kinderlosen relevant zu sein scheint. Vor allem Kinderlose gehören zu den beiden anspruchsvollen Gruppen, während Eltern eher bei den Gelassenen zu finden sind. Aufgrund des Querschnittdesigns des Fragebogens lässt sich an dieser Stelle die Frage nicht beantworten, ob dies ein Erfahrungseffekt ist oder ob Gelassene eher Eltern werden, was beides möglich ist.

Tabelle 5: Familiengründungstypen nach sozialstrukturellen Merkmalen (in Prozent)

\begin{tabular}{lcccrr}
\hline & \multicolumn{4}{c}{ Familiengründungstypen } \\
& $\begin{array}{c}\text { Anspruchs- } \\
\text { voll }\end{array}$ & $\begin{array}{c}\text { Anspruchsvoll } \\
\text { Pragmatisch }\end{array}$ & $\begin{array}{c}\text { Gelassen } \\
\text { Egalitär }\end{array}$ & Gelassen & Gesamt \\
\hline Anteile der jeweiligen Gruppe am Typ & & & & \\
Frauen & $56,9^{* * *}$ & $34,4^{* * *}$ & $59,5^{* * *}$ & $44,2^{* * *}$ & 49,5 \\
20- bis 29-Jährige & $54,9^{* * *}$ & $52,5^{* * *}$ & $35,7^{* * *}$ & $37,0^{* * *}$ & 50,4 \\
Eltern & $35,2^{* * *}$ & $35,8^{* * *}$ & $58,1^{* * *}$ & $58,7^{* * *}$ & 40,3 \\
Hohe Bildung & $26,8^{*}$ & $25,3^{*}$ & $31,9^{*}$ & $26,2^{*}$ & 26,7 \\
West & $80,5^{* *}$ & $80,9^{* *}$ & $74,2^{* *}$ & $77,1^{* *}$ & 79,7 \\
Kinderwunsch & $1,94^{* * *}$ & $2,05^{* * *}$ & $2,20^{* * *}$ & $2,33^{* * *}$ & 2,04 \\
Idealalter Familiengründung Frauen & $27,26^{* * *}$ & $26,64^{* * *}$ & $27,04^{* * *}$ & $26,24^{* * *}$ & 26,95 \\
Idealalter Familiengründung Männer & $29,30^{* * *}$ & $28,47^{* * *}$ & $29,03^{* * *}$ & $27,99^{* * *}$ & 28,89 \\
\hline Gesamt & 52,1 & 26,9 & 8,4 & 12,7 & 100 \\
N & 2.594 & 1.338 & 417 & 634 & 4.982 \\
\hline
\end{tabular}

Quelle: FLB (2012), gewichtete Daten, eigene Berechnungen

Anm.: ${ }^{a}$ Die Differenz zu 100 \% entfällt auf die jeweilige Referenzgruppe Männer, (30- bis 39-Jährige, Kinderlose, niedrig bzw. mittel Qualifizierte und Ost); Signifikante Differenzen: Chi²-Test für Anteile, ANOVA für Mittelwerte, Signifikanzniveau: ${ }^{* * *} \mathrm{p} \leq 0,001 ;{ }^{* *} \mathrm{p} \leq 0,01 ;{ }^{*} \mathrm{p} \leq 0,05$.

\section{$4 \quad$ Diskussion und Fazit}

Am Anfang des Fazits möchten wir ein Ergebnis herausstellen, das auf den ersten Blick banal erscheinen mag. In den Analysen zu den Gründen, warum man Kinder bekommt, hat sich der Grund „weil Kinder einfach zum Leben dazu gehören“ als außerordentlich bedeutsam herausgestellt. Ausgehend von dieser Normalität Kinder zu haben ist zusammen mit der Aussage, dass Kinder das Leben bereichern, ein positiv besetztes „Leitbild der Selbstverständlichkeit des Kinderhabens“ formuliert worden. Dieses Ergebnis ist insofern hervor- 
zuheben, da im Kontext der Diskussion um gewollte Kinderlosigkeit, Deinstitutionalisierung der Familie und dem Entstehen familienalternativer Lebensformen bei jüngeren Frauen und Männern eine allgemeine positive Kinderorientierung gemessen wird. Allerdings bleibt ein Anteil von ca. $10 \%$ der Befragten, die keine Kinder haben und keine Kinder möchten, also bewusst kinderlos bleiben wollen.

Diesem Ergebnis entsprechen die gemessenen Kinderwünsche größer zwei. Allerdings ist zu beachten, dass in der Fragestellung kein Bezug zu den aktuellen Lebensumständen hergestellt wurde. Bestätigt wurde mit den erzielten Resultaten, dass seit der Jahrtausendwende ein Wiederanstieg der Kinderwünsche zu beobachten ist. Auffällig ist, dass die Paritätsstruktur der Kinderwünsche von der realen am Ende des gebärfähigen Alters abweicht. Niedrige Paritäten werden häufiger realisiert als gewünscht, während höhere Paritäten häufiger nur Wunsch bleiben.

Was das ideale Alter bei der Erstgeburt anbelangt, werden frühe und späte Geburten nur von ganz Wenigen als Idealalter angesehen. Hervorzuheben ist an dieser Stelle, dass das durchschnittlich angegebene Idealalter niedriger ist als das tatsächliche Erstgebäralter. Diejenigen, die es als ideal ansehen, ihre Kinder früher zu bekommen, wünschen sich für ihre Familie auch mehr Kinder.

Bei der Entscheidung für Kinder wurden zwei Leitbilder aufgefunden. Einerseits wurde als wichtigste Bedingung für die Erfüllung des Kinderwunsches das Vorhandensein von genügend Geld eingestuft, dieses Ergebnis führte zur Formulierung des Leitbildes der materiell gesicherten Familiengründung. Über den Bezug zum Kinderwunsch konnte ermittelt werden, dass hohe Ansprüche an die Bedingungen für die Geburt eines Kindes die als ideal angesehene Kinderzahl begrenzen. Ob davon ein Einfluss auf das generative Verhalten ausgeht, kann an dieser Stelle nicht beurteilt werden. Andererseits ist bei den Gründen für Kinder einem Item am häufigsten zugestimmt worden, das einen immanenten Nutzen zum Ausdruck bringt. Kinder werden gewünscht, weil das Leben durch Kinder bunter und vielfältiger wird. Eltern müssen sich dem stellen und sich weiterentwickeln. Insgesamt lassen sich vier Familiengründungstypen unterscheiden: die Anspruchsvollen, die AnspruchsvollPragmatischen, die Gelassenen und die Gelassen-Egalitären. Diese sind einerseits nach ihren Ansprüchen an eine materielle Sicherung der Familiengründung zu unterscheiden und andererseits nach der Bedeutung, die der beruflichen Eigenständigkeit der Frau zugeschrieben wird. Während die materiellen Ansprüche den Kinderwunsch direkt senken, geschieht dies bei der Forderung nach beruflicher Eigenständigkeit der Frau vermittelt über ein höheres ideales Familiengründungsalter, das wiederum den Kinderwunsch senkt. Eine gesicherte Aussage über derartige Zusammenhänge können aber erst multivariate Analysen bieten.

Als Fazit lässt sich an dieser Stelle festhalten, dass Kinderhaben durchaus als eine Selbstverständlichkeit angesehen und auch gewünscht wird. Allerdings kann diese Lebensorientierung in den Augen der jungen Deutschen nicht einfach in die Tat umgesetzt werden, sondern ist an vielfältige Bedingungen geknüpft, die dafür erfüllt sein müssen. Dabei gilt, je höher die Ansprüche sind, umso niedriger fällt der als möglich angesehene Kinderwunsch aus. Da vor allem Kinderlose diese hohen Ansprüche haben, sollten an dieser Stelle finanzielle Hürden verringert und die Vereinbarkeit von Familie und Beruf erhöht werden. Dem Umstand folgend, dass bei Familien mit mehreren Kindern die aktive fertile Lebensphase früher beginnt und nicht etwa später endet, eröffnet sich somit eine familienpolitische Option. Die Möglichkeit, schon zwischen dem 25. und 30. Lebensjahr materiell gesichert eine Familie gründen zu können, könnte sich positiv auf die Geburtenrate auswirken. Schließlich könnte beispielsweise auf gesellschaftlicher Ebene die Botschaft stärker vertreten werden, dass eine Familiengründung weit weniger Voraussetzungen braucht, als häufig angenommen wird. 


\section{Literatur}

Ajzen, Icek (1985): From Intensions to Actions: A Theory of Planned Behavior. In: Kuhi, Julius; Beckmann, Jürgen (Hrsg.): Action Control: From Cognition to Behavior. Heidelberg: Springer, S. 11-39.

Ajzen, Icek; Klobas, Jane (2013): Fertility intentions: An approach based on the theory of planned behavior. In: Demographic Research, 29, S. 203-232.

Berntsen, Dorthe; Rubin, David C. (2002): Emotionally charged autobiographical memories across the lifespan: The recall of happy, sad, traumatic and involuntary memories. In: Psychology and Aging, 17, S. 636-652.

Bertram, Hans (2012): Keine Zeit für Liebe - oder: Die Rushhour des Lebens als Überforderung der nachwachsenden Generation? In: Bertram, Hans; Bujard, Martin (Hrsg.): Zeit, Geld, Infrastruktur - zur Zukunft der Familienpolitik. Soziale Welt, Sonderband 19, S. 25-44.

Block, Karin; Schmidt, Gunter (2009): Jugendliche Schwangere und ihre Partner. In: Bundeszentrale für gesundheitliche Aufklärung (BZgA) (Hrsg.): Schwangerschaft und Schwangerschaftsabbruch bei minderjährigen Frauen. Köln: Bundeszentrale für gesundheitliche Aufklärung, S. 41-57.

Blossfeld, Hans-Peter; Rohwer, Götz (1995):West Germany. In: Blossfeld, Hans-Peter (Hrsg.): The New Role of Women: Family Formation in Modern Societies. Boulder et al.: Westview Press, S. 56-76.

BMFSFJ (Bundesministerium für Familie, Senioren, Frauen und Jugend) (Hrsg.) (2005): Das subjektive Zeitfenster für die Elternschaft. Eine Repräsentativbefragung der Bevölkerung zwischen 16 und 44 Jahren. Allensbach: Institut für Demoskopie Allensbach.

Bundeszentrale für gesundheitliche Aufklärung (2009): Teenager-Schwangerschaften in Deutschland. Köln: Bundeszentrale für gesundheitliche Aufklärung.

Buss, David M. (2003): The Evolution of Desire. Strategies of Human Mating. Revised edtion 4. New York: Basic Books.

Diabaté, Sabine, Lück, Detlev, (2014): Familienleitbilder - Identifikation und Wirkungsweise auf generatives Verhalten. In: Zeitschrift für Familienforschung, 26, 1, S. 49-69.

Dorbritz, Jürgen (2010): Bilokale Paarbeziehungen. Die Bedeutung und Vielfalt einer Lebensform. In: Zeitschrift für Bevölkerungswissenschaft, 34, S.31-56.

Dorbritz, Jürgen; Naderi, Robert (2013): Trendwende beim Kinderwunsch? In: Bevölkerungsforschung Aktuell, 4, S. 2-7.

Eckhard, Jan; Klein, Thomas (2006): Männer, Kinderwunsch und generatives Verhalten: Eine Auswertung des Familiensurvey zu Geschlechterunterschieden. Wiesbaden: VS Verlag.

Fitzner, Kristin; Klaus, Daniela; Kopp, Johannes; Nauck, Bernhard; Seidel, Jan; Steinbach, Anja; Suckow, Jana (2007): Die Instrumente zur Erfassung der, Value of Children' und der ,intergenerationalen Beziehungen' der 1. und 2. Welle des PAIRFAM Minipanels. Arbeitspapier des DFGSchwerpunktes „Beziehungs- und Familienentwicklungspanel“ [http://www.pairfam.de/uploads/ tx_sibibtex/arbeitspapier_07p.pdf, 01.12.2014].

Fthenakis, Wassilios (1999): Engagierte Vaterschaft. Die sanfte Revolution in der Familie. Opladen: Leske + Budrich.

Hasenkamp, Andrea; Kümmerling, Angelika; Hassebrauck, Mannfred (2005): Blinder Mann sucht schöne Frau? Evolutionspsychologische und soziokulturelle Erklärungsansätze der Partnerwahl. In: Zeitschrift für Sozialpsychologie, 36, S. 77-89.

Höhn, Charlotte, Ette, Andreas, Ruckdeschel, Kerstin (2006): Kinderwünsche in Deutschland. Konsequenzen für eine nachhaltige Familienpolitik, Stuttgart: Robert-Bosch-Stiftung.

Höhn, Charlotte; Avramov, Dragana; Kotowska, Irena E. (Hrsg.) (2008): People, Population Change and Policies. Volume 1 und 2. Dordrecht: Springer.

Hoffman, Lois W.; Hoffman, Martin L. (1973): The Value of Children to Parents. In: Fawcett, James T. (Hrsg): Psychological Perspektives on Population. New York: Basic Books, S. 19-76. 
Huinink, Johannes (1995a): Familienentwicklung und Haushaltsgründung in der DDR: Vom traditionellen Muster zur instrumentellen Lebensplanung? In: Nauck, Bernhard; Schneider, Norbert S.; Tölke, Angelika (Hrsg.): Familie und Lebensverlauf im gesellschaftlichen Umbruch. Stuttgart: Enke, S. 39-55.

Huinink, Johannes (1995b): Warum noch Familie? Zur Attraktivität von Partnerschaft und Elternschaft in unserer Gesellschaft. Frankfurt/M: Campus Verlag.

Huinink, Johannes; Konietzka, Dirk (2007): Familiensoziologie. Eine Einführung. Frankfurt/New York: Campus Verlag.

Janssen, Steve M. J.; Rubin, David C. (2011): Age Effects in Cultural Life Scripts. In: Applied Cognitive Psychology, 25, S. 291-298.

Kaufmann, Franz-Xaver (1990): Zukunft der Familie. Stabilität, Stabilitätsrisiken und Wandel der familialen Lebensformen sowie ihre gesellschaftlichen und politischen Bedingungen. Perspektiven und Orientierungen. Schriftenreihe des Bundeskanzleramtes 10. München: Beck.

Klein, Thomas; Eckhard, Jan (2006): Männer, Kinderwunsch und generatives Verhalten. Eine Auswertung des Familiensurvey zu Geschlechterunterschieden in der Motivation zur Elternschaft. Wiesbaden: VS Verlag für Sozialwissenschaften.

Konietzka, Dirk; Kreyenfeld, Michaela (Hrsg.) (2013): Ein Leben ohne Kinder. Ausmaß, Strukturen und Ursachen von Kinderlosigkeit. 2. Auflage. Wiesbaden: Springer VS.

Meulemann, Heiner (2007): Sind Kinder den Deutschen weniger wert geworden? Entwicklungen zwischen 1979 und 2005 in West- und Ostdeutschland. In: Soziale Welt, 58, 29-52.

Miller, Warren B. (2011): Differences between fertility desires and intentions: implications for theory, research, and policy. In: Vienna Yearbook of Population Research, 9, S. 75-98.

Nauck, Bernhard (2001): Der Wert von Kindern für ihre Eltern. In: Kölner Zeitschrift für Soziologie und Sozialpsychologie, 53, S. 407-435.

Neugarten, Bernice L.; Moore, Joan W.; Lowe, John C. (1965). Age norms, age constraints, and adult socialization. In: American Journal of Sociology, 70, S. 710-717.

PAIRFAM (2008-2022): Beziehungs- und Familienpanel - Panel Analysis of Intimate Relationships and Family Dynamics. [http://PAIRFAM.de/]

Peukert, Rüdiger (2008): Familienformen im sozialen Wandel. Wiesbaden: VS Verlag für Sozialwissenschaften.

Philipov, Dimiter; Bernardi, Laura (2011): Concepts and Operationalisation of Reproductive Decisions Implementation in Austria, Germany and Switzerland. In: Comparative Population Studies, 36, S. 495-530.

Ruckdeschel, Kerstin (2007): Der Kinderwunsch von Kinderlosen. In: Zeitschrift für Familienforschung, 19, S. 210-230.

Ruckdeschel, Kerstin; Ette, Andreas; Hullen, Gerd; Leven, Ingo (2006): Generations and Gender Survey. Dokumentation der ersten Welle der Hauptbefragung in Deutschland. Materialien zur Bevölkerungsforschung 121a. Wiesbaden: Bundesinstitut für Bevölkerungsforschung.

Rubin, David C.; Berntsen Dorthe (2003): Life scripts help to maintain memoires of highly positive, but not highly negative, events. In: Memory and Cognitio, 31, S. 1-14.

Rupp, Marina (2005): Kinderlosigkeit in stabilen Ehen. In: Zeitschrift für Familienforschung, 17, S. 21-40.

Schaeper, Hildegard (2013). Familiengründung von Hochschulabsolventinnen. Eine empirische Untersuchung verschiedener Examenskohorten. In: Kreyenfeld, Michaela; Konietzka, Dirk (Hrsg.): Ein Leben ohne Kinder. Ausmaß, Strukturen und Ursachen von Kinderlosigkeit. 2. Auflage. Wiesbaden: Springer VS, S. 47-80.

Schmitt, Christian (2004): Kinderlose Männer in Deutschland - Eine sozialstrukturelle Bestimmung auf der Basis des Sozio-oekonomischen Panels (SOEP). DIW Materialien 34 Berlin: DIW.

Schmitt, Christian; Winkelmann, Ulrike (2005): Wer bleibt kinderlos? Sozialstrukturelle Daten zur Kinderlosigkeit von Frauen und Männern. DIW-Diskussionspapier 473. Berlin: DIW.

Schneider, Norbert F., Dorbritz, Jürgen (2011): Wo bleiben die Kinder? Der niedrigen Geburtenrate auf der Spur. In: Politik und Zeitgeschichte, 10-11, S. 26-34.

Schröder, Jette; Brüderl, Josef (2008): Der Effekt der Erwerbstätigkeit von Frauen auf die Fertilität: Kausalität oder Selbstselektion? In: Zeitschrift für Soziologie, 37, S. 117-136. 
Statistisches Bundesamt (2014): 682000 Kinder kamen im Jahr 2013 zur Welt. Pressemitteilung vom 8.12.2014. Wiesbaden: Statistisches Bundesamt.

Statistisches Bundesamt (Hrsg.) (2007): Geburten in Deutschland. Wiesbaden: Statistisches Bundesamt. Statistisches Bundesamt (Hrsg.) (2013): Geburtentrends und Familiensituation in Deutschland. Wiesbaden: Statistisches Bundesamt.

Tölke, Angelika; Diewald, Martin (2003): Berufsbiographische Unsicherheiten und der Übergang zur Elternschaft bei Männern. In: Bien, Walter; Marbach, Jan H. (Hrsg.): Partnerschaft und Familiengründung. Ergebnisse der dritten Welle des Familien-Survey. Opladen: Leske + Budrich, S. 349-384.

Trommsdorf, Gisela; Nauck, Bernhard (Hrsg.) (2005): The Value of Children in Cross-Cultural Perspective. Case Studies From Eight Societies. Lengerich et al.: Pabst.

Zerle, Claudia; Cornelißen, Waltraud; Bien, Walter (2012): Das Timing der Familiengründung und dessen Folgen für Familien. In: Zeitschrift für Familienforschung, 24, S. 46-66. 


\title{
Mütter in Ost- und Westdeutschland: Wie wichtig sind regionalspezifische Leitbilder für Elternschaft?
}

\author{
Katrin Schiefer \& Robert Naderi ${ }^{*}$
}

\section{Zusammenfassung}

Wie die Forschungsergebnisse und Zahlen zum generativen Verhalten belegen, lassen sich nach wie vor Unterschiede zwischen Ost- und Westdeutschen identifizieren - die allerdings nicht zwangsläufig nur auf die unterschiedliche Regimetradition zurückgeführt werden können. Es stellt sich deshalb die Frage, welche Faktoren hier zum Tragen kommen. Es wird vermutet, dass neben sozioökomischen Rahmenbedingungen auch die kulturelle Prägung der Bürger eine Rolle spielt.

Ziel dieses Beitrages ist es, den kulturellen Verankerungen anhand der jeweiligen Leitbilder zur Bedeutung eigener Kinder sowie zu vermuteten Anforderungen an eine Elternschaft nachzugehen und diese dabei mit anderen relevanten Variablen zur Sozialisation, zur Partnerschaft und zur sozioökonomischen Lage zu vergleichen. Hierzu wird die Kinderlosigkeit im Verhältnis zur Elternschaft als besonders auffällige Merkmale der Fertilitätsunterschiede zwischen alten und neuen Bundesländern betrachtet. Erstere ist im Westen nach wie vor stärker ausgeprägt, die TFR ist aber insgesamt in beiden Regionen nahezu gleich niedrig.

In Bezug auf die betrachteten Facetten von Leitbildern zeigen sich v. a. bei der Wichtigkeit eigener Kinder sowie der Kleinkindbetreuung erhebliche Ost-West-Unterschiede. Mittels logistischer Regressionsmodelle wird untersucht, welchen Einfluss die familienbezogenen Leitbilder im Vergleich zu anderen Faktoren auf eine Elternschaft haben. Die Ergebnisse belegen u. a., dass Leitbilder im Westen Deutschlands eine in etwa gleich hohe Erklärungskraft aufweisen wie individuelle, sozioökonomische Rahmenbedingungen - im Osten sind sie wichtiger als alle anderen möglichen Faktoren.

\section{$1 \quad$ Einleitung}

Seit vielen Jahren gehört Deutschland bereits zu den Niedrigfertilitätsländern; im europäischen Vergleich rangiert es u. a. zusammen mit Portugal $(1,28)$, Polen $(1,30)$, Spanien $(1,32)$ und Ungarn $(1,34)$ auf den hinteren Rängen des Fertilitätsspektrums (BiB 2012). Bei einer genaueren Analyse der Geburtenrate in Deutschland wird deutlich, dass hier nicht nur verhältnismäßig wenige Kinder geboren werden, sondern dass auch erhebliche regionale Unterschiede bestehen (siehe zu dieser Thematik auch Bujard et al. 2012). Das Geburtenniveau fällt zwar deutschlandweit niedrig aus, die dahinter liegenden Muster sind allerdings nicht in allen Regionen gleich. Bei der Suche nach möglichen Erklärungen für dieses

Wir danken den Herausgebern sowie der anonymen Gutachterin bzw. dem anonymen Gutachter für die wertvollen Hinweise und die hilfreiche Kritik. 
Phänomen müssen demnach zwei Aspekte berücksichtigt werden: Zum einen geht es um die niedrige Anzahl an Geburten und deren Begründung, zum anderen stellt sich aber auch die Frage nach der Ursache für die unterschiedlichen Fertilitätsmuster innerhalb Deutschlands.

Betrachtet man Ost- und Westdeutschland getrennt, weisen zwar beide Teile Deutschlands eine ähnlich niedrige zusammengefasste Geburtenziffer auf (1,37 Kinder pro Frau im Westen und 1,42 im Osten im Jahr 2012), dennoch sind die jeweiligen Verteilungen sehr unterschiedlich. So sind z. B. die Paritäten verschieden: Im Westen hat die Kinderlosigkeit stetig zugenommen, dafür gibt es wenige Mütter mit nur einem Kind, die wiederum häufiger im Osten vorkommen (Dorbritz 2010). Mehrkindfamilien (mit drei und mehr Kindern) sind indes häufiger im Westen als im Osten zu finden, werden aber insgesamt immer seltener (BMFSFJ 2013: 13ff.). Bezüglich des Alters bei der Geburt des ersten Kindes lassen sich heute zwar immer noch Unterschiede zwischen Ost und West finden, hier handelt es sich nach unserer Einschätzung aber um einen konvergierenden Trend: Denn mit 29,4 (Westdeutschland) und 27,9 (Ostdeutschland) Jahren im Durchschnitt nähert sich das Erstgeburtsalter in beiden Landesteilen weiter an (Statistisches Bundesamt 2012). Insofern ist dieser Aspekt für ein unterschiedliches generatives Verhalten heute vermutlich weniger von Bedeutung. Es lassen sich aber auch Beispiele für eine gegenteilige Entwicklung finden: So verstärkt sich die Divergenz zwischen Ost- und Westdeutschland z. B. in Bezug auf die Nichtehelichenquote über die Zeit sogar. Im Westen werden Fertilitätsentscheidungen regelmäßig mit der Institutionalisierung der Paarbeziehung verknüpft, im Osten scheint dies nicht so stark zusammenzuhängen (siehe dazu auch Kreyenfeld et al. 2011). Nach über zwei Jahrzehnten der Wiedervereinigung ist die regionale Differenzierung Deutschlands in Ost und West in der bevölkerungswissenschaftlichen und familiensoziologischen Literatur nach wie vor präsent (Krause/Ostner 2010; Goldstein/Kreyenfeld 2011).

Angesichts der gezeigten Entwicklungen stellt sich die Frage, warum sich nach wie vor unterschiedliche Fertilitätsmuster in Ost- und Westdeutschland finden lassen. Warum gibt es scheinbar immer noch typische ostdeutsche und typische westdeutsche Verhaltensweisen, obwohl heute beide Bevölkerungsteile in einem Staat zusammenleben? Welche Rolle spielt in diesem Kontext die kulturelle Prägung der Bürger?

Wir vermuten u. a., dass das Aufwachsen in unterschiedlichen politischen Regimen mit grundlegend verschiedenen Weltanschauungen und unterschiedlichen strukturellen Rahmenbedingungen einen Einfluss auf die Ansichten und das Verhalten der Menschen auch in Bezug auf eine mögliche Elternschaft hat und somit auch zur Erklärung der unterschiedlichen Fertilitätsmuster in Ost und West beiträgt (siehe dazu auch Bauernschuster/Rainer 2012). Dieser Effekt wird zusätzlich durch die Weitergabe von Einstellungen und Werten von Generation zu Generation manifestiert. Auf diesen Aspekt werden wir in Abschnitt 2 noch genauer eingehen. Als empirische Annäherung wird die Bündelung spezifischer Vorstellungen und Ansichten (Leitbilder) als möglicher Haupterklärungsfaktor betrachtet. Analysiert wird dabei der Zusammenhang zwischen familienbezogenen Leitbildern und Elternschaft - unter Kontrolle wesentlicher sozioökonomischer Merkmale, welche theoretisch in beiden Teilen Deutschlands von unterschiedlicher Bedeutung für die Familiengründung und -erweiterung sein könnten. Es handelt sich um einen Vergleich zwischen kinderlosen Frauen und Müttern, weil hier eine nach wie vor auffällige regionale Unterschiedlichkeit vorliegt. In unseren Analysen konzentrieren wir uns ausschließlich auf Frauen, weil die Datenlage zur Elternschaft bei Männern im Vergleich wesentlich schlechter ausfällt.

1 Bei Männern kommen bei der Erklärung einer Elternschaft andere Faktoren zum Tragen, der Unterschied zwischen Mann und Frau steht hier nicht im Fokus. 
Im Folgenden werden wir zunächst einen Überblick über den aktuellen Forschungsstand geben. Danach werden die theoretischen Grundlagen unserer Analysen dargelegt und unsere Annahmen formuliert. Es folgt eine kurze Beschreibung der verwendeten Daten und ein ausführlicher Abschnitt zur methodischen Vorgehensweise sowie eine Darstellung der abhängigen und unabhängigen Variablen. Danach gehen wir auf die Ergebnisse ein und diskutieren diese. Am Ende unserer Betrachtungen und Analysen wird schließlich ein Fazit gezogen und ein Ausblick gegeben.

\section{Forschungsstand und theoretische Grundlagen}

Wenn hier von Familienbildungsprozessen gesprochen wird, beziehen wir uns auf die Geburt von Kindern. Wir interessieren uns also für die Einflussgrößen einer Elternschaft. Unsere Analysen basieren auf der Grundannahme, dass gewisse Erwartungen mit der Familiengründung bzw. Elternschaft verknüpft sind. Doch woher kommen diese Erwartungen eigentlich? Wir nehmen an, dass jeder Mensch konkrete Vorstellungen hat, wie eine Familiengründung idealerweise ablaufen sollte und welche Aspekte dabei von zentraler Bedeutung sind (vgl. Dorbritz/Ruckdeschel, Kap. 9). Diese individuellen Vorstellungen und Erwartungen unterscheiden sich innerhalb der Gesellschaft, aber es gibt auch größere Schnittmengen, d. h. Menschen teilen in verschiedenen Bereichen die gleichen Vorstellungen. In diesem Kontext definieren wir Leitbilder als ,,...] Bündel aus kollektiv geteilten bildhaften Vorstellungen des „Normalen“, das heißt von etwas Erstrebenswertem, sozial Erwünschtem und/oder mutmaßlich weit Verbreitetem, also Selbstverständlichem“ (Diabaté/Lück 2014: 56; siehe dazu auch: Giesel 2007). Nach dieser Definition geben Leitbilder wichtige Hinweise auf die kulturelle Prägung einer Gesellschaft und sollten somit einen wichtigen Beitrag zur Erklärung der unterschiedlichen Fertilitätsmuster in Ost und West leisten.

Diese unterschiedlichen Muster haben viele Wissenschaftler beschäftigt: Kurz nach der Wende nahmen Experten noch an, dass sich die neuen Bundesländer allmählich in Richtung der alten Länder entwickeln würden und es zu einer Homogenisierung der Fertilitätsmuster kommen würde (u. a. Pfau-Effinger/Geissler 2002: 78). Über 25 Jahre nach der Wiedervereinigung ist klar, dass das Bild sehr viel differenzierter ausfällt. Schneider et al. 2012 sprechen in diesem Zusammenhang von Hybridisierung, denn wie eingangs bereits dargelegt werden konnte, kommt es in einigen Bereichen nachweislich zur Annäherung (Konvergenz) der demografischen Muster, in anderen Bereichen aber auch zu einer weiteren Etablierung bestehender unterschiedlicher Muster oder sogar $\mathrm{zu}$ sich auseinander bewegenden Trends (Divergenz).

In vielen Studien konnte gezeigt werden, dass diese genannten Unterschiede nicht allein durch sozioökonomische Faktoren erklärt werden können. So beziehen jüngste Studien (Bernardi/Keim 2013; Boehnke 2013; Arránz Becker et al. 2010; Bernardi et al. 2007; Alheit 2005 u. a.) neben strukturellen auch kulturelle Faktoren zur Erklärung der unterschiedlichen familialen Muster in Ost- und Westdeutschland ein.

Bernardi und Keim (2013) setzen der Hypothese der Angleichung einen vermuteten Trägheitseffekt entgegen, danach bestehen die Unterschiede in Ost- und Westdeutschland aufgrund der Sozialisation in unterschiedlichen Regimen fort. Durch die Prägekraft der eigenen Familie werden typische ostdeutsche und typische westdeutsche Verhaltensweisen und Werte auch an die nächste Generation weitergegeben, so dass die alten Werte fortbestehen, obwohl heute alle in einem vereinigten Deutschland zusammenleben (eine ähnliche 
Argumentationslinie findet sich auch bei Bernardi et al. 2007). Die Autoren betonen, dass die Lebensbedingungen zwar für alle Bürger gleich seien, die Bewältigungsstrategien seien allerdings sehr unterschiedlich. Auch Boehnke (2013) stützt sich bei der Erklärung von deutsch-deutschen Unterschieden bei der Kinderlosigkeit von Akademikerinnen auf den Zusammenhang von Struktur und Kultur. Dabei versteht die Autorin unter Kultur v. a. das allgemeine gesellschaftliche Meinungsklima zum Thema Elternschaft. Hier findet Boehnke erhebliche Unterschiede zwischen Ost- und Westdeutschland, auch sie geht davon aus, dass Sozialisationserfahrungen hierbei eine entscheidende Rolle spielen.

In vielen dieser Studien zu den Ursachen der Unterschiede zwischen Ost und West wird allerdings die Datenlage bemängelt, empirische Daten seien nicht detailliert genug, man müsse sich die Informationen häufig aus verschiedenen Studien herausziehen und stoße schnell an Fallzahlprobleme. Die Studie zu Familienleitbildern (FLB 2012) versucht, diese Forschungslücke zu schließen.

Da Einstellungen und Werte von Generation zu Generation (über Sozialisation) weitergegeben werden, sind sie über die Zeit relativ stabil. Huinink et al. (2012) sprechen in diesem Zusammenhang allerdings von unterschiedlichen Dynamiken des sozialen Wandels und differenzieren zwischen einer Kohortensukzession und kurzfristigen Verhaltensänderungen. Dabei lässt die Kohortensukzession auf der einen Seite einen eher langsamen Wandel vermuten, da Werte, Einstellungen und Erfahrungen von der Eltern- an die Kinderkohorte weitergegeben werden und sich dementsprechend eher langfristig verändern. Auf der anderen Seite kann struktureller Wandel auch zu kurzfristigen Verhaltensänderungen führen, wenn sich beispielsweise institutionelle und ökonomische Rahmenbedingungen ändern und dadurch andere Verhaltensweisen nahelegen oder es gar zu einem Systembruch kommt, wie das in der ehemaligen DDR der Fall war. Beide „Meta-Mechanismen“ sind stark interdependent und entwickeln so eigene Geschwindigkeiten, was wiederum den stattfindenden gesellschaftlichen Wandel beeinflusst. Deshalb vermuten wir, dass man innerhalb Deutschlands kein einheitliches Familienleitbild vorfindet, sondern dass unterschiedliche Leitbilder in Ost und West identifiziert werden können (Grundannahme). Diese sollten zur Erklärung der Existenz unterschiedlicher Familienregime in beiden Landesteilen beitragen.

Nach der deutschen Wiedervereinigung Anfang der 1990er Jahre haben sich die Einstellungen der Ostdeutschen - entgegen der Erwartungen - nicht einfach an die der Westdeutschen angepasst, stattdessen haben sie zum Teil eigene Leitbilder verinnerlicht, die nach wie vor das kulturelle Erbe der DDR erahnen lassen. So wird beispielsweise die Bedeutung eigener Kinder stark betont (Hypothese H1) und stellt damit ein wichtiges persönliches Lebensziel für viele ostdeutsche Bürger (insbesondere Frauen) dar (Boehnke 2013). Allerdings geht damit nicht ein Rückzug aus der Arbeitswelt einher, wie dies in Westdeutschland zu beobachten ist. Deshalb gehen wir davon aus, dass die Berufstätigkeit der Frau in Ostdeutschland bei der Entscheidung für die Familiengründung eine untergeordnete Rolle spielt und demnach weniger als Hinderungsgrund eingestuft wird als im Westen Deutschlands. Für ostdeutsche Frauen gehören Kinder genauso zum Leben dazu wie ihre eigene Berufstätigkeit. Wir führen dies darauf zurück, dass die Erwerbstätigkeit der Frau in der ehemaligen DDR aktiv gefördert wurde und nicht in direkter Konkurrenz zur Mutterschaft stand. Durch umfassende Betreuungsmöglichkeiten kam es nicht zu einer Vereinbarkeitsproblematik, die eine Frau möglicherweise vor die Wahl Kind oder Karriere ${ }^{2}$ stellte. Hier hieß das Motto vielmehr: Kind und Karriere (Ruckdeschel 2009: 111-112).

2 Wenn hier von Kind und Karriere gesprochen wird, meinen wir den Vereinbarkeitskonflikt zwischen Elternschaft und Erwerbstätigkeit. 
Außerdem bot die Familie eine Rückzugsmöglichkeit ins Private und damit einen Gegenpol zum ansonsten stark überwachenden Staat. Familie bezieht sich hier vor allem auf die Kernfamilie, also den Partner und die eigenen Kinder (siehe dazu u. a. Stöbel-Richter 2010: 49ff.).

Im Westen sollte sich ein anderes Bild abzeichnen: Hier werden Mütter mit starken normativen gesellschaftlichen Erwartungen konfrontiert. Eine Mutterschaft wird vielfach als Vollzeitjob angesehen, der somit in Konkurrenz zu Karriereambitionen von Frauen tritt. In Kontrast zum Doppelverdienermodell der DDR wurde in der BRD vielmehr das Alleinernährermodell unterstützt und durch politische Maßnahmen gefördert. Nach diesem Modell erfolgt eine klare Aufgabenteilung innerhalb der Familie: Der Vater ist demnach für die materielle Absicherung der Familie zuständig, während die Mutter Kindererziehung und Haushalt managt. Das daraus resultierende stark mutterzentrierte Erziehungsbild scheint nach wie vor in den Köpfen verankert zu sein und wandelt sich erst allmählich, siehe dazu auch Diabaté, Kap. 13. Für das Wohl des Kindes und dessen Entwicklung spielt die Betreuung durch die Mutter sowie ihre Präsenz zu Hause nach wie vor eine wichtige Rolle (Hypothese H2). Vor allem einer Kleinkindbetreuung außerhalb der Familie dürften immer noch viele kritisch gegenüberstehen. So wird Elternschaft zu einer Art Profession, die entsprechende Vorbereitung und Aufopferung erfordert (siehe dazu auch Ruckdeschel, Kap. 12). Wir vermuten deshalb, dass die wahrgenommenen Anforderungen vor allem in Westdeutschland einen negativen Einfluss auf eine Elternschaft haben (Hypothese H3).

\section{Vorgehensweise}

Die aufgestellten Hypothesen H1 bis H3 sollen im Folgenden empirisch überprüft werden. Wie bereits kurz erwähnt wurde, arbeiten wir dazu mit der Familienleitbildstudie (FLB 2012), da diese eine detaillierte Analyse der Vorstellungen von Familie in Ost- und Westdeutschland ermöglicht. Erstmalig wird hier der Versuch unternommen, gesellschaftlich vorherrschende Leitbilder rund um das Thema Familie in einer repräsentativen quantitativen Umfrage zu erfassen. Auch auf der individuellen Ebene wurden neue Einstellungsitems erhoben. Unser Modell enthält neben soziodemografischen Merkmalen auch weitere relevante Variablen, die in einem engen Zusammenhang zur Elternschaft stehen (Komponenten verschiedener individueller Leitbilder).

Im Folgenden liefern wir zunächst einige Erläuterungen zur verwendeten Stichprobe: Die Befragten wurden anhand ihres aktuellen Wohnortes in die Gruppen Ost- und Westdeutschland unterteilt (der Ort des Aufwachsens wurde in der Studie ebenfalls erfasst und geht als unabhängige Variable in die Modelle ein). Für die Auswertung wurde Berlin ausgeschlossen, weil aus unserer Sicht für die hier vorliegende Fragestellung die Sonderstellung als Großstadt und die ehemalige Teilung zu Verzerrungen bei einer rein geografischen Zuordnung zu Ostdeutschland führen würde. Die ursprüngliche Stichprobe, welche ansonsten nur noch auf Frauen begrenzt wird, erfährt sonst keine weiteren Einschränkungen. In Tabelle 1 wird diese nach zentralen Merkmalen beschrieben.

Die dargestellten Verteilungen der einzelnen Merkmale nach Ost- und Westdeutschland weisen nur bei wenigen Variablen deutliche und in einigen leichte Unterschiede zwischen den beiden Regionen auf. Die Altersstruktur der beobachteten Gruppe von Frauen ist nahezu identisch. Die Abweichung des Sozialisationsortes (Ort des Aufwachsens) und des derzeitigen Wohnortes ist in Ostdeutschland mit $8 \%$ erwartungsgemäß etwas höher als in Westdeutschland mit 3,4\%. Dies lässt sich vermutlich auf die höhere Binnenwanderung 
von Ost nach West zurückführen. Umgekehrt, also Wanderungen von West nach Ost, gibt es kaum. Im Westen zeigt sich ein leicht höherer Anteil von Frauen ohne Partner in der Stichprobe - der Unterschied beträgt rund sechs Prozentpunkte. Bei der ISCED-Zuordnung lassen sich im Westen etwas häufiger niedrige Abschlüsse zu Lasten mittlerer identifizieren. Die hohen ISCED-Stufen sind gleich hoch in beiden Regionen. Der Anteil an Hausfrauen ist im Westen mehr als doppelt so hoch. Bei der Selbsteinschätzung der eigenen finanziellen Lage findet man im Osten Deutschlands häufiger eine mittlere Zuordnung, die positive Einschätzung ist seltener.

Tabelle 1: Verschiedene Merkmale der verwendeten Stichprobe (nur Frauen), nach Wohnort in Ost- oder Westdeutschland, ohne Berlin

\begin{tabular}{lrrrr}
\hline & $\begin{array}{r}\text { West } \\
\text { (in \%) }\end{array}$ & N & $\begin{array}{c}\text { Ost } \\
\text { (in \%) }\end{array}$ & N \\
\hline Altersgruppen & & & & \\
20 - 29 Jahre & 49,5 & 899 & 48,9 & 157 \\
30 - 39 Jahre & 50,5 & 1.263 & 51,1 & 293 \\
Aufgewachsen in & & & & \\
$\quad$ Westdeutschland & 92,0 & 1.862 & 3,4 & 12 \\
$\quad$ Ostdeutschland & 8,0 & 185 & 96,6 & 424 \\
Partnerschaft & & & & \\
$\quad$ Ohne Partner & 26,5 & 538 & 20,9 & 88 \\
$\quad$ Mit Partner & 73,5 & 1.622 & 79,1 & 362 \\
Bildungsstand (ISCED97) & & & & \\
$\quad$ Niedrig (1-2) & 7,5 & 92 & 3,4 & 6 \\
$\quad$ Mittel (3-4) & 67,9 & 1.393 & 72,1 & 311 \\
$\quad$ Hoch (5-6) & 24,6 & 638 & 24,5 & 127 \\
Erwerbssituation & & & & \\
$\quad$ Erwerbstätig & 64,0 & 1.481 & 66,5 & 333 \\
$\quad$ Hausfrau & 10,9 & 232 & 4,8 & 23 \\
$\quad$ Sonstige Nichterwerbstätige (alle anderen) & 25,1 & 447 & 28,8 & 92 \\
Zurechtkommen mit dem zur Verfügung stehenden Geld & & & & \\
$\quad$ Schlecht & 7,5 & 129 & 7,2 & 32 \\
Einigermaßen & 34,3 & 702 & 40,6 & 168 \\
$\quad$ Gut & 58,2 & 1.315 & 52,2 & 249 \\
\hline
\end{tabular}

Quelle: FLB 2012, gewichtete Daten, eigene Berechnungen

Im Anschluss an die Beschreibung der Stichprobe kommen wir nun zum weiteren Vorgehen: Nach der kurzen Darstellung deskriptiver Befunde werden mithilfe einer binären logistischen Regression kinderlose Frauen (kodiert mit 0) und Mütter (kodiert mit 1) miteinander verglichen. Wir rechnen jeweils separate Regressionen für West- und Ostdeutschland, so dass für die Analyse zwei Regressionsmodelle interpretiert werden. Da es sich hier um Querschnittsanalysen handelt, können keine Aussagen zu Kausalitäten getroffen werden. Die Regressionen ermöglichen aber die Identifizierung von Faktoren, die für die Effekte hinter bivariaten Korrelationen verantwortlich sind. Unsere unabhängigen Variablen nehmen wir nacheinander in verschiedenen Teilmodellen in die Regressionen auf. 
Abschließend wird noch ein Gesamtmodell berechnet, das alle Variablen enthält. Die Fallzahlen in den Teilmodellen wurden an die Maximalzahl im vollständigen Modell angeglichen, also unter gemeinsamer Berücksichtigung aller fehlenden Werte. Dies sind für Westdeutschland 1.947 und für die neuen Bundesländer ohne Berlin 424 Frauen.

In unser erstes Teilmodell werden lediglich die beiden Variablen Alter und Sozialisationsort aufgenommen. Vom Alter wissen wir, dass es einen positiven Einfluss auf die Wahrscheinlichkeit einer Elternschaft hat, denn mit zunehmendem Alter werden Kinderwünsche konkreter und dementsprechend eher umgesetzt. Auch hinsichtlich des Sozialisationsortes, also des Ortes des Aufwachsens, erwarten wir einen Zusammenhang mit der Elternschaft. Dahinter steckt die Annahme unterschiedlicher kultureller Leitbilder in Ostund Westdeutschland, die eine Elternschaft mehr oder weniger begünstigen. Unsere Referenzkategorie ist in diesem Fall allerdings Westdeutschland, so dass wir im ersten Regressionsmodell den Effekt des Aufwachsens in Ostdeutschland für in Westdeutschland lebende Befragte isolieren. Im zweiten Regressionsmodell wird allerdings nur der Effekt für ostdeutsche Befragte gemessen, die ebenfalls dort aufgewachsen sind. Das zweite Teilmodell enthält nur die Partnerschaftsvariable als Voraussetzung einer Elternschaft, auch hier sollte sich ein deutlicher, positiver Zusammenhang mit der Elternschaft identifizieren lassen. Das dritte Teilmodell umfasst sozioökonomische Variablen, die wir für relevant erachten. Es handelt sich dabei um die Bildung, den Erwerbsstatus (vgl. z. B. Dorbritz/Ruckdeschel 2013) sowie die persönliche Einschätzung der eigenen ökonomischen Lage. Letzteres wurde über die Frageformulierung „Wie gut kommen Sie mit diesem Einkommen zurecht?“ erfasst. Diese Frage wurde auch denjenigen gestellt, die zuvor keine Angabe zum Einkommen gemacht haben. Die Verwendung dieser Variable hat den Vorteil, dass man hier im Vergleich zur Einkommensvariable mit weniger Ausfällen (Missing Values) konfrontiert wird und die für uns wichtige subjektive Einschätzung der ökonomischen Lage erfasst wird. Hier erwarten wir unterschiedliche Effekte: Eine höhere Bildung sollte vor allem in Westdeutschland einen negativen Einfluss ausüben, da diese mit längeren Ausbildungszeiten und einem späten Berufseinstieg einhergeht, was wiederum zum Aufschub der Elternschaft führt (u. a. Huinink 1995: 295ff.; Boehnke 2013). Eine bessere finanzielle Situation, gemessen über die Frage nach dem Zurechtkommen mit dem zur Verfügung stehenden Einkommen, sollte hingegen einen positiven Effekt haben, weil die Kosten für das Kind sowie der (vorübergehende) Verdienstausfall der Mutter dann leichter ausgeglichen werden können (u. a. Ziefle 2009: v. a. Kap. 2) und so finanzielle Ängste minimiert werden. ${ }^{3}$ Im Falle der Erwerbssituation testen wir v. a. den Zusammenhang einer Hausfrauentätigkeit und einer Mutterschaft, da hier der größte Effekt zu erwarten ist. Das nächste Teilmodell enthält verschiedene Variablen, die den Einfluss familienbezogener Leitbilder auf unsere abhängige Variable messen, dies wurde in den Hypothesen bereits angesprochen. Wir erfassen hier verschiedene Facetten von Familienleitbildern, bei denen ein Zusammenhang mit der Geburt von Kindern zu erwarten ist. Diese unterschiedlichen Bestandteile von Leitbildern werden auf der Individualebene gemessen. ${ }^{4}$ Es handelt sich hier zum einen um die (H1) Bedeutung eigener Kinder (Bedeutung von Kindern für die Partnerschaft, Wichtigkeit eigener Kinder, Akzeptanz von Kinderlosigkeit) sowie die antizipierten Anforderungen an eine Elternschaft, die sich aufteilen in (H3) die sogenannte verantwortete Elternschaft sowie (H2) die Präsenz der Mutter (Betreuung und Unterstützung der Kinder durch die

Dies trifft v. a. zu, wenn man annimmt, dass Männer den größeren Beitrag zum Haushaltseinkommen leisten. Die vierstufige Antwortskala von „Stimme voll und ganz zu“ über „Stimme eher zu“ bis „Stimme eher nicht zu“ und „Stimme überhaupt nicht zu“ wurde für die Analyse jeweils zu den beiden Kategorien „Zustimmung“ und „Ablehnung“ zusammengefasst, wobei „Ablehnung“ die jeweilige Referenzkategorie bildet. 
eigene Mutter). Unser Leitbild-Modell enthält somit Facetten unterschiedlicher familienbezogener Leitbilder, die bereits Gegenstand der einzelnen Kapitel dieses Sammelbands sind und dort ausführlich diskutiert werden.

\section{$4 \quad$ Ergebnisse}

\subsection{Deskriptive Analysen}

Bevor wir zu den Regressionsmodellen kommen, möchten wir zunächst die Zahlen für den Kinderwunsch und die realisierte Kinderzahl von ost- und westdeutschen Frauen gegenüberstellen, um die unterschiedlichen Muster anhand konkreter Zahlen aufzuzeigen, die dann im Folgenden erklärt werden sollen. Abbildung 1 zeigt die Kinderzahl und als zusätzliche Information den Kinderwunsch von kinderlosen Frauen in Ost- und Westdeutschland getrennt für zwei Altersgruppen.

Abbildung 1: Kinderzahl von in West- oder Ostdeutschland (ohne Berlin) lebenden bzw. aufgewachsenen $^{\text {a }}$ Frauen nach Altersgruppen (in Prozent)

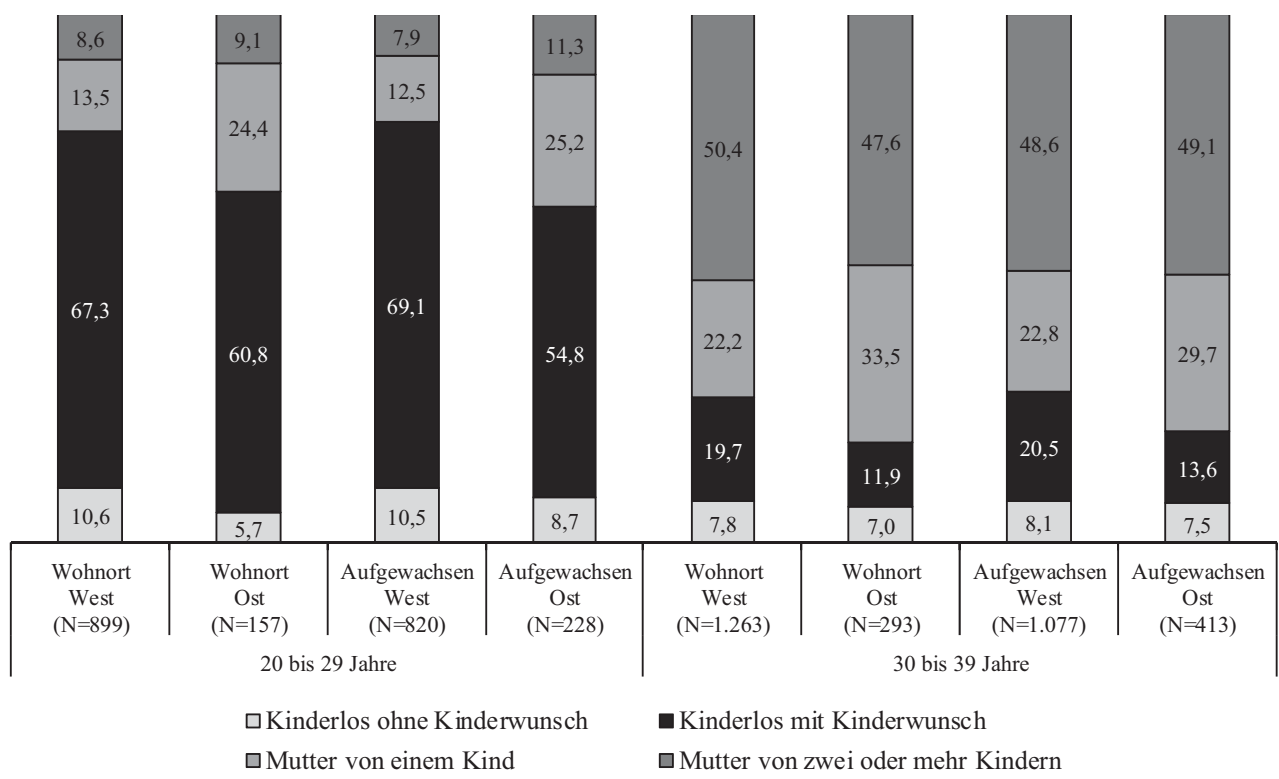

Quelle: FLB 2012, gewichtete Daten, eigene Berechnungen

Anm.: ${ }^{a}$ Hier wurde gefragt, wo die Person den überwiegenden Teil ihrer Kindheit und Jugend verbracht hat. Es konnte das Bundesland oder „ehemalige DDR“ bzw. Westdeutschland angegeben werden.

Die Zahlen, welche auf der Familienleitbildstudie basieren, decken sich mit den Befunden auf der Makroebene bzw. der amtlichen Statistik, lässt man dabei den Kinderwunsch außer Acht, der in jener nicht erfasst sein kann. Unter Berücksichtigung des Kinderwunsches werden die ohnehin relativ hohen Unterschiede zwischen Ost und West bezüglich des fakti- 
schen generativen Verhaltens allerdings noch deutlicher. Betrachtet man den Wohnort zum Zeitpunkt der Befragung so zeigt sich, dass der Anteil der 20- bis 29-jährigen Frauen, die kinderlos und ohne Kinderwunsch sind, im Westen fast doppelt so hoch ausfällt wie im Osten. Die „Ost-Sozialisation“ scheint dies nur geringfügig zu kompensieren. Die stärkste Annäherung wird hierbei durch das Alter erreicht. Ab dem Zeitpunkt, wenn Kinderwünsche konkreter werden (ab dem 30. Lebensjahr), verringert sich wohnortbezogen im Westen der Anteil der Frauen ohne Kinder und ohne Kinderwunsch, während er sich im Osten fast auf das Westniveau erhöht. Noch gravierender sind die Unterschiede zwischen west- und ostdeutschen Frauen im Hinblick auf die Realisierung der Kinderwünsche. Deutlich mehr Frauen im Westen und vor allem jene, die den Großteil ihrer Kindheit im Westen verbracht haben, sind kinderlos, obwohl sie einen Kinderwunsch haben. Dabei sollte das Augenmerk auf die 30- bis 39-jährigen Frauen gerichtet werden, weil die Kinderwunschformulierung im Leitbildsurvey verhältnismäßig abstrakt gehalten ist (Dorbritz/Naderi 2013; Testa 2012). In dieser Gruppe zeigen sich sowohl beim Wohn- als auch beim Sozialisationsort ungefähr sieben bis acht Prozentpunkte Unterschied zwischen Ost (11,9 bzw. 13,6 \%) und West (19,7 bzw. 20,5\%). Ostdeutsche Frauen haben zum gleichen Zeitpunkt seltener einen unerfüllten Kinderwunsch, dafür aber bereits häufiger Kinder. Wie gezeigt werden konnte, ergeben sich hinsichtlich der Kinderzahl (aber auch beim Kinderwunsch) nach wie vor erhebliche Unterschiede zwischen ost- und westdeutschen Frauen. Bevor wir jedoch zu den multivariaten Modellen kommen, möchten wir die Unterschiede in den Einstellungen in Bezug auf die gewählten Leitbildfacetten darstellen (Tabelle 2).

Tabelle 2: Zustimmung zu den verwendeten Leitbildaussagen, nach Wohnort ${ }^{\mathrm{a}}$

\begin{tabular}{|c|c|c|c|c|}
\hline & $\begin{array}{l}\text { West } \\
(\text { in } \%)\end{array}$ & Phi & $\begin{array}{l}\text { Ost } \\
(\text { in } \%)\end{array}$ & Phi \\
\hline \multicolumn{5}{|l|}{$\begin{array}{l}\text { „Eine Partnerschaft funktioniert gut, wenn man } \\
\text { gemeinsame Kinder hat.“ }\end{array}$} \\
\hline $\begin{array}{l}\text { Kinderlos } \\
\text { Mutter }\end{array}$ & $\begin{array}{l}49,7 \\
66,5\end{array}$ & $-0,17^{* * *}$ & $\begin{array}{l}57,0 \\
67,3\end{array}$ & $-0,11^{*}$ \\
\hline \multicolumn{5}{|l|}{ „Es ist persönlich wichtig, eigene Kinder zu haben.“ } \\
\hline $\begin{array}{l}\text { Kinderlos } \\
\text { Mutter }\end{array}$ & $\begin{array}{l}77,6 \\
97,9\end{array}$ & $-0,30^{* * *}$ & $\begin{array}{r}83,7 \\
100,0\end{array}$ & $-0,32^{* * *}$ \\
\hline \multicolumn{5}{|l|}{$\begin{array}{l}\text { "Heutzutage ist es etwas ganz Normales, keine Kinder } \\
\text { zu haben.“ }\end{array}$} \\
\hline $\begin{array}{l}\text { Kinderlos } \\
\text { Mutter }\end{array}$ & $\begin{array}{l}64,3 \\
56,6\end{array}$ & $0,08^{* *}$ & $\begin{array}{l}59,2 \\
54,3\end{array}$ & 0,05 \\
\hline \multicolumn{5}{|l|}{$\begin{array}{l}\text { „Eltern können bei der Erziehung vieles falsch machen, } \\
\text { daher müssen sie sich gut informieren." }\end{array}$} \\
\hline $\begin{array}{l}\text { Kinderlos } \\
\text { Mutter }\end{array}$ & $\begin{array}{l}85,5 \\
75,8\end{array}$ & $0,12^{* * *}$ & $\begin{array}{l}85,6 \\
71,4\end{array}$ & $0,17^{* *}$ \\
\hline \multicolumn{5}{|l|}{$\begin{array}{l}\text { „Mütter sollten nachmittags Zeit haben, um ihren } \\
\text { Kindern beim Lernen zu helfen.“ }\end{array}$} \\
\hline $\begin{array}{l}\text { Kinderlos } \\
\text { Mutter }\end{array}$ & $\begin{array}{l}80,0 \\
88,1\end{array}$ & $-0,11^{* * *}$ & $\begin{array}{l}73,5 \\
88,0\end{array}$ & $-0,19^{* * *}$ \\
\hline \multicolumn{5}{|l|}{$\begin{array}{l}\text { „Für ein Kind zwischen } 1 \text { und } 3 \text { Jahren ist es das Beste, } \\
\text { wenn es nur von der Mutter betreut wird.“ }\end{array}$} \\
\hline $\begin{array}{l}\text { Kinderlos } \\
\text { Mutter }\end{array}$ & $\begin{array}{l}17,4 \\
28,7\end{array}$ & $-0,14^{* * *}$ & $\begin{array}{l}7,2 \\
9,0\end{array}$ & $-0,03$ \\
\hline
\end{tabular}

Quelle: FLB 2012, gewichtete Daten, eigene Berechnungen

Anm.: ${ }^{* * *} \mathrm{p} \leq 0,001 ;{ }^{* *} \mathrm{p} \leq 0,01 ;{ }^{*} \mathrm{p} \leq 0,05 .{ }^{a}$ ohne Berlin. 
Die Verteilung zeigt, dass auch hier zum Teil Unterschiede zwischen ost- und westdeutschen Frauen bestehen. So stimmen sowohl ostdeutsche kinderlose Frauen $(83,7 \%)$ als auch Mütter $(100 \%)$ zu einem höheren Anteil der Aussage zu, dass ihnen eigene Kinder wichtig sind. In Westdeutschland ist die Differenz in Prozentpunkten größer, kinderlose Frauen stimmen hier mit 77,6\% sehr viel seltener zu als Mütter (97,9\%). Die Akzeptanz von Kinderlosigkeit scheint bei westdeutschen Frauen (sowohl Kinderlose als auch Mütter) tendenziell etwas höher zu sein als bei ostdeutschen. Bei der Frage nach der Unterstützung der Kinder beim Lernen findet sich interessanterweise aber kein Unterschied zwischen ostund westdeutschen Müttern (88,0 und 88,1 \% Zustimmung), kinderlose ostdeutsche Frauen stimmen hier zu einem geringeren Anteil zu (73,5\%) als westdeutsche (80\%). Bei der leicht zugespitzten Frage, ob es für ein Kleinkind am besten sei, nur von der eigenen Mutter betreut zu werden, lassen sich allerdings erhebliche Ost-West-Unterschiede entdecken. Die Zustimmung ist unter ostdeutschen Frauen (Kinderlose und Mütter) wesentlich niedriger als unter den westdeutschen, wobei auch hier lediglich eine Minderheit zustimmt.

\subsection{Multivariate Analysen}

Zunächst möchten wir auf die Regressionsergebnisse für westdeutsche Frauen Bezug nehmen (Tabelle 3). Einen wichtigen Erklärungsgehalt für die Wahrscheinlichkeit einer Elternschaft hat das Vorhandensein einer Partnerschaft. Da dieser Zusammenhang „trivial“ ist, muss hier nicht stärker darauf eingegangen werden. Modell 2 ist nur zur Abschätzung der Bedeutung der anderen Effekte einbezogen worden. Ebenfalls trivial, aber notwendig, ist der Zusammenhang mit dem Alter. Sieht man folglich von Modell $1 \mathrm{ab}$, zeigt sich in Westdeutschland im Vergleich in Modell $4\left(\mathrm{R}^{2}=0,21\right)$, das die gewählten Facetten von Leitbildern enthält, eine ähnliche, aber leicht geringere Erklärungskraft als in Modell 3 zur tatsächlichen sozioökonomischen Situation $\left(\mathrm{R}^{2}=0,23\right)$. Im Hinblick auf die sozioökonomische Situation wird deutlich, dass Mutterschaft in Westdeutschland stark mit einem Hausfrauenstatus (-dasein) korreliert. ${ }^{5}$ Der entscheidende Faktor ist hier vermutlich die damit verbundene Präsenz zu Hause und die Möglichkeit, für den Nachwuchs da zu sein. Der zentrale Konflikt, zwischen Beruf und Familie entscheiden zu müssen, stellt sich hier nicht bzw. wurde schon zu einem früheren Zeitpunkt entschieden. Die Effekte zur finanziellen Situation fallen anders aus als erwartet, denn eine positive Einschätzung der finanziellen Lage steht offenbar in einem negativen Zusammenhang zur Mutterschaft. Auf dieses Ergebnis gehen wir noch einmal genauer ein. Bei den Leitbildkomponenten sind die Effekte bei jenen Aussagen ausgeprägter, die auf die Wichtigkeit einer Elternschaft für das eigene Leben (H1) rekurrieren. Ein deutlich negativer Effekt auf die Wahrscheinlichkeit Mutter zu werden, geht im Westen Deutschlands von der Befürchtung der Frauen aus, bei der Erziehung der Kinder etwas falsch zu machen (H3 zur Verantworteten Elternschaft).

5 Es ist sicherlich auch so, dass Hausfrauen mit einer höheren Wahrscheinlichkeit Kinder bekommen bzw. Mütter Hausfrauen sind, weil sie Kinder haben - beide Erklärungen sind denkbar. Hinter dem Effekt verbirgt sich vermutlich beides. Zu kausalen Bezügen können wir hier keine Aussagen treffen. 
Tabelle 3: Binär logistische Regression, abhängige Variable Kinderlosigkeit (0) versus Elternschaft (1), Wohnort Westdeutschland (Odds Ratio)

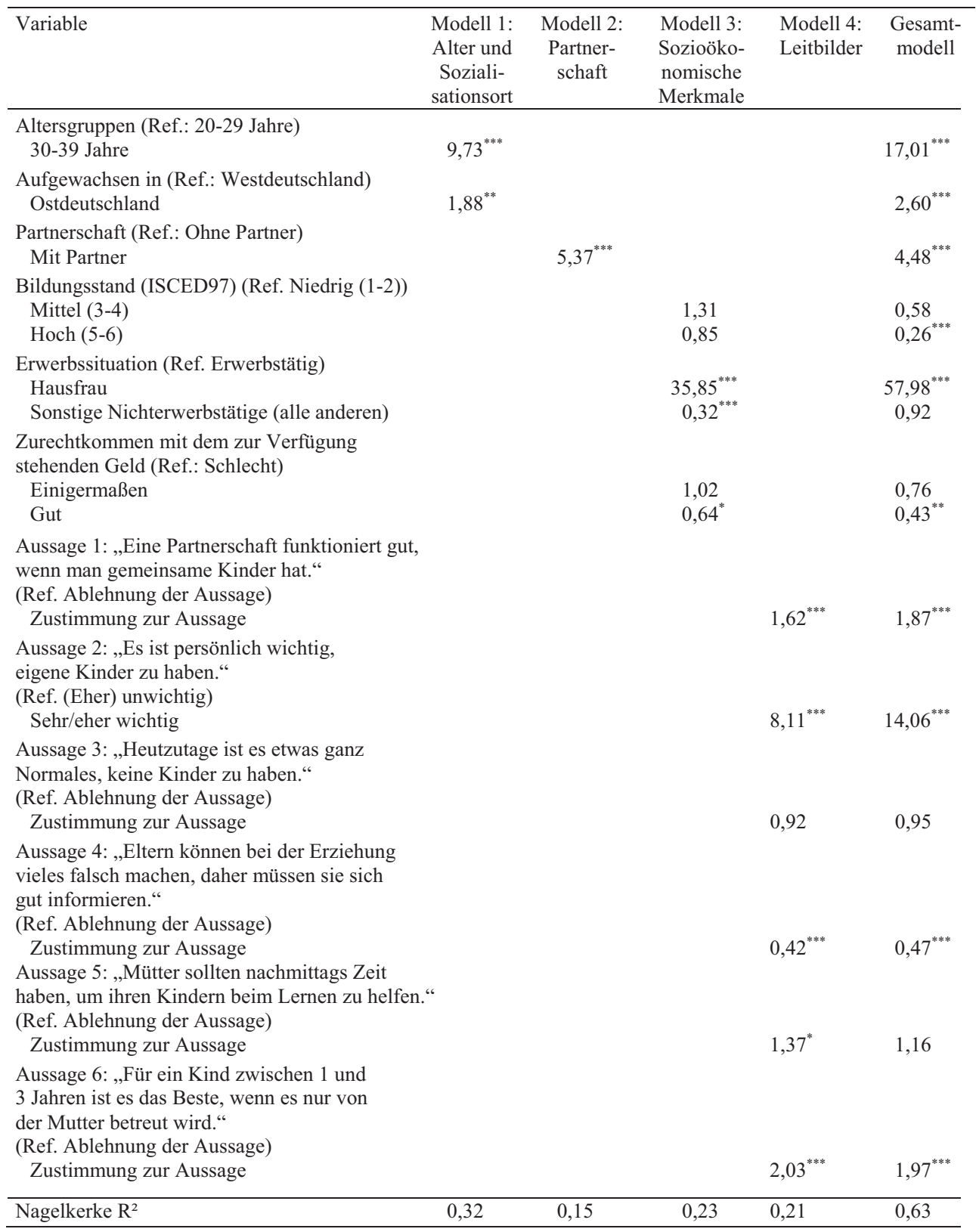

Quelle: FLB 2012, eigene Berechnungen mit ungewichteten Daten, N=1.947

Anm.: ${ }^{* * *} \mathrm{p} \leq 0,001 ;{ }^{* *} \mathrm{p} \leq 0,01 ;{ }^{*} \mathrm{p} \leq 0,05$. 
Tabelle 4: Binär logistische Regression, abhängige Variable Kinderlosigkeit (0) versus Elternschaft (1), Wohnort Ostdeutschland (Odds Ratio)

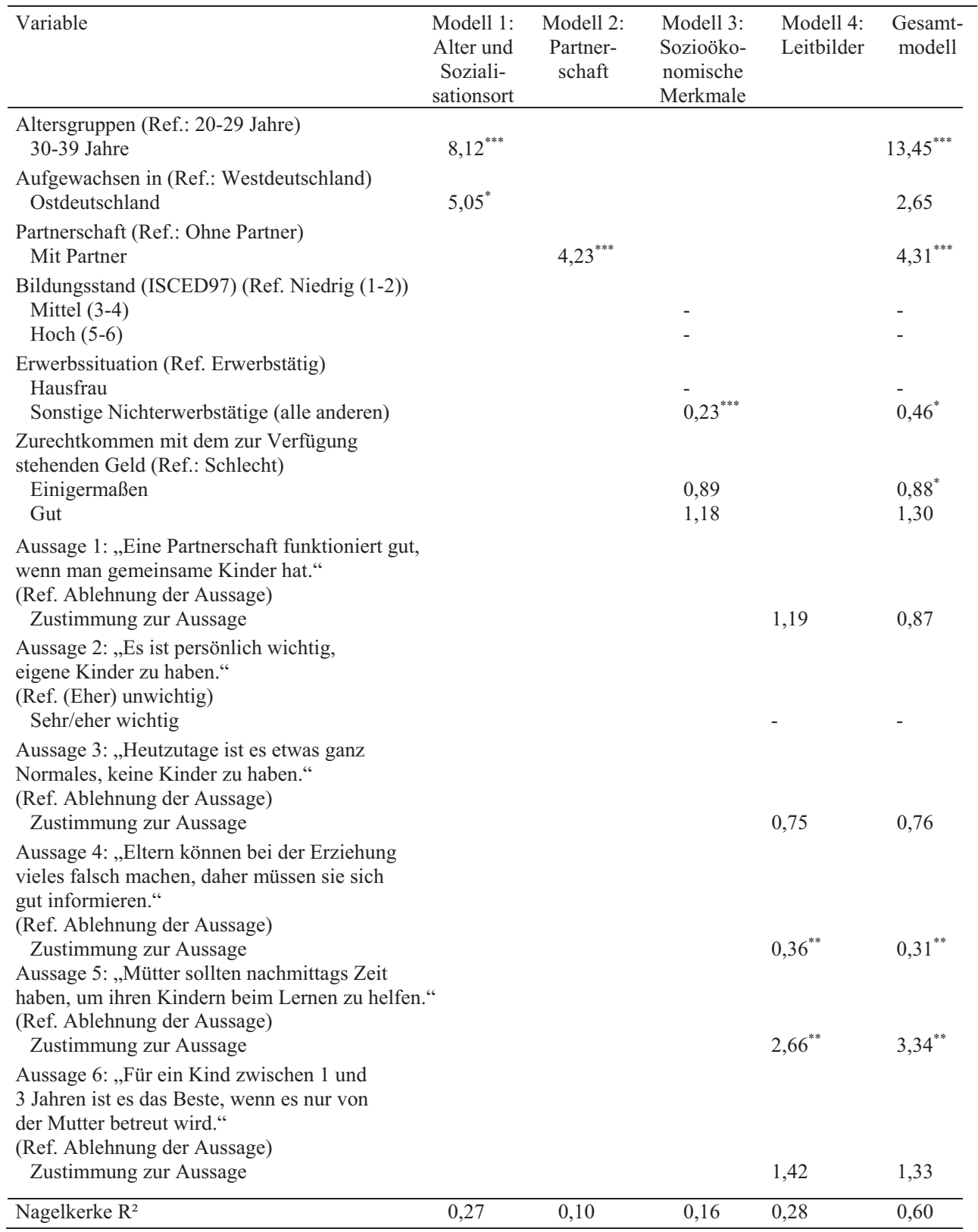

Quelle: FLB 2012, eigene Berechnungen mit ungewichteten Daten, N=424

Anm.: ${ }^{* * *} \mathrm{p} \leq 0,001 ;{ }^{* *} \mathrm{p} \leq 0,01 ;{ }^{*} \mathrm{p} \leq 0,05$. 
In Tabelle 4 werden die Ergebnisse der Regressionsanalyse für ostdeutsche Frauen dargestellt. Im ersten Teilmodell hat das Alter den größten Effekt, dieser ist gleichzeitig hoch signifikant. Auch das Vorhandensein einer Partnerschaft (Modell 2) hat einen signifikanten positiven Einfluss. Während im Westen das Modell mit den sozioökonomischen Variablen für die Erklärung einer Elternschaft etwas wichtiger zu sein scheint als die gemessenen Facetten von Leitbildern, so ist im Osten das Muster deutlicher in Richtung der gemessenen Leitbildkomponenten ausgerichtet $\left(\mathrm{R}^{2}=0,16\right.$ in Modell 3 und $\mathrm{R}^{2}=0,28$ in Modell 4). Wie erwartet, übt die Erwerbssituation der Frau (Hausfrauentätigkeit) keinen Einfluss auf eine Elternschaft aus, zumindest lässt sich kein Effekt messen. Dies kann damit zusammenhängen, dass eine Hausfrauentätigkeit in unseren Daten seltener vorkommt (nur 4,8 \% der ostdeutschen Frauen geben an, Hausfrau zu sein, siehe Tabelle 1). Auch in Bezug auf die Bildung kann kein Effekt gemessen werden. Der Effekt, der von der Variablen ausgeht, die die subjektive Einschätzung der finanziellen Situation misst, ist zwar nicht bzw. nur schwach signifikant, aber dennoch sehr interessant. Er verläuft nämlich entgegengesetzt zum Effekt, der sich im Modell für Westdeutschland abzeichnet. Dort zeigt sich ein negativer Effekt auf die Mutterschaft, wenn man angibt, gut mit dem Geld auszukommen, was darauf hindeutet, dass ein enger Zusammenhang zur Bildung besteht. Mehr Geld und mehr Bildung scheinen dort $\mathrm{u}$. U. dazu zu führen, dass die Unvereinbarkeit von Mutterschaft und Beruf zum Tragen kommt (Eckhard/Klein 2013: 327). Klein/Eckhard (2005: 160ff.) weisen ferner darauf hin, dass Bildungsunterschiede bei Frauen auch mit differierenden Werteinstellungen in Bezug auf den immateriellen Nutzen einer Elternschaft einhergehen. Im Modell für die ostdeutschen Frauen ist es umgekehrt: Eine bessere finanzielle Situation hat, wie von uns erwartet, einen positiven Einfluss. Hier scheint es folglich, einen entscheidenden Unterschied zwischen Ost und West zu geben. Dazu passen auch die Befunde, dass ostdeutsche Akademikerinnen tendenziell sogar mehr Kinder bekommen haben als Nichtakademikerinnen, dahinter könnte folglich auch ein generationenübergreifendes Leitbildelement stecken. Die Erfahrung hat in der ehemaligen DDR gezeigt, dass ein akademischer Abschluss, Berufstätigkeit und Kinder durchaus vereinbar sind.

Bei den Einzeleffekten der überprüften Leitbild-Aussagen (Modell 4) gibt es prinzipiell keinen Unterschied zwischen beiden deutschen Regionen, wobei im Gegensatz zum Westen die Frage der Kleinkindbetreuung keinen statistisch signifikanten (aber gleichgerichteten) Effekt aufweist. Die Wichtigkeit überhaupt Kinder zu haben (H1), rückt im Osten in den Hintergrund, ist statistisch nicht messbar, wahrscheinlich weil hier Kinderlosigkeit wegen einer starken Familienorientierung und einem hohen Stellenwert von Kindern prinzipiell unerwünscht ist, auch in jungen Altersgruppen (Arránz Becker et al. 2010: 13; Buhr et al. 2011: 177f., 180). Statistisch gesehen gibt es hier keine Varianz, was auch die Ergebnisse in Tabelle 2 belegen: $100 \%$ der ostdeutschen Mütter bejahen die Wichtigkeit eigener Kinder. Es kann vermutet werden, dass Kinder im Osten stärker verinnerlicht sind und hier in einem stärkeren Maße zum Leben dazu gehören, was bedeutet, dass die Familiengründung weniger bewusst reflektiert wird als das vielleicht im Westen der Fall ist. Gemeinsam haben beide Regionen die wichtige Bedeutung der sogenannten verantworteten Elternschaft (H3). Die Sorge um den richtigen Umgang mit dem Nachwuchs, und dass genügend Zeit für die Kinder da ist (H2), eint beide Teile Deutschlands. Entsprechend zeigt sich im Osten zwar eine geringer ausgeprägte Ablehnung der Kleinkinderbetreuung (siehe Tabelle 2), der Zusammenhang ist jedoch, wie bereits erwähnt, nicht signifikant. Der Wunsch nach ausgeprägter persönlicher Verantwortung der Mutter für das Kind existiert allerdings in beiden Teilen Deutschlands gleichermaßen, denn die Zustimmung zu Aussage 5 ist in beiden Teilmodellen signifikant. 
Fasst man die Ergebnisse der beiden Regressionsanalysen noch einmal kurz zusammen, zeigt sich ein wichtiger Unterschied zwischen Ost- und Westdeutschen in der Erklärungskraft der gerechneten Teilmodelle. Im Westen zeigt sich ein ausgewogeneres Bild beim Vergleich der sozioökonomischen Rahmenbedingungen und dem Einfluss der kulturellen Leitbilder. Hier sind beide Teilmodelle etwa gleich wichtig für die Erklärung einer Elternschaft. Im Osten fallen die Ergebnisse etwas differenzierter aus, hier ist das Modell mit den Leitbildfacetten wichtiger als das sozioökonomische Modell.

Es kann festgehalten werden, dass Leitbilder (bzw. die gewählten Elemente von Leitbildern) neben der soziökonomischen Situation einen eigenständigen Erklärungsgehalt bei der Untersuchung einer Eltern- bzw. Mutterschaft haben. Leitbilder fungieren hier als sogenannter „Missing Link“, dabei bleiben die Effekte auch im Gesamtmodell signifikant. Bemerkenswert ist ebenfalls, dass Ost- und Westdeutsche offenbar die gleichen Sorgen teilen, was sich an den nicht immer signifikanten, aber gleichgerichteten Effekten zeigt (siehe auch Ruckdeschel, Kap. 12).

\section{$5 \quad$ Fazit}

In unserem Beitrag gingen wir den beiden Fragen nach, ob wir in den neuen und alten Bundesländern unterschiedliche Familienleitbilder (bzw. unterschiedlich ausgeprägte Facetten von Leitbildern) vorfinden und ob diese für die unterschiedlichen Fertilitätsregime in beiden Regionen mitverantwortlich sind.

Unterschiede zwischen ost- und westdeutschen Frauen ließen sich v. a. hinsichtlich der Wichtigkeit eigener Kinder, der Akzeptanz von Kinderlosigkeit sowie bei der Frage der Kleinkindbetreuung durch die Mutter identifizieren. Ostdeutsche Frauen sind stärker kindorientiert, lehnen eine alleinige Kleinkindbetreuung durch die Mutter allerdings häufiger ab als westdeutsche Frauen. Einig sind sich die Frauen bezüglich der Einstufung der Erziehung als anspruchsvolle Aufgabe, bei der man als Eltern vieles falsch machen kann. Hier teilen die Befragten offenbar die gleichen Sorgen.

In einem zweiten Analyseschritt wurden kinderlose Frauen mit Müttern auf Basis binominaler logistischer Regressionen verglichen, dabei wurden den Elementen verschiedener Leitbilder zur Bedeutung eigener Kinder sowie zu vermuteten Anforderungen an eine Elternschaft sozioökonomische Individualmerkmale gegenübergestellt. Die Ergebnisse belegen, dass Leitbilder mit Kinderlosigkeit und Elternschaft zusammenhängen, v. a. die Sorge um die richtige Erziehung (verantwortete Elternschaft) hat einen signifikanten negativen Effekt auf eine Mutterschaft. Hinsichtlich der Gesamterklärungskraft der gerechneten Teilmodelle existiert im Westen eine relative Ausgewogenheit zwischen Leitbildern und sozioökonomischen Merkmalen und im Osten sogar eine stärkere Tendenz zur Erklärung durch Leitbildfacetten.

Ein Paneldesign wäre für weiterführende Analysen wünschenswert, um dann auch Aussagen zu kausalen Bezügen treffen zu können. Ein solches Design hätte den Vorteil, dass man die Leitbilder von Personen in verschiedenen Altersgruppen zu unterschiedlichen Zeitpunkten im Lebensverlauf erfassen könnte, um so Verlaufsanalysen durchzuführen und den jeweiligen Einfluss der Leitbilder auf generatives Verhalten zu messen. Dies ist auf Basis unserer Daten leider nicht möglich, da wir nur Aussagen zum Ist-Zustand der Befragten treffen können.

Allerdings weisen unsere Befunde darauf hin, dass die Berücksichtigung der prägenden Auffassungen (Leitbilder bzw. Leitbildfacetten) von Ost- und Westdeutschen zum Verständ- 
nis von Eltern- bzw. Mutterschaft und der daraus resultierenden Unterschiede äußerst vielversprechend ist. Daneben spielt aber auch die individuelle tatsächliche Lebenssituation eine wichtige Rolle und sollte auf keinen Fall vernachlässigt werden.

Die Leitbilder zeichnen sich durch ihre relative Unabhängigkeit von den tatsächlichen institutionellen Rahmenbedingungen aus, was auch dadurch erklärt werden kann, dass Einstellungen aber auch konkrete Verhaltensweisen von den Müttern an ihre Töchter weitergegeben werden und diese ähnliche Einstellungen entwickeln und ein ähnliches Verhalten erkennen lassen. Wenn beispielsweise die eigene Mutter erwerbstätig war (was in der ehemaligen DDR quantitativ häufiger zu finden war als in der BRD), strebt die Tochter $u$. U. auch nach einem ähnlichen Vereinbarkeitsmodell (vgl. Diabaté, Kap. 13). Es ist deshalb davon auszugehen, dass Leitbilder über die Zeit hinweg relativ stabil bleiben werden und sich nur allmählich wandeln. Deshalb werden sich auch noch in den nächsten Jahren unterschiedliche Einstellungen in Ost- und Westdeutschland finden lassen.

\section{Literatur}

Alheit, Peter (2005): Modernisierungsblockaden in Ostdeutschland? In: Aus Politik und Zeitgeschichte (APuZ), 40, S. 32-40.

Arránz Becker, Oliver; Lois, Daniel; Nauck, Bernhard (2010): Differences in Fertility Patterns between East and West German Women. Disentangling the roles of cultural background and of the transformation process. In: Comparative Population Studies 35, 1, S. 7-34.

Bauernschuster, Stefan; Rainer, Helmut (2012): Political regimes and the family: how sex-role attitudes continue to differ in reunified Germany. In: Journal of Population Economics 25, 1, S. 5-27.

Bernardi, Laura; Keim, Sylvia (2013): Anfang dreißig und noch kinderlos? Lebenswege und Familienmodelle berufstätiger Frauen aus Ost- und Westdeutschland. In: Konietzka, Dirk; Kreyenfeld, Michaela (Hrsg.): Ein Leben ohne Kinder. Ausmaß, Strukturen und Ursachen von Kinderlosigkeit. Wiesbaden: Springer VS, S. 173-190.

Bernardi, Laura; Keim, Sylvia; Von der Lippe, Holger (2007): Social Influences on Fertility. A Comparative Mixed Methods Study in Eastern and Western Germany. In: Journal of Mixed Methods Research 1, 1, S. 23-47.

Boehnke, Mandy (2013): Hochschulbildung und Kinderlosigkeit. Deutsch-deutsche Unterschiede. In: Konietzka, Dirk; Kreyenfeld, Michaela (Hrsg.): Ein Leben ohne Kinder. Ausmaß, Strukturen und Ursachen von Kinderlosigkeit. Wiesbaden: Springer VS, S. 81-100.

Buhr, Petra; Huinink, Johannes; Boehnke, Mandy; Maul, Katharina (2011): Kinder oder keine? Institutionelle Rahmenbedingungen und biographische Voraussetzungen für die Familiengründung und -erweiterung in Ost- und Westdeutschland. In: Brüderl, Josef; Castiglioni, Laura; Schumann, Nina (Hrsg.): Partnerschaft, Fertilität und intergenerationale Beziehungen. Würzburg: Ergon Verlag, S. 175-201.

Bujard, Martin; Dorbritz, Jürgen; Grünheid, Evelyn; Kühntopf, Stephan; Lück, Detlev; Naderi, Robert; Passet, Jasmin; Ruckdeschel, Kerstin (2012): (Keine) Lust auf Kinder? Geburtenentwicklung in Deutschland. Wiesbaden: Bundesinstitut für Bevölkerungsforschung [urn:nbn:de: bib-var-2012-011].

BiB (Bundesinstitut für Bevölkerungsforschung) (2012): Zusammengefasste Geburtenziffer in europäischen Ländern, 1960 bis 2012 [http://www.bib-demografie.de/DE/ZahlenundFakten/06/Tabellen/ t_06_01_zusgef_geburtenziffer_europ_laender_ab1960.html?nn=3071458, 14.11.2014].

BMFSFJ (Bundesministerium für Familie, Senioren, Frauen und Jugend) (2013): Mehrkindfamilien in Deutschland. Berlin.

Diabaté, Sabine; Lück, Detlev (2014): Familienleitbilder - Identifikation und Wirkungsweise auf generatives Verhalten. In: Zeitschrift für Familienforschung 26, 1, S. 49-69. 
Dorbritz, Jürgen (2010): Kinderzahlen und Lebensformen im West-Ost-Vergleich - Ergebnisse des Mikrozensus 2008. In: Bevölkerungsforschung Aktuell 31, 1, S. 11-15.

Dorbritz, Jürgen; Naderi, Robert (2013): Trendwende beim Kinderwunsch? In: Bevölkerungsforschung Aktuell 34, 4, S. 2-7.

Dorbritz, Jürgen; Ruckdeschel, Kerstin (2013): Kinderlosigkeit - differenzierte Analysen und europäische Vergleiche. In: Konietzka, Dirk; Kreyenfeld, Michaela (Hrsg.): Ein Leben ohne Kinder - Ausmaß, Strukturen und Ursachen von Kinderlosigkeit. Wiesbaden: Springer VS, S. 253-278.

Eckhard, Jan; Klein, Thomas (2013): Die Motivation zur Elternschaft. Unterschiede zwischen Männern und Frauen. In: Konietzka, Dirk; Kreyenfeld, Michaela (Hrsg.): Ein Leben ohne Kinder. Ausmaß, Strukturen und Ursachen von Kinderlosigkeit. Wiesbaden: Springer VS, S. 311-331.

Giesel, Katharina D. (2007): Leitbilder in den Sozialwissenschaften. Wiesbaden: VS Verlag für Sozialwissenschaften.

Goldstein, Joshua R.; Kreyenfeld, Michaela (2011): Has East Germany Overtaken West Germany? Recent Trends in Order-Specific Fertility. In: Population and Development Review 37, 3, S. 453-472.

Huinink, Johannes (1995): Warum noch Familie? Zur Attraktivität von Partnerschaft und Elternschaft in unserer Gesellschaft. Frankfurt a.M.: Campus-Verlag.

Huinink, Johannes; Kreyenfeld, Michaela; Trappe, Heike (2012): Familie und Partnerschaft in Ostund Westdeutschland. Eine Bilanz. In: Huinink, Johannes; Kreyenfeld, Michaela; Trappe, Heike (Hrsg.): Familie und Partnerschaft in Ost- und Westdeutschland. Ähnlich und doch immer noch anders. In: Zeitschrift für Familienforschung, Sonderheft 9, S. 9-28.

Klein, Thomas; Eckhard, Jan (2005): Bildungsbezogene Unterschiede des Kinderwunschs und des generativen Verhaltens. Eine kritische Analyse der Opportunitätskostenhypothese. In: Steinbach, Anja (Hrsg.): Generatives Verhalten und Generationenbeziehungen. Wiesbaden: VS-Verlag für Sozialwissenschaften, S. 151-174.

Krause, Peter; Ostner, Ilona (2010): Leben in Ost- und Westdeutschland. Eine sozialwissenschaftliche Bilanz der deutschen Einheit 1990-2010. Frankfurt a. M.: Campus Verlag.

Kreyenfeld, Michaela; Konietzka, Dirk; Walke, Rainer (2011): Dynamik und Determinanten nichtehelicher Mutterschaft in Ost- und Westdeutschland. In: Brüderl, Josef; Castiglioni, Laura; Schumann, Nina (Hrsg.): Partnerschaft, Fertilität und intergenerationale Beziehungen. Würzburg: Ergon Verlag, S. 155-174.

Pfau-Effinger, Birgit; Geissler, Birgit (2002): Cultural change and family policies in East and West Germany. In: Carling, Alan; Duncan, Simon; Edwards, Rosalind (Hrsg.): Analysing Families. Morality and Rationality in Policy and Practice. London: Routledge, S. 75-84.

Ruckdeschel, Kerstin (2009): Rabenmutter contra Mère Poule: Kinderwunsch und Mutterbild im deutsch-französischen Vergleich. In: Zeitschrift für Bevölkerungswissenschaft 34, 1-2, S. 105-134.

Schneider, Norbert F.; Naderi, Robert; Ruppenthal, Silvia (2012): Familie in Deutschland nach dem gesellschaftlichen Umbruch. Sind Ost-West-Differenzierungen in der Familienforschung zwanzig Jahre nach der Wiedervereinigung noch sinnvoll? In: Huinink, Johannes; Kreyenfeld, Michaela; Trappe, Heike (Hrsg.): Familie und Partnerschaft in Ost- und Westdeutschland. Ähnlich und doch immer noch anders. In: Zeitschrift für Familienforschung, Sonderheft 9, S. 29-53.

Statistisches Bundesamt (2012): Durchschnittliches Alter der Mütter bei der Geburt des Kindes (biologische Geburtenfolge) [https://www.destatis.de/DE/ZahlenFakten/GesellschaftStaat/ Bevoelkerung/Geburten/Tabellen/GeburtenMutterBiologischesAlter.html, 06.11.2014]

Stöbel-Richter; Yve (2010): Fertilität und Partnerschaft. Eine Längsschnittstudie zu Familienbildungsprozessen über 20 Jahre. Gießen: Psychosozial-Verlag.

Testa, Maria Rita (2012): Family Sizes in Europe: Evidence from the 2011 Eurobarometer Survey. Wien: Vienna Institute of Demography [http://www.oeaw.ac.at/vid/download/edrp_2_2012.pdf, 29.01.2014].

Ziefle, Andrea 2009: Familienpolitik als Determinante weiblicher Lebensverläufe? Die Auswirkungen des Erziehungsurlaubs auf Familien- und Erwerbsbiographien in Deutschland. Wiesbaden: VSVerlag für Sozialwissenschaften. 


\title{
Familie XXL: Leitbild Kinderreichtum?
}

\author{
Sabine Diabaté, Kerstin Ruckdeschel, Jürgen Dorbritz \& Linda Lux*
}

\section{Zusammenfassung}

Kinderreiche Familien sind in Deutschland zu einer immer kleineren Gruppe geworden. Die Sichtweisen auf die Familien mit drei oder mehr Kindern sind ambivalent: Einerseits werden aus der individuellen Sicht viele Kinder als etwas ganz Wundervolles gesehen. Andererseits wird in der Gesellschaft ein negatives Image gegenüber Großfamilien wahrgenommen. Dies kann mit den hohen und teilweise überfrachteten Erwartungen an die Elternrolle erklärt werden. Dabei entstehen Ängste, den individuellen und gesellschaftlichen Ansprüchen an eine ,ideale' Elternschaft nicht gerecht werden zu können, wodurch die Entscheidung für eine Familiengründung oder -erweiterung erschwert werden könnte. Von den Kinderreichen wird außerdem angenommen, dass sie zu wenig Zeit und Geld für jedes ihrer Kinder aufbringen können und damit die Normen des Leitbildes der Verantworteten Elternschaft verletzt werden.

Bezüglich des Kinderreichtums konnten drei gesellschaftlich wahrgenommene Leitbilder identifiziert werden. Das erste wurde mit dem Begriff der Distanz gegenüber Kinderreichen bezeichnet. Bestandteile sind eine negative Sicht auf Kinder, die Einstellung, mehrere Kinder sollten nur die haben, die es sich leisten können, das Empfinden, dass Kinderreiche ,asozial“ sind und die Überzeugung, dass in kinderreichen Familien die Kinder nicht genügend Aufmerksamkeit erhalten. Ein zweites Leitbild bringt eine gemischtgeschlechtliche Geschwisterorientierung zum Ausdruck. Ein weiteres Kind wird angestrebt, da das erste kein Einzelkind bleiben und das Geschwisterkind ein anderes Geschlecht haben soll, ein Stammhalter erwünscht ist und/oder die Öffentlichkeit positiv reagiert, wenn zu einer Familie Mädchen und Jungen gehören. Ein drittes Leitbild entspricht einem positiv konnotiertem Kinderreichtum: Viele Kinder werden als etwas Wundervolles gesehen und von der Allgemeinheit wird angenommen, dass Kinder zu haben als sehr wichtig angesehen wird.

\footnotetext{
* Wir danken der anonymen Gutachterin bzw. dem anonymen Gutachter für die wertvollen Hinweise und die hilfreiche Kritik. Ein besonderer Dank geht an Robert Herter-Eschweiler für die Mikrozensusauswertungen.
} 


\section{$1 \quad$ Einleitung}

Kinderreichtum ${ }^{1}$ erzeugt verschiedene Assoziationen, die auch durch die mediale Inszenierung geprägt sind: Ob es Doku-Soaps im deutschen Privatfernsehen sind wie „XXL-Abenteuer Großfamilie“ oder aber Filme über die „Flodders“ - das Alltagsleben in Großfamilien wird häufig als etwas sehr Lebhaftes, Chaotisches und Abenteuerliches dargestellt. Oftmals wird ein extremes Bild gezeichnet, welches negative Stereotype von kinderreichen Familien in sich bündelt. Betrachtet man die Darstellung von kinderreichen Familien in den deutschen Printmedien, dann dominieren Probleme wie finanzielle Belastungen, innerfamiliäre Konflikte oder unangemessene Wohnverhältnisse (Schulten-Jaspers et al. 2013). In einer Kölner Studie ${ }^{2}$ wurden über 1.000 Texte in den deutschen Printmedien der Jahre 2011 und 2012 dahingehend analysiert, wie darin über kinderreiche Familien und über Familie im Allgemeinen berichtet wurde. Negativ-Klischees wie solche, dass Kinderreiche „asozial“" seien oder „nur Menschen mit Migrationshintergrund viele Kinder“ hätten, werden laut Schulten-Jaspers et al. (2013) eher selten dargestellt. Jedoch haftet solchen Familien ein „Exoten- oder Freak-Image“ an (ebd.), eine große Familie zu haben grenzt an ein Abenteuer, resümieren die Autorinnen der Medienuntersuchung.

Insgesamt zeigen die beschriebenen Beispiele die Faszination, die von kinderreichen Familien ausgeht. In der sozialwissenschaftlichen Forschung jedoch sind sie kaum Gegenstand großer repräsentativer Untersuchungen. Noch weniger ist über die Akzeptanz von kinderreichen Familien in Deutschland bekannt. Aus einigen Studien ist ersichtlich (Keddi et al. 2010, Höhn et al. 2006; Walla et al. 2006), dass sich kinderreiche Familien benachteiligt und diskriminiert fühlen. Eltern negativ bewertet wird. Wie aber steht es insgesamt aus Sicht junger Menschen in Deutschland, die im Familiengründungsalter sind, um das Image von Großfamilien? Ab wann gilt eine Familie hierzulande als kinderreich? Gibt es in Deutschland so etwas wie eine Kultur des Kinderreichtums, ein Milieu, in dem viele Kinder als etwas Positives betrachtet werden? Und wie hängt Kinderreichtum mit Kinderwunsch, Kinderzahl, Bildungshintergrund, Geschlecht, Religion etc. zusammen? Diese Fragen sollen im Folgenden beleuchtet werden.

1 Der Begriff „Kinderreichtum“ wird in der Literatur durchaus kritisch diskutiert, da die implizierte Verbindung von vielen Kindern und Reichtum nicht mehr angemessen erscheint (Bierschock 2010). Im vorliegenden Beitrag wird der Begriff jedoch völlig wertfrei als Alternative zum Begriff „Mehrkindfamilie“ verwendet. „Kinderreich“ wird in diesem Beitrag (vgl. Eggen 2014) als „,...) »zahlreich« begriffen. Dies entspricht der Bezeichnung „familles nombreuses“, der in Frankreich gebräuchlichen Bezeichnung für den schlichten quantitativen Sachverhalt, dass die Familie drei, vier oder mehr Kinder hat" (Eggen 2014: 145).

2 Die inhaltsanalytische Untersuchung von einem Kölner Forscherinnen-Team umfasst 24 deutsche Printmedien, darunter überregionale und regionale Tageszeitungen, Sonntagszeitungen, Wochenzeitungen, Nachrichtenmagazine sowie Zeitschriften, die sich speziell an Familien richten. Ergänzend dazu wurden 14 Experteninterviews mit Wissenschaftlern, Journalisten und Verbandsvertretern geführt. 


\section{Stand der Forschung und theoretische Grundlagen}

\subsection{Kinderreichtum in Deutschland-die demografische Situation}

Nachfolgend werden Ergebnisse aus der Forschung und eigene Datenanalysen auf der Basis des Mikrozensus 2012 vorgestellt. Ziel ist es, vor den soziologischen Analysen einen Eindruck über das demografische Phänomen, die Ist-Situation und die Trends der Vergangenheit zu vermitteln.

Kinderreiche Familien mit mindestens drei Kindern sind in Deutschland inzwischen eine eher seltene Familienform. Im Jahr 2013 gab es insgesamt rund 8,1 Millionen Familien $^{3}$ mit mindestens einem minderjährigen Kind im Haushalt, davon 1,2 Millionen Familien (15\%) mit mindestens drei oder mehr Kindern (Statistisches Bundesamt 2013: 50f.), somit ist jede siebte Familie kinderreich. Dabei leben in den meisten dieser kinderreichen Familien drei Kinder im Haushalt $(77 \%)$, bereits vier Kinder sind wesentlich seltener $(17 \%)$ und mehr als vier (6\%) stellen die absolute Ausnahme dar (Statistisches Bundesamt 2013: 51).

Im Familienleitbildsurvey beträgt der Anteil Kinderreicher an allen Befragten in der Altersgruppe 20 bis 39 Jahre weniger als ein Zehntel (7,1\%), betrachtet man nur die Eltern, dann sind es darunter 17,7 \% Kinderreiche. Die Mehrheit der kinderreichen Eltern (75,7 \%) hat drei Kinder, 24,3\% haben vier oder mehr Kinder, dabei sind in 17,3\% aller Fälle nicht alle Kinder und in 6,4 \% keines dieser Kinder aus der gegenwärtigen Beziehung.

Der allgemeine Trend

Einen anderen Blickwinkel erhält man, wenn die Trends bei den kinderreichen Familien nach Geburtskohorten analysiert werden (vgl. Abbildung 1). Die Ergebnisse unterscheiden sich deutlich von der Querschnittsbetrachtung. Es macht dabei Sinn, nur Frauen zu betrachten, die mit sehr großer Wahrscheinlichkeit kaum noch Kinder bekommen werden. Im Jahr 2012 lagen relativ sichere Daten zum Kinderreichtum für die Frauen der Geburtsjahrgänge 1933 bis 1972 vor. Die Frauen des Jahrgangs 1972 sind im Erhebungsjahr des Mikrozensus 40 Jahre alt geworden. Nach dem 40. Lebensjahr werden nur noch sehr wenige Kinder geboren - das trifft auch auf dritte und vierte Kinder zu.

Es zeigt sich ein deutlicher Trend eines rückläufigen Anteils kinderreicher Familien. Innerhalb der betrachteten Kohorten weist der Jahrgang 1933 mit $\approx 33 \%$ die höchsten Anteile kinderreicher Frauen auf. Der niedrigste Wert wurde für den Jahrgang 1967 mit $\approx 16 \%$ ermittelt. Betrachtet man die etwas jüngeren Jahrgänge, die in gewissem Umfang noch Kinder bekommen werden, deutet sich an, dass der Rückgang des Anteils Kinderreicher sich nicht weiter fortsetzt. Der rückläufige Trend ist sowohl bei den Frauen mit drei als auch mit vier und mehr Kindern zu beobachten. Allerdings sind die Anteile der Parität 3 weniger schnell zurückgegangen. In der Kohorte 1933 waren die Anteile der Frauen mit 3 bzw. 4 und mehr Kindern mit 16,9\% (3 Kinder) und 16,5\% noch sehr ähnlich (vgl. auch Eggen 2014: 151).

3 Ehepaare, Lebensgemeinschaften, alleinerziehende Elternteile. 
Abbildung 1: Anteile von Frauen mit drei bzw. vier und mehr Kindern für ausgewählte Geburtsjahrgänge in Deutschland (in Prozent)

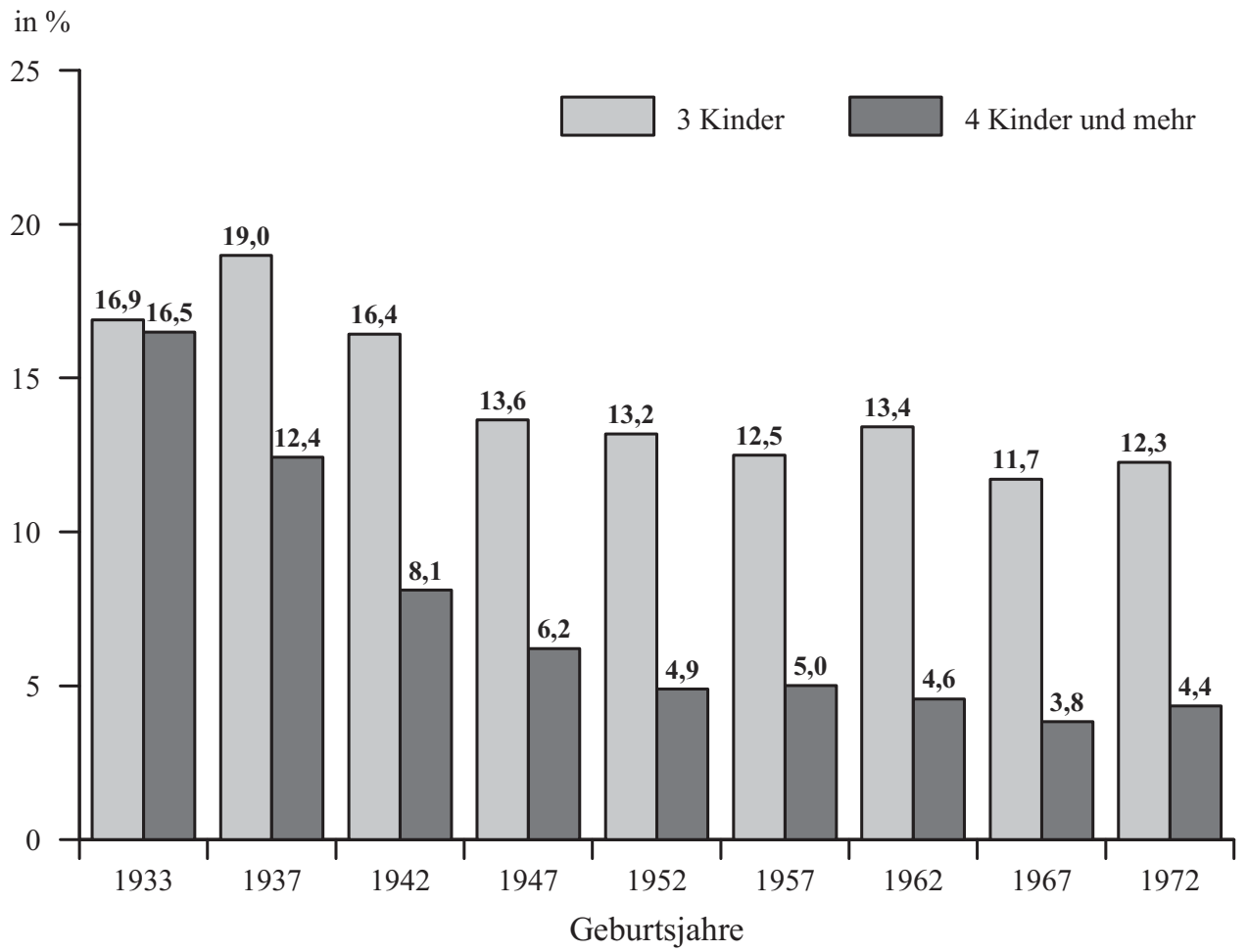

Quelle: Statistisches Bundesamt, Mikrozensus 2012, eigene Berechnungen und Darstellung.

Im Jahrgang 1972 beträgt der Anteil der Frauen mit drei Kindern $\approx 12 \%$ und der mit vier und mehr Kindern $\approx 4 \%$. Familien mit vier und mehr Kindern sind in Deutschland demnach eine tatsächliche Rarität geworden.

\section{Sozialstrukturelle Differenzierungen}

Die Anteile kinderreicher Frauen haben sich sozialstrukturell und regional sehr unterschiedlich entwickelt. Es sind zum Teil erhebliche Unterschiede nach der Lebensform, der beruflichen Bildung, dem Migrationshintergrund, der Erwerbssituation sowie zwischen West- und Ostdeutschland zu finden (vgl. Tabelle 1). 
Tabelle 1: Anteile kinderreicher Frauen in den Geburtsjahrgängen 1969 - 1973 nach ausgewählten sozialstrukturellen Merkmalen in Deutschland

\begin{tabular}{lc}
\hline Merkmale & Anteile in Prozent \\
\hline Lebensform & 19,9 \\
Verheiratet & 8,5 \\
In nichtehelicher Lebensgemeinschaft lebend & 11,3 \\
Ohne Partner im Haushalt & \\
Berufliche Bildung & 33,3 \\
ohne Ausbildung & 13,0 \\
Lehre/Anlernausbildung & 10,9 \\
(Fach-)Hochschule & \\
Erwerbskonstellation & 32,4 \\
Mann Vollzeit / Frau nicht erwerbstätig & 7,9 \\
Mann Vollzeit / Frau Vollzeit & 17,7 \\
Mann Vollzeit / Frau Teilzeit & \\
Migrationshintergrund & 25,3 \\
Ja & 13,0 \\
Nein & \\
Region & \\
West & 16,5 \\
Ost & 12,8 \\
\hline
\end{tabular}

Quelle: Statistisches Bundesamt, Mikrozensus 2012, eigene Berechnungen

Anm.: ${ }^{a}$ jeweils ohne Berlin.

Es gelten folgende Zusammenhänge:

- Verheiratete Frauen in Deutschland haben häufiger drei oder mehr Kinder als Frauen, die zum Befragungszeitpunkt zu einer nichtehelichen Lebensgemeinschaft gehörten oder alleinlebend waren. Das Beispiel der Frauen der Geburtsjahrgänge 1969-1973 veranschaulicht den Zusammenhang. Verheiratete in Deutschland waren zu 19,9 \% kinderreich. Auf Frauen in einer nichtehelichen Lebensgemeinschaft bzw. ohne Partner trifft dies nur zu 8,5 bzw. 11,3 \% zu. Diese Differenzierungen sind im Westen stärker als im Osten ausgeprägt.

- Für die berufliche Bildung gilt: Je höher der Abschluss, desto geringer ist der Anteil kinderreicher Frauen. Besonders auffällig sind dabei die mit 33,3 \% sehr hohen Anteile der Kinderreichen bei den Frauen ohne beruflichen Ausbildungsabschluss. Verfügen die Frauen über eine Lehr- oder Anlernausbildung, sinkt der Anteil auf $13 \%$. Bei einem Fach- oder Hochschulabschluss tritt ein weiterer Rückgang auf 10,9\% ein.

- Einen deutlich differenzierenden Einfluss übt die paarspezifische Erwerbssituation aus. Drei der möglichen Konstellationen sind in der Tabelle 1 dargestellt. Die traditionelle Erwerbskonstellation - Mann in Vollzeit, Frau nicht erwerbstätig - begünstigt Kinderreichtum oder vice versa: Kinderreichtum verhindert Erwerbstätigkeit (bei Müttern). $32,4 \%$ der Frauen haben 3 oder mehr Kinder. Dagegen begrenzt die Vollzeiterwerbs- 
tätigkeit der Frau den Kinderreichtum (oder umgekehrt). In der Konstellation, in der beide Partner vollerwerbstätig sind, beträgt der Anteil dritter und weiterer Kinder nur 7,9 \%.

- Das Vorhandensein eines Migrationshintergrunds geht mit einem höheren Anteil kinderreicher Frauen einher. Verfügen Frauen über einen Migrationshintergrund, dann ist der Anteil Kinderreicher mit 25,3 \% nahezu doppelt so hoch wie bei Frauen ohne Migrationshintergrund mit 13,0 \% (vgl. auch BMFSFJ 2013: 7).

- Aus der regionalen Sicht sind die West-Ost-Unterschiede zu betonen. Der Anteil kinderreicher Frauen ist generell in Westdeutschland höher als in den neuen Bundesländern. Die Frauen der Geburtsjahrgänge 1969-1973 sind im Osten zu 12,8 \% und im Westen zu 16,5 \% kinderreich. Das ist zum einen darauf zurückzuführen, dass in Westdeutschland der enge Zusammenhang von Ehe und Elternschaft stärker als im Osten erhalten geblieben ist und dadurch die Verbreitung von Familien mit 3 oder mehr Kindern begünstigt wird (Dorbritz/Schneider 2012: 30ff.). Zum anderen ist auf die hohe Erwerbsorientierung der ostdeutschen Frauen zu verweisen, die aufgrund dieser Situation viel öfter nur ein Kind und nicht drei oder mehr zur Welt gebracht haben.

Verglichen mit Familien mit einem oder zwei Kindern weisen kinderreiche Familien einige besondere Merkmale auf: Eltern von später mindestens drei Kindern ziehen deutlich früher aus dem elterlichen Haushalt aus, als andere Männer und Frauen (Keddi et al. 2010; Bien/Marbach 2007). Zudem sind die Mütter bei der Geburt des ersten Kindes wesentlich jünger. Im Jahr 2012 lag das durchschnittliche Alter von 35- bis 44-jährigen Müttern bei Geburt des ersten Kindes bei 30 Jahren, wenn sie ein Kind geboren hatten, bei 25 Jahren, wenn sie 3 Kinder geboren hatten und bei 23 Jahren, wenn sie mehr als drei Kinder hatten (Statistisches Bundesamt 2013: 21). Zusätzlich weisen Mehrkindfamilien in Industrieländern ein „verdichtetes“ Muster der Familiengründung auf, d. h. die Kinder werden in engem zeitlichem Abstand geboren (Keddi et al. 2010, Nauck 1995: 139).

\subsection{Forschungsstand zu kinderreichen Familien}

In der Forschungsliteratur werden Familien mit drei und mehr Kindern als kinderreich oder als „große Familien“ definiert (vgl. Eggen/Leschhorn 2004). Ab wann eine Familie als kinderreich gilt, ist dabei immer im historischen Kontext zu betrachten: Während um das Jahr 1900 Familien mit drei bis fünf Kindern als durchschnittlich und damit als „normal“ galten (Eggen 2014; Eggen/Rupp 2006; Eggen/Leschhorn 2004), bedeutet dies heute ein Abweichen von der bundesweiten Norm. Berens (2004) kommt in seiner Analyse ebenfalls zu dem Schluss, dass gegenwärtig Familien in Deutschland ab drei Kindern als kinderreich angesehen werden.

Aus der demografischen Sicht wird vor allem auf den Rückgang von Mehrkindfamilien und dessen Folgen für das Geburtenniveau fokussiert. Ein Trend zu kleineren Familien besteht europaweit, jedoch liegt Deutschland zusammen mit einigen süd- und osteuropäischen Ländern unter dem gesamteuropäischen Durchschnitt (Rost 2009). Demografisch gesehen schlagen sich die Folgen des Rückgangs in einer geringeren Geburtenrate nieder, wobei Bertram et al. (2005: 7) diesen sinkenden Anteil Kinderreicher und nicht die steigende Kinderlosigkeit als Hauptursache für die niedrige Geburtenrate benennen. Länder mit ähnlich hoher Kinderlosigkeit wie z. B. Finnland oder die USA hätten wegen eines wesentlich höheren Anteils an Mehrkindfamilien auch eine deutlich höhere Geburtenrate als 
Deutschland (ebenda: 11). Die Entstehung von Mehrkindfamilien steht seltener im Mittelpunkt des Interesses. Es existiert aber z. B. ein Forschungsstrang, der die Geschlechterpräferenz von Eltern für ihre Kinder mit der Parität in Zusammenhang bringt. Dabei lässt sich mit relativ großer Sicherheit sagen, dass in den westeuropäischen Ländern, wenn überhaupt, für Kinder überwiegend ein Geschlechtermix gewünscht wird. Inwiefern dies tatsächlich Auswirkungen auf die Kinderzahl hat, bleibt allerdings umstritten (z. B. Gray/ Evans 2004; Hank/Kohler 2003; Hank/Kohler 2000). Werthaltungen beeinflussen auch in anderer Hinsicht die Entstehung von kinderreichen Familien. So unterscheiden sich die Eltern in Mehrkindfamilien schon vor ihrer Familiengründung von anderen Eltern bzw. Paaren mit Kinderwunsch, da sie Familie und Kinder wesentlich höher schätzen als Freizeit, Beruf und Konsum (Keddi et al. 2010: 77; Eggen/Rupp 2006; Bruchholz et al. 2002). Rupp und Blossfeld (2008: 164) sprechen von einer hohen intrinsischen Motivation zur Elternschaft. Für einen Teil der Mehrkindfamilien verlief die Entwicklung zu einer solchen allerdings auch völlig ungeplant. Ein zusätzliches Indiz für die hohe Bedeutung von Werteinstellungen bei der Entstehung von kinderreichen Familien ist schließlich die starke Bedeutung von Religion und Religiosität in diesem Kontext (Keddi et al. 2010: 83; Bien/ Marbach 2007; Blume et al. 2006; Bruchholz et al. 2002).

Ein weiterer häufiger Untersuchungsgegenstand in Bezug auf Mehrkindfamilien sind deren wirtschaftliche und sozioökonomische Lebensbedingungen (siehe auch Keddi et al. 2010). Neben Einkommenslage, Bildung und Migrationshintergrund zählen dazu z. B. die Wohnverhältnisse von kinderreichen Familien oder deren Alltagsgestaltung, aber auch die Erwerbskonstellationen, wobei mit zunehmender Kinderzahl eine doppelte Erwerbstätigkeit der Eltern immer schwieriger zu realisieren ist (Eggen/Rupp 2006). Auch der Einfluss der Herkunftsfamilie wird in den Blick genommen (Keddi et al. 2010; Rille-Pfeiffer et al. 2009; Bien/Marbach 2007). Eine höhere Geschwisterzahl der Eltern hat einen positiven Effekt auf die eigene Kinderzahl und erhöht die Wahrscheinlichkeit von Mehrkindfamilien. Von psychologischer Seite her wird schließlich vor allem die Bedeutung von Familiengröße und Geschwisterkonstellation, z. B. für die Bildungs- und Entwicklungschancen der Kinder betrachtet. Für den Zusammenhang zwischen Geschwisterzahl und Bildungserfolg beispielsweise liegen dabei überwiegend negative Befunde vor (Keddi et al. 2010; BMFSFJ 2007).

In der Literatur wird auch immer wieder auf die besondere finanzielle Situation kinderreicher Familien hingewiesen, die in der Regel ein vergleichsweise niedrigeres Pro-KopfEinkommen aufweisen als kleinere Familien. Viele Kinder zu haben stellt einen wesentlichen Faktor für Einkommensarmut dar (BMFSFJ 2007; Eggen/Leschhorn 2004), laut Allmendinger und Hinz (1998) liegt der Anteil der Haushalte mit weniger als $50 \%$ des Nettoäquivalenzeinkommens bei drei und mehr Kindern bei 21,2 \%. Über ein höheres Einkommen verfügen dagegen unterdurchschnittlich viele Mehrkindfamilien, d. h. $5 \% \mathrm{im}$ Vergleich zu 8 \% der Gesamtbevölkerung (Eggen/Rupp 2006).

Aufgrund der These, dass Stieffamilien häufiger größer als andere Familien sind, da die neue Paarbeziehung mit einem weiteren gemeinsamen Kind manifestiert und nach außen demonstriert werden soll, sind Stieffamilien auch hinsichtlich des Kinderreichtums zu betrachten. Sie entstehen heute vor allem als Scheidungsfolge und weisen verschiedene Formen auf. Es können eheliche und nichteheliche Stieffamilien unterschieden werden, wobei auch die bilokalen Paarbeziehungen zu beachten sind. Daneben werden sie nach der Zugehörigkeit der Kinder zu den Partnern klassifiziert. Aus dem Blickwinkel des Kinderreichtums sind vor allem die komplexen Stieffamilien interessant, da zu den in die Bezie- 
hung mitgebrachten Kindern noch leibliche Kinder beider Partner hinzukommen. Anhand der von Thomson mit dem Family and Fertility Survey erzielten Ergebnisse weist Peukert (2008: 214) darauf hin, dass Paare in dieser Lebensform ,einen starken Drang nach (mindestens) einem gemeinsamen Kind verspüren, auch wenn bereits ein Kind oder mehrere Kinder aus einer früheren Beziehung vorhanden sind". In Deutschland haben sich insbesondere Klein und Eckard (2004: 91) mit dem generativen Verhalten in Stieffamilien beschäftigt. Sie kommen bei der Analyse zur Wahrscheinlichkeit einer Geburt des ersten gemeinsamen Kindes zu diesem Ergebnis:

„Eine zunächst festgestellte geringere Neigung zur Familiengründung durch Geburt eines ersten gemeinsamen Kindes in Stieffamilien ist im Wesentlichen auf das höhere Durchschnittsalter der Frau in Stieffamilien zurückzuführen.“(ebd.)

\section{Ergebnisse}

Im Folgenden werden die verschiedenen inhaltlichen Dimensionen von Kinderreichtum analysiert (3.1), die gesellschaftlich zugeschriebene Bedeutung von Geschwistern erläutert (3.2) und schließlich geprüft, ob in der Gesellschaft kollektiv wahrgenommene Leitbilder zum Kinderreichtum existieren und wie sie nach sozialstrukturellen Merkmalen verteilt sind (3.3).

\subsection{Dimensionen von Kinderreichtum}

Bevor die Fragen zum Kinderreichtum gestellt wurden, war eine Frage zur Definition von Kinderreichtum aus Sicht der Befragten vorgeschaltet: Es wurde gefragt: „Ab welcher Anzahl ist eine Familie kinderreich?". Hierzu konnte sowohl eine genaue Angabe als auch eine Spanne (von ...bis ...) abgegeben werden. Insgesamt definierten die Befragten eine Familie mit durchschnittlich vier Kindern als kinderreich. Die Einstellungen zu Familien mit vielen Kindern wurden im Familienleitbildsurvey über vier verschiedene Aussagen gemessen, die mit einer vierstufigen Zustimmungsskala erfasst wurden.

Kinderreichtum als positives Phänomen

Zunächst wurde auf einer vierstufigen Zustimmungsskala erhoben, inwiefern die Befragten selbst der Aussage zustimmen, dass viele Kinder etwas Wundervolles sind (vgl. Tabelle 2).

Eine Mehrheit von $72,4 \%$ hat dieser Aussage auf der individuellen Ebene zugestimmt. Geschlechterunterschiede konnten dabei nicht festgestellt werden. Jedoch sind Personen in den alten Bundesländern positiver gegenüber vielen Kindern eingestellt $(73,8 \%)$ als solche aus den neuen Bundesländern (66,7\%). Gleichermaßen verschieden bewerten Eltern $(75,7 \%)$ im Vergleich zu Kinderlosen $(70,1 \%)$ große Familien. Aufgeschlüsselt nach Kinderzahl ergeben sich weitere Unterschiede (nicht tabellarisch dargestellt): So glauben Elternteile mit einem oder zwei Kindern (76 \%), dass große Familien ein schlechtes Image in der Gesellschaft haben, Menschen mit mindestens drei Kindern stimmen dieser Aussage sogar zu 81 \% zu. Dahinter stecken vermutlich Erfahrungswerte von Menschen mit großer Familie: Eltern von Mehrkindfamilien sind wesentlich häufiger als andere davon überzeugt, 
dass Familien mit drei oder mehr Kindern gesellschaftlich benachteiligt werden (Keddi et al. 2010: 85). Ähnlich bewerten auch Personen mit Migrationshintergrund ${ }^{4}$ Kinderreichtum deutlich positiver $(76,6 \%)$ als solche ohne Migrationshintergrund (71,0\%). Die Altersunterschiede sind hingegen nur schwach ausgeprägt.

Tabelle 2: Zustimmung ${ }^{5}$ auf persönlicher und Allgemeinheits-Ebene zu verschiedenen Dimensionen des Kinderreichtums nach soziodemografischen Merkmalen (in Prozent)

\begin{tabular}{|c|c|c|c|c|c|c|c|}
\hline \multirow[t]{2}{*}{ Merkmal } & \multirow[t]{2}{*}{ Ausprägung } & \multicolumn{2}{|c|}{$\begin{array}{c}\text { Viele Kinder } \\
\text { sind etwas } \\
\text { Wundervolles. }\end{array}$} & \multicolumn{2}{|c|}{$\begin{array}{c}\text { Kinderreiche } \\
\text { gelten als } \\
\text { asozial. }\end{array}$} & \multirow{2}{*}{$\begin{array}{l}\text { Nur Familien, } \\
\text { die genügend } \\
\text { Geld haben, } \\
\text { sollten sich viele } \\
\text { Kinder leisten. } \\
\text { Allg. }\end{array}$} & \multirow{2}{*}{$\begin{array}{l}\text { Wer viele Kinder } \\
\text { hat, kann sich um } \\
\text { das einzelne Kind } \\
\text { nicht mehr richtig } \\
\text { kümmern. } \\
\text { Allg. }\end{array}$} \\
\hline & & Pers. & Allg. & Pers. & Allg. & & \\
\hline \multirow[t]{2}{*}{ Geschlecht } & Männer & 71,4 & $41,3^{* * *}$ & 8,0 & $63,9^{* * *}$ & $75,0^{* * *}$ & $67,7^{* * *}$ \\
\hline & Frauen & 73,4 & $32,9^{* * *}$ & 9,0 & $80,3^{* * *}$ & $82,3^{* * *}$ & $77,8^{* * *}$ \\
\hline \multirow[t]{2}{*}{ Wohnort } & West & $73,8^{* * *}$ & $37,9^{*}$ & $7,9^{*}$ & 72,3 & 79,0 & 72,7 \\
\hline & Ost (inkl. Berlin) & $66,7^{* * *}$ & $34,3^{*}$ & $11,0^{*}$ & 70,9 & 77,0 & 72,7 \\
\hline \multirow{3}{*}{$\begin{array}{l}\text { Bildungs- } \\
\text { niveau }^{\text {a }}\end{array}$} & Niedrig & $75,1^{* * *}$ & $48,8^{* * *}$ & 8,1 & $54,8^{* * *}$ & $68,0^{* * *}$ & 69,8 \\
\hline & Mittel & $70,5^{* * *}$ & $35,7^{* * *}$ & 8,3 & $73,7^{* * *}$ & $77,8^{* * *}$ & 73,8 \\
\hline & Hoch & $76,4^{* * *}$ & $37,0^{* * *}$ & 9,0 & $73,4^{* * *}$ & $84,7^{* * *}$ & 71,4 \\
\hline \multirow{2}{*}{$\begin{array}{l}\text { Haben Sie } \\
\text { Kinder? }^{\text {b }}\end{array}$} & $\mathrm{Ja}$ & $75,7^{* * *}$ & $30,4^{* * *}$ & 9,4 & $77,1^{* * *}$ & $77,0^{*}$ & $76,3^{* * *}$ \\
\hline & Nein & $70,1^{* * *}$ & $41,7^{* * *}$ & 7,9 & $68,6^{* * *}$ & $79,7^{*}$ & $70,2^{* * *}$ \\
\hline \multirow{2}{*}{$\begin{array}{l}\text { Migrations- } \\
\text { hintergrund }^{\mathrm{c}}\end{array}$} & $\mathrm{Ja}$ & $76,6^{* * *}$ & $45,7^{* * * *}$ & 8,3 & $65,2^{* * *}$ & $74,5^{* * *}$ & $69,7^{* *}$ \\
\hline & Nein & $71,0^{* * *}$ & $34,4^{* * *}$ & 8,6 & $74,1^{* * *}$ & $79,9^{* * *}$ & $73,6^{* *}$ \\
\hline \multirow[t]{2}{*}{ Altersgruppe } & $20-29$ & 71,4 & $40,3^{* * *}$ & 8,3 & $67,3^{* * *}$ & 78,9 & $70,9^{* *}$ \\
\hline & $30-39$ & 73,4 & $33,9^{* * *}$ & 8,7 & $76,7^{* * *}$ & 78,4 & $74,4^{* *}$ \\
\hline Gesamt & & 72,4 & 37,1 & 8,5 & 72,0 & 78,6 & 72,7 \\
\hline
\end{tabular}

Quelle: FLB (2012), gewichtete Daten, eigene Berechnungen

Anm.: ${ }^{* * *} \mathrm{p} \leq 0,001 ;{ }^{* *} \mathrm{p} \leq 0,01 ;{ }^{*} \mathrm{p} \leq 0,05$ (Chi ${ }^{2}$-Test). Pers.: Persönliche Sicht; Allg.: Wahrgenommene Sicht in der Gesellschaft. ${ }^{a}$ Die Zuordnung des Bildungsniveaus erfolgt nach ISCED 97: Low (1-2), Medium (3A-4A), High (5B-6). ${ }^{\mathrm{b}}$ „Haben Sie Kinder? Ich meine sowohl Ihre eigenen Kinder als auch Adoptivkinder, egal, wo sie momentan wohnen." c Personen, die selbst und/oder deren Elternteil(e) eingewandert sind bzw. Personen mit ausländischer Staatsbürgerschaft (zur Erfassung wurde das Geburtsland des Befragten, dessen Staatsangehörigkeit sowie das Geburtsland der Eltern und deren Staatsangehörigkeit hinzugezogen). Demnach sind in dieser Kategorie Personen mit Migrationshintergrund, zugewanderte Ausländer, in Deutschland geborene Ausländer, eingebürgerte Ausländer, Spätaussiedler und Personen mit zumindest einem Elternteil, der eines der genannten Merkmale erfüllt.

4 Innerhalb der Befragtengruppe mit Migrationshintergrund verbirgt sich eine heterogene Zusammenstellung, am häufigsten haben die Personen einen türkischen Migrationshintergrund.

5 Alle in diesem Beitrag verwendeten Items wurden über eine vierstufige Zustimmungsskala ohne Mitte erhoben. Die Antwortkategorien wurden für sämtliche Tabellen dichotomisiert, d. h. 1=,,Stimme voll und ganz zu“ und 2=,Stimme eher zu“ sind zusammengefasst zum Wert 1, auf den Wert 0 umkodiert wurden die Antwortkategorien 3="Stimme eher nicht zu" sowie 4="Stimme überhaupt nicht zu". 
Des Weiteren wurde gemessen, wie die Befragten die Sicht der Gesellschaft auf die Aussagen zum Kinderreichtum wahrnehmen: Dass viele Kinder etwas Wundervolles sind, sehen insgesamt rund ein Drittel der Befragten in der Allgemeinheit, wobei signifikant mehr Männer (41,3\%) als Frauen (32,9\%) diese Auffassung vertreten. Niedrig gebildete Befragte $(48,8 \%)$ finden signifikant häufiger, dass in der Gesellschaft Kinderreichtum geschätzt wird als mittel $(35,7 \%)$ und höher Qualifizierte $(37,0 \%)$. Sehr viel stärker als Eltern $(30,4 \%)$ sehen Kinderlose $(41,7 \%)$ eine große allgemeine Wertschätzung von Kinderreichtum. Vermutlich basieren diese Differenzen auf negativen Alltagserfahrungen von Eltern, die schon bei einem oder zwei Kindern gemacht werden. Der Wert von vielen Kindern in der Gesellschaft wird auch aus Sicht der Personen mit Migrationshintergrund deutlich positiver eingestuft (45,7\% vs. 34,4\%), und auch die ältere Altersgruppe (30- bis 39-Jährige) steht dem gesellschaftlichen Image von Kinderreichtum skeptischer gegenüber als die jüngere (20- bis 29-Jährige).

Dies liegt daran, dass die älteren Befragten häufiger schon eine Familie gegründet haben und eine realistischere Sichtweise auf ein Leben mit Kindern und die Anforderungen an Eltern im Lebensalltag aufweisen. Individuelle Erfahrungen färben vermutlich auch auf die Gesamtsicht der Gesellschaft ab, persönliche Erfahrungen und die in der Öffentlichkeit wahrgenommene Stimmung gegenüber Großfamilien sind schwer trennbar.

\section{Negativ-Image von kinderreichen Familien}

Eine weitere Dimension von Kinderreichtum ist das Image von Kinderreichen. Zunächst wurde dies aus der individuellen Perspektive gefragt, mit der Aussage „Kinderreiche gelten als asozial“". Dem stimmen knapp ein Zehntel der männlichen und weiblichen Befragten zu. Der gewählte Stimulus „asozial“ macht eine persönliche Zustimmung vermutlich etwas schwieriger, da hier möglicherweise auch Effekte der sozialen Erwünschtheit wirken. Auch im Hinblick auf Ost- und Westdeutschland, Bildungsabschluss, Elternschaft, Migrationshintergrund und nach Alter lassen sich keine nennenswerten Unterschiede feststellen.

Deutlich differenzierter sind die Aussagen bei der Sicht der Befragten auf die Gesellschaft: Hier nehmen fast drei Viertel der Befragten in der Gesellschaft wahr, dass Kinderreiche als „asozial“" bewertet werden. Wesentlich mehr Frauen (80,3\%) als Männer $(63,9 \%)$ glauben, dass Kinderreiche in der Gesellschaft als ,asozial“" gelten. Gleiches denken auch mittel $(73,7 \%)$ und hoch Gebildete $(73,4 \%)$, und zwar signifikant häufiger als niedrige Gebildete $(54,8 \%)$. Auch drei Viertel der Eltern sehen in der Gesellschaft ein negatives Image von Familien mit vielen Kindern (77,1 \% vs. 68,6 \%), ähnliches gilt für Menschen ohne Migrationshintergrund (74,1\%) eher als für welche mit $(65,2 \%)$ und für Ältere $(76,7 \%)$ im Vergleich wesentlich häufiger als für Jüngere $(67,3 \%)$.

\section{Bedingungen für Kinderreichtum: Finanzielle Ausstattung}

Des Weiteren wurde gemessen, wie die Befragten die Gesellschaft hinsichtlich der Aussage einschätzen „Nur Familien, die genügend Geld haben, sollten sich viele Kinder leisten.“ Diese Einstellung sahen Frauen (82,3 \% vs. Männer = 75,0 \%) und Hochgebildete $(84,7$ \% im Vergleich zu mittel $=77,8 \%$; niedrig $=68,0 \%$ ) sowie Personen ohne Migrationshintergrund (79,9\% vs. 74,5 \%) signifikant häufiger in der Gesellschaft verbreitet. Die Gründung einer Großfamilie ist demnach gesellschaftlich als finanziell voraussetzungsvoll zu bewerten. 
Betreuungsprobleme bei Kinderreichen

Nachfolgend wird dargestellt, wie aus Sicht der Befragten die Gesellschaft einschätzt, ob sich Eltern mit vielen Kindern um das einzelne Kind ausreichend kümmern können. Deutlich mehr Frauen $(77,8 \%$ ) als Männer $(67,7 \%)$ nehmen in der Gesellschaft wahr, dass Kinderreichen unterstellt wird, sich nicht mehr richtig um das einzelne Kind kümmern zu können. Ähnlich häufig sehen das auch Eltern so (76,3\% vs. Kinderlose: 70,2 \%) und die 30- bis 39-Jährigen (74,4 \% vs. 20 - bis 29-Jährige: 70,9 \%).

\subsection{Die Rolle von Geschwistern}

Für die Entscheidung, wie viele Kinder ein Paar bekommen möchte, spielt auch die Wertigkeit von Geschwisterkindern eine Rolle. Im Leitbildsurvey wurde dies ausschließlich auf gesellschaftlicher Ebene gefragt, da wir davon ausgehen, dass es soziale Normen gibt, wie viele Kinder eine Familie umfassen soll und welches Geschlecht Kinder idealerweise haben sollten. Diese Geschwisterleitbilder wiederum nehmen vielleicht auch einen Einfluss darauf, ob sich Paare zusätzlich ein zweites, drittes Kind oder noch mehr Kinder wünschen.

In der Gesellschaft wird häufig (51,6\%) ein negatives Bild von Einzelkindern gesehen. Auch die Sichtweise, dass eine Familie einen Stammhalter benötigt, ist mit rund 23,2 \% eine recht häufig wahrgenommene Norm innerhalb der Gesellschaft. Die überwiegende Mehrheit $(77,8 \%)$ stimmt zudem der Aussage zu, dass es in der Allgemeinheit die Vorstellung gibt, dass sowohl ein Mädchen als auch ein Junge unter den Geschwistern vorhanden sein sollte.

Betrachtet man die Geschwisternormen differenziert nach soziodemografischen Merkmalen, dann ergeben sich einige signifikante Unterschiede: Eine negative Wahrnehmung von Einzelkindern in der Gesellschaft kommt in den alten Bundesländern signifikant häufiger vor $(53,6 \%)$ als in den neuen Bundesländern (43,5\%). Es bleibt zu klären, ob die tatsächlichen Familienformen einen Einfluss auf das Image von Einzelkindern haben, denn in Ostdeutschland gibt es wesentlich häufiger Ein-Kind-Familien als in Westdeutschland (Bujard et al. 2012). Oder ob das Image dazu führt, dass es regionale Unterschiede hinsichtlich des generativen Verhaltens gibt. Außerdem nehmen niedrig $(56,7 \%)$ und hoch Qualifizierte (56,4\%) häufiger als mittel Qualifizierte (49,6\%) wahr, dass es für die Entwicklung von Kindern schlecht ist, wenn sie ohne Geschwister aufwachsen. Die Stammhalter-Norm sehen etwas mehr Männer (25,6\%) als Frauen (20,7\%) sowie etwas mehr ältere Befragte (30- bis 39-Jährige: 25,6\%) als jüngere Befragte (20- bis 29-Jährige: $20,8 \%$ ) weit verbreitet. Dass ein Junge und ein Mädchen unter den Geschwisterkindern dabei sein sollte, das sehen etwas mehr männliche $(80,0 \%)$ als weibliche Befragte $(75,5 \%)$ für allgemein gültig ${ }^{6}$. Niedrig Qualifizierte $(87,3 \%)$ sind wesentlich häufiger als mittel $(77,7 \%)$ oder hoch Qualifizierte (76,2\%) der Ansicht, dass die Allgemeinheit ein geschlechter-heterogenes Geschwisterpaar wichtig findet. Ähnlich verhält es sich, wenn Personen mit und ohne Migrationshintergrund gegenübergestellt werden: Mit 81,8\% sind Befragte mit Migrationshintergrund häufiger als solche ohne (76,6 \%) der Meinung, dass gemischte Geschwisterkonstellationen in der Öffentlichkeit als „gut“ bewertet werden.

6 An dieser Stelle wäre es gewinnbringend, auch die individuelle Einstellung zu den Geschwistern zu kennen, um sie gegenüberstellen zu können. Dies soll in einer geplanten Wiederholungsbefragung untersucht werden. 


\subsection{Wahrgenommenes gesellschaftliches Leitbild des Kinderreichtums}

Die gesellschaftlich ${ }^{7}$ wahrgenommenen Einstellungs-Items wurden mittels einer Hauptkomponentenanalyse untersucht, um Leitbilder zu identifizieren. Es ergeben sich drei Leitbilder, zwei davon treten markanter in Erscheinung: Ein ,gesellschaftliches Leitbild der Distanz zum Kinderreichtum“ und eines der ,gemischtgeschlechtlichen Geschwisterorientierung“. Neben diesen beiden zeigt sich auch ein „Leitbild des Kinderreichtums“ (vgl. Tabelle 3).

Das erste gesellschaftlich wahrgenommene Leitbild der Distanz gegenüber Kinderreichtum besteht (Komponente 1) aus vier Items und umfasst die gesellschaftlich wahrgenommene Ablehnung, dass viele Kinder etwas Wundervolles seien. Gleichermaßen verbunden ist damit ein hoher gefühlter Anspruch, dass zur Gründung einer Großfamilie auch ausreichend finanzielle Kapazitäten vorhanden sein sollten. Damit einhergeht auch die Empfindung, dass Kinderreiche von der Allgemeinheit als ,asozial“ verurteilt werden, u. a. vermutlich auch deswegen, weil das Vorurteil gesehen wird, dass sich in kinderreichen Familien die Eltern nicht mehr ausreichend um die vielen Kinder kümmern können.

Tabelle 3: Hauptkomponentenanalyse zu verschiedenen Dimensionen des Kinderreichtums auf gesellschaftlicher Ebene (basierend auf Wahrnehmung der Befragten)

\begin{tabular}{lrrr}
\hline & \multicolumn{3}{c}{ Komponente } \\
& 1 & 2 & \multicolumn{1}{c}{3} \\
\hline Anteil erklärter Varianz (in Prozent) & 24,6 & 16,9 & 12,8 \\
\hline Cronbach's Alpha $\alpha$ &, 61 &, 42 &, 29 \\
\hline Viele Kinder sind etwas Wundervolles ${ }^{\text {a. }}$ &,- 536 & &, 448 \\
Nur Familien, die genügend Geld haben, sollten sich viele Kinder leisten. &, 670 & & \\
Kinderreiche gelten als asozial. &, 754 & \\
Wer viele Kinder hat, kann sich um das einzelne Kind nicht mehr &, 705 & \\
richtig kümmern. & &, 529 \\
\hline Es ist schlecht, wenn ein Kind als Einzelkind aufwächst. &, 755 & \\
Eine Familie braucht einen Jungen als Stammhalter. &, 731 & \\
Am besten ist es, wenn ein Junge und ein Mädchen dabei sind. & &, 875 \\
Wichtigkeit, Kinder zu haben. & & \\
\hline Quelle: FLB (2012), gewichtete Daten, eigene Berechnungen & & \\
Anm.: Extraktionsmethode: Hauptkomponentenanalyse, Varimax, rotierte Komponentenmatrix, die ersten \\
drei Komponenten erklären 54,3 \% der Gesamtvarianz, basierend auf allen Befragten, die die Sicht \\
der Allgemeinheit eingeschätzt haben. Werte ab ,4 werden angezeigt. ${ }^{2}$ Dieses Item wurde für die \\
Indexbildung umkodiert.
\end{tabular}

Das zweite Leitbild (Komponente 2) beinhaltet drei Items: Darin gehen die Befragten davon aus, dass Einzelkinder in der Gesellschaft als kritisch betrachtet werden, dass es kollektiv verbreitet noch Stammhalter-Wünsche gibt beim Geschlecht des Kindes und dass es die Öffentlichkeit als positiv bewertet, wenn unter Geschwisterkindern sowohl ein Mädchen als auch ein Junge vertreten ist. Daher wird dies als gesellschaftlich wahrgenommenes Leitbild der gemischtgeschlechtlichen Geschwisterorientierung bezeichnet.

$7 \quad$ Für die Identifikation von individuellen Leitbildern zum Kinderreichtum sind lediglich zwei Items im Fragebogen enthalten, so dass diese nicht möglich ist. 
Die dritte Dimension (Komponente 3), die extrahiert wurde, umfasst lediglich zwei Items und hat eine geringe interne Konsistenz. Dennoch werden die beiden Variablen zusammengefasst, um sie inhaltlich weiter bearbeiten zu können. Wir bezeichnen dies als das gesellschaftlich wahrgenommene Leitbild des Kinderreichtums. Es beinhaltet die positive Faktorladung hinsichtlich der Bewertung, dass viele Kinder etwas Wundervolles sind und die gesellschaftlich wahrgenommene Wichtigkeit, Kinder zu haben.

Für alle drei Dimensionen wurden Summenindizes gebildet, die jeweils durch die Anzahl ihrer einbezogenen Variablen dividiert wurden. In einem weiteren Schritt wurden aus den drei Indizes dichotome Variablen gebildet.

\section{Kinderreichtumsleitbilder: Zwischen Ablehnung und Befürwortung}

Eine überwiegende Mehrheit der Befragten (71,4\%) nimmt in Deutschland ein negatives Leitbild des Kinderreichtums wahr (vgl. Tabelle 4), welches verbunden ist mit sozialen Abstiegssorgen, sozialem Statusverlust und Überforderungsproblemen. Die Hälfte (52,3\%) sieht die Zwei- bzw. Mehr-Kind-Norm (Geschwisternorm = „man sollte mit Geschwistern aufwachsen") weit verbreitet. Rund ein Drittel $(38,1 \%)$ nimmt ein vorbehaltlos positives Leitbild des Kinderreichtums in der Gesellschaft wahr.

In Tabelle 4 sind die drei gesellschaftlichen Leitbilder zum Kinderreichtum nach soziodemografischen Aspekten untergliedert, deutliche signifikante Unterschiede zeichnen sich ab: Mehr Frauen (78,4 \%) als Männer (64,7\%) sehen in der Gesellschaft das Leitbild der Distanz zum Kinderreichtum. Dies könnte damit zusammenhängen, dass Frauen im Sample häufiger Mutter sind und daher auch eher die Erfahrung machen, dass Kinder in der Öffentlichkeit abgelehnt werden (Erfahrungseffekt). Auch mittel und hoch Qualifizierte nehmen häufiger die Ablehnung wahr (beide Gruppen $\approx 73 \%$ ). Ein weiteres Argument für den Erfahrungseffekt erweist sich bei den Eltern, die mit 76,3\% sehr viel häufiger die Ablehnung von Kinderreichtum beobachten als Kinderlose (68,2\%). Uneindeutig ist das Ergebnis hinsichtlich der Herkunft: Personen mit Migrationshintergrund (welche in der Stichprobe häufig türkische Wurzeln haben) sehen signifikant weniger $(64,6 \%)$ als Befragte ohne Migrationshintergrund (73,6 \%) ein negatives Image von Kinderreichen. Dies könnte damit zu tun haben, dass solche Personen sich eher in (innerethnischen) Milieus bewegen, in denen (viele) Kinder zu haben, kulturell akzeptierter ist. Laut der amtlichen Zahlen kommen bei Personen mit Migrationshintergrund etwas häufiger Mehrkindfamilien vor, zudem könnte es sein, dass sich diese Personengruppe eher mit Verwandten und Freunden aus ihrem Herkunftsland vergleicht. Dies würde die Theorie des sozialen Vergleichs stützen: Aufgrund dieser (Kessler/Harth 2008, Kessler et al. 2000) kann vermutet werden, dass insbesondere durch den Status der Migration und durch die engen innerethnischen Netzwerkstrukturen von hier bei Personen mit türkischem Migrationshintergrund in Deutschland die unmittelbare soziale Umgebung eine zentrale Rolle für die Beurteilung von Kinderreichtum spielen könnte. Es ist zudem denkbar, dass diese Personen zur Beurteilung von Familiengrößen andere Maßstäbe anlegen, die sich eher an ihrer eigenen Bezugsgruppe orientieren. Ein weiteres Kriterium, welches die Annahme des Erfahrungseffekts stützt, ist, dass auch die Gruppe der 30- bis 39-Jährigen häufiger ein gesellschaftliches Leitbild der Distanz zum Kinderreichtum sieht (Ältere $=75,1 \%$ vs. Jüngere $=67,8 \%$ ).

Hinsichtlich der Wahrnehmung des gesellschaftlichen Leitbildes der gemischtgeschlechtlichen Geschwisterorientierung, das indirekt auch Aspekte des Kinderreichtums in sich trägt, nehmen die Befragten aus den alten Bundesländern die Allgemeinheit etwas aufge- 
schlossener (53,3\%) wahr als diejenigen aus den neuen Bundesländern (48,0\%). Niedrig Gebildete $(63,8 \%)$ und Personen mit Migrationshintergrund $(55,9 \%$ vs. ohne $=51,2 \%)$ empfinden in der Öffentlichkeit deutlich häufiger eine positive Haltung gegenüber Mehrkindfamilien als solche mit höheren Abschlüssen ( $\approx 51-52 \%)$.

Das gesellschaftliche Leitbild des Kinderreichtums sehen signifikant mehr niedrig Gebildete $(50,0 \%)$ gesellschaftlich weit verbreitet als mittel $(35,8 \%)$ oder hoch Gebildete $(39,8 \%)$. Wesentlich mehr Kinderlose $(43,7 \%)$ als Eltern $(29,8 \%)$ sowie mehr Personen mit (43,5\%) als ohne Migrationshintergrund (36,4\%) empfinden das ebenso. Auch Kohorteneffekte werden sichtbar: Häufiger sind jüngere Befragte $(41,5 \%$ vs. Ältere: $34,6 \%$ ) der Ansicht, dass Kinderreichtum in der Allgemeinheit befürwortet wird.

Tabelle 4: Gesellschaftliche Leitbilder des Kinderreichtums nach soziodemografischen Merkmalen (Zustimmung)

\begin{tabular}{|c|c|c|c|c|c|}
\hline & & & $\begin{array}{c}\text { Distanz zum } \\
\text { Kinderreichtum }\end{array}$ & $\begin{array}{c}\text { Gemischt- } \\
\text { geschlechtliche } \\
\text { Kindorientierung }\end{array}$ & Kinderreichtum \\
\hline \multirow{4}{*}{ Geschlecht } & Männlich & $\mathrm{N}$ & 1.615 & 1.350 & 1.007 \\
\hline & & $\%$ & $64,7^{* * *}$ & $53,8^{*}$ & $40,0^{*}$ \\
\hline & Weiblich & $\mathrm{N}$ & 1.912 & 1.238 & 889 \\
\hline & & $\%$ & $78,4^{* * *}$ & $50,6^{*}$ & $36,2^{*}$ \\
\hline \multirow[t]{4}{*}{ Wohnort } & West & $\mathrm{N}$ & 2.815 & 2.103 & 1.517 \\
\hline & & $\%$ & 71,6 & $53,3^{* * *}$ & 38,3 \\
\hline & Ost (inkl. & $\mathrm{N}$ & 712 & 484 & 379 \\
\hline & Berlin) & $\%$ & 71,0 & $48,0^{* * *}$ & 37,5 \\
\hline \multirow{6}{*}{$\begin{array}{l}\text { ISCED } \\
\text { Bildung }\end{array}$} & Niedrig & $\mathrm{N}$ & 206 & 219 & 173 \\
\hline & & $\%$ & $60,1^{* * *}$ & $63,8^{* * *}$ & $50,0^{* * *}$ \\
\hline & Mittel & $\mathrm{N}$ & 2.330 & 1.663 & 1.158 \\
\hline & & $\%$ & $72,7^{* * *}$ & $51,8^{* * *}$ & $35,8^{* * *}$ \\
\hline & Hoch & $\mathrm{N}$ & 942 & 667 & 519 \\
\hline & & $\%$ & $72,9^{* * *}$ & $51,3^{* * *}$ & $39,8^{* * *}$ \\
\hline \multirow{4}{*}{$\begin{array}{l}\text { Haben Sie } \\
\text { Kinder }\end{array}$} & $\mathrm{Ja}$ & $\mathrm{N}$ & 1.519 & 1.069 & 2.002 \\
\hline & & $\%$ & $76,3^{* * *}$ & 53,4 & $29,8^{* * *}$ \\
\hline & Nein & $\mathrm{N}$ & 2.007 & 1.518 & 2.949 \\
\hline & & $\%$ & $68,2^{* * *}$ & 51,5 & $43,7^{* * *}$ \\
\hline \multirow{4}{*}{$\begin{array}{l}\text { Migrations- } \\
\text { hintergrund }\end{array}$} & $\mathrm{Ja}$ & $\mathrm{N}$ & 758 & 657 & 516 \\
\hline & & $\%$ & $64,6^{* * *}$ & $55,9^{* *}$ & $43,5^{* * *}$ \\
\hline & Nein & $\mathrm{N}$ & 2.763 & 1.927 & 1.379 \\
\hline & & $\%$ & $73,6^{* * *}$ & $51,2^{* *}$ & $36,4^{* * *}$ \\
\hline \multirow{4}{*}{$\begin{array}{l}\text { Alters- } \\
\text { gruppen }\end{array}$} & $20-29$ & $\mathrm{~N}$ & 1.686 & 1.272 & 1.039 \\
\hline & & $\%$ & $67,8^{* * *}$ & 51,1 & $41,5^{* * *}$ \\
\hline & $30-39$ & $\mathrm{~N}$ & 1.840 & 1.315 & 857 \\
\hline & & $\%$ & $75,1^{* * *}$ & 53,4 & $34,6^{* * *}$ \\
\hline \multirow[t]{2}{*}{ Gesamt } & & $\mathrm{N}$ & 3.497 & 2.573 & 1.885 \\
\hline & & $\%$ & 71,4 & 52,3 & 38,1 \\
\hline
\end{tabular}

Quelle: FLB (2012), gewichtete Daten, eigene Berechnungen

Anm.: ${ }^{* * *} \mathrm{p} \leq 0,001 ;{ }^{* *} \mathrm{p} \leq 0,01 ;{ }^{*} \mathrm{p} \leq 0,05$; Signifikanzen wurden mit dem $\mathrm{Chi}^{2}$-Test getestet. 
Die in der Tabelle 4 nicht aufgeführten Ergebnisse nach Stärke der subjektiv eingestuften Religiosität ${ }^{8}$ ergeben keine größeren Unterschiede für die ersten beiden Leitbilder, sie spielen offenbar für die Wahrnehmung gesellschaftlicher Leitbilder zur Distanz gegenüber Kinderreichtum und zur gemischtgeschlechtlichen Kindorientierung keine Rolle. Jedoch nehmen sehr viel weniger hoch Religiöse $(14,4 \%)$ als mittel $(25,2 \%)$ oder wenig/nicht Religiöse $(60,5 \%)$ in der Gesellschaft ein vorbehaltloses kinderreichtum-freundliches Klima wahr. Das liegt vermutlich daran, dass kinderreiche Eltern (ab 3 Kindern) tendenziell religiöser sind (siehe Abschnitt 2.2) und im Alltag auch häufiger erleben, dass ihre Familiengröße nicht nur positiv bewertet wird (s. o.).

Auch die Zugehörigkeit zu einer Konfession ${ }^{9}$ ist bedeutsam bei der Einschätzung der Allgemeinheit durch die Befragten (vgl. Tabelle 5):

- Protestanten nehmen am häufigsten wahr (74,8 \%), dass Kinderreiche in der Gesellschaft negativ gesehen werden. Die anderen Gruppen liegen etwa gleich hoch: Es folgen die Katholiken (70,6\%) und die anderen Religionsgemeinschaften (70,5\%). Ähnlich bewerten dies Konfessionslose (69,6 \%) und Muslime (68,7 \%).

- Bei der Betrachtung des gesellschaftlichen Leitbildes der gemischtgeschlechtlichen Geschwisterorientierung stimmen die muslimischen Befragten $(63,3 \%)$ am häufigsten $\mathrm{zu}$, dies in der Allgemeinheit wahrzunehmen. Katholiken (54,3\%) und Protestanten $(53,0 \%)$ unterscheiden sich dabei kaum. Konfessionslose und Angehörige anderer Religionsgemeinschaften hingegen sind noch etwas seltener der Ansicht, dass in der Gesellschaft Stammhalter, gemischtgeschlechtliche Geschwisterkonstellationen oder generell Geschwister zu haben als wichtig erachtet wird.

- Bei denjenigen, die das Leitbild des Kinderreichtums in der Allgemeinheit weit verbreitet sehen, stechen die Muslime mit 42,0\% deutlich hervor im Vergleich zu den anderen Konfessionen.

Bei der Konfessionszugehörigkeit deutet sich ein Umgebungseffekt an, dass nämlich in bestimmten religiösen Milieus auch bestimmte Einstellungen bezüglich der Zusammensetzung einer Familie bestehen. In den abrahamitischen Religionen spielen generell die Eheschließung und die damit normativ verknüpfte Familiengründung eine größere Rolle, demnach sind auch solche gesellschaftlichen Gruppen kindorientierter, so dass die Befragten diesen Ausschnitt der Öffentlichkeit als Teil ihres gesellschaftlichen Stimmungsbildes einordnen. Im Leitbildsurvey haben auch Muslime und Angehörige anderer (überwiegend christlicher) Religionsgemeinschaften tendenziell etwas häufiger Mehrkindfamilien. Darüber hinaus sind hier auch Einflüsse aus der Herkunftsfamilie der Befragten wirksam (siehe Dietrich/Gies, Kap. 4). Deren Werthaltungen spielen bei der Bewertung der Allgemeinheit eine wesentliche Rolle.

8 „Als wie religiös würden Sie sich selbst bezeichnen?“ Hier gab es die Antwortkategorien „Sehr religiös“, „Religiös“, „Mittel religiös“, „Wenig religiös“ und „Nicht religiös“. Diese Frage wurde nur solchen Personen gestellt, die nicht explizit die Antwort zur Religionsgemeinschaft verweigert haben.

9 „Darf ich Sie fragen, ob Sie einer Religionsgemeinschaft angehören?“ Die Antwortmöglichkeiten wurden zunächst mit einem „Ja, ...“ eingeleitet gefolgt von „,der römisch-katholischen Kirche“, „der evangelischen Kirche (ohne Freikirchen)“, „,dem Islam“, „dem Judentum“, „einer anderen christlichen Religionsgemeinschaft" und ,einer anderen nicht-christlichen Religionsgemeinschaft". Konfessionslose wurden der Kategorie „Keine Religionsgemeinschaft“ zugeordnet. Für die folgenden Analysen wurden Juden, andere christliche Religionsgemeinschaften und andere nicht-christliche Religionen in eine Kategorie („Andere“) zusammengefasst. Ein Großteil darin gehört anderen christlichen Religionsgemeinschaften an. 
Tabelle 5: Gesellschaftlich wahrgenommene Leitbilder des Kinderreichtums nach Konfession (Zustimmung)

\begin{tabular}{lcccc}
\hline & Distanz gegenüber & Kinderreichtum & $\begin{array}{c}\text { Gemischtgeschlechtliche } \\
\text { Geschwisterorientierung }\end{array}$ & Kinderreichtum \\
\hline Katholiken & $\mathrm{N}$ & 919 & 710 & 510 \\
& $\%$ & 70,6 & 54,3 & 38,8 \\
Protestanten & $\mathrm{N}$ & 1.050 & 750 & 526 \\
& $\%$ & $\mathbf{7 4 , 8}$ & 53,0 & 37,1 \\
Muslime $^{\mathrm{a}}$ & $\mathrm{N}$ & 103 & 95 & 63 \\
& $\%$ & 68,7 & $\mathbf{6 3 , 3}$ & $\mathbf{4 2 , 0}$ \\
Konfessionslose & $\mathrm{N}$ & 1.320 & 948 & 735 \\
& $\%$ & 69,6 & 49,9 & 38,5 \\
\multirow{2}{*}{ Andere RG } & $\mathrm{N}$ & 105 & 70 & 51 \\
& $\%$ & 70,5 & 47,0 & 32,9 \\
\hline
\end{tabular}

Quelle: FLB (2012), gewichtete Daten, eigene Berechnungen

Anm.: $\quad R G=$ Religionsgemeinschaften, ${ }^{a}$ Unterscheidungen in die verschiedenen Strömungen des Islam sind im Fragebogen nicht gemessen worden. Fett gedruckt $=$ höchster Wert innerhalb der LeitbildKategorie.

\section{Diskussion und Fazit}

Ziel der spezifischen Leitbildanalysen war es zu klären, wer momentan unter den jüngeren Personen in Deutschland als kinderreich gilt. Des Weiteren sollte untersucht werden, wie Kinderreichtum individuell beurteilt und gesellschaftlich wahrgenommen wird und ob sich gesellschaftliche Leitbilder identifizieren lassen.

Heutzutage wird unter den 20- bis 39-Jährigen davon ausgegangen, dass Familien ab vier Kindern als kinderreich gelten. Dieses Ergebnis überrascht, da die Zwei-Kind-Familie als die Normalfamilie gilt und somit angenommen wurde, dass Familien bereits darüber hinaus $-\mathrm{d}$. h. ab dem dritten Kind - als kinderreich gelten.

Zur Beurteilung der Frage, ob Kinderreiche ein Negativimage haben, wurden zwei Thesen aufgestellt. Erstens, dass kinderreiche Familien auf individueller Ebene positiv besetzt sind. Auf der gesellschaftlichen Ebene hingegen wurde angenommen, dass hier ein eher negativ konnotiertes Bild von Großfamilien vorherrscht. Beide Annahmen lassen sich mit den Leitbilddaten gut replizieren. Die erste Annahme auf der individuellen Ebene lässt sich über eine weitgehende Zustimmung zu der Aussage, dass viele Kinder etwas Wundervolles sind und in der nahezu vollständigen Ablehnung der Aussage, nach der Kinderreiche ,asozial“" sind, gut bestätigen. Auf der gesellschaftlichen Ebene bietet sich ein konträres Bild. Von der Allgemeinheit nehmen die Befragten an, dass sie vielen Kindern in der Familie eher ablehnend gegenüber steht. Bemerkenswert ist, dass nahezu drei Viertel der jungen Menschen in Deutschland glauben, dass Kinderreiche in Deutschland negativen Vorurteilen ausgesetzt sind. Woher kommen die Unterschiede in der Bewertung auf der 
individuellen und gesellschaftlichen Ebene? Es ist davon auszugehen, dass diese Differenz aus dem Nebeneinander von nach wie vor existierenden negativen Klischees (die immer noch von den Medien bedient werden und auch innerhalb der Gesellschaft vorkommen ${ }^{10}$ ) auf der gesellschaftlichen Ebene und persönlichen Erfahrungen entsteht, die diesem Negativimage nicht entsprechen.

Über das Entstehen des schlechten Rufs besteht keine endgültige Klarheit. Drei Thesen sollen an dieser Stelle in die Diskussion gebracht werden. In der ersten Annahme wird davon ausgegangen, dass das Negativimage in der Zeit des ersten Geburtenrückgangs entstanden ist. Der Rückgang der Kinderzahlen hat zunächst im Bildungsbürgertum stattgefunden und sich erst später als gesellschaftlicher Mainstream durchgesetzt.

„An Bildung und Wohlstand reiche Eltern begannen früher, die Zahl ihrer Kinder zu verringern als Eltern in wirtschaftlichen Notlagen. Ein Vorreiter der Entwicklung war die in der Stadt lebende bürgerliche Familie. Dazu gehörten der neue Mittelstand, Beamte, freie Berufe, Angestellte. (...) Die „respektable“ Arbeiterschaft, geleitet vom bürgerlichen Familienideal, betrachtete Kinder nicht mehr als Schicksal und widmete dem einzelnen Kind eine größere Aufmerksamkeit. Die Arbeiterfamilie im Allgemeinen jedoch zog ihre normativen Orientierungen weiterhin aus den traditionellen Moral- und Ordnungsvorstellungen.“ (Eggen und Rupp 2006: 27)

Die kinderreiche Arbeiterschicht mit niedriger Bildung und geringem Einkommen erhielt im Zuge dieses Wandels den Stempel des Asozialen. Die zweite These basiert auf der Überlegung, dass das Negativimage auf die überfrachteten Erwartungen an die Elternrolle zurückzuführen ist und es sich dabei um ein typisch deutsches Phänomen handelt. Schon Eltern von ein oder zwei Kindern fürchten, dem Elternideal nicht gerecht werden zu können. Dazu stellt Schneider fest:

„Natürlich wollen Eltern überall auf der Welt das Beste für ihre Kinder - aber nicht in einem solchen Maße wie bei uns. Die Erwartungen an die Erfolge der eigenen Kinder sind enorm, und damit steigt auch das Risiko des Scheiterns. Die Gelassenheit und das Vertrauen, dass unsere Kinder ihren Weg gehen, egal, ob wir sie nun in die Klavierstunde fahren oder täglich mit ihnen Vokabeln lernen, fehlen uns." (FAZ, 09.01.2013)

Von den Kinderreichen wird deshalb noch mehr angenommen, dass sie viel zu wenig Zeit und Aufmerksamkeit für jedes Kind erübrigen können, damit ihre Elternrolle nur unzureichend wahrnehmen und sich dadurch eben ,asozial“ verhalten.

Drittens kann davon ausgegangen werden, dass die pronatalistischen Missbrauchserfahrungen des Dritten Reiches in Deutschland dazu beigetragen haben, dass Kinderreichtum in der Gesellschaft als negativ bewertet wird, wie auch in anderen Ländern mit ähnlichen Erfahrungen (Bujard 2011: 212, 279ff., 364). Die finanzielle und ideelle Unterstützung von kinderreichen Familien zielte darauf ab, die NS-Ideologie zu realisieren (Kramer 2014).

Sozialstrukturelle Unterschiede sind auf der individuellen Leitbildebene relativ schwach ausgeprägt. Im Trend sehen Westdeutsche, Personen mit Migrationshintergrund und Eltern Kinder etwas häufiger als etwas Wundervolles an. Zur Aussage, „Kinderreiche gelten als asozial“, gibt es auf niedrigem Niveau keine Zustimmungsunterschiede. Deutlich differenzierter fallen die Bewertungen auf der gesellschaftlichen Ebene aus. Hervorzuheben sind drei Unterschiede. Erstens beurteilen die Befragten das Ansehen, das große Familien in der Öffentlichkeit haben, in Abhängigkeit von der eigenen Kinderzahl. Kinderreiche Familien, so die Annahme, nehmen die Gesellschaft aufgrund eigener Erfahrungen also

10 Wie von kinderreichen Eltern aus ihrem Alltag berichtet wird, siehe Gierth 2014: 18; Thiede 2014: 7ff. 
deutlich kinderfeindlicher wahr als andere. Das deckt sich mit den Ergebnissen anderer Studien (z. B. Kaufmann 1995: 169ff.), in denen Kinderreiche über diskriminierende Äußerungen klagen. Da in einem weiteren Beitrag dieses Bandes (Dorbritz/Diabaté, Kap. 8) eine kulturelle Verankerung der Kinderlosigkeit als etwas „Normales“ in Teilen der jüngeren Bevölkerung nachgewiesen werden konnte, kann davon ausgegangen werden, dass sich dies auch in einem Unverständnis gegenüber Kinderreichtum äußert. Zweitens ist darauf hinzuweisen, dass die Befragten mit einer niedrigeren Bildung signifikant seltener denken, dass die Allgemeinheit Kinderreiche als asozial ansieht. Kinderreichtum kommt, wie eingangs gezeigt, bei niedriger Qualifizierten häufiger vor und könnte daher stärker als Normalität empfunden werden. Drittens fällt auf, dass Männer deutlich seltener in der Allgemeinheit wahrnehmen, dass Kinderreichtum als ,,asozial“ eingestuft wird.

Auf der Suche nach Leitbildern, die mit dem Kinderreichtum verknüpft sind, konnten drei in der Gesellschaft wahrgenommene identifiziert werden. Das erste Leitbild ist das der Distanz gegenüber Kinderreichen, das sich aus vier Items zusammensetzt: 1. Ablehnung der Aussage, dass Kinder etwas Wunder-volles sind auf der gesellschaftlichen Ebene, 2. die Auffassung, dass nur die kinderreich werden sollten, die es sich auch leisten können, 3. das Empfinden, dass die Allgemeinheit Kinderreiche als „asozial“ empfindet und 4. die Vorstellung, dass sich kinderreiche Eltern nicht ausreichend um die Kinder kümmern können. Das zweite Leitbild wird als Leitbild der gemischtgeschlechtlichen Geschwisterorientierung bezeichnet. Es basiert auf der Annahme, dass Einzelkinder ein Negativimage haben, dem Stammhalterdenken (nach der Geburt von zwei Mädchen wird ein drittes Kind in der Hoffnung gewünscht, dass es ein Junge wird) und der Vorstellung, dass es in der Öffentlichkeit positiv gesehen wird, wenn zu einer Familie sowohl Mädchen als auch Jungen gehören. Das dritte Leitbild, das gesellschaftliche Leitbild des Kinderreichtums, besteht aus nur zwei Items. Die Träger dieses Leitbilds sehen Kinder in der gesellschaftlichen Perspektive als etwas Wundervolles an und gehen davon aus, dass die Allgemeinheit Kindern eine hohe Wichtigkeit beimisst. Vom zweiten und dritten Leitbild ist anzunehmen, dass sie positiv auf die Entscheidung für (weitere) Kinder wirken.

Als zentrales Fazit der Analysen ist festzuhalten, dass Kinderreichtum in Deutschland nicht grundsätzlich negativ besetzt ist. Gefunden wurde ein Dualismus in den Bewertungen auf der individuellen und der gesellschaftlichen Leitbildebene. Dieser wird interpretiert als individuelle Toleranz gegenüber Kinderreichtum bei einem vermuteten gesellschaftlichen Negativbild, das nicht durchgängig existent ist, aber verstärkt wahrgenommen wird. Diese wahrgenommene Meinung der Öffentlichkeit - Diskriminierung von kinderreichen Familien - wirkt sich möglicherweise über die Norm der verantworteten Elternschaft negativ auf den Kinderwunsch aus bzw. auf die Entscheidung für ein drittes oder weiteres Kind. Durch diese Situation könnte die deutsche Niedrig-Fertilitäts-Situation gestützt sein, was weiterführende Analysen zeigen könnten.

\section{Literatur}

Allmendinger, Jutta; Hinz, Thomas (1998): Frauen, Alleinerziehende und kinderreiche Familien. In: Familie, Frauen und Gesundheit. Bayerisches Staatsministerium für Arbeit und Sozialordnung (Hrsg.): Material- und Analyseband zur sozialen Lage in Bayern. München, S. 433-458. 
Berens, Petra (2004): Der Grundrechtsschutz der Familie unter besonderer Berücksichtigung der kinderreichen Familie. Köln: Kleikamp Druck.

Bertram, Hans; Rösler, Wiebke; Ehlert, Nancy (2005): Nachhaltige Familienpolitik. Zukunftssicherung durch einen Dreiklang von Zeitpolitik, finanzieller Transferpolitik und Infrastrukturpolitik. (Hrsg.): Bundesministerium für Familie, Senioren, Frauen und Jugend.

Bien, Walter; Marbach, Jan (2007): Mehrkindfamilien. Erkenntnisse aus den Daten des Familiensurvey - Wellen 1988, 1994 und 2004. München.

Bierschock, Kurt (2010): Kinderreiche Familien - ein Überblick. [http://www.familienhandbuch.de/ elternschaft/familie/kinderreiche-familien-ein-uberblick, 16.10.2014]

Blume, Michael; Ramsel, Carsten; Graupner, Sven (2006): Religiosität als demographischer Faktor Ein unterschätzter Zusammenhang? In: Marburg Journal of Religion, Volume 11, No. 1, S. 1-24.

BMFSFJ (Bundesministerium für Familie, Senioren, Frauen und Jugend) (Hrsg.) (2007): Monitor Familienforschung 10. Kinderreichtum in Deutschland. Berlin.

BMFSFJ (Bundesministerium für Familie, Senioren, Frauen und Jugend) (Hrsg.) (2013): Monitor Familienforschung 28. Alleinerziehende in Deutschland. Lebenssituation und Lebenswirklichkeit von Müttern und Kindern. Beiträge aus Forschung, Statistik und Familienpolitik.

Bruchholz, Roswitha; Fügemann, Christiane; Minsel, Wolf-Rüdiger (2002): Der Übergang zur DreiKind-Familie. Eine Befragung von Müttern zu Motivation, Veränderungen, Anforderungen und Bewältigung. In: systhema, 16 (1), S. 42-49.

Bujard, Martin (2011): Geburtenrückgang und Familienpolitik. Ein verschiedene Theorien integrierender Erklärungsansatz und dessen empirische Überprüfung im OECD-Länder-Vergleich 19702006. Baden-Baden: Nomos.

Bujard, Martin; Dorbritz, Jürgen; Grünheid, Evelyn; Kühntopf, Stefan; Lück, Detlev; Naderi, Robert; Passet, Jasmin; Ruckdeschel, Kerstin (2012): (Keine) Lust auf Kinder? - Geburtenentwicklung in Deutschland. Wiesbaden: Bundesinstitut für Bevölkerungsforschung.

Diabaté, Sabine; Lück, Detlev (2014): Familienleitbilder. Identifikation und Wirkungsweise auf generatives Verhalten. In: Zeitschrift für Familienforschung, 26 (1). Opladen:Verlag Barbara Budrich.

Dorbritz, Jürgen; Schneider, Norbert F. (2012): Familiendemografische Trends in Deutschland Herausforderungen für zukünftiges politisches Handeln. In: Hüther, Michael; Naegele, Gerhard (Hrsg.): Demografiepolitik - Herausforderungen und Handlungsfelder. Wiesbaden: Springer VS, S. 142-162.

Dürnberger, Andrea; Rupp, Marina (2006): Entstehung und Entwicklung kinderreicher Familien. In: Eggen, Bernd; Rupp, Marina (Hrsg.): Kinderreiche Familien. Wiesbaden: VS Verlag für Sozialwissenschaften, S. 129-166.

Eggen, Bernd (2014): Kinderreiche Familien: Ein Weg durch die Zeiten. In: Thiede, Rocco: Kinderglück. Leben in großen Familien. Bundeszentrale für politische Bildung, Schriftenreihe, Band 1448. Bonn.

Eggen, Bernd; Rupp, Marina (Hrsg.) (2006): Kinderreiche Familien. Wiesbaden: VS Verlag für Sozialwissenschaften.

Eggen, Bernd; Leschhorn, Harald (2004): Kinderreiche Familien und ihre Haushaltsformen. In: Statistisches Monatsheft Baden-Württemberg, Heft 5, S. 18-21.

Gierth, Matthias (2014): Jedes Kind ein Wunschkind. In: Thiede, Rocco: Kinderglück. Leben in großen Familien. Bundeszentrale für politische Bildung, Schriftenreihe, Band 1448. Bonn.

Gray, Edith; Evans, Ann (2004): Sex composition of children as a determinant of parity progression.

Hank, Karsten; Kohler, Hans-Peter (2000): Gender Preferences for Children in Europe: Empirical Results from 17 FFS Countries. In: Demographic Research 2, 1.

Hank, Karsten; Kohler, Hans-Peter (2003): Sex Preferences for Children Revisited: New Evidence from Germany. In: Population - English Edition 58 (1), S. 133-144.

Höhn, Charlotte; Ette, Andreas; Ruckdeschel, Kerstin (2006): Kinderwünsche in Deutschland: Konsequenzen für eine nachhaltige Familienpolitik. (Hrsg.): Robert-Bosch-Stiftung. Stuttgart.

Kaufmann, Franz Xaver (1995): Zukunft der Familie. München: Beck C. H. 
Keddi, Barbara; Zerle, Claudia; Lange, Andreas; Cornelißen, Waltraud (2010): Der Alltag von Mehrkinderfamilien - Ressourcen und Bedarfe. Forschungsbericht DJI. (Hrsg.): Deutsches Jugendinstitut München.

Kessler, Thomas; Mummendey, Amelié; Leisse, Utta-Kristin (2000): The Personal-Group Discrepancy: Is there a common information basis of personal and group judgment? In: Journal of Personality and Social Psychology 79, S. 95-109.

Kessler, Thomas; Harth, Nicole Syringa (2008): Die Theorie relativer Deprivation. In: Stereotype, Vorurteile und soziale Diskriminierung. 1. Auflage. Weinheim, Basel: Beltz PVU, S. 249-259

Klein, Thomas; Eckard, Jan (2004): Fertilität in Stieffamilien. In: Kölner Zeitschrift für Soziologie und Sozialpsychologie 56/1, S. 71-94.

Kramer, Nicole (2014): Anmerkungen zur Einführung des Mutterkreuzes. In: Zeitgeschichte-online, Mai 2014. [http://www.zeitgeschichte-online.de/kommentar/anmerkungen-zur-einfuehrung-desmutterkreuzes-im-mai-1939, 19.09.2014]

Nauck, Bernhard (1995): Lebensbedingungen von Kindern in Einkind-, Mehrkind- und Vielkindfamilien. In: Nauck, Bernhard; Bertram, Hans (Hrsg.): Kinder in Deutschland. Opladen: Westdeutscher Verlag, S. 137-169.

Peuckert, Rüdiger (2008): Familienformen im sozialen Wandel. VS Verlag für Sozialwissenschaften, 7. vollständige überarbeitete Auflage. Wiesbaden: GWV Fachverlage GmbH.

Rille-Pfeiffer, Christiane; Kaindl, Markus; Klepp, Doris; Fröhlich, Elisabeth (2009): Der Übergang zur Dreikind-Familie. Eine qualitative Untersuchung von Paaren mit zwei und drei Kindern. (Hrsg.): Österreichisches Institut für Familienforschung. Wien.

Rost, Harald (2009): Familienhaushalte im europäischen Vergleich. In: Familie und Frauen. Bayerisches Staatsministerium für Arbeit und Sozialordnung (Hrsg.): ifb-Familienreport Bayern 2009. Schwerpunkt: Familie in Europa. ifb Staatsinstitut für Familienforschung an der Universität Bamberg. München, S. 9-32.

Rupp, Marina; Blossfeld, Hans-Peter (2008): Familiale Übergänge: Eintritt in nichteheliche Lebensgemeinschaften, Heirat, Trennung und Scheidung, Elternschaft. In: Schneider, Norbert F. (Hrsg.): Lehrbuch Moderne Familiensoziologie. Opladen: B. Budrich, S. 139-166.

Schneider, Norbert F. (2013): Nirgendwo sonst stehen Eltern so unter Druck. In: Frankfurter Allgemeine, 09.01.2013. [http://www.faz.net/-1v0-751jz]

Schulten-Jaspers, Yasmin; Tank, Jennifer; Prinzing, Marlis (2013): Der politische Restposten. Presseberichterstattung über kinderreiche und andere Familien. Studie (Abschlussbericht) im Auftrag des Bundesministeriums für Familie, Senioren, Frauen und Jugend. [http://issuu.com/ prinzing_tank_schulten-jaspers/docs/der_politische_restposten, 19.09.2014]

Statistisches Bundesamt (Hrsg.) (2013): Geburtentrends und Familiensituation in Deutschland. Unter Mitarbeit von Olga Pötzsch, Julia Weinmann und Thomas Haustein. Wiesbaden: Statistisches Bundesamt. [https://www.destatis.de/DE/Publikationen/Thematisch/Bevoelkerung/Haushalte Mikrozensus/Geburtentrends5122203129004.pdf?_blob=publicationFile, 24.06.2014].

Thiede, Rocco (2014): Kinderglück. Leben in großen Familien. Bundeszentrale für politische Bildung, Schriftenreihe, Band 1448. Bonn.

Walla, Wolfgang; Eggen, Bernd; Lipinski, Heike (2006): Der demographische Wandel: Herausforderung für Politik und Wirtschaft. Stuttgart: W. Kohlhammer Verlag. 


\title{
Verantwortete Elternschaft: „Für die Kinder nur das Beste“
}

\author{
Kerstin Ruckdeschel*
}

\section{Zusammenfassung}

Das Leitbild Verantwortete Elternschaft ist in Deutschland stark präsent und prägt die Vorstellungen von Elternschaft. In diesem Leitbild findet sich einerseits das Fördergebot, d. h. Kinder sollten beim Aufwachsen intensiv begleitet werden, sie werden also nicht „von alleine groß“. Diese Leitbilddimension findet individuell breite Zustimmung und wird auch in der Gesellschaft häufig wahrgenommen. Sie verbindet verschiedenste Elterntypen und wird als gesellschaftlicher Anspruch an Eltern empfunden. Insgesamt kann von einem stark in der Gesellschaft verankerten Leitbildbestandteil gesprochen werden.

Auf der anderen Seite steht das Gebot der Mutternähe und Aufopferung, das Fremdbetreuung von unter 3-Jährigen ablehnt und die Rückstellung eigener Bedürfnisse hinter die des Kindes fordert. Diese Leitbilddimension differiert sowohl zwischen individueller und gesellschaftlicher Ebene als auch zwischen einzelnen gesellschaftlichen Subpopulationen. Dabei werden die Ansprüche vor allem als gesellschaftliche Erwartungen wahrgenommen, die auf individueller Ebene aber überwiegend abgelehnt werden.

Eltern, die vor allem das Fördergebot bejahen, stellen die größte Gruppe dar, gefolgt von Eltern, die zusätzlich eine Kinderbetreuung innerhalb der Familie einer externen vorziehen. Die beiden extremen Typen, d. h. Eltern, die dem Leitbild der Verantworteten Elternschaft sehr distanziert gegenüberstehen genauso wie diejenigen, die es in allen seinen Aspekten bejahen, sind ebenfalls relativ stark vertreten.

\section{$1 \quad$ Einleitung}

Elternschaft hat sich gesellschaftlich zu einem Thema entwickelt, das eher mit Überforderung und Überanstrengung als mit Glück und Zufriedenheit assoziiert ist. In den Medien wird von der „großen Erschöpfung“ (Voigt 2013) gesprochen und davon, dass man „wahnsinnig sein (muss), heute ein Kind zu bekommen" (Baum 2014). Elternschaft wird gegenwärtig als eine verantwortungsvolle Aufgabe begriffen, die anstrengend ist und eine Menge Arbeit und Zeit abverlangt. Dies bestätigen auch Ergebnisse einer Umfrage der Zeitschrift „Eltern“ aus dem Jahr 2010, bei der über die Hälfte der 30- bis 34-Jährigen angegeben haben, sie würden keine Kinder wollen, weil andere Eltern auf sie so gestresst wirken. Und fast drei Viertel der 25- bis 29-Jährigen befürchtet, mit Kindern keine Zeit mehr für sich zu haben (Eltern 2010). Für diese zunehmende Selbst- und Fremdwahrnehmung von Eltern als Leistungsträger der Erziehung, hat sich der von Kaufmann geprägte Begriff der „Verantworteten Elternschaft" herausgebildet. Er besagt, dass man nur dann Kinder in die Welt

* Ich danke meiner Kollegin Sabine Diabaté und den anonymen Gutachtern für hilfreiche Kritik und wertvolle Anmerkungen. 
setzen sollte, wenn man auch bereit ist, sich ausreichend um sie zu kümmern, sowohl materiell als auch zeitlich (Kaufmann 1990). Diese Maxime, die Mitte des letzten Jahrhunderts in Europa aufkam, führte zu einer Entwicklung, in deren Verlauf die "Ansprüche an die Erziehung, die sich die Eltern selbst stellten und die von ihrer Umgebung an sie gestellt wurden, (...) erheblich an(stiegen)" (Kaelble 2007: 41), mit der Folge, dass die "Familie (...) unter einem Erziehungsdruck (steht), der historisch seinesgleichen sucht" (Kaufmann et al. 1982: 530).

Im folgenden Beitrag soll deshalb die Frage erörtert werden, ob heute junge Menschen in Deutschland im Familiengründungsalter Elternschaft ebenfalls vor allem als Anstrengung und Verpflichtung sehen. Gleichzeitig soll geklärt werden, wie gesellschaftliche Erwartungen an Eltern wahrgenommen werden und inwieweit es hier Übereinstimmungen und Diskrepanzen zwischen einzelnen gesellschaftlichen Gruppen gibt. Dazu wird zunächst ein kurzer theoretischer Überblick über das Thema „Verantwortete Elternschaft" gegeben, um im Anschluss daran dessen Operationalisierung in der Studie „Familienleitbilder in Deutschland" und das methodische Vorgehen vorzustellen. Im Ergebnisteil werden vor allem deskriptive Befunde dargestellt, die zu Typen zusammengefasst und nach sozialstrukturellen Merkmalen verglichen werden. Im Schlussteil folgt eine kurze Zusammenfassung der Ergebnisse in Hinblick auf die eingangs gestellten Fragen.

\section{Forschungsstand und theoretische Grundlagen}

\subsection{Zum Normenkomplex der ,,Verantworteten Elternschaft “}

Elternrollen und Erziehungsgeschehen sind soziale Konstruktionen, die sich wandeln können und die durch kulturelle und strukturelle Einflüsse geprägt sind (Seiffge-Krenke/ Schneider 2012: 98f.). Entsprechend hat sich die Elternrolle historisch stark verändert, als im Laufe der Zeit immer mehr Funktionen aus der Familie ausgelagert wurden und die Familie sich in exklusiver Weise zur primären Sozialisationsinstanz für die nachfolgende Generation entwickelt hat (Huinink 1997: 80). Die Folge ist, dass noch „nie zuvor in der Geschichte unseres Kulturkreises die frühkindliche Erziehung den Eltern so exklusiv überantwortet und von ihnen als zu verantwortende Aufgabe gesehen (wurde)“ (Nave-Herz 2012: 35; Kaufmann et al. 1982: 530).

Durch die massenhafte Verbreitung von Verhütungsmitteln seit Mitte des letzten Jahrhunderts ist Elternschaft „,von einem oft ungewollten Schicksal mehr und mehr zu einer Lebensentscheidung geworden oder wird zum mindesten sozial als solche gewertet" (Kaufmann 1990: 101) und entsprechend hat man zu den Konsequenzen seiner freiheitlichen Entscheidung zu stehen. Das beinhaltet eine bewusste Übernahme von Elternpflichten, wobei diese zunehmend anspruchsvoller wurden (Kaufmann 1990), mit dem Ziel einer "bestmögliche(n) Förderung der Fähigkeiten des Kindes" (Beck-Gernsheim 1991: 59). Verschiedene Entwicklungen seit den 1950er und 1960er Jahren haben diesen Prozess beeinflusst und beschleunigt: Fortschritte in der Medizin, der Psychologie und Pädagogik betonen die Bedeutung der ersten Lebensjahre des Kindes für seine gesamte weitere Entwicklung, so dass unterlassene oder falsche Förderung mit verpassten Chancen für das gesamte weitere Leben gleichgesetzt wird. Der allgemeine Anstieg der Einkommen hat gleichzeitig Förderung für breite Schichten finanzierbar gemacht. Verstärkt wurden diese beiden Aspekte durch eine breite politische Werbung für mehr Bildung (Beck-Gernsheim 1991: 60f.). Erziehung wurde zu einer immer anspruchsvolleren Aufgabe. Zusätzlich er- 
schwert der demografische Wandel die Erziehungsarbeit, denn Kinder wachsen häufiger ohne Geschwister auf, wodurch das einzelne Kind mehr Aufmerksamkeit auf sich zieht und die Erwachsenen mehr in Anspruch nimmt, während gleichzeitig die Geschwistergemeinschaft als Entlastung der Eltern fehlt. Auch die fehlende Erfahrung im alltäglichen Umgang mit Kleinkindern, sowohl in der eigenen Familie als auch in der Öffentlichkeit, erschwert die Entscheidung für eine Elternschaft, die aus Sicht der potentiellen Eltern einer immer intensiveren Vorbereitung bedarf (Nave-Herz 2012; Quaiser-Pohl 2001: 307f.).

Den aus diesen Entwicklungen resultierenden Wandel der Elternrolle fasst Meyer (2002: 40) in fünf Aspekten zusammen:

- Vorverlagerte Elternschaft: Förder- und Kontrollansprüche werden bereits auf die Schwangerschaft ausgeweitet.

- Professionalisierte Elternschaft: Der Anspruch auf best- und frühestmögliche Förderung erfordert zunehmendes Expertenwissen der Eltern, das erworben werden muss. Zudem werden Experten und deren Ratschläge immer wichtiger in der Erziehung.

- Bildungsengagierte Elternschaft: Schule hat einen großen Bedeutungsanstieg erfahren - auch innerhalb der Familie. Das stellt Eltern durch eine teilweise Funktionsrückverlagerung via Hausaufgabenkontrolle ebenfalls unter erhöhten Anforderungsdruck (Kaufmann 1990: 108; Nave-Herz 2012: 44).

- Kommunikative Elternschaft: In der Erziehung hat sich der Schwerpunkt weg vom „Befehlshaushalt hin zum Verhandlungshaushalt (Du Bois-Reymond et al. 1994) verlagert, was das Erziehungsgeschehen verkompliziert und Eltern vor neue kommunikative Herausforderungen stellt.

- Organisierende Elternschaft: Fehlende nachbarschaftliche Spielgruppen führen zu einem Prozess der Verhäuslichung und Verinselung von Kindheit, der Eltern teilweise große logistische Herausforderungen beim Familienzeitmanagement abverlangt. Als Stichworte seien hier die Mutter als Transporteur und Zeitmanagerin genannt (NaveHerz 2012: 44).

Das Aufwachsen eines Kindes bedarf also einer intensiven Begleitung und Betreuung durch die Eltern, was im Idealfall die ständige Verfügbarkeit mindestens eines Elternteils für das Kind bedeutet (z. B. Nave-Herz 2012; Meyer 2002). Lange Zeit wurde diese Anforderung durch Positionen aus Psychologie, Pädagogik und anverwandten Wissenschaften bestärkt, die die ständige Präsenz der Mutter in den ersten Lebensjahren des Kindes als wichtige Voraussetzung für dessen gesundes Aufwachsen postulierten (Schneider 2002, BeckGernsheim 1991), was vor allem im Westen Deutschlands großen Anklang fand. Von Seiten der Wissenschaft werden inzwischen neue Befunde präsentiert, die weiterhin die Bedeutung der Eltern für Babys und Kleinkinder betonen, die aber gleichzeitig eine frühe Inanspruchnahme von Tagesbetreuung als nicht grundsätzlich schlecht für die Entwicklung des Kindes bewerten (z. B. Ahnert 2014; Becker-Stoll et al. 2010). Auch die Familienpolitik hat sich verstärkt die Förderung der Berufstätigkeit von Müttern zum Ziel gesetzt und versucht mit dem neuen, zeitlich enger begrenzten Elterngeld oder dem Ausbau von Krippenplätzen entsprechende Anreize zu schaffen. Die daraus resultierende zunehmende Berufstätigkeit von Frauen mit Kindern unter drei Jahren verstärkt allerdings die Debatte um das Wohl des Kindes, da sie von vielen als tendenziell eher nachteilig für das Kind wahrgenommen wird (Scheuer/Dittmann 2007; Mühling et al. 2006; siehe auch Diabaté, Kap. 6), was wiederum Eltern weiter verunsichert. Mit den zunehmenden Anforderungen sind Eltern entsprechend mehr und mehr überfordert, aber man geht davon aus, dass sie sie mit der Entscheidung für das Kind grundsätzlich akzeptieren. Allerdings mit relativ hoher Verunsicherung, die sich unter anderem in einem großen Informationsbedarf manifestiert, gerade 
bei Eltern mit kleinen Kindern (Kaufmann 1990). Eltern versuchen sowohl den objektiven als auch den subjektiven Ansprüchen gerecht zu werden, da ,eine Elternschaft umso befriedigender ist, je erfolgreicher in die Entwicklung, Bildung, Kreativität und in den persönlichen Erfolg der Kinder investiert wird" (Huinink 1997: 87, s. a. Kaufmann 1990: 145). Sie möchten ihren Kindern einen möglichst guten Platz in der Gesellschaft sichern und investieren entsprechend. Gleichzeitig wird dieser Wunsch der Eltern durch gesellschaftliche Leitbilder bestärkt, die dazu führen, dass "Eltern praktisch von allen Seiten vom Gebot bestmöglicher Förderung umstellt (sind), von Fernsehen bis Zeitschriften, von Werbung bis Schule" (Beck-Gernsheim 1991: 63). Auch gesellschaftlich betrachtet sind die Erwartungen an den Erziehungserfolg der Eltern sehr hoch, während gleichzeitig nur wenig Zugeständnisse an die Bedürfnisse von Eltern gemacht werden - mit der steigenden Gefahr einer Überforderung (Schneider et al. 2014; Schneider 2002: 14). Das Phänomen der Verantworteten Elternschaft lässt sich also so zusammenfassen, dass sich "das individuelle und gesellschaftliche Aspirationsniveau in Bezug auf die Qualität der Kinder erhöht hat" (Huinink 1997: 88).

\section{2 „Verantwortete Elternschaft“ als Leitbild}

Eltern stehen gesellschaftlich unter Druck, möglichst viel Zeit und Energie in die Erziehung ihrer Kinder zu investieren, um ein optimales Ergebnis zu erreichen. Das hat sich auch im Selbstverständnis der meisten Mütter und Väter niedergeschlagen und prägt deren individuelle Sichtweise auf die Elternrolle. Es stellt sich deshalb die Frage, wie sich dieses Elternleitbild beschreiben lässt, welche Dimensionen es hat und ob es eher gesellschaftlich oder individuell motiviert ist. Gemäß dem Leitbildkonzept von Diabaté und Lück (2014) (auch Lück/Diabaté, Kap. 2) wurden Leitbilder als themenbezogene, multidimensionale „Bündel aus kollektiv geteilten Vorstellungen einer Normalität" definiert, die für eine bestimmte Lebenssituation eine Art „Gesamtprogramm“ zur Handlungsorientierung anbieten. Sie umfassen verschiedene, thematisch aufeinander bezogene Aspekte, die im Falle von Verantworteter Elternschaft das Fördergebot für das Kind mit einer bestimmten Vorstellung von der Verfügbarkeit der Eltern verbinden, und zwar dahingehend spezifiziert, ob das die Mutter oder der Vater sein sollte. Je nach gesellschaftlicher Subpopulation, können die einzelnen Aspekte unterschiedlich gewichtet oder zusammengesetzt sein, so dass sich die Leitbilder unterscheiden können und Erklärungen für unterschiedliche Verhaltensmuster anbieten.

Des Weiteren sind Leitbilder sowohl auf der Makroebene als auch auf der Mikroebene $\mathrm{zu}$ verorten. Auf der Makroebene werden sie vom Individuum als handlungsweisende „Normalität“ wahrgenommen. Auf der Mikroebene werden sie von Individuen entweder reproduziert, indem sie sich ihnen gemäß verhalten, oder verändert, indem sie (unbewusst oder bewusst) an die aktuelle Lebenssituation angepasst werden. So wird Eltern der Anspruch vermittelt, dass man seine Kinder fördern sollte und mit der Akzeptanz dieses Anspruches wird wiederum das Leitbild verfestigt, da es sich anderen Eltern als Normalität darstellt. Andererseits kann z. B. die zunehmende Realität berufstätiger Mütter auch zu einer langsamen Umgestaltung von Leitbildern führen. Im Zuge der Anforderungen und Wünsche an steigende Erwerbsbeteiligung von Frauen, werden auch die Bedürfnisse des Kindes entsprechend neu interpretiert: "Heute ist vom 'robusten Kind' die Rede" und die 'Verwöhnung' der Mütter der 68er Generation wird missbilligt und statt dessen auf das Bedürfnis von Eltern zur Selbstbehauptung gesetzt" (Zeiher 2005: 80).

Für den folgenden Beitrag ergibt sich aus diesen Befunden eine Reihe von Fragen. In der Forschung wurden die vielen verschiedenen Facetten dieses Leitbildes bisher nur getrennt dargestellt und nicht in ihrem Zusammenwirken. Auch eine Quantifizierung des 
Phänomens ist bislang nicht erfolgt. So ist zunächst zu klären, ob das Leitbild der Verantworteten Elternschaft in der postulierten Form tatsächlich in der Bevölkerung zu finden ist und welche Unterschiede zwischen allgemeinem und individuellem Leitbild herrschen. Außerdem sollte das Zusammenspiel der verschiedenen Aspekte des Leitbildes und dessen konkrete Ausgestaltung in verschiedenen gesellschaftlichen Subpopulationen untersucht werden. Schließlich werden erste Schlussfolgerungen zur Verbreitung und zur Stabilität der gefundenen Leitbilder gezogen.

\section{Ergebnisse}

Zur Beantwortung der Forschungsfragen werden im Ergebnisteil zunächst bivariate Resultate vorgestellt, die die Bestandteile „Erziehungsleistung“ und „Verfügbarkeit der Eltern“ des Leitbildes, Verantwortete Elternschaft" darstellen. Im Anschluss wird eine Elterntypologie erstellt und analysiert. Begonnen werden soll aber mit wenigen, die Methoden und den Datensatz betreffenden, Vorbemerkungen.

\subsection{Methodische Vorbemerkungen}

Die Familienleitbildstudie bietet für obige Fragestellungen die ideale Datengrundlage (siehe Lück/Naderi/Ruckdeschel, Kap. 3). Um die Multidimensionalität von Leitbildern abzubilden, wurden für das Thema verantwortete Elternschaft zunächst drei Items ausgewählt, die vor allem die Anforderungen an die Erziehungsleistung, die sich aus diesem Leitbild ergeben, abbilden sollen: 1) „Eltern sollten ihre eigenen Bedürfnisse für ihre Kinder komplett zurückstellen“, 2) „Kinder werden sowieso groß, da muss man sich nicht so viele Gedanken machen“ und 3) „Eltern können bei der Erziehung vieles falsch machen, daher müssen sie sich gut informieren." Items 1) und 3) wurden auf der allgemeinen Ebene gespiegelt, d. h. dass sie in einem allgemeinen Kontext wiederholt wurden, um perzipierte gesellschaftliche Leitbilder abzubilden (Lück/Naderi/Ruckdeschel, Kap. 3). Da Verantwortete Elternschaft auch die Verfügbarkeit der Eltern mit einschließt, wurden des Weiteren vier Items zur Bewertung verschiedener Betreuungsformen für Ein- bis Dreijährige ausgewählt: 1) „Für ein Kind zwischen 1 und 3 Jahren ist es das Beste, wenn es nur von der Mutter betreut wird“, 2) „Kinder zwischen 1 und 3 Jahren leiden, wenn sie überwiegend von den Großeltern betreut werden“, 3) „Kinder zwischen 1 und 3 Jahren leiden, wenn sie überwiegend von einer Tagesmutter betreut werden“ und 4) „Kinder zwischen 1 und 3 Jahren leiden, wenn sie überwiegend in einer Kindertagesstätte oder Krippe betreut werden, also in einer Einrichtung für Kinder unter drei Jahren“. Auch hier wurden wieder zwei Items auf die Ebene der Allgemeinheit gespiegelt, Item 1) und 4). Alle Items konnten auf einer 4-stufigen Skala von 1 bis 4, d. h. von „Stimme sehr zu“ bis „Stimme überhaupt nicht zu“ beantwortet werden, wobei keine sogenannte Mittelkategorie für Unentschiedene angeboten wurde. Mit Hilfe einer Hauptkomponentenanalyse wurden die Items zu verschiedenen Dimensionen zusammengefasst, die als Summenindex dargestellt wurden. Dafür wurden die einzelnen Ausprägungen zunächst quadriert, um bei der nachfolgenden Aufsummierung eindeutige Ergebnisse zu erzielen, indem Antwortmuster identifiziert werden können. Die Resultate werden im Folgenden dargestellt. 


\subsection{Anforderungen an die Erziehungsleistung von Eltern}

Elternschaft wird individuell als verantwortungsvolle Aufgabe wahrgenommen, das bestätigen auch unsere Ergebnisse. So stimmen nur 10,6\% der Befragten der Aussage zu, Kinder würden sowieso groß, da müsse man sich nicht so viele Gedanken machen (vgl. Tabelle 1). Im Umkehrschluss heißt dies, dass fast $90 \%$ der jungen Deutschen der Meinung sind, man muss Kindern beim „Großwerden“ helfen, d. h. sie fördern und begleiten. Diese Einstellung herrscht in allen Bevölkerungsgruppen vor, unabhängig von Geschlecht, Elternschaft, regionaler Herkunft oder Bildung.

Tabelle 1: Anforderungen an die Erziehungsleistung von Eltern

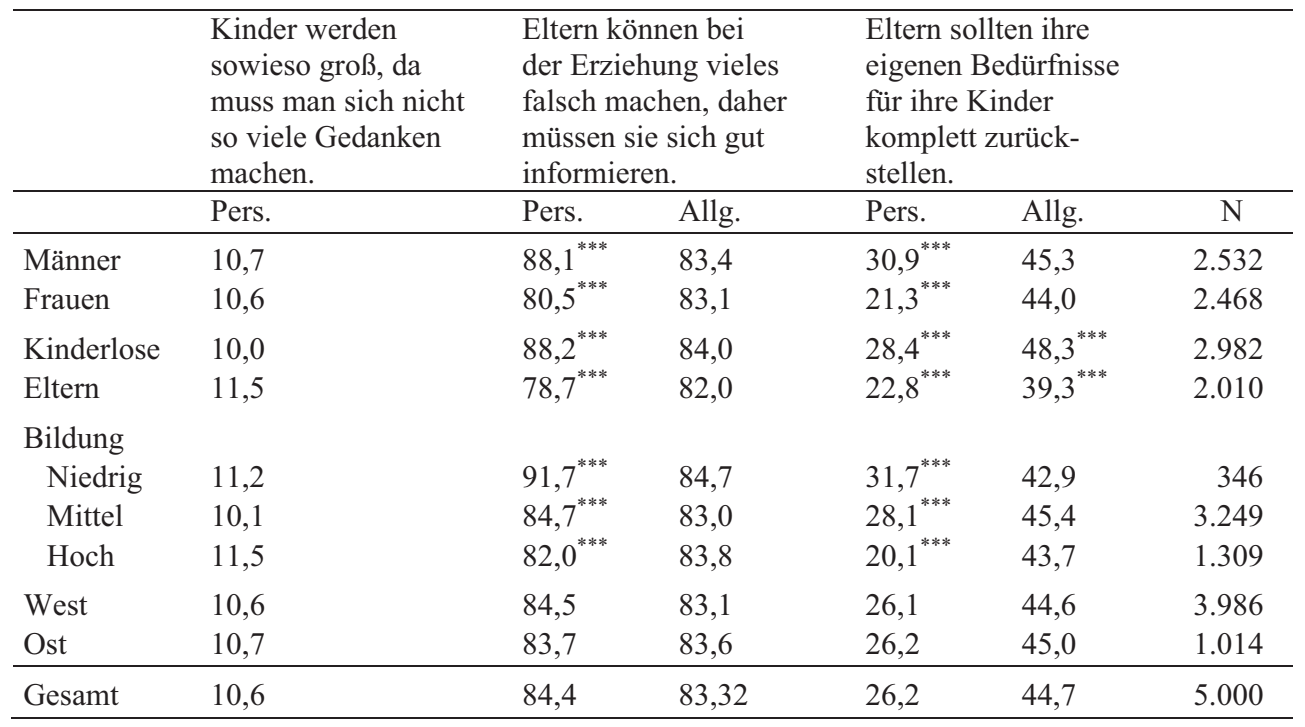

Quelle: FLB (2012), gewichtete Daten, eigene Berechnungen.

Anm.: Zustimmung: „Stimme sehr zu“ und „Stimme zu“ zusammengefasst, in \%; Chi'-Test, Signifikanzniveau: ${ }^{* * *} \leq 0,001$; Pers.: Persönliche Sicht; Allg.: Wahrgenommene Sicht in der Gesellschaft.

Damit korrespondiert die Ansicht, dass Eltern bei der Erziehung vieles falsch machen können und sich deshalb gut informieren müssen. Die Norm verantworteter Elternschaft scheint sich diesbezüglich auf breiter Basis durchgesetzt zu haben und wird mit einer $\mathrm{Zu}$ stimmung von $84,4 \%$ sowohl von den Befragten persönlich akzeptiert als auch als Teil des gesellschaftlichen Leitbildes gesehen (83,2\%) (vgl. Tabelle 1). Während aber in allen Populationsgruppen das gesellschaftliche Leitbild relativ einheitlich wahrgenommen wird, die Zustimmung schwankt zwischen $82 \%$ und $85 \%$, sieht dies auf der persönlichen Ebene der Befragten anders aus. Es finden sich klare Unterschiede zwischen Männern und Frauen $(88,1 \% \mathrm{zu} 80,5 \%)$ und zwischen Kinderlosen und Eltern $(88,2 \%$ zu 78,7\%), wobei Männer und Kinderlose den Informationsbedarf als wichtiger einschätzen. Diejenigen, die normalerweise im Alltag den Großteil der Kindererziehung bewältigen, also mehr Erfahrung damit haben, bejahen das Informationsgebot signifikant seltener, allerdings sind es auch bei Frauen und Müttern immer noch rund $80 \%$. An dieser Stelle lohnt ein differenzierterer Blick auf die Ergebnisse, da in der vorliegenden Studie ein starker Zusammenhang zwischen Geschlecht und Elternschaft vorliegt - der überwiegende Teil der Männer ist 
kinderlos (68\%), was sich auch auf die Ergebnisse auswirkt. Nach Geschlecht und Elternschaft gleichzeitig betrachtet bestätigen sich die bivariaten Ergebnisse jedoch. Den größten Informationsbedarf haben kinderlose Männer (90\%), gefolgt von kinderlosen Frauen und Vätern (86\% bzw. $84 \%$ ), während nur $75 \%$ der Mütter dieser Aussage zustimmen. Dass zunehmender Informationsbedarf mit abnehmender Alltagserfahrung in der Kindererziehung einhergeht, zeigt sich hier noch deutlicher und bestätigt, was bereits aus anderen Studien bekannt ist: Der fehlende Alltag mit Kindern in unserer Gesellschaft führt dazu, dass tradiertes Wissen über den Umgang mit Kindern verloren gegangen ist und Eltern zunehmend verunsichert sind (Nave-Herz 2012; Quaiser-Pohl 2001). Diese Verunsicherung ist bei niedriger Gebildeten wesentlich stärker ausgeprägt als bei höher Gebildeten $(91,7 \%$ zu 82,0\%) und führt dazu, dass sich Eltern mit geringerem Bildungsgrad professionellen Erziehern gegenüber oft unterlegen fühlen (Kaufmann 1990: 108).

Die größten Diskrepanzen, sowohl zwischen persönlicher Ebene und Allgemeinheit als auch zwischen einzelnen gesellschaftlichen Gruppen, finden sich jedoch bei der Frage, ob Eltern ihre Bedürfnisse für ihre Kinder komplett zurückstellen sollten. Nur ein gutes Viertel der Befragten ist persönlich der Meinung, dass Kinder absolut an erster Stelle stehen sollten, als gesellschaftliches Leitbild wird dies dagegen von 44,7\% wahrgenommen. Der Druck auf Eltern, einem Ideal der aufopfernden Eltern zu entsprechen, scheint damit größtenteils gesellschaftlich bedingt zu sein und weniger individuell motiviert. Die größten Unterschiede finden sich auf der persönlichen Ebene: Männer fordern signifikant häufiger als Frauen eigene Bedürfnisse für Kinder völlig zurückzustellen (30,9\% zu 21,3\%), während sich Kinderlose und Eltern hier weniger unterscheiden $(28,4 \%$ zu 22,8 \%). Der Trend mit zunehmender Erziehungserfahrung realistischere Ansprüche an die Elternrolle zu stellen zeigt sich aber auch hier. Differenziert man noch weiter, dann findet sich eine klare Geschlechtergrenze. Sowohl kinderlose Männer als auch Väter (32\% und 28 \%) verlangen wesentlich häufiger, Kinder als oberste Priorität zu sehen als kinderlose Frauen oder Mütter (23\% und $19 \%$ ).

Da immer noch die Mutter als wichtigste Bezugsperson für das Kind gilt (z. B. Mantl 2006; Herwartz-Emden 1995 und Diabaté, Kap. 13), weist dieses Ergebnis darauf hin, dass implizit davon ausgegangen wird, dass es Frauen bzw. Mütter sind, die ihre eigenen Bedürfnisse zurückstellen sollten, was für Männer wesentlich einfacher zu fordern ist als für Frauen. Schließlich zeigen sich auch zwischen den verschiedenen Bildungsgruppen große Unterschiede. Im Gegensatz zu höher Gebildeten verbinden Personen mit niedrigerem Bildungsgrad die Elternrolle häufiger mit persönlichen Opfern und sehen auch einen höheren Informationsbedarf. Als gesellschaftliches Leitbild wird die Aufopferung für das Kind vor allem von Kinderlosen und Eltern unterschiedlich wahrgenommen $(48,3 \% \mathrm{zu}$ $39,3 \%$ ), was an den unterschiedlichen persönlichen Umfeldern liegen könnte. Eltern haben vermehrt mit anderen Eltern Kontakt, die ihre Alltagserfahrungen mit Kindern teilen, was sich letztlich auch in ihren wahrgenommenen Leitbildern widerspiegelt und diese bestärkt (siehe Gies/Dietrich, Kap. 4).

\subsection{Anforderungen an die Verfügbarkeit von Eltern}

Als wesentliches Merkmal Verantworteter Elternschaft wurde die ständige Verfügbarkeit der Eltern genannt, die eine umfassende Förderung des Nachwuchses ermöglichen und sichern soll. In den vorliegenden Analysen beschränken wir uns auf 1- bis 3-jährige Kinder, für die die Frage nach der Betreuung angesichts der verschiedenen Möglichkeiten als besonders interessant erscheint. 
Auf der individuellen Ebene erfährt die ausschließliche Betreuung durch die Mutter die größte Ablehnung, nur 15,2 \% der Befragten halten sie für gut (vgl. Tabelle 2). Neben der Betreuung durch die Mutter sollten sich nach Meinung der Befragten also auch noch andere um ein Kleinkind kümmern. Dabei werden Großeltern bevorzugt, allerdings nicht als überwiegende Betreuung, denn ein relativ großer Anteil der Befragten von 37,2\% geht davon aus, dass Kinder dann leiden. Noch mehr, 41,3\%, halten eine überwiegende Betreuung in einer Kindertagesstätte für schlecht und Tagesmütter werden von über der Hälfte, 54,0 \%, abgelehnt. Die ausschließliche Betreuung durch die Mutter stellt ein Thema dar, bei dem individuelle Vorstellungen und das wahrgenommene Leitbild sehr stark voneinander abweichen. Den 15,2 \%, die dieses Modell persönlich befürworten stehen 57,4 \% gegenüber, die es als gesellschaftliches Leitbild wahrnehmen. Obwohl also kaum jemand von diesem Modell überzeugt ist, denkt weit mehr als die Hälfte, es werde gesellschaftlich erwartet. Das Thema polarisiert auch zwischen gesellschaftlichen Gruppen, weit mehr Frauen als Männer sind davon überzeugt, dass Kinder zur Mutter gehören (19,8 \% zu 10,7 \%), ebenso mehr Eltern als Kinderlose (19,3 \% zu 12,4 \%). Differenzierter nach Geschlecht und Elternschaft gleichzeitig betrachtet, sind vor allem die Mütter selbst dieser Meinung (24,7\%), während Männer eher ablehnend sind und sich nicht nach Elternschaftsstatus unterscheiden (Väter: 11,4 \%, kinderlose Männer: 10,3 \%). Auch kinderlose Frauen vertreten eher die Meinung, Kleinkinder sollten nicht ausschließlich von der Mutter betreut werden (15,2 \%). Die unterschiedlichen Einstellungen spiegeln sich zum Teil auch auf der Ebene der gesellschaftlichen Leitbilder wider: Vor allem Frauen nehmen die exklusive mütterliche Kinderbetreuung auch als gesellschaftliches Leitbild wahr (61,0 \% zu 53,8 \%) und ebenso Kinderlose $(59,1 \% \mathrm{zu} 54,9 \%)$. Bildungsunterschiede sind nicht besonders ausgeprägt.

Tabelle 2: Anforderungen an die Verfügbarkeit von Eltern

\begin{tabular}{|c|c|c|c|c|c|c|c|}
\hline & \multicolumn{2}{|c|}{$\begin{array}{l}\text { Für ein Kind zw. } \\
1 \text { und } 3 \text { Jahren } \\
\text { ist es das Beste, } \\
\text { wenn es nur von } \\
\text { der Mutter } \\
\text { betreut wird. }\end{array}$} & $\begin{array}{l}\text { Kinder zwische } \\
\text { von den } \\
\text { Großeltern } \\
\text { betreut werden. }\end{array}$ & $\begin{array}{l}\text { von einer } \\
\text { Tagesmutter } \\
\text { betreut werden. }\end{array}$ & $\begin{array}{l}\text { in einer } \\
\text { oder } \mathrm{Kr} \\
\text { betreut }\end{array}$ & $\begin{array}{l}\text { Kita } \\
\text { ppe } \\
\text { verden. }\end{array}$ & \\
\hline & Pers. & Allg. & Pers. & Pers. & Pers. & Allg. & $\mathrm{N}$ \\
\hline Männer & $10,7^{* * *}$ & $53,8^{* * *}$ & $41,8^{* * *}$ & $58,1^{* * *}$ & 42,3 & $42,3^{* * *}$ & 2.532 \\
\hline Frauen & $19,8^{* * *}$ & $61,0^{* * *}$ & $32,6^{* * *}$ & $49,8^{* * *}$ & 40,2 & $48,3^{* * *}$ & 2.468 \\
\hline Kinderlose & $12,4^{* * *}$ & $59,1^{* *}$ & $36,1^{*}$ & $56,4^{* * *}$ & $44,1^{* * *}$ & $46,8^{* *}$ & 2.982 \\
\hline Eltern & $19,3^{* * *}$ & $54,9^{* *}$ & $38,9^{*}$ & $50,5^{* * *}$ & $37,1^{* * *}$ & $42,9^{* *}$ & 2.010 \\
\hline Bildung & & & & & & & \\
\hline Niedrig & $15,0^{* *}$ & 54,5 & $53,2^{* * *}$ & $70,9^{* * *}$ & $52,3^{* * * *}$ & $44,5^{* * * *}$ & 346 \\
\hline Mittel & $16,3^{* *}$ & 56,9 & $39,4^{* * *}$ & $57,6^{* * *}$ & $43,0^{* * *}$ & $43,2^{* * *}$ & 3.249 \\
\hline Hoch & $12,7^{* *}$ & 59,6 & $27,6^{* * *}$ & $40,3^{* * *}$ & $33,7^{* * *}$ & $51,3^{* * *}$ & 1.309 \\
\hline West & $17,2^{* * *}$ & $59,6^{* * *}$ & 37,0 & $56,8^{* * *}$ & $46,7^{* * *}$ & $47,6^{* * *}$ & 3.986 \\
\hline Ost & $7,0^{* * *}$ & $48,9^{* * *}$ & 38,0 & $42,8^{* * *}$ & $20,0^{* * *}$ & $35,9^{* * *}$ & 1.014 \\
\hline Gesamt & 15,2 & 57,4 & 37,2 & 54,0 & 41,3 & 45,2 & 5.000 \\
\hline
\end{tabular}

Quelle: FLB (2012), gewichtete Daten, eigene Berechnungen

Anm.: Zustimmung,zusammengefasst „Stimme sehr zu“ und „Stimme zu“ in \%; Pers.: Persönliche Sicht; Allg.: Wahrgenommene Sicht in der Gesellschaft. Signifikanz (Chi $\left.{ }^{2}-T e s t\right):{ }^{* * *} \mathrm{p} \leq 0,001 ;{ }^{* *} \mathrm{p} \leq 0,01$; $\mathrm{p} \leq 0,05$. 
Im Gegensatz zu den bisher betrachteten Themen finden sich bei der Betreuung auch sehr große Ost-West-Unterschiede. Die Zustimmung zu einer ausschließlichen Kinderbetreuung durch die eigene Mutter ist im Westen wesentlich höher als in den neuen Bundesländern $(17,2 \%$ zu 7,0 \%). Ein Ergebnis, das aufgrund der unterschiedlichen historischen Entwicklung nicht überrascht. In der ehemaligen DDR war externe Kinderbetreuung der Normalfall und wurde auch gesellschaftlich gewünscht, während sich in den alten Bundesländern lange Zeit die Norm hielt, dass eine Mutter für ihr Kind da zu sein habe, was sich erst langsam ändert (z. B. Kreyenfeld/Geisler 2006; Schneider 1994). Diese unterschiedlichen Entwicklungen spiegeln sich sowohl in den individuellen Einstellungen wider, als auch im gesellschaftlich wahrgenommen Leitbild, die sich jeweils um 10 Prozentpunkte unterscheiden. Das gleiche Ergebnis zeigt sich auch bei der Frage, bei welcher externen Betreuungsform kleine Kinder am wenigsten leiden. Nur in den neuen Bundesländern steht die Kindertagesstätte an erster Stelle ${ }^{1}$ gefolgt von den Großeltern und der Tagesmutter, wobei die Werte auch bei der Tagesmutter signifikant niedriger liegen als im Durchschnitt. Im Gegensatz dazu stehen bei anderen Gruppen meist die Großeltern auf dem ersten Platz, gefolgt von der Kindertageseinrichtung. Die zweite Ausnahme stellen Eltern dar, bei denen Großeltern mit der Kindertageseinrichtung fast gleichauf liegen, aber nur den zweiten Platz belegen, was möglicherweise auf gute Erfahrungen mit solchen Einrichtungen schließen lässt. Generell spiegelt sich auch in diesen Ergebnissen wieder die „Praxisnähe“ zur Kindererziehung wider. Männer sind externer Kinderbetreuung wesentlich kritischer gegenüber eingestellt als Frauen. Kinderlose dagegen misstrauen vor allem Tagesmüttern und Kindertagesstätten, während die Großeltern noch am ehesten akzeptiert werden. Differenziert man nach Bildung, dann stehen niedriger Gebildete unabhängig von der Betreuungsart externer Betreuung grundsätzlich kritischer gegenüber, während höher Gebildete vergleichsweise offen sind. Da Kinder höher gebildeter Mütter häufiger extern betreut werden (Kreyenfeld 2004), könnte dieses Ergebnis auf positive Erfahrungen zurückgeführt werden. Gleichzeitig nehmen höher Gebildete das gesellschaftliche Leitbild kritischer wahr, d. h. über die Hälfte denkt dass externe Kinderbetreuung von der Allgemeinheit negativ bewertet wird (51,3\%) im Gegensatz zu 44,5 \% der Personen mit niedrigerer und 43,2 \% derer mit mittlerer Bildung.

\subsection{Die Dimensionen , Verantworteter Elternschaft“}

\section{Verantwortete Elternschaft auf persönlicher Ebene}

Die unterschiedlichen persönlichen Meinungen und Aussagen lassen sich mittels Hauptkomponentenanalyse zu drei Dimensionen zusammenfassen, die zusammen $61 \%$ der Gesamtvarianz erklären und die unterschiedlichen Aspekte des persönlichen Leitbildes benennen. Aus den entsprechenden Items wurde jeweils ein Index gebildet (vgl. Abschnitt 3.1), der umso kleiner wird, je höher die Zustimmung ist:

1. Externe Kinderbetreuung ist schlecht für das Kind. Zu dieser Dimension gehören die drei Items, die die externe Betreuung durch Großeltern, Tagesmutter und Kindertagesstätte bewerten lassen. Der Index setzt sich aus den drei entsprechenden Items zusammen und weist eine eher gleichmäßige Verteilung auf. Bei 48 möglichen Punkten, was

1 „Erste Stelle“ bedeutet, dass der Anteil der Befragten, die der Meinung sind, dass ein Kind bei überwiegender Betreuung in der jeweiligen Form leidet, am geringsten ist im Vergleich zu den anderen beiden Betreuungsformen. 
die völlige Ablehnung aller drei Items bedeuten würde 2 , liegt der Median bei 22, also relativ in der Mitte. Der Modus ist 27, was eine eindeutige, aber nicht übermäßige Ablehnung des behaupteten Leidensdruckes von extern betreuten Kindern bedeutet ${ }^{3}$.

2. Kinder als oberste Priorität. Die Dimension wird aus den Items „Eltern sollten ihre Bedürfnisse komplett zurückstecken“ und „Für das Kind ist eine Betreuung durch die eigene Mutter am besten“" gebildet. Der Index aus den beiden Items ist eher rechtsschief verteilt. Bei 32 möglichen Punkten liegt der Median bei 18, der Modus bei 25, d. h. insgesamt ist die Ablehnung dieser Dimension bei den Befragten relativ stark ausgeprägt. Dies manifestiert sich auch in einer starken Besetzung der extremen Ablehnungskategorie 32 , in der $11,2 \%$ der Befragten zu finden sind.

3. Elternschaft bedeutet Arbeit. In dieser Dimension finden sich die Items, „Kinder werden nicht von alleine groß ${ }^{“ 4}$ und „Als Eltern kann man vieles falsch machen und muss sich gut informieren“. Der Index dieser Dimension ist sehr stark linksschief verteilt, der Median liegt bereits bei der Kategorie 5, d. h. $50 \%$ der Befragten haben beiden Aussagen ,sehr“ zugestimmt bzw. bei maximal einer nur ,eher“" zugestimmt.

Verantwortete Elternschaft auf gesellschaftlicher Ebene

Dem gesellschaftlichen Leitbild lassen sich zwei Dimensionen mit einem - bedingt durch weniger Items - etwas anders gearteten Schwerpunkt zuordnen ${ }^{5}$, der mehr auf die Bedeutung der Mutter abstellt, was sich auch in den bivariaten Analysen bereits angedeutet hat:

1. Mutternähe: Dazu zählt, dass kleine Kinder von der Mutter betreut werden sollten und dass sie bei überwiegender Betreuung in einer Kindertagesstätte leiden. Der Index ist stark linksschief, bei 32 möglichen Punkten liegt der Median bei 13, was mit dem Modus übereinstimmt. Der Großteil der Befragten nimmt die Erwartungen der Allgemeinheit derart wahr, dass Mütter bei ihren Kindern bleiben sollten, weil Kinder bei überwiegend externer Betreuung leiden würden. Dieses Ergebnis steht in starkem Gegensatz zu den persönlichen Ansichten der Befragten.

2. Elternschaft bedeutet Verzicht und Arbeit: Die Meinung, dass Eltern bei der Erziehung vieles falsch machen können und sie ihre Bedürfnisse komplett hinter die des Kindes zurückstellen sollten, bildet die Kernaussage dieser Leitbilddimension. Auch dieser Index ist stark linksschief verteilt, mit einem Median von 10 bei 32 möglichen Punkten. Auch in dieser Hinsicht werden die Erwartungen der Allgemeinheit von den Befragten so wahrgenommen, dass Eltern vieles falsch machen können und ihre Bedürfnisse hinter die der Kinder zurückstellen sollten. Während der Notwendigkeit einer Förderung von Kindern auch individuell stark zugestimmt wird, steht das wahrgenommene Leitbild, was die Bedürfnishierarchie betrifft, in starkem Gegensatz zu den eigenen Ansichten der Befragten.

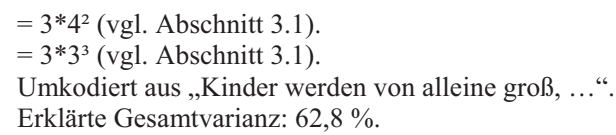




\subsection{Elterntypen}

In einem nächsten Schritt wurden aus den persönlichen Einstellungsdimensionen Typen gebildet $^{6}$, von denen vier jeweils mindestens $10 \%$ der Befragten umfassen (vgl. Tabelle 3 ). Die resultierenden Elterntypen lassen sich in Relation zur Norm der Verantworteten Elternschaft benennen, wobei darauf hingewiesen werden muss, dass alle Befragten aufgrund ihrer Angaben einem Elterntyp zugwiesen wurden, unabhängig von einer tatsächlichen Elternschaft. Der häufigste Typus mit $34 \%$ stellt in gewisser Weise die gesellschaftliche Normalität dar, denn schon in den bivariaten Analysen wurde deutlich, dass das Fördergebot des Leitbildes Verantwortete Elternschaft gesellschaftlich mehr oder weniger umfassend akzeptiert ist. Insofern ist dieser Typus der Meinung, dass Elternschaft anstrengend ist, d. h. man soll seine Kinder fördern und muss sich dafür auch informieren. Gleichzeitig werden aber weder externe Kinderbetreuung negativ bewertet noch Kinder als absolute Priorität gesetzt. Wir bezeichnen den Elterntyp als engagiert, er entspricht dem Leitbild der Verantworteten Elternschaft im wichtigsten Punkt, nimmt sich selbst aber nicht völlig zurück. Der Typ engagierte Eltern zeichnet sich vor allem durch überdurchschnittlich viele höher Gebildete und durch relativ viele Befragte aus den neuen Bundesländern aus. Ambitionierte Eltern (23\%) ähneln den engagierten Eltern sehr, bewerten aber externe Kinderbetreuung negativ. Das subjektive Leitbild von Elternschaft wird insofern anstrengender, da nicht nur das Fördergebot anerkannt wird, sondern auch möglichst von den Eltern selbst erfüllt werden sollte. Anders als der erste Typus ist dieser wesentlich stärker durch Männer und Kinderlose geprägt, also Personen, die in der Regel weniger in die alltägliche Kindererziehung involviert sind (s. o.). Zudem sind wesentlich häufiger Befragte aus den alten Bundesländern und niedrig bis mittel Gebildete vertreten, was mit den bivariaten Analysen übereinstimmt und vor allem auf die Bewertung externer Kinderbetreuungseinrichtungen zurückzuführen ist.

Als dritthäufigster Typus (13\%) wurden die distanzierten Eltern gefunden, die dem gesellschaftlichen Leitbild der Verantworteten Elternschaft distanziert gegenüberstehen und alle drei individuellen Dimensionen verneinen. Sie nehmen Elternschaft nicht als Arbeit wahr, externe Kinderbetreuung wird positiv bewertet und Kinder werden nicht als absolute Priorität bewertet. Distanzierte Eltern sind wesentlich häufiger Frauen als Männer und mehr tatsächliche Eltern, also im Gegensatz zu den ambitionierten Eltern eher diejenigen, die den Alltag der Kindererziehung gestalten.

Der letzte häufigere Typus nimmt im Gegensatz zu den distanzierten Eltern die Verantwortung, die gesellschaftlich mit Elternschaft assoziiert wird umso ernster, d. h. Elternschaft wird mit Anstrengung gleichsetzt, die möglichst die Eltern selbst leisten sollen und dem Kind wird absolute Priorität eingeräumt. Diese Eltern wurden als protektive Eltern bezeichnet und umfassen $10 \%$ der Befragten. Diesem Typus gehören signifikant häufiger niedrig und mittel Gebildete an sowie Personen aus den alten Bundesländern.

Vergleicht man die wahrgenommenen gesellschaftlichen Leitbilder zwischen den Typen, dann bestätigt sich ein weiteres Mal, dass die Leitbilddimension der Verantworteten Elternschaft im engeren Sinne, d. h. die Maxime, dass Kinder Förderung brauchen, allgemein anerkannt ist, entsprechend ist auch die Varianz zwischen den Gruppen nur gering. Anders dagegen wird die Dimension der Mutternähe gesehen, die die Kleinkindbetreuung

6 Dafür wurden die Indizes 0-1 kodiert. Aus Verteilungsgründen wurden für die Dimension „Elternschaft gleich Arbeit“ nur die Ausprägungen „1-stimme sehr zu“ oder „2-stimme zu“ als „,1“ kodiert, für die beiden anderen Dimensionen konnte zusätzlich eine Ausprägung „,3-stimme eher nicht zu“ sein. Aus den drei bipolaren Indizes mit jeweils zwei Ausprägungen ergeben sich insgesamt acht mögliche Typen. 
möglichst ausschließlich der Mutter zuweist, hier findet man starke Varianz zwischen den Gruppen. Dabei nehmen diejenigen, die diesen Leitbildbestandteil individuell nicht favorisieren, also engagierte und distanzierte Eltern, dies verstärkt von außen wahr, möglicherweise als Anspruchshaltung ihrer Umgebung. Ambitionierte und protektive Eltern dagegen, die diese Dimension aufgrund ihrer individuellen Einstellungen eher unterstützen, nehmen sie gesellschaftlich seltener wahr. In diesem Fall möglicherweise als Diskrepanz zwischen einer gesellschaftlich etwas lockereren Haltung und eigenen höheren Ansprüchen.

Tabelle 3: Elterntypen nach soziodemografischen Merkmalen und allgemeinen Leitbilddimensionen

\begin{tabular}{|c|c|c|c|c|c|}
\hline & \multicolumn{5}{|c|}{ Elterntypen } \\
\hline & Engagiert & Ambitioniert & Distanziert & Protektiv & Gesamtdatensatz \\
\hline \multicolumn{6}{|l|}{ Leitbilddimension } \\
\hline Elternschaft gleich Arbeit & ja & ja & nein & ja & \\
\hline $\begin{array}{l}\text { Externe Kinderbetreuung } \\
\text { schlecht f. Kind }\end{array}$ & nein & ja & nein & ja & \\
\hline Kind sollte Priorität haben & nein & nein & nein & ja & \\
\hline $\mathrm{N}$ & 1.682 & 1.165 & 655 & 522 & 5.000 \\
\hline$\%$ & 34,0 & 23,0 & 13,0 & 10,0 & 100,0 \\
\hline Anteil Frauen & $49,1^{* * *}$ & $41,5^{* * *}$ & $58,9^{* * *}$ & $52,3^{* * *}$ & $49,4^{* * *}$ \\
\hline Anteil Eltern & $37,3^{* * *}$ & $34,4^{* * *}$ & $52,1^{* * *}$ & $40,4^{* * *}$ & $40,3^{* * *}$ \\
\hline Anteil höher Gebildete & $33,4^{* * *}$ & $20,2^{* * *}$ & $35,9^{* * *}$ & $15,8^{* * *}$ & $26,7^{* * *}$ \\
\hline Anteil West & $74,0^{* * *}$ & $84,9^{* * *}$ & $74,4^{* * *}$ & $84,5^{* * *}$ & $79,7^{* * *}$ \\
\hline $\begin{array}{l}\text { Allgemeine Leitbilddimension } \\
\text { Mutternähe }\end{array}$ & $36,5^{* * *}$ & $25,2^{* * *}$ & $34,5^{* * *}$ & $27,8^{* * *}$ & $32,2^{* * *}$ \\
\hline $\begin{array}{l}\text { Allgemeine Leitbilddimension } \\
\text { Verantwortete Elternschaft }\end{array}$ & $36,3^{* * *}$ & $36,0^{* * *}$ & $40,9^{* * *}$ & $40,6^{* * *}$ & $39,0^{* * *}$ \\
\hline
\end{tabular}

Quelle: FLB (2012), gewichtete Daten, eigene Berechnungen

Anm.: 4 häufigste Typen + Verteilung des jeweiligen Merkmals im Gesamtdatensatz. Zur Kodierung von Zustimmung und Ablehnung siehe Fußnote 6; Chi $^{2}$-Test, Signifikanzniveau: ${ }^{* * *} \leq 0,001$.

Lesebeispiel: Engagierte Eltern stimmen zu, dass ,Elternschaft gleich Arbeit“" ist, sie stimmen nicht $\mathrm{zu}$, dass „Externe Kinderbetreuung schlecht für das Kind ist“" und sie stimmen nicht zu, dass das „Kind oberste Priorität haben sollte“.

\section{$4 \quad$ Zusammenfassung}

Im vorliegenden Beitrag sollte geklärt werden, ob sich in Deutschland ein Leitbild der Verantworteten Elternschaft ausmachen lässt, wie es ausgestaltet ist und ob man eher von gesellschaftlicher oder individueller Motivation sprechen sollte. Dafür wurden zwei Bereiche des Normenkomplexes Verantwortete Elternschaft, das Fördergebot und das Gebot der Mutternähe, näher betrachtet und auf ihre Verteilung in verschiedenen gesellschaftlichen Subpopulationen hin untersucht.

Als Ergebnis kann dabei zunächst festgehalten werden, dass der hier als Fördergebot bezeichnete Teil des Leitbildes sowohl allgemein als auch individuell breite Zustimmung 
findet. Die jungen Deutschen sind weit überwiegend der Meinung, dass man Kinder beim Aufwachsen intensiv begleiten sollte und dass man sich dafür gut informieren muss, dass Kinder also nicht „,von alleine groß werden“. Diese Einstellung verbindet verschiedenste Elterntypen und wird auch als gesellschaftlicher Anspruch an Eltern wahrgenommen. Sowohl auf individueller wie auch auf gesamtgesellschaftlicher Ebene finden sich nur graduelle Unterschiede zwischen gesellschaftlichen Subgruppen, die auf einen Zusammenhang mit der Alltagserfahrung im Umgang mit Kindern hinweisen, d. h. je weniger jemand im Alltag mit Kindern zu tun hat, desto höher sind die Ansprüche und desto größer ist die Verunsicherung. Insgesamt kann man aber von einer stark in der Gesellschaft verankerten Leitbilddimension sprechen.

Anders stellt sich die Situation bei der Frage nach der Verfügbarkeit der Mutter dar. Die Leitbilddimension der Mutternähe differiert sowohl zwischen individueller und gesellschaftlicher Ebene als auch zwischen einzelnen gesellschaftlichen Subpopulationen. Dabei werden die Ansprüche vor allem als gesellschaftliche Erwartungen wahrgenommen, die auf individueller Ebene aber überwiegend abgelehnt werden. Interessant sind hier vor allem die Geschlechterdifferenzen, Männer stimmen dieser Leitbilddimension mit ihrer expliziten Zuweisung der Betreuungsarbeit an die Mutter wesentlich stärker zu als Frauen. Die Akzeptanz bei Frauen dagegen schwindet zunächst auf der individuellen Ebene, aber dort nicht in allen Aspekten gleichzeitig oder gleich stark. So sind vor allem Mütter der Meinung, Kleinkinder sollten von der Mutter betreut werden, möchten aber gleichzeitig nicht, dass man für Kinder alle Bedürfnisse komplett zurückstecken sollte. Die verschiedenen nicht kongruenten Teile dieser Leitbilddimension können auf einen Umbruch hinweisen, der die veränderte Realität von Frauen mit der Mutterrolle neu verknüpft (Zeiher 2005). Dabei findet der Wandel zunächst auf der Individualebene statt, während der Anpassungsprozess der Leitbilder auf der Makroebene verzögert erfolgt. Neben den typischen Geschlechterdifferenzen finden sich bei diesem Leitbildbestandteil auch erwartbare Unterschiede zwischen den alten und den neuen Bundesländern, was die Permanenz gesellschaftlicher Leitbilder in bestimmten Kontexten nochmals zeigt.

Zusammenfassend lässt sich festhalten, dass das Leitbild Verantworteter Elternschaft in Deutschland stark präsent ist und die Vorstellungen von Elternschaft prägt. Da Leitbilder multidimensional sind, findet Wandel durch Veränderungen in einzelnen Teilaspekten statt, wodurch ein kontinuierlicher Anpassungsprozess an die Lebenswelt der Individuen gewährleistet ist. Im Falle der Verantworteten Elternschaft findet sich einerseits das Fördergebot, dessen implizite Anforderungen vom überwiegenden Teil der jungen Deutschen bejaht werden und das einen stabilen Bestandteil des Leitbildes darstellt. Auf der anderen Seite steht das Gebot der Mutternähe und Aufopferung, das sowohl in sich inkongruent ist, weil einzelne Gruppen gleichzeitig einander ausschließende Ansichten haben, als auch zwischen den Ebenen stark differiert, was auf einen Wandel dieses Leitbildaspektes schließen lässt. Betrachtet man verschiedene Elterntypen, dann bestätigen sich die Ergebnisse, d. h. Eltern, die vor allem das Fördergebot bejahen, stellen die größte Gruppe dar, gefolgt von Eltern, die zusätzlich eine Kinderbetreuung innerhalb der Familie einer externen vorziehen. Relativ stark vertreten sind auch die beiden extremen Typen, d. h. Eltern, die dem Leitbild der Verantworteten Elternschaft sehr distanziert gegenüberstehen und Eltern, die es in allen seinen Aspekten bejahen. Interessanterweise nehmen diese Elterntypen die allgemeinen Leitbilder eher als Gegensatz zu ihren individuellen Auffassungen wahr. Insofern bleiben noch viele Fragen offen, die es sich lohnt, in weiteren Analysen intensiver zu klären. 


\section{Literatur}

Ahnert, Liselotte (2014): Frühe Bindung: Entstehung und Entwicklung. München: Ernst Reinhardt. Baum, Antonia (2014): Man muss wahnsinnig sein, heute ein Kind zu kriegen. In: Frankfurter Allgemeine, 16.09.2013.

Beck-Gernsheim, Elisabeth (1991): Was Eltern das Leben erschwert: Neue Anforderungen und Konflikte in der Kindererziehung. In: Teichert, Volker (Hrsg.): Junge Familien in der Bundesrepublik. Familienalltag - Familienumwelt - Familienpolitik. Wiesbaden: VS Verlag für Sozialwissenschaften, S. 55-73.

Becker-Stoll, Fabienne; Berkic, Julia; Kalicki, Bernhard (Hrsg.) (2010): Bildungsqualität für Kinder in den ersten drei Jahren. Berlin, Düsseldorf, Mannheim: Cornelsen Scriptor.

Diabaté, Sabine; Lück, Detlev (2014): Familienleitbilder. Identifikation und Wirkungsweise auf generatives Verhalten. In: Zeitschrift für Familienforschung 26 (1), S. 49-69.

Du Bois-Reymond, Manuela; Büchner, Peter; Krüger, Heinz-Hermann; Ecarius, Jutta; Fuhs, Burkhard (1994): Kinderleben. Modernisierung von Kindheit im interkulturellen Vergleich. Wiesbaden: VS Verlag für Sozialwissenschaften.

Eltern (2010): [http://www.eltern.de/c/pdf/gesamt-studie-zgf-forsa.pdf, 17.04.2014].

Herwartz-Emden, Leonie (1995): Mutterschaft und weibliches Selbstkonzept. Eine interkulturell vergleichende Untersuchung. Weinheim/München: Juventa.

Huinink, Johannes (1997): Elternschaft in der modernen Gesellschaft. In: Gabriel, Karl; Herlth, Alois; Strohmeier, Klaus Peter (Hrsg.): Modernität und Solidarität. Konsequenzen gesellschaftlicher Modernisierung. Freiburg: Herder, S. 79-90.

Kaelble, Hartmut (2007): Sozialgeschichte Europas. 1945 bis zur Gegenwart. München: Beck.

Kaufmann, Franz-Xaver (1990): Zukunft der Familie. Stabilität, Stabilitätsrisiken und Wandel der familialen Lebensformen sowie ihre gesellschaftlichen und politischen Bedingungen. Perspektiven und Orientierungen. Schriftenreihe des Bundeskanzleramtes 10. München: Beck.

Kaufmann, Franz-Xaver; Herlth, Alois; Quitmann, Joachim; Simm, Regina; Strohmeier, Klaus Peter (1982): Familienentwicklung - generatives Verhalten im familialen Kontext. In: Zeitschrift für Bevölkerungswissenschaft 8 (4), S. 523-545.

Kreyenfeld, Michaela (2004): Soziale Ungleichheit und Kinderbetreuung - eine Analyse der sozialen und ökonomischen Determinanten der Nutzung von Kindertageseinrichtungen. In: Becker, Rolf; Lauterbach, Wolfgang (Hrsg.): Bildung als Privileg? Erklärungen und Befunde zu den Ursachen der Bildungsungleichheit. Wiesbaden: VS Verlag für Sozialwissenschaften, S. 99-125.

Kreyenfeld, Michaela; Geisler, Esther (2006): Müttererwerbstätigkeit in Ost- und Westdeutschland. In: Zeitschrift für Familienforschung 18 (3), S. 333-360.

Mantl, Elisabeth (2006): Gute Mütter - gute Töchter. Konzepte - Visionen - Lebenswirklichkeit. Zur Kulturalität deutscher Erfahrungen seit 1870. In: Bertram, Hans; Krüger, Helga; Spieß, Katharina C. (Hrsg.): Wem gehört die Familie der Zukunft? Expertisen zum 7. Familienbericht der Bundesregierung. Opladen: Barbara Budrich, S. 235-257.

Meyer, Thomas (2002): Moderne Elternschaft - neue Erwartungen, neue Ansprüche. In: Aus Politik und Zeitgeschichte. Beilage zur Wochenzeitung „Das Parlament“ (22-23), S. 40-46.

Mühling, Tanja; Rost, Harald; Rupp, Marina; Schulz, Florian (2006): Kontinuität trotz Wandel. Die Bedeutung traditioneller Familienleitbilder für die Berufsverläufe von Müttern und Vätern. Weinheim/München: Juventa.

Nave-Herz, Rosemarie (2012): Familie im Wandel? - Elternschaft im Wandel? In: Böllert, Karin; Peter, Corinna (Hrsg.): Mutter + Vater=Eltern? Sozialer Wandel, Elternrollen und soziale Arbeit. Wiesbaden: Springer VS, S. 33-49.

Quaiser-Pohl, Claudia (2001): Deutsche Eltern im interkulturellen Vergleich. In: Nickel, Horst; Quaiser-Pohl, Claudia (Hrsg.): Junge Eltern im kulturellen Wandel. Untersuchungen zur Familiengründung im internationalen Vergleich. Weinheim: Juventa, S. 301-310.

Scheuer, Angelika; Dittmann, Jörg (2007): Berufstätigkeit von Müttern bleibt kontrovers: Einstellungen zur Vereinbarkeit von Beruf und Familie in Deutschland und Europa. In: Informationsdienst Soziale Indikatoren 38, 1-5. 
Schneider, Norbert F. (1994): Familie und private Lebensführung in West- und Ostdeutschland. Eine vergleichende Analyse des Familienlebens 1970-1992. Stuttgart: Enke.

Schneider, Norbert F. (2002): Elternschaft heute. Gesellschaftliche Rahmenbedingungen und individuelle Gestaltungsaufgaben - einführende Betrachtungen. In: Schneider, Norbert F.; MatthiasBleck, Heike (Hrsg.): Elternschaft heute. Gesellschaftliche Rahmenbedingungen und individuelle Gestaltungsaufgaben. Zeitschrift für Familienforschung, Sonderheft 2. Opladen: Leske + Budrich, S. 9-21.

Schneider, Norbert F.; Diabaté, Sabine; Lück, Detlev (2014): Familienleitbilder in Deutschland. Ihre Wirkung auf Familiengründung und Familienentwicklung. Konrad-Adenauer-Stiftung e.V., Sankt Augustin/Berlin.

Seiffge-Krenke, Inge; Schneider, Norbert F. (2012): Familie - nein danke?! Familienglück zwischen neuen Freiheiten und alten Pflichten. Göttingen: Vandenhoeck \& Ruprecht.

Voigt, Claudia (2013): Die große Erschöpfung. In: Der Spiegel 48/2013, S. 156-157.

Zeiher, Helga (2005): Neue Zeiten - neue Kindheiten? Wandel gesellschaftlicher Zeitbedingungen und die Folgen für Kinder. In: Mischau, Anina; Oechsle, Mechtild (Hrsg.): Arbeitszeit - Familienzeit - Lebenszeit: Verlieren wir die Balance? Zeitschrift für Familienforschung, Sonderheft 5. Wiesbaden: VS Verlag für Sozialwissenschaften, S. 74-91. 



\title{
Mutterleitbilder: Spagat zwischen Autonomie und Aufopferung
}

\author{
Sabine Diabaté*
}

\section{Zusammenfassung}

Mutterleitbilder sind in der empirischen Sozialforschung in Deutschland eher selten untersucht worden, eine Analyse mit quantitativen und repräsentativen Daten steht bislang aus. Ziel dieses Beitrags ist die Identifikation und Beschreibung von Leitbildern zur Mutterschaft in Deutschland. Des Weiteren soll untersucht werden, welche Mutterleitbilder auf individueller und gesellschaftlicher Ebene existieren und wie diese inhaltlich ausgestaltet sind. Die Ergebnisse spiegeln den hohen Qualitätsanspruch an Mütter, die sowohl die Erziehung („Fürsorge“) als auch ihre „Selbstsorge“ parallel als zentrale Lebensziele verfolgen sollen. Gleichzeitig wird auch deutlich, dass das allgemein als vorherrschend wahrgenommene Mutterleitbild aufgrund der Widersprüchlichkeit und Komplexität der Anforderungen besonders in Westdeutschland überfrachtet erscheint.

Bei der hauptkomponenten- und indexbasierten Typisierung kristallisierten sich vier Mutterleitbilder heraus, die gegenwärtig in Deutschland bei den 20- bis 39-Jährigen existieren. Es wurden zwei Reintypen, das berufs- und das kindorientierte Mutterleitbild sowie zwei Mischtypen, das moderate und das vereinbarkeitsorientierte Mutterleitbild identifiziert. Auch eine stärkere Kindorientierung bei in Westdeutschland lebenden Personen zeigte sich. Interessanterweise hängen vor allem die kind- und berufsorientierten Mutterleitbilder eng mit dem Lebensmodell der Mütter der Befragten zusammen. Hierbei erscheinen u. a. die gesellschaftlichen Systeme (alte vs. neue Bundesländer) prägend, in denen die Mütter sozialisiert wurden und die in die nachfolgende Generation der Befragten hineinwirken.

\section{$1 \quad$ Einleitung}

Ob „Rabenmutter“, „Familienmanagerin“ oder „Muttertier“, die Assoziationen, wie eine „gute Mutter" sein sollte, sind vielfältig und für die meisten Menschen verbunden mit Attributen wie z. B. Fürsorglichkeit und Hingabe. Deutlich wird dies auch an den Begrifflichkeiten: Während das „Bemuttern“ im alltäglichen Sprachgebrauch üblich ist, hat sich der Begriff „Bevatern“ bislang nicht durchgesetzt. Mutterleitbilder haben eine lange Tradition, sie sind historisch gewachsen und in allen Kulturen mit besonderen Eigenschaften verbunden. Diese kulturellen Aspekte von Mutterschaft beeinflussen laut verschiedener Studien neben sozialstrukturellen Rahmenbedingungen und den individuellen Kosten-Nutzen-Abwägungen die private Lebensführung (z. B. Pfau-Effinger et al. 2009) und den Kinderwunsch (z. B. Ruckdeschel 2009). Es ist daher anzunehmen, dass individuelle Vorstellungen zur Mutterrolle, aber auch die in der Gesellschaft wahrgenommenen Ansprüche an

\footnotetext{
* Ich danke Kerstin Ruckdeschel sowie der anonymen Gutachterin bzw. dem anonymen Gutachter für die
} wertvollen Hinweise und die hilfreiche Kritik. 
Mütter mit familialem Verhalten in Verbindung stehen. Mutterleitbilder sind in den vergangenen Jahrzehnten, seit der Bildungsexpansion, facettenreicher geworden und verbunden mit einer Reihe normativer Aspekte (Schütze 2010). Sie reflektieren dadurch gesellschaftliche Erwartungen und Normalitätsvorstellungen an die Mutter-Kind-Beziehung zu einem bestimmten Zeitpunkt.

Welche Mutterleitbilder es gegenwärtig in Deutschland gibt und wie sie in verschiedenen sozialen Teilpopulationen (z. B. unterschieden nach Geschlecht, alte/neue Bundesländer etc.) verbreitet sind, dazu ist bislang erst wenig bekannt. Zudem ist ungewiss, welche Mutterleitbilder auf individueller sowie auf gesellschaftlicher Ebene existieren und wie gut diese miteinander korrespondieren. Im Familienleitbild-Survey (Lück et al. 2013) werden daher persönliche sowie erstmals allgemein wahrgenommene Vorstellungen zur Mutterrolle gemessen. Die Ergebnisse zu diesem Themengebiet sind Gegenstand dieses Beitrags. Ziel ist es, zu klären, wie viele und welche Leitbilder es zur Mutterschaft in Deutschland gibt. Des Weiteren soll untersucht werden, welche auf individueller und welche auf gesellschaftlicher Ebene existieren und wie diese inhaltlich ausgestaltet sind.

\section{Stand der Forschung und theoretische Grundlagen}

Mutterschaft und Geschlechterrollen sind seit langem Gegenstand der quantitativen und qualitativen Forschung (Überblick zu Familienleitbildern bei Diabaté/Lück 2014). Die soziologische Forschung zur Mutterschaft beschäftigt sich dabei vor allem mit der Frage nach einer angemessenen Verteilung der Familien- und Erwerbsarbeit innerhalb von Partnerschaften. Zentrale Pole in der wissenschaftlichen und auch öffentlichen Debatte sind einerseits das Kindeswohl und andererseits das Mutterwohl (Bedürfnis nach beruflicher Entwicklung und Etablierung/Karriere, finanzieller Absicherung und Selbstentfaltung jenseits der häuslichen/familiären Sphäre) bzw. auf partnerschaftlicher Ebene das Elternund Partnerwohl.

In verschiedenen Studien werden ,gender systems“ (siehe Norlander 2003: 3ff., Hirdman 1988 ) erforscht oder in „doing gender“-Ansätzen die Verteilung von bezahlter und nichtbezahlter Arbeit durch kulturelle Konzepte begründet (Klement/Rudolph 2003). Geissler und Oechsle (1996) untersuchen mit leitfadengestützten Interviews die Risiken und Optionen in den Übergangsphasen von Frauen, die in das Erwachsenenleben führen. Sie identifizieren verschiedene elterliche Leitbilder wie das ,historisch überlieferte und modifizierte Leitbild“ der „guten Mutter“ und das Leitbild des Familienernährers. Vorstellungen zur „guten Mutter" (Merkle/Wippermann 2008) und Mutterleitbilder werden darüber hinaus z. B. bei Schütze (2010) oder bei Herwartz-Emden (1995) thematisiert. Mutterschaft wird als äußerst anspruchsvolle und stark auf das Kind fokussierte Lebensweise konstruiert, die sich in verschiedenen sozialen Milieus laut Merkle und Wippermann (2008: 37ff.) gleichermaßen verfestigt hat. Sie untersuchen die milieu-spezifischen Mutterrollenbilder mittels der Sinus-Milieus in einer qualitativen Studie durch narrative Einzelinterviews.

Weitere neuere Studien thematisieren den Einfluss von Geschlechterarrangements und -regimes, von Familien- und Integrationsmodellen sowie kulturellen Geschlechterrollenorientierungen (z. B. Pfau-Effinger et al. 2009, Pfau-Effinger 2000, 1996; Beier/Rupp 2009; Lück/Hofäcker 2008; Beckmann 2007). In diesem Kontext werden auch Konzepte wie das des „normative climate“ (Butler 2002) oder die „,cultural patterns“ (Heut 2004) als kulturelle Erklärungsgrößen für die Arbeitsteilung zwischen den Geschlechtern aufgeführt. Insbesondere in den quantitativen und z. T. auch internationalen Analysen von Pfau- 
Effinger (2000, 1996; Pfau-Effinger et al. 2009) wird die Bedeutung von „Geschlechterkultur" für die Teilnahme von Frauen am Arbeitsmarkt deutlich.

Im Wesentlichen sind zwei miteinander konkurrierende Prinzipien zu erkennen (Schneider et al. 2014): Das Prinzip der Gleichberechtigung innerhalb der Partnerschaft (Erwerbs-, Haus- und Familienarbeit werden zwischen den Elternteilen gleich verteilt) steht dem Prinzip der „,verantworteten Elternschaft““ (Kaufmann 1990) und dem „Mythos Mutterliebe“ (Schütze 1986) gegenüber. Das Konzept der Mutterliebe beinhaltet, dass eine leibliche Mutter quasi per Geburt stärker mit ihrem Kind verbunden ist, größere Fürsorglichkeit besitzt als der Vater und intuitiv daher auch besser weiß, was es braucht. Diese natürliche Verbindung zwischen Mutter und Kind sei unauflöslich und exklusiv (gegenüber dem Vater). Daher besteht aus normativer Sicht die Erwartung, dass sich Mütter in besonderer Art und Weise für ihr Kind aufopfern sollen und ihre Bedürfnisse (stärker als der Vater) denen des Kindeswohls unterordnen müssen. Aus dieser Vorstellung nährt sich die normative Erwartung, dass sich Mütter (mehr als der Vater) in die Erziehung des Kindes einbringen und engagieren „sollen“. In Deutschland ist anzunehmen, dass sich das Mutterleitbild stark am beschriebenen Konzept der Mutterliebe orientiert, in den alten Bundesländern stärker als in den neuen. Das Bürgerliche Gesetzbuch (BGB) definiert „Mutter“ dementsprechend als erste Bezugsperson vor dem Vater, da sie durch die Geburt dem Kind eindeutiger zugeordnet werden kann. Demnach hat die Mutter von Geburt an das (alleinige) Sorgerecht für ihr Kind. Im Gegensatz dazu ist der Vater nach $\S 1592$ Nr. 1 BGB der Ehemann der Mutter, zunächst unabhängig davon, ob er auch der biologische Vater ist. ${ }^{1}$

Ein weiterer Beleg für die exklusive Rolle von Müttern für die Erziehungsarbeit ist, dass der überwiegende Anteil von Alleinerziehenden in Deutschland Frauen sind ${ }^{2}$ (BMFSFJ 2012a). Dieses Phänomen verdeutlich die zentrale Bedeutung, die einer Mutter gesellschaftlich zugesprochen wird, und das Selbstverständnis von vielen Müttern und Vätern, im Falle einer Trennung der Eltern den Lebensmittelpunkt der Kinder bei der Mutter zu belassen (Diabaté 2014). Außerdem wird Frauen eine höhere Erziehungskompetenz zugeschrieben, das zeigen die Ergebnisse im International Social Survey Program (ISSP 2012): Tendenziell betrachten die Befragten aller Altersgruppen eher zwei Frauen als zwei Männer als gleich „gute“ Eltern wie heterosexuelle Eltern. Die Forschung bietet Ansatzpunkte, wonach insbesondere in Deutschland ein spezielles Mutterleitbild vorherrscht (Ruckdeschel 2009): In einem deutsch-französischen Vergleich wird nachgewiesen, dass Kinderwünsche mit divergierenden Mutterbildern zusammenhängen (deutsche „Rabenmutter“ vs. französische Gluckenmutter „mère poule“).

Es gibt daher eine Reihe von Phänomenen, die darauf hindeuten, dass es in Deutschland eine besondere Form des Mutterleitbildes gibt, die sich stärker am Prinzip der „Verantworteten Mutterschaft" (siehe Ruckdeschel, Kap. 12) orientiert. Es ist davon auszugehen, dass dies in den alten Bundesländern noch stärker in Erscheinung tritt als in den neuen Bundesländern.

Bei der Betrachtung des Forschungsstandes zeigt sich insgesamt, dass bislang eine systematische Suche und falls möglich, Deskription von Mutterleitbildern und deren Ver-

1 Ohne bestehende Ehe muss die Vaterschaft anerkannt werden, entweder durch den Erzeuger selbst oder durch eine gerichtliche Feststellung. Erst durch die Eheschließung vor der Geburt oder die Anerkennung der Vaterschaft nach der Geburt bei nicht verheirateten Elternpaaren kann der biologische Vater auch gesetzlich als Vater anerkannt werden und damit das Sorgerecht erhalten (Diabaté 2014).

2 „In neun von zehn Fällen ist der alleinerziehende Elternteil die Mutter. Bei jeder zehnten Ein-Eltern-Familie ist der Vater der alleinerziehende Elternteil. Alleinerziehende Mütter betreuen häufiger jüngere Kinder, alleinerziehende Väter dagegen eher bereits ältere Kinder.“(BMFSJ 2012a: 7) 
teilung in verschiedenen sozialen Gruppen, basierend auf quantitativen repräsentativen Daten, fehlt. Es wurden lediglich einzelne Bestandteile von Mutterleitbildern untersucht. Tiefgreifende inhaltliche Analysen dieser Leitbilder finden sich vor allem in der qualitativen Forschung. Um sich dem komplexen Konstrukt Mutterschaft empirisch und quantitativ weiter anzunähern und der Komplexität bzw. Vielschichtigkeit besser gerecht zu werden, verfolgt dieser Beitrag das Ziel, Mutterschaft als ein Konstrukt aus verschiedenen kulturellen Normalitätsvorstellungen zu umrahmen, die in ihrer Verbindung kulturelle Leitbilder $^{3}$ ergeben (vgl. Diabaté/Lück 2014). Demnach ist ein Mutterleitbild ein Bündel aus verschiedenen Normalitätsvorstellungen zur Lebensführung von Müttern. Die dargestellten Studien zur Wirkung von kulturellen Mustern auf das Familienleben verdeutlichen, dass Mutterleitbilder einen zusätzlichen Erkenntnisgewinn liefern können, um familiales Verhalten besser als bislang verstehen zu können. Auf diese Weise kann einerseits auf Individualebene gezeigt werden, wie weit bestimmte Normalitätsvorstellungen zur Mutterschaft verbreitet sind. Andererseits kann erstmals beleuchtet werden, welche Vorstellungen innerhalb der Gesellschaft als existent wahrgenommen werden. Eine grundlegende Annahme in der Leitbildforschung ist es, dass nicht nur die persönliche Vorstellung und das eigene Leitbild zur Mutterschaft, sondern auch die in der sozialen Umgebung (vermeintlich) vorherrschenden Mutterleitbilder einen Einfluss auf die private Lebensführung nehmen (Diabaté/Lück 2014).

Ein weiterer theoretischer Ausgangspunkt für diesen Beitrag ist das Konzept der preference theory von Hakim (2000). Darin werden drei Dimensionen thematisiert: Die Kindorientierung und Berufsorientierung (family- vs. work-centered) sowie ein Mischtypus (adaptive), der beide Dimensionen verbindet. Hakim (2002) belegt, dass diese Typen das Verhalten von Frauen hinsichtlich ihrer Haus-/Familien- und Erwerbsarbeit erklären. Basierend auf der Dimension „family-centered“ von Hakim und dem Konzept der „Verantworteten Elternschaft" (vgl. Ruckdeschel, Kap. 12) wird im Folgenden ein Konzept der Verantworteten Mutterschaft vorgeschlagen: Hierin sind gesellschaftlich weit verbreitete Vorstellungen davon, wie die Mutter-Kind-Beziehung heutzutage idealerweise aussehen sollte, miteinander verknüpft. Grundlegende Voraussetzung ist eine starke Kind- und Familienarbeitsorientierung, wie sie Hakim (2000: 159) auch mit „home-centered“ umschreibt. Frauen mit dieser Orientierung („Homemakers“, Hakim 2000) favorisieren eine klassische Arbeitsteilung mit ihrem Partner, bevorzugen es, nicht erwerbstätig zu sein und haben die Familienarbeit als oberste Priorität. Zahlreiche Ansprüche, wie ein risikoarmes Aufwachsen mit gesunder Ernährung, schadstofffreien Spielzeugen und frühzeitiger Förderung sowie mütterliche Präsenz zuhause, gehören gleichermaßen dazu. Damit verbunden ist naturgemäß ein hohes Informationsbedürfnis seitens vieler (auch werdender) Mütter, sichtbar durch das große Angebot an Internetforen und in der Vielfalt von Ratgeberliteratur. Die darin z. T. auch kontrovers diskutierten Ratschläge und formulierten multidimensionalen Anforderungen an Mutterschaft, um eine ,glückliche Kindheit" zu gewährleisten, erzeugen, so die Vermutung, bei (werdenden) Müttern heutzutage einen besonders hohen Druck. Mutterschaft ist in diesem Konzept als eine höchst anspruchsvolle Aufgabe zu verstehen, die keineswegs allein intuitiv, d. h. ,aus dem Bauch heraus“, erfolgreich bewältigt werden kann.

Diese Facetten stehen in Verbindung mit der Vorstellung, die Mutterrolle zu professionalisieren. Komplementär dazu existiert das Vaterleitbild des Familienernährers, damit die Mutter auf Erwerbsarbeit verzichten und sich ganz um die Kinder kümmern kann. Aus diesem, vor allem in den 1950er und danach folgenden Jahrzehnten weit verbreitetem, Mutterleitbild haben sich verschiedene Varianten von Mutterleitbildern entwickelt: Während

3 Vgl. die Ausführungen zum theoretischen Konzept von Lück/Diabaté, Kap. 2. 
in den Wirtschaftswunder-Jahren das Mutterleitbild in Deutschland mit Haus- und Familienarbeit verbunden war, sind die heutigen Leitbilder der „guten Mutter“" vielfältiger geworden. Außerdem gibt es eine immer größer werdende Gruppe von Müttern, die erwerbstätig sind, zumeist als Hinzuverdienerin, was dem ,,adaptive“-Typus von Hakim (2002) entspricht. Die vollzeiterwerbstätige Mutter lässt sich mit Hakims Bezeichnung „workcentered" verbinden.

Ziel dieses Beitrages ist es, die theoretisch postulierten Mutterleitbilder auf Individualebene zu identifizieren. Im Folgenden soll daher untersucht werden:

1. Wie verbreitet verschiedene Vorstellungen zur Mutterrolle sind,

2. wie diese Vorstellungen aus individueller Sicht der Befragten bewertet werden und ob sie von den Befragten innerhalb der Gesellschaft wahrgenommen werden,

3. ob signifikante Differenzen hinsichtlich der sozialen Gruppen existieren,

4. inwieweit persönliche und in der Gesellschaft subjektiv wahrgenommene Vorstellungen zur Mutterschaft miteinander korrespondieren,

5. ob sich daraus verschiedene Mutterleitbilder bilden lassen,

6. wo sich Mutterleitbilder in Deutschland auf der Achse zwischen Kind- und Berufsorientierung konzentrieren,

7. und ob bestimmte Personen bestimmte Mutterleitbilder vertreten.

Die beschriebenen Forschungsfragen werden deskriptiv, bivariat und mit einer hauptkomponenten-basierten Indexbildung analysiert. In einem weiteren Schritt soll geprüft werden, ob sich aus den Indizes verschiedene Mutterleitbilder identifizieren lassen.

\section{Ergebnisse}

Zunächst wird es in diesem Kapitel darum gehen, die inhaltliche Ausgestaltung von Mutterleitbildern in Deutschland genauer zu beschreiben. Dazu werden in einem ersten Schritt die Verbreitung und Ausdifferenzierung der persönlichen und allgemeinen MutterleitbildDimensionen, auch nach verschiedenen sozialen Teilpopulationen, analysiert (3.1). Im Anschluss daran wird geprüft, wie gut die persönlichen Vorstellungen mit den (mutmaßlich) in der Gesellschaft verbreiteten korrespondieren (3.2). In einem weiteren Schritt wird überprüft, ob sich die theoretisch postulierte Annahme der Polarisierung von Mutterleitbildern in den Daten widerspiegelt (3.3). Danach wird untersucht, ob und falls vorhanden, wie viele und welche Mutterleitbilder es gegenwärtig in Deutschland gibt (3.4).

\subsection{Verbreitung und Ausdifferenzierung der Vorstellungen zur Mutterschaft}

In der Befragung wurde zunächst hinsichtlich der verschiedenen Aussagen zur Mutterschaft nach der persönlichen Meinung gefragt. Die Aussagen wurden bei der FragebogenKonzeption basierend auf den beiden in der Theorie thematisierten Aspekten Kind- und Berufsorientierung formuliert. Dementsprechend wurden Aussagen zur Kinderbetreuung und zur Familienarbeit gestellt sowie zur Bewertung der Erwerbstätigkeit von Müttern. Die Item-Bildung verfolgte damit das Ziel, das Spannungsfeld von Mutterschaft zwischen Familien- und Erwerbsarbeit zu erfassen. Die Items basieren auf den dargestellten Überlegungen zu einem Konzept der „Verantworteten Mutterschaft“. 
Nachfolgend sind für jedes Item ${ }^{4}$ die Anteile der Zustimmung und Ablehnung dargestellt (vgl. Abbildung 1). Die Ergebnisse sind nicht eindeutig interpretierbar: Fragt man die 20- bis 39-Jährigen persönlich nach ihren Vorstellungen, dann zeichnen sich sowohl für die berufs- als auch für die kindorientierten Aussagen hohe Zustimmungswerte ab: Ein Großteil der Befragten stimmt dem Item „Mütter sollten einem Beruf nachgehen, um unabhängig vom Mann zu sein.“ zu. Gleichzeitig befürwortet eine überwiegende Mehrheit (über $80 \%$ ) die Aussage: „Mütter sollten nachmittags Zeit haben, um ihren Kindern beim Lernen zu helfen.“

Abbildung 1: Individuelle Vorstellungen und Vorstellungen der Allgemeinheit über Mütter (in Prozent)

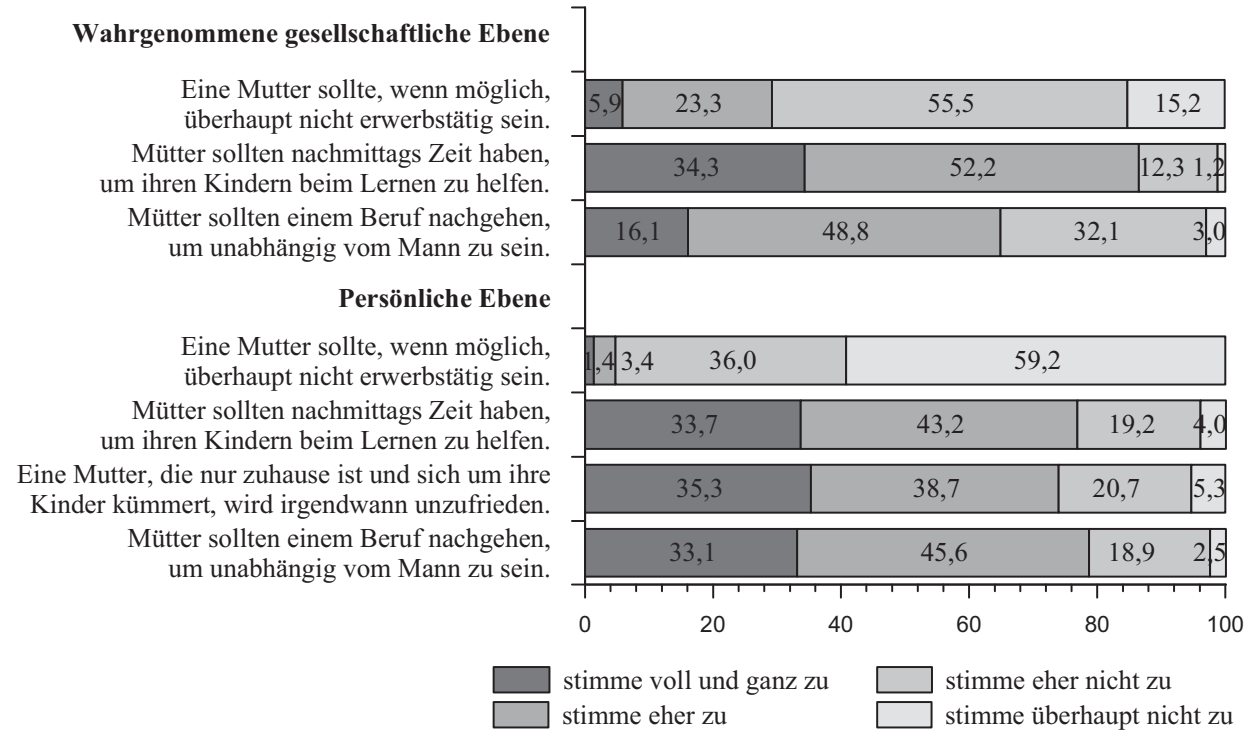

Quelle: FLB (2012), gewichtete Daten, eigene Berechnungen

Lediglich das Item „Eine Mutter sollte, wenn möglich, überhaupt nicht erwerbstätig sein.“ wird überwiegend abgelehnt. Es zeigt sich, dass sich auf individueller Ebene sowohl kindbezogenes als auch außerhäusliches bzw. berufliches Engagement als MutterleitbildDimensionen ähnlich hoch bewertet gegenüberstehen.

Fragt man danach, was in der Allgemeinheit als Normalitätsvorstellung zur Mutterschaft wahrgenommen wird, ergibt sich ein Bild, das für eine starke und dominierend wahrgenommene Kindorientierung spricht, wenngleich auch eine gesellschaftliche Erwar-

4 Wortlaut im Fragebogen (FLB 2012): „Jetzt zu ihrer persönlichen Meinung: Stimmen Sie persönlich (voll und ganz/eher/eher nicht/überhaupt nicht) zu...: 1. Mütter sollten nachmittags Zeit haben, um ihren Kindern beim Lernen zu helfen. 2. Mütter sollten einem Beruf nachgehen, um unabhängig vom Mann zu sein. 3. Eine Mutter, die nur zuhause ist und sich um ihre Kinder kümmert, wird irgendwann unzufrieden. 4. Eine Mutter sollte, wenn möglich, überhaupt nicht erwerbstätig sein.“ Die wahrgenommene Meinung innerhalb der Gesellschaft wurde eingeleitet durch „Jetzt zur Allgemeinheit: ...“. Hier wurden drei Aussagen erneut im selben Wortlaut an die Befragten gerichtet. Das vierte Item zur Mutterschaft wurde nicht auf AllgemeinheitsEbene gespiegelt gemessen, da aufgrund der für Telefoninterviews empfohlenen max. Dauer an verschiedenen Stellen Kürzungen vorgenommen werden mussten. 
tung zur mütterlichen Erwerbsarbeit gesehen wird. Jedoch besitzt die Müttererwerbstätigkeit $(64,9 \%)$ aus Sicht der Befragten innerhalb der Allgemeinheit einen etwas geringeren Stellenwert, im Vergleich zur persönlichen Ebene. Und mit 86,5 \% nimmt eine überwiegende Mehrheit seitens der Allgemeinheit die Erwartung an Mütter wahr, nachmittags die Kinder beim Lernen zu unterstützen. Zudem glaubt ein Drittel, dass die Gesellschaft es befürwortet, wenn Mütter nach Möglichkeit überhaupt nicht arbeiten gehen.

In einem weiteren Schritt stellt sich die Frage, ob sich die beschriebenen Vorstellungen über Mütter sozialstrukturell, z. B. nach Bildungsniveau, unterscheiden. In Tabelle 1 sind die Zustimmungswerte ${ }^{5}$ in Prozent auf persönlicher Ebene zu den verschiedenen Dimensionen von Mutterschaft abgetragen, differenziert nach soziodemografischen Merkmalen.

Tabelle 1: Zustimmung auf persönlicher Ebene zu Dimensionen von Mutterschaft nach soziodemografischen Merkmalen (in Prozent)

\begin{tabular}{llccccc}
\hline & $\begin{array}{c}\text { Mutter sollte } \\
\text { nachmittags } \\
\text { beim Lernen } \\
\text { helfen. }\end{array}$ & $\begin{array}{c}\text { Mütter sollten } \\
\text { einem Beruf } \\
\text { nachgehen, um } \\
\text { unabhängig vom } \\
\text { Mann zu sein. }\end{array}$ & $\begin{array}{c}\text { Eine Mutter, die } \\
\text { nur zuhause ist, } \\
\text { wird irgendwann } \\
\text { unzufrieden. }\end{array}$ & $\begin{array}{c}\text { Eine Mutter } \\
\text { sollte, wenn } \\
\text { möglich, über- } \\
\text { haupt nicht } \\
\text { erwerbstätig sein. }\end{array}$ & Gesamt \\
\hline Merkmal & Kategorie & $\%$ & $\%$ & $\%$ & $\%$ & N \\
\hline Geschlecht & Männlich & $70,7^{* * *}$ & $73,3^{* * *}$ & $71,8^{* * *}$ & 5,2 & 2.532 \\
Bildung & Weiblich & $83,1^{* * *}$ & $84,2^{* * *}$ & $76,4^{* * *}$ & 4,4 & 2.468 \\
ISCED97 & Niedrig & $90,7^{* * *}$ & $77,9^{*}$ & $65,4^{* * *}$ & $7,6^{* * *}$ & 346 \\
$(2002)$ & Mittel & $80,2^{* * *}$ & $77,5^{*}$ & $75,8^{* * *}$ & $5,3^{* * *}$ & 3.249 \\
Kind & Hoch & $63,8^{* * *}$ & $81,5^{*}$ & $72,1^{* * *}$ & $2,8^{* * *}$ & 1.309 \\
& Eltern & $82,1^{* * *}$ & $76,7^{* *}$ & $78,6^{* * *}$ & $5,9^{* *}$ & 2.012 \\
Kinderlose & $73,3^{* * *}$ & $80,0^{* *}$ & $71,0^{* * *}$ & $4,0^{* *}$ & 2.986 \\
\hline Gesamt & West & $77,6^{*}$ & $76,7^{* * *}$ & 73,4 & $5,3^{* *}$ & 3.986 \\
\hline
\end{tabular}

Quelle: FLB (2012), gewichtete Daten, eigene Berechnungen

Anm.: ${ }^{* * *} \mathrm{p} \leq 0,001 ;{ }^{* *} \mathrm{p} \leq 0,01 ;{ }^{*} \mathrm{p} \leq 0,05$ (Signifikanzen nach $\mathrm{Chi}^{2}$-Test), die Ausfallquote lag bei den einbezogenen Variablen bei unter $3 \%$. ${ }^{a}$ Die Zuordnung erfolgt nach: Low (1-2), Medium (3A-4A), High (5B-6). " „Haben Sie Kinder? Ich meine sowohl Ihre eigenen Kinder als auch Adoptivkinder, egal, wo sie momentan wohnen."

Den beiden Items, dass Mütter nachmittags beim Lernen helfen und auch einem Beruf nachgehen sollten, stimmen signifikant ${ }^{6}$ mehr Frauen (über $80 \%$ ) als Männer (70,7 \%) zu. Verstärkend für diesen Geschlechtereffekt könnte sein, dass in der Stichprobe ein hoher Anteil von Müttern und kinderlosen Männern vertreten ist (48,4 \% aller Frauen sind Mütter aber nur 32,4 \% aller Männer sind Väter). Bei der Kontrolle von Kinderlosigkeit hinsichtlich des Zusammenhangs von Geschlecht und persönlicher Vorstellung bleiben die

5 Die Antwortkategorien der vierstufigen Zustimmungsskala wurden dichotomisiert, d. h. 1=,Stimme voll und ganz zu“ und 2=,Stimme eher zu“ sind zusammengefasst zum Wert 1, auf den Wert 0 umkodiert wurden die Antwortkategorien 3="Stimme eher nicht zu" sowie 4="Stimme überhaupt nicht zu".

6 Signifikanzen wurden mit dem $\mathrm{Chi}^{2}$-Test getestet. 
Geschlechterunterschiede jedoch bestehen. Statistisch kaum mehr relevant ist die ablehnende Vorstellung zur Müttererwerbstätigkeit. Es fällt zudem auf, dass dem Item „Mütter sollten nachmittags Zeit haben, um ihren Kindern beim Lernen zu helfen." besonders von niedrig Gebildeten zugestimmt wird. Mittlere und höhere Bildung erhöht auch die Zustimmung dazu, dass das Hausfrauendasein allein nicht zufrieden macht, niedrigere Bildung erzeugt eine etwas höhere Zustimmung zum Hausfrauen-Modell (7,6 \% vs. 2,8 \%), wenngleich die Ablehnung deutlich überwiegt.

Zudem sind Eltern signifikant häufiger der Ansicht, dass die mütterliche Unterstützung für schulische Belange wichtig ist, umgekehrt denken sie etwas seltener, dass mütterliche Unabhängigkeit vom Partner bedeutsam ist. Jedoch teilen sie häufiger als Kinderlose die Überzeugung, dass die ständige Präsenz von Müttern zuhause irgendwann unzufrieden macht. Dahinter steckt eventuell die Vorstellung, dass Mütter vielleicht nicht unbedingt erwerbstätig zu sein brauchen, sondern auch in anderem z. B. ehrenamtlichem Engagement (in Schule, Kindergarten, Gemeindearbeit) ihren Ausgleich zur häuslichen Familienarbeit finden können. Beim Vergleich nach Wohnort der Befragten fällt auf, dass Ostdeutsche viel häufiger (86,3\% vs. 76,7\%) ein Unabhängigkeitsideal der Mutter vertreten als Westdeutsche. Eine Erklärung dafür könnten die unterschiedlichen politischen Systeme sein, in denen die Befragten sozialisiert bzw. wodurch ihre Eltern geprägt wurden. Ansonsten lassen sich an dieser Stelle für die anderen Items keine weiteren markanten Differenzen hinsichtlich alter und neuer Bundesländer bei den Vorstellungen zur Mutterschaft feststellen. Um später individuelle mit gesellschaftlich wahrgenommenen Vorstellungen vergleichen zu können, werden im nächsten Schritt drei Items dargestellt, die die Sicht des Einzelnen auf die Allgemeinheit in Deutschland widerspiegeln.

In Tabelle 2 sind die von den Befragten in der Gesellschaft wahrgenommenen Vorstellungen nach Zustimmung dargestellt. Der überraschende Befund ist, dass sich über Geschlecht, Bildung, familiäre Situation (Eltern vs. Kinderlose) und auch Wohnort hinweg fast keine hoch signifikanten Differenzen zeigen. Am häufigsten wird innerhalb der Gesellschaft wahrgenommen, dass Kinder nachmittags mütterliche Unterstützung benötigen (86,5 \%). Mit 64,9 \% sehen rund zwei Drittel der Befragten die Erwerbstätigkeit von Müttern in der Öffentlichkeit positiv konnotiert.

Gleichzeitig nimmt aber auch knapp ein Drittel wahr, dass es in der Gesellschaft den Anspruch an Mütter gibt, nach Möglichkeit nicht arbeiten zu gehen. Hoch signifikante Unterschiede lassen sich hinsichtlich der Bildung feststellen: Je niedriger der Bildungsabschluss, desto höher ist der Anteil der Personen, die der Aussage zustimmen, dass die Allgemeinheit Müttererwerbstätigkeit befürwortet. Daraus lässt sich schlussfolgern, dass von den niedriger gebildeten Befragten der höchste externe normative Druck empfunden wird, dass Mütter erwerbstätig sein sollten. Zwischen allen drei Bildungsgruppen gibt es signifikante Differenzen, die Zustimmung steigt von 59,7\% der hoch gebildeten, über $65,3 \%$ bei Personen mit mittlerem Abschluss bis hin zu von 80,0\% bei denjenigen mit niedrigem Abschluss. Da formale Bildungsabschlüsse und Einkommen hoch miteinander korrelieren, könnte man diesen Befund als Ergebnis von Erfahrungswerten einordnen.

Zwei Interpretationen sind denkbar: Erstens haben Befragte mit niedrigerem Bildungsabschluss vermutlich häufiger direkt oder in ihrem Umfeld ökonomische Engpässe erlebt. Haushalte mit geringqualifizierten Personen, in denen beide Elternteile arbeiten gehen, sind finanziell besser ausgestattet als Alleinernährer-Haushalte. Zusätzlich verschärfend kann eine Trennung wirken, da in den niedrigen Einkommensgruppen der Unterhalt oftmals kaum ausreicht. Dies führt vermutlich dazu, dass besonders niedrig Qualifizierte in der Allgemeinheit einen hohen Erwartungsdruck von ihrer direkten Umgebung (Familie, Freunde, Nachbarn) empfinden, arbeiten zu gehen, da ihnen sonst finanzielle Abhängigkeit und damit auch 
soziale Sanktionen aus direkter Umgebung drohen. Zweitens könnte der wahrgenommene Erwartungsdruck durch gering Qualifizierte auch bedeuteten, dass die mögliche Abhängigkeit von Sozialleistungen (und damit von der Solidargemeinschaft) als Stigmata wahrgenommen wird, welches durch die Medien oder durch andere (statushöhere Personen) verbreitet wird. Diese Vermutung wird dadurch gestärkt, dass es keine Geschlechterunterschiede gibt. Auch hier nimmt der soziale Erwartungsdruck, dass Mütter arbeiten gehen sollten, graduell zu mit sinkender Qualifikation. Wenn es hingegen einen Geschlechtereffekt gäbe, dann müssten Frauen eher die negativen Folgen von erziehungsbedingten Auszeiten im Job antizipieren und dementsprechend eher Müttererwerbstätigkeit befürworten.

Tabelle 2: Wahrnehmung der gesellschaftlichen Meinung - Zustimmung ${ }^{\mathrm{a}}$ zu Dimensionen von Mutterschaft nach soziodemografischen Merkmalen (in Prozent)

\begin{tabular}{|c|c|c|c|c|c|}
\hline & & $\begin{array}{c}\text { Mutter sollte } \\
\text { nachmittags } \\
\text { beim Lernen } \\
\text { helfen } \\
\end{array}$ & $\begin{array}{l}\text { Mütter sollten einem } \\
\text { Beruf nachgehen, um } \\
\text { unabhängig vom } \\
\text { Mann zu sein }\end{array}$ & $\begin{array}{c}\text { Eine Mutter sollte, } \\
\text { wenn möglich, } \\
\text { überhaupt nicht } \\
\text { erwerbstätig sein }\end{array}$ & Gesam \\
\hline Merkmal & Kategorie & $\%$ & $\%$ & $\%$ & $\mathrm{~N}$ \\
\hline \multirow[t]{2}{*}{ Geschlecht } & Männlich & $85,1^{* *}$ & 64,5 & 28,4 & 2.532 \\
\hline & Weiblich & $88,0^{* *}$ & 65,3 & 30,1 & 2.468 \\
\hline \multirow{3}{*}{$\begin{array}{l}\text { Bildung } \\
\text { ISCED97 }^{\text {b }} \\
(2002)\end{array}$} & Niedrig & $84,5^{* * *}$ & $80,0^{* * *}$ & 30,1 & 346 \\
\hline & Mittel & $86,2^{* * *}$ & $65,3^{* * *}$ & 29,2 & 3.249 \\
\hline & Hoch & $87,4^{* * *}$ & $59,7^{* * *}$ & 29,5 & 1.309 \\
\hline \multirow[t]{2}{*}{ Kind $^{\mathrm{c}}$} & Eltern & 86,2 & $66,9^{*}$ & $27,4^{*}$ & 2.012 \\
\hline & Kinderlose & 86,7 & $63,6^{*}$ & $30,5^{*}$ & 2.986 \\
\hline \multirow[t]{2}{*}{ Wohnort } & West & $85,6^{* * *}$ & 64,5 & $30,1^{* *}$ & 3.986 \\
\hline & Ost (inkl. Berlin) & $89,9^{* * *}$ & 66,4 & $25,8^{* *}$ & 1.014 \\
\hline Gesamt & & 86,5 & 64,9 & 29,2 & 5.000 \\
\hline
\end{tabular}

Quelle: FLB (2012), gewichtete Daten, eigene Berechnungen

Anm.: ${ }^{* * *} \mathrm{p} \leq 0,001 ;{ }^{* *} \mathrm{p} \leq 0,01 ;{ }^{*} \mathrm{p} \leq 0,05$ (Signifikanzen nach $\mathrm{Chi}^{2}$-Test), die Ausfallquote lag bei den einbezogenen Variablen bei unter $3 \%$. " "Stimme voll und ganz zu“ sowie „Stimme eher zu“ wurden ebenso zusammengefasst wie „Stimme eher nicht zu“ und „Stimme überhaupt nicht zu“, d. h. die Items wurden dichotomisiert. ${ }^{b}$ Die Zuordnung erfolgt nach: Low (1-2), Medium (3A-4A), High (5B-6). ${ }^{c}$ „Haben Sie Kinder? Ich meine sowohl Ihre eigenen Kinder als auch Adoptivkinder, egal, wo sie momentan wohnen."

Abgesehen von diesem Aspekt werden offenbar in der Allgemeinheit von fast allen Befragten einheitliche Vorstellungsmuster bezüglich der Mutterrolle in Deutschland wahrgenommen, welche stark kindorientiert anmuten. Die große Homogenität bestätigt die Annahme, dass es kollektive stereotype Mutterleitbilder innerhalb der Gesellschaft gibt, die von einer breiten Masse gleichermaßen wahrgenommen werden, unabhängig vom Erfahrungshintergrund und dem sozialen Kontext. Daraus könnte man schließen, dass das negativ konnotierte Leitbild der „Rabenmutter“, der ganztags berufstätigen Mutter, immer noch aus Sicht der Befragten innerhalb der Gesellschaft weit verbreitet ist. Ob die gesellschaftliche Referenzgruppe ältere oder einfach andere Personen sind, bleibt dabei diffus (siehe Lück/Naderi/Ruckdeschel, Kap. 3). Wie gut (durch den Einzelnen) in der Gesellschaft wahrgenommene Leitbilder mit den persönlichen Einstellungen zur Mutterschaft übereinstimmen, wird im Folgenden näher untersucht. 


\subsection{Zusammenhang von persönlichen und gesellschaftlichen Vorstellungen zur Mutterschaft}

Nun soll geklärt werden, wie gut die persönlichen Vorstellungen zur Mutterschaft mit den (mutmaßlich) in der Gesellschaft verbreiteten korrespondieren. Es wird daher überprüft, inwieweit persönliche Vorstellungen mit denen, die in der Allgemeinheit in Deutschland wahrgenommen werden, miteinander übereinstimmen. Die Ergebnisse zeigen, dass einige theoretisch angenommene Zusammenhänge nicht mit den Daten verifizierbar sind. Die häufig hoch signifikanten Zahlen sind das Ergebnis der Stichprobengröße, zugleich sind die Koeffizienten überwiegend gering bis moderat. ${ }^{7}$ Dadurch ist ersichtlich, dass persönliche Vorstellungen häufig nicht deckungsgleich mit solchen sind, die in der Gesellschaft wahrgenommen werden. Oder in der Gesellschaft wahrgenommene Vorstellungen stehen im Widerspruch zu den eigenen Vorstellungen. Die persönliche Vorstellung, dass Mütter nachmittags beim Lernen helfen sollen, korreliert leicht $\left(, 207^{* *}\right)$ mit der in der Allgemeinheit wahrgenommenen Ansicht, dass Mütter einem Beruf nachgehen sollen, um unabhängig vom Mann zu sein. Daraus folgt, dass der persönliche Anspruch an Mütter, Kinder nachmittags zu unterstützen auf einen in der Gesellschaft wahrgenommenen Anspruch prallt, gleichzeitig auch einem Beruf nachzugehen. Diese individuellen und gesellschaftlichen Ansprüche sind in der Praxis oftmals wohl nur mit einer Teilzeit-Tätigkeit der Mutter vereinbar. Die persönliche Überzeugung, dass Müttererwerbstätigkeit von Vorteil für Frauen ist, hängt signifikant mit der in der Gesellschaft beobachteten Meinung zusammen, dass Mütter nachmittags beim Lernen helfen sollten $\left(, 217^{* *}\right)$.

Tabelle 3: Zusammenhang zwischen persönlichen und (mutmaßlich) verbreiteten gesellschaftlichen Vorstellungen zur Mutterschaft

\begin{tabular}{lcccc}
\hline \multicolumn{4}{c}{ Persönliche Ebene } \\
\hline & $\begin{array}{c}\text { Mutter sollte } \\
\text { nachmittags beim } \\
\text { Lernen helfen. }\end{array}$ & $\begin{array}{c}\text { Mütter sollten einem } \\
\text { Beruf nachgehen, um } \\
\text { unabhängig vom } \\
\text { Mann zu sein. }\end{array}$ & $\begin{array}{c}\text { Eine Mutter, die } \\
\text { nur zuhause ist, } \\
\text { wird irgendwann } \\
\text { unzufrieden. }\end{array}$ & $\begin{array}{c}\text { Eine Mutter sollte, } \\
\text { wenn möglich, } \\
\text { überhaupt nicht } \\
\text { erwerbstätig sein. }\end{array}$ \\
\hline Allgemeinheits-Ebene &, $194^{* *}$ & Spearman's r &,$- 053^{* *}$ \\
\hline $\begin{array}{l}\text { Mutter sollte nachmittags } \\
\text { beim Lernen helfen. }\end{array}$ &, $207^{* *}$ &, $217^{* *}$ &, $093^{* *}$ \\
$\begin{array}{l}\text { Mütter sollten einem Beruf } \\
\text { nachgehen, um unabhängig } \\
\text { vom Mann zu sein. }\end{array}$ & &, $069^{* *}$ &,- 010 &, $085^{* *}$ \\
$\begin{array}{l}\text { Eine Mutter sollte, wenn } \\
\text { möglich, überhaupt nicht } \\
\text { erwerbstätig sein. }\end{array}$ &,$- 113^{* *}$ &, $064^{* *}$ &, $048^{* *}$ &, \\
\hline
\end{tabular}

Quelle: FLB (2012), gewichtete Daten, eigene Berechnungen

Anm.: ${ }^{* * *} \mathrm{p} \leq 0,001 ;{ }^{* *} \mathrm{p} \leq 0,01 ;{ }^{*} \mathrm{p} \leq 0,05$. Signifikanzniveau (Rangkorrelationen nach Spearman)

Dahinter könnte sich eine Präferenz für das weibliche Hinzuverdiener-Modell verbergen, eventuell jedoch auch das Modell von mütterlicher Vollzeit-Erwerbstätigkeit. Außerdem könnten diese Ergebnisse auch den inneren Konflikt von Menschen abbilden, dass das

7 Nachfolgend werden lediglich solche Werte interpretiert, die größer als ,2 sind. 
eigene Lebens-Modell nicht als „richtig“ empfunden wird, im Sinne von „wie man es macht, ist es verkehrt" aus Sicht der Allgemeinheit. Das heißt, der Spagat zwischen Job und Kind impliziert für viele ein ständiges Zerrissensein zwischen den eigenen Ansprüchen, denen des anderen Elternteils, denen des Kindes, denen der Verwandten/Freunde, denen des Arbeitsgebers und der Kolleginnen/Kollegen und schließlich den gesellschaftlichen insgesamt. An dieser Stelle lässt sich nicht präzisieren, welches Arbeitsmodell für Mütter genau erstrebenswert ist, jedoch impliziert die Umschreibung „um unabhängig vom Mann zu sein", dass es wohl mindestens Teilzeit sein sollte, denn sonst wird mit durchschnittlichen Berufseinkommen bei Teilzeitbeschäftigung kein existenzsichernder Verdienst für Frauen möglich sein, der ihnen eine Unabhängigkeit vom Partner gewährleistet. Das Item ist in dieser Hinsicht leider schwer interpretierbar, da unklar ist, auf welchen Aspekt es sich mehr bezieht, auf Erwerbstätigkeit oder Unabhängigkeit.

Beide Zusammenhänge zeigen den Widerspruch zwischen dem persönlichen Anspruch an Mütter und den wahrgenommenen gesellschaftlichen Erwartungen: Der Anspruch der mütterlichen Präsenz zuhause konterkariert den Anspruch mütterlicher Berufstätigkeit, die zur Unabhängigkeit vom Partner beitragen soll. Um nachmittags zuhause zu sein, ist eine Vollzeiterwerbstätigkeit nicht möglich. Eine Mehrheit der Befragten (67,8 \%, N=2.588), die finden, dass Mütter nachmittags beim Lernen helfen sollten, nehmen in der Gesellschaft die Vorstellung wahr, dass Mütter einem Beruf nachgehen sollten, um unabhängig vom Mann zu sein. Fast alle Personen (89,0 \%, N=3.478), die persönlich der Ansicht sind, dass Müttererwerbstätigkeit wichtig ist, glauben gleichzeitig, dass die Gesellschaft von Müttern erwartet, dass sie ihren Kindern nachmittags beim Lernen helfen. Offenbar bestehen hier starke Diskrepanzen zwischen den persönlichen und den gesellschaftlich wahrgenommenen normativen Vorstellungen gegenüber Müttern. Diese Kluft könnte erstens dadurch erklärt werden, dass die befragten Personen auf Mutterleitbilder ihrer Mütter bzw. Großmütter Bezug nehmen oder generell auf andere ältere Personen in ihrer Umgebung, die in der Studie nicht befragt wurden. Zweitens könnte die Wahrnehmung der gesellschaftlichen Mutterleitbilder z. T. nicht mehr der Realität entsprechen: Vielleicht wirken sie aus den vergangenen Jahrzehnten nach (z. B. durch institutionelle Regelungen wie kostenlose Mitversicherung der nicht erwerbstätigen Ehefrau oder durch Darstellungen von Müttern in der Werbung). Unabhängig davon, ob ein Mutterleitbild tatsächlich von vielen geteilt wird oder nicht, ist davon auszugehen, dass es allein durch die Wahrnehmung und die Annahme, es sei gesellschaftlich weit verbreitet, handlungsrelevant für den Einzelnen wird (siehe Lück/Diabaté, Kap. 2).

\subsection{Persönliche Mutterleitbilder: Zwischen Kindes- und Berufsorientierung}

In einem nächsten Schritt soll untersucht werden, inwiefern sich die verschiedenen Aspekte zur Mutterschaft in Leitbilder zusammenfassen lassen und ob sich darin die theoretisch postulierten inhaltlichen Dimensionen der Kindeswohl- und Berufsorientierung widerspiegeln. Konkret soll also geprüft werden, wie viele und welche Mutterleitbilder es gegenwärtig in Deutschland gibt. Mit einer Hauptkomponentenanalyse werden die MutterleitbildItems auf persönlicher Ebene analysiert (Tabelle 4).

1. Die Variablen „Mütter sollten einem Beruf nachgehen, um unabhängig vom Mann zu sein.“ und „Eine Mutter, die nur zuhause ist und sich um ihre Kinder kümmert, wird irgendwann unzufrieden." messen die Berufsorientierung und laden hoch mit ,7 auf dem Faktor „Berufsorientiertes Mutterleitbild“ (siehe Komponente 1). Damit tragen sie mit rund $26 \%$ (Cronbach's Alpha ,423) zur Erklärung dieses Leitbildes bei. Bei beiden Dimensionen lädt die Variable „Eine Mutter sollte, wenn möglich, überhaupt nicht 
erwerbstätig sein“ mit rund ,5 mit positivem Vorzeichen auf dem kindorientierten, und mit negativem Vorzeichen auf dem berufsorientierten Mutterleitbild.

2. Für die Bedeutung von Kindern als Lebensinhalt wird als Proxy die Frage „Finden Sie es für sich selbst wichtig, eigene Kinder zu haben?" verwendet, um die Kindorientierung näher fassen zu können. Rund 85,1 \% finden es eher bis sehr wichtig, eigene Kinder zu haben (sehr wichtig=53,8 \%; eher wichtig=31,2\%). Als Indikator für die primär weibliche Zuständigkeit der Kinderbetreuung durch die Mutter wird die Variable „Für ein Kind zwischen 1 und 3 Jahren ist es das Beste, wenn es nur von der Mutter betreut wird." hinzugezogen. Dieser Aussage stimmen insgesamt 15,2 \% zu (Stimme voll und ganz zu=6,0 \%; Stimme eher zu=9,1\%), die überwiegende Mehrheit lehnt dies tendenziell ab (Stimme eher nicht zu=40,0 \%; Stimme überhaupt nicht $\mathrm{zu}=44,8 \%$ ). Die Items „Mütter sollten nachmittags Zeit haben, um ihren Kindern beim Lernen zu helfen.“, „Wichtigkeit von Kindern“ und „beste Betreuung eines Kindes durch die Mutter" laden wie inhaltlich erwartet auf einem Faktor. Die aufgenommenen Variablen erklären rund $21 \%$ der Varianz der Dimension „Kindorientiertes Mutterleitbild“ (siehe Komponente 2). Die interne Konsistenz dieser drei Variablen (Cronbach's Alpha ,332) ist passabel, jedoch nicht hoch.

Tabelle 4: Hauptkomponentenanalyse auf persönlicher Ebene zu verschiedenen Dimensionen des Mutterleitbildes, Betreuung und Bedeutung von Kindern

\begin{tabular}{lcc}
\hline Persönliche Ebene & $\begin{array}{c}\text { Berufsorientiertes } \\
\text { Mutterleitbild (1) }\end{array}$ & $\begin{array}{c}\text { Kindorientiertes } \\
\text { Mutterleitbild (2) }\end{array}$ \\
\hline erklärte Varianz (in Prozent) & 26,3 & 21,2 \\
\hline Cronbach's Alpha &, 423 &, 332 \\
\hline $\begin{array}{l}\text { Mütter sollten nachmittags Zeit haben, um ihren } \\
\text { Kindern beim Lernen zu helfen. }\end{array}$ &, 751 \\
$\begin{array}{l}\text { Mütter sollten einem Beruf nachgehen, um } \\
\text { unabhängig vom Mann zu sein. }\end{array}$ &, 753 & \\
$\begin{array}{l}\text { Eine Mutter, die nur zuhause ist und sich um ihre } \\
\text { Kinder kümmert, wird irgendwann unzufrieden. }\end{array}$ &, 735 & \\
$\begin{array}{l}\text { Eine Mutter sollte, wenn möglich, überhaupt nicht } \\
\text { erwerbstätig sein. }\end{array}$ &,$- 530(+)$ &, $469(+)$ \\
$\begin{array}{l}\text { Es ist wichtig, eigene Kinder zu haben. } \\
\text { Für ein Kind zwischen 1 und 3 Jahren ist es das } \\
\text { Beste, wenn es nur von der Mutter betreut wird. }\end{array}$ & &, 476 \\
\hline
\end{tabular}

Quelle: FLB (2012), gewichtete Daten, eigene Berechnungen

Anm.: Varimax, rotierte Komponentenmatrix. (+) Zwar lädt dieses Item auf beiden Dimensionen, was sich mit den theoretischen Annahmen deckt, jedoch wird es aufgrund seiner stark schiefen Verteilung nicht in den Summenindex aufgenommen. Denn für die Typenbildung „stark orientiertes Mutterleitbild“ lagen beim Einbezug der Variable in den Index lediglich $2 \%$ der Fälle in dieser Kategorie. Auch bei der Berechnung von Cronbach's Alpha wurde er daher nicht einbezogen. Diese Dimensionen erklären 48 \% der Gesamtvarianz. Werte ab ,4 werden angezeigt.

Beide Ergebnisse decken sich mit der inhaltlichen Annahme, dass die Berufsaufgabe der Mutter zugunsten der Kinderbetreuung noch eine gewisse Gültigkeit hat. Es zeigt sich daher, dass ungeachtet der Vielschichtigkeit der heutigen Mutterrolle es zwei zentrale Dimensionen gibt, auf denen sich die Vorstellungen zur Mutterschaft verorten lassen. 


\section{Typenbildung zur Identifikation der Mutterleitbilder}

Im Folgenden werden zwei Indizes aus den Variablen mit hohen Faktorladungen gebildet, um die verschiedenen Indikatoren der beiden Dimensionen Berufs- und Kindorientierung zusammenzufassen. In einem ersten Schritt wurden die Items quadriert, um die dahinter liegenden Antwortmuster besser interpretieren zu können. Anschließend erfolgte eine Summierung. Je kleiner der Indexwert ausfällt, desto höher ist die Zustimmung. Die beiden Indizes setzen sich wie folgt zusammen:

Der Index „Kindorientierung“ umfasst drei Items, die jeweils mit einer 4-stufigen Zustimmungs-Skala ohne Mitte erfasst wurden. Daraus folgt für die Interpretation: Je geringer der Indexwert ausfällt, desto höher ist die Zustimmung dazu, dass sich Mütter auf die Kinderbetreuung konzentrieren sollten. Der Wertebereich des Indexes liegt zwischen 3 und $48^{8}$ und er ist rechtsschief verteilt. ${ }^{9}$ Es zeigt sich dabei, dass ein großer Teil der Befragten tendenziell eher zustimmt, jedoch sind die Extremwerte, die volle Zustimmung bei allen aufgenommenen Variablen bedeuten, nicht stark besetzt. Auch beim Index zur „Berufsorientierung“ bedeuten geringe Werte Zustimmung, hohe Werte Ablehnung der Berufsorientierung von Müttern. Er wurde aus zwei Variablen gebildet und umfasst insgesamt den Wertebereich 2 bis $32 .{ }^{10}$ Insgesamt ist er stärker rechtschief verteilt als der erste Index. ${ }^{11}$ Demnach erachtet eine überwiegende Zahl der befragten Personen die Müttererwerbstätigkeit für wichtig.

In einem nächsten Schritt wurden für die Indizes „Kindorientierung“ und „Berufsorientierung“ je zwei dichotome Variablen gebildet, um sie für weitere Analysen verwenden zu können. Die Dichotomisierung erfolgt auf Basis inhaltlicher Aspekte, hinsichtlich der Zustimmung der Befragten, beide Indizes werden nach denselben Kriterien eingeteilt. Die weitere Aufteilung ist hilfreich, um in einem nachfolgenden Schritt eine Typisierung von Leitbildern vornehmen zu können:

Der Index „Kindorientierung“ wurde in zwei Bereiche gesplittet, die neue Variable „starke Kindorientierung“ umfasst die Summenindex-Werte 3 bis 12, d. h. bei den drei aufgenommenen Items werden die Personen erfasst, die eher sowie voll und ganz zugestimmt haben. In einer weiteren Variable „moderate Kindorientierung“ sind diejenigen enthalten, die bei mindestens zwei der drei aufgenommenen Items mindestens zugestimmt und bei einem weiteren eher abgelehnt haben. ${ }^{12}$ Ähnlich wurde mit dem Index zur „Berufsorientierung“ verfahren: Die Variable „starke Berufsorientierung“ wurde gebildet, bei der die zwei Indikatoren im Wertebereich zwischen nur Zustimmung, d. h. Indexwert 2, so dass aufsummiert und quadriert maximal der Wert 8 alle möglichen Antwortmuster „Stimme voll und ganz zu“ sowie „stimme eher zu“ wiedergibt (d. h. Wertebereich geht von 2 bis 8). Für eine weitere Variable „moderate Berufsorientierung“" wurde der Wertebereich ${ }^{13}$ gemäßigter definiert.

Hieraus ergeben sich vier Variablen für die Orientierung am Kind oder am Beruf, je nach Stärke der Zustimmung:

- $\quad$ starke Kindorientierung,

- moderate Kindorientierung,

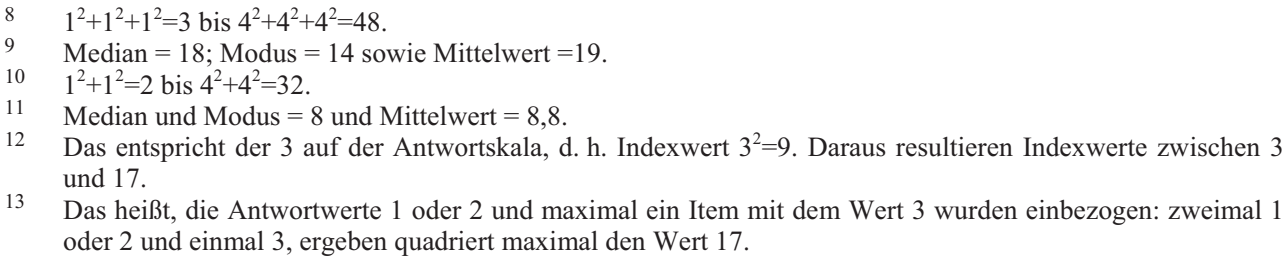

13 Das heißt, die Antwortwerte 1 oder 2 und maximal ein Item mit dem Wert 3 wurden einbezogen: zweimal 1 oder 2 und einmal 3, ergeben quadriert maximal den Wert 17. 
- starke Berufsorientierung,

- moderate Berufsorientierung.

In einem weiteren Schritt wurden diese Variablen zu einer zusammengefasst, mit den o. g. vier Ausprägungen. Die untersuchten Personen verteilen sich unterschiedlich über die nachfolgende Vierfelder-Tafel (vgl. Tabelle 5), so dass vier Typen von Mutterleitbildern ersichtlich werden: Ein Drittel der Befragten (31,0\%), die ein stark berufsorientiertes Mutterleitbild ablehnen, lehnen auch eine starke Kindorientierung ab, sie $(\mathrm{N}=1.548)$ vertreten damit ein „moderates Mutterleitbild“, in dem extrem hohe Ansprüche an Mütter hinsichtlich ihrer Verantwortung für die Familien- und Erwerbsarbeit abgelehnt werden.

Tabelle 5: Kreuztabelle zur Zustimmung „Berufs- und Kindorientierung“

\begin{tabular}{llccc}
\hline & & \multicolumn{2}{c}{ Starke Berufsorientierung } \\
& & & Ablehnung & Zustimmung \\
\hline Starke Kindorientierung & Ablehnung $^{\mathrm{a}}$ & $\mathrm{N}$ & 1.548 & 2.418 \\
& & $\%$ & 31,0 & 48,4 \\
& \multirow{2}{*}{ Zustimmung $^{\mathrm{b}}$} & $\mathrm{N}$ & 426 & 608 \\
& & $\%$ & 8,4 & 12,2 \\
\hline
\end{tabular}

Quelle: FLB (2012), gewichtete Daten, eigene Berechnungen

Anm.: ${ }^{a}$ Entspricht den zusammengefassten beiden Antwortkategorien „stimme (eher/überhaupt) nicht zu“.

${ }^{\mathrm{b}}$ Entspricht den zusammengefassten beiden Antwortkategorien „stimme (sehr) zu“. Die Anteilswerte sind gerundet, so dass die Gesamtsumme über $100 \%$ liegt.

Lediglich 8,4\% der Personen, die eine starke Berufsorientierung von Müttern ablehnen, stimmen einer hohen mütterlichen Kindorientierung zu. Das bedeutet, rund ein Zwölftel der Befragten $(\mathrm{N}=426)$ vertritt ein ,kindorientiertes Mutterleitbild“, bei dem der Fokus der Mutter auf der Familien- und Hausarbeit liegt. Knapp die Hälfte aller jungen Deutschen $(48,4 \%)$ mit stark berufsorientiertem Mutterleitbild lehnen eine starke Kindorientierung ab und vertreten damit ein „, berufsorientiertes Mutterleitbild“. Es zeigt sich, dass es vielen jungen Befragten wichtig erscheint, dass Mütter trotz Familiengründung berufstätig bleiben und damit auch unabhängig von ihren Partnern. Lediglich ein Fünftel der Befragten, die stark berufsorientiert denken, haben gleichzeitig auch ein stark kindorientiertes, und damit ein ,vereinbarkeitsorientiertes Mutterleitbild“ (12,2\%), in dieser Extremgruppe prallen z. T. widersprüchliche Ansprüche aufeinander.

Verbreitung der Mutterleitbilder nach sozialstrukturellen Merkmalen

Es stellt sich die Frage, wie die identifizierten Mutterleitbilder hinsichtlich ihrer Verteilung auf verschiedene soziale Teilpopulationen aussehen.

In Tabelle 6 fällt zunächst auf, dass das berufsorientierte Mutterleitbild (48,4\%) am häufigsten vorkommt, gefolgt vom moderaten $(31,0 \%)$, dem vereinbarkeitsorientierten $(12,2 \%)$ und schließlich dem kindorientierten Mutterleitbild (8,4\%). Betrachtet man die Verteilung der beiden Altersgruppen innerhalb der vier Leitbild-Typen, zeigt sich, dass etwa gleich viele Jüngere (20- bis 29-Jährige) wie Ältere (30- bis 39-Jährige) die moderaten und berufsorientierten Mutterleitbilder vertreten. Es kann außerdem festgestellt werden, dass es einen Erfahrungseffekt gibt: Die 30- bis 39-Jährigen haben etwas häufiger ein kindorientiertes (53,6\%) oder vereinbarkeits-orientiertes Mutterleitbild (56,1\%), da sie 
auch häufiger schon Eltern sind als die jüngere Gruppe der 20- bis 29-Jährigen. Auch der Migrationshintergrund ${ }^{14}$ spielt eine Rolle: Innerhalb des kindorientierten $(31,3 \%)$ und vereinbarkeitsorientierten Mutterleitbildes (35,6\%) sind die Anteile der Personen mit Migrationshintergrund etwas höher. Das moderate Mutterleitbild wird überwiegend von Männern vertreten, lediglich 37,0 \% sind Frauen. 82,7 \% der Personen, die diesen Typus tragen, leben in den alten Bundesländern und es sind vor allem Personen mit mittlerem Bildungsabschluss (nicht in Tabelle 6: 64,3\%).

Tabelle 6: Typenbildung zu den Mutterleitbildern nach sozialstrukturellen Merkmalen (in Prozent)

\begin{tabular}{|c|c|c|c|c|c|c|}
\hline \multirow[b]{2}{*}{$\begin{array}{l}\text { Anteile der } \\
\text { jeweiligen Gruppe }\end{array}$} & \multicolumn{6}{|c|}{ Mutterleitbild } \\
\hline & & Moderat & Kindorientiert & $\begin{array}{c}\text { Berufs- } \\
\text { orientiert }\end{array}$ & $\begin{array}{c}\text { Vereinbarkeits- } \\
\text { orientiert }\end{array}$ & $\begin{array}{c}\text { Anteil } \\
\text { Gesamt }\end{array}$ \\
\hline 20- bis 29-Jährige & $\%$ & $51,3^{* * *}$ & $46,4^{* * *}$ & $52,0^{* * *}$ & $43,9^{* * *}$ & 50,3 \\
\hline Frauen & $\%$ & $37,0^{* * *}$ & $61,0^{* * *}$ & $49,4^{* * *}$ & $72,4^{* * *}$ & 49,3 \\
\hline West & $\%$ & $82,7^{* * *}$ & $86,6^{* * *}$ & $74,7^{* * *}$ & $87,5^{* * *}$ & 79,7 \\
\hline Hohe Bildung & $\%$ & $28,0^{* * *}$ & $21,1^{* * *}$ & $29,7^{* * *}$ & $15,2^{* * *}$ & 26,7 \\
\hline Eltern & $\%$ & $33,8^{* * *}$ & $53,8^{* * *}$ & $36,5^{* * *}$ & $62,1^{* * *}$ & 40,3 \\
\hline \multirow[t]{2}{*}{ Gesamt } & $\%$ & 31,0 & 8,4 & 48,4 & 12,2 & 100,0 \\
\hline & $\mathrm{N}$ & 1.548 & 426 & 2.418 & 608 & 5.000 \\
\hline
\end{tabular}

Quelle: FLB (2012), gewichtete Daten, eigene Berechnungen

Anm.: ${ }^{* * *} \mathrm{p} \leq 0,001 ;{ }^{* *} \mathrm{p} \leq 0,01 ;{ }^{*} \mathrm{p} \leq 0,05$. In der Tabelle 5 werden die vier Leitbilder nach Verteilung charakteristischer Merkmale dargestellt; als Referenz ist innerhalb jedes Leitbildes die Verteilung des jeweiligen Merkmals im Gesamtdatensatz gewählt. ${ }^{\text {a }}$ Die Differenz zu $100 \%$ entfällt auf die jeweilige Referenzgruppe (30- bis 39-Jährige, Männer, Ost, niedrig bzw. mittel Qualifizierte und Kinderlose).

Auch befinden sich in dieser Kategorie überwiegend Kinderlose. Hinsichtlich der Geschlechterverteilung heißen ungefähr gleich viele Frauen wie Männer ein berufsorientiertes Mutterleitbild für erstrebenswert. Drei Viertel leben in Westdeutschland und sind zu knapp einem Drittel nach ISCED-Klassifikation hochgebildet, immerhin 36,5 \% haben Kinder.

Das kindorientierte Mutterleitbild erscheint ein eher weiblich konnotiertes Phänomen $(61,0 \%)$, noch mehr ein westdeutsches $(86,6 \%)$ und wie erwartet seltener eines der Menschen mit hohem Bildungsabschluss (21,1\%). Das könnte damit zusammenhängen, dass die Opportunitätskosten insbesondere für gut ausgebildete Frauen hoch sind. Naheliegend ist, dass rund die Hälfte der Befragten mit kindorientiertem Mutterleitbild bereits eine Familie gegründet hat. Es bleibt offen, was zuerst da war: Das Kind bzw. die Kinder oder zuerst das kindorientierte Mutterleitbild, welches impliziert, dass solche Personen auch ihren Kinderwunsch eher realisieren als solche mit anderen Mutterleitbildern.

Das Leitbild der vereinbarkeitsorientierten Mutter, die sowohl hohe kind- als auch berufsorientierte Anforderungen erfüllen soll, ist zu zwei Drittel (72,4 \%) eine Normalitätsvorstellung von jungen Frauen und von überwiegend Westdeutschen $(87,5 \%)$. Personen mit hoher formaler Bildung teilen dieses Leitbild eher selten, auch hier sind vor allem Befragte mit mittleren Bildungsabschlüssen vertreten (nicht in Tabelle 6: 77,2 \%). Über-

14 Er wurde aus Übersichtsgründen nicht in der Tabelle aufgeführt. 
raschenderweise sind 62,1 \% Eltern. Die konkurrierenden Ansprüche an Elternschaft führen also nicht unbedingt dazu, dass keine Familie gegründet wird. Vielleicht aber führen die gemachten Erfahrungen dazu, dass weder „nur“ Kindorientierung noch „,nur“ Berufsorientierung zielführend sind für das kindliche und elterliche Wohl vieler Befragter. Hierin spiegelt sich der elterliche Wunsch oder vielleicht auch die Zerrissenheit, den Anforderungen beider Welten (besser) gerecht zu werden. Es wird in diesem Kontext angenommen, dass Leitbilder durch die eigenen Eltern geprägt werden (vgl. Dietrich/Gies, Kap. 4). Daher wurde im FLB gefragt, ob und in welchem Umfang die Mutter der Befragten während deren Grundschulzeit einer Erwerbstätigkeit nachgegangen ist. Überwiegend Vollzeit erwerbstätig waren die Mütter der ostdeutschen Befragten während der Grundschulzeit $(67,8 \%)$, bei den westdeutschen waren es lediglich $28,9 \%$. Teilzeit-Erwerbstätigkeit hingegen war mit 28,8\% bei den westdeutschen Müttern häufiger das präferierte Modell als bei den ostdeutschen mit 18,0\%. Das Hausfrauen-Modell lebten 36,2 \% der westdeutschen Mütter der Befragten, im Gegensatz dazu 10,5 \% der ostdeutschen Mütter.

In Tabelle 7 sind die Mutterleitbilder gekreuzt mit den vier Beschäftigungsmodellen der Mütter der befragten Personen abgebildet. Das realisierte Arbeitsmodell der Mutter während der Grundschulzeit ist hier mit dem Mutterleitbild der Befragten eng verknüpft. Es kann angenommen werden, dass das elterliche (mütterliche) Lebensmodell das Antwortverhalten der Befragten mitgeprägt hat: Hier wird in den Rein-Typen deutlich, dass berufsorientierte Befragte mit 43,5\% vor allem auch selbst Vollzeit berufstätige Mütter hatten. Kindorientierte wurden hingegen fast zur Hälfte zuhause von ihren Müttern direkt nach der Schule betreut (46,9\%). Teilzeiterwerbstätigkeit der Mutter oder solche im geringen Umfang hingegen stehen offenbar in keinem Zusammenhang mit dem Mutterleitbild der Befragten. Das Lernen am Modell der eigenen Mutter im Grundschulalter hängt demnach stark mit dem späteren Mutterleitbild der jungen Befragten zusammen.

Tabelle 7: Zusammenhang von Mutterleitbildern auf Individualebene und Sozialisation durch Mutter der befragten Person (Anteile in Prozent)

\begin{tabular}{lcccc}
\hline & Moderat & $\begin{array}{c}\text { Mutterleitbild } \\
\text { Berufsorientiert }\end{array}$ & Kindorientiert & $\begin{array}{c}\text { Vereinbarkeits- } \\
\text { orientiert }\end{array}$ \\
\hline $\begin{array}{l}\text { überwiegend Vollzeit } \\
\text { erwerbstätig }\end{array}$ & 31,5 & $\mathbf{4 3 , 5}$ & 23,3 & 32,9 \\
$\begin{array}{l}\text { überwiegend Teilzeit } \\
\text { erwerbstätig }\end{array}$ & 27,0 & 26,9 & 23,6 & 26,5 \\
$\begin{array}{l}\text { gelegentlich / in } \\
\text { geringem Umfang }\end{array}$ & 5,0 & 4,9 & 5,7 & 5,2 \\
nie & 35,9 & 24,1 & $\mathbf{4 6 , 9}$ & 34,8 \\
\hline Gesamt & 1.532 & 2.401 & 420 & 601 \\
& 100 & 100 & 100 & 100 \\
\hline
\end{tabular}

Quelle: FLB (2012), gewichtete Daten, eigene Berechnungen

Anm.: ${ }^{a}$ Weitere nicht aufgeführte Kategorien „Meine Mutter war damals bereits verstorben.“ und „Ich hatte keinen Kontakt zu ihr." Fett gedruckt = vollständige Übereinstimmung von Mutterleitbild und Erwerbsverhalten der Mutter der Befragten. 


\section{$4 \quad$ Zusammenfassung und Fazit}

Erstes Ziel dieses Beitrags war es, die Verbreitung und inhaltlichen Bestandteile von Mutterleitbildern zu untersuchen. Persönliche Vorstellungen wie, dass Mütter zuhause ihren Kindern helfen sollen, jedoch auch erwerbstätig sein sollten, um unabhängig vom Partner zu sein, sind weit verbreitet und werden von fast drei Viertel der Befragten geteilt. Die Ablehnung der Muttererwerbstätigkeit hingegen wird von wenigen vertreten, jedoch ist eine weit verbreitete mütterlich-häusliche Präsenzkultur auf individueller Ebene ersichtlich. Die Ergebnisse spiegeln den hohen Qualitätsanspruch an Mütter, sowohl die Erziehung („Fürsorge“) als auch ihre „Selbstsorge“ parallel als zentrale Lebensziele zu verfolgen. Die hohe Zustimmung zu den verschiedenen Dimensionen der Mutterschaft, die die Lebensführung von Müttern thematisieren, zeigt ungelöste Widersprüche (vgl. Oechsle 1998).

Bei der Ursachensuche spielt die historische Entwicklung des Mutterleitbildes eine wesentliche Rolle. Henry-Huthmacher (2008) interpretiert, dass junge Frauen und Mütter zwischen den tradierten und heutigen Vorstellungen hin- und hergerissen sind:

„So sind die Normbilder der 1950er/1960er Jahre, die ihrer Großeltern, aber auch die ihrer Eltern aus den 1970er Jahren eine starke Referenz zur Orientierung, aber gleichzeitig auch zur Abgrenzung. Gerade dadurch ist das Bild der guten Mutter außerordentlich wirkmächtig. Die Bedeutung dieser Norm für das Fremdbild, aber auch für das Selbstbild junger Mütter ist nicht zu unterschätzen und prägt ihr Verhalten. (...) So ist das normative Ausmaß an Verzicht und Aufopferung, das Mütter für ihre Kinder zu erbringen haben, heute zwar wesentlich geringer als vor 30 oder 40 Jahren, dennoch sind die Erwartungen an die mütterliche Zuwendungsbereitschaft und Versorgung der Kinder nicht wesentlich geringer geworden“ (Henry-Huthmacher 2008: 10).

Gleichzeitig wird auch deutlich, dass das allgemein vorherrschend wahrgenommene Mutterleitbild aufgrund der Widersprüchlichkeit und Komplexität der Anforderungen überfrachtet erscheint. Die Vermutung liegt nah, dass Mütter sich leicht dem gesellschaftlichen Vorwurf ausgesetzt sehen könnten, eine „Rabenmutter“ zu sein, wenn sie sich zu stark auf ihr Berufsleben konzentrieren. Dies resümieren auch Merkle und Wippermann (2008: 44). Ob dieser in der Gesellschaft wahrgenommene normative Druck Frauen (und auch Männer) in ihrer privaten Lebensführung (Arbeitsteilung von Familien- und Erwerbsarbeit) und in ihrer Familienplanung beeinflusst, muss weiteren Untersuchungen überlassen werden.

Während auf persönlicher Ebene die Vorstellungen je nach sozialer Gruppe stark variieren, hierbei sind besonders Geschlechter- und Ost-West-Unterschiede hervorzuheben, sind die wahrgenommenen Leitbilder in der Gesellschaft fast unabhängig und flächendeckend gleichermaßen über die verschiedenen Gruppen präsent und erlangen damit quasi eine universale Gültigkeit, deren Wirkung es in Zukunft zu untersuchen gilt.

Ein weiteres Ziel war die Analyse der Passung von persönlichen Mutterleitbild-Aspekten mit den gesellschaftlich wahrgenommenen: Das allgemeine Mutterleitbild ist stärker kindorientiert und am Ideal der teilzeit- oder nicht arbeitenden Mutter ausgerichtet als die persönlichen Mutterleitbilder, welche eher ausgleichend zwischen Berufs- und Kindorientierung ausgerichtet sind. Es wird demnach ein gewisser Druck in der Öffentlichkeit wahrgenommen, als Mutter zuhause zu bleiben und keinem Beruf nachzugehen. Das NegativLeitbild der „Rabenmutter“ hat demnach noch immer Gültigkeit. Insgesamt wurde beim Abgleich der persönlichen und allgemeinen Vorstellungen wesentlich mehr Dissonanz gefunden als zuvor angenommen. Es bestätigt sich die Vermutung, dass Abweichungen des eigenen Leitbildes von gesellschaftlichen Leitbildern genau wahrgenommen werden und hier ein Spannungsfeld besteht. Auch wird deutlich, dass Befragte ihre Abweichungen von der Allgemeinheit eher wahrnehmen als solche, bei denen sie übereinstimmen, da wahrgenommene Dissonanzen Konfliktpotenzial bergen: Dies wird vermutlich konkreten Beobach- 
tungen der Befragten geschuldet sein, in denen persönliche und Umgebungs-Meinungen oder die daraus entstehenden Verhaltensweisen miteinander kollidieren. Oder der Allgemeinheit wird vermeintlich ein Leitbild unterstellt, welches jedoch auf Individualebene gar nicht mehr so weit verbreitet ist, weil diese Wahrnehmung aus medialen oder institutionellen Kontexten ${ }^{15}$ stammt. Die große Diskrepanz zwischen Selbst- und Gesellschaftsleitbildern könnte als „cultural lag“ gedeutet werden: Individuelle Ansichten wandeln sich schneller als die gesellschaftlich gültigen, das allgemeine Mutterleitbild ist dahingehend starrer, zumal es auch stärker durch institutionelle Rahmungen fixiert ist (z. B. Gesetze, Familien- und Sozialpolitik) und dadurch tradierte Rollenbilder ${ }^{16}$ von Müttern weiterhin reproduziert werden (BMFSFJ 2012b: 53ff.). Drei miteinander in Wechselwirkung stehende Aspekte - individuelles Verhalten, kulturelle Leitbilder und institutionelle Rahmenbedingungen - können für diese Kluft zwischen individueller und allgemein wahrgenommener Sichtweise eine Rolle spielen: Kulturelle Vorstellungen zur Mutterschaft sind historisch gewachsen (werden z. T. intergenerational übertragen) und sind daher per se nicht schnell wandelbar. Familienpolitische Instrumente wie z. B. das Elterngeld und der Ausbau der Kinderbetreuungsinfrastruktur führen jedoch unter Umständen dazu, dass sich kulturelle und tradierte Vorstellungen zur Mutterschaft bzw. Elternschaft (schrittweise) verändern können (siehe Lück/Diabaté, Kap. 2; Diabaté/Lück 2014).

Bei der hauptkomponenten- und indexbasierten Typisierung konnten vier Mutterleitbilder herausgearbeitet werden, die gegenwärtig in Deutschland bei den 20- bis 39-Jährigen existieren. Es wurden zwei Reintypen, das berufs- und das kindorientierte Mutterleitbild sowie zwei Mischtypen, das moderate und das vereinbarkeitsorientierte Mutterleitbild identifiziert. In Anlehnung an Hakims drei Typen wurde somit noch ein vierter Typus manifestiert, der die widersprüchlichen Anforderungen an Mütter in sich vereint. Zudem wurden Alters- und Geschlechterdifferenzen festgestellt, was auf einen Erfahrungseffekt schließen lässt: Ältere und Frauen vertreten häufiger kindorientierte und vereinbarkeitsorientierte Mutterleitbilder. Dahinter könnte jedoch auch ein Kohorteneffekt stecken, nämlich dass die 30- bis 39-Jährigen tendenziell etwas traditioneller denken als die Jüngeren. Gleichermaßen sind in diesen beiden Leitbildern weniger hoch Qualifizierte vertreten. Auch zeigte sich eine stärkere Kindzentrierung bei in Westdeutschland lebenden Personen. Interessanterweise hängen vor allem die kind- und berufsorientierten Mutterleitbilder eng mit dem Lebensmodell der Mütter der Befragten zusammen. Hierbei prägend erscheinen die politischen Systeme, in denen die Mütter sozialisiert wurden und die in die nachfolgende Generation hineinwirken. Wie genau diese intergenerationale Transmission von Mutterleitbildern erfolgt, bleibt unklar.

Insgesamt hat sich die Messung der Mutterleitbilder im Leitbildsurvey als valide erwiesen. Zudem ergibt die konstruierte Item-Batterie in sich konsistente Muster. Mit den vorliegenden Daten lassen sich die zuvor theoretisch angenommenen inhaltlichen Dimensionen gut replizieren. Hinsichtlich der Gültigkeit der Mutter-Items für Väter müssten bei einer Wiederbefragung die Fragen identisch für Väter gespiegelt werden. Jedoch (siehe Lück, Kap. 14) legen die Ergebnisse hinsichtlich der Vaterleitbilder nahe, dass die Leitbilder nicht identisch sind und es z. T. andere, nicht zu Müttern kongruente, Anforderungen gibt. Wie sich Mutter- und Vaterleitbilder zu elterlichen Leitbild-Konstellationen zusammenfügen lassen, wird in einem gesonderten Beitrag dargestellt (siehe Diabaté/Lück/Schneider, Kap. 15). Offen bleibt schließlich, wie die vier Mutterleitbilder in Wechselwirkung zu sozial-

15 Gies und Dietrich (vgl. Kap. 4) gehen der Frage nach, woher die Leitbilder stammen könnten.

16 Beispiele dafür sind das Ehegattensplitting oder die kostenlose Mitversicherung der Ehefrau in der Krankenkasse des Partners. 
strukturellen Faktoren und institutionellen Rahmenbedingungen stehen und wie stabil sie im Lebensverlauf sind. Ein zweiter Messzeitpunkt könnte an dieser Stelle gewinnbringend sein. Aussichtsreich erscheint vor dem Hintergrund der hier erzielten Ergebnisse auch zu untersuchen, wie sich die Mutterleitbilder auf die Familiengründungs- und Familienerweiterungsprozesse auswirken und wie stabil sie im Lebensverlauf sind.

\section{Literatur}

Beckmann, Sabine (2007): Die geteilte Arbeit? Möglichkeiten einer sozialpolitischen Steuerung des Careverhaltens von Männern. In: Zeitschrift für Familienforschung 19 (3), S. 371-392.

Beier, Loreen; Rupp, Marina (2009): Rollenbilder und Arbeitsteilung in den Ländern der EU. In: Mühling, Tanja; Rost, Harald (Hrsg.): ifb-Familienreport Bayern 2009. Schwerpunkt: Familien in Europa. Staatsinstitut für Familienforschung an der Universität Bamberg. Bamberg: ifb-Materialien 6, S. 121-141.

BMFSFJ (Bundesministerium für Familie, Senioren, Frauen und Jugend) (Hrsg.) (2012a): Alleinerziehende in Deutschland - Lebenssituationen und Lebenswirklichkeiten von Müttern und Kindern. Monitor Familienforschung. Beiträge aus Forschung, Statistik und Familienpolitik. Nr. 28.

BMFSFJ (Bundesministerium für Familie, Senioren, Frauen und Jugend) (Hrsg.) (2012b): Neue Wege - gleiche Chancen. Gleichstellung von Frauen und Männern im Lebensverlauf. Stellungnahme der Bundesregierung zum Gutachten der Sachverständigenkommission. Drucksache 17/6240.

Butler, Amy C. (2002): Welfare, Premarital Childbearing, and the Role of Normative Climate: 1968-1994. In: Journal of Marriage and Family 64 (2), S. 295-313.

Diabaté, Sabine; Lück, Detlev (2014): Familienleitbilder. Identifikation und Wirkungsweise auf generatives Verhalten. In: Zeitschrift für Familienforschung 26, 1, S. 49-69.

Diabaté, Sabine (2014): Mütter heute: Leitbilder, Lebensrealitäten und Wünsche. In: Bundeszentrale für politische Bildung. [http://www.bpb.de/politik/innenpolitik/familienpolitik/191689/muetterheute? $\mathrm{p}=$ all, 14.01.2015]

Geissler, Birgit; Oechsle, Mechtild (1996): Lebensplanung junger Frauen. Zur widersprüchlichen Modernisierung weiblicher Lebensläufe. Weinheim: Beltz Deutscher Studienverlag

Hakim, Catherine (2000): Work-Lifestyle Choices in the 21st Century: Preference Theory. Oxford: University Press.

Hakim, Catherine (2002): Lifestyle preferences as determinants of women's differentiated labour market careers. In: Work and Occupations 29, S. 428-459.

Henry-Huthmacher, Christine (2008): Eltern unter Druck. Zusammenfassung der wichtigsten Ergebnisse der Studie. In: Merkle, Tanja; Wippermann, Carsten (2008): Eltern unter Druck. Selbstverständnisse, Befindlichkeiten und Bedürfnisse von Eltern in verschiedenen Lebenswelten. Eine sozialwissenschaftliche Untersuchung von Sinus Sociovision GmbH. Stuttgart: Lucius \& Lucius, S. 1-25.

Herwartz-Emden, Leonie (1995): Mutterschaft und weibliches Selbstkonzept. Eine interkulturell vergleichende Untersuchung. Weinheim und München: Juventa Verlag.

Heut, Michael (2004): Familienleitbilder. Die sozialethische Dimension des Leitbildes für die Institution Familie. Studien zur Familienforschung 14. Hamburg: Verlag Dr. Kovac.

Hirdman, Yvonne (1988): Genussystemet - reflexioner kring kvinnors sociala underordning. In: Kvinnovetenskaplig tidskrift 9 (3), S. 49-63.

Kaufmann, Franz-Xaver (1990): Zukunft der Familie. Stabilität, Stabilitätsrisiken und Wandel der familialen Lebensformen sowie ihre gesellschaftlichen und politischen Bedingungen. München: C.H. Beck.

Klement, Carmen; Rudolph, Brigitte (2003): Auswirkungen staatlicher Rahmenbedingungen und kultureller Leitbilder auf das Geschlechterverhältnis. Deutschland und Finnland. In: Aus Politik und Zeitgeschichte 44, S. 23-30. 
Lück, Detlev; Gründler, Sabine; Naderi, Robert; Dorbritz, Jürgen; Schiefer, Katrin; Ruckdeschel, Kerstin; Hiebl, Johannes; Wolfert, Sabine; Stadler, Manuela; Pupeter, Monika (2013): Familienleitbilder 2012. Methodenbericht zur Studie. BiB Working Paper 2/2013. Wiesbaden: Bundesinstitut für Bevölkerungsforschung.

Lück, Detlev; Hofäcker, Dirk (2008): The Values of Work and Care among Women in Modern Societies. In: Oorschot, Wim van; Opielka, Michael; Pfau-Effinger, Birgit (Hrsg.): Culture and Welfare State. Values of Social Policy from a Comparative Perspective. Cheltenham, GB \& Northampton, USA: Edward Elgar, S. 289-313.

Merkle, Tanja; Wippermann, Carsten (2008): Eltern unter Druck. Selbstverständnisse, Befindlichkeiten und Bedürfnisse von Eltern in verschiedenen Lebenswelten. Eine sozial-wissenschaftliche Untersuchung von Sinus Sociovision. Stuttgart: Lucius \& Lucius.

Norlander, Kerstin (2003): Some Reflections on Gender Relations. Presented at "Gender and Power in the New Europe", the 5th European Feminist Research Conference. August 20-24, 2003, Lund University, Sweden.

Oechsle, Mechthild (1998): Ungelöste Widersprüche. Leitbilder für die Lebensführung von Frauen. In: Oechsle, Mechthild; Geissler, Birgit (Hrsg.): Die ungleiche Gleichheit. Junge Frauen und der Wandel im Geschlechterverhältnis. Opladen: Leske + Budrich Verlag, S. 185-200.

Pfau-Effinger, Birgit (1996): Analyse internationaler Differenzen in der Erwerbsbeteiligung von Frauen. Theoretischer Rahmen und empirische Ergebnisse. In: Kölner Zeitschrift für Soziologie und Sozialpsychologie 48 (3), S. 462-492.

Pfau-Effinger, Birgit (2000): Kultur und Frauenerwerbstätigkeit in Europa. Theorie und Empirie des internationalen Vergleichs. Opladen: Leske + Budrich Verlag.

Pfau-Effinger, Birgit; Flaquer, Lluis; Jensen, Per H. (Hrsg.) (2009): Formal and Informal Work in Europe. The Hidden Work Regime. New York: Routledge.

Ruckdeschel, Kerstin (2009): Rabenmutter contra Mère Poule. Kinderwunsch und Mutterbild im deutsch-französischen Vergleich. In: Zeitschrift für Bevölkerungswissenschaft/Comparative Population Studies 34 (1-2), S. 105-134.

Schneider, Norbert; Diabaté, Sabine; Lück, Detlev (2014): Familienleitbilder in Deutschland. Ihre Wirkung auf Familiengründung und Familienentwicklung. Herausgegeben von der KonradAdenauer-Stiftung e.V., Sankt Augustin/Berlin.

Schütze, Yvonne (1986): Die gute Mutter. Zur Geschichte des normativen Musters „Mutterliebe“. Bielefeld: Kleine.

Schütze, Yvonne (2010): Mutterbilder in Deutschland. In: Psychoanalyse - Texte zur Sozialforschung $25(2 / 3)$, S. 179 


\title{
Vaterleitbilder: Ernährer und Erzieher?
}

\author{
Detlev Lück*
}

\section{Zusammenfassung}

Unter den Vorstellungen, wie ein Vater sein sollte, lassen sich zwei Leitbilder entdecken: zum einen das des ,Familienernährers“, der für das Bestreiten des Haushaltseinkommens Verantwortung übernimmt und Familienarbeit der Mutter überlässt. Dieses Leitbild ist auf gesellschaftlicher Ebene relevant: als Vorstellung davon, was anderen denken. Zum zweiten lässt sich das Leitbild des ,,aktiven Vaters “ identifizieren, der seine Erwerbsarbeit reduziert und sich aktiv in die Erziehung einbringt. Dieses ist unter den persönlichen Leitbildern dominant. Daneben gibt es eine relevante Gruppe, die beide Leitbilder verinnerlicht hat und sich vereinbarkeitsorientiert zeigt.

Ungeachtet gesellschaftsweit ähnlicher Größenordnungen sind bestimmte Vaterleitbilder in bestimmten Teilen der Gesellschaft graduell stärker verbreitet. Das Leitbild des ,aktiven Vaters" ist in Ostdeutschland, im akademischen Milieu sowie unter Konfessionslosen besonders häufig; das des „Familienernährers “ ist charakteristisch für Frauen, niedrig Gebildete, Protestanten und religiöse Menschen. Das vereinbarkeitsorientierte Leitbild ist typisch für Männer, niedrig Gebildete und Katholiken. Genau genommen ist das Leitbild des ,,aktiven Vaters" mit kinderlosen Frauen assoziiert und das vereinbarkeitsorientierte Leitbild mit kinderlosen Männern, die somit zur Selbstüberforderung neigen. Für Väter und Mütter gelten diese Charakteristika kaum, so dass von einer Desillusionierung im Zuge der Familiengründung auszugehen ist.

\section{$1 \quad$ Einleitung}

,Wann ist der Mann ein Mann? “ fragt Herbert Grönemeyer in einem 1984 veröffentlichten Lied. Natürlich ist die Frage nicht tautologisch zu verstehen, sondern meint eigentlich: Wann ist ein Mann ein ,richtiger“ Mann? Wann entspricht er der allgemein verbreiteten Vorstellung davon, wie ein Mann üblicherweise ist oder sein sollte? Oder auch: Wie sieht das Leitbild des Mannseins aus? Der Eindruck, dass sich dieses wandelt und dabei viele Männer orientierungslos zurücklässt, beschäftigt seit mindestens drei Jahrzehnten die öffentliche Diskussion. Zuletzt etwa widmete die ZEIT ihr Magazin zur Ausgabe 14/2014 dem Thema „Der Mann von heute“.

Die Vermutung liegt nahe, dass sich auch und gerade die Vorstellungen davon gewandelt haben, wie ein Mann im Kreise seiner Familie sein sollte und was er dort zu tun und zu lassen hat. Wie also steht es um das Leitbild des Vaters? Welche Vorstellungen haben

\footnotetext{
* Ich danke der anonymen Gutachterin bzw. dem anonymen Gutachter für die wertvollen Hinweise und die hilfreiche Kritik.
} 
Menschen in Deutschland heute davon, was es bedeutet, Vater bzw. ein „richtiger“ und „guter" Vater sein? Sind diese Vorstellungen einheitlich? Sind sie untereinander konsistent? Wann ist der Vater ein Vater?

Der wissenschaftliche Forschungsstand zu dieser Frage ist uneindeutig, und es deutet sich an, dass das auch für die Leitbilder der Vaterschaft selbst gilt: Zwar dominiert heute in den Einstellungen, wie Familien-, Haus- und Erwerbsarbeit zwischen Frau und Mann in einer Partnerschaft aufgeteilt sein sollten, die Forderung nach gleicher und geteilter Verantwortung in allen Bereichen; doch vor allem Männer glauben, dass sie nach wie vor in erster Linie an ihrer Fähigkeit gemessen werden, mit ihrem Einkommen einen Familienhaushalt zu unterhalten. Befunde zeigen sowohl Unterschiede in den Vorstellungen verschiedener Teile der Gesellschaft als auch Widersprüche in den Vorstellungen einzelner (vgl. ausführlicher den nachfolgenden Abschnitt). Dieser Beitrag analysiert die Daten der Studie Familienleitbilder (FLB 2012) im Hinblick auf Vaterleitbilder in dem Bemühen, damit zur Entwirrung der unübersichtlichen Befundlage beizutragen.

\section{$2 \quad$ Stand der Forschung}

Spiegelungen solcher Leitbilder finden sich zuweilen in soziologischen Theorien und in ihren idealtypischen Überzeichnungen der Realität. Eine besonders gängige Beschreibung der bürgerlichen Kernfamilie in der Industriegesellschaft zeichnet Talcott Parsons (1973) in den 1950er Jahren. Er weist dem Vater die ,instrumentelle“ Rolle zu, also jene Funktionen, die die Beziehung zwischen der Familie und ihrer Umwelt betreffen. Dazu gehören vor allem das Erwirtschaften des Haushaltseinkommens sowie die Repräsentanz der Familie nach außen, gegenüber dem Staat und anderen Institutionen. Für den sozialen und emotionalen Zusammenhalt zu sorgen, (die „expressive“ Rolle) bleibt Aufgabe der Mutter. Diese beiden Zuständigkeiten sind in Parsons' Theorie klar voneinander getrennt - was wahrscheinlich auch dem Leitbild seiner Zeit entspricht (Peitz 2006: 30).

\subsection{Entwicklung des Vaterleitbildes in den letzten fünf Jahrzehnten}

Dieses normative Muster und das damit verbundene Leitbild des Vaters als Oberhaupt und Ernährer der Familie finden sich um die Mitte des 20. Jahrhunderts in nahezu allen industrialisierten Staaten wieder, jedoch in unterschiedlichen Abstufungen. Während es in Westdeutschland stark präsent ist, gehört es zur Normalität Ostdeutschlands, dass Mütter nahezu vollständig in die Erwerbsarbeit und Väter vergleichsweise stark in die Haus- und Familienarbeit eingebunden sind. Diese Unterschiede bestehen der Tendenz nach bis heute fort (Pollmann-Schult 2012; Goldstein et al. 2010).

Vieles spricht dafür, dass sich das Vaterleitbild der 1950er Jahre über das vergangene halbe Jahrhundert hinweg entweder graduell verschoben oder dass es Konkurrenz bekommen hat. Denn in den 1980er Jahren wird in der Familienforschung der „,neue Vater“ entdeckt (Schneider 1989; Nave-Herz 1988; Schweizer 1986), der sich nicht mehr auf die Erwerbsarbeit beschränkt, sondern aktiv in die Kinderbetreuung und Erziehung einmischt. Das Bild dieses ,neuen Vaters“, der zuweilen auch ,aktiver Vater“ heißt $\mathrm{t}^{1}$, beschreibt - so

1 Beide Begriffe werden im Folgenden synonym verwendet. 
die eine Diagnose - zunehmend die Lebensrealität und das Selbstverständnis der Väter in Deutschland. Allerdings gibt es an dieser Diagnose Zweifel, denn beim Vergleich von Einstellungen und faktischem Engagement von Vätern in Haus- und Familienarbeit zeigt sich eine bereits viel zitierte „verbale Aufgeschlossenheit bei gleichzeitiger Verhaltensstarre“ (Beck/Beck-Gernsheim 1990: 31): Männer sprechen sich zwar (ähnlich wie Frauen) in Umfragen zunehmend deutlich für eine egalitäre Aufteilung von Erwerbs-, Haus- und Familienarbeit in der Partnerschaft aus; sie verändern jedoch ihr Verhalten diesbezüglich kaum: Ihr Engagement im Beruf bleibt unvermindert hoch; ihr Engagement in Haus- und Familienarbeit wächst dementsprechend verhalten und bleibt vorzugsweise auf Wochenenden und angenehme Tätigkeiten wie z. B. das Spielen mit Kindern beschränkt (Lück 2009; Hofäcker 2007; Oberndorfer/Rost 2005; Fthenakis/Minsel 2002).

Die Reform der Elternzeit zu Beginn des Jahres 2007 stellte einen deutlicheren Entwicklungsschub in Aussicht. Die beiden „Vätermonate“, die nur vom zweiten Elternteil in Anspruch genommen werden können, führten dazu, dass der Anteil der Väter, die sich an der Elternzeit beteiligen, tatsächlich kontinuierlich stieg: Wurde das Erziehungsgeld im Jahr 2006 noch zu weniger als 4\% von Männern beantragt, wuchs der Anteil der Kinder, deren Vater Elterngeld bezog, von 15,4 \% im Jahr 2007 auf zuletzt 29,6 \% im vierten Quartal 2012 (Statistisches Bundesamt 2014: 33; Statistisches Bundesamt 2008: 1). Allerdings bleibt es weiterhin die Ausnahme, dass ein Vater über die beiden „Vätermonate“ hinaus Elternzeit in Anspruch nimmt (Statistisches Bundesamt 2014: 28). Inwieweit die Reform nachhaltige Auswirkungen auf die Arbeitsteilung in Elternpaaren hat, ist bislang nicht hinreichend erforscht.

Einer Egalisierung steht unter anderem die Neigung vieler Paare im Weg, die Arbeitsteilung im Beziehungsverlauf nach und nach (wieder) stärker komplementär aufzuteilen, selbst wenn Haus- und Erwerbsarbeit zum Beziehungsbeginn einmal gleich verteilt waren. Gerade im Zuge der Familiengründung - dann, wenn männliche Partner zu Vätern werden - beginnen sich Paare wieder stark an den Mustern partnerschaftlicher Arbeitsteilung zu orientieren, die Parsons Mitte des 20. Jahrhunderts beschrieb (Huinink/Reichart 2008; Grunow 2007; Schulz/Blossfeld 2006). Als Gründe werden unter anderem Effizienzvorteile sowie Schwierigkeiten bei der Vereinbarkeit von Familie und Beruf genannt.

\subsection{Ambivalenz und Widersprüchlichkeit als Status Quo}

Tatsächlich gibt es Hinweise darauf, dass Paare zuweilen bei der Umsetzung ihrer Vorstellungen einer idealen Arbeitsteilung an den strukturellen Barrieren scheitern. Dies gilt beispielsweise für drohende Einkommenseinbußen bei Arbeitszeitreduktion oder Inanspruchnahme von Elternzeit und für fehlende Möglichkeiten flexibler Arbeitszeitgestaltung (Merkle/Wippermann 2008: 32f., 226f.; Vaskovics/Rost 1999: 43f., 156f.). Mindestens ebenso relevant scheinen in dieser Hinsicht sozio-kulturelle Barrieren zu sein, wie die fehlende soziale Anerkennung von Familienarbeit oder die Anwesenheitskultur in Unternehmen (Merkle/Wippermann 2008: 34f., 224f.). Trotz neuer Erwartungshaltungen an Männer, sich auch in Haus- und Familienarbeit zu engagieren, wird eine Vernachlässigung ihrer Ernährerrolle sozial wenig akzeptiert (Peitz 2006: 35f.)

Doch es sind nicht nur äußere Hürden, die dem Siegeszug des „,neuen Vaters“ im Wege stehen. Auch das Selbstbild von Männern hat sich nicht so grundlegend gewandelt, wie die Zustimmungswerte einzelner Einstellungsfragen zuweilen mutmaßen lassen. Zwar nennen Männer als Eigenschaften, die sie an anderen Männern sympathisch finden, heute häufig auch weiblich konnotierte Eigenschaften - wie etwa liebevolle Fürsorge für Kinder (57 \%), Hilfsbereitschaft (43\%), Zärtlichkeit (43\%) oder Gefühle zeigen (42\%); doch die Eigen- 
schaften, die einem männlichen Familienernährer entsprechen, erhalten weiterhin die höchsten Zustimmungswerte: die Familie gut zu versorgen (76\%), berufliche Kompetenz (60\%) sowie Leistungsorientierung und Ehrgeiz (57\%) (Wippermann 2013: 32f.). Offenbar treten zum Bild eines guten Vaters eher neue Fähigkeiten hinzu, als dass die Vorstellung des Familienernährers durch die des egalitären Partners ersetzt würde (Fthenakis/Minsel 2002: 67). Widersprüche, Rollenkonflikte und Überforderung sind mögliche Konsequenzen.

Um eine differenzierte empirische Beschreibung des Mannes bemüht sich unter anderem die Studie von Rollet und Werneck (2002). Ihr zufolge machen in Österreich sowohl „Neue Väter“ als auch „Familienorientierte Väter“, die eine komplementäre Geschlechterrollenteilung vor Augen haben, jeweils eine Minderheit aus. Eine Mehrheit der Väter, die im Zuge von Familiengründung und Dauer der Elternschaft weiter zunimmt, ist zu den „Eigenständigen Vätern“ zu zählen, die zwar eine geteilte Verantwortung sehen, sich aber wenig in der Familienarbeit engagieren und dem Umgang mit dem Kind wenig Bedeutung beimessen (ebd.: 332f.).

Eine ähnlich differenzierte Beschreibung finden Volz und Zulehner (2009) für Deutschland. Basierend auf repräsentativen Einstellungsdaten aus den Jahren 1998 und 2008 teilen sie Männer (ebenso wie Frauen) in vier Typen ein (ebd.: 28f.): in „teiltraditionelle“ (1998: $30 \%$, 2008: $27 \%)$, in „balancierende“ (23\%, $24 \%)$, in „suchende“ $(29 \%$, $30 \%)$ und in „moderne“ (17\%, $19 \%$ ) Männer (ebd.: 34). Zwei der vier Typen markieren die Pole „(teil-)traditionell“" und „modern“ auf einer Achse sozialen Wandels, die einerseits durch den Mann als Familienernährer und andererseits durch den Mann als gleichberechtigtem Partner gekennzeichnet sind, der sich die Verantwortungen mit seiner Partnerin teilt und sozial mit ihr auf einer Stufe steht. Demnach ist der „Neue Mann“ bzw. der „Neue Vater" bis heute noch eine Minderheit. Zur Minderheit ist demnach aber auch der „traditionelle Mann“ bzw. der „Familienernährer“ geworden. Eine Mehrheit zeigt ambivalente Vorstellungen: Teilweise werden Zuschreibungen beider Vaterleitbilder verknüpft und im Alltag pragmatisch „(aus-)balanciert“, teilweise werden beide Vaterleitbilder abgelehnt, was eine verunsicherte „Suche“ nach der richtigen Gestaltung von Vaterschaft erahnen lässt.

Auch die Untersuchung von Merkle und Wippermann (2008) findet Ambivalenzen und unterschiedliche Schattierungen von Vaterleitbildern. Diese korrespondieren der Tendenz nach mit den Sinus-Milieus und der sozialen Schicht, wobei das Rollenbild des Vaters, der präsent ist und sich aktiv um seine Kinder kümmert, charakteristisch für die obere Mittelschicht ist und das Rollenbild des „Geldverdieners und Chefs“ für die Unterschicht. Dazwischen liegt unter anderem der ,aktive Feierabend-Papa“" (ebd.: 46f.).

Für Männer stellt sich die Situation widersprüchlich dar: Einerseits sehen sie sich aufgefordert, ihren fairen Anteil an Haus- und Familienarbeit zu übernehmen und möchten zudem häufig dem Bild eines „aktiven Vaters“ entsprechen. Andererseits empfinden sie nach wie vor die Erwartung, zusätzlich auch die Rolle des Familienernährers zu erfüllen, und tun sich schwer, diesen Anspruch aufzugeben oder zu relativieren. Das gilt insbesondere für junge Männer (Zerle/Krok 2008). Männer richten dabei an sich deutlich höhere Erwartungen in Bezug auf ihre Rolle als Familienernährer und etwas niedrigere Erwartungen in Bezug auf ihr Engagement in der Haus- und Familienarbeit, als Frauen dies von ihnen erwarten (Institut für Demoskopie Allensbach 2013: 17f.). So glauben beispielsweise $71 \%$ der Männer zwischen 18 und 34, von ihnen werde erwartet, für das Familieneinkommen zu sorgen, obwohl diese Erwartung ,lediglich“ von $60 \%$ der Frauen im gleichen Alter artikuliert wird. Dass sie viele Aufgaben im Haushalt und in der Familie übernehmen sollten, erwarten $66 \%$ der Frauen, und $62 \%$ der Männer nehmen dies auch wahr (ebd.). So oder so werden viele Väter ihren eigenen Ansprüchen nicht gerecht. Seine Schwierigkeiten, den Vorsatz, Erwerbs- und Familienarbeit mit seiner Partnerin fair zu teilen, in die Tat 
umzusetzen, erläutert ein junger Vater in einem Selbsterfahrungsbericht in der ZEIT mit einem Fortbestehen des Leitbildes des Familienernährers: „Wir werden in unserem Umfeld anders wahrgenommen und haben ein Bild von uns, in das eine Teilzeitstelle nicht hineinpasst" (von Daniels 2014).

Vor dem Hintergrund dieser vielschichtigen Befunde stellt sich die Frage, ob sich mit Daten des FLB 2012 überhaupt ein gegenwärtiges konsistentes Leitbild der Vaterschaft identifizieren lässt. Falls dies möglich ist, schließen sich weitere Fragen an: Gibt es ein Leitbild der Vaterschaft oder mehrere konkurrierende? Wir klar konturiert - wie diffus sind sie? Inwieweit bilden sie den Typus des „Familienernährers“, inwieweit den des „neuen Vaters" und inwieweit Mischformen dieser Typen ab?

\section{Vorstellungen zu Vätern und Vaterschaft}

Leitbilder bündeln Vorstellungen zu einem Lebensbereich. Bevor nach solchen Bündeln gesucht wird, bietet es sich daher an, die einzelnen Vorstellungen zu Vaterschaft zu betrachten, die als Bausteine von Leitbildern der Vaterschaft in Betracht kommen.

\subsection{Vater sein - ein Leitbild für Männer?}

Bevor es um die Leitbilder der Vaterschaft im eigentlichen Sinne geht - also um die Frage, welche Vorstellungen es davon gibt, wie Väter sein sollten -, soll zunächst gefragt werden, ob es überhaupt die Vorstellung gibt, dass Männer Väter sein sollten: Sollte ein Mann in der Vorstellung der Deutschen (ungeachtet davon, ob er auch ein Haus baut oder einen Baum pflanzt) im Laufe seines Lebens ein Kind zeugen?

Diese Frage lässt sich mit den Daten des FLB 2012 nur bedingt beantworten, denn nach dieser Normalitätsvorstellung wurde nicht gefragt. Hinweise kann aber die Frage geben, wie wichtig es für den Befragten persönlich ist, ,eigene Kinder zu haben“. Demnach ist es eine sehr deutliche Mehrheit von 82,2 \% der Männer, denen es persönlich wichtig ist, einmal Kinder zu haben: Fast jeder zweite Mann (47,5\%) beantwortet die Frage sogar mit einem ,sehr wichtig“, ein weiteres Drittel (34,7 \%) antwortet moderater mit „eher wichtig“. Diese Anteilswerte sinken auch nur leicht, wenn man diejenigen aus der Analyse ausschließt, die bereits Kinder haben und nun möglicherweise eine sozial erwünschte Antwort geben: Drei Viertel (75,3 \%) der kinderlosen Männer empfinden es als wichtig, einmal eine Familie zu gründen; etwa die Hälfte von ihnen $(35,7 \%)$ empfindet es sogar als sehr wichtig. Trotz (oder wegen) der allgemeineren Formulierung der Frage liegen diese Zustimmungswerte tendenziell über der Verbreitung persönlicher Kinderwünsche (Ruckdeschel 2007; Eckhard/Klein 2006: 34f.) und deuten darauf hin, dass es durchaus Teil eines Leitbildes des Mannes ist, früher oder später Vater zu werden.

\subsection{Vorstellungen über Väter und Vaterschaft}

Wenn ein Paar eine Familie gründet, ändert sich vieles: Ein Kind benötigt viel Aufmerksamkeit, und es bringt für die jungen Eltern neue Aufgaben mit sich, wie Stillen oder Windeln wechseln. Außerdem belastet es den Haushalt durch zusätzliche Kosten und eventuell durch Einkommensausfälle infolge einer Reduktion der Erwerbsarbeit. Das Paar 
muss innerhalb kurzer Zeit seinen Alltag neu regeln, und zwar wahrscheinlich auf eine einschneidendere Art als jemals sonst im Leben (Rost/Schneider 1995). Dabei helfen kulturell vermittelte Vorstellungen davon, welche Aufgaben auf Eltern im Einzelnen zukommen (vgl. Ruckdeschel, Kap. 12), in welcher Weise diese die Mutter betreffen (vgl. Diabaté, Kap. 13) und auf welche Weise den Vater. Ungeachtet dessen, was für einen Mann im Allgemeinen als normal gilt, wird jetzt relevant, welche elterlichen Aufgaben der Vater eines Kindes normalerweise übernimmt und wie er sie idealerweise ausfüllt.

Den aus der Theorie und Empirie bekannten Leitbildern eines Vaters entsprechend, sind im FLB 2012 solche Vorstellungen über Väter bzw. über Vaterschaft erhoben worden, die sich zum einen auf deren (alleinige) Verantwortung für die Sicherung des Haushaltseinkommens und zum anderen auf deren (Mit-)Verantwortung für die Betreuung und Erziehung der Kinder beziehen. Von den vier erhobenen Items entsprechen jeweils zwei der Rolle des Vaters als alleiniger Einkommensbezieher und „Ernährer“ der Familie und zwei der des ,neuen“ oder ,aktiven Vaters", der sich gemeinsam mit seiner Partnerin die Familienarbeit teilt. Alle vier Items wurden auf individueller Ebene erfasst, d. h. es wurde gefragt, inwieweit sie der persönlichen Vorstellung des Befragten entsprechen. Drei dieser Items sind außerdem auf gesellschaftlicher Ebene erfasst, d. h. es wurde gefragt, inwieweit diese Vorstellungen (der Wahrnehmung des Befragten zufolge) in der Gesellschaft verbreitet sind. ${ }^{2}$ Im Folgenden sind für jedes Item die Anteile der Zustimmung und Ablehnung dargestellt (vgl. Abbildung 1).

Es zeigen sich recht unterschiedliche Befunde für persönliche und für mutmaßlich gesellschaftliche Leitbilder. Persönlich befragt, stimmen Menschen eher den Vorstellungen $\mathrm{zu}$, die einem aktiv in die Familienarbeit eingebundenen Vater entsprechen: So erhält die Aussage „Für ein Kind ist es nicht gut, wenn der Vater die Erziehung allein der Mutter überlässt." mit 75,3 \% den höchsten Zustimmungswert. (Mehr als die Hälfte der Befragten stimmt diesem Item sogar ,voll und ganz $\mathrm{zu}^{\prime}$.) Immer noch eine deutliche Mehrheit (58,0 \%) identifiziert sich mit der Aussage: „Väter sollten für ihre Kinder beruflich kürzer treten." Die Aussagen „Es liegt nicht in der Natur des Mannes, Hausmann zu sein." (26,4\%) und „Ein Mann muss seine Familie allein ernähren können.“ (28,9\%) stoßen dagegen nur bei etwas mehr als einem Viertel der Befragten auf Zustimmung.

Beinahe spiegelverkehrt sieht das Antwortverhalten aus, wenn es darum geht, die in der Gesellschaft verbreiteten Vorstellungen zu beurteilen. Obwohl die Ansicht, dass es „für ein Kind nicht gut [ist], wenn der Vater die Erziehung allein der Mutter überlässt", unter den Befragten selbst sehr verbreitet ist, hat kaum mehr als ein Drittel $(37,8 \%)$ den Eindruck, dass auch ihre Mitmenschen sie mehrheitlich teilen. Umgekehrt erfahren nun die beiden Items mehrheitlich Zustimmung, die den Vater in der Rolle des „Familienernährers“ sehen: Etwa zwei Drittel (67,0\%) glauben, dass es der Vorstellung der Allgemeinheit zufolge „,nicht in der Natur des Mannes [liegt], Hausmann zu sein“. Und drei Viertel der Befragten $(75,4 \%)$ nehmen wahr, dass ihre Mitmenschen glauben, dass „ein Mann seine Familie allein ernähren können [muss]“. Zusammenfassend lässt sich sagen: Persönliche Leitbilder der Vaterschaft orientieren sich überwiegend an einer egalitären Arbeitsteilung in der Partnerschaft, die mutmaßlich gesellschaftlichen Leitbilder überwiegend an einer komplementären.

2 Eine Spiegelung aller vier Items fiel der verfügbaren Interviewdauer zum Opfer. 
Abbildung 1: Individuelle Vorstellungen und Vorstellungen der Allgemeinheit über Väter

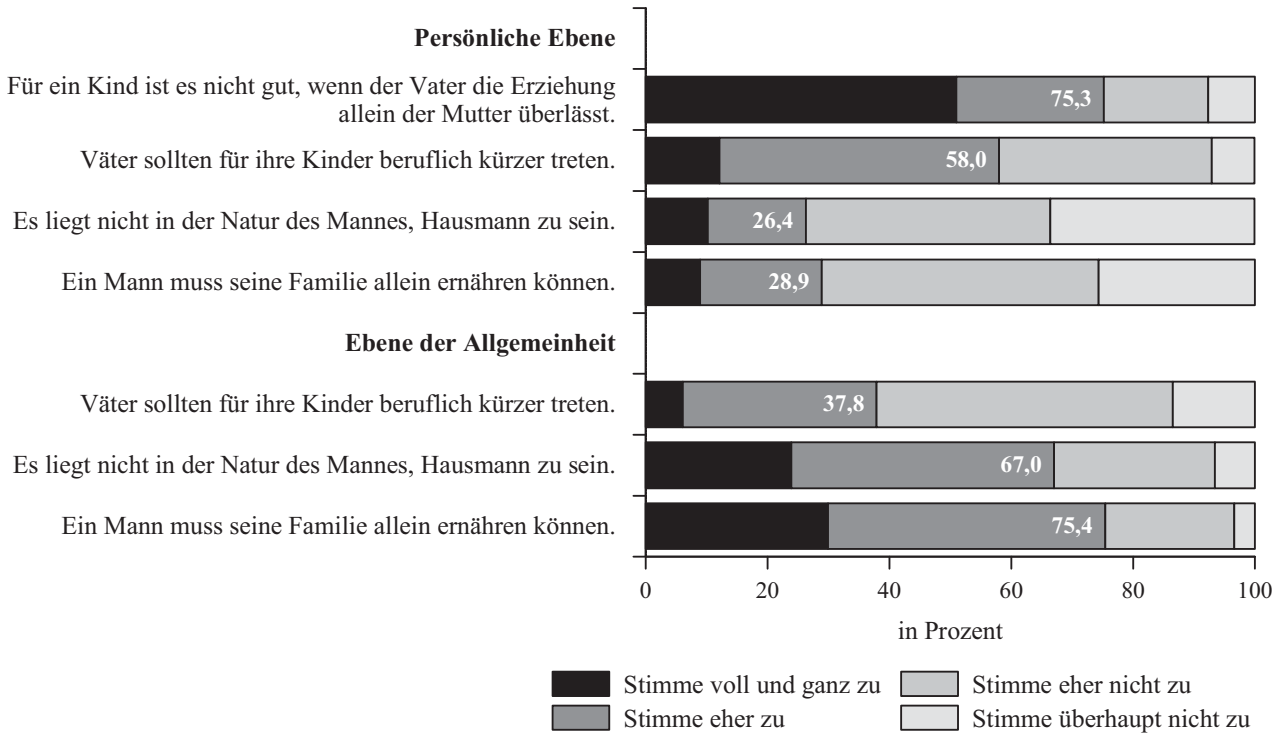

Quelle: FLB (2012), gewichtete Daten, eigene Berechnungen

Anm.: Die angegebenen Prozentwerte geben jeweils an, welcher Anteil der Befragten insgesamt zugestimmt hat (,stimme voll und ganz zu“ plus „stimme eher zu“).

\subsection{Individuelle Vorstellungen-gesellschaftliche Vorstellungen}

Der Gegensatz zwischen persönlichen und gesellschaftlichen Normalitätsvorstellungen wirft die Frage auf, wie dieser zustande kommt. Immerhin sind die in Abbildung 1 dargestellten - statistisch aggregierten - persönlichen Zustimmungswerte ihrerseits Ausdruck kollektiv verbreiteter Vorstellungen (vgl. auch Junck/Lück 2015 sowie Lück/Naderi/Ruckdeschel, Kap. 3). Warum also haben Menschen, die den Vater mehrheitlich als aktiven Elternteil sehen wollen, den Eindruck, dass die Mehrheit den Vater als bloßen „Familienernährer" sieht? Angeschlossen werden soll die allgemeinere Frage: Welche Beziehung gibt es zwischen den persönlichen und den gesellschaftlichen Vorstellungen von Vaterschaft?

Offenkundig gibt es unterschiedliche und konkurrierende Leitbilder der Vaterschaft in der Gesellschaft. Sowohl das Bild des ,aktiven Vaters“ als auch das des „Familienernährers“ wird wahrgenommen. Eine Erklärung für die Differenz zwischen den Ebenen kann unterschiedliche Gründe haben, die sich hier empirisch nicht überprüfen lassen: Eine erste Möglichkeit besteht darin, dass das Leitbild des „Familienernährers“ außerhalb der im FLB 2012 erfassten Teilpopulation der 20- bis 39-Jährigen, also in früher geborenen Generationen, tatsächlich von einer Mehrheit geteilt wird, deren Normalitätsvorstellungen aber aufgrund des Stichprobenzuschnitts nur indirekt - eben in Form der gesellschaftlichen Ebene - erfasst werden (ebd.). Eine zweite Möglichkeit ist es, dass hinter den auf gesellschaftlicher Ebene erfassten Leitbildern gar keine größere Bevölkerungsgruppe (mehr) steht. Sie wirken entweder dadurch nach, dass sie einmal mehrheitlich geteilt wurden und ihr Verblassen noch nicht in vollem Umfang aufgefallen ist. Oder sie wirken, indem 
sie im öffentlichen Diskurs großes Gewicht haben - sei es, dass Medien sie als Normalität darstellen, sei es, dass gesellschaftliche Eliten sie vorleben, sei es, dass institutionelle Regelungen sich an ihnen orientieren (ebd.). Dann gewinnt ein kulturelles Leitbild ein Eigenleben, unabhängig von der Zahl der Menschen, die hinter ihm stehen, und übt Einfluss auf das Handeln der Menschen aus, ähnlich wie Durkheim (1984: 109) es allgemein für soziale Tatbestände beschreibt. Auch kann der Eindruck entstehen, eine Mehrheit der Gesellschaft stünde persönlich hinter dem Leitbild, ähnlich wie es NoelleNeumann in ihrem Modell der Schweigespirale für öffentliche Meinung im Kontext von politischen Einstellungen beschreibt (Noelle-Neumann 1980). Wirkungsmächtig können Leitbilder dennoch sein.

Eine weitere Frage schließt sich an: In welcher Beziehung stehen persönliche und gesellschaftliche Leitbilder empirisch? Gibt es einen statistischen Zusammenhang zwischen der persönlichen Zustimmung zu einer bestimmten Normalitätsvorstellung und der Zustimmung, dass diese Vorstellung (auch) in der Gesellschaft insgesamt weit verbreitet sei? Es gibt diesen Zusammenhang, wenn auch sehr schwach ausgeprägt: Der Tendenz nach sehen Menschen die gleichen Vorstellungen als etabliert an, die sie auch persönlich teilen. Für alle drei Items, die Vorstellungen von Vaterschaft messen und auf beiden Ebenen gespiegelt wurden, ergibt sich eine hochsignifikante positive Korrelation (vgl. Tabelle 1).

Tabelle 1: Korrelation zwischen persönlicher und gesellschaftlicher Vorstellung

\begin{tabular}{lc}
\hline & Spearman's Rho \\
\hline Ein Mann muss seine Familie allein ernähren können. &, $130^{* * *}$ \\
Es liegt nicht in der Natur des Mannes, Hausmann zu sein. &, $144^{* * *}$ \\
Väter sollten für ihre Kinder beruflich kürzer treten. &, $140^{* * *}$ \\
\hline
\end{tabular}

Quelle: FLB (2012), gewichtete Daten, eigene Berechnungen

Anm.: ${ }^{* * *}=$ signifikant auf $0,1 \%$-Niveau.

Allerdings sind alle drei Korrelationen vergleichsweise schwach, was angesichts der sehr gegensätzlichen Verteilungen (siehe Abbildung 1) nicht überraschen kann. Die Vorstellungen, die auf persönlicher Ebene starke Zustimmungswerte erhalten, werden auf gesellschaftlicher Ebene mehrheitlich abgelehnt, und umgekehrt. Allein daraus ergibt sich notwendigerweise, dass es auch einen relativ großen Bevölkerungsanteil gibt, der seine persönlichen Vorstellungen im Widerspruch zu denen seiner Mitmenschen sieht. Möglicherweise wird auch gerade durch diese Gegensätzlichkeit das Bewusstsein dafür geschärft, dass es ein Leitbild auf gesellschaftlicher Ebene gibt. Die Frage, wie ein Vater zu sein hat, birgt demnach gesellschaftlichen Konfliktstoff.

\section{Identifikation von Vaterleitbildern}

Inhaltlich lassen sich die Items relativ plausibel zwei theoretisch erwartbaren gegensätzlichen Leitbildern der Vaterschaft zuordnen. Aber tun sie das auch empirisch? Gibt es tatsächlich Bündel aus bestimmten Normalitätsvorstellungen zu Vaterschaft, die typischerweise gemeinsam, also jeweils von den gleichen Menschen verinnerlicht wurden? Heruntergebrochen auf die Analyseebene stellt sich damit die Frage nach den Korrelationen zwischen den verschiedenen Normalitätsvorstellungen jeweils einer Ebene. Die Struktur aus 
einem oder mehreren „Bündeln“ von Vorstellungen verdeutlicht eine Hauptkomponentenanalyse (Tabelle 2).

Tabelle 2: Dimensionale Struktur individueller Vorstellungen über Väter

\begin{tabular}{lcc}
\hline & Dimension 1 & Dimension 2 \\
\hline $\begin{array}{l}\text { Cronbach's Alpha } \\
\text { erklärte Varianz }\end{array}$ &, 429 &, 201 \\
\hline Ein Mann muss seine Familie allein ernähren können. & $32,1 \%$ & $28,0 \%$ \\
Es liegt nicht in der Natur des Mannes, Hausmann zu sein. &, 792 & \\
$\begin{array}{l}\text { Für ein Kind ist es nicht gut, wenn der Vater die Erziehung } \\
\text { allein der Mutter überlässt. }\end{array}$ & &, 781 \\
Väter sollten für ihre Kinder beruflich kürzer treten. & &, 703 \\
\hline
\end{tabular}

Quelle: FLB (2012), gewichtete Daten, eigene Berechnungen

Anm.: Hauptkomponentenanalyse, Varimax-Rotation, rotierte Komponentenmatrix.

Unter den Vorstellungen von Vaterschaft, die die Befragten selbst äußern, lassen sich tatsächlich zwei Leitbilder identifizieren. Sie entsprechen den aus der Literatur bekannten „Reinformen“ eines Vaterschaftsbildes: zum einen das Leitbild des „Familienernährers“, der dafür verantwortlich ist, die ökonomische Existenz der Familie zu sichern, aber zur Haus- und Familienarbeit nicht geeignet ist, zum anderen das des „aktiven Vaters“, der für die Betreuung und Erziehung seines Kindes mit verantwortlich ist und sich engagiert. Die dimensionale Struktur, die durch Hauptkomponentenanalyse dargestellt wird, entspricht einer Einfachstruktur, bei der jedes Item auf genau einer Dimension eine hohe Faktorladung aufweist und somit eindeutig zuzuordnen ist.

Während die erste Dimension durch die beiden Items „Ein Mann muss seine Familie allein ernähren können.“ und „Es liegt nicht in der Natur des Mannes, Hausmann zu sein.“ vergleichsweise gut abgebildet wird (Cronbach's Alpha = ,429), erreicht die zweite Dimension, gemessen durch die Items „Für ein Kind ist es nicht gut, wenn der Vater die Erziehung allein der Mutter überlässt.“ und „Väter sollten für ihre Kinder beruflich kürzer treten.“, nur eine sehr schwache Reliabilität (,201). Das könnte daran liegen, dass für das Leitbild der aktiven Vaterschaft weniger gut geeignete Items erhoben wurden. Es könnte auch ein Hinweis darauf sein, dass das Leitbild selbst vergleichsweise diffus ist und die darin gebündelten Vorstellungen nicht von allen Menschen konsistent zusammen gedacht werden. (Dass das Leitbild ungeachtet dessen auf persönlicher Ebene stärker verbreitet ist, lässt sich anhand der Zustimmungswerte der einzelnen Items bereits erahnen - vgl. Abbildung 1.)

Die Items, die der Hauptkomponentenanalyse zufolge auf einer Dimension liegen, lassen sich als ein Leitbild deuten: Wer hohe Zustimmungswerte auf der Dimension hat, teilt das Leitbild, wer eher Ablehnung artikuliert, teilt es nicht.

Da eine Dimension zwei Pole besitzt - Zustimmung und Ablehnung - kann sie grundsätzlich auch zwei Leitbilder erfassen, denn auch die Ablehnung eines Items kann unter Umständen eine inhaltliche Vorstellung zu Vaterschaft zum Ausdruck bringen: Beispielsweise könnte die Ablehnung der Aussage „Väter sollten für ihre Kinder beruflich kürzer treten." auch gelesen werden als: Väter sollten ihr Engagement voll und ganz dem Beruf widmen, um die Familie ernähren zu können (vgl. Lück/Naderi/Ruckdeschel, Kap. 3). Allerdings wäre die Deutung der Ablehnungen der beiden Dimensionen als jeweils eigenes Leitbild in diesem Fall nicht plausibel, zumal diese inhaltlich relativ genau der Zustimmung 
der jeweils anderen Dimension entsprächen. Sie wäre also nur dann plausibel, wenn alle vier Items auf einer einzigen Dimension lägen und die Zustimmung zu aktiver Vaterschaft stark negativ mit der Zustimmung zum „Familienernährer“ korrelieren würde. Das ist nicht der Fall - beide Leitbilder werden durch unterschiedliche Dimensionen gemessen. Das spricht dafür, dass eine Ablehnung auf einer Dimension zunächst nur heißt, dass das entsprechende Leitbild nicht geteilt wird - und nicht, dass ein gegenteiliges Leitbild verinnerlicht ist. Es spricht jedoch auch dafür, dass Individuen beide Leitbilder von Vaterschaft gleichzeitig verinnerlicht haben können - oder keines von beiden. Offenbar kann es bei der Wahrnehmung von Vaterleitbildern innere Widersprüche und Ambivalenzen geben, so wie es vorangegangene Forschungsbefunde erwarten lassen (vgl. Wippermann 2013; Volz/Zulehner 2009; Zerle/Krok 2008).

Die gleiche Analyse für die Normalitätsvorstellungen auf gesellschaftlicher Ebene ergibt ein ähnliches Bild (Tabelle 3). Zwar vereint die Hauptkomponentenanalyse hier zunächst alle drei Items auf einer Dimension. Doch die Faktorladung des einen Items, das nicht in dieses Leitbild passt („Väter sollten für ihre Kinder beruflich kürzer treten.“), ist sehr niedrig. Zudem ergibt die Reliabilitätsanalyse, dass die gefundene Dimension konsistenter ist, wenn dieses Item weggelassen wird: Dann steigt Cronbach's Alpha von ,192 auf ,476. Auch auf gesellschaftlicher Ebene treten also der „Familienernährer“ und der „aktive Vater“ als zwei Leitbilder auf, denen zuweilen gleichzeitig zugestimmt wird bzw. die zuweilen beide abgelehnt werden. Dass die Hauptkomponentenanalyse nur eine der beiden Dimensionen identifizieren kann, dürfte damit zusammenhängen, dass ein Item zu aktiver Vaterschaft („Für ein Kind ist es nicht gut, wenn der Vater die Erziehung allein der Mutter überlässt.") auf gesellschaftlicher Ebene nicht gespiegelt wurde. Ein Vergleich der Befunde aus Tabelle 2 und 3 ist daher nur eingeschränkt möglich.

Tabelle 3: Dimensionale Struktur gesellschaftlicher Vorstellungen über Väter

\begin{tabular}{lc}
\hline & Dimension 1 \\
\hline Cronbach's Alpha &, 476 \\
Varianz & $44,9 \%$ \\
\hline Ein Mann muss seine Familie allein ernähren können. &, 801 \\
Es liegt nicht in der Natur des Mannes, Hausmann zu sein. &, 766 \\
Väter sollten für ihre Kinder beruflich kürzer treten. & $(-, 344)$ \\
\hline
\end{tabular}

Quelle: FLB (2012), gewichtete Daten, eigene Berechnungen

Anm.: Hauptkomponentenanalyse, Komponentenmatrix.

\section{Verbreitung von Vaterleitbildern in der Gesellschaft}

Auch wenn mit zwei Items je Dimension die Datengrundlage zur Messung von Leitbildern schmal ist, so soll sie dennoch genutzt werden, um eine Beschreibung der Verbreitung bestehender Vaterleitbilder in Deutschland vorzunehmen. Dazu wird zunächst für jede der zuvor identifizierten Dimensionen (vgl. Tabellen 2 und 3) jeweils ein binärer Indikator 
gebildet, der tendenzielle Zustimmung von tendenzieller Ablehnung der zu dieser Dimension gehörenden Vorstellungen unterscheidet. ${ }^{3}$ So ergeben sich drei Indikatoren: (1) Zustimmung zum Leitbild des „,Familienernährers “ auf persönlicher Ebene, (2) Zustimmung zum Leitbild des ,,aktiven Vaters" auf persönlicher Ebene sowie die (3) Zustimmung zum Leitbild des „,Familienernährers “ auf gesellschaftlicher Ebene.

Anschließend werden die Befragten auf Grundlage ihres Antwortverhaltens in eine Typologie eingeteilt. Diese ergibt sich aus den vier Kombinationsmöglichkeiten der beiden binären Indikatoren zur Messung von Zustimmung oder Ablehnung jeweils eines Vaterleitbildes (vgl. Tabelle 4): Wer das Leitbild des Vaters als Hauptverdiener und „Ernährer“ der Familie teilt, nicht aber das des Vaters als aktiv an der Kindererziehung mitwirkender Elternteil, erhält die Ausprägung (1): Orientierung am Leitbild des Vaters als „Familienernährer". Die spiegelverkehrte Kombination führt zu der Ausprägung (2): der Orientierung am Leitbild des Vaters als ,,aktiver Vater“. Zustimmung zu beiden Leitbildern ergeben die Ausprägung (3), die im Folgenden als Vereinbarkeitsorientierung gedeutet werden soll: als Orientierung an beiden Leitbildern mit dem Bestreben, eine Synthese aus ihnen zu finden. Die Ablehnung von beiden Leitbildern ergibt schließlich die Ausprägung (4), die Menschen ohne bzw. mit geringer Orientierung an (den von uns gemessenen) Vaterleitbildern entspricht. Die Typologie lässt sich für Leitbilder auf persönlicher Ebene mithilfe der zwei dichotomen Indikatoren bilden, die Zustimmung oder Ablehnung zu den entsprechenden Leitbildern messen. Für Leitbilder auf gesellschaftlicher Ebene muss neben dem einen verfügbaren Indikator auf die Zustimmung oder Ablehnung zu dem Item „Väter sollten für ihre Kinder beruflich kürzer treten.“ zurückgegriffen werden.

Tabelle 4: Typologie zur Orientierung an Vaterleitbildern

\begin{tabular}{|c|c|c|c|c|c|}
\hline \multirow[t]{2}{*}{ Leitbild des Vaters als ... } & & \multicolumn{4}{|c|}{... „Familienernährer“ } \\
\hline & & & Zustimmung & & Ablehnung \\
\hline \multirow[t]{2}{*}{..., ,aktiver Vater“ } & Zustimmung & (3) & $\begin{array}{l}\text { Vereinbarkeits- } \\
\text { orientierung }\end{array}$ & (2) & $\begin{array}{l}\text { Orientierung am } \\
\text { Leitbild des } \\
\text { „aktiven Vaters“ }\end{array}$ \\
\hline & Ablehnung & (1) & $\begin{array}{l}\text { Orientierung am } \\
\text { Leitbild des } \\
\text { „Familienernährers“ }\end{array}$ & (4) & $\begin{array}{l}\text { keine bis geringe } \\
\text { Orientierung an } \\
\text { Vaterleitbildern }\end{array}$ \\
\hline
\end{tabular}

Quelle: eigene schematische Darstellung

Diese Typologisierung sowie die beiden ihr zugrundeliegenden Indikatoren erlauben es nun, die Verbreitung verschiedener Vaterleitbilder einerseits sowie die Verbreitung verschiedener Typen der Orientierungen an Vaterleitbildern andererseits hinsichtlich ihrer zahlenmäßigen Verbreitung in der Gesellschaft zu beschreiben (vgl. Abbildung 2). Demnach teilt das Leitbild des Vaters als „Familienernährer" auf persönlicher Ebene etwa ein Drittel (33,9\%) der Deutschen im Alter zwischen 20 und 39. Dies spiegelt die eher verhaltenen Zustimmungswerte zu den beiden einzelnen Items wider, die zu diesem Leitbild ge-

3 Genau genommen werden die beiden Items, die jeweils eine Dimension messen, in einem ersten Schritt quadriert und danach aufsummiert, so dass sich Ausprägungen zwischen $2(1+1)$ und $32(16+16)$ ergeben. Im dritten Schritt werden die so gebildeten Indizes dichotomisiert. Dabei erlaubt es die vorherige Quadrierung, die mittleren Summenwerte differenziert zu behandeln: Werte von 2 bis 13 werden als Zustimmung codiert, wobei die Kombination ,stimme eher zu“ bei einem Item und „stimme eher nicht zu“ beim anderen Item berücksichtigt wird $(4+9=13)$. Werte von 15 bis 32 werden als Ablehnung codiert, wobei die Kombination „stimme voll und ganz zu“ plus ,stimme überhaupt nicht zu“ berücksichtigt wird (1+16=17). 
hören (vgl. Abbildung 1). Das Leitbild des Vaters als „,aktiver Vater" teilen etwa vier von fünf Befragten (79,1\%), also eine deutliche Mehrheit. Einen noch höheren Zustimmungswert erhält mit 81,6\% die Wahrnehmung eines gesellschaftlichen Leitbildes des Vaters als „Familienernährer" auf gesellschaftlicher Ebene.

Betrachtet man nicht die Leitbilder und ihre Zustimmung, sondern die Individuen und deren Orientierung an den beiden Vaterleitbildern, so ergeben sich etwas unterschiedliche Zahlen, weil nun auch berücksichtigt werden muss, dass sich manche Menschen an beiden Vaterleitbildern orientieren oder keinem von beiden (vgl. Abbildung 2), vergleichbar der Typologisierung von Volz und Zulehner (2009). Entsprechend sind die Anteilswerte tendenziell kleiner.

Betrachtet man die persönlichen Vaterleitbilder, an denen sich Menschen orientieren, so ist nach wie vor die Orientierung am Leitbild des ,aktiven Vaters“ dominant: Etwa die Hälfte $(51,9 \%)$ der Deutschen zwischen 20 und 39 Jahren orientiert sich daran, und damit weitaus mehr, als aufgrund der Befunde von Volz und Zulehner (2009) zu erwarten wären. Daneben gibt es aber auch eine relativ große Bevölkerungsgruppe $(27,3 \%)$, die sich an beiden Vaterleitbildern orientiert und sie zu vereinen sucht. Das Bild von Vaterschaft dieser Gruppe entspricht dem des „balancierenden Mannes“ (ebd.), wobei eine Neigung zur (Selbst-)Überforderung von Vätern zu erwarten ist. Menschen, die beide Leitbilder zurückweisen, machen mit 14,1\% eine Minderheit aus. Noch seltener sind solche, die sich lediglich am Leitbild des „Familienernährers“ orientieren (6,7 \%).

Abbildung 2: Verbreitung von Vaterleitbildern

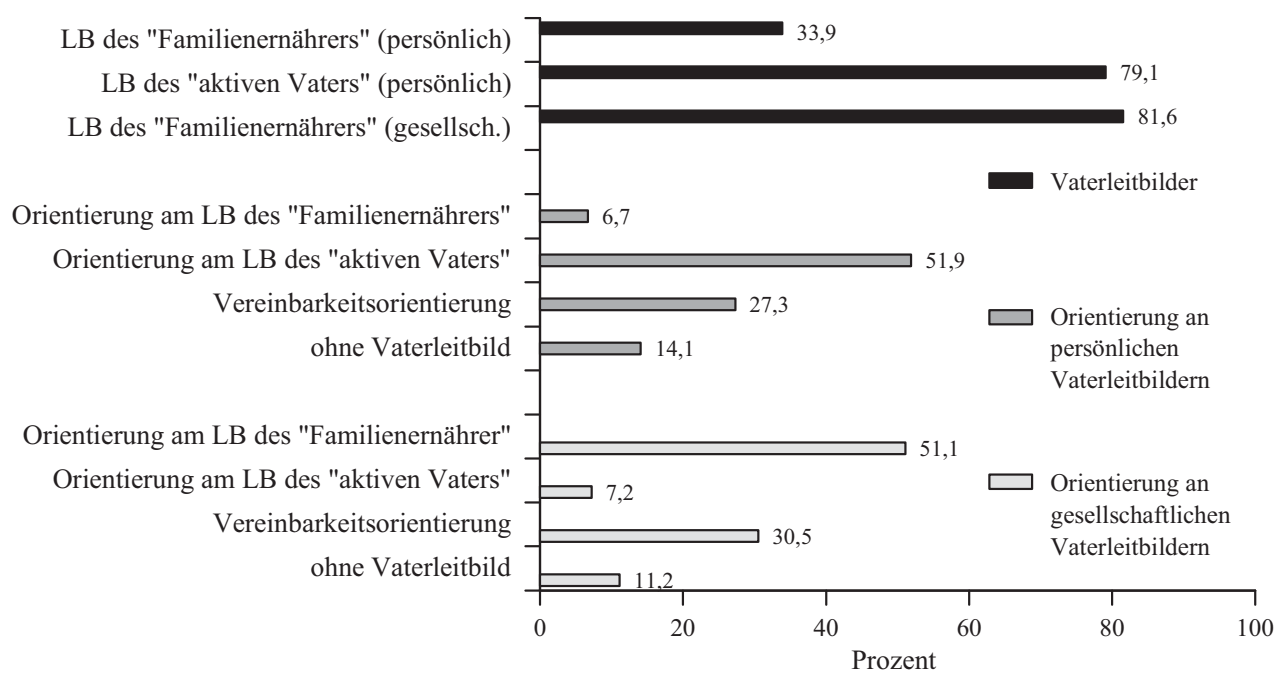

Quelle: FLB (2012), gewichtete Daten, eigene Berechnungen

Der Blick auf die Wahrnehmung von gesellschaftlichen Vaterleitbildern ergibt ein gewissermaßen symmetrisches Bild: Nun ist die Wahrnehmung des Leitbildes des „Familienernährers“ als alleinigem gesellschaftlich verbreitetem Vaterleitbild mit $51,1 \%$ am häufigsten. Die Wahrnehmung beider Leitbilder und einer dementsprechenden Vereinbarkeitsorientierung ist immerhin bei 30,5 \% vorzufinden. Die Wahrnehmung, dass auf gesellschaftlicher Ebene keines der beiden Vaterleitbilder (11,2\%) oder nur das des ,aktiven Vaters“ (7,2\%) etabliert sei, hat jeweils nur eine Minderheit. 


\section{Sozialräumliche und regionale Verteilung der Orientierungen an Vaterleitbildern}

Es ist davon auszugehen, dass Vaterleitbilder nicht in jedem Teil der Gesellschaft gleich stark verbreitet sind und dass die Orientierung der Menschen am einen oder am anderen Leitbild zwischen Regionen, Generationen, sozialen Schichten und sozio-demografischen Gruppen variiert. Oder andersherum gewendet: Wahrscheinlich rekrutieren sich Menschen, die sich an unterschiedlichen Vaterleitbildern orientieren, auch tendenziell aus unterschiedlichen sozialen Gruppen. Inwieweit das zutrifft, soll anhand der ersten drei Typen der Orientierung an persönlichen Vaterleitbildern überprüft werden: anhand derer, die sich nur am Leitbild des „Familienernährers “ orientieren, anhand derer, die allein das des ,, aktiven Vaters " verinnerlicht haben, und anhand derer, die ,vereinbarkeitsorientiert" sind (vgl. Abbildung 3 und 4).

Abbildung 3: Soziodemografische Profile verschiedener Typen der Orientierung an persönlichen Vaterleitbildern

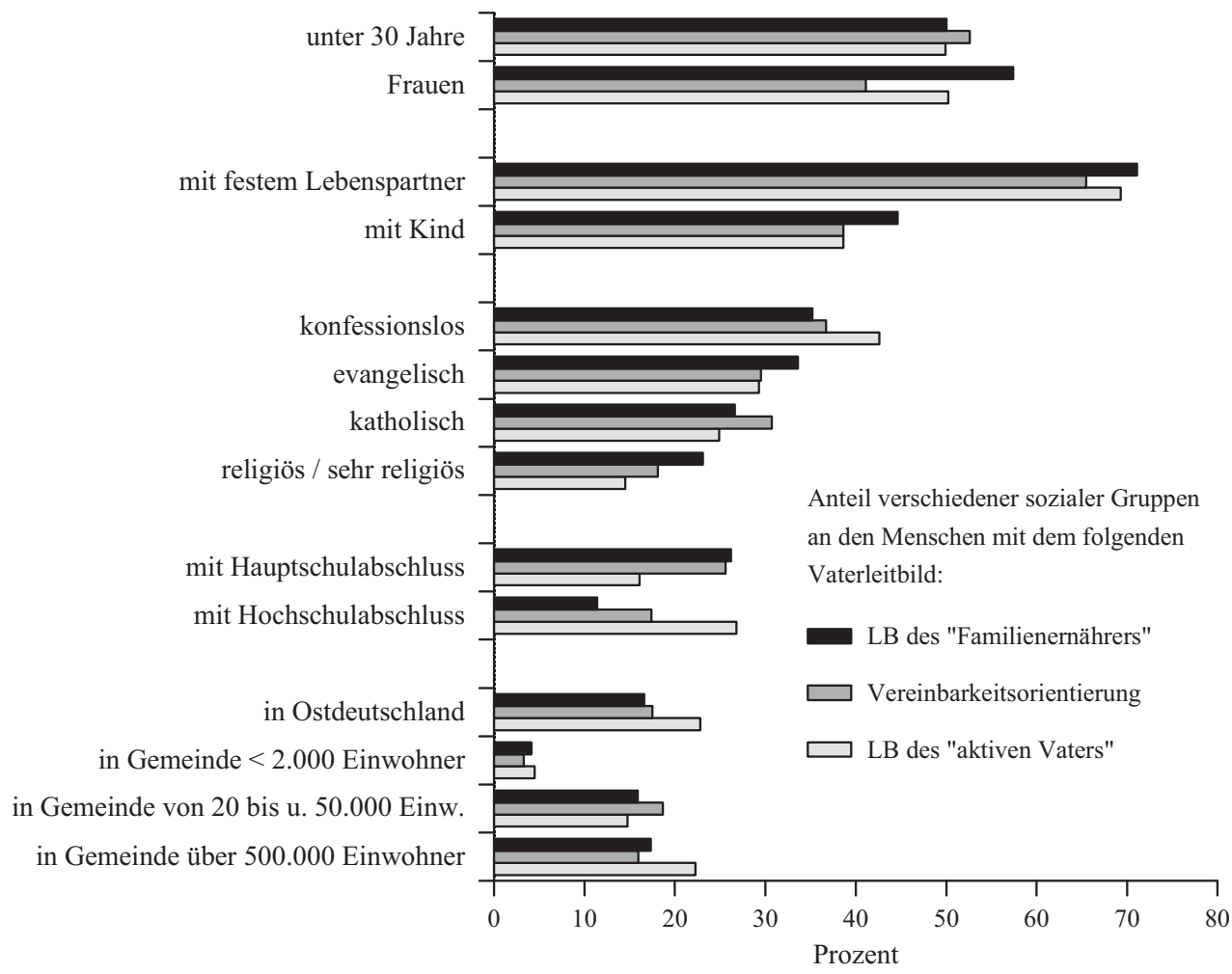

Quelle: FLB (2012), gewichtete Daten, eigene Berechnungen

Anm.: Unter den Konfessionen, den höchsten Bildungsabschlüssen sowie unter den Gemeindegrößenklassen sind lediglich ausgewählte Kategorien dargestellt.

Lesebeispiel: „Von allen Personen mit dem Vaterleitbild des ,Familienernährers“ sind 50 \% jünger als 30 Jahre und 57 \% Frauen. 71 \% haben einen festen Lebenspartner, und 45\% sind Eltern eines Kindes.“ 
Ähnliche Analysen lassen sich auch für die gesellschaftliche Ebene oder anhand der Indikatoren zur Messung der einzelnen Leitbilder durchführen. Da diese aber wenig zusätzlichen Erkenntnisgewinn beisteuern, soll auf einer Präsentation dieser Analysen an dieser Stelle verzichtet werden.

Menschen, die sich ausschließlich am Leitbild des „Familienernährers “ orientieren, sind überdurchschnittlich häufig religiös oder sehr religiös. Unter den Konfessionen ist die evangelische überproportional vertreten. Auch Westdeutsche und Menschen mit niedrigem Bildungsniveau neigen eher zu diesem Vaterleitbild als Ostdeutsche und höher Gebildete. Hinter diesen Befunden lassen sich Einflüsse einer milieuspezifischen Sozialisation vermuten. Anders ist zu bewerten, dass Menschen mit dem Vaterleitbild des „Familienernährers" etwas überdurchschnittlich einen festen Lebenspartner und etwas häufiger Kinder haben. Hier könnte eine situationsspezifische Anpassung der eigenen Leitbilder an eine veränderte Lebensrealität zu beobachten sein. Denn auch faktisch verfallen Männer typischerweise stärker in die Rolle des Familienernährers, nachdem sie Vater geworden sind. Bemerkenswert ist ferner, dass besonders Frauen zu diesem Vaterleitbild neigen, was den Ergebnissen des Instituts für Demoskopie Allensbach (2013) tendenziell widerspricht. Auf diesen Befund soll später eingegangen werden.

In mancher Hinsicht spiegelbildlich gestaltet sich das Profil derjenigen, die das Leitbild des ,aktiven Vaters "verinnerlicht haben: Hier sind Konfessionslose und Akademiker überrepräsentiert. Ebenso Ostdeutsche sowie die Bewohner von Großstädten ab 500.000 Einwohnern. In der Neigung der Großstädter zu diesem Vaterleitbild könnten sich diverse Effekte überlagern: Es könnte sowohl einen spezifischen Sozialisationseffekt durch das urbane Milieu geben als auch einen Selektionseffekt derart, dass Menschen mit dem Bild eines ,, aktiven Vaters “ häufiger in die Großstädte ziehen. Ein Teil des Befundes erklärt sich dadurch, dass der Wohnort Großstadt mit anderen Merkmalen statistisch zusammenhängt, beispielsweise dem hohen Bildungsgrad oder der Kinderlosigkeit.

Beachtenswert ist die Gruppe derjenigen, die beide Vaterleitbilder verinnerlicht haben und sich vereinbarungsorientiert zeigen. Ihr Profil reiht sich keineswegs zwischen den übrigen beiden ein. Menschen mit einem vereinbarkeitsorientierten Vaterleitbild rekrutieren sich stärker aus den alten Bundesländern und aus der niedrigen Bildungsschicht ähnlich wie diejenigen mit einem „Familienernährer"-Leitbild. Sie sind überdurchschnittlich häufig katholisch. Und sie wohnen überproportional in mittleren Kleinstädten (zwischen 20.000 und 50.000 Einwohnern). Auch für dieses Vaterleitbild scheint es also spezifische „günstige“ soziale Milieus zu geben, in denen es eher vermittelt wird als in anderen. Dieses ähnelt dem Milieu, in dem das „konservativere“ Leitbild des Familienernährers typischerweise zu finden ist. Eine mögliche Deutung lautet: Es gibt es einen allgemeinen Wandel des Vaterleitbildes hin zum aktiven Familienarbeiter, der alle Teile der Gesellschaft erfasst hat. In den Teilen, in denen diese Entwicklung zuletzt angekommen ist, behält jedoch die Vorstellung, dass ein Vater seine Familie ernähren muss, vorläufig zusätzlich Gültigkeit.

Doch auch mit dem Leitbild des ,aktiven Vaters“ hat das vereinbarungsorientierte Vaterleitbild eine Gemeinsamkeit: Es scheint im Zuge einer Biografie unter Druck zu geraten, wenn Menschen eine Familie gründen und sich im Alltag eine relativ akzentuierte Rolle des Vaters als „Familienernährer“ einstellt, denn ähnlich wie unter Vertretern des Leitbilds des „,aktiven Vaters“ sind auch unter den Vereinbarkeitsorientierten Eltern unterproportional vertreten.

Ein weiteres Spezifikum der Vereinbarkeitsorientierten liegt darin, dass besonders Männer zu diesem Leitbild neigen. Anders als Frauen, die eine überproportionale Neigung zum Leitbild des „Familienernährers“ zeigen, ist es demnach für Männer charakteristisch, 
sich (zukünftig) zumindest auch als ,aktiven Vater“ zu sehen. Männer stimmen nicht seltener als Frauen dem Leitbild des „Familienernährers“ zu, sondern häufiger (zusätzlich) dem des ,aktiven Vaters“. Hier deutet sich an, dass der Anspruch, den Männer an sich selbst haben, höher ist als der, den Frauen an Männer richten.

Abbildung 4: Orientierung an persönlichen Vaterleitbildern in unterschiedlichen Teilen der Gesellschaft

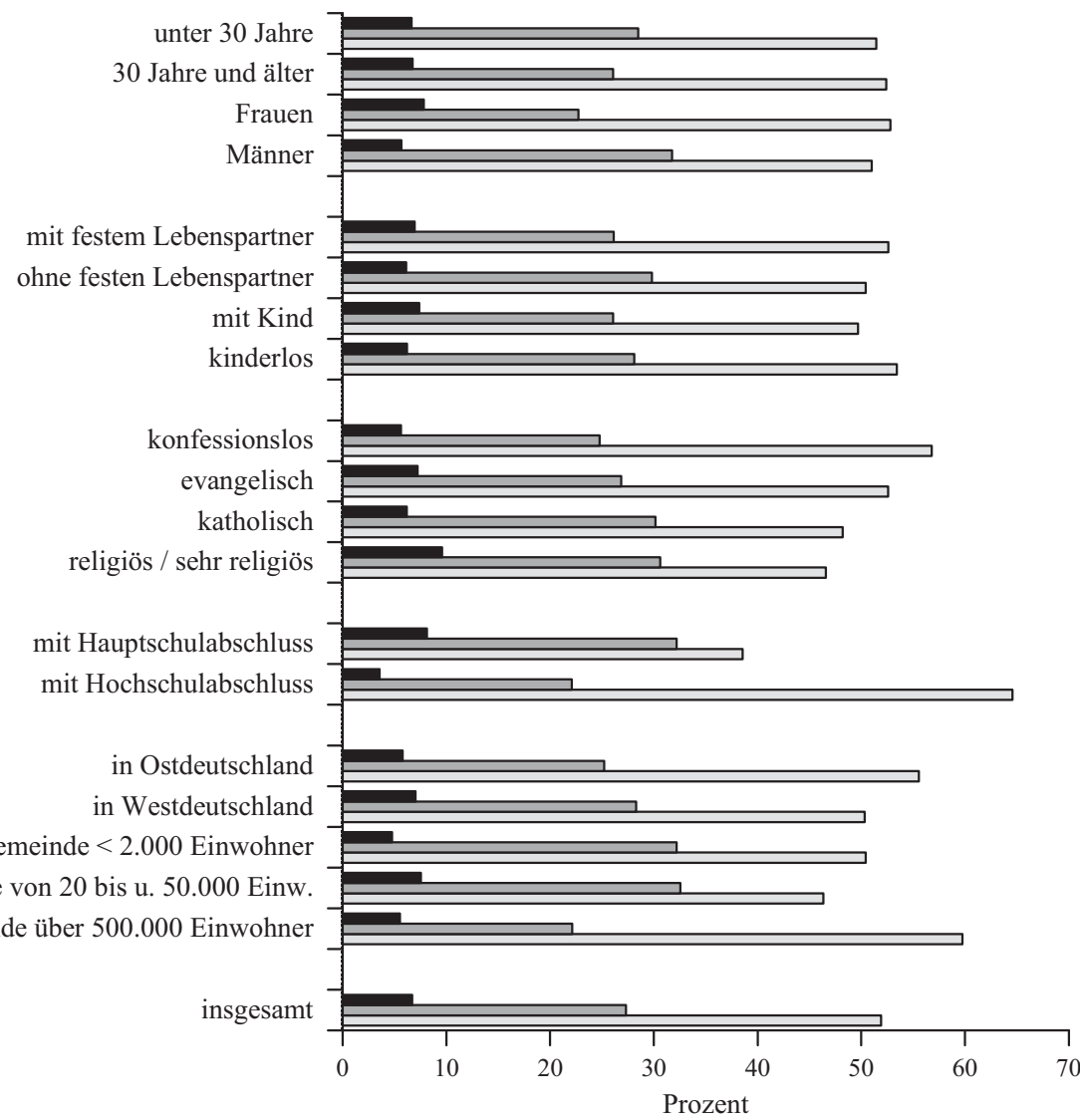

in Gemeinde von 20 bis u. 50.000 Einw.

in Gemeinde über 500.000 Einwohne

nsgesamt

LB des "Familienernährers"

Vereinbarkeitsorientierung

LB des "aktiven Vaters"

Quelle: FLB (2012), gewichtete Daten, eigene Berechnungen

Anm.: Unter den Konfessionen, den höchsten Bildungsabschlüssen sowie unter den Gemeindegrößenklassen sind lediglich ausgewählte Kategorien dargestellt.

Lesebeispiel: „Von allen Personen unter 30 Jahre haben $7 \%$ das Vaterleitbild des ,Familienernährers', unter allen, die 30 oder älter sind, ebenso viele. Unter den Frauen haben 8 \% das Vaterleitbild des ,Familienernährers', unter den Männern sind dies $6 \%$.“

Bemerkenswert ist auch die Interaktion von Geschlecht und Elternschaft (nicht in den Abbildungen 3 und 4 dargestellt). Kinderlose Frauen, kinderlose Männer, Mütter und Väter unterscheiden sich in ihrer Orientierung an Väterleitbildern. Dabei sind zum einen die 
Geschlechterunterschiede in der Ausgangssituation der Kinderlosen von Interesse, die sowohl auf geschlechtsspezifische Sozialisationseinflüsse als auch auf unterschiedliche Interessen in der Planung der Familienbiografie zurückzuführen sein können. Zum anderen müssen die geschlechtsspezifischen Reaktionen auf die Familiengründung beachtet werden. Die Ausgangssituation der Kinderlosen zeichnet sich, wie bereits dargestellt, vor allem dadurch aus, dass Männer stärker zu einem vereinbarkeitsorientierten Leitbild neigen (33,5\% gegenüber $20,8 \%$ bei kinderlosen Frauen), während Frauen sowohl unter den Befürwortern des „Familienernährers" als auch unter denen des „, aktiven Vaters “ überproportional vertreten sind. Mit der Familiengründung vollzieht sich bei Frauen eine Verlagerung weg vom Leitbild des ,aktiven Vaters“, und bei Männern weg vom vereinbarkeitsorientierten Leitbild, hin zu den jeweils übrigen Leitbildern. Die faktische Retraditionalisierung in der partnerschaftlichen Aufgabenteilung, die typischerweise mit der Familiengründung einhergeht, scheint also zu einer Desillusionierung zu führen: Bei Frauen wird die Vorstellung eines Vaters, der sich aktiv in die Familienarbeit einbringt und dafür beruflich kürzer tritt, von der Realität des Familienlebens eingeholt, bei Männern die Vorstellung, sie könnten „Familienernährer“ und ,aktiver Vater“ gleichzeitig sein.

Die Charakteristika der Profile zeichnen sich nur durch über- bzw. unterproportionale Häufigkeiten bestimmter sozialer Gruppen aus. In keinem Teil der Gesellschaft weicht die Rangfolge der Vaterleitbilder oder die Größenordnung ihrer Häufigkeit ab (vgl. Abbildung 4): Stets ist das Leitbild des ,,aktiven Vaters “ mit ungefähr $50 \%$ Zustimmung das am weitesten verbreitete und das des „Familienernährers“ mit unter $10 \%$ Zustimmung das seltenste.

Die Charakteristika lassen sich derart zusammenfassen, dass das Leitbild des ,,aktiven Vaters " besonders in Ostdeutschland, im akademischen Milieu sowie unter Konfessionslosen verbreitet ist. Das vereinbarkeitsorientierte Leitbild ist typischer für Männer, niedriger Gebildete und Katholiken. Das Leitbild des „Familienernährers “ ist charakteristisch für Frauen, niedriger Gebildete, Protestanten und religiöse Menschen. Mit der Familienplanung und der Familiengründung - sowie mit den faktischen Verschiebungen der partnerschaftlichen Arbeitsteilung, die damit häufig einhergehen - nimmt auch das „Familienernährer"-Leitbild an Bedeutung zu, wenn auch auf sehr niedrigem Niveau.

\section{$7 \quad$ Fazit}

In der Forschungsliteratur ist einerseits ein allgemeiner Entwicklungstrend vom Bild des Vaters als Familienernährer zu dem des „,neuen Vaters“ zu erkennen, der sich Haus-, Familien- und Erwerbsarbeit gleichberechtigt mit seiner Partnerin aufteilt. Andererseits gibt es zahlreiche Hinweise auf Verunsicherung, innere Widersprüche und „Kompromisse“ bei der subjektiven Definition dessen, was einen guten Vater ausmacht. Beides zeigt sich auch in den Daten des FLB 2012.

Einerseits lassen sich zwei Leitbilder der Vaterschaft identifizieren - was angesichts der vorhandenen Ambivalenzen nicht selbstverständlich ist. Sie markieren den Anfangsund den Endpunkt des allgemeinen Entwicklungstrends: das Leitbild des „Familienernährers " und das des ,,aktiven Vaters". Andererseits haben Menschen nicht unbedingt (nur) eines dieser beiden Leitbilder der Vaterschaft verinnerlicht, sondern unter Umständen auch keines von beiden oder (noch häufiger) beide. Es gibt also bei vielen Menschen die Vorstellung, ein Vater sollte idealerweise sowohl seine Familie mit seinem Erwerbs- 
einkommen ökonomisch absichern als auch im Haushalt helfen und aktiv bei der Betreuung und Erziehung der Kinder beteiligt sein. Allerdings macht die Gruppe der Vereinbarkeitsorientierten nur etwa ein Viertel der 20- bis 39-Jährigen aus.

Widersprüche zeigen sich vor allem auch in dem Kontrast zwischen persönlichen und mutmaßlichen gesellschaftlichen Leitbildern. Während auf persönlicher Ebene das Leitbild des ,aktiven Vaters" deutlich weiter verbreitet ist, dominiert auf gesellschaftlicher Ebene das des ,Familienernährers“. Obwohl sich also die Menschen in ihren subjektiven Vorstellungen dessen, was ein guter Vater tun sollte, heute stark an einer gemeinsamen partnerschaftlichen Zuständigkeit für Beruf und Familie orientieren, existiert das Bild des exklusiv auf die Erwerbsarbeit fokussierten bürgerlichen Familienvaters noch deutlich in ihren Köpfen. Das Bild mag negativ gesehen werden, aber es ist deutlicher ausgeprägt als das des „aktiven Vaters“. Zudem wird vom Leitbild des „Familienernährers" angenommen, dass eine Mehrheit der Gesellschaft hinter ihm stünde, weshalb es sehr wahrscheinlich auch in hohem Maße handlungsrelevant ist - vergleichbar einer Geschwindigkeitsbegrenzung für einen Autofahrer, die zwar aufgehoben ist, deren Aufhebung der Autofahrer aber übersehen hat.

Die Leitbilder der Vaterschaft sind grundsätzlich in ganz Deutschland in ähnlicher Weise anzutreffen. Sie ziehen sich durch alle sozialen Schichten und Milieus. Doch es gibt graduelle Unterschiede in ihrer Verbreitung. Teilweise deuten diese darauf hin, dass bestimmte Leitbilder in bestimmten sozialen Milieus eine größere Chance haben, auf dem Weg der Sozialisation weitergegeben zu werden. Das gilt etwa für Unterschiede zwischen Konfessionen, Bildungsschichten oder Regionen. Teilweise lassen die Unterschiede erahnen, dass Leitbilder zuweilen aufgrund prägender Erfahrungen im Lebenslauf individuell angepasst werden, wenn sie sich als unrealistisch erweisen. Für das Leitbild der Vaterschaft ist dabei die Erfahrung der Familiengründung maßgeblich.

\section{Literatur}

Beck, Ulrich; Beck-Gernsheim, Elisabeth (1990): Das ganz normale Chaos der Liebe. Frankfurt a. M.: Suhrkamp.

Daniels, Justus von (2014): Ich habe es versucht. In: DIE ZEIT, 27/2014 sowie ZEIT-Online vom 11.07.2014. [http://www.zeit.de/2014/27/erfahrungsbericht-vater-teilzeit, 07.10.2014]

Durkheim, Émile (1984): Die Regeln der soziologischen Methode. Frankfurt a. M.: Suhrkamp.

Eckhard, Jan; Klein, Thomas (2006): Männer, Kinderwunsch und generatives Verhalten. Eine Auswertung des Familiensurvey zu Geschlechterunterschieden in der Motivation zur Elternschaft. Wiesbaden: VS Verlag für Sozialwissenschaften.

Fthenakis, Wassilios E.; Minsel, Beate (2002): Die Rolle des Vaters in der Familie. Schriftenreihe des Bundesministeriums für Familie, Senioren, Frauen und Jugend, Band 213. Stuttgart: Kohlhammer.

Goldstein, Joshua; Kreyenfeld, Michaela; Huinink, Johannes; Konietzka, Dirk; Trappe, Heike (2010): Familie und Partnerschaft in Ost- und Westdeutschland. Ergebnisse im Rahmen des Projektes „demographic Differences in Life Course Dynamics in Eastern and Western Germany“. Rostock: Max-Planck-Institut für Demografische Forschung.

Grunow, Daniela (2007): Wandel der Geschlechterrollen und Väterhandeln im Alltag. In: Mühling, Tanja; Rost, Harald (Hrsg.): Väter im Blickpunkt. Perspektiven der Familienforschung. Opladen: Verlag Barbara Budrich, S. 49-76.

Hofäcker, Dirk (2007): Gut gemeint ist noch lange nicht getan. Eine international vergleichende Analyse zur partnerschaftlichen Arbeitsteilung im Haushalt. In: Informationsdienst Soziale Indikatoren 37, S. 12-15. 
Huinink, Johannes; Reichart, Elisabeth (2008): Der Weg in die traditionelle Arbeitsteilung - eine Einbahnstraße? In: Bien, Walter; Marbach, Jan H. (Hrsg.): Familiale Beziehungen, Familienalltag und soziale Netzwerke. Ergebnisse der drei Wellen des Familiensurvey. Wiesbaden: VS Verlag für Sozialwissenschaften, S. 43-79.

Institut für Demoskopie Allensbach (2013): Der Mann 2013. Arbeits- und Lebenswelten - Wunsch und Wirklichkeit. Herausgegeben durch Bild der Frau. Hamburg: Axel Springer AG.

Junck, Sara; Lück, Detlev (2015): Die Älteren setzen den Standard. Untersuchung von Altersunterschieden in persönlichen Leitbildern als Ansatz zur Erklärung von Diskrepanzen zwischen den vorherrschenden gesellschaftlichen und den vorherrschenden persönlichen Leitbildern in der Studie „Familienleitbilder“ (FLB 2012). Wiesbaden: Bundesinstitut für Bevölkerungsforschung. [http://www.bib-demografie.de/leitbild]

Lück, Detlev (2009): Der zögernde Abschied vom Patriarchat. Der Wandel von Geschlechterrollen im internationalen Vergleich. Berlin: edition sigma.

Merkle, Tanja; Wippermann, Carsten (2008): Eltern unter Druck. Selbstverständnisse, Befindlichkeiten und Bedürfnisse von Eltern in verschiedenen Lebenswelten. Eine sozialwissenschaftliche Untersuchung von Sinus Sociovision GmbH im Auftrag der Konrad-Adenauer-Stiftung e.V. Stuttgart: Lucius \& Lucius.

Nave-Herz, Rosemarie (1988): Zum Wandel der Vaterrolle. In: Zeitschrift für Sozialisationsforschung und Erziehungssoziologie, Jg. 8 (4), S. 242-245.

Noelle-Neumann, Elisabeth (1980): Die Schweigespirale. Öffentliche Meinung - unsere soziale Haut. München: Langen Müller.

Oberndorfer, Rotraut; Rost, Harald (2005): Neue Väter - Anspruch und Realität. In: Zeitschrift für Familienforschung 17 (1), S. 50-65.

Parsons, Talcott (1973 orig. 1954): Beiträge zur soziologischen Theorie. Herausgegeben von Rüschemeyer, Dietrich. Neuwied: Luchterhand, S. 109-122.

Peitz, Gabriele (2006): Vaterbilder - Erwartungen und Vorstellungen von der Vaterrolle. In: Fthenakis, Wassilios E. (Hrsg.): Facetten der Vaterschaft - Perspektiven einer innovativen Väterpolitik. Herausgegeben vom Bundesministerium für Familie, Senioren, Frauen und Jugend. Berlin: BMFSFJ, S. 29-38.

Pollmann-Schult, Matthias (2012): Das Erwerbsverhalten von Vätern in Ost- und Westdeutschland. In: Huinink, Johannes; Kreyenfeld, Michaela; Trappe, Heike (Hrsg.): Familie und Partnerschaft in Ost- und Westdeutschland. Ähnlich und doch immer noch anders. Zeitschrift für Familienforschung, Sonderheft 9, S. 79-93.

Rollet, Brigitta; Werneck, Harald (2002): Die Vaterrolle in der Kultur der Gegenwart und die väterliche Rollenentwicklung in der Familie. In: Walter, Heinz (Hrsg.): Männer als Väter. Sozialwissenschaftliche Theorie und Empirie. Gießen: Psychosozial-Verlag, S. 323-343.

Rost, Harald; Schneider, Norbert F. (1995): Differentielle Elternschaft. Auswirkungen der ersten Geburt für Männer und Frauen. In: Nauck, Bernhard; Onnen-Isemann, Corinna (Hrsg.): Familie im Brennpunkt von Wissenschaft und Forschung. Neuwied: Luchterhand, S. 177-194.

Ruckdeschel, Kerstin (2007): Der Kinderwunsch von Kinderlosen. In: Zeitschrift für Familienforschung, 19 (2), S. 210-230.

Schneider, Werner (1989): Die neuen Väter - Chancen und Risiken. Augsburg: AV-Verlag.

Schulz, Florian; Blossfeld, Hans-Peter (2006): Wie verändert sich die häusliche Arbeitsteilung im Eheverlauf? Eine Längsschnittstudie der ersten 14 Ehejahre in Westdeutschland. In: Kölner Zeitschrift für Soziologie und Sozialpsychologie, 58 (1), S. 23-49.

Schweizer, Herbert (1986): Strukturwandel der Vaterrolle in der häuslichen Gruppe: „neue Väter"? In: Gabriel, Karl; Sauer, Paul Ludwig; Vieth, Willi (Hrsg.): Sinnfragen sozialer Arbeit. Hildesheim: Bernward, S. 91-113.

Statistisches Bundesamt (2008): Elterngeld - Eine erste Bilanz. STATmagazin. Wiesbaden.

Statistisches Bundesamt (2014): Öffentliche Sozialleistungen. Statistik zum Elterngeld. Beendete Leistungsbezüge für im 4. Vierteljahr 2012 geborene Kinder. Oktober 2012 bis März 2014. Wiesbaden.

Vaskovics, Laszlo A.; Rost, Harald (1999): Väter und Erziehungsurlaub. Schriftenreihe des Bundesministeriums für Familie, Senioren, Frauen und Jugend, Nr. 179. Stuttgart: Kohlhammer. 
Volz, Rainer; Zulehner, Paul M. (2009): Männer in Bewegung. Zehn Jahre Männerentwicklung in Deutschland. Forschungsreihe des Bundesministeriums für Familie, Senioren, Frauen und Jugend, Band 6. Baden-Baden: Nomos.

Wippermann, Carsten (2013): Jungen und Männer im Spagat: Zwischen Rollenbildern und Alltagspraxis. Eine sozialwissenschaftliche Untersuchung zu Einstellungen und Verhalten. Herausgegeben vom Bundesministerium für Familie, Senioren, Frauen und Jugend. Berlin: BMFSFJ.

ZEIT-Magazin (2014): Der Mann von heute. Ausgabe 14.

Zerle, Claudia; Krok, Isabelle (2008): Null Bock auf Familie? Der schwierige Weg junger Männer in die Vaterschaft. Gütersloh: Verlag Bertelsmann-Stiftung. 



\title{
Leitbilder der Elternschaft: Zwischen Kindeswohl und fairer Aufgabenteilung
}

\author{
Sabine Diabaté, Detlev Lück \& Norbert F. Schneider ${ }^{*}$
}

\section{Zusammenfassung}

Die Vorstellungen, wie Elternschaft idealerweise gestaltet werden sollte, sind vielfältig. Mit den Daten der Familienleitbildstudie (FLB) konnten mittels Hauptkomponentenanalyse auf individueller wie auf gesellschaftlicher Ebene jeweils zwei Elternschaftsleitbilder identifiziert werden: Im Kern des einen Leitbildes steht die Vorstellung einer komplementären Geschlechterrollenteilung der Eltern (Leitbild der komplementären Elternrollen), verbunden mit der Ablehnung externer Kinderbetreuung. Das andere Leitbild ist durch reduziertes berufliches Engagement des Vaters bei hohen Ansprüchen an eine verantwortete Elternschaft gekennzeichnet (Leitbild der kindzentrierten Erziehungsarbeit). Während die Verbreitung der Elternschaftsleitbilder auf individueller Ebene sozialstrukturell durchaus variiert, bestehen bei den gesellschaftlich wahrgenommenen Leitbildern diese Unterschiede nicht.

Sowohl im Hinblick auf individuelle wie auf gesellschaftliche Leitbilder ist das auf einer stärkeren Väterbeteiligung in der Erziehung basierende Leitbild weiter verbreitet. Festzustellen ist jedoch auch, dass die Elternschaftsleitbilder ambivalent und widersprüchlich sind. Rund die Hälfte der 20- bis 39-Jährigen stimmt entweder beiden oder keinem der beiden Leitbilder zu.

Beim Abgleich zwischen Leitbild und faktischer Lebensrealität zeichnet sich eine Tendenz ab, dass Menschen Leitbild und reale Ausgestaltung der Elternschaft in Einklang bringen. Allerdings lebt auch ein relevanter Teil nicht konform mit dem eigenen Leitbild.

\section{$1 \quad$ Einleitung}

Trotz der in den letzten Jahrzehnten rasch gestiegenen Verbreitung dauerhafter Kinderlosigkeit wünschen sich die meisten Menschen weiterhin Kinder, und eine große Mehrheit bekommt sie auch. Mit der Familiengründung kommt auf die jungen Eltern ein grundlegend neuer Lebensabschnitt mit vielen zusätzlichen Herausforderungen und Aufgaben zu. Das war schon immer so. Neu ist, dass sich Eltern in Deutschland heute häufig einem starken Druck ausgesetzt fühlen, in der Erziehung und im Beruf alles richtig zu machen.

„Eltern stehen heute unter enormem Druck, sowohl hinsichtlich ihrer subjektiven Befindlichkeit als auch hinsichtlich der objektiv gestiegenen Anforderungen. (...) Familiäre Werte stehen dem Leitbild der wettbewerbsorientierten Wirtschaft entgegen, in der sich jeder Einzelne als Wettbewerbsteilnehmer begreifen muss.“ (Henry-Huthmacher 2008: 23)

Für die wertvollen Anmerkungen und die hilfreiche Kritik möchten wir unserer anonymen Gutachterin bzw. unserem anonymen Gutachter danken. 
Daher sind viele Eltern in Deutschland im Erziehungsalltag gestresst und erleben ihre Kinder häufiger als Belastung als in anderen Ländern (Quaiser-Pohl 2001: 301). Sowohl der hohe Anteil dauerhaft Kinderloser hierzulande als auch die (in Westdeutschland) vergleichsweise niedrige Vollzeiterwerbsquote von Müttern signalisieren, dass es gerade in Deutschland schwer ist, Beruf und Familie erfolgreich zu vereinbaren. Die Begründung dafür wird häufig in den besonderen ökonomischen Belastungen gesehen, die jedoch auch in vielen anderen Ländern bestehen, oder in der unzureichenden Infrastruktur, die die Vereinbarkeit von Familie und Beruf erschwert. Eine alternative oder zusätzliche Erklärung zielt auf die gesellschaftlichen Erwartungen ab, die an junge Eltern gerichtet werden, und auf die subjektiven Leitvorstellungen an gelingende Elternschaft. Wie aber sehen kulturelle Leitbilder der Elternschaft in Deutschland aus?

Die primäre Zielsetzung dieses Beitrages ist es, Leitbilder der Elternschaft zu identifizieren und zu beschreiben. Dabei sollen die in diesem Band zuvor behandelten Leitbilder der Mutter- und der Vaterschaft, der verantworteten Elternschaft sowie der Kinderbetreuung $^{1}$ zu einem übergeordneten Sinnzusammenhang auf Elternebene verbunden werden.

Unter Elternschaftsleitbildern verstehen wir einen weit gefassten Komplex an Bezügen zwischen den Eltern (leibliche und soziale) und den Kindern. Elternschaft adressiert die Beziehungen zwischen den Eltern sowie zwischen den Eltern und den Kindern. Elternschaft umfasst aber auch ihre Einbettung in den sozialen Kontext, dazu zählt die Beschaffenheit sozialer Netzwerke ebenso wie die strukturellen Gegebenheiten, etwa bzgl. externer Betreuungseinrichtungen, Schule oder Arbeitswelt. In diesem Geflecht aus sozialen Bezügen und subjektiven Ansprüchen findet Elternschaft statt. Sie hat dabei neben ihren „natürlichen“ Grundlagen immer auch eine gesellschaftliche Komponente. Elternschaft ist Gegenstand fortwährender sozialer Konstruktionsprozesse und unterliegt darüber hinaus auch dem sozialen Wandel (Schneider 2002).

Basierend auf der Entwicklung der faktischen Gestaltung von Elternschaft und der Aufgabenteilung zwischen Müttern und Vätern sowie auf den Befunden der vorangegangenen Kapitel lässt sich erwarten, dass Elternschaft als eine höchst voraussetzungsvolle (Lebens-)Aufgabe gilt, die im Wesentlichen durch drei Varianten elterlicher Aufgabenteilung erfüllt werden kann:

1. Beide Eltern sorgen in gleicher Weise für Kind und Familieneinkommen; dazu sind sie auf externe Kinderbetreuung angewiesen.

2. Eltern verteilen Haus-, Erziehungs- und Erwerbsarbeit komplementär; ein Partner ist Alleinverdiener, der andere für Haushalt und Kindererziehung zuständig; externe Kinderbetreuung ist verzichtbar, wenn sie nicht gar als unverantwortbar gilt.

3. Eltern verteilen die Zuständigkeiten der Tendenz nach komplementär, wobei meist die Mutter durch zeitlich begrenzte Erwerbsarbeit hinzuverdient und der Vater sie (insbesondere am Wochenende) bei der Erziehungsarbeit entlastet. Auch hier ist die Fremdbetreuung von kleinen Kindern ein möglicher Bestandteil des Modells.

Bei diesen drei Varianten handelt es sich nicht nur um Modelle der Alltagsgestaltung, sie haben gleichzeitig auch den Charakter von leitbildhaften Orientierungsrahmen, wie es Forschungsergebnisse aus der Perspektive von Müttern nahelegen (Pfau-Effinger 2004: 383).

$1 \quad$ Verantwortete Elternschaft und Kinderbetreuung bei Ruckdeschel (Kap. 12), Mutterleitbilder bei Diabaté (Kap. 13), Vaterleitbilder bei Lück (Kap. 14). 
Dieser Beitrag adressiert mehrere Fragestellungen:

1. Sind individuelle Elternschaftsleitbilder nachweisbar? (Abschnitt 3.1)

2. Wie sind die verschiedenen Normalitätsvorstellungen zur Elternschaft verbreitet? (Abschnitt 3.1.1)

3. Unterscheidet sich ihr Verbreitungsgrad nach sozialstrukturellen Merkmalen? (Abschnitte 3.1.2 und 3.1.3)

4. Lassen sich gesellschaftliche Elternschaftsleitbilder identifizieren? Wie viele und welche wahrgenommene Elternschaftsleitbilder gibt es, und welche Normalitätsvorstellungen fassen sie jeweils zusammen? (Abschnitt 3.2)

5. Wie sind sie in unterschiedlichen sozialstrukturellen Gruppen verteilt? (Abschnitt 3.2.1)

6. Inwieweit korrespondieren individuelle und gesellschaftlich wahrgenommene Elternschaftsleitbilder miteinander? (Abschnitt 3.2.1)

7. Stimmen individuelle Leitbilder mit der faktisch gelebten Alltagsrealität überein? (Abschnitt 3.3).

\section{Theoretische Grundlagen, Stand der Forschung und methodisches Vorgehen}

Die Vorstellung, wie Elternschaft idealerweise gestaltet werden kann, hat sich in den vergangenen Jahrzehnten auf zwei Aspekte fokussiert: Beim ersten Aspekt steht die Wahrung und Förderung des Kindeswohls als Aufgabe der Eltern im Mittelpunkt. Dazu zählt die Idee, dass das Kind als schwach und besonders schützenswert gilt und einer umfassenden Förderung und Begleitung bedarf. Demnach soll möglichst viel in Kinder und ihre Entwicklung investiert werden. Zudem wird erwartet, dass Eltern ihre Bedürfnisse hinter die des Kindes zurückstellen (Schneider et al. 2014; Ruckdeschel, Kap. 12). Kinder und Kindheit werden mythologisiert und zum „Projekt Zukunft” stilisiert (Honig 1992). Im Zuge dieses Prozesses sehen sich Eltern mit erhöhten Erwartungen an ihre Professionalität als Erzieher und mit neuen Normen konfrontiert.

„Verantwortete Elternschaft und kindgerechte Erziehung sind Normkomplexe, die Elternschaft zu einer zunehmend schwieriger zu bewältigenden Gestaltungsaufgabe machen." (Schneider et al. 2014: 17)

Der zweite Aspekt der optimalen Gestaltung von Elternschaft ist darauf gerichtet, wie eine als fair wahrgenommene Arbeitsteilung zwischen den Elternteilen auszusehen hat, wobei Fairness (equity) eine subjektiv als gerecht eingeschätzte Aufgabenteilung meint. Dabei ist es nicht bedeutsam, ob sie faktisch betrachtet, d. h. anhand objektiver Kriterien, zwischen den Elternteilen gleich verteilt ist.

In der Familienforschung ist es Tradition, die elterliche Lebenswelt hinsichtlich ihres Funktionierens und ihrer Ausgestaltung zu beleuchten (z. B. Berk 1985; Goffman 1977; Stafford et al. 1977). Dabei hat vor allem die erhöhte Erwerbsbeteiligung von Frauen seit den fünfziger Jahren eine Neuverteilung von Familienarbeit nötig gemacht (Stauder 2002: 15). Bei der Erklärung der Ausgestaltung elterlicher Arbeitsteilung hat ein Paradigmenwechsel stattgefunden: Waren zu Beginn vor allem rollentheoretische Überlegungen und somit gesellschaftlich vordefinierte Positionen bedeutsam (Dahrendorf 1965), verlagerte sich die Argumentation später auf Verhandlungs- und Ressourcentheorien (Ott 1998; Ross 1987; Coverman 1985): Die Gestaltung der Arbeitsteilung erschien als offener, verhandelbarer Prozess, in dem rationale Argumente ausschlaggebend sind (Becker 1993). Daneben 
spielt vor allem auch der Equity-Ansatz eine bedeutende Rolle zur Erklärung der elterlichen Aufgabenteilung (Walster et al. 1978): Demnach ist die Verteilung der Zuständigkeiten auch eine moralische Frage der Gerechtigkeit und der Akzeptanz durch die Beteiligten.

Wenngleich Beziehungen bis vor wenigen Jahrzehnten noch mit einer gewissen Selbstverständlichkeit eher patriarchalisch organisiert waren, überwiegt heute das Beziehungsideal der Gleichberechtigung, in dem die Aufgaben partnerschaftlich ausgehandelt und einvernehmlich gelöst werden. Dabei müssen die Bedürfnisse beider Partner in Einklang gebracht werden. Heute werden anstelle von „Befehlshaushalten“ „Verhandlungshaushalte" ${ }^{\text {"2 }}$ angestrebt (du Bois-Reymond 1994; de Swaan 1982: 50f.). Ebenso hat sich die Idealvorstellung der Arbeitsteilung innerhalb der Partnerschaft verschoben: Ein komplementäres Lebensmodell, in dem die Haus- und Erziehungsarbeit von der Mutter und die Erwerbsarbeit vom Vater übernommen wird, galt früher als selbstverständlich. Heute überwiegt das Bedürfnis nach egalitärer Aufteilung, nicht zuletzt durch die Bildungs- und Modernisierungsgewinne der Frauen in den vergangenen vier Jahrzehnten. Junge Frauen sind heute weniger dazu bereit, eigene berufliche Lebensziele, das Anbringen der eigenen Fachkompetenz, ökonomische Unabhängigkeit, beruflichen Aufstieg und den damit verbundenen sozialen Status zugunsten von Partnerschaft, Familienplanung oder Familie zurückzustellen (Allmendinger 2008; Beck-Gernsheim 1992, 2008). Auch Männer artikulieren zunehmend öfter, dass sie eine gleiche Aufteilung von Haus-, Familien- und Erwerbsarbeit für richtig halten und mehr Zeit mit dem eigenen Kind verbringen wollen (Oberndorfer/ Rost 2005).

Empirische Befunde zeigen, dass in der Lebensrealität das Komplementärmodell noch vielfach dominiert und sich bei Elternpaaren nach der Familiengründung meist wieder komplementäre Formen der Aufgabenteilung stabilisieren (Henry-Huthmacher/Borchard 2008; Grunow et al. 2007; Schulz/Blossfeld 2006). Dennoch könnte angenommen werden, dass sich zumindest auf kulturell-normativer Ebene ein egalitäres Elternschaftsleitbild etabliert hat und dass Menschen versuchen, daran ihre Lebensgestaltung auszurichten, wenn auch nicht immer mit Erfolg. Es ist jedoch unklar, inwiefern sich elterliche Leitbilder inhaltlich tatsächlich entwickelt haben - angesichts der stärkeren weiblichen Partizipation am Arbeitsmarkt, dem Wunsch nach Selbstverwirklichung (insbesondere für Frauen), der Maxime der verantworteten Elternschaft (Kind als oberste Priorität) und hinsichtlich der Forderung eines stärkeren väterlichen Engagements für die Erziehungsarbeit. Dabei ist zum einen ungeklärt, wie Normalitätsvorstellungen zur Elternschaft heutzutage auf der individuellen Ebene aussehen. Zum anderen ist unbekannt, welche Normalitätsvorstellungen innerhalb der Gesellschaft wahrgenommen werden.

Um die eingangs formulierten Ziele zu erreichen, werden Items aus der Studie „Familienleitbilder in Deutschland“" (FLB 2012) verwendet, die überwiegend bereits in anderen Beiträgen in diesem Band analysiert wurden: Items zu Mutterschaft (siehe Diabaté, Kap. 13), zu Vaterschaft (siehe Lück, Kap. 14) sowie zu Kinderbetreuung und zu verantworteter Elternschaft (siehe Ruckdeschel, Kap. 12). Allerdings werden in den Analysen diese Items erstmals gleichzeitig berücksichtigt und miteinander in Verbindung gesetzt. So können übergeordnete Leitbilder der Elternschaft sichtbar werden, die eine bestimmte Konstellation aus Mutter- und Vaterschaft, aus Ansprüchen an Eltern und Inanspruchnahme von Unterstützung durch externe Betreuung beschreiben. Das bedeutet konkret, dass alle Items, die die verschiedenen Facetten von Elternschaft im FLB (2012) messen, mittels einer Hauptkomponentenanalyse auf mögliche inhaltliche Dimensionen untersucht werden.

2 Zur empirischen Evidenz von familienökonomischen Verhandlungsmodellen in der „Verhandlungsfamilie“ siehe Althammer et al. (2010). 


\section{Ergebnisse}

Im Folgenden werden die Ergebnisse zu den eingangs gestellten Forschungsfragen dargestellt. Dabei werden zunächst die persönlichen (Abschnitt 3.1) und danach die allgemein wahrgenommenen Vorstellungen (Abschnitt 3.2) zur Elternschaft thematisiert. In Abschnitt 3.3 erfolgt der Abgleich von Elternschaftsleitbildern und Alltagsgestaltung.

\subsection{Persönliche Elternschaftsleitbilder}

Wie bereits dargestellt, lassen sich diverse Leitbilder der Mutter- und der Vaterschaft sowie der verantworteten Elternschaft identifizieren. Sind diese Leitbilder logisch konsistent aufeinander abgestimmt? Lassen sie sich zu übergeordneten Leitbildern der Elternschaft zusammenfassen - beispielsweise zu einem Leitbild der komplementären oder der egalitären Aufgabenteilung? Dies soll hier zunächst für individuelle Leitbilder getestet werden. Dafür werden sämtliche vier Items zur Mutterschaft ${ }^{3}$ (Verteilung der Variablen siehe Diabaté, Kap. 13) und zur Vaterschaft ${ }^{4}$ (siehe Lück, Kap. 14) zueinander in Beziehung gesetzt, um die Normalitätsvorstellungen zu beiden Elternteilen auf persönlicher Ebene abzubilden.

Eine erste Überprüfung durch eine Korrelationsmatrix ${ }^{5}$ macht deutlich, dass die einzelnen Normalitätsvorstellungen nicht stark, jedoch überwiegend hoch signifikant und stets in inhaltlich erwarteter Richtung miteinander korrelieren. Die Items hängen logisch widerspruchsfrei in einer Weise zusammen, wie es beim Vorhandensein übergeordneter Konzepte zu einer komplementären oder egalitären Aufgabenteilung zu erwarten wäre: Demnach korrelieren z. B. wie erwartet Items, die das Male-Breadwinner/Female-HomemakerModell umschreiben, miteinander. Das spricht zunächst für die Existenz solcher Elternschaftsleitbilder, die die Aufgaben von Mutter und Vater im Zusammenhang definieren.

Allerdings sind die Korrelationen weitaus moderater, als zu erwarten wäre, wenn man davon ausginge, dass die meisten Menschen vollständig widerspruchsfreie Leitbilder der Elternschaft haben. Vielleicht liegt das daran, dass vor allem jenseits der Vorstellungen einer komplementären Arbeitsteilung (die tendenziell die stärksten bivariaten Zusammenhänge aufweisen) alternative Modelle partnerschaftlicher Arbeitsteilung und elternschaftlicher Organisation nicht sehr stark durchreflektiert sind. Das erscheint insofern plausibel, als gerade egalitäre Modelle der Arbeitsteilung in der Alltagspraxis schwieriger zu realisieren und daher weniger stark präsent sind. Zudem sind sie als Leitbild relativ neu und hatten wenig Zeit, sich zu bewähren und zu etablieren. Bei der separaten Betrachtung von Eltern und von Kinderlosen variieren die Zusammenhänge zwar etwas, aber es ergibt sich in keiner der beiden Teilgruppen ein grundlegend anderes Bild.

In einem zweiten Schritt werden die Normalitätsvorstellungen zur Mutterschaft, Vaterschaft, verantworteten Elternschaft und Kinderbetreuung in einer Hauptkomponenten-

3 Pers.: Mütter sollten nachmittags Zeit haben, um ihren Kindern beim Lernen zu helfen. / Mütter sollten einem Beruf nachgehen, um unabhängig vom Mann zu sein. / Eine Mutter, die nur zuhause ist und sich um ihre Kinder kümmert, wird irgendwann unzufrieden. / Eine Mutter sollte, wenn möglich, überhaupt nicht erwerbstätig sein. $($ Pers. $=$ Was denkt der/die Befragte/r persönlich)

4 Pers.: Für ein Kind ist es nicht gut, wenn der Vater die Erziehung allein der Mutter überlässt. / Väter sollten für ihre Kinder beruflich kürzer treten. / Es liegt nicht in der Natur des Mannes, Hausmann zu sein. / Ein Mann muss seine Familie allein ernähren. (Pers. = Was denkt der/die Befragte/r persönlich)

5 Die Korrelationsmatrix ist auf Anfrage bei den Autoren erhältlich. Aus Gründen der Übersicht wurde auf eine grafische Darstellung verzichtet. 
analyse auf eine gemeinsame dimensionale Struktur hin untersucht. Dabei werden in der folgenden Analyse lediglich solche Items verwendet, die analog auch auf Ebene der Allgemeinheit gemessen wurden, um später einen von methodischen Einflüssen bereinigten Vergleich zwischen individuellen und gesellschaftlich wahrgenommenen Elternschaftsleitbildern vornehmen zu können.

In Tabelle 1 sind die Ergebnisse für die persönlichen Elternschaftsleitbilder dargestellt: Die erste inhaltliche Dimension (s. Komponente 1) zeigt eine Aufgabenteilung zwischen Vater und Mutter, die sich an dem Modell der männlichen Alleinverdiener-Ehe orientiert. Dabei wird außerdem jegliche Fremdbetreuung von Kindern in Krippen bzw. Kitas abgelehnt. Kinderbetreuung sollte demnach in der Familie stattfinden und allein durch die Mutter geleistet werden. Im Ergebnis entspricht dies einem Modell, wie es die bürgerliche Kernfamilie in den 1950er Jahren überwiegend gelebt hat. Die einzelnen Normalitätsvorstellungen im Kontext Mutterschaft, Vaterschaft und externe Kinderbetreuung lassen sich also tatsächlich nicht nur zu Mutter- oder Vaterleitbildern, sondern auch zu einem in sich stimmigen übergeordneten Leitbild der Elternschaft zusammensetzen. Das erste hier sichtbare Elternschaftsleitbild gibt klar komplementäre Elternrollen wieder.

Tabelle 1: Hauptkomponentenanalyse zur Identifikation von Elternschaftsleitbildern auf individueller Ebene

\begin{tabular}{|c|c|c|c|}
\hline & \multicolumn{3}{|c|}{ Komponente } \\
\hline & 1 & 2 & 3 \\
\hline Cronbach's Alpha $\alpha$ &, 55 &, 37 &, 25 \\
\hline Anteil erklärter Varianz (in Prozent) & 21,5 & 12,1 & 11,2 \\
\hline $\begin{array}{l}\text { Mütter sollten nachmittags Zeit haben, um ihren Kindern } \\
\text { beim Lernen zu helfen. }\end{array}$ & 649 & & \\
\hline Es liegt nicht in der Natur des Mannes, Hausmann zu sein. &, 594 & & \\
\hline $\begin{array}{l}\text { Für ein Kind zwischen } 1 \text { und } 3 \text { Jahren ist es das Beste, } \\
\text { wenn es nur von der Mutter betreut wird. }\end{array}$ &, 571 & & \\
\hline Ein Mann muss seine Familie allein ernähren können. &, 511 & & \\
\hline $\begin{array}{l}\text { Kinder zwischen } 1 \text { und } 3 \text { Jahren leiden, wenn sie } \\
\text { überwiegend in einer Kita oder Krippe betreut werden. }\end{array}$ &, 452 & & \\
\hline $\begin{array}{l}\text { Mütter sollten einem Beruf nachgehen, } \\
\text { um unabhängig vom Mann zu sein. }\end{array}$ & &,- 813 & \\
\hline $\begin{array}{l}\text { Eine Mutter sollte, wenn möglich, } \\
\text { überhaupt nicht erwerbstätig sein. }\end{array}$ & &, 542 & \\
\hline Väter sollten für ihre Kinder beruflich kürzer treten. & & & ,661 \\
\hline $\begin{array}{l}\text { Eltern sollten ihre eigenen Bedürfnisse für ihre } \\
\text { Kinder komplett zurückstellen. }\end{array}$ & & &, 584 \\
\hline $\begin{array}{l}\text { Eltern können bei der Erziehung vieles falsch machen, } \\
\text { daher müssen sie sich gut informieren. }\end{array}$ & & & ,582 \\
\hline
\end{tabular}

Quelle: FLB (2012), gewichtete Daten, eigene Berechnungen

Anm.: Varimax, rotierte Komponentenmatrix, Werte ab 0,4 werden angezeigt, insgesamt werden $45 \%$ Varianz erklärt. 
Eine zweite Dimension (s. Komponente 2) spiegelt im Wesentlichen ein Fragment des Leitbildes der Mutterschaft wider, das bereits im entsprechenden Beitrag identifiziert und dargestellt wurde (siehe Diabaté, Kap. 13): Es umschreibt das Leitbild der kindorientierten Mutter, die nach Möglichkeit nicht erwerbstätig sein sollte. Ihre Unabhängigkeit vom Partner wird abgelehnt. Bemerkenswert ist, dass dieser Aspekt der Organisation von Elternschaft nicht mit den übrigen Aspekten Teil des ersten vorgefundenen Leitbildes ist, obwohl ein Arrangement, in dem der Vater das Haushaltseinkommen bestreitet, die Mutter für die Kinderbetreuung allein verantwortlich ist und eine externe Betreuung abgelehnt wird, die Nichterwerbstätigkeit der Mutter beinahe logisch zwingend erfordert. Dennoch ist die Vorstellung, ob eine Mutter einem Beruf nachgehen sollte, von komplexeren Leitbildern der Elternschaft abgekoppelt. Dies könnte daran liegen, dass sich die Frage, ob eine Mutter einem Beruf nachgeht, in der Erfahrung der Menschen häufig auch nach ökonomischen Notwendigkeiten zu richten hat und losgelöst von den allgemeinen Organisationsprinzipien von Elternschaft entschieden werden muss. Außerdem spielt vermutlich auch die mediale Darstellung von Mutterschaft eine Rolle, die häufig unabhängig vom übrigen Kontext thematisiert wird.

In einem dritten Leitbild (s. Komponente 3) ist zum einen - ähnlich wie im von Lück (Kap. 14) vorgefundenen Vaterleitbild - die Vorstellung eines ,aktiven Vaters“ enthalten, der sich in seiner Erwerbsarbeit einschränkt, um gemeinsam mit seiner Partnerin an der Kindererziehung mitwirken zu können. Zudem laden die beiden Items zur „Verantworteten Elternschaft" auf derselben Dimension. Auch dieses Leitbild ist also eines, das Elternschaft in einem übergeordneten Zusammenhang beschreibt. Es deutet sich darin ein Elternschaftsleitbild der kindzentrierten Erziehungsarbeit an, das zum Leitbild der „komplementären Elternrollen“" in Konkurrenz tritt. Es betont die Wichtigkeit eines starken elterlichen Engagements für die Entwicklung ihrer Kinder und sieht beide Eltern (insbesondere den Vater) in der Pflicht, dieses Engagement zu leisten. Allerdings weist die Reliabilitätsanalyse lediglich ein Cronbach's Alpha von ,25 aus, d. h. die Vorstellungen, die dieses Leitbild ausmachen, sind vergleichsweise lose aneinander gebunden; das Leitbild ist weniger klar konturiert als die übrigen.

Im Folgenden werden das erste sowie das dritte Elternschaftsleitbild der „komplementären Elternrollen“" sowie der „kindzentrierten Erziehungsarbeit“ näher beschrieben. Auf eine Darstellung der zweiten Komponente, die Teile eines Mutterleitbildes abbildet, wird verzichtet; weitere Ausführungen dazu befinden sich bei Diabaté (Kap. 13).

\subsubsection{Verbreitung von individuellen Elternschaftsleitbildern}

Um die Verbreitung der verschiedenen Elternschaftsleitbilder auf individueller Ebene zu messen, wurde jeweils zunächst aus den Items, die zu einem Leitbild zusammengefasst sind, ein Summenindex gebildet. Dieser wurde anschließend durch die Anzahl der einbezogenen Variablen dividiert, so dass sich (genau wie bei jedem einzelnen Item) eine Spanne der Zahlenwerte von 1 (größte Zustimmung) bis 4 (starke Ablehnung) ergibt. Die Ausprägungen des Summenindexes wurden anschließend dichotomisiert. Werte von 1 bis 2,49 wurden als Zustimmung gewertet, Werte von 2,5 bis 4,0 als Ablehnung. Somit werden auch solche Antwortmuster als Zustimmung gezählt, bei denen einzelne Items abgelehnt wurden, so dass genau genommen von ,überwiegender Zustimmung“ gesprochen werden muss. Zum Vergleich wurden auch Indizes gebildet, die starke oder reine Zustimmung messen. Für das Elternschaftsleitbild der komplementären Elternrollen ergibt sich somit eine überwiegende Zustimmung von 31,0\% (N=1.527); eine deutliche Mehrheit von 69,0\% $(\mathrm{N}=3.398)$ lehnt dieses Leitbild ab. Zu diesem Befund ist festzuhalten, dass Zustimmung 
und Ablehnung jeweils moderat ausfallen; starke Zustimmung und Ablehnung kommen nur selten vor. Zu beobachten ist eine glockenförmige Verteilung um den Indexmittelwert, die inhaltlich so zu deuten ist, dass das Leitbild der „komplementären Elternrollen“ bei den meisten Menschen graduell abgelehnt oder bejaht wird und starke Positionierungen nur selten vorkommen.

Für das Elternschaftsleitbild der kindzentrierten Erziehungsarbeit ergibt sich eine überwiegende Zustimmung von 64,5\% (N=3.207) sowie eine überwiegende Ablehnung von $35,5 \%(\mathrm{~N}=1.762)$. Damit findet dieses Leitbild doppelt so häufig Zustimmung wie das der ,komplementären Elternrollen“. Ähnlich wie beim Leitbild der komplementären Elternrollen überwiegen auch hier mittlere Zustimmungswerte. Doch die graduell unterschiedlichen Haltungen neigen insgesamt eher ins Positive.

Betrachtet man Zustimmung und Ablehnung der beiden Elternschaftsleitbilder gleichzeitig auf einer zweidimensionalen Verteilung (Abbildung 1), so zeigt sich, dass nur knapp ein Zehntel (8,9\%) ausschließlich dem Leitbild der „komplementären Elternrollen“ und immerhin knapp die Hälfte (42,3 \%) ausschließlich dem der „,kindzentrierten Erziehungsarbeit" zustimmen.

Abbildung 1: Zustimmung und Ablehnung zu den individuellen Elternschaftsleitbildern „komplementäre Elternrollen“ und „kindzentrierte Erziehungsarbeit“" (in Prozent)

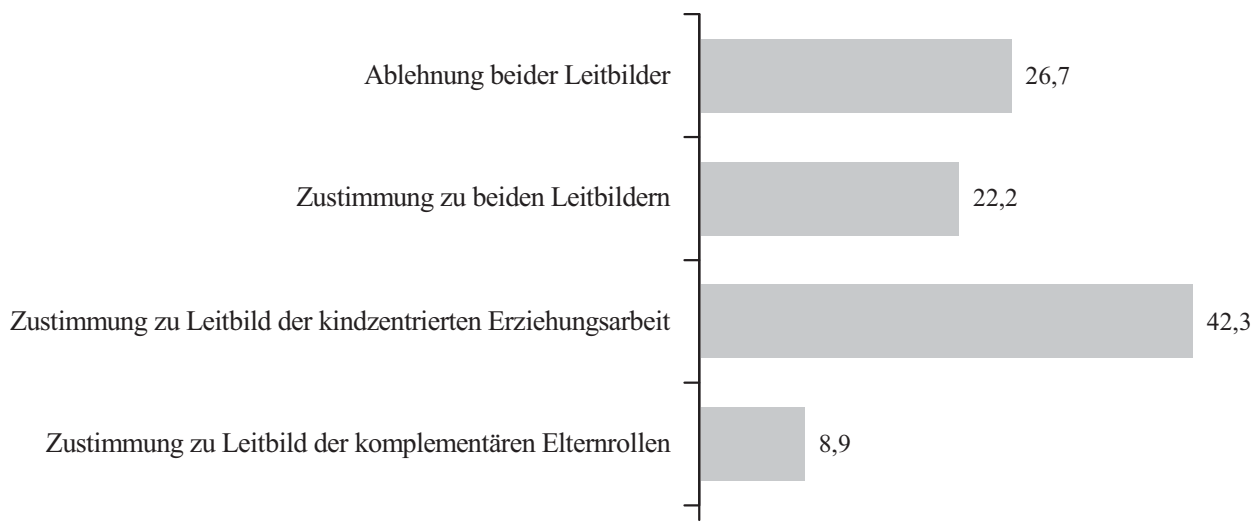

Quelle: FLB (2012), gewichtete Daten, eigene Berechnungen

Anm.: Die Anteilswerte sind auf eine Nachkommastelle gerundet, so dass die Summe der vier Werte $100,1 \%$ ergibt.

Das heißt, selbst wenn man vernachlässigt, dass es sich in beiden Fällen nur um überwiegende Zustimmungen und Ablehnungen handelt, die bereits für sich genommen keine starke Positionierung darstellen, weist nur etwa die Hälfte der Befragten (51,2 \%) konsistente Leitbilder zur Elternschaft auf. Dem gegenüber steht die andere Hälfte der Befragten: Innerhalb dieser weisen 22,2\% in dem Sinne widersprüchliche Leitbilder auf, als sie beiden Elternschaftsleitbildern zustimmen. Das bedeutet, dieser Personenkreis vereint in sich sowohl Normalitätsvorstellungen einer komplementären bürgerlichen Arbeitsteilung als auch Vorstellungen eines väterlichen Engagements in der Erziehungsarbeit, die miteinander konkurrieren. In dieser Widersprüchlichkeit und Inkonsistenz manifestiert sich möglicherweise Unsicherheit über eine für das Eltern- und Kindeswohl angemessene elterliche Aufgabenteilung. Möglicherweise zeigt sich darin auch der unrealistisch erscheinende und zur Selbstüberforderung neigende Anspruch, zwei Maßstäben gleichzeitig zu genügen, weil in 
der Priorisierung des komplementären Elternschaftsleitbildes eine Gewährleistung des Kindeswohl gesehen wird und in der Priorisierung des kindzentrierten Elternschaftsleitbildes eine Realisierung der elterlichen Bedürfnisse nach (finanzieller und persönlicher) Autonomie und Selbstentfaltung jenseits der häuslichen Sphäre. Eine noch etwas größere Gruppe $(26,7 \%)$ lehnt beide Elternschaftsleitbilder ab. Dies kann als ein Hinweis auf eine subjektiv wahrgenommene Überforderung verstanden werden oder als Indiz dafür, dass hinsichtlich der elterlichen Aufgabenteilung eine größere Gruppe der Befragten Schwierigkeiten hat, sich für eine eindeutige Aufgabenteilung in Richtung Egalität oder Komplementarität zu entscheiden und daher beides tendenziell ablehnt, vielleicht weil sie in beiden Modellen Probleme sehen.

\subsubsection{Differentielle Beschreibung des individuellen Elternschaftsleitbildes der komplementären Elternrollen nach sozialstrukturellen Merkmalen}

In Tabelle 2 wird zunächst die Verbreitung des Elternschaftsleitbildes der „komplementären Elternrollen“" differenziert nach sozialstrukturellen Merkmalen beschrieben. Dahinter steht die Annahme, dass unterschiedliche Leitbilder in unterschiedlichen Teilen der Gesellschaft stärker oder schwächer verbreitet sind. Bei der Interpretation der nachfolgenden Tabellen 2 und 3 ist zu beachten, dass sich hinter der Zusammenfassung der Zustimmung, wie oben beschrieben, unterschiedliche Grade an Zustimmung verbergen, unter denen die schwachen überwiegen. Eine differenzierte Auswertung mit starker oder reiner Zustimmung kommt hinsichtlich der Unterschiede zwischen verschiedenen sozialen Kategorien zu gleichen Befunden, weist jedoch wegen der allgemein niedrigen Zustimmungswerte weniger signifikante Unterschiede aus.

Insgesamt stimmen 31,0 \% dem Leitbild persönlich zu. Hinsichtlich des Geschlechts zeigt sich kein signifikanter Unterschied. Jeweils fast ein Drittel der befragten Frauen und Männer sieht es als leitbildhaft an, dass die Familien- und Erwerbsarbeit zwischen den Partnern komplementär aufgeteilt wird. Ebenfalls keine Unterschiede sind hinsichtlich des Alters erkennbar. Zunächst könnte man vermuten, dass daher ein rascher Wandel der Elternschaftsleitbilder entlang der Geburtskohorten nicht zu erwarten ist. Die Befragtengruppe ist insgesamt jedoch relativ jung, so dass eine Ausweitung der Befragtengruppe durch ältere Kohorten (40+) Unterschiede hervorbringen könnte.

Bei allen anderen Merkmalen zeigen sich dagegen signifikante Unterschiede: So stimmen deutlich mehr Westdeutsche (33,9\%) als Ostdeutsche (19,8\%) dem Elternschaftsleitbild der „komplementären Elternrollen“ zu. Dies ist nicht erstaunlich, da eine Beschränkung der Frau auf Familien- und Hausarbeit in Ostdeutschland kaum verbreitet war oder ist. Nicht nur zu DDR-Zeiten wurde hier das Selbstverständnis einer berufstätigen Mutter etabliert. Schon für die Zeit davor lassen sich entsprechende Mentalitätsdifferenzen zwischen Ost und West nachweisen (Schneider et al. 2012; Dorbritz/Ruckdeschel 2009), die sich in den Ansprüchen der Frauen durch die DDR-Ära hinweg bis heute fortschreiben. Laut Bauernschuster und Rainer (2012) divergieren die Geschlechterrollen sogar nach der Wiedervereinigung.

Entlang der Dimension des Bildungsniveaus wird deutlich, dass die Zustimmung zum Elternschaftsleitbild der „komplementären Elternrollen“ mit steigendem Bildungsniveau stark abnimmt. Während knapp die Hälfte der Menschen mit niedrigem Bildungsniveau (43,8 \%) zustimmt, tut dies nur ein Drittel der Befragten mit mittlerem Bildungsniveau (33,8\%) und ein Fünftel derjenigen mit hohem Bildungsniveau (20,6 \%). Da hoch gebildete Paare (vor allem Frauen) höhere Opportunitätskosten tragen, wenn sie auf ein Erwerbseinkommen verzichten, ist es plausibel, dass sie Zweiverdiener-Modelle bevorzugen. Sehr deutlich fällt 
auch der Unterschied zwischen Personen mit (42,1\%) und ohne Migrationshintergrund $(27,5 \%)$ aus. Diese Befunde lassen sich vermutlich zum Teil als kulturelle Milieuunterschiede interpretieren und zum Teil auf Unterschiede in der soziodemografischen Zusammensetzung der Populationen zurückführen.

Tabelle 2: Zustimmung zum individuellen Elternschaftsleitbild der „,komplementären Elternrollen“ nach sozialstrukturellen Merkmalen (in Prozent)

\begin{tabular}{llcr}
\hline Merkmal & Ausprägung & Überwiegende Zustimmung & $\mathrm{N}$ \\
\hline Geschlecht & Männlich & 29,3 & 2.190 \\
& Weiblich & 32,8 & 2.735 \\
Wohnort $^{\mathrm{a}}$ & Westdeutschland & $33,9^{* * *}$ & 3.848 \\
& Ostdeutschland & $19,8^{* * *}$ & 1.077 \\
Bildung $^{\mathrm{b}}$ & niedrig & $43,8^{* * *}$ & 200 \\
& mittel & $33,8^{* * *}$ & 3.092 \\
& hoch & $20,6^{* * *}$ & 1.564 \\
Kind $^{c}$ & Ja & $34,1^{* * *}$ & 2.166 \\
& Nein & $28,9^{* * *}$ & 2.757 \\
Migrationshintergrund $^{\mathrm{d}}$ & Ja & $42,1^{* * *}$ & 1.032 \\
& Nein & $27,5^{* * *}$ & 3.885 \\
Altersgruppe & $20-29$ & 31,2 & 2.128 \\
& $30-39$ & 30,8 & 2.797 \\
\hline
\end{tabular}

Quelle: FLB (2012), gewichtete Daten, eigene Berechnungen

Anm.: ${ }^{* * *} \mathrm{p} \leq 0,001 ;{ }^{* *} \mathrm{p} \leq 0,01 ;{ }^{*} \mathrm{p} \leq 0,05$ (Ergebnis des $\mathrm{Chi}^{2}$-Tests); die Prozentwerte entsprechen dem Anteil an $\mathrm{N}$ der jeweiligen Zeile; ${ }^{\mathrm{a}}$ West: Befragte Person wohnt in alten Bundesländern; Ost: Befragte Person wohnt in neuen Bundesländern inklusive Berlin; ${ }^{b}$ Bildungsniveau nach ISCED97, Klassifikation nach UNESCO (gemäß der demografischen Standards des Statistischen Bundesamtes (2010): Die Zuordnung erfolgt nach: Low (1-2), Medium (3A-4A), High (5B-6); ${ }^{\mathrm{c}}$ „Haben Sie Kinder? Ich meine sowohl Ihre eigenen Kinder als auch Adoptivkinder, egal, wo sie momentan wohnen.“; ${ }^{\mathrm{d}}$ Personen, die selbst eingewandert sind und/oder deren Elternteil(e) eingewandert sind bzw. Personen mit ausländischer Staatsbürgerschaft (zur Erfassung wurde das Geburtsland des Befragten, dessen Staatsangehörigkeit sowie das Geburtsland der Eltern und deren Staatsangehörigkeit hinzugezogen). Demnach sind in dieser Kategorie Personen mit Migrationshintergrund, zugewanderte Ausländer, in Deutschland geborene Ausländer, eingebürgerte Ausländer, Spätaussiedler und Personen mit zumindest einem Elternteil, der eines der genannten Merkmale erfüllt.

Ein weniger deutlicher, aber ebenfalls signifikanter Unterschied zeigt sich zwischen Eltern und Kinderlosen: Etwas mehr Eltern $(34,1 \%)$ als Kinderlose $(28,9 \%)$ orientieren sich am „klassischen“ Modell, Elternschaft zu gestalten. Hier können Kausalitäten in beide Richtungen vorliegen: Einerseits neigen Menschen mit dem Elternschaftsleitbild der „komplementären Elternrollen" möglicherweise eher dazu, eine Familie zu gründen, andererseits könnte die Erfahrung der Elternschaft (und die einer lückenhaften Kinderbetreuungsinfrastruktur) auch zu einer Umorientierung bei den entsprechenden Leitbildern führen. Da Menschen versuchen, kognitive Dissonanzen zu vermeiden, passen sie sich mit ihren Normalitätsvorstellungen ein Stück weit an die Realität an, die sie vorfinden. 


\subsubsection{Differentielle Beschreibung des individuellen Elternschaftsleitbildes der kindzentrierten Erziehungsarbeit nach sozialstrukturellen Merkmalen}

In Tabelle 3 sind die Zustimmungswerte für das Elternschaftsleitbild der „kindzentrierten Erziehungsarbeit" dargestellt. Synonym zu Tabelle 2 wird die Zustimmung nach denselben soziodemografischen Merkmalen vorgenommen. Auch hier würden mit anderen Indikatoren, die nur starke oder reine Zustimmung messen, gleiche Unterschiede auf niedrigerem Niveau ausgewiesen.

Von allen Befragten stimmen 64,5 \% dem Leitbild auf persönlicher Ebene zu. Insgesamt wird deutlich, dass die Unterschiede zwischen den verschiedenen sozialen Gruppen geringer ausgeprägt sind, als beim „Leitbild der komplementären Elternrollen“. Zwar sind viele Differenzen hoch signifikant, aber nur schwach relevant. Dies verweist möglicherweise auf eine vergleichsweise ähnlich weit fortgeschrittene Diffusion dieses Leitbildes durch alle hier untersuchten Gesellschaftsschichten. Möglicherweise kann das Leitbild aber auch deswegen weniger stark zwischen sozialen Gruppen differenzieren, weil es weniger akzentuiert ist und weniger stark als einheitliches Leitbild wahrgenommen wird, das polarisieren könnte. Bemerkenswert ist, dass zwischen Ost- und Westdeutschland, sowie zwischen Personen mit und ohne Migrationshintergrund keine Unterschiede bei der Zustimmung zu diesem Leitbild bestehen.

Tabelle 3: Zustimmung zum individuellen Elternschaftsleitbild der „kindzentrierten Erziehungsarbeit" nach sozialstrukturellen Merkmalen (in Prozent)

\begin{tabular}{|c|c|c|c|}
\hline Merkmal & Ausprägung & Überwiegende Zustimmung & $\mathrm{N}$ \\
\hline \multirow[t]{2}{*}{ Geschlecht } & Männlich & $72,8^{* * *}$ & 2.215 \\
\hline & Weiblich & $56,1^{* * *}$ & 2.750 \\
\hline \multirow[t]{2}{*}{ Wohnort $^{\mathrm{a}}$} & Westdeutschland & 64,7 & 3.879 \\
\hline & Ostdeutschland & 64,0 & 1.086 \\
\hline \multirow[t]{3}{*}{ Bildung $^{b}$} & niedrig & $74,1^{* * *}$ & 205 \\
\hline & mittel & $64,4^{* * *}$ & 3.120 \\
\hline & hoch & $63,6^{* * *}$ & 1.573 \\
\hline \multirow[t]{2}{*}{ Kind $^{\mathrm{c}}$} & $\mathrm{Ja}$ & $56,3^{* * *}$ & 2.175 \\
\hline & Nein & $70,1^{* * *}$ & 2.788 \\
\hline \multirow[t]{2}{*}{ Migrationshintergrund $^{d}$} & $\mathrm{Ja}$ & 66,5 & 1.040 \\
\hline & Nein & 63,9 & 3.917 \\
\hline \multirow[t]{2}{*}{ Altersgruppe } & $20-29$ & $68,6^{* * *}$ & 2.145 \\
\hline & $30-39$ & $60,4^{* * *}$ & 2.820 \\
\hline
\end{tabular}

Quelle: FLB (2012), gewichtete Daten, eigene Berechnungen

Anm.: ${ }^{* * *} \mathrm{p} \leq 0,001 ;{ }^{* *} \mathrm{p} \leq 0,01 ;{ }^{*} \mathrm{p} \leq 0,05$ (Ergebnis des $\mathrm{Chi}^{2}$-Tests); die Prozentwerte entsprechen dem Anteil an $\mathrm{N}$ der jeweiligen Zeile; Erläuterungen siehe Anmerkungen von Tabelle 2.

Dagegen zeigt sich, dass die Geschlechter in diesem Fall unterschiedliche Vorstellungen haben: Deutlich mehr Männer (72,8 \%) als Frauen (56,1\%) stimmen diesem Leitbild zu. Dieser Befund (der auch bei Kontrolle anderer soziodemografischer Merkmale Bestand hat) mag überraschen, da eine Reduktion der Erwerbsarbeit seitens des Vaters eine egalitärere Aufgabenteilung ermöglichen würde, von der vor allem Mütter profitieren würden. 
Ausschlaggebend ist hier jedoch, dass Männer den elterlichen Verpflichtungen im Sinne einer ,verantworteten Elternschaft“ stärker zustimmen.

Beiden Items zur verantworteten Elternschaft stimmen Männer häufiger zu als Frauen - möglicherweise deshalb, weil sich ihre Ansprüche aufgrund der faktischen Arbeitsteilung seltener in der Alltagspraxis bewähren müssen (vgl. Ruckdeschel, Kap. 12). Der Bildungsunterschied deckt sich im Wesentlichen mit den Erkenntnissen von Ruckdeschel (ebd.), die feststellt, dass ,protektive Eltern“ signifikant häufiger niedrig und mittel gebildet sind. Das ist der Typus, der dem Konzept der verantworteten Elternschaft am nächsten ist. Dementsprechend neigen Menschen mit niedriger Bildung (74,1\%) eher zum Leitbild der ,kindzentrierten Erziehungsarbeit" als jene mit mittlerer (64,4 \%) oder höherer Bildung (63,6 \%). Das könnte daran liegen, dass gering Gebildete mit der Erziehung ihrer Kinder am ehesten einen Wunsch nach sozialem Aufstieg verbinden. Das Investment in die eigenen Kinder wird vielleicht noch stärker als bei mittel und hoch Gebildeten als Pflicht betrachtet, um dem Nachwuchs ein besseres Leben zu ermöglichen. Die Vorstellung, dass der Vater dazu seine Erwerbsarbeit einschränken sollte, mag dazu teilweise im Widerspruch stehen, weil es die Chancen schmälert, die Kinder materiell gut ausstatten zu können. In der Tat stimmen diesem Item für sich genommen Akademiker am häufigsten zu. Die in Tabelle 3 sichtbaren Bildungsunterschiede der Dimension gehen also allein auf die Items zur Verantworteten Elternschaft zurück.

Ein deutlicher Unterschied ist beim Vergleich von Eltern und Kinderlosen zu beobachten: Während das Leitbild unter Kinderlosen mit 70,1\% sehr stark verbreitet ist, ist der Zustimmungswert unter Eltern (56,3\%) vergleichsweise niedrig. Hier könnte wiederum entweder ein Selektionseffekt derart vorliegen, dass diejenigen, die dieses Leitbild verinnerlicht haben, seltener Kinder bekommen. Man könnte von einem „Abschreckungseffekt“ durch allzu anspruchsvolle Vorstellungen von Elternschaft sprechen, der ggf. vermutlich vor allem über Männer wirkt. Oder es könnte ein Effekt derart vorliegen, dass die Erfahrung der Elternschaft die Orientierung an Leitbildern verändert. In dem Fall läge ein Effekt der „Desillusionierung“ vor. Der Altersunterschied, demzufolge 68,6\% der Menschen unter 30 aber nur (noch) 60,4\% der älteren Befragungsteilnehmer dem Leitbild der ,kindzentrierten Erziehungsarbeit" zustimmen, hängt überwiegend mit den vorher beschriebenen Unterschieden zusammen und reduziert sich stark, wenn Elternschaft kontrolliert wird.

Insgesamt zeigt sich, dass die vorgefundenen Leitbilder der „komplementären Elternrollen“ und der „kindzentrierten Erziehungsarbeit“ eine gewisse Ähnlichkeit haben und durchaus gemeinsam vorstellbar sind, da sich einige Elemente der jeweiligen Leitbilder ergänzen. Insofern ist es kaum verwunderlich, dass sich die soziodemografischen Profile ähneln. Außerdem ist dazu festzustellen, dass starke Positionierungen seltener vorkommen und eine Mehrheit zur gleichzeitigen graduellen Zustimmung und Ablehnung neigt, was für eine gewisse Unsicherheit in den Leitvorstellungen zur Elternschaft spricht.

\subsection{Gesellschaftliche Elternschaftsleitbilder}

Als nächstes steht die Frage im Mittelpunkt, welche Leitbilder zur Elternschaft in der Gesellschaft wahrgenommen werden. Dazu werden die zu Abschnitt 3.1 inhaltlich analogen Items analysiert, die Normalitätsvorstellungen messen, die (aus Sicht der befragten Person) in Deutschland mutmaßlich weit verbreitet sind. Ähnlich wie zuvor werden in einer Hauptkomponentenanalyse drei Dimensionen identifiziert (Tabelle 4), basierend auf den Vor- 
stellungen zur Mutterschaft ${ }^{6}$, Vaterschaft ${ }^{7}$ und Kinderbetreuung. Das Ergebnis ähnelt der dimensionalen Struktur auf individueller Ebene in vieler Hinsicht:

Tabelle 4: Hauptkomponentenanalyse zur Identifikation von Elternschaftsleitbildern auf gesellschaftlicher Ebene (Wahrnehmung der Allgemeinheit)

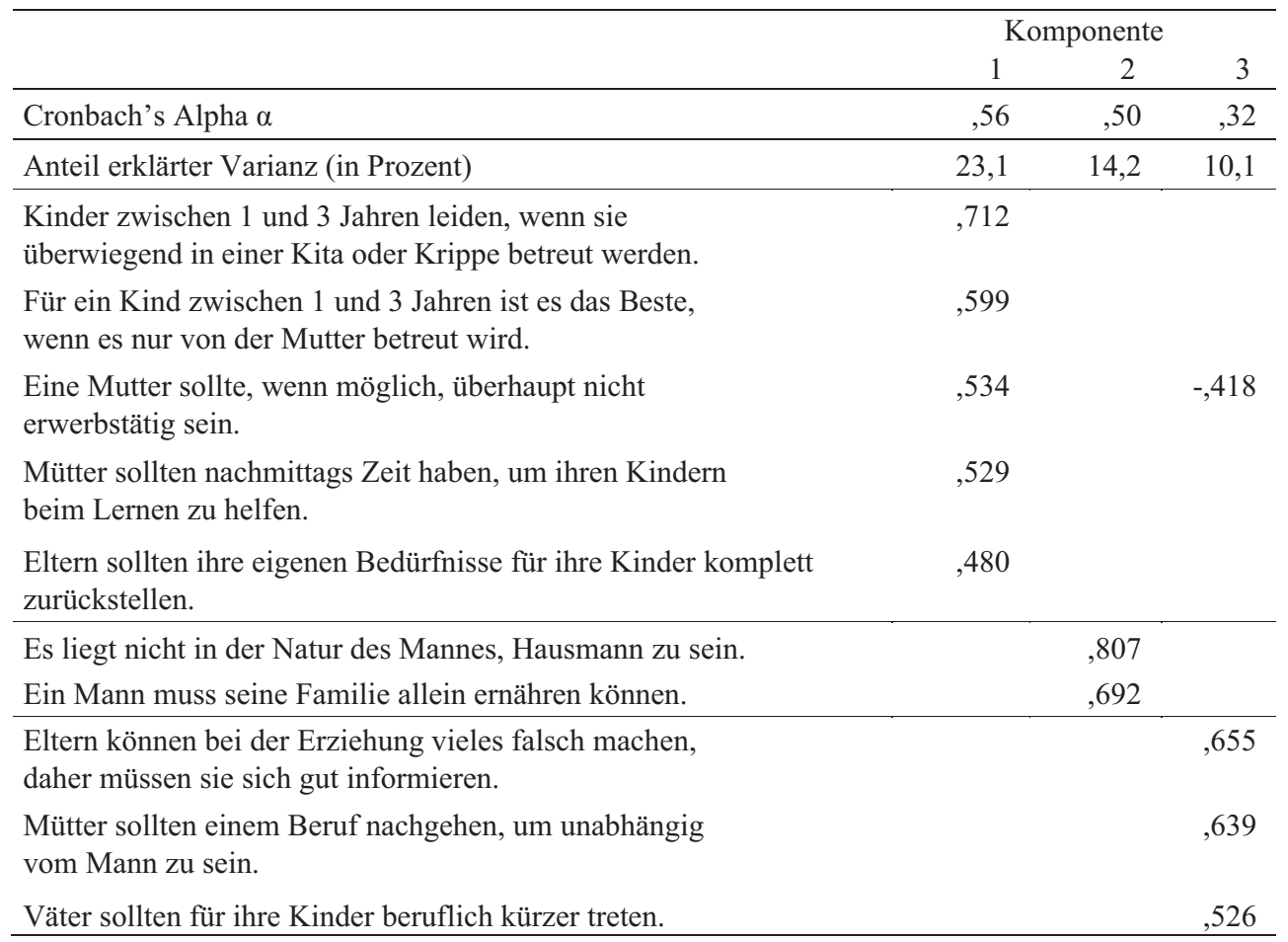

Quelle: FLB (2012), gewichtete Daten, eigene Berechnungen

Anm.: Varimax, rotierte Komponentenmatrix, Werte ab 0,4 werden angezeigt, insgesamt werden $47 \%$ Varianz erklärt.

Auch hier entspricht die erste vorgefundene Dimension einem Elternschaftsleitbild (s. Komponente 1), das Vorstellungen zu verschiedenen Facetten der Elternschaft vereint und im Sinne komplementärer Elternrollen interpretiert werden kann. Wieder ist die Verantwortung der Mutter enthalten, nachmittags zuhause zu sein, um dem Kind bei den Hausaufgaben helfen zu können. Zudem ist die Ablehnung externer Kinderbetreuung Teil des Leitbildes. Unvollständig ist die Spiegelung des Leitbildes auf individueller Ebene dadurch,

$6 \quad$ Allg.: Mütter sollten nachmittags Zeit haben, um ihren Kindern beim Lernen zu helfen. / Mütter sollten einem Beruf nachgehen, um unabhängig vom Mann zu sein. / Eine Mutter, die nur zuhause ist und sich um ihre Kinder kümmert, wird irgendwann unzufrieden. / Eine Mutter sollte, wenn möglich, überhaupt nicht erwerbstätig sein. (Allg. = Was denkt der/die Befragte, wie die Allgemeinheit in Deutschland zu diesen Aussagen steht?)

7 Allg.: Für ein Kind ist es nicht gut, wenn der Vater die Erziehung allein der Mutter überlässt. / Väter sollten für ihre Kinder beruflich kürzer treten. / Es liegt nicht in der Natur des Mannes, Hausmann zu sein. / Ein Mann muss seine Familie allein ernähren. (Allg. = Was denkt der/die Befragte, wie die Allgemeinheit in Deutschland zu diesen Aussagen steht?) 
dass sich die Vorstellungen des Vaters als Familienernährer auf einer separaten Dimension wiederfinden. Das heißt: Während in den individuellen Vorstellungen von Elternschaft Hausfrau und Familienernährer tendenziell zwei logisch miteinander verknüpfte Aspekte des gleichen Leitbildes sind, scheint in den Wahrnehmungen der Leitideen, die einem im sozialen Umfeld bzw. in den öffentlichen Diskursen begegnen, Hausfrau und Familienernährer weniger aufeinander bezogen zu werden. Insofern ist das erste vorgefundene Leitbild ein Stück weit auf das Mutterleitbild der „Hausfrauen-Rolle“ reduziert. Allerdings ist, anders als auf individueller Ebene, auch eine Vorstellung aus dem Kontext der verantworteten Elternschaft Teil des ersten Leitbilds: das Item „Eltern sollten ihre eigenen Bedürfnisse für ihre Kinder komplett zurückstellen“. Die Maxime nach elterlicher Selbstaufopferung hängt demnach - in der öffentlichen Diskussion - eng mit der traditionellen Hausfrauenrolle zusammen.

Als zweites Leitbild (s. Komponente 2) kann das bereits bei Lück (Kap. 14,) beschriebene Leitbild des „Vaters als Familienernährer“ identifiziert werden, demzufolge sich Väter auf ihre „klassische“ Zuständigkeit für Erwerbsarbeit beschränken sollen. Dieses Leitbild ist, wie eben ausgeführt, aus dem Elternleitbild der komplementären Elternrollen herausdifferenziert.

In der dritten vorgefundenen Dimension (s. Komponente 3) zeichnen sich erneut die Konturen eines Elternleitbildes der kindzentrierten Erziehungsarbeit ab. Es beinhaltet wiederum die Erwartung, dass Paare eine egalitäre Arbeitsteilung pflegen und verantwortungsvolle Eltern sind. Zur Vorstellung, dass Männer ihre Erwerbsarbeit zugunsten ihrer Kinder reduzieren, wenn sie Vater werden, gesellt sich nun auch die Vorstellung der erwerbstätigen und ökonomisch eigenständigen Mutter.

\subsubsection{Verbreitung und differentielle Beschreibung der gesellschaftlichen Elternschaftsleitbilder}

Während die zweite Dimension bereits bei Lück (Kap. 14) beschrieben wird, folgt hier eine detaillierte Darstellung des ersten und dritten auf gesellschaftlicher Ebene vorgefundenen Leitbildes. Analog zu Abschnitt 3.1 werden auch hier dazu Summenindizes gebildet und dichotomisiert.

Demzufolge sieht etwas mehr als die Hälfte, 56,6 \% ( $\mathrm{N}=2.804)$, in der Gesellschaft das Elternleitbild der „komplementären Elternrollen“ etabliert und damit deutlich weniger als das Elternleitbild der „kindzentrierten“ Erziehungsarbeit, das von 69,8\% (N=3.475) als gesellschaftlich verbreitetes Leitbild wahrgenommen wird. Demnach ist - genau wie auf individueller Ebene - das Leitbild der „,kindzentrierten Erziehungsarbeit“ präsenter als das der „komplementären Elternrollen“. Beide werden jedoch auf gesellschaftlicher Ebene als weiter verbreitet angenommen, als sie es auf individueller Ebene sind. Auch hier ist die Verteilung der Summenindizes glockenförmig, d. h. Antwortmuster, die starke Zustimmung oder starke Ablehnung zeigen, sind selten. Auch hinsichtlich der Wahrnehmung von gesellschaftlichen Leitbildern besteht also eine gewisse Unsicherheit oder Ambivalenz.

Im Folgenden werden beide Leitbilder hinsichtlich ihrer Konzentration auf bestimmte soziale Gruppen untersucht. Dazu werden, zunächst für das Leitbild der „komplementären Elternrollen“" (Tabelle 5), und anschließend für das der „kindzentrierten Erziehungsarbeit“ (Tabelle 6), jeweils die Zustimmungswerte in verschiedenen gesellschaftlichen Teilgruppen verglichen. Signifikante Unterschiede finden sich nur wenige, und sie sind nicht sehr groß: 
Tabelle 5: Zustimmung zum gesellschaftlich wahrgenommenen Elternschaftsleitbild der „komplementären Elternrollen“ nach sozialstrukturellen Merkmalen (in Prozent)

\begin{tabular}{llcr}
\hline Merkmal & Ausprägung & Überwiegende Zustimmung & $\mathrm{N}$ \\
\hline Geschlecht & Männlich & $53,5^{* * * *}$ & 2.211 \\
& Weiblich & $59,8^{* * *}$ & 2.748 \\
Wohnort $^{\mathrm{a}}$ & West & $58,0^{* * *}$ & 3.879 \\
& Ost & $51,0^{* * *}$ & 1.080 \\
Bildung $^{\mathrm{b}}$ & niedrig & 55,1 & 204 \\
& mittel & 56,9 & 3.116 \\
& hoch & 56,5 & 1.570 \\
Kind $^{c}$ & Ja & $52,2^{* * *}$ & 2.179 \\
& Nein & $59,6^{* * *}$ & 2.778 \\
Migrationshintergrund $^{\mathrm{d}}$ & Ja & 54,3 & 1.035 \\
& Nein & 57,3 & 3.916 \\
Altersgruppe & $20-29$ & 54,9 & 2.141 \\
& $30-39$ & 58,3 & 2.818 \\
\hline
\end{tabular}

Quelle: FLB (2012), gewichtete Daten, eigene Berechnungen

Anm.: ${ }^{* * *} \mathrm{p} \leq 0,001 ;{ }^{* *} \mathrm{p} \leq 0,01 ;{ }^{*} \mathrm{p} \leq 0,05$ (Ergebnis des $\mathrm{Chi}^{2}$-Tests), die Prozentwerte entsprechen dem Anteil an $\mathrm{N}$ der jeweiligen Zeile; Erläuterungen siehe Anmerkungen von Tabelle 2.

Tabelle 6: Zustimmung zum gesellschaftlich wahrgenommenen Elternschaftsleitbild der „kindzentrierten Erziehungsarbeit“" nach sozialstrukturellen Merkmalen (in Prozent)

\begin{tabular}{llcr}
\hline Merkmal & Ausprägung & Überwiegende Zustimmung & $\mathrm{N}$ \\
\hline Geschlecht & Männlich & $71,3^{*}$ & 2.225 \\
& Weiblich & $68,3^{*}$ & 2.751 \\
Wohnort $^{\mathrm{a}}$ & Westdeutschland & 69,4 & 3.889 \\
& Ostdeutschland & 71,3 & 1.087 \\
Bildung $^{\mathrm{b}}$ & niedrig & $81,4^{* * *}$ & 206 \\
& mittel & $70,8^{* * *}$ & 3.125 \\
Kind $^{\text {c }}$ & hoch & $64,1^{* * *}$ & 1.577 \\
& Ja & 69,0 & 2.176 \\
Migrationshintergrund $^{\mathrm{d}}$ & Nein & 70,4 & 2.798 \\
& Ja & $74,2^{* * *}$ & 1.038 \\
Altersgruppe & Nein & $68,5^{* * *}$ & 3.930 \\
& $20-29$ & 70,9 & 2.151 \\
\hline
\end{tabular}

Quelle: FLB (2012), gewichtete Daten, eigene Berechnungen

Anm.: ${ }^{* * *} \mathrm{p} \leq 0,001 ;{ }^{* *} \mathrm{p} \leq 0,01 ;{ }^{*} \mathrm{p} \leq 0,05$ (Ergebnis des $\mathrm{Chi}^{2}$-Tests), die Prozentwerte entsprechen dem Anteil an N der jeweiligen Zeile; Erläuterungen zu Merkmalen siehe Anmerkungen von Tabelle 2. 
Etwas mehr Frauen (59,8 \%) als Männer (53,5 \%) nehmen in der Öffentlichkeit ein Leitbild der komplementären Elternrollen wahr (Tabelle 5). Dasselbe gilt für Befragte, die in den alten Bundesländern leben: Sie stimmen der Existenz eines solchen Leitbildes etwas häufiger $(58,0 \%)$ zu als Bewohner der neuen Bundesländer (51,0 \%). Auch mehr Kinderlose $(59,6 \%)$ als Eltern $(52,2 \%)$ sehen das Alleinverdienermodell in der Gesellschaft als etabliert an.

Ähnlich wenig Varianz gibt es bei der Verbreitung des Elternleitbildes der kindzentrierten Erziehungsarbeit, welches gruppenübergreifend zu einer Zustimmung von etwa 70 Prozent führt (Tabelle 6). Hier erzeugen zwei der sozialstrukturellen Merkmale einen signifikanten Unterschied. Demzufolge nehmen die gering Gebildeten $(81,4 \%)$ dieses Leitbild in der Gesellschaft deutlicher wahr als die mittel (70,8\%) und die höher Gebildeten $(64,1 \%)$. Auch gibt es etwas mehr Zustimmung unter Menschen mit Migrationshintergrund $(74,2 \%)$ als unter jenen ohne $(68,5 \%)$.

Im Vergleich zu den individuellen Leitbildern, die zwischen den gesellschaftlichen Gruppen viel stärker variieren, sind die Gruppenunterschiede hier zwar der Tendenz nach ähnlich, aber, vom Bildungsunterschied in Tabelle 6 abgesehen, deutlich schwächer ausgeprägt. Offenbar werden gesellschaftliche Elternschaftsleitbilder von Angehörigen aller sozialen Gruppen in ähnlicher Weise wahrgenommen.

Auffälligster Kontrast zwischen individuellen und gesellschaftlichen Elternschaftsleitbildern ist (Abbildung 2), dass das Elternschaftsleitbild der „komplementären Elternrollen“ auf gesellschaftlicher Ebene gruppenübergreifend von einer Mehrheit (fast $\approx 60 \%$ ) wahrgenommen, als individuelles Leitbild jedoch nur von etwa $\approx 30 \%$ geteilt wird.

Abbildung 2: Zustimmung zu den Elternschaftsleitbildern „komplementäre Elternrollen“ und „kindzentrierte Erziehungsarbeit" auf individueller und allgemeiner Ebene (in Prozent)

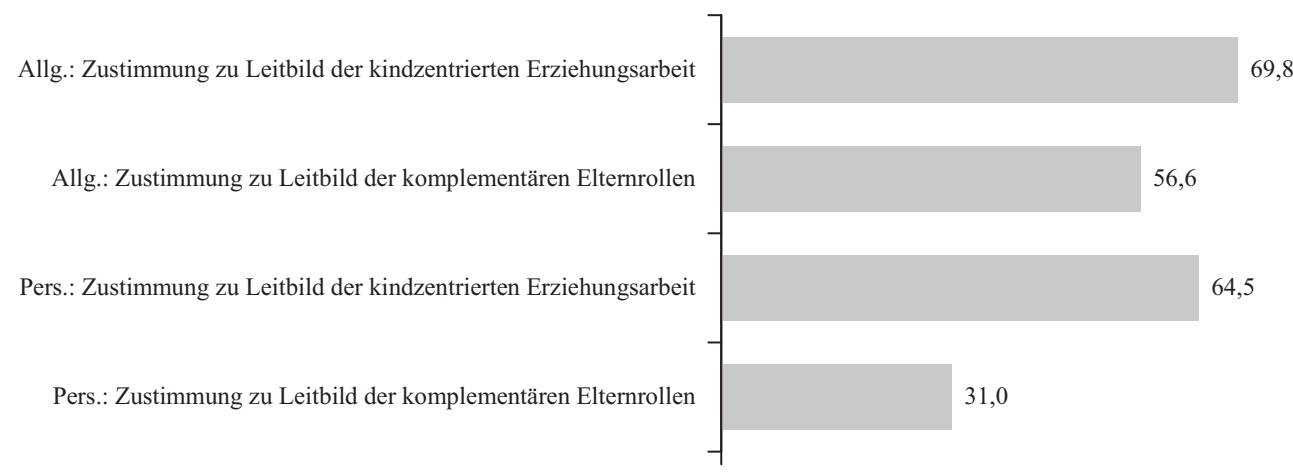

Quelle: FLB (2012), gewichtete Daten, eigene Berechnungen

Anm.: Pers.: Was denkt der/die Befragte/r persönlich; Allg.: Was denkt der/die Befragte, wie die Allgemeinheit in Deutschland zu diesen Aussagen steht?

Das Elternschaftsleitbild der „kindzentrierten Erziehungsarbeit“ ist sowohl auf gesellschaftlicher Ebene (mit etwa $\approx 70 \%$ ) stärker dominant als auch auf persönlicher Ebene, mit knapp $\approx 65 \%$ Zustimmung. 


\subsection{Abgleich von Elternschaftsleitbildern und Alltagsgestaltung}

Bislang ist ungeklärt, inwieweit Leitbilder das Handeln von Individuen beeinflussen. Auch ist nicht bekannt, in welchem Umfang Leitbilder durch Alltagserfahrungen beeinflusst werden und sich variabel der jeweils aktuellen Lebenssituation eines Menschen anpassen. Zwar lassen sich diese beiden Einflüsse mit Hilfe einer Querschnittserhebung nicht voneinander unterscheiden, doch kann ein Abgleich der Leitbilder mit der tatsächlich gelebten Alltagsrealität Hinweise darauf geben, in welchem Umfang solche Effekte in der einen oder in der anderen Richtung existieren könnten. Gefragt wird daher: Gibt es einen Zusammenhang zwischen der faktischen Alltagsgestaltung und den Leitbildern von Elternschaft? Inwieweit verhält sich das eine zum anderen konsistent? Wie häufig sind Abweichungen anzutreffen?

Dazu wurde für alle Befragten, die Kinder haben und mit einem Partner bzw. einer Partnerin im Haushalt leben, eine Variable konstruiert, die die verschiedenen Erwerbsarrangements zwischen den Partnern klassifiziert. In Tabelle 7 ist zu erkennen, dass die häufigste Erwerbskonstellation das weibliche Hinzuverdienermodell ist, bei dem der Mann Vollzeit und die Frau Teilzeit arbeitet ${ }^{8}(42,7 \%)$. Etwas seltener ist das männliche Alleinverdienermodell (31,6 \%), bei dem die Frau nicht erwerbstätig ist. In einem Zweiverdienerpaar $^{9}$ lebt etwa jeder Fünfte im Alter zwischen 20 und 39 (19,1\%). Damit entspricht die Verteilung der Erwerbskonstellationen relativ gut den Ergebnissen des Mikrozensus 2012 (vgl. Keller/Haustein 2013: 870f.).

Tabelle 7: Erwerbskonstellation bei Elternpaaren nach Zustimmung zu bzw. Ablehnung von bestimmten Elternleitbildern auf Individualebene (in Prozent)

\begin{tabular}{|c|c|c|c|c|c|}
\hline \multirow[t]{3}{*}{ Erwerbskonstellation } & \multicolumn{4}{|c|}{ Elternschaftsleitbilder } & \multirow{3}{*}{$\begin{array}{c}\text { Elternpaare } \\
\text { insgesamt }\end{array}$} \\
\hline & \multicolumn{2}{|c|}{$\begin{array}{l}\text { Komplementäre } \\
\text { Elternrollen }\end{array}$} & \multicolumn{2}{|c|}{$\begin{array}{l}\text { Kindzentrierte } \\
\text { Erziehungsarbeit }\end{array}$} & \\
\hline & Zustimmung & Ablehnung & Zustimmung & Ablehnung & \\
\hline $\begin{array}{l}\text { Zweiverdiener-Modell } \\
\text { (Mann und Frau Vollzeit erwerbstätig) }\end{array}$ & 10,3 & 23,8 & 19,3 & 19,0 & 19,1 \\
\hline $\begin{array}{l}\text { weibliches Hinzuverdiener-Modell } \\
\text { (Mann Vollzeit, Frau Teilzeit erwerbstätig) }\end{array}$ & 44,1 & 41,5 & 38,5 & 47,9 & 42,7 \\
\hline $\begin{array}{l}\text { männliches Alleinverdiener-Modell } \\
\text { (Mann Vollzeit, Frau nicht erwerbstätig) }\end{array}$ & 38,1 & 28,5 & 33,2 & 29,6 & 31,6 \\
\hline Sonstige & 7,4 & 6,2 & 8,9 & 3,5 & 6,6 \\
\hline \multirow[t]{2}{*}{ gesamt } & 100 & 100 & 100 & 100 & 100 \\
\hline & (611) & $(1.128)$ & (916) & $(825)$ & $(1.753)$ \\
\hline
\end{tabular}

Quelle: FLB (2012), gewichtete Daten, eigene Berechnungen

Anm.: Befragte, die Kinder haben und mit Partner/in im selben Haushalt leben. Als „Vollzeit“ gilt ein Erwerbsumfang von mehr als 30 Std./Woche; Unter „Sonstige“ befinden sich auch die Familienernährerinnen, auf die hier aus Fallzahl-Gründen nicht näher eingegangen werden kann (Überblick dazu bei Klenner/Klammer 2009). 8 Der Mann arbeitet über 30 Stunden/Woche, die Frau 30 Stunden/Woche oder weniger.
9 Mann und Frau arbeiten über 30 Stunden/Woche. 
Unter „Sonstiges“ zusammengefasst sind jene, die kein geregeltes Einkommen haben $(2,2 \%)$, Erwerbskonstellationen, in denen nur die Mutter erwerbstätig ist $(3,3 \%)$ sowie Menschen in gleichgeschlechtlichen Partnerschaften, die sich in die Typologie nicht einfügen lassen $(1,1 \%)$. Diese Erwerbskonstellationen werden in Tabelle 7 mit der Zustimmung zu den persönlichen Elternschaftsleitbildern der „,komplementären Elternrollen“ sowie der ,kindzentrierten Erziehungsarbeit“" verglichen: Für alle vier Teilgruppen - für jene, die dem ersten Leitbild zustimmen, für jene, die es ablehnen, für jene, die sich am zweiten Leitbild orientieren, und für jene, die das nicht tun - wird jeweils die Verteilung auf die verschiedenen Erwerbskonstellationen dargestellt.

Wie zu erwarten, leben Befragte, die dem Leitbild der „komplementären Elternrollen“ zustimmen, vergleichsweise selten in einer Zweiverdiener-Konstellation: Lediglich auf ein Zehntel (10,3\%) trifft das $\mathrm{zu}$ - und damit nur auf etwa halb so viele wie unter den Elternpaaren insgesamt (19,1\%). Das weibliche Hinzuverdienermodell $(44,1 \%)$ ist unter denen, die dem Leitbild zustimmen, etwa durchschnittlich weit verbreitet. Das männliche Alleinverdienermodell hingegen, das einer faktischen Umsetzung des Leitbildes am ehesten gerecht wird, wird mit 38,1 \% deutlich häufiger gelebt. Abweichungen finden sich bei jenen, die das Elternschaftsleitbild der „komplementären Elternrollen“ ablehnen. So zeigt sich insgesamt ein deutlicher Zusammenhang zwischen Leitbild und gelebter Alltagsrealität. Weniger deutlich zeigt sich dieser Zusammenhang beim Leitbild der „kindzentrierten Erziehungsarbeit"“. Aufgrund der Befürwortung einer egalitären Aufteilung der elterlichen Aufgaben würde dieses Leitbild eher eine Neigung zu einer mehr oder weniger starken Erwerbsbeteiligung der Mutter erwarten lassen. Stattdessen unterscheidet sich diese Gruppe kaum vom Durchschnitt der Befragten. Der einzige auszumachende Unterschied besteht in einem leicht unterproportionalen Anteil des weiblichen Hinzuverdiener-Modells (38,5\%) und einem leicht überproportionalen Anteil des Alleinverdiener-Modells (33,2\%). Das Leitbild der „kindzentrierten Erziehungsarbeit“ lässt sich demnach möglicherweise weniger gut in der alltäglichen Realität des Familienlebens umsetzen. Vielleicht spielen hierbei die hohen Ansprüche an eine verantwortungsvolle Elternschaft eine Rolle.

Insgesamt zeigt der Abgleich, dass es zwar eine Tendenz gibt, Leitbild und gelebte Realität in Einklang zu bringen (ungeachtet dessen, in welche Richtung diese Anpassung erfolgt), dass dies aber durchaus nicht in jedem Fall gelingt und dass es für unterschiedliche Leitbilder ungleich häufig gelingt. Es gibt eine größere Gruppe von Personen, die nicht leitbild-konform lebt bzw. leben kann: Betrachtet man beispielsweise die Gruppe der Zweiverdiener mit dem Leitbild der „komplementären Elternrollen“, so zeigt sich, dass überdurchschnittlich viele dieser Menschen einen Migrationshintergrund haben (33,9\% gegenüber 25,0\% insgesamt) und dass viele von ihnen die Frage „Wie gut kommen Sie mit Ihrem Einkommen zurecht?" 10 mit „schlecht" beantworten (14\% gegenüber 7,1 \% insgesamt). Möglicherweise führt in diesem Fall ökonomischer Druck dazu, dass Menschen nicht leitbild-konform leben (Schulz/Hummer 2005: 355f.). Diejenigen, die in der Praxis ein Alleinverdienermodell bzw. ein männliches Versorgermodell leben, jedoch dem Leitbild der „kindzentrierten Erziehungsarbeit“ zustimmen, sind tendenziell jung und leben in Westdeutschland. Das stünde im Einklang mit einem Scheitern an fehlenden öffentlichen Betreuungsangeboten als Hintergrund für die Inkonsistenz.

10 Statt des Einkommens wurde die aussagekräftiger erscheinende subjektive Einschätzung gewählt. Zudem gibt es dort weniger fehlende Werte. 


\section{$4 \quad$ Fazit}

Im Zentrum dieses Beitrags stand die Identifikation von Leitbildern der Elternschaft. Dazu bilden die im FLB 2012 gemessenen Vorstellungen eine passende Datengrundlage. Es wurden sowohl auf individueller als auch, aus Sicht der Befragten, auf gesellschaftlicher Ebene Elternschaftsleitbilder gefunden. Dieser Befund spiegelt über vielfältige sozialstrukturelle Unterschiede hinweg eine Art schichtübergreifenden common sense hinsichtlich verschiedener Normalitätsvorstellungen. Dies ist keineswegs selbstverständlich und spricht dafür, dass zentrale Leitbilder der Elternschaft gesellschaftsweit in der jüngeren Bevölkerung etabliert sind. Das Leitbild, das bei hoher Ausrichtung an verantworteter Elternschaft gleichermaßen ein berufliches Zurückstecken des Vaters vorsieht, ist weiter verbreitet als das, das an einer komplementären Rollenteilung ausgerichtet ist. Das trifft sowohl für die individuelle als auch für die gesellschaftliche Ebene zu.

Für alle identifizierten Leitbilder gilt - wenn auch in graduell unterschiedlichem Maße: Extreme Zustimmung oder Ablehnung sind selten, moderate Zustimmungswerte sind dagegen die Regel. Das heißt, dass die Menschen entweder zu den mittleren Kategorien neigen und nur „eher“ zustimmen oder ablehnen; oder sie stimmen den Items einer Dimension nicht durchgängig zu, sondern lehnen einzelne davon ab. Dies spricht dafür, dass hinsichtlich der Elternschaftsleitbilder Verunsicherung, Ambivalenz und Indifferenz existieren.

Stärkster Kontrast zwischen individuellen und gesellschaftlichen Elternschaftsleitbildern ist, dass das Elternschaftsleitbild der komplementären Elternrollen auf gesellschaftlicher Ebene gruppenübergreifend von mehr als der Hälfte der Menschen zwischen 20 und 39 Jahren wahrgenommen, als individuelles Leitbild jedoch nur von weniger als einem Drittel geteilt wird. Das Elternschaftsleitbild der kindzentrierten Erziehungsarbeit ist sowohl auf gesellschaftlicher Ebene als auch auf persönlicher Ebene dominant. Diese Ergebnisse könnten als ein Zeichen für einen vorangeschrittenen sozialen Wandel interpretiert werden, der jedoch sehr wahrscheinlich noch nicht abgeschlossen ist. Dafür spricht beispielsweise der Befund, dass etwa die Hälfte der jüngeren Bevölkerung sowohl das komplementäre als auch das kindzentrierte Elternschaftsleitbild befürwortet oder beide gleichermaßen ablehnt. Dahinter könnten sowohl innere Konflikte bzw. eine Verunsicherung stehen, welches der Leitbilder aktuell Gültigkeit besitzt, als auch eine Überforderung derart, dass sich die 20- bis 39-Jährigen dem Anspruch ausgesetzt sehen, die vielfältigen Ansprüche an Elternschaft gleichzeitig zu erfüllen. Dabei konkurriert die Maxime der Förderung und Sicherung des Kindeswohls und das damit verbundene hohe Maß an elterlicher (insbesondere mütterlicher) Betreuung und Beschäftigung mit dem Kind - oft durch nicht ausreichende Betreuungsinfrastruktur weiter intensiviert als gewünscht - mit dem Leitgedanken nach Gleichberechtigung und fairer Aufgabenteilung zwischen den Eltern sowie mit dem Streben nach Selbstbestimmung und Selbstentfaltung beider Eltern. Außerdem spiegeln die Ergebnisse die Diskussionen um die sich in den ökonomischen Anreizen widersprechenden familienpolitischen Instrumente „Rechtsanspruch auf einen Kitaplatz“ und „Betreuungsgeld“ (Müller/Wrohlich 2014) wider, die beide jeweils auf eines der beiden Leitbilder abzielen. Die beiden Elternschaftsleitbilder auf persönlicher Ebene erscheinen symptomatisch für die bisher unzureichende Definition der familienpolitischen Ziele in Deutschland.

Des Weiteren wurden die individuellen mit den gesellschaftlich wahrgenommenen Elternschaftsleitbildern verglichen. Es zeigt sich unter den individuellen Leitbildern deutlich mehr Varianz als unter den gesellschaftlichen. Das ist zu erwarten gewesen, wenn man annimmt, dass auch in einer Gesellschaft mit individuell unterschiedlichen Leitbildern ein gesellschaftsweit dominantes Leitbild existiert, das jeder kennt und einheitlich berichten kann (auch wenn sein persönliches Leitbild davon abweicht). 
Manche Leitbilder sind mit der faktischen Familienrealität konsistent, andere nicht. Die Ergebnisse verweisen darauf, dass Menschen zwar versuchen, Leitbild und Realität in Einklang zu bringen - sei es, dass sie ihre Leitbilder in die Realität umsetzen, sei es, dass sie sie der Realität anpassen. Sie scheitern aber vermutlich zuweilen daran, etwa weil fehlende Betreuungsmöglichkeiten oder die Notwendigkeit eines zweiten Erwerbseinkommens sie daran hindern. Oder sie scheitern, weil sie mit dem gesellschaftlichen Leitbild konform sein wollen, obwohl sie auf persönlicher Ebene ein gegensätzliches Leitbild vertreten. Die Ursachen für solche Diskrepanzen müssen in weiteren Untersuchungen tiefgreifend analysiert werden.

\section{Literatur}

Allmendinger, Jutta (2008): Frauen auf dem Sprung. Die Brigitte-Studie 2008. Die Lebensentwürfe junger Frauen in Deutschland im Alter von 17 bis 19 und 27 bis 29 Jahren. Hamburg: Gruner und Jahr.

Althammer, Jörg; Ott, Notburga; Dudel, Christian (2010): Die Verhandlungsfamilie: empirische Evidenz familienökonomischer Verhandlungsmodelle. In: Walper, Sabine; Wendt, Eva-Verena (Hrsg.): Partnerschaften und die Beziehungen zu Eltern und Kindern. Befunde zur Beziehungsund Familienentwicklung in Deutschland. Würzburg: Ergon, S. 113-133.

Bauernschuster, Stefan; Rainer, Helmut (2012): Political Regimes and the Family: How Sex Role Attitudes Continue to Differ in Reunified Germany. In: Journal of Population Economics 25, 1, S. 5-27.

Beck-Gernshein, Elisabeth (1992): Arbeitsteilung, Selbstbild und Lebensentwurf: Neue Konfliktlagen in der Familie. In: Kölner Zeitschrift für Soziologie und Sozialpsychologie 2, S. 273-291.

Beck-Gernsheim, Elisabeth (2008): Vom „Dasein für andere“ zum Anspruch auf ein Stück „eigenes Leben“: Individualisierungsprozesse im weiblichen Lebenszusammenhang. In: Wilz, Sylvia M. (Hrsg.): Geschlechterdifferenzen - Geschlechterdifferenzierungen. Wiesbaden: VS, S. 19-61.

Becker, Gary S. (1993): A treatise on the family. Cambridge, Mass: Harvard University Press.

Berk, Sarah F. (1985): The gender factory. The apportionment of work in American households. New York: Plenum Press.

Coverman, Shelley (1985): Explaining husbands' participation in domestic labor. In: Sociological Quarterly 26, S. 81-97.

Dahrendorf, Ralf (1965): Homo Sociologicus. Opladen: Westdeutscher Verlag.

de Swaan, Abram (1982): Vom Ausgehverbot zur Angst vor der Straße. In: PÄD extra 2: S. 48-55.

Dorbritz, Jürgen; Ruckdeschel, Kerstin (2009): Die langsame Annäherung. Demografisch relevante Einstellungsunterschiede und der Wandel in den Lebensformen in West- und Ostdeutschland. In: Cassens, Insa; Luy, Marc; Scholz, Rembrandt (Hrsg.): Die Bevölkerung in Ost- und Westdeutschland. Wiesbaden: VS Verlag, S. 265-298.

du Bois-Reymond, Manuela (1994): Die moderne Familie als Verhandlungshaushalt. Eltern-KindBeziehungen in West- und Ostdeutschland und in den Niederlanden. In: du Bois-Reymond, Manuela; Büchner, Peter; Krüger, Heinz-Hermann; Ecarius, Jutta; Fuhs, Burkhard (Hrsg.): Kinderleben. Modernisierung von Kindheit im interkulturellen Vergleich. Opladen: Leske + Budrich, S. $137-219$.

Goffman, Erving (1977): The arrangement between the sexes. In: Theory and Society 4, S. 301-331.

Grunow, Daniela; Schulz, Florian; Blossfeld, Hans-Peter (2007): Was erklärt die Traditionalisierungsprozesse häuslicher Arbeitsteilung im Eheverlauf: soziale Normen oder ökonomische Ressourcen? In: Zeitschrift für Soziologie 36, 3, S. 162-181. 
Henry-Huthmacher, Christine; Borchard, Michael (2008): Eltern unter Druck. Selbstverständnisse, Befindlichkeiten und Bedürfnisse von Eltern in verschiedenen Lebenswelten. Eine sozialwissenschaftliche Untersuchung von Sinus Sociovision GmbH im Auftrag der Konrad-AdenauerStiftung. Stuttgart: Lucius \& Lucius.

Henry-Huthmacher, Christine (2008): Eltern unter Druck. Zusammenfassung der wichtigsten Ergebnisse der Studie. In: Henry-Huthmacher, Christine; Borchard, Michael (Hrsg.): Eltern unter Druck. Stuttgart: Lucius \& Lucius.

Honig, Michael-Sebastian (1992): Verhäuslichte Gewalt. Sozialer Konflikt, wissenschaftliche Konstrukte, Alltagswissen, Handlungssituationen. Frankfurt a. M.: Suhrkamp.

Keller, Matthias; Haustein, Thomas (2013): Vereinbarkeit von Familie und Beruf. Ergebnisse des Mikrozensus 2012. In: Wirtschaft und Statistik 12/2013. Wiesbaden: Statistisches Bundesamt, S. 1079-1100.

Klenner, Christina; Klammer, Ute (2009): Weibliche Familienernährerinnen in West- und Ostdeutschland - Wunschmodell oder neue Prekarität? In: Rollenleitbilder und -realitäten in Europa: rechtliche, ökonomische und kulturelle Dimensionen, Forschungsreihe Band 8 des BMFSFJ, Nomos, S. 62-84.

Müller, Kai-Uwe; Wrohlich, Katharina (2014): Two Steps Forward - One Step Back? Evaluating Contradicting Child Care Policies in Germany. Diskussionspapiere/Discussion Papers 1396. Deutsches Institut für Wirtschaftsforschung, DIW: Berlin.

Oberndorfer, Rotraut; Rost, Harald (2005): Neue Väter - Anspruch und Realität. In: Zeitschrift für Familienforschung 17, 1, S. 50-65.

Ott, Notburga (1998): Der familienökonomische Ansatz von Gary S. Becker. In: Pies, Ingo; Leschke, Martin (Hrsg.): Gary Beckers ökonomischer Imperialismus. Tübingen: Mohr Siebeck, S. 63-90.

Pfau-Effinger, Birgit (2004): Socio-historical paths of the male breadwinner model - an explanation of cross-national differences: In: The British Journal of Sociology 55, 3, S. 377-399.

Quaiser-Pohl, Claudia (2001): Deutsche Eltern im interkulturellen Vergleich. In: Nickel, Horst; Quaiser-Pohl, Claudia (Hrsg.): Junge Eltern im kulturellen Wandel: Untersuchungen zur Familiengründung im internationalen Vergleich. Weinheim, München: Beltz Juventa, S. 301-311.

Ross, Catherine E. (1987): The division of labor at home. Social Forces 65, S. 816-833.

Schneider, Norbert F. (2002): Elternschaft heute. Gesellschaftliche Rahmenbedingungen und individuelle Gestaltungsaufgaben. Einführende Betrachtungen. In: Schneider, Nobert F.; MatthiasBleck, H. (Hrsg.): Elternschaft heute. Opladen, Leske + Budrich, S. 9-21.

Schneider, Norbert F., Naderi, Robert; Ruppenthal, Silvia (2012): Familie in Deutschland nach dem gesellschaftlichen Umbruch. Sind Ost-West-Differenzierungen in der Familienforschung zwanzig Jahre nach der Wiedervereinigung noch sinnvoll? In: Huinink, Johannes; Kreyenfeld, Michaela; Trappe, Heike (Hrsg.): Familie und Partnerschaft in Ost- und Westdeutschland. Ähnlich und doch immer noch anders. In: Zeitschrift für Familienforschung, Sonderheft 9, S. 29-53.

Schneider, Norbert F.; Diabaté, Sabine; Lück, Detlev (2014): Familienleitbilder in Deutschland. Ihre Wirkung auf Familiengründung und Familienentwicklung. In: Henry-Huthmacher, Christine (Hrsg.): Konrad-Adenauer-Stiftung e.V., Sankt Augustin, Berlin.

Schulz, Florian; Blossfeld, Hans-Peter (2006): Wie verändert sich die häusliche Arbeitsteilung im Eheverlauf? Eine Längsschnittstudie der ersten 14 Ehejahre in Westdeutschland. In: Kölner Zeitschrift für Soziologie und Sozialpsychologie 58, 1, S. 23-49.

Schulz, Wolfgang; Hummer, Christian (2005): Veränderungen in den Formen des Zusammenlebens und Wandel der Einstellungen zu Ehe und Familie. In: Schulz, Wolfgang; Haller, Max; Grausgruber, Alfred (Hrsg.): Österreich zur Jahrhundertwende. Wiesbaden: VS, S. 343-366.

Stafford, Rebecca; Backman, Elaine; Dibona, Pamela (1977): The division of labor among cohabitating and married couples. In: Journal of Marriage and the Family 39, S. 43-57.

Stauder, Johannes (2002): Eheliche Arbeitsteilung und Ehestabilität. Eine Untersuchung mit den Daten der Mannheimer Scheidungsstudie 1996 unter Verwendung ereignisanalytischer Verfahren. Würzburg: Ergon-Verlag.

Statistisches Bundesamt (2010). Statistik und Wissenschaft. Demographische Standards. Nr. 17.

Walster, Elaine; Walster, G. William; Berheide, Ellen (1978): Equity: Theory and Research. Boston. 



\title{
Leitbildforschung: Befunde, Potenziale und Impulse
}

\author{
Sabine Diabaté, Kerstin Ruckdeschel \& Norbert F. Schneider*
}

\section{Zusammenfassung}

In diesem Beitrag werden die theoretischen Eckpfeiler der Leitbildstudie sowie deren zentrale Ergebnisse erläutert und zusammengefasst. Zudem werden die Potenziale und Grenzen der Leitbildforschung diskutiert, vor allem im Hinblick auf die wissenschaftliche Erklärung von generativen Handlungen und die Gestaltung des Familienlebens. Aus den Befunden werden abschließend Impulse für die politische Debatte abgeleitet. Die Studie hat gezeigt, dass familiäre Beziehungen, Partnerschaften und eigene Kinder bei den meisten 20- bis 39-Jährigen eine hohe Wertschätzung erfahren und eine breite Anerkennung für die vielfältigen Lebensformen in Deutschland existiert. Vier Kernaussagen lassen sich aus den Befunden ableiten: (1) Pluralität: $\mathrm{Zu}$ vielen Aspekten des Familienlebens gibt es zwar vorherrschende, aber keine einheitlichen Leitbilder in der Gesellschaft. (2) Diskrepanz: Die Befragten nehmen in der Gesellschaft konservativere Leitbilder wahr, als die, denen sie persönlich folgen (möchten). (3) Mismatch: Elterliche Leitbilder und Alltagspraxis sind häufig widersprüchlich. (4) Verantwortete Elternschaft: Das Leitbild der „Verantworteten Elternschaft“" erhöht die Opportunitätskosten für Elternschaft, leistet einer Ablehnung von externer Kinderbetreuung Vorschub, und begünstigt dadurch den Aufschub oder sogar die Ablehnung der Familiengründung. Insgesamt geht von diesem Leitbild ein ungünstiger Impuls für die Geburtenentwicklung in Deutschland aus.

\section{$1 \quad$ Einleitung}

Kulturelle Faktoren erfahren in der jüngeren demografischen Forschung größere Aufmerksamkeit, da sie zusätzlich zu strukturellen Determinanten das Geburtengeschehen beeinflussen. Innerhalb der diversen etablierten kulturellen Ansätze (z. B. Arránz Becker et al. 2010; Billari et al. 2009; McDonald 2000; Pfau-Effinger 1996, 2004, 2005; Böllert 1993; Lesthaeghe 1992; van de Kaa 1987) soll der Leitbild-Ansatz einen weiteren Zugang bieten, um Kultur innerhalb der bevölkerungswissenschaftlichen und familiensoziologischen Forschung zu operationalisieren. Zentral am Leitbild-Ansatz ist, dass neben dem Einfluss von sozialen Normen und von subjektiven Überzeugungen vor allem den subtileren, unreflektierten und häufig unbewussten Einflüssen von Normalitätsvorstellungen bzw. von Frame-Skript-Verbindungen (Esser 1990) eine größere Bedeutung beigemessen wird als bisher. Demnach sind Familienleitbilder gebündelte Systeme, die sich aus verschiedenen Normalitätsvorstellungen zusammensetzen, die den Themenbereich Familie beinhalten. Dabei bestehen sie auf zwei Ebenen: Es gibt sowohl die individuellen als auch die in der Gesellschaft wahrgenommenen kulturellen Leitbilder.

Die AutorInnen danken den Kollegen Detlev Lück und Robert Naderi sowie der anonymen Gutachterin bzw. dem anonymen Gutachter für die hilfreichen Kommentare und die konstruktive Kritik. 
Der Mehrwert des Leitbild-Konzeptes besteht darin, dass jenseits der Einstellungen und Werthaltungen zum Thema Familie zusätzlich eine repräsentative und quantitative Datenbasis geschaffen wurde, um die Vorstellungen, die junge Menschen in der Phase vor und während der Familiengründung und -erweiterung von einem „,normalen“ und „guten“ Familienleben haben, zu ermitteln. Zentrale Annahme dieses methodischen Zugangs ist es, dass die kulturellen Normalitätsvorstellungen, die in ihrer Summe ein Leitbild repräsentieren, handlungsleitend sind. Vice versa ist davon auszugehen, dass bestimmte Lebenssituationen und strukturelle Rahmenbedingungen wiederum diese Leitbilder (um-)formen oder verstetigen. Um persönliche und gesellschaftlich wahrgenommene Leitbilder im Kontext der Familie systematisch quantitativ zu erforschen, bietet die bundesweite Studie „Familienleitbilder" (FLB) 2012 eine neukonzipierte und aktuelle Datengrundlage. Die Ergebnisse der ersten Welle, die in diesem Sammelband aufgeführt sind, werden in diesem abschließenden Beitrag aggregiert und dargestellt.

Für die Messung der Leitbilder wurden Vorstellungen zum normalen oder ,guten“ Familienleben bei 5.000 Personen im Alter von 20 bis 39 erhoben. Die Zustimmung der Befragten wurde dabei sowohl auf persönlicher Ebene gemessen, als auch auf allgemeiner Ebene, d. h. ob sie annehmen, dass die Allgemeinheit der Menschen in Deutschland den Vorstellungen zustimmen würde. Dadurch erweitert die Studie die Vorgehensweise der klassischen Einstellungs- und Werteforschung, da sie zwar auch Einstellungen, jedoch zusätzlich Normalitätsvorstellungen misst.

Familienleitbilder sind Kombinationen aus individuellen sowie auch in der Gesellschaft wahrgenommenen Vorstellungen zum „normalen“ Familienleben, die im Hinblick auf Elternschaft oft, aber nicht immer, in einem Zusammenhang mit der tatsächlich gelebten Alltagswirklichkeit stehen. Leitbilder vereinen in sich überlagernde Wertvorstellungen, Frames und Normen und sind mal mehr oder weniger konkret und trennscharf. Sie können dabei unbewusst, aber auch so bewusst und konkret sein, dass sie bildhaft-kognitiv Gestalt annehmen. Die Vorstellungen können, mit unterschiedlichen Anteilen, einem Wert (das Gewünschte), einer Norm (das Erwünschte) oder einem Frame (das Selbstverständliche) entsprechen. Üblicherweise beinhalten Leitbilder alle drei Eigenschaften gleichzeitig. Familienleitbilder fokussieren verschiedene Lebensbereiche: Sie lassen sich beispielsweise für eine „vorbildliche” Elternschaft (strukturbezogen) oder für das „richtige” Timing von Familienbiografien (prozessbezogen) beschreiben. Eingelagert in diese Leitbilder finden sich Vorstellungen, die sich wiederum auf Teilaspekte von Partnerschaft oder Elternschaft beziehen. Im Wesentlichen lassen sich die genannten Leitbilder unter die Themenfelder Partnerschaft, Elternschaft und Familienleben subsummieren. Die Familie stellt dabei einen Möglichkeitsraum mit vielfältigen Dimensionen dar und ein zentrales Ergebnis ist, dass es nicht das Leitbild der Familie gibt, sondern mannigfaltige Manifestationen. Hierin spiegelt sich die Vielfalt in der Gesellschaft.

Um Familienleitbilder einer empirischen Untersuchung zugänglich zu machen, wurde im Leitbildsurvey des Bundesinstituts für Bevölkerungsforschung eine neuartige Form der Operationalisierung gewählt. Dafür wurden neue Items konstruiert, die individuelle Vorstellungen davon messen, wie Familienleben normalerweise aussieht und die sich zu gesellschaftlichen Leitbildern der jüngeren Wohnbevölkerung Deutschlands (20 bis 39 Jahre) aggregieren lassen. Zusätzlich wurde ein Zugang geschaffen, um gesellschaftlich wahrgenommene Leitbilder standardisiert zu erfassen. Die einzelnen Befragten sollten einschätzen, wie „die Allgemeinheit“ die Items beantworten würde, die sie zuvor persönlich beantwortet hatten. Die meisten Items auf persönlicher Ebene wurden in diesem Sinne für die gesellschaftliche Ebene ,gespiegelt". Dadurch sollte die Vielschichtigkeit kultureller Normalitätsvorstellungen erfasst werden, die als komplexe Visualisierungen den Menschen als 
Orientierung dienen. Leitbilder können demnach als persönliche Leitbilder auf individueller Ebene vorgefunden werden; sie existieren jedoch auch als kulturelle Leitbilder auf der Meso- und Makro-Ebene. Denn sie werden intersubjektiv geteilt und sind für bestimmte gesellschaftliche Bereiche charakteristisch. In der Gesamtgesellschaft ist, insbesondere innerhalb bestimmter sozialer Milieus, Generationen und Regionen, eine stärkere Homogenität individueller Leitbilder gefunden worden. Jedoch sind kulturelle Leitbilder nicht durch die Summe der individuellen Leitbilder innerhalb einer Gesellschaft bestimmt. Sie gewinnen durch Institutionalisierung (z. B. durch Gesetze) und auch durch das Alltagshandeln verschiedener Akteure eine eigene, unabhängige, sozial reproduzierte Realität.

Mit den Ergebnissen aus der ersten Welle, die in diesem Sammelband vorgestellt werden, soll kulturellen Aspekten bei der Erklärung der privaten Lebensführung und des Geburtenverhaltens mehr Gewicht verschafft werden. Dafür wurde geprüft, welche Leitbilder der Familie existieren, wie sie beschaffen sind, auf welchen Ebenen sie anzusiedeln sind und wie sie inhaltlich gefüllt werden. Zudem wurde nach sozialstrukturellen Variationen gesucht und analysiert, inwiefern individuelle Vorstellungen mit den in der Gesellschaft wahrgenommenen Leitbildern korrespondieren. Im Anschluss sollen erste Schlussfolgerungen zum Zusammenhang zwischen Leitbildern und generativem Verhalten gezogen. Abschließend folgen Anknüpfungspunkte für die zukünftige Leitbildforschung und Impulse für die gesellschafts- und familienpolitische Debatte.

\section{Befunde: Inhalt und Verbreitung von Familienleitbildern}

Familienleitbilder sind komplexe Konstrukte, in denen verschiedene Teilaspekte von Familie eingebettet sind. Im Mittelpunkt dieser Leitbilder stehen die Vorstellungen über die Zusammensetzung von Familie. Hinsichtlich der Frage, was eine Familie ist bzw. aus welchen Personen sich eine Familie idealerweise zusammensetzt, haben die Menschen ein im Kern einheitliches, aber in seiner äußeren Abgrenzung sehr heterogenes Leitbild: Universell ist die Klassifizierung eines zusammenwohnenden heterosexuellen Paares mit Kindern als Familie. Allerdings gelten für die meisten Menschen auch viele nichtkonventionelle Lebensformen als Familie, diejenigen, die ausschließlich Kernfamilien als Familie wahrnehmen, sind eine kleine Minderheit. Die Beurteilung der übrigen Lebensformen scheint sich an ihrer Ähnlichkeit zur Normalfolie einer solchen Kernfamilie zu orientieren (vgl. Lück/Ruckdeschel, Kap. 5). Entscheidend sind letztlich die Dimensionen Partnerschaft und Elternschaft, aber auch die Ausgestaltung des Familienlebens. Im Folgenden werden daher, diesen Dimensionen folgend, zentrale Befunde aus den drei Themenfeldern zusammengefasst.

\subsection{Partnerschaft}

In einer Partnerschaft zu leben ist ein entscheidender Bestandteil von Familienleitbildern, was sich u. a. darin zeigt, dass das Leben von Singles zumindest in Teilen der Gesellschaft eher als Negativfolie präsent ist: Laut einem Großteil der jungen Deutschen finden Menschen nur in einer festen und stabilen Partnerschaft ihr Glück. Hinsichtlich der Frage, wie eine Partnerschaft aussehen sollte, dominieren assoziativ-orientierte über fusions-orientierte Normalitätsvorstellungen (vgl. Diabaté, Kap. 6): Statt zu einer Einheit mit übereinstimmenden Ansichten und deckungsgleichen Interessen zu „verschmelzen“, bleiben die Partner 
demnach zwei miteinander verbundene (aber nicht vereinigte) Individuen, die sich gewisse Freiräume lassen (Schneider et al. 2002). Außerdem sind die Vorstellungen zur Partnerschaft eher ,egalitär-modern“ als „klassisch-komplementär“ ausgerichtet.

Mit einer erfolgreichen Partnerschaft werden als erstes gegenseitige Liebe, danach erfüllte Sexualität und schließlich persönliche Freiräume assoziiert, jedoch auch Sicherheitsbedürfnisse, wie eine finanzielle Absicherung. Entgegen dieser vergleichsweise progressiven persönlichen Sicht wird in der Gesellschaft ein eher konservatives Bild von Partnerschaft wahrgenommen.

Insgesamt wurden vier individuelle Partnerschaftsleitbilder identifiziert: Personen des ersten Leitbildes (assoziativ-modern) gehen davon aus, dass Partnerschaften funktionieren, wenn man sich liebt, eine erfüllte Sexualität miteinander hat, dem anderen Freiräume lässt, beide Partner assoziativ Seite an Seite leben und finanziell abgesichert sind. Das zweite (fusionsorientiert) bezieht sich auf einen Glauben an das Lebensglück durch Partnerschaft, auf eine hohe Eheorientierung, die Verfolgung gemeinsamer Ziele sowie die Familiengründung als Stabilitätsgarant für die Partnerschaft. Im dritten (bürgerlich-konventionell) ist gleichermaßen die Eheschließung wichtig, jedoch wird es abgelehnt, wenn die Frau höher gebildet ist als der Partner. Und es wird befürwortet, wenn Männer die Entscheidungsmacht haben und es finanzielle Sicherheit gibt. Zudem beinhaltet dieses Partnerschaftsleitbild, dass Frauen nach der Hochzeit den Namen ihres Mannes tragen sollten und dass Paare ohne gemeinsamen Haushalt keine richtigen Paare sind. Ein viertes Leitbild (eheablehnendinstabil) ergibt sich aus einer geringen Eheorientierung und aus Pessimismus gegenüber der Dauerhaftigkeit von Partnerschaften generell. Eine vertiefende Analyse der Partnerschaftsleitbilder (vgl. Naderi, Kap. 7) zeigt zudem einen engen Zusammenhang zwischen Leitbildern, Religiosität und Religionszugehörigkeit, wobei eine selbsteingeschätzte, stärkere Religiosität eher mit einem bürgerlich-konventionellen Leitbild korreliert. Dahinter steht eine hohe Orientierung an der Ehe, die Bedeutsamkeit der Familiengründung und des Führens des männlichen Nachnamens als Familienname, die Ablehnung von bildungshypogamen ${ }^{1}$ Paaren, die Entscheidung für die männliche Entscheidungsmacht in Partnerschaften und das Führen eines gemeinsamen Haushaltes.

\subsection{Elternschaft}

Trotz aller Diskussionen um den Wandel der Familie in Deutschland und die in Teilen der Gesellschaft vorhandene Kinderlosigkeit gehören Kinder selbstverständlich zum Leben dazu (vgl. Dorbritz/Ruckdeschel, Kap. 9). Kinder sind ein fester Bestandteil der Normalitätsvorstellung von Familie und stellen für die überwiegende Mehrheit der jungen Deutschen einen wichtigen Lebensinhalt dar. Elternschaft ist ein zentrales Lebensziel, wovon ausgehend das Leitbild der „Selbstverständlichkeit des Kinderhabens“ formuliert wurde. Was die Kinderzahl betrifft, fokussiert das Leitbild der idealen Familiengröße auf die ZweiKind-Familie. Kinderlos wollen knapp $10 \%$ bleiben, beachtenswert ist, dass mit einem Viertel der Befragten ein relativ hoher Anteil gerne drei oder mehr Kinder hätte. Kinder sind also ein selbstverständliches Lebensziel, aber es werden hohe persönliche Ansprüche an die Bedingungen gestellt, die erfüllt sein müssen, um sich für ein Kind zu entscheiden. Hervorzuheben sind dabei eine solide materielle Grundlage und eine stabile Partnerschaft, aber auch die berufliche Etablierung der Frau. Elternschaft ist demnach zu einem sehr voraussetzungsvollen Lebensziel geworden, wobei gilt, dass, je höher die Ansprüche sind,

1 Bildungsniveau des Mannes geringer als das der Frau. 
desto niedriger der Kinderwunsch ausfällt. Von der hohen Bedeutung der „Verantworteten Elternschaft" geht insgesamt ein negativer Impuls für die Geburtenentwicklung aus, da sie die Opportunitätskosten von Elternschaft erhöht und den Aufschub oder auch die Ablehnung der Familiengründung begünstigt.

Bemerkenswert ist, dass das als ideal angesehene Alter für die Geburt des ersten Kindes niedriger ist als das tatsächliche: So wird das ideale Familiengründungsalter für Frauen von den 20- bis 39-Jährigen mit 27 Jahren angegeben, während das tatsächliche Alter von Müttern bei der Geburt ihres ersten Kindes im Jahr 2012 bei 29,2 Jahren lag (Statistisches Bundesamt 2014). Frühe und späte Geburten vor dem 20. bzw. nach dem 35. Lebensjahr werden kaum akzeptiert. Häufig liegt der Zeitpunkt, zu dem die als notwendig empfundenen Voraussetzungen für eine Familiengründung erfüllt sind, in einem vergleichsweise späten Lebensabschnitt, so dass es faktisch zur Verkürzung der reproduktiven Lebensphase kommt. Außerdem fallen dadurch entscheidende berufliche und familiäre Entwicklungen oft in einen engen gemeinsamen Zeitkorridor (Rushhour des Lebens), wodurch die Lebensqualität von jungen Erwachsenen beeinträchtigt wird und es zu einem (unfreiwilligen) Verzicht auf die Familiengründung oder -erweiterung kommen kann.

Neben Leitbildern zur Familiengründung finden sich auch sehr klare gesellschaftliche Vorstellungen davon, ab welcher Anzahl von Kindern die Familienerweiterung abgeschlossen sein sollte. Als ideale Kinderzahl für Familien in Deutschland wurden überwiegend zwei Kinder genannt. Am Beispiel kinderreicher Familien (Kinderreichtum wurde von den Befragten ab vier Kindern definiert) ist es möglich zu untersuchen, wie eine Abweichung von diesem Ideal bewertet wird. Es treten große Unterschiede zwischen individueller und gesellschaftlicher Ebene zu Tage: Während kinderreiche Familien auf individueller Ebene positiv besetzt sind, herrscht auf der gesellschaftlichen Ebene ein eher negativ konnotiertes Bild von Mehrkindfamilien vor (vgl. Diabaté/Ruckdeschel/Dorbritz/Lux, Kap. 11).

Ein weiterer Schwerpunkt der Studie war die Untersuchung von regionalen Unterschieden. So bestehen zwischen Ost- und Westdeutschland weiterhin erhebliche Unterschiede beim Fertilitätsgeschehen. In Ostdeutschland bleiben weniger Frauen kinderlos, Mütter sind häufiger erwerbstätig und der Anteil nichtehelicher Geburten ist sehr viel höher. Zudem liegen die Kinderbetreuungsquoten für unter 3-Jährige in Ostdeutschland höher als in Westdeutschland. Für diese Differenzen werden zwei Erklärungen angeboten (vgl. Schneider et al. 2012): Erstens wirken die unterschiedlichen politischen Orientierungen aus der Zeit der Teilung nach. Zweitens gab es bereits vor der Teilung Deutschlands ähnliche Unterschiede, die u. a. auf historische und kulturell-konfessionell bedingte Besonderheiten zurückzuführen sind, was vor allem die höhere Nichtehelichenquote in Ostdeutschland erklären könnte.

Eine grundlegende Annahme der Leitbildforschung, wie wir sie betreiben, lautet, dass sowohl das vom Individuum individuell verinnerlichte Leitbild als auch das in seinem sozialen Umfeld (vermeintlich) vorherrschende kollektiv geteilte Leitbild (die nicht deckungsgleich sein müssen) individuelles Verhalten beeinflussen. Ziel der Analysen war es daher auch, die Familienleitbilder der Befragten mit der faktischen Gestaltung ihres Familienlebens abzugleichen, um Hinweise auf mögliche Einflüsse zu erhalten.

Für die Untersuchung dieses Zusammenhangs (vgl. Schiefer/Naderi, Kap. 10) wurde die Kinderlosigkeit im Verhältnis zur Elternschaft betrachtet. Erstere ist im Westen nach wie vor stärker ausgeprägt, die zusammengefasste Geburtenziffer (TFR) ist aber insgesamt in beiden Regionen nahezu gleich. Es zeigt sich, dass individuelle Familienleitbilder einen relevanten zusätzlichen Beitrag zur Erklärung von Elternschaft liefern, sofern unterstellt werden darf, dass sie in Bezug auf Elternschaft vorwiegend erklärende Variable sind. Für Elternschaft bei Frauen ist es sowohl in West- als auch in Ostdeutschland signifikant 
ungünstig, wenn sie einer Leitbild-Facette (Informationsgebot) der „Verantworteten Elternschaft“ zustimmen. Es ist, wie bereits angesprochen, unklar, ob das Leitbild die Lebensführung oder die Lebensführung das Leitbild formt. Im Sinne der Sozialisation könnte man davon ausgehen, dass das Leitbild zuerst da ist, im Laufe des Lebens mit den gesammelten Erfahrungen abgeglichen wird und gegebenenfalls eine Anpassung erfährt, um kognitive Dissonanzen zu vermeiden.

Ähnlich dazu manifestiert sich der ungünstige Einfluss der „Verantworteten Elternschaft“ auf generative Entscheidungen bei der Identifikation der Familiengründungstypen (vgl. Dorbritz/Ruckdeschel, Kap. 9). Die gemessenen Ansprüche an Elternschaft sind aus Sicht der Befragten zu hoch, um in die Elternphase eintreten zu können. Demnach wirkt das Leitbild der verantworteten Elternschaft als Verzögerung oder sogar als Barriere. Über die Hälfte der jungen Erwachsenen entsprechen dem anspruchsvollen Familiengründungstypus, bei dem sowohl die materielle Absicherung als auch die berufliche Etablierung der Frau als notwendige Voraussetzungen für die Familiengründung bewertet werden. Aus den Ergebnissen wird deutlich: Je höher das Anspruchsniveau an Elternschaft ist, desto niedriger ist der Kinderwunsch. Die Befunde zeigen auch, dass Kinder eine hohe individuelle Bedeutung haben und eine große Wertschätzung erfahren. Jedoch scheint es so, als ob die Anforderungen so hoch ausfallen, dass sie eine Realisierung der hohen Kinderwünsche be- bzw. sogar verhindern.

Die Untersuchungen zum sozialen Kontext (Dorbritz/Diabaté, Kap. 8) zeigen zudem, dass es lohnenswert ist, die soziale Umgebung von Menschen vor und während der Familiengründungs- und -erweiterungsphase stärker in den Blick zu nehmen, um generatives Verhalten zu erklären. Zielführend hierfür ist das Konzept der „linked lives“ (Elder/Shanahan 2006), nach dem Individuen in Abhängigkeit von ihren sozialen Kontexten Entscheidungen treffen und sich danach verhalten.

Insgesamt erscheint es vielversprechend, Leitbilder als zusätzlichen missing link für die Erklärung von Fertilitätsentscheidungen hinzuzuziehen. Mit Längsschnittdaten wäre es außerdem möglich, die Wirkungsrichtung und Stärke des Einflusses von Leitbildern auf die Familiengründung ansatzweise entschlüsseln zu können.

\subsection{Familienleben: Eltern-Kind-Beziehung und Elternrollen}

Mit dem Thema Elternschaft eng verbunden sind die Vorstellungen von einer guten Ausgestaltung des Familienlebens und der Elternrollen. Das Leitbild Verantwortete Elternschaft ist in Deutschland stark präsent. Dieser Befund spiegelt sich auch in einer aktuellen Umfrage von EMNID wider, bei der $70 \%$ der Befragten angeben, dass der Druck auf Eltern, perfekt zu sein, gewachsen ist (BamS 2014). Dies beeinflusst nicht nur die Entscheidung für oder gegen Elternschaft, sondern prägt auch die Vorstellungen davon, wie Elternschaft gestaltet werden sollte (vgl. Ruckdeschel, Kap. 12). Zu nennen ist hier vor allem das Fördergebot: Die jungen Deutschen sind überwiegend der Meinung, dass man Kinder beim Aufwachsen intensiv begleiten sollte, dass Kinder also nicht „,von alleine groß werden“. Dies ist eine Einstellung, die verschiedenste Elterntypen verbindet und auch als gesellschaftlicher Anspruch an Eltern wahrgenommen wird. Hinzu kommt das Gebot der Mutternähe und Aufopferung, die vor allem als gesellschaftliche Erwartung wahrgenommen, aber auf individueller Ebene überwiegend abgelehnt wird.

Bei der Betrachtung der Mutterleitbilder zeigen sich gleichermaßen hohe Ansprüche: Mütter sollen sowohl die Erziehung („Fürsorge“) als auch ihre „Selbstsorge“ parallel als zentrale Lebensziele verfolgen. Die Erwartungen von Präsenz der Mutter zuhause und gleichzeitig im Beruf erscheinen schwer vereinbar. Zudem existieren vier verschiedene Leitbild-Typen: Das berufsorientierte, das kindorientierte sowie das moderate und das ver- 
einbarkeitsorientierte Mutterleitbild. Insgesamt lässt sich eine stärkere Kindzentrierung bei in Westdeutschland lebenden Personen diagnostizieren. Interessanterweise hängen vor allem das kind- und das berufsorientierte Mutterleitbild eng mit dem Lebensmodell der Mütter der Befragten zusammen. Hierbei sind vermutlich u. a. die unterschiedlichen Rahmenbedingungen in Ost- und Westdeutschland (vor der Wiedervereinigung) prägend, in denen die Mütter der Befragten sozialisiert wurden und die in die nachfolgende Generation hineinwirken (vgl. Diabaté, Kap. 13).

Unter den Vorstellungen, wie ein Vater sein sollte, lassen sich grundsätzlich zwei Leitbilder entdecken: Zum einen das des „Familienernährers“, der sich um das Haushaltseinkommen kümmert und die Familienarbeit der Mutter überlässt. Dieses Leitbild wird in der Gesellschaft als weit verbreitet wahrgenommen. Zum anderen lässt sich das Leitbild des „aktiven Vaters“ identifizieren, der mit der Familiengründung seine Erwerbsarbeit reduziert und sich an der Familienarbeit beteiligt. Dieses Leitbild dominiert auf persönlicher Ebene. Für Männer ist typisch, dass sie von einem vereinbarkeitsorientierten Leitbild (Vereinbarkeit hinsichtlich Beruf und Familie) ausgehen und somit zu einer gewissen Selbstüberforderung neigen, die jedoch von den Alltagserfahrungen im Zuge der Familiengründung desillusioniert wird. Für Frauen ist typisch, dass sich ihre Orientierung im Zuge der Familiengründung weg von einem Leitbild des ,aktiven Vaters“ hin zu den übrigen eher traditionell geprägten Leitbildern verlagert, die den Vater stärker als Ernährer und weniger als Erzieher definieren. Auch hier ist von einer Desillusionierung durch Alltagserfahrungen auszugehen. Dahinter steht die Anpassung der Leitbilder an eine (durch Elternschaft veränderte) Realität, womit dann der Widerspruch zwischen vorherigem Leitbild und der Lebenswirklichkeit aufgehoben wird (vgl. Lück, Kap. 14). Die Ergebnisse zur Ausgestaltung der mütterlichen und väterlichen Rollen insgesamt verweisen auf eine gewisse Unsicherheit, z. T. auch auf eine hohe Zwiespältigkeit und Dissonanz von Leitvorstellungen sowie auf deren Konkurrenzhaftigkeit.

Die vorgestellten Leitbilder der Mutter- und Vaterschaft lassen sich teilweise zu Elternschaftsleitbildern zusammenfassen (vgl. Diabaté/Lück/Schneider, Kap. 15). Dabei sind zwei Leitbilder bedeutsam: Im Mittelpunkt des einen Leitbilds steht die Vorstellung einer komplementären Arbeitsverteilung, die sich am male-breadwinner/female-homemakerModell orientiert (Leitbild der komplementären Elternrollen), verknüpft mit der Ablehnung externer Kinderbetreuung. Das andere Leitbild ist durch das Kürzertreten des Vaters im Beruf mit hohen Ansprüchen an eine „Verantwortete Elternschaft“ gekennzeichnet (Leitbild der kindzentrierten Erziehungsarbeit). Während die individuellen Elternschaftsleitbilder sozialstrukturell teilweise unterschiedlich verteilt sind, werden die allgemeinen in allen Teilen der Gesellschaft ähnlich wahrgenommen, unabhängig z. B. von Bildung oder Geschlecht. Sowohl im Hinblick auf individuelle wie auf gesellschaftliche Leitbilder ist das einer kindzentrierten Erziehungsarbeit weiter verbreitet. Die überwiegend moderaten Zustimmungs- und Ablehnungswerte sprechen dafür, dass es eine gewisse Verunsicherung hinsichtlich der idealen Ausgestaltung von Elternschaft in Deutschland gibt.

Beim Abgleich zwischen Leitbild und Lebensrealität zeichnet sich eine Tendenz ab, dass Menschen Leitbild und reale Ausgestaltung der Elternschaft in Einklang bringen. Jedoch leben auch einige nicht leitbildkonform, d. h. sie können weder ihr Leitbild im Alltag verwirklichen noch ihr Leitbild an ihre Lebensrealität anpassen. Der Abgleich gelingt für unterschiedliche Leitbilder ungleich häufig. Mehr als ein Viertel derjenigen, die komplementäre Elternrollen tendenziell ablehnen, praktizieren sie in ihrer Beziehung in Form eines „klassischen“ männlichen Alleinverdiener-Modells (mit Vollzeit erwerbstätigem Mann und nichterwerbstätiger Frau). Umgekehrt arbeitet bei der Hälfte derjenigen, die diesem Leitbild zustimmen, die Mutter in Teil- oder Vollzeit. Dies ist ein überraschender 
Befund, wenn man von der Annahme ausgeht, dass Menschen kognitive Dissonanzen vermeiden möchten und daher Wunsch und Wirklichkeit nach Möglichkeit synchronisieren.

Insgesamt lässt sich anhand der verschiedenen Leitbilder übergreifend feststellen, dass bei der Zustimmung zu bestimmten Leitbild-Dimensionen einige interessante geschlechtsspezifische Differenzen festzustellen sind. Hinsichtlich der Müttererwerbstätigkeit haben Männer ein traditionelleres Geschlechterbild. In gewisser Hinsicht haben, umgekehrt, auch weibliche Befragte konservativere Haltungen in Bezug auf die Vaterrolle: Sie erwarten von Vätern seltener, dass sie sich um die Kinder kümmern und in der Erwerbsarbeit kürzer treten, als Männer das tun. Das Vaterleitbild des „Familienernährers“ ist unter Müttern relativ häufig. Allerdings ist unter kinderlosen Frauen das Leitbild des „aktiven Vaters“ dominant und weiter verbreitet als unter kinderlosen Männern. Kinderlose Männer wiederum, und zu einem gewissen Grad auch noch Väter, neigen vor allem zu einem vereinbarkeitsorientierten Vaterleitbild. Bezogen auf die Beurteilung der Mutterrolle könnte man geneigt sein, sich der Interpretation von Grunow und Baur anzuschließen:

„dass normative Stereotype (...) stärker auf das andere Geschlecht angewendet werden als auf das eigene [, weil die] Lebenszusammenhänge, die man selbst als Außenstehender wahrnimmt, weniger komplex und ambivalent erscheinen als der eigene Lebenszusammenhang" (Grunow/Baur 2014: 512).

Bezogen auf die Beurteilung der Vaterrolle erscheint dies jedoch nur teilweise hilfreich zu sein. Eher scheint es so, dass Männer generell höhere Ansprüche an sich selbst richten, als Frauen dies an die Vaterrolle tun, und dass die Praxiserfahrung nach der Familiengründung einerseits die Ansprüche von Männern an sich selbst senkt und andererseits die der Frauen an Väter konservativer werden lässt. Die Ergebnisse werfen die Frage auf, ob die Geschlechterunterschiede in den Leitbildern einen „Mismatch“ auf dem Partnermarkt erzeugen, weil die Vorstellungen sowohl von der Vater- als auch von der Mutterrolle nicht deckungsgleich sind. Unabhängig davon, ob die unterschiedlichen Vorstellungen bereits bei der Partnerfindung Schwierigkeiten verursachen, lässt sich fragen, ob es aufgrund dessen Partnerschaftskonflikte im Zuge der Familiengründung und in den ersten Monaten der Elternschaft gibt (sowie eine erhöhte Trennungs-/Scheidungswahrscheinlichkeit). Die Befunde verweisen außerdem darauf, dass es lohnend sein kann, das Konzept der Leitbilder auch auf Auswertungen zur Zeitverwendung von jungen Eltern anzuwenden, um die faktische Arbeitsaufteilung im Haushalt besser zu verstehen.

Trotz der überragenden Bedeutung von Elternschaft konnte auch eine spezielle, eher kleine Gruppe der Kinderlosen innerhalb der 20- bis 39-Jährigen identifiziert werden, die keine Kinder hat und sich auch keine Kinder wünscht (vgl. Dorbritz/Diabaté, Kap. 8). Sie umfasst rund ein Zehntel der jüngeren Bevölkerung. Es wurden zwei gesellschaftlich wahrgenommene Leitbilder identifiziert, weshalb einige Menschen in Deutschland aus Sicht der Befragten keine Kinder bekommen möchten: Ein „Leitbild der risiko-vermeidenden Kinderlosigkeit“ und das „Leitbild der autonomie-betonten Kinderlosigkeit“" werden als Ursachen gesehen. Das erste beinhaltet vor allem Ängste vor der Zukunft und vor der Verantwortung, die ein Kind im Alltag bedeutet. Das zweite betont die Selbstbestimmung bzw. die Autonomie der Kinderlosen. Vor allem in weiten Teilen der jüngeren Gesellschaft wird Kinderlosigkeit als selbstverständlicher Bestandteil des Lebens wahrgenommen und ist sozial akzeptiert - zumindest als Lebensentwurf der anderen. Insgesamt wird das Thema von den 20- bis 39-Jährigen jedoch ambivalent gesehen, denn eine größere Gruppe der Befragten (vor allem Eltern) empfindet die Kinderlosen auch als egoistisch und sehen sie deshalb in der Pflicht, höhere Abgaben zu leisten. 


\section{Woher kommen Leitbilder?}

Wo liegt der Ursprung von individuellen und allgemein wahrgenommenen Leitbildern und welche Orientierungsgeber liegen den Familienleitbildern zugrunde? Welche Faktoren haben dabei die größte Bedeutung? Die Herkunft von Familienleitbildern lässt sich mit den vorliegenden Querschnittsdaten nicht abschließend klären, wenngleich sich die ältesten Befragten im Jahr der Wiedervereinigung im Teenageralter befanden.

Die Ergebnisse lassen aber den Schluss zu, dass persönliche Leitbilder durch politische Systeme (West- und Ostdeutschland vor der Wende) und durch regionalspezifische kulturelle Eigenheiten indirekt und mittelbar mit beeinflusst werden (vgl. Bauernschuster/Rainer 2012). Kulturelle gesamtgesellschaftlich verbreitete Leitbilder erscheinen langlebig und finden ihren Ausdruck auch in den Institutionen (z. B. Ost-West-Unterschiede in der Kinderbetreuungsinfrastruktur). Zudem sind gesamtgesellschaftliche Leitbilder durch regionale, kulturelle und religiöse Komponenten und gleichzeitig auch durch politische Systeme mit geformt und wirken auf persönliche Leitbilder. Ostdeutschland weist demnach signifikant häufiger egalitäre Leitbildmuster auf als Westdeutschland, was vermutlich nicht nur durch das spezifische Mutterleitbild des DDR-Regimes, sondern auch durch seine historisch eher protestantische beeinflusste Kultur aus der Zeit bereits vor der Teilung Deutschlands bedingt ist (Schneider et al. 2012).

Am Beispiel der Mutterleitbilder (vgl. Diabaté, Kap. 13) wird deutlich, dass sie auch durch intergenerationale Transmission mit geprägt werden: Hier konnte ein Zusammenhang zwischen dem persönlichen Mutterleitbild und der Erwerbstätigkeit der Mutter der Befragten nachgewiesen werden, um Unterschiede zwischen west- und ostdeutschen jungen Erwachsenen zu erklären. Sie kopieren das Lebensmodell ihrer Eltern, welches sie in ihrer Entwicklung erlebt und als selbstverständliche Lebensweise internalisiert haben. Neben der Prägung im Elternhaus und der späteren, weiteren Sozialisation spielen auch die Konfessionszugehörigkeit und die Religiosität sowie die kulturelle Herkunft (Migrationshintergrund) eine Rolle. Befragte, die ihre eigene Religiosität als hoch einstufen, weisen beispielsweise eine deutlich höhere Chance auf, ein bürgerlich-konventionelles Partnerschaftsleitbild zu vertreten. Einschränkend für die Beurteilung ist die betrachtete Altersgruppe und somit die mangelnde Berücksichtigung verschiedener Lebensphasen oder Kohorten. Dazu zählen im Lebenslauf gemachte Erfahrungen und die eigene Lebenssituation (z. B. „Desillusionierungseffekte“ durch Elternschaft).

Es zeigt sich außerdem, dass sich die Befragten bei der Bildung ihrer AllgemeinheitsMeinung vor allem durch Personen aus ihrem direkten und auch indirekten Umfeld beeinflussen lassen und man sich weniger (zumindest bewusst) an Institutionen orientiert (vgl. Gies/Dietrich, Kap. 4). Dadurch kann die Theorie von Noelle-Neumann $(1996,1994)$ zur Entstehung der öffentlichen Meinung nur teilweise als Erklärung hilfreich sein. Trotz der Allgegenwart der öffentlichen Medien, die tagtäglich gesellschaftliche Normen und Werte verbreiten, haben diese bei der Reflektion der Herkunft von Familienleitbildern einen weniger starken Einfluss auf die Meinungsbildung bei den jungen Erwachsenen als die zwischenmenschlichen Kontakte. Die Themenfelder Familie und Partnerschaft sind sehr persönlich, weshalb an dieser Stelle die medialen Einflüsse weniger präsent sind bzw. unterschwellig wirken. Inwieweit sich unterbewusst der Prozess der Leitbild-Entstehung und Modifizierung bzw. Verstetigung vollzieht (auch unter dem Einfluss der Massenmedien), bleibt dabei unklar. Hierzu ist mehr Forschung nötig, um solche latenten und unterschwelligen Prozesse besser zu verstehen. Methoden und theoretische Ansätze aus der Entwicklungspsychologie sowie aus der Medienwirkungsforschung könnten ein sinnvoller Anknüpfungspunkt für künftige Forschungsvorhaben sein. 
Bei diesem Prozess der Beeinflussung und Reproduktion von Normalitätsvorstellungen kommt den Medien insofern eine besondere Bedeutung zu, da dort die gängigen und etablierten Normalitätsvorstellungen aus Politik und Gesellschaft vermittelt werden (Neidhardt 1994). Leitbilder der Familie werden in diesem Kontext nicht nur positiv, sondern auch negativ dargestellt und wirken dabei mutmaßlich anziehend oder abstoßend für das Alltagshandeln. Wie genau der Einfluss im Vergleich zu den anderen Faktoren aussieht (z. B. institutionelle Rahmenbedingungen), lässt sich aufgrund von fehlenden Längsschnittdaten sowie zusätzlicher Analysemethoden (z. B. Medienanalyse von Familienleitbildern) nicht belegen. Weitere Untersuchungen $^{2}$ zur Herkunft von Familienleitbildern sind daher notwendig.

\section{Inwieweit korrespondieren individuelle und gesellschaftliche Leitbilder?}

Zur Messung der gesellschaftlichen Leitbilder wurden zwei Zugänge genutzt: Erstens wurde gefragt, wie die Befragten bestimmte Vorstellungen in ihrer Umgebung wahrnehmen. Zweitens können die persönlichen Leitbilder zu gesellschaftlichen Leitbildern aggregiert werden, die innerhalb der Gruppe der 20- bis 39-Jährigen repräsentativ sind. Beim Vergleich dieser beiden Arten der Leitbild-Messung differierten die Ergebnisse teilweise erheblich (z. B. bezüglich der Vorstellungen zum Kinderreichtum), manche korrespondierten eng miteinander (z. B. hinsichtlich der Meinung, dass eine Mutter nachmittags ihre Kinder beim Lernen unterstützen soll). Persönliche und allgemeine Leitbilder unterscheiden sich z. T. signifikant, häufig ist das individuelle Leitbild „,moderner“ bzw. „liberaler“ als das in der Gesellschaft wahrgenommene Leitbild. Vier mögliche Ursachen wurden diskutiert (vgl. Lück/Naderi/Ruckdeschel, Kap. 3): Soziale Erwünschtheit; Vorurteile gegenüber der Allgemeinheit, die grundsätzlich pessimistischer wahrgenommen wird als die eigene Person (Terwey 1990: 149-151); Alters- bzw. Selektionseffekte, da das Alter der Befragten lediglich einen Ausschnitt der Bevölkerung abdeckt und die Jüngeren liberalere Vorstellungen zum Thema Familie haben als Ältere (Junck/Lück 2015); sowie zeitlich versetzter sozialer Wandel.

Nach Diabaté und Lück (2014) sind Familienleitbilder durch verschiedene Formen der Institutionalisierung, wie beispielsweise durch Gesetze, verfestigt und geradezu greifbar fixiert. Dadurch kann es passieren, dass sich innerhalb der Gesellschaft bereits ein Wandel vollzieht z. B. hinsichtlich der Mutter- und Vaterleitbilder hin zu einem egalitären Engagement beider Elternteile für das Kind, jedoch institutionelle Rahmenbedingungen sich (noch) nicht gewandelt haben (institutional lag). Zusätzlich können auch die innerhalb der Institutionen zugrunde liegenden kulturellen Leitbilder denen der Individuen mehrheitlich nicht (mehr) entsprechen. Dementsprechend können auch politisch neu geschaffene Strukturen, etwa der Ausbau von Krippenplätzen in Westdeutschland, Leitbilder beeinflussen und gesellschaftlichen Wandel initiieren. Denn einerseits sind die neuen Strukturen Folge eines verbreiteten vereinbarkeitsorientierten Mutterleitbildes, andererseits wiederum wird diese Leitvorstellung verstetigt und damit auch zur Verbreitung der Akzeptanz von Müttererwerbstätigkeit bei unter 3-Jährigen beigetragen.

2 In diesem Zusammenhang ist das Forschungsprojekt APPARENT zu nennen, welches auf die Erforschung aktuell vorherrschender Elternschaftsnormen, und dabei speziell auf deren Entstehung, Verbreitung und praktische Relevanz für geschlechtsspezifisches Handeln in sieben europäischen Ländern abzielt. Untersucht werden dabei die verschiedenen Akteure, die zur Konstruktion und Verfestigung bestimmter Elternschaftsnormen beitragen wie z. B. ExpertInnen, FamilienberaterInnen, Hebammen), Wohlfahrtsstaaten und Massenmedien. 
Dass sich kulturell und institutionell ein Wandel im Kontext von Familie vollzieht, erscheint offensichtlich (Nave-Herz 2012). Die These, dass dabei Ungleichzeitigkeiten zwischen verschiedenen Ebenen (zwischen der Geschlechterkultur und der Geschlechterordnung) auftreten, liegt nahe und ist nicht neu (z. B. Pfau-Effinger 1996: 467). Angesichts der ebenfalls deutlich erkennbaren Altersunterschiede (beim Vergleich der Leitbildstudienteilnehmer mit den älteren Befragten aus anderen Surveys, vgl. Kap. 3) lässt sich annehmen, dass es sich bei diesen Altersunterschieden nicht - oder nicht nur - um Lebenslaufeffekte, sondern - zumindest teilweise auch - um Generationenunterschiede handelt und dass sich dieser Wandel zumindest teilweise durch die Abfolge der Generationen vollzieht. Dies wiederum bedingt, dass die Allgemeinheitsmeinung auch die tendenziell weniger liberale Meinung der „Älteren“ ist, wahrgenommen von den „Jüngeren“, die liberalere persönliche Leitbilder zu Protokoll geben (vgl. These 3: Alters- bzw. Selektionseffekte). Auch ist unklar, ob sich diese Differenz im Laufe einiger Generationen auflösen und in einem gesellschaftsweit einheitlicheren Leitbild münden wird oder ob sie ein „normales“ gesellschaftliches Spannungsfeld darstellt, das dauerhaft fortbesteht. In einem größeren Interpretationskontext könnte die große Kluft aber auch als Schritt hin zu einer Individualisierung und Liberalisierung von Familienleitbildern verstanden werden. Der stetige Anstieg von familialen Lebensformen in Deutschland in den vergangenen Jahrzehnten (Wagner 2008), die im Kontext der Familienbildungsprozesse und der strukturellen Zusammensetzung nicht dem „Normalitätsmuster“ von Familie (Nave-Herz 2012) entsprechen, hat vermutlich den Vorstellungshorizont von Individuen erweitert und auf der Einstellungsebene ein Stück weit zu einer Egalisierung und Entdiskriminierung der häufigsten alternativen Familienformen (im Bezug zur klassischen Kernfamilie) geführt.

\section{$5 \quad$ Limitationen, Potenziale und Perspektiven für künftige Forschung}

\subsection{Limitationen der ersten Welle des Leitbildsurveys}

Die in diesem Band präsentierten Ergebnisse zeigen die vielfältigen Potenziale der vorliegenden Daten und der identifizierten Familienleitbilder, jedoch auch die Begrenzungen u. a. durch den methodischen Zugang und durch die Verfügbarkeit nur von Querschnittsdaten. Es besteht die Notwendigkeit, in Zukunft Leitbilder in multivariaten Modellen zu analysieren. Zudem sollten stärker prozessorientiert (im Lebensverlauf) die Zusammenhänge von Familienleitbildern und individuellem Verhalten untersucht werden, um sowohl Familienbildungsprozesse als auch demografische Entwicklungen besser zu verstehen. Darüber hinaus könnten Panel-Studien Hinweise auf kausale Beziehungen und Wechselwirkungen sowie auf die Stabilität von Leitbildern liefern. Außerdem sind durch die standardisierte Messung von Leitbildern immer auch Vorstellungen ausgeklammert. Schließlich ergibt sich naturgemäß eine Verzerrung durch soziale Erwünschtheit.

\subsection{Potenziale der zukünftigen empirischen Leitbildforschung}

Trotz der Limitationen sind die aufgeführten Erkenntnisse eine Motivation, die leitbildbezogene Perspektive auf bevölkerungswissenschaftliche Phänomene theoretisch und methodisch hinsichtlich des Designs weiterzuentwickeln. Vielfältige Entwicklungspotenziale sind denkbar: 
1. Durch die Entwicklung neuer standardisierter Messinstrumente können weitere Leitbilder zum Thema Familie untersucht werden, die z. B. auf die Generationenbeziehungen fokussieren.

2. Mittels der Bildanalyse, einem qualitativen Zugang, soll künftig ${ }^{3}$ unbewusstes Alltagswissen (Bilder sozialer Praktiken vom Familienleben) zugänglich gemacht werden, das mangels Reflexion im Zuge einer Befragung nicht geäußert werden könnte. Die Bildanalyse kann in ihren Ergebnissen nicht den Grad an Objektivität erreichen, der standardisierte Erhebungsinstrumente auszeichnet. Und sie ist ungeeignet, über große Fallzahlen statistisch belastbare Befunde zu liefern. Sie eignet sich jedoch dafür, das Leitbild der „normalen“ Familie präziser als bisher zu beschreiben bzw. neue, bislang unbekannte Aspekte daran explorativ zu entdecken.

3. Leitbilder entstehen, verändern und verstetigen sich u. a. auch durch die alltägliche Interaktion auf der Paarebene. Partnerschaft kann als zentraler Ort der Konstruktion einer idealisierten Wirklichkeit betrachtet werden. Für die künftige Leitbildforschung ist es bedeutsam, detaillierte Informationen über den Partner bzw. die Partnerin zu gewinnen sowie deren Leitbilder zu erfassen, sowohl auf standardisiertem Weg als auch auf qualitativer Basis. Auf diese Weise können Übereinstimmungen und Diskrepanzen auf dyadischer Ebene erfasst und deren Wechselwirkung mit der partnerschaftlichen Entwicklung (wechselseitige Anpassung der Leitbilder, Umsetzung von Kinderwunsch, Wirkung auf Partnerschaftszufriedenheit) abgeglichen werden.

4. Mittels der Zugänge aus der Medienwirkungsforschung könnten Familienleitbilder in ihrer visualisierten Form erfasst und in ihrer Wirkung auf die Konsumenten sowie ihre Entscheidungen analysiert werden. Zudem könnte die Verbreitung spezifischer Leitbilder in den Massenmedien (besonders Internet/TV) inhaltsanalytisch untersucht werden.

5. Eine unserer Annahmen lautet, dass Familienleitbilder u. a. vom Sozialisationshintergrund abhängen, demnach müsste es z. B. Unterschiede hinsichtlich des Migrationshintergrundes geben. Dies könnte insgesamt hilfreich sein, um interkulturelle Vergleiche hinsichtlich der Familiengründung durchführen zu können. Die Studie „Familienleitbilder“ bietet für die Untersuchung des türkischen Migrationshintergrundes eine geeignete Datengrundlage, andere Herkunftsländer sind allerdings nicht ausreichend vertreten.

6. Auch sind internationale Vergleiche vielversprechend: In industrialisierten, individualisierten Gesellschaften findet man eventuell eine größere Vielfalt von Familienleitbildern als in eher traditionell-orientierten, weniger industrialisierten Gesellschaften. Über Leitbilder im internationalen Vergleich, gerade auch zwischen Industrie-, Schwellen- und Entwicklungsländern (insbesondere solchen Ländern mit höheren Fertilitätsniveaus) ist jedoch wenig bekannt. Es lohnt sich ein Blick auf die womöglich international unterschiedlichen Familienleitbilder z. B. in Richtung Subsahara-Afrika und nach Asien oder Lateinamerika. Die Ergebnisse der international vergleichenden Value-of-ChildrenForschung (z. B. Mayer/Trommsdorff 2010) verweisen auf die Bedeutsamkeit der kulturellen Besonderheiten, um den Kinderwunsch und das generative Verhalten - und damit das Geburtenniveau in verschiedenen Ländern - zu erklären.

7. Jenseits der Untersuchung über kontinentale Grenzen hinweg, erweist sich vermutlich auch schon ein binationaler Vergleich zwischen Industrieländern als erkenntnisreich: Analysen zum Einfluss von kulturellen Vorstellungen auf Familiengründungs- und Erweiterungsprozesse im deutsch-französischen Ländervergleich können als vielversprechend gelten, denn Frankreich hat eine der höchsten und Deutschland eine der nied-

3 Forschungsprojekt „Familienleitbilder in Familienbildern“ am Bundesinstitut für Bevölkerungsforschung. 
rigsten Geburtenraten innerhalb der EU. Das INED ${ }^{4}$ hat Teile des deutschen Fragebogens der Familienleitbild-Studie in seine Panelstudie (ELIPSS ${ }^{5}$ 2015) integriert.

\subsection{Perspektiven für die Leitbildforschung im Längsschnitt}

Die Ergebnisse der ersten Welle sind vielversprechend, es ergeben sich jedoch weitere Anschlussfragen, die durch eine Wiederbefragung bearbeitet werden könnten:

1. Wie stabil sind Familienleitbilder im Lebensverlauf: Bestehen diese Leitbilder fort bzw. sind sie modifiziert (angepasst an die Lebensumstände) oder sogar aufgegeben worden? Es können neue Erkenntnisse darüber gewonnen werden, unter welchen Bedingungen kulturelle Vorstellungen zum Familienleben konstant erhalten bleiben oder im Laufe der Zeit eine Korrektur bzw. Anpassung erfahren. In diesem Kontext erscheint es gewinnbringend, für die künftige Leitbildforschung im Feld der Bevölkerungs- und Familienwissenschaft stärker die Lebensverlaufsperspektive zu integrieren.

2. Neben der Dauerhaftigkeit soll auch die Wirksamkeit von Leitbildern analysiert werden: Sind die in der Erstbefragung geäußerten Vorstellungen zur Ausgestaltung des Familienlebens hinsichtlich gewünschter Kinderzahl u. ä. realisiert worden? Wie (stark) beeinflussen Familienleitbilder die Partnerschafts- und Familienentwicklungsprozesse? Ein weites Feld stellen ferner Analysen zu den Wechselwirkungen zwischen Leitbildern, Strukturen und der Handlungsebene dar: Welche sozialstrukturellen Faktoren und auch familienpolitischen Rahmenbedingungen beeinflussen die Stabilität bzw. den Wandel von Familienleitbildern? Welche Faktoren haben die Umsetzung der in der Erstbefragung geäußerten Leitbilder gefördert oder gehemmt?

Für die künftige Leitbildforschung ergeben sich demnach vielfältige Forschungsfragen: Ein zentrales Ziel für die Zukunft der Leitbildforschung sind daher längsschnittliche Betrachtungen. Nur auf diese Weise kann untersucht werden, unter welchen Bedingungen Leitbilder stabil bleiben oder wie schnell sich Leitbilder verändern und wie sie wirken. Dieser Wandel ist auf verschiedenen Ebenen denkbar:

Auf individueller Ebene: Dabei stellt sich die Forschungsfrage, ob ein Leitbild die Familienbildung/-entwicklung oder die Familienbildung/-entwicklung das Leitbild moderiert. Der Erfahrungseffekt im Lebensverlauf bzw. der Desillusionierungseffekt könnten z. T. auch Kohorteneffekte sein, die sich aktuell nicht überprüfen lassen.

Auf gesellschaftlicher Ebene: Es ist bislang unbekannt, wie sich der Wandel über die verschiedenen sozialen Gruppen vollzieht und wer die Pioniere sind.

Insgesamt lässt sich feststellen, dass ein tieferes Verständnis von der Stabilität bzw. dem Wandel der Familienleitbilder eine sinnvolle Gestaltung und Ausrichtung der Familienpolitik ermöglichen kann. So könnte man den Aufschub der Erstgeburt sowie auch die Entscheidung für Kinderlosigkeit und die Entscheidung gegen ein drittes oder gegen weitere Kinder besser verstehen.

Institut National d'Études Démographiques, Paris, Frankreich

Étude Longitudinale par Internet Pour les Sciences Sociales 


\section{Impulse für die politische Debatte}

Warum sind Familienleitbilder und die Erkenntnisse aus der Leitbildforschung interessant für die Politik? Politische Maßnahmen, die auf die Familie abzielen, sollen u. a. die Lebensqualität von Menschen verbessern und sie bei der Verwirklichung ihrer Lebensplanung unterstützen, beispielsweise bei Kinderwünschen, die an fehlenden Möglichkeiten der Vereinbarkeit von Beruf und Familie zu scheitern drohen. Dennoch bleibt ungeklärt, warum die Akzeptanz für die Betreuung von unter Dreijährigen immer noch regional stark differiert. Zudem ist es ein eher westdeutsches Phänomen, dass viele hochqualifizierte Frauen nach einer Familiengründung bewusst einseitige Karriereeinbußen hinnehmen, ohne von ihren männlichen Partnern das gleiche Engagement einzufordern. Warum verfallen Elternpaare nach der Geburt des Kindes in die klassischen Rollenmuster, obwohl sie das vorher gar nicht wollten? Die Antwort darauf ist vielschichtig und basiert auf einem Zusammenspiel von sozialstrukturellen Rahmenbedingungen, Infrastruktur und Kultur, wobei die Erkenntnisse der Leitbildforschung nahelegen, dass Kultur und kulturelle Leitbilder einen eigenen Beitrag zur Erklärung von familialem Verhalten leisten und daher die Wirkung von familienpolitischen Maßnahmen schwächen könnten, was bisher in der Politik vernachlässigt wurde. Unsere Ergebnisse spiegeln zudem die sich widersprechenden familienpolitischen Instrumente „Rechtsanspruch auf einen Kitaplatz“ und „Betreuungsgeld“ (vgl. auch Müller/ Wrohlich 2014) wider, die auf zwei gegensätzliche inhaltliche Dimensionen innerhalb der elternschaftsbezogenen Leitbilder abzielen. Die Inkonsistenz der verschiedenen persönlichen Leitbilder zur Mutter-, Vater- und Elternschaft insgesamt erscheint symptomatisch für die bisher widersprüchliche familienpolitische Strategie in Deutschland.

Das Einbeziehen kultureller Leitbilder könnte die Familienpolitik effektiver machen und einen Schlüssel zum Verständnis darstellen, warum Lebenspläne und Lebenswirklichkeit bei der Gestaltung des Familienlebens nach wie vor häufig divergieren. Politisches Handeln muss einbeziehen, dass bestimmte Maßnahmen Menschen u. U. zu Lebensmodellen nötigen, die nicht ihren Lebensplänen und Alltagsabläufen entsprechen. Zwei Konsequenzen sind denkbar: Erstens kann die Politik versuchen, der Vielfalt an Ansprüchen und an Familienleitbildern gerecht zu werden, indem sie dafür auch eine Vielfalt an Maßnahmen bereithält, die teilweise auch widersprüchlich sein können. Zentral dabei sollte jedoch der Abbau von infrastrukturellen Restriktionen sein, um mehr Handlungsspielräume und Lebensqualität für Familien zu schaffen. Zweitens kann Politik versuchen, die bestehenden kulturellen Leitbilder zu beeinflussen und zu modernisieren, beispielsweise im Sinne der Gleichstellungspolitik und durch strategische Kommunikation. Die hierzu auf den Weg gebrachten Quotenregelungen mit dem parallel betriebenen flächendeckenden Ausbau der Kinderbetreuungsinfrastruktur und der Ganztagsschulen (vgl. Berkemeyer et al. 2014) sind Maßnahmen in diese Richtung, um die Gleichstellung und auch die Chancengleichheit für Eltern und Kinder zu forcieren. Dies könnte dabei helfen, auf längere Sicht die kulturellen Leitbilder zu modifizieren und so das Wohlergehen von Familien zu fördern. Gleichermaßen muss auch die Legislative in Deutschland kritisch hinterfragen, ob sie in ihrer Rechtsprechung die vielfach nichtehelichen Lebensgemeinschaften den ehelichen gleichstellen will. Auch hier lassen sich leitbild-geprägte Urteile finden wie die Rechtsprechung für eine künstliche Befruchtung. Ein aktuelles Urteil sieht vor, dass die gesetzlichen Krankenkassen bei unverheiratet zusammenlebenden Paaren die Kosten nicht übernehmen. Die Urteilsbegründung wurde wie folgt formuliert: 
„Die Begrenzung auf miteinander verheiratete Eheleute und eine homologe Insemination prägt den gesetzlichen Anspruch auf künstliche Befruchtung. Ihm liegt verfassungskonform die Ehe als rechtlich verfasste Paarbeziehung von Mann und Frau zugrunde, in der gegenseitige Solidarität nicht nur faktisch gelebt wird, solange es gefällt, sondern rechtlich eingefordert werden kann.“6 (6. B 1 A 1/14 R; LSG BerlinBrandenburg)

Angesichts der heute sehr hohen Anforderungen, die viele Eltern in Deutschland an die Erziehung stellen, sollte sich die Politik noch viel stärker als bisher um die Gewährleistung qualitativ hochwertiger öffentlicher Betreuung kümmern, so dass deren Akzeptanz steigt. Bislang wird beispielsweise die Optimierung der ErzieherInnen-Ausbildung bzw. der Entwicklung von Studiengängen, wie sie in Schweden bereits üblich sind, in Deutschland vernachlässigt. Außerdem fehlt eine angemessene Bezahlung und hohe soziale Anerkennung für die Fürsorgearbeit, um Berufe in diesem Sektor für motivierte und gut ausgebildete Fachkräfte als attraktives Beschäftigungsfeld (für Frauen und Männer) zu etablieren. Nur so kann sich langfristig eine qualitativ hochwertige Kinderbetreuung entwickeln und gewährleistet werden, so dass Eltern nicht nur operativ, sondern auch in ihrem Verantwortungsdruck ein stückweit entlastet werden. Dies ist zudem die Möglichkeit zur flächendeckenden Frühförderung, um die Chancengleichheit von Kindern für ihren späteren Bildungsweg zu verbessern.

Aus den Ergebnissen des Leitbildsurveys lassen sich verschiedene Impulse ableiten, die sowohl auf familienpolitischer als auch auf gesellschaftlicher Ebene Berücksichtigung finden könnten: Die gegenwärtige Familienpolitik basiert auf den Handlungsebenen Geld-, Infrastruktur- und Zeitpolitik (BMFSFJ 2006; Stock et al. 2012). Bisher bildeten finanzund steuerpolitische Instrumente den Schwerpunkt familienpolitischen Handelns, seit einiger Zeit wurde zudem auf den Ausbau der Infrastruktur gesetzt, die jedoch in vielen Kommunen noch nicht die Nachfrage decken kann. Die Trias der Handlungsfelder sollte um zwei weitere Dimensionen erweitert werden (vgl. Schneider et al. 2014): um eine nachhaltige Gleichstellungspolitik, die proaktiv zu betreiben ist und um eine Kommunikationspolitik, die zur Dekonstruktion von Mythen und Klischees beiträgt, die das Denken und Handeln rund um Familie in Deutschland nach wie vor in ungünstiger Weise prägen. Gleichzeitig sind auf dieser Dimension familienbezogene Handlungsziele der Politik transparenter zu machen. Bedeutsam ist dabei eine noch gezieltere Frauenförderung innerhalb der Arbeitswelt bei gleichzeitiger strategischer öffentlicher Kommunikation, die sich sowohl auf das Kindeswohl als auch auf das Elternwohl fokussiert. Dabei können politische Akteure dazu beitragen, die Maximen der „Verantworteten Elternschaft“ ein stückweit zu relativieren, um damit zur Absenkung all zu hoher Erwartungen (an Eltern und an Kinder) und zu einer Reduktion der Opportunitätskosten von Elternschaft beizutragen. Politische Kommunikation hat an dieser Stelle auf „Kampfbegriffe“ zu verzichten und zu vermeiden, verschiedene Lebensmodelle zu diskreditieren oder gegeneinander auszuspielen. Zielführend ist dafür eine noch stärker am Bedürfnis von Eltern ausgerichtete strategische Gleichstellungs- und Arbeitsmarktpolitik, in der die Idee des Familien-Mainstreaming fest zu verankern wäre (BMFSFJ 2006; Stock et al. 2012).

6 L 1 KR 435/12 KL: „Das Gesetz durfte die Ehe als eine Lebensbasis für ein Kind ansehen, die den Kindeswohlbelangen mehr Rechnung trägt als eine nichteheliche Partnerschaft. Hiervon weicht die betroffene Satzungsregelung grundlegend ab“ (Terminbericht des BSG Nr. 51/14 zur Sitzung vom 18.11.2014, in der er über sieben Revisionen in Angelegenheiten der gesetzlichen Krankenversicherung (GKV) zu entscheiden hatte). Online verfügbar unter: https://www.juris.de/jportal/portal/page/homerl.psml?nid=jnachr-JUNA $141102956 \& \mathrm{cmsuri}=\% 2 \mathrm{Fjuris} \% 2 \mathrm{Fde} \% 2 \mathrm{Fnachich}$ ten $\% 2$ Fzeigenachricht.jsp 
Adressaten zum Abbau normativer Schranken sind alle gesellschaftlichen Bereiche und Akteure: Von Medien, Kirchen, Politik und anderen relevanten Akteuren (z. B. ExpertInnen), die Geschlechterrollen und das Familienleben indirekt mit beeinflussen, ist kritisch zu hinterfragen, welche Vorstellungen eines „normalen“ Familienlebens transportiert werden und wie ideologiefrei familienpolitisch relevante Sachverhalte debattiert und in die Öffentlichkeit hineingetragen werden.

Modernes familienpolitisches Handeln ist strategisch anzulegen sowie konsistent und auf klare Ziele hin auszurichten (Schneider et al. 2014). Es sollte keine Leitbilder vorgeben - auch nicht implizit. Vielmehr geht es darum, die Vielfalt des Familienlebens und der dahinter stehenden Leitbilder vorbehaltlos zu akzeptieren.

\section{Literatur}

Arránz Becker, Oliver; Lois, Daniel; Nauck, Bernhard (2010): Unterschiede in den Fertilitätsmustern zwischen ost- und westdeutschen Frauen. In: Zeitschrift für Bevölkerungswissenschaft, 35, 1, S. 35-64.

BamS, BILD am SONNTAG (2014). 23.11.2014, EMNID-Umfrage unter 502 Befragten, S. 6-7.

Bauernschuster, Stefan; Rainer, Helmut (2012): Political regimes and the family: How sex-role attitudes continue to differ in reunified Germany. In: Journal of Population Economics, 25, 1, S. 5-27.

Berkemeyer, Nils; Bos, Wilfried; Manitius, Veronika; Hermstein, Björn; Bonitz, Melanie; Semper, Ina (2014): Chancenspiegel. Regionale Disparitäten in der Chancengerechtigkeit und Leistungsfähigkeit der deutschen Schulsysteme. Gütersloh: Bertelsmann Stiftung (Hrsg.).

Billari, Francesco C.; Philipov, Dimiter; Testa, Maria R. (2009): Attitudes, Norms and Perceived Behavioural Control: Explaining Fertility Intentions in Bulgaria. In: European Journal of Population, 25, 4, S. 439-465.

BMFSFJ, Bundesministerium für Familie, Senioren, Frauen und Jugend (2006): Siebter Familienbericht. Familie zwischen Flexibilität und Verlässlichkeit - Perspektiven für eine lebenslaufbezogene Familienpolitik.

Böllert, Karin (1993): Die „,wiedervereinigte“ Familie. Zur Dominanz herkömmlicher Familienleitbilder. In: Böllert, Karin; Otto, Hans-Uwe Otto (Hrsg.): Die neue Familie. Lebensformen und Familiengemeinschaften im Umbruch. Bielefeld: Böllert, KT-Verlag, S. 112-125.

Diabaté, Sabine; Lück, Detlev (2014): Familienleitbilder - Identifikation und Wirkungsweise auf generatives Verhalten. In: Zeitschrift für Familienforschung, 26, 1, S. 49-69.

Elder, Glen H.; Shanahan, Michael J. (2006): The life course and human development. In: Damon, William; Lerner, Richard M. (Hrsg.): Handbook of Child Psychology: Theoretical Models of Human Development, New York: Wiley and Stone, S. 665-715.

Esser, Harmut (1990): „Habits“, „Frames“ und „Rational Choice“. In: Zeitschrift für Soziologie, 4, S. 231-247.

Grunow, Daniela; Baur, Nina (2014): Die Korrespondenz von normativen Vorstellungen und Handeln. Das Beispiel männlicher Hausarbeit. In: Comparative Population Studies, 39, 3, S. 479-520.

Grunow, Daniela; Schulz, Florian; Blossfeld, Hans-Peter (2007): Was erklärt die Traditionalisierungsprozesse häuslicher Arbeitsteilung im Eheverlauf: soziale Normen oder ökonomische Ressourcen? In: Zeitschrift für Soziologie, 36, 3, S. 162-181.

Henry-Huthmacher, Christine (2008): Eltern unter Druck. Zusammenfassung der wichtigsten Ergebnisse der Studie. In: Merkle, Tanja; Wippermann, Carsten (2008): Eltern unter Druck. Selbstverständnisse, Befindlichkeiten und Bedürfnisse von Eltern in verschiedenen Lebenswelten. Eine sozial-wissenschaftliche Untersuchung von Sinus Sociovision GmbH im Auftrag der KonradAdenauer-Stiftung. Stuttgart: Lucius \& Lucius. 
Junck, Sara; Lück, Detlev (2015): Die Älteren setzen den Standard. Untersuchung von Altersunterschieden in persönlichen Leitbildern als Ansatz zur Erklärung von Diskrepanzen zwischen den vorherrschenden gesellschaftlichen und den vorherrschenden persönlichen Leitbildern in der Studie „Familienleitbilder“ (FLB 2012). Wiesbaden: Bundesinstitut für Bevölkerungsforschung. [http://www.bib-demografie.de/leitbild]

Kaina, Viktoria; Deutsch, Frankziska (2006): Verliert die „Stille Revolution” ihren Nachwuchs? Wertorientierungen in Deutschland im Kohorten- und Zeitvergleich. In: Roller, Edeltraud; Brettschneider, Frank; van Deth, Jan W. (Hrsg.): Jugend und Politik: „Voll normal!”. Wiesbaden, S. 157-181.

Lesthaeghe, Ron (1992): Der zweite demographische Übergang in den westlichen Ländern. Eine Deutung. In: Zeitschrift für Bevölkerungswissenschaft, 18, 3, S. 313-354.

Mayer, Boris; Trommsdorff, Gisela (2010): Adolescents' value of children and their intentions to have children: A cross-cultural and multilevel analysis. In: Journal of Cross-Cultural Psychology, 41, S. 671-689.

McDonald, Peter (2000): Gender equity, social institutions and the future of fertility. In: Journal of Population Research, 17, 1, S. 1-16.

Müller, Kai-Uwe; Wrohlich, Katharina (2014): Two Steps Forward - One Step Back? Evaluating Contradicting Child Care Policies in Germany. Diskussionspapiere 1396. Deutsches Institut für Wirtschaftsforschung, DIW: Berlin.

Nave-Herz, Rosemarie (2012): Familie heute. Wandel der Familienstrukturen und Folgen für die Erziehung. 5. Auflage. Darmstadt: WBG.

Neidhardt, Friedhelm (1994): Öffentlichkeit, öffentliche Meinung, soziale Bewegungen. In: Neidhardt, Friedhelm (Hrsg.): Öffentlichkeit, öffentliche Meinung, soziale Bewegungen. In: Kölner Zeitschrift für Soziologie und Sozialpsychologie, Sonderheft 34. Opladen: Westdeutscher Verlag, S. 7-41.

Noelle-Neumann, Elisabeth (1994): Wirkung der Massenmedien auf die Meinungsbildung. In: Noelle-Neumann, Elisabeth; Schulz, Winfried; Wilke, Jürgen (Hrsg.): Publizistik. Massenkommunikation. Frankfurt am Main, S. 518-571.

Noelle-Neumann, Elisabeth (1996): Öffentliche Meinung. Die Entdeckung der Schweigespirale. Erweiterte Ausgabe. Frankfurt/Main: Ullstein.

Pfau-Effinger, Birgit (1996): Analyse internationaler Differenzen in der Erwerbsbeteiligung von Frauen. Theoretischer Rahmen und empirische Ergebnisse. In: Kölner Zeitschrift für Soziologie und Sozialpsychologie, 48, S. 462-492.

Pfau-Effinger, Birgit (2004): Socio-historical paths of the male breadwinner model - an explanation of cross-national differences. In: The British Journal of Sociology, 55, 3, S. 377-399.

Pfau-Effinger, Birgit (2005): Wandel der Geschlechterkultur und Geschlechterpolitiken in konservativen Wohlfahrtsstaaten - Deutschland, Österreich und Schweiz [http://www.fu-berlin.de/sites/gpo/ tagungen/Kulturelle_Hegemonie_und_Geschlecht_als_Herausforderung/Birgit_Pfau-Effinger Wandel_der_Geschlechterkultur_und_Geschlechterpolitiken_in_konservativen_Wohlfahrtsstaaten Deutschland sterreich_und_Schweiz/index.html, 16.12 .2014$]$

Pokorny, Sabine (2013): Wo bitte geht's zum Generationenkonflikt. Politische Einstellungen Jugendlicher und junger Erwachsender in Deutschland. Konrad-Adenauer-Stiftung e.V. (Hrsg.), Sankt Augustin/Berlin.

Schneider, Norbert F.; Limmer, Ruth; Ruckdeschel, Kerstin (2002): Mobil, flexibel, gebunden. Beruf und Familie in der mobilen Gesellschaft. Frankfurt am Main: Campus.

Schneider, Norbert F.; Naderi, Robert; Ruppenthal, Silvia (2012): Familie in Deutschland nach dem gesellschaftlichen Umbruch. Sind Ost-West-Differenzierungen in der Familienforschung zwanzig Jahre nach der Wiedervereinigung noch sinnvoll? In: Huinink, Johannes; Kreyenfeld, Michaela; Trappe, Heike (Hrsg.): Familie und Partnerschaft in Ost- und Westdeutschland. Ähnlich und doch immer noch anders. In: Zeitschrift für Familienforschung, Sonderheft 9, 29-53

Schneider, Norbert F., Diabaté, Sabine; Lück, Detlev (2014): Familienleitbilder in Deutschland. Ihre Wirkung auf Familiengründung und Familienentwicklung. In: Veröffentlichungen der KonradAdenauer-Stiftung e.V. (Hrsg.), Sankt Augustin/Berlin [http://www.kas.de/wf/de/33.38060/, 16.12.2014]. 
Schnell, Rainer; Hill, Paul B.; Esser, Elke (2005): Methoden der empirischen Sozialforschung. München: Oldenbourg (7. Auflage).

Statistisches Bundesamt (2014): [https://www.destatis.de/DE/ZahlenFakten/GesellschaftStaat/Bevoel kerung/Geburten/Tabellen/GeburtenMutterBiologischesAlter.html, 02.12.2014].

Stock, Günter; Bertram, Hans; Fürnkranz-Przskawetz, Alexia; Holgreve, Wolfgang; Kohli, Martin; Staudinger; Ursula M. (2012): Zukunft mit Kindern. Fertilität und gesellschaftliche Entwicklung in Deutschland, Österreich und der Schweiz. Campus Verlag, Frankfurt.

Terwey, Michael (1990): Zur Wahrnehmung von wirtschaftlichen Lagen in der Bundesrepublik. In: Müller, Walter; Mohler, Peter Ph.; Erbslöh, Barbara; Wasmer, Martina (Hrsg.): Einstellungen und Verhalten der Bundesbürger. Opladen: Westdeutscher Verlag, S. 144-171.

van de Kaa, Dirk J. (1987): Europe's second demographic transition. In: Population Bulletin, 41, 1, S. 3-57.

Wagner, Michael (2008): Entwicklung und Vielfalt der Lebensformen. In: Schneider, Norbert F. (Hrsg.): Lehrbuch Moderne Familiensoziologie. Opladen: Barbara Budrich, S. 99-121. 


\section{Autorinnen und Autoren}

Dr. Sabine Diabaté

Bundesinstitut für Bevölkerungsforschung (BiB)

Friedrich-Ebert-Allee 4

65185 Wiesbaden

sabine.diabate@bib.bund.de

Dorothee Dietrich (extern)

c/o Bundesinstitut für Bevölkerungsforschung (BiB)

Friedrich-Ebert-Allee 4

65185 Wiesbaden

doro.dietrich@web.de

Dr. Jürgen Dorbritz

Bundesinstitut für Bevölkerungsforschung (BiB)

Friedrich-Ebert-Allee 4

65185 Wiesbaden

juergen.dorbritz@bib.bund.de

Nadine Gies (extern)

c/o Bundesinstitut für Bevölkerungsforschung (BiB)

Friedrich-Ebert-Allee 4

65185 Wiesbaden

nadinegies@gmx.de

Dr. Detlev Lück

Bundesinstitut für Bevölkerungsforschung (BiB)

Friedrich-Ebert-Allee 4

65185 Wiesbaden

detlev.lueck@bib.bund.de

Linda Lux

Bundesinstitut für Bevölkerungsforschung (BiB)

Friedrich-Ebert-Allee 4

65185 Wiesbaden

linda.lux@bib.bund.de

Robert Naderi

Bundesinstitut für Bevölkerungsforschung (BiB)

Friedrich-Ebert-Allee 4

65185 Wiesbaden

robert.naderi@bib.bund.de 
Kerstin Ruckdeschel

Bundesinstitut für Bevölkerungsforschung (BiB)

Friedrich-Ebert-Allee 4

65185 Wiesbaden

kerstin.ruckdeschel@bib.bund.de

Katrin Schiefer

Bundesinstitut für Bevölkerungsforschung (BiB)

Friedrich-Ebert-Allee 4

65185 Wiesbaden

katrin.schiefer@bib.bund.de

Prof. Dr. Norbert F. Schneider

Bundesinstitut für Bevölkerungsforschung (BiB)

Friedrich-Ebert-Allee 4

65185 Wiesbaden

norbert.schneider@bib.bund.de 